Ralf Ueding

\title{
Management von Messebeteiligungen
}




\section{Ralf Ueding}

\section{Management von Messebeteiligungen}

Die Bedingungen für Messebeteiligungen haben sich in den vergangenen Jahren gravierend verändert. Messen und Ausstellungen stehen vermehrt in einem Substitutionswettbewerb mit den neueren Instrumenten der Unternehmenskommunikation. Die seit den 80er Jahren anhaltende Diskussion um die Leistungsfähigkeit und Transparenz von Messebeteiligungen für das Marketing macht deutlich, daß die Notwendigkeit einer konzeptionellen Auseinandersetzung mit dem Themenkomplex besteht. Die Arbeit verfolgt das Ziel, das messespezifische Ausstellerverhalten systematisch zu beschreiben und $\mathrm{zu}$ erklären. Hierzu werden messespezifische Grundhaltungen identifiziert und detailliert hinsichtlich der strategischen und operativen Ausgestaltung sowie Erfolgswirkungen der Messebeteiligungen analysiert. Der empirischen Analyse liegt eine schriftliche Befragung von 602 Unternehmen zugrunde.

Ralf Ueding wurde 1964 in Altenberge/Westfalen geboren. Nach der Ausbildung zum Verlagskaufmann studierte er von 1986 bis 1992 Betriebswirtschaftslehre an der Universität Münster. Von 1992 bis 1997 war er wissenschaftlicher Mitarbeiter am Institut für Marketing der Universität Münster. Seither ist er als Marketingmanager in einem Münchener Verlagshaus tätig. 


\section{Management von Messebeteiligungen}




\section{SCHRIFTEN ZU \\ MARKETING UND MANAGEMENT \\ Herausgegeben von Prof. Dr. Dr. h.c. Heribert Meffert}

Band 34

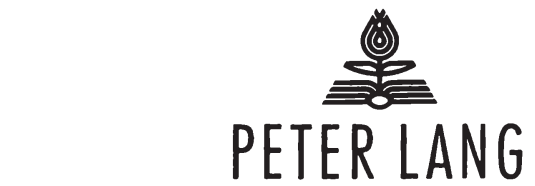

Frankfurt am Main - Berlin - Bern - New York - Paris - Wien 


\title{
Ralf Ueding
}

\author{
Management \\ von Messebeteiligungen \\ Identifikation und Erklärung \\ messespezifischer Grundhaltungen \\ auf der Basis einer empirischen Untersuchung
}

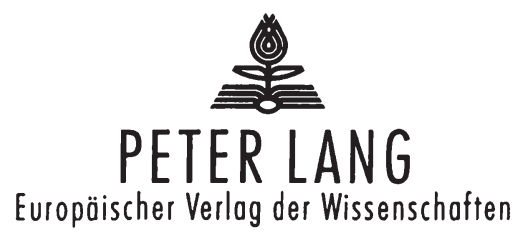


Die Deutsche Bibliothek - CIP-Einheitsaufnahme

Ueding, Ralf:

Management von Messebeteiligungen : Identifikation und Erklärung messespezifischer Grundhaltungen auf der Basis einer empirischen Untersuchung / Ralf Ueding. - Frankfurt am Main ; Berlin ; Bern ; New York ; Paris ; Wien : Lang, 1998

(Schriften zu Marketing und Management ; Bd. 34)

Zugl.: Münster (Westfalen), Univ., Diss., 1997

ISBN 3-631-32999-7

Open Access: The online version of this publication is published on www.peterlang.com and www.econstor.eu under the international Creative Commons License CC-BY 4.0. Learn more on how you can use and share this work: http://creativecommons. org/licenses/by/4.0.

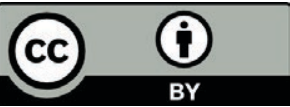

This book is available Open Access thanks to the kind support of ZBW - Leibniz-Informationszentrum Wirtschaft.

D 6

ISSN 0176-2729

ISBN 3-631-32999-7

ISBN 978-3-631-75073-5 (eBook)

(C) Peter Lang $\mathrm{GmbH}$

Europäischer Verlag der Wissenschaften

Frankfurt am Main 1998

Alle Rechte vorbehalten.

Das Werk einschließlich aller seiner Teile ist urheberrechtlich geschützt. Jede Verwertung außerhalb der engen Grenzen des

Urheberrechtsgesetzes ist ohne Zustimmung des Verlages

unzulässig und strafbar. Das gilt insbesondere für

Vervielfältigungen, Übersetzungen, Mikroverfilmungen und die Einspeicherung und Verarbeitung in elektronischen Systemen.

Printed in Germany 124567 
Meiner Familie

Ralf Ueding and Universität Münster - 978-3-631-75073-5

Downloaded from PubFactory at 01/11/2019 08:38:01AM

via free access 
Ralf Ueding and Universität Münster - 978-3-631-75073-5

Downloaded from PubFactory at 01/11/2019 08:38:01AM

via free access 


\section{Vorwort des Herausgebers}

Messebeteiligungen haben in der jüngeren Vergangenheit für viele Unternehmen einen wachsenden Stellenwert im Rahmen ihrer kommunikationspolitischen Überlegungen erreicht. Dies insbesondere deshalb, da der Einsatz von Messen als face to face-Medium in besonderem Maße einem Wandel der Kommunikationsbedürfnisse sowohl in der konsumentengerichteten als auch in der Business to Business-Kommunikation Rechnung trägt, der sich in dem Wunsch nach individueller Beratung und Betreuung sowie einem steigendem Anspruchsspektrum gegenüber der Erlebnis- und Informationsqualität der Unternehmenskommunikation ausdrückt. Steigende Messekosten sowie das Aufkommen neuerer Kommunikationsinstrumente stellen ausstellende Unternehmen andererseits zunehmend vor ein Spannungsfeld. Angesichts begrenzter Budgets und expandierendem Messeangebot ergibt sich verstärkt die Notwendigkeit zu einem systematischen Management der Messebeteiligungen. Erfahrungen im Bereich der Planung und Durchführung von Messebeteiligungen zeigen aber, daß Entscheidungen in der Unternehmenspraxis heute vielfach noch in Form von „ad-hoc-Maßnahmen“ umgesetzt werden.

Es ist aber ebenfalls darauf hinzuweisen, daß auch die betriebswirtschaftliche Forschung sich mit Fragen des Management von Messebeteiligungen bislang nur in Ansätzen auseinandergesetzt hat. So werden grundlegende Fragestellungen der Ausgestaltung von Planungs- und Umsetzungsprozessen bei Messebeteiligungen vergleichsweise stiefmütterlich behandelt. Vielmehr beschränken sich die wenigen bisher vorliegende Untersuchungen überwiegend auf die Fokussierung branchenspezifischer Problemstellungen.

Vor diesem Hintergrund wird in der vorliegenden Arbeit eine theoretische Aufarbeitung und empirische Fundierung des Untersuchungskomplexes geleistet. Die generelle Zielsetzung besteht dabei in der Entwicklung eines generellen Typologisierungsansatzes zur Identifikation, Beschreibung und Erklärung von messespezifischen Grundhaltungen sowie der darauf aufbauenden empirischen Analyse der Ausgestaltung von Messebeteiligungen am Beispiel ausstellender Unternehmen auf Messeveranstaltungen in Deutschland. Der empirischen Analyse liegt eine schriftliche Befragung von 602 Ausstellern zugrunde.

Den Ausgangspunkt der Ausführungen bildet die konzeptionelle Ableitung der

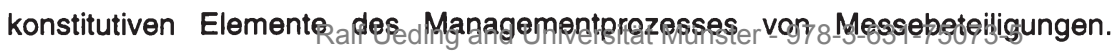


Hierbei wird zudem deutlich, daß sowohl Veränderungen im kommunikativen Umfeld der Unternehmen als auch Anpassungsprozesse im Messewesen bei der Planung und Ausgestaltung von Messebeteiligungen zu berücksichtigen sind. Auf diese Weise folgt die Arbeit konsequent einem kontingenztheoretischen Ansatz.

Einen wesentlichen Schwerpunkt der Arbeit bildet die Herausarbeitung messespezifischer Grundhaltungen bei Ausstellern. Als strategische Dimensionen werden dabei die Messebeteiligungsziele, die Messeselektion und die messespezifische Positionierung des ausstellenden Unternehmens identifiziert und operationalisiert. In der sich anschließenden empirischen Analyse können fünf Grundhaltungs-Cluster identifiziert und differenziert charakterisiert werden.

Auf der Grundlage der Ergebnisse der Typenbildung werden die grundhaltungsspezifische Ausgestaltung und der Erfolg von Messebeteiligungen analysiert. Hierzu werden zunächst relevante Maßnahmen zur Ausgestaltung von Messebeteiligungen, die eine typenspezifische Differenzierung erwarten lassen, konzeptionell abgeleitet und als Grundlage für die sich anschließende empirische Analyse herangezogen. Weiterhin werden auch die Erfolgswirkungen der grundhaltungsspezifischen Ausgestaltung von Messebeteiligungen anhand von ausgewählten Erfolgsmaßen untersucht.

Im Rahmen der empirischen Analyse der grundhaltungstypenbezogenen Besonderheiten in der Ausgestaltung der Messebeteiligungen wird gezeigt, daß die Aktivitäten der fünf Grundhaltungstypen sich vor allem in der Vor-Messe-Phase unterscheiden, während die Unterschiede in der Messe- und Nach-Messe-Phase zunehmend abnehmen. Bei der empirischen Untersuchung der Durchführung von Erfolgskontrollen werden typenübergreifende Schwächen identifiziert. Des weiteren zeigen die empirischen Ergebnisse, daß die messespezifischen Grundhaltungen sich weniger aus einer gemeinschaftlich-branchengerichteten, sondern eher aus einer unternehmensindividuell-kommunikationspolitischen Perspektive heraus erklären lassen.

Angesichts der Tatsache, daß die empirischen Analysen zur Erfolgskontrolle große Schwächen bei nahezu allen befragten Ausstellem offenbarten, entwickelt der Autor einen Ansatz zur systematischen Kontrolle von Messebeteiligungen. Zentrale Bestandteile dieses Ansatzes sind das Messebeteiligungs-Audit und die Ergebniskontrolle i. S. e. Effizienzbewertung. Die Durchführbarkeit dieses Ansatzes zur Ergebnis- und Rrozesskentrolle, heim Management, yon Masssebetgiligun- 
gen wird exemplarisch anhand der identifizierten Grundhaltungstypen dargestellt. Auf der Grundlage der gewonnenen Erkenntnisse leitet der Verfasser schließlich sowohl für ausstellende Unternehmen, als auch für die Messeveranstalter eine Reihe strategischer Implikationen und Empfehlungen ab.

Die vorliegende Untersuchung liefert mit ihrer empirisch fundierten Analyse der Bildung und Ausgestaltung messespezifischer Grundhaltungen eine umfassende Bestandsaufnahme des strategischen und operativen Verhaltens ausstellender Unternehmen auf Messen in Deutschland. Darüber hinaus konnten grundlegende Erkenntnisse über den Einfluß interner und externer Situationsbedingungen auf die messespezifische Grundhaltung ausstellender Unternehmen gewonnen werden. Mit dem Forschungsansatz, diese Untersuchung branchen- und unternehmensgrößenübergreifend zu gestalten, kommt der vorliegenden Arbeit der Charakter einer Basisuntersuchung zu. Es bleibt daher zu wünschen, daß die Arbeit eine entsprechende Resonanz in Wissenschaft und Praxis findet.

Die empirische Basis der vorliegenden Untersuchung gründet sich auf die Zusammenarbeit mit dem Ausstellungs- und Messe-Ausschuss der Deutschen Wirtschaft e. V. (AUMA). Für die Unterstützung und Kooperationsbereitschaft gilt mein besonderer Dank.

Münster, im September 1997

Prof. Dr. Dr. h.c. H. Meffert 
Ralf Ueding and Universität Münster - 978-3-631-75073-5

Downloaded from PubFactory at 01/11/2019 08:38:01AM

via free access 


\section{Vorwort des Verfassers}

Das Marketing vieler Unternehmen wird heute durch eine komplexer werdende Aufgabenumwelt und Diskontinuitäten vor Herausforderungen gestellt, die sich insbesondere auch auf Fragen der Unternehmenskommunikation erstrecken. Gerade die Bedingungen für Messebeteiligungen haben sich in den vergangenen Jahren in gravierender Weise verändert. Messen und Ausstellungen, die zu den ältesten Marketinginstrumenten zählen, stehen in den letzten Jahren vermehrt in einem Substitutionswettbewerb mit den neueren Instrumenten der Unternehmenskommunikation. Nicht zuletzt auch die seit Mitte der 80 er Jahre vor diesem Hintergrund geführte Diskussion um die Leistungsfähigkeit und Transparenz von Messebeteiligungen für das Marketing ausstellender Unternehmen hat deutlich gemacht, daß die Notwendigkeit einer konzeptionellen Auseinandersetzung mit dem Management von Messebeteiligungen besteht. Aktuelle Entwicklungen im Bereich der Planung und Durchführung von Messebeteiligungen zeigen aber nicht nur Defizite in der Unternehmenspraxis, sondern auch in der wissenschaftlichen Auseinandersetzung mit dem Themenkomplex. Hier ist insbesondere die Marketingforschung aufgefordert, vorhandene Lücken zu schließen und auf diese Weise das Management von Messebeteiligungen wissenschaftlich stärker zu fundieren.

Die vorliegende Arbeit verfolgt das Ziel, das messespezifische Ausstellerverhalten auf der Basis eines Typologisierungsansatzes zu beschreiben und zu erklären. Hierzu werden messespezifische Grundhaltungstypen identifiziert und detailliert auf ihre Besonderheiten hinsichtlich der strategischen und operativen Ausgestaltung sowie der Erfolgswirkungen der Messebeteiligungen analysiert. Darauf aufbauend werden Möglichkeiten zur Feststellung, Optimierung und Vergleichbarkeit des Messebeteiligungserfolges diskutiert. Zur Erklärung der messespezifischen Grundhaltungstypen wird insbesondere der Einfluß situativer Rahmenbedingungen untersucht.

Die Erstellung der Arbeit war nur mit der Unterstützung zahlreicher Personen möglich. Hier gilt mein besonderer Dank zunächst meinem akademischen Lehrer, Herm Prof. Dr. Dr. h.c. Heribert Meffert, der nicht nur die Themenstellung anregte, sondern auch die empirischen Untersuchungen sowie die Fertigstellung der Arbeit förderte. Herm Prof. Dr. Klaus Backhaus möchte ich für die Übernahme des Zweitgutachtens danken. Ferner bin ich zahlreichen Vertretern der Unternehmenspraxis und insbesondere den Repräsentanten des Ausstellungs- und Messe-

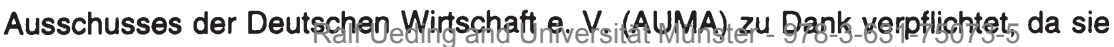


die komplexe empirische Erhebung durch ihre kompetente und kooperative Unterstützung erst möglich werden ließen.

Mein besonderer Dank gilt aber vor allem allen Kollegen, die mich während der Abfassung der Arbeit entlastet und vielfältig unterstützt haben. Hervorheben möchte ich insbesondere Dr. Manfred Kirchgeorg und Dipl.-Kfm. Jesko Perrey. Ohne ihr fachliches Engagement und ihre uneingeschränkte Bereitschaft zu kritischen Diskussionen wäre die vorliegende Arbeit nicht in dieser Form realisierbar gewesen. Ferner danke ich meinen Ex-Kollegen und Freunden Dr. Kai Laakmann und Dr. Michael Szeliga, deren fachlicher Rat und Unterstützung mir vor allem in der Schlußphase der Arbeit den Blick für das Wesentliche offen hielten.

Für die perfekte Erstellung der Abbildungen und Tabellen möchte ich Frau cand. rer. pol. Christa Grothenrath und Frau cand. rer. pol. Piera Kaempf herzlich danken, die meine ständigen Änderungswünsche mit unerschütterlicher Geduld ertrugen. Ferner möchte ich allen Beteiligten der "letzten Nacht" für ihren besonderen Einsatz danken. Hervorheben möchte ich dabei Frau Dipl.-Kfm. Silvia Danne und Herm Dipl.-Ing. Marcus Doemer, die die Arbeit akribisch durchgesehen und die vorhandenen Fehler ausgemerzt haben sowie Herm Dipl.-Kfm. Christian Böing, der mit bewundernswerter Präzision die Gestaltung des Layouts und das Zusammenstellen der Endfassung der Arbeit übernommen hat.

Dank schulde ich meinen Eltern, die mich in allen Phasen meiner Ausbildung unterstützt und damit den Grundstein für diese Arbeit gelegt haben. Mein ganz besonderer Dank gilt schließlich meiner Frau Silke und meinen Kindern Julian und Nele, die mir während der gesamten Abfassung der Arbeit ein Höchstmaß an Verständnis entgegengebracht haben und an zahllosen Abenden und Wochenenden auf mich verzichten mußten. Ohne ihren familiären Rückhalt und ihre liebevolle Geduld wäre der erfolgreiche Abschluß dieser Arbeit nicht vorstellbar gewesen.

Münster, im September 1997

Ralf Ueding 


\section{Inhaltsverzeichnis}

\section{A. Messebeteiligungen als Herausforderung an das}

\section{Kommunikationsmanagement.}

1. Bedeutung von Messebeteiligungen für das Marketing ausstellender Unternehmen.

2. Management von Messebeteiligungen als Gegenstand der Untersuchung...

3. Zielsetzung und Gang der Untersuchung

4. Design der empirischen Untersuchung

B. Ableitung und Identifikation von messespezifischen Grundhaltungstypen.

1. Ableitung messespezifischer Grundhaltungen

1.1 Kennzeichnung der Dimensionen einer messespezifischen Grundhaltung

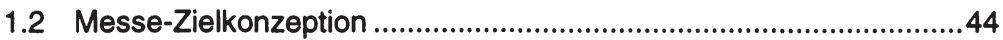

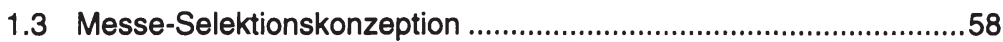

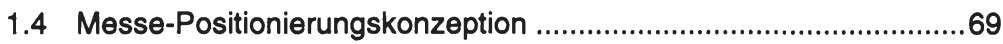

2. Identifikation messespezifischer Grundhaltungstypen auf der Basis einer empirischen Analyse

2.1 Konfirmatorische Faktorenanalyse für die messespezifische Zielkonzeption von ausstellenden Unternehmen ....

2.2 Ermittlung der Anzahl verschiedener Grundhaltungstypen ..............86

2.3 Darstellung der Grundhaltungstypen ...........................................92

2.4 Diskriminanzanalytische Überprüfung der Clusterlösung und Ermittlung der zur Typendifferenzierung zentralen Grundhaltungsdimensionen

2.5 Zusammenfassende Würdigung der empirischen Grundhaltungstypen 
C. Grundhaltungsspezifische Ausgestaltung und Erfolg von Messebeteiligungen........................................................................................107

1. Kennzeichnung der Maßnahmen zur Ausgestaltung von Messebeteiligungen

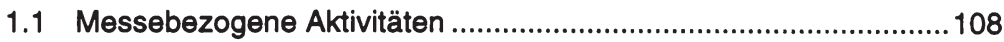

1.11 Aktivitäten in der Vor-Messe-Phase .................................109

1.12 Aktivitäten in der Messe-Phase .......................................110

1.13 Aktivitäten in der Nach-Messe-Phase...............................113

1.2 Erfolgskontrolle von Messebeteiligungen.....................................116

1.21 Methoden zur Erfolgsprognose in der Vor-Messe-Phase.... 122

1.22 Methoden zur Erfolgskontrolle in der Messe-Phase .............125

1.23 Methoden zur Erfolgskontrolle in der Nach-Messe-Phase .. 132

1.24 Verwendung der Ergebnisse der Erfolgskontrolle ................143

1.3 Messebeteiligungsorganisation ....................................................144

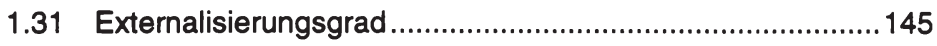

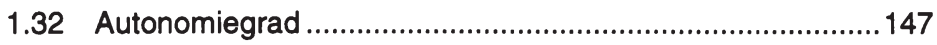

2. Empirische Analyse der Maßnahmen zur Ausgestaltung von Messebeteiligungen. 149

2.1 Analyse der messebezogenen Aktivitäten ..................................150

2.11 Aktivitäten in der Vor-Messe-Phase .................................150

2.12 Aktivitäten in der Messe-Phase .........................................154

2.13 Aktivitäten in der Nach-Messe-Phase.................................158

2.2 Analyse der Erfolgskontrolle von Messebeteiligungen ...................160

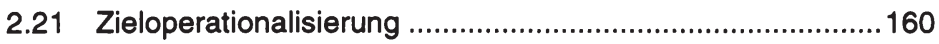

2.22 Stellenwert der Erfolgskontrolle .........................................163

2.23 Einsatz der Methoden zur Kontrolle des

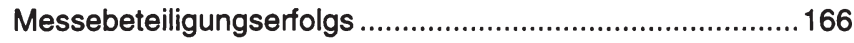

2.24 Verwendung der Ergebnisse der Erfolgskontrolle ................178

2.3 Analyse der Messebeteiligungsorganisation .................................. 182

2.4 Analyse des Erfolgs von Messebeteiligungen .................................185

2.5 Zusammenfassende Würdigung der empirischen Ergebnisse....... 189 
D. Grundhaltungsspezifische Analyse der situativen Einflußfaktoren .192

1. Identifikation von Einflußfaktoren des Management von Messebeteiligungen 192

2. Markt- und Wettbewerbsbedingungen als zentrale Einflußfaktoren der Aufgabenumwelt. 195

3. Interne Einflußfaktoren der messespezifischen Grundhaltung 199

3.1 Stellenwert und Integration im Kommunikations-Mix 199

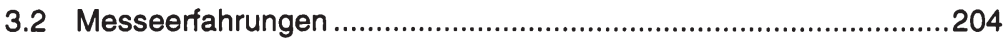

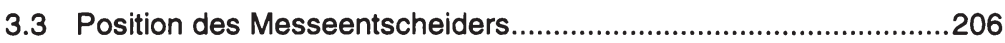

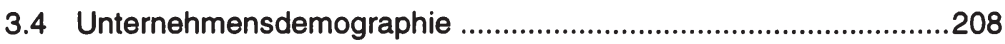

4. Empirische Analyse von Einflußfaktoren auf die messespezifische Grundhaltung.

4.1 Analyse des Einflusses der Markt- und Wettbewerbssituation ......212

4.2 Analyse des Einflusses unternehmensinterner Faktoren 221

4.21 Einfluß des Stellenwerts und der Integration von Messebeteiligungen im Kommunikations-Mix. 221

4.22 Einfluß der Messeerfahrungen. 225

4.23 Einfluß der Position des Messeentscheiders 228

4.24 Einfluß der Unternehmensdemographie 231

4.3 Integrierte Analyse der Einflußfaktoren auf die messespezifische Grundhaltung

E. Ansatzpunkte für eine systematische Messebeteiligungskontrolle und Implikationen der Untersuchung.

1. Zusammenfassende Würdigung der Untersuchungsergebnisse. 239

2. Ableitung eines Ansatzes zur Kontrolle von Messebeteiligungen. 251

3. Implikationen für die weiterführende Forschung zum Management von Messebeteiligungen sowie für ausstellende Unternehmen und Messeveranstalter

Gesamtverzeichnis des Anhangs.

Literaturverzeichnis. 306 


\section{Abbildungsverzeichnis:}

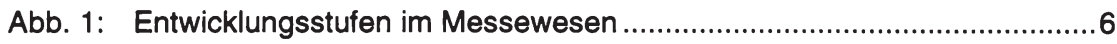

Abb. 2: Überblick über ausgewählte Forschungsarbeiten zum Management von Messebeteiligungen .......................................................................13

Abb. 3: Stellung von Messebeteiligungen im Marketing-Mix................................17

Abb. 4: Managementprozeß der Messebeteiligung ….....................................19

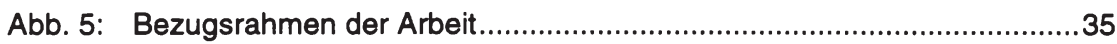

Abb. 6: Zusammensetzung der Stichprobe........................................................38

Abb. 7: Konzept messespezifischer Grundhaltungen von Ausstellern.................43

Abb. 8: Aufstellung von Messebeteiligungszielen nach Zielkategorien................51

Abb. 9: Bedeutung von Messebeteiligungszielen .......................................52

Abb. 10: Bestandteile der messespezifischen Zielkonzeption von Ausstellem.....56

Abb. 11: Entscheidungsprozeß der Messeselektion ...........................................59

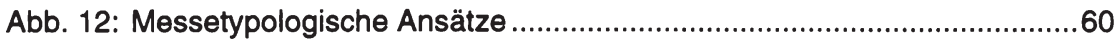

Abb. 13: Operationalisierungsansatz zur Erfassung der Messe-Selektion ...........67

Abb. 14: Selektionsprofil von Messen in Deutschland .......................................68

Abb. 15: Strategische und operative Entscheidungsfelder der MessePositionierungskonzeption....................................................................76

Abb. 16: Stellenwert der Positionierungskonzeptionen bei Messebeteiligungen ..78

Abb. 17: Konfirmatorische Faktorenanalyse für das Modell der MesseZielkonzeption

Abb. 18: Fusionierungsproze $B$ des Ward-Verfahrens und Varianzkriterium zur Bestimmung der Clusterlösung

Abb. 19: Klassifikationsmatrix der diskriminanzanalytischen Untersuchung der messespezifischen Grundhaltungstypen.........................................100

Abb. 20: Synopse phasenspezifischer Messe-Aktivitäten .................................115

Abb. 21: Systematisierung der Methoden zur Messeerfolgskontrolle ..................121

Abb. 22: Messebeteiligungsintensität differenziert nach messespezifischen Grundhaltungstypen ........................................................................226

Abb. 23: Mitarbeiterzahl und Umatz differenziert nach messespezifischen

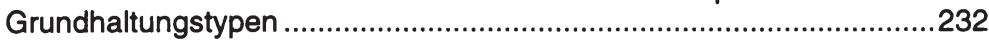

Abb. 24: Das Messebeteiligungs-Audit im Phasenüberblick............................253

Abb. 25: Synoptische Darstellung des Messebeteiligungs-Audits am Beispiel der messespezifischen Grundhaltungstypen......................................257

Abb. 26: Vergleichsobjekte zur Effizienzbewertung von Messebeteiligungen ....262

Abb. 27: Grundhaltungspezifische Darstellung des MessebeteiligungsNutzen-Index 


\section{Tabellenverzeichnis:}

Tab. 1: Clusterbildende Merkmale der ermittelten Grundhaltungstypen............91

Tab. 2: Mittelwerte für die clusterbildenden Merkmale der ermittelten Grundhaltungstypen

Tab. 3: Beiträge der Grundhaltungsdimensionen für die Diskriminierung der ermittelten Grundhaltungstypen

Tab. 4: Erfolgskriterien und Methoden für die Kontrolle von Informationszielen

Tab. 5: Erfolgskriterien und Methoden für die Kontrolle von Motivationssowie markt- und anspruchsgruppenbezogenen Beeinflussungszielen

Tab. 6: Erfolgskriterien und Methoden für die Kontrolle von Verkaufs- und Kontaktzielen.

Tab. 7: Varianzanalytische Prüfung auf Unterschiede der Vor-MesseAktivitäten zwischen den messespezifischen Grundhaltungstypen

Tab. 8: Varianzanalytische Prüfung auf Unterschiede der Messe-Aktivitäten zwischen den messespezifischen Grundhaltungstypen ....

Tab. 9: Varianzanalytische Prüfung auf Unterschiede der Nach-MesseAktivitäten zwischen den messespezifischen Grundhaltungstypen

Tab. 10: Varianzanalytische Prüfung auf Unterschiede der Zieloperationalisierung zwischen den messespezifischen Grundhaltungstypen.

Tab. 11: Zusammenhang zwischen Intensität der Erfolgskontrolle und den messespezifischen Grundhaltungstypen....

Tab. 12: Varianzanalytische Prüfung auf Unterschiede beim Einsatz der Methoden der Erfolgskontrolle in der Vor-Messe- und Messe-Phase zwischen den messespezifischen Grundhaltungstypen

Tab. 13: Varianzanalytische Prüfung auf Unterschiede beim Einsatz der Methoden der Erfolgskontrolle in der Nach-Messe-Phase zwischen den messespezifischen Grundhaltungstypen

Tab. 14: Gegenüberstellung der Messebeteiligungsziele und dem Einsatz der Methoden zur Erfolgskontrolle in den Grundhaltungsclustern

Tab. 15: Varianzanalytische Prüfung auf Unterschiede bei der Verwendung der Ergebnisse der Erfolgskontrolle zwischen den messespezifischen Grundhaltungstypen

Tab. 16: Varianzanalytische Prüfung auf Unterschiede bei der Organisation der Messebeteiligung zwischen den messespezifischen Grundhaltungstypen

Tab. 17: Varianzanalytische Prüfung auf Unterschiede bei der Zielerreichung und der generellen Erfolgseinschätzung bei Messebeteiligungen zwischen den messespezifischen Grundhaltungstypen 
Tab. 18: Einfluß der Markt- und Wettbewerbssituation auf die messespezifische Grundhaltung

Tab. 19: Clusterbildende Merkmale der ermittelten Kontexttypen ......................215

Tab. 20: Zusammenhang zwischen Kontext- und messespezifischen Grundhaltungstypen

Tab. 21: Einfluß des Stellenwerts und der Integration von Messebeteiligungen auf die messespezifische Grundhaltung.....

Tab. 22: Kontingenzanalytische Untersuchung des Zusammenhangs zwischen der Position des Messeentscheiders und der messespezifischen Grundhaltung.....

Tab. 23: Zusammenhang zwischen Branchenzugehörigkeit und messespezifischer Grundhaltung

Tab. 24: Diskriminatorische Bedeutung der Einflußfaktoren für die Bildung messespezifischer Grundhaltungen

Tab. 25: Synopse der clusterbildenden Dimensionen der messespezifischen Grundhaltungstypen

Tab. 26: Synopse der Ausgestaltungsanalyse von Messebeteiligungen für die messespezifischen Grundhaltungstypen

Tab. 27: Synopse der Ausgestaltungsanalyse von Messebeteiligungen für die messespezifischen Grundhaltungstypen

Tab. 28: Synopse der Einflußfaktoren für die ermittelten messespezifischen Grundhaltungstypen 


\section{Abkürzungsverzeichnis:}

a.a.O.

Abb.

a.M.

asw

Aufl.

AUMA

Bd.

BddW

bspw.

bzw.

ca.

$\mathrm{Cl}$

d.h.

DBW

Diss.

DWG

Ed.

einschl.

EJoM

EMJ

erwt. Aufl.

et al.

etc.

e.V.

f.

ff.

FAMAB

FAZ

FKM

Fn.

FQS

GfK

$\mathrm{GmbH}$

GWA

ggfs.

GewO am angegebenen Ort

Abbildung

am Main

Absatzwirtschaft

Auflage

Ausstellungs- und Messeausschuss der Deutschen Wirtschaft e.V.

Band

Blick durch die Wirtschaft

beispielsweise

beziehungsweise

cirka

Corporate Identity

das heißt

Die Betriebswirtschaft

Dissertation

Deutsche Werbewissenschaftliche Gesellschaft $\Theta . \mathrm{V}$.

Editor

einschließlich

European Journal of Marketing

European Marketing Journal

erweiterte Auflage

et alteri

et cetera

eingetragener Verein

folgende

fortfolgende

Fachverband Messe- und Ausstellungsbau e.V.

Frankfurter Allgemeine Zeitung

Gesellschaft zur freiwilligen Kontrolle von Messe- und Ausstellungszahlen

Fußnote

Fehlerquadratsumme

Gesellschaft für Konsum-, Markt- und Absatzforschung

Gesellschaft mit beschränkter Haftung

Gesamtverband Werbeagenturen GWA e.V.

gegebenenfalls

Gewerbe Ordnung 
$\mathrm{H}$.

HB

HBR

HdWW

HM

Hrsg.

i.e.S.

i.d.R.

i.d.S.

IMM

insbes.

i.S.

i.S.d.

i.w.S.

JAVF

$\mathrm{Jg}$.

JoM

JoR

jr.

k.A.

Kap.

LISREL

It.

Mass.

MJ

MM

MS

Nr.

No.

n.s.

o.g.

o.J.

o.Nr.

0.0 .

o.V.

p.a.

rd.
Heft

Handelsblatt

Harvard Business Review

Handwörterbuch der Wirtschaftswissenschaften

Harvard Manager

Herausgeber

im engeren Sinne

in der Regel

in diesem Sinne

International Marketing Management

insbesondere

im Sinne

im Sinne der

im weiteren Sinne

Jahrbuch für Verkaufsförderung

Jahrgang

Journal of Marketing

Journal of Retailing

junior

keine Angabe

Kapitel

Linear Structural Relations System

laut

Massachusetts

Marketing Journal

Manager Magazin

Marketing Science

Nummer

Number

nicht signifikant

oben genannte(r)

ohne Jahrgang

ohne Nummer

ohne Ortsangabe

ohne Verfasser

per anno

rund 
S.

s.o.

sog.

SMJ

Sp.

SPSS

Tab.

Tsd.

TDM

u.a.

u.ä.

u.a.m.

usw.

u.U.

vgl.

VM

Vol.

vs.

WiSt

WiWo

z.B.

ZfB

$\mathrm{ZfbF}$

ZfM

ZFP

z.T.

z.Zt.
Seite

siehe oben

sogenannte

Strategic Management Journal

Spalte

Superior Performance Software System

Tabelle

Tausend

Tausend Deutsche Mark

und andere, unter anderem

und ähnliche

und andere mehr

und so weiter

unter Umständen

vergleiche

Verkaufsmanagement

Volume

versus

Wirtschaftswissenschaftliches Studium

Wirtschaftswoche

zum Beispiel

Zeitschrift für Betriebswirtschaft

Zeitschrift für betriebswirtschaftliche Forschung

Zeitschrift für Marketing

Zeitschrift für Forschung und Praxis

zum Teil

zur Zeit 
Ralf Ueding and Universität Münster - 978-3-631-75073-5

Downloaded from PubFactory at 01/11/2019 08:38:01AM

via free access 


\section{A. Messebeteiligungen als Herausforderung an das Kommuni- kationsmanagement}

\section{Bedeutung von Messebeteiligungen für das Marketing ausstellender Unternehmen}

In den letzten zwei Jahrzehnten haben Messebeteiligungen im Rahmen kommunikationspolitischer Überlegungen einen wachsenden Stellenwert erreicht. ${ }^{1}$ Die Ursachen für diese seit Jahren andauernde dynamische Entwicklung sind vielschichtig und bestehen in nachhaltigen Veränderungen des unternehmenspolitischen Umfeldes sowie nicht zuletzt auch in einem starken Wandel im Messewesen.

Mit Blick auf die wirtschaftlichen Rahmenbedingungen ist das Marketing vieler Unternehmen heute durch Diskontinuitäten und eine komplexer werdende Aufgabenumwelt vor neue Herausforderungen gestellt. ${ }^{2}$ Dabei gestalten sich vor allem die Wettbewerbsbedingungen zunehmend intensiver. ${ }^{3}$ Diese resultieren insbesondere auch aus der Verschärfung des den klassischen Produktwettbewerb ${ }^{4}$ ergänzenden Kommunikationswettbewerbs. ${ }^{5}$

$1 \quad$ Neuere Untersuchungen bei deutschen Ausstellern machen deutlich, daß Messen und Ausstellungen eine hohe Priorität für das absatzmarktgerichtete Marketing eingeräumt wird. Messebeteiligungen rangieren branchenübergreifend unmittelbar hinter dem persönlichen Verkauf sowie dem Kundenservice und vor der klassischen Werbung, Verkaufsförderung und den PR. Vgl. Ausstellungs- und Messe-Ausschuss der Deutschen Wirtschaft e. V. (AUMA) (Hrsg.), Die Messewirtschaft 1995/96, Bergisch Gladbach 1996, S. 19 ff. Ähnliche Ergebnisse erbrachte eine 1990 durchgeführte Untersuchung bei ausstellenden Unternehmen. Messen und Ausstellungen nehmen nach Vertreterbesuchen den zweiten Bedeutungsrang ein und sind deutlich vor Anzeigen-, Katalog- oder Prospektwerbung positioniert. Vgl. Küng. P., Der Einsatz des Verkaufs an Messen, in: VM international, Heft 3, 1990, S. $45 \mathrm{ff}$.

Zum aktuellen Stellenwert sowie zu den Entwicklungslinien des Marketing als Ausdruck einer konsequent marktgerichteten Unternehmensführung vgl. Meffert. $H_{1}$, Marketing: Retrospektive und Perspektive der marktorientierten Unternehmensführung, in: Wissenschaftliche Gesellschaft für Marketing und Unternehmensführung e. V. (Hrsg.), Marketing im Dialog neue Herausforderungen an die marktorientierte Führung, Münster 1995, S. 1-13; ders., Marketing-Management: Analyse-Strategie-Implementierung, Wiesbaden 1994, S. 3 ff.; ders., Marktorientierte Unternehmensführung im Umbruch: Entwicklungsperspektiven des Marketing in Wissenschaft und Praxis, in: Bruhn, M. Meffert. H. Wehrle. F. (Hrsg.), Marktorientierte Unternehmensführung im Umbruch, Stuttgart 1994, S. 5 ff.

3 Zu den Bestimmungsformen der Wettbewerbsintensität vgl. Porter. M. E., Wettbewerbsstrategien, Frankfurt a.M. 1992, S. 26 ff.

4 Vgl. Meyer, A., Produktdifferenzierung, in: Marketing ZFP, Heft 2, 7. Jg., 1985, S. 99.

5 Vgl. Bruhn, M., Integrierte Unternehmenskommunikation, 2. Aufl., Stuttgart 1995, S. 2. 
Sowohl in der konsumentengerichteten als auch in der "Business to Business"Kommunikation sind eine wachsende Informationsüberlastung und Reizüberflutung der potentiellen Botschaftsempfänger auszumachen, die eine Wahrnehmung der Kommunikationsinhalte immer mehr erschweren. ${ }^{6}$ Neben der Informationsüberlastung ist gleichzeitig auf ein sinkendes Interesse der Adressaten gegenüber der klassischen Kommunikation über die sog. Massenmedien zu verweisen. ${ }^{?}$

Der Einsatz von Messen als sog. „face to face“-Medium ${ }^{8}$ trägt dabei in besonderem Maße einem Wandel der Kommunikationsbedürfnisse aktueller und potentieller Kunden Rechnung, der sich in dem Wunsch nach individueller Betreuung und Beratung sowie einem steigenden Anspruchsspektrum gegenüber der Erlebnis- und Informationsqualität der Kommunikationsmaßnahmen der Unternehmen ausdrückt. ${ }^{9}$ In diesem Zusammenhang konnten bei empirischen Untersuchungen die folgenden zentralen Bedürfniskategorien der Botschaftsempfänger sowie die sich daraus ergebenden Konsequenzen für die Ausgestaltung der Marktkommunikation herausgearbeitet werden: ${ }^{10}$

6 Empirischen Untersuchungen zufolge werden lediglich ca. 2 bis $5 \%$ der im Rahmen der Marktkommunikation angebotenen Informationen überhaupt durch die Adressaten wahrgenommen und haben damit die Chance der intrapersonalen Weiterverarbeitung i. S. psychographischer und ökonomischer Kommunikationswirkungen. Vgl. hierzu Arnold, U., Zur Informationsüberlastung von Konsumenten, in: JAVF, Heft 4, 1989, S. 387-401; Kroeber-Riel, W., Informationsüberlastung durch Massenmedien in Deutschland, in: DBW, Heft 3, 1987, S. 257264; Dallmer. H., System des Direct-Marketing - Entwicklung und Zukunftsperspektiven, in: Dallmer. H. (Hrsg.), Handbuch Direct-Marketing, 6. Aufl., Wiesbaden 1991, S. 3-16.

Vgl. Kroeber-Riel. W., Strategie und Technik der Werbung. Verhaltenswissenschaftliche Ansätze, 4. Aufl., Stuttgart u.a. 1993, S. 5 f.

8 Vgl. Meffert. $H_{\text {. }}$ Messen als Marketinginstrument, in: Bayerisch-Schwäbische Wirtschaft, Heft 11, 1995, S. 27; o.V., Messe-Marketing - Face-to-Face ist Trumpf, in: Pro, Heft 9, 1995, S. $26-$ 30.

9 Vgl. Weinhold-Stünzi. H., Die Rolle der Messen im Marketing der neunziger Jahre, in: Thexis, Heft 2, 1989, S. 1.

10 Vgl. McQuail. R. Blumler, J. G. Brown, J. R., The Television Audience: A Revised Perspective, in: McQuail, R. (Ed.), Sociology of Mass Communication, Harmondsworth 1972, S. $135-165$. 
- Informationsbedürfnis,

d. h. schnelle und umfassende Übermittlung von Informationen über Produkte und Leistungen, Anwendungserläuterungen sowie bspw. Preis- und Garantieinformationen,

- Unterhaltungsbedürfnis,

d. h. Notwendigkeit des Aufbaus von Erlebniswelten um das Produktangebot"

- Integrationsbedürfnis,

d. h. Ausrichtung der Unternehmenskommunikation an den Bedürfnissen verschiedener Zielgruppen ${ }^{12}$,

- Interaktionsbedürfnis,

d. h. Dialogorientierung der Unternehmenskommunikation ${ }^{13}$,

- Identitätsbedürfnis,

d. h. Individualisierung der Unternehmenskommunikation. ${ }^{14}$

Messen sind aufgrund ihres kommunikativen Leistungsspektrums bzw. ihrer "Multifunktionalität" ${ }^{\text {"15 }}$ in besonderem Maße in der Lage, auf diese genannten Kommunikationsanforderungen einzugehen. Sie zeichnen sich vor allem durch ein hohes $\mathrm{Maß}$ an persönlichem Kontakt und Interaktivität zwischen Ausstellern und Messebesuchern aus, bieten gute Möglichkeiten zur Besichtigung und Erklärung von Ausstellungsobjekten, haben nicht nur einen hohen Erlebniswert für Aussteller und Besucher, sondern verfügen für beide Gruppen auch über einen hohen Informationsnutzen aufgrund der unmittelbaren und i. d. R. umfassenden Mög-

11 Vgl. auch Kroeber-Riel, W., Erlebnisbetontes Marketing, in: Belz. C. (Hrsg.), Realisierung des Marketing, Savosa, St. Gallen 1986, S. 1137-1151; Weinberg. P., Erlebnismarketing, München 1992, S. 34 f. und die dort angegebene Literatur.

12 Vgl. auch Bruhn, M., Integrierte Unternehmenskommunikation, 2. Aufl., a.a.O., S. 4.

$13 \mathrm{Vgl}$. auch Meffert, H., Eröffnungsrede anläßlich des 30. Münsteraner Führungsgesprächs am 22./23. Februar 1996 in Gütersloh, in: Meffert. H. Wagner. H., Backhaus, K. (Hrsg.), Mediale Welt von morgen - neue Chancen für das Marketing?, Dokumentationspapier Nr. 104 der Wissenschaftlichen Gesellschaft für Marketing und Unternehmensführung e. V., Münster 1996, S. 2.

14 Vgl. auch Bolz. N., Individualität als Medieneffekt, in: planung \& analyse, Heft 1, 1996, S. 911.

15 Multifunktionalität sei hier verstanden als die Möglichkeit zur persönlichen und unpersönlichen Kommunikation zwischen Aussteller und Messebesucher. 
lichkeit zu Konkurrenzvergleichen. ${ }^{16}$ Messen sind nicht zuletzt deshalb in den letzten Jahren vermehrt als "Markt für Informationen" in den Vordergrund gerückt. ${ }^{17}$

Die Kommunikationspotentiale, die Messebeteiligungen bieten, sind von den ausstellenden Unternehmen - deren Perspektive dieser Arbeit zugrunde gelegt werden soll - in den letzten Jahren zunehmend erkannt worden. Allein am Messeplatz Deutschland ${ }^{18}$ steigerte sich bspw. in den letzten zehn Jahren die Zahl der Aussteller von ca. 88.000 in 1985 um fast $61 \%$ auf über 141.000 in $1995 .{ }^{19} \mathrm{Im}$ gleichen Zeitraum wuchs die Zahl der Messebesucher um nahezu $32 \%$ von knapp 8 Mio. auf über 10 Mio. $^{20}$

Diese bemerkenswerte Entwicklung geht mit mehreren parallel verlaufenden und teilweise interdependenten Anpassungsprozessen im Messewesen einher. Messen haben in der jüngsten Vergangenheit sowohl eine Angebotserweiterung ${ }^{21}$ als

16 Vgl. Rost. D., Messen als Kommunikations-Problem, in: DWG Deutsche Werbewissenschaftliche Gesellschaft e. V. (Hrsg.), Messen als Marketing-Instrument, Bonn 1983, S. 35 ff.; Backhaus. K., Messen als Institutionen der Informationspolitik, in: Strothmann. K.-H. Busche. M. (Hrsg.), Handbuch Messemarketing, Wiesbaden 1992, S. $91 \mathrm{ff}$.

17 Vgl. Henkel. H. O., Neue Funktionen der Messen, in: Der Handel, Heft 11, 1995, S. 31; Backhaus. K., Messen als Institutionen der Informationspolitik, a.a.O., S. 89; Meffert, H. Gass, C., Messen und Ausstellungen im System des Kommunikationsmix, in: Meffert. $H$. (Hrsg.), Arbeitspapier des Instituts für Marketing der Universität Münster, Nr. 33, Münster 1985, S. 7.

18 Das Messewesen in Deutschland nimmt im internationalen Vergleich der Messeplätze eine führende Position ein. Unter den zehn größten Messeplätzen dieser Welt (gemessen in Flächenkapazität) sind allein vier deutsche Plätze (Hannover, Frankfurt, Köln und Düsseldorf). Von den ca. 150 internationalen Leitmessen finden 100 (das entspricht einem Anteil von ca. $70 \%$ ) in Deutschland statt. Deutschland hat daher im internationalen Messewesen eine Schrittmacherfunktion. Vgl. Lückmann. R., Messewirtschaft 1995: Schub durch Flaggschiffe, in: HB, Nr. 11, vom 16.01.1996, Sonderteil Internationale Messen, S. B1.

19 Vgl. Q.V., Bewegung bei den Riesen, in: asw, Heft 8, 1996, S. 102 f.

20 Die genannten Besucherzahlen beziehen sich 1995 ausschließlich auf 114 überregionale Messeveranstaltungen. Vgl. Ausstellungs- und Messe-Ausschuss der Deutschen Wirtschaft e. V. (AUMA) (Hrsg.), Die Messewirtschaft 1995/96, a.a.O., S. 7-12.

21 In Deutschland wurden allein in 1995 insgesamt 135 Neuanmeldungen von Messeveranstaltungen registriert. Hierzu zählen allerdings nur 14 Veranstaltungen, die einen überregionalen Anspruch erheben. Immer dominierender werden allerdings Fachmessen. Vgl. Ausstellungsund Messe-Ausschuss der Deutschen Wirtschaft e. V. (AUMA) (Hrsg.), Die Messewirtschaft 1995/96, a.a.O., S. 16. Eine direkte Folge der Angebotsausdehnung im Messewesen ist die damit einhergehende Ausdehnung der Standortkapazitäten der Messegesellschaften. Allein in Deutschland werden die überregional bedeutenden Messegesellschaften innerhalb der nächsten Jahre über 6 Mrd. DM investieren, um die Hallenkapazitäten von heute 2,1 auf über 2,3 Mio. Quadratmeter bis zum Jahr 2000 auszubauen. Vgl. Wettwer. B., Warmer Regen - Die Messestandorte investieren Milliarden in ihre Zukunft, in: Wirtschaftswoche, Nr. 1/2, vom 4.1.1996, S. $62 \mathrm{ft}$. 
auch eine Internationalisierung ${ }^{22}$ erfahren. Des weiteren werden bestehende Messen einer Differenzierung durch Abspaltung bestimmter Aussteller- und vor allem Besuchersegmente in eigens konzipierte Messeveranstaltungen unterzogen. ${ }^{23}$ Als Folge dieser Entwicklungen existieren heute für nahezu alle Wirtschaftsbereiche Messeveranstaltungen mit jeweils spezifischen Messethemen, so daß sich die oben skizzierte Potentialausdehnung für Messen auf der Ausstellerund Besucherseite ergeben hat. ${ }^{24}$

Eine Betrachtung der Entwicklungsstufen von Messen macht zudem deutlich, daß das Anspruchsspektrum an Messen in der jüngsten Vergangenheit vor allem aus der Ausstellerperspektive starken Veränderungen unterlag. ${ }^{25}$ Mit dem Wandel von Verkäufer- zu Käufermärkten hat sich auch ein inhaltlicher Wandel der Messen in verschiedenen Etappen vollzogen. Die Entwicklungsstufen im Messewesen werden in Abb. 1 graphisch dargestellt.

22 Weltweit werden jährlich von den ca. 5000 zur Auswahl stehenden Messeveranstaltungen etwa 500 Messen und Ausstellungen von deutschen Unternehmen in größerem Umfang beschickt. Vgl. Ausstellungs- und Messe-Ausschuss der Deutschen Wirtschaft e. V. (AUMA) (Hrsg.), Erfolg auf Auslandsmessen, Bergisch Gladbach 1994, S. 4. Dieser Trend wird auch dadurch verstärkt, daß in Deutschland erfolgreiche Messekonzepte durch die Messeveranstalter ins Ausland - meist in die Wachstumsregionen von Asien und Nordamerika - exportiert werden. Vgl. Winter. St. F. Wengler. St., Das Unternehmen "Deutschland" darstellen, in: BddW, Nr. 27, vom 7.2.1989, S. 7; 0.V., Messe Frankfurt sieht inr Wachstumsfeld im Ausland, in: HB, Nr. 233, vom 1./2.12.1995, S. 17.

23 Als jüngste Beispiele können für diesen Entwicklungstrend die Abspaltung der CeBIT HOME von der CeBIT sowie die Abtrennung des Nutzfahrzeugebereichs von der Internationalen Automobil-Ausstellung (IAA) in eine eigene IAA-Nutzfahrzeuge angeführt werden. Beide Veranstaltungsdifferenzierungen können als Schritt zu einer stärkeren Fachbesucherorientierung interpretiert werden. Vgl. stellvertr. Goehrmann, K., Vortrag anläßlich des Besuchs von Mitgliedern der Wissenschaftlichen Gesellschaft für Marketing und Unternehmensführung e. V. auf der CeBIT HOME, Hannover, am 29.08.1996, S. 1.

24 Vgl. zum Angebot an überregionalen Messen Ausstellungs- und Messe-Ausschuss der Deutschen Wirtschaft e. V. (AUMA) (Hrsg.), AUMA-Handbuch Messeplatz Deutschland '97, Bergisch Gladbach 1996; zum Angebot regionaler Messeveranstaltungen ders., AUMA-Handbuch Regionale Ausstellungen '97, Bergisch Gladbach 1996; zum Angebot von Fachmessen ders., Fachmessen made in Germany '97, Bergisch Gladbach 1996.

Zu einem umfassenderen Abriß der geschichtlichen Entwicklungen von Messen vgl. Fischer, W., Zur Geschichte der Messen in Europa, a.a.O., S. 3-14; Strothmann, K.-H., Historischer Abriß über die Entstehung von Messen, in: Strothmann.K.-H. (Hrsg.), Arbeitspapier des Instituts für Markt- und Verbrauchsforschung der Freien Universität Berlin, Berlin 1979/80; Maurer. E., Missa profana. Geschichte und Morphologie der Messen und Fachausstellungen, Stuttgart 1973; Roth. G. D., Messen und Märkte. Aus der Geschichte des Handels: Von der Warenmesse zur Mustermesse, München 1965; Heil. E. B., Entwicklung und Ausgestaltung des Messe- und Ausstellungswesens in Deutschland nach dem zweiten Weltkrieg, Nürnberg 1966; Möller. H., Das deutsche Messe- und Ausstellungswesen, Berlin 1989. 


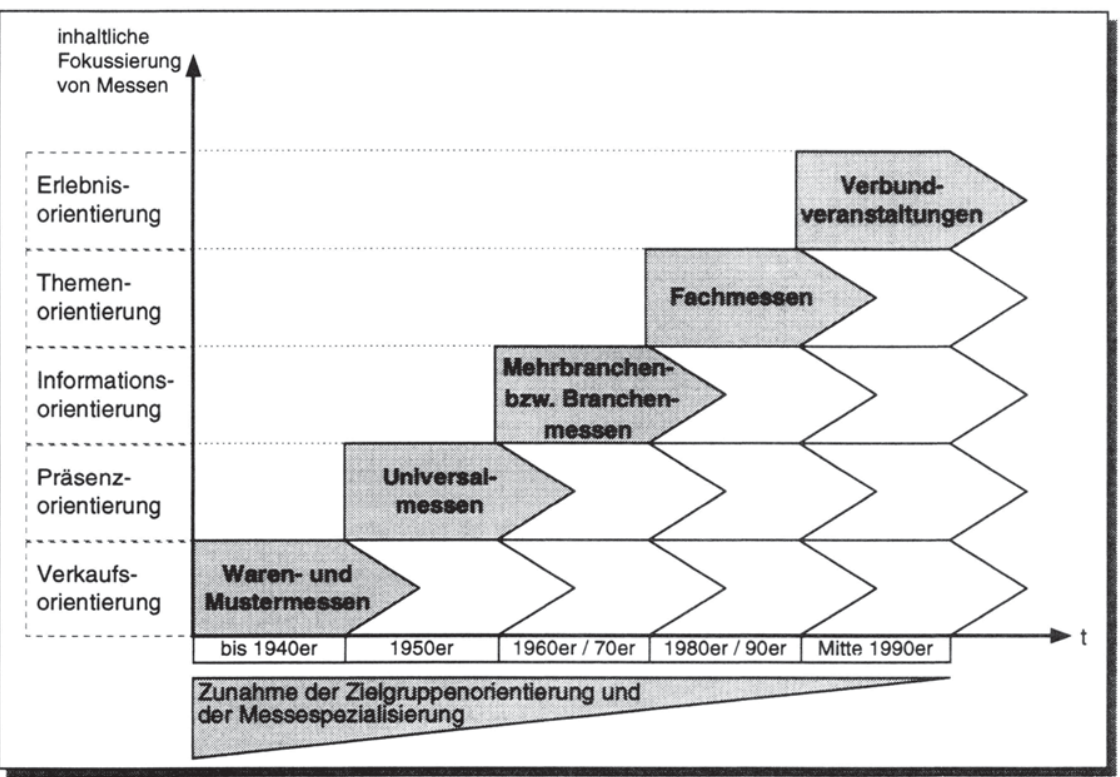

Abb. 1: Entwicklungsstufen im Messewesen

Während bis in die 40er Jahre die Messeform der Waren- und Mustermesse vorwiegend vertreten war, deren Aufgabe fast ausschließlich in der Verkaufs- und Orderfunktion bestand, dominierten in den 50er Jahren Universalmessen das Messegeschehen. Da das Angebot auf diesen Messen weder auf Branchen noch auf bestimmte Produktgruppen festgelegt war, wies der inhaltliche Fokus der Veranstaltungen zunächst eine Präsenzorientierung mit Leistungsschaucharakter gegenüber einem anonymen Massenpublikum auf.

Aufgrund des vielfach verwirrenden und sowohl für Aussteller als auch Messebesucher nicht mehr zu überblickenden Angebotes an und auf Messen wurden in den 60 er und 70er Jahren die Universalmessen durch Branchen- bzw. Mehrbranchenmessen abgelöst. Nicht zuletzt aufgrund der zunehmenden Produktdifferenzierung in den verschiedenen Märkten wurde eine stärkere Konzentration auf das Angebot einzelner Wirtschaftszweige notwendig. Damit ging gleichzeitig eine erste Anwendungs- und Zielgruppenfokussierung einher, die ihre Entsprechung in einer stärkeren Informationsorientierung der Messeveranstaltungen fand. Bis heute haben sich diese Messekonzepte ihren Platz im Veranstaltungsgefüge erhalten können. 
Zum dominierenden Messetyp hat sich seit den 80er Jahren allerdings die Fachmesse entwickelt. ${ }^{26}$ Das Angebot auf einer Fachmesse richtet sich hauptsächlich an einen begrenzten Kreis von gewerblichen Besuchern und ist daher durch eine besonders hohe Angebotstiefe bei gleichzeitig geringerer Angebotsbreite gekennzeichnet. Fachmessen sind dabei i. d. R. themenorientiert, d. h. sie widmen sich spezifischen Anwendungsproblemen und bieten einen Überblick über mögliche Problemlösungskonzepte. Die Zusammensetzung des Ausstellerkreises unterliegt daher vielfach einer Auswahl, die sich vor allem aus der thematischen Affinität der Unternehmen zum Thema der Messeveranstaltung ergibt.

Verbundveranstaltungen stellen die jüngste Form von Messen dar. Ihr Charakter liegt im wesentlichen in der Verbindung von Fachmessen und begleitenden bzw. weiterbildenden Veranstaltungsarten zu einer Hauptveranstaltung. ${ }^{27}$ Die Messeveranstalter reagieren mit einer stärkeren Erlebnisorientierung der Veranstaltungen durch das zusätzliche Angebot von Diskussionsforen, Symposien, Kongressen und Fachvorträgen auf das veränderte Informations- und Kommunikationsverhalten der Aussteller und Messebesucher.

Gleichzeitig mit der thematischen Messespezialisierung und zunehmenden Besucherzielgruppenfokussierung verschwimmen im Messewesen vermehrt die Grenzen zu messeähnlichen Veranstaltungsformen, wie z. B. Verbandsmessen, Kongreßausstellungen, Hausmessen, Road Shows, virtuellen Messen. ${ }^{28}$ Das

26 Vgl. Neglein, H.-G., Das Messewesen in Deutschland, a.a.O., S. 19; Meffert. H., Messen und Ausstellungen als Marketinginstrument, in: Goehrmann. K. E. (Hrsg.), Politmarketing auf Messen, Düsseldorf 1993, S. 73; Groth. C., Das Leistungspotential von Fachmessen, in: DWG Deutsche Werbewissenschaftliche Gesellschaft e. V. (Hrsg.), Messen als Marketing-Instrument, Bonn 1983, S. 57; Hassmann, V., Fachmessen: Mehr Zeit für Ihre Kunden, in: Sales Profi, Heft 1, 1996, S. 18-19; o.V., Wo liegt die Zukunft der deutschen Messen?, in: Der Handel, Heft 11, 1995, S. 29-30; $\underline{\underline{0 . V} .}$., Verschärtter Kampf um die Marktpositionen, in: pro, Heft 7 8, 1995, S. 32-36.

Vgl. Strothmann, K.-H., Verbundveranstaltungen des Messe- und Kongresswesens im Investitionsgüter-Marketing, in: Rost, D. Strothmann, K.-H. (Hrsg.), Handbuch Werbung für Investitionsgüter, Wiesbaden 1983, S. 399-425; ‥V., Messe mit Effekt, in: Asw, Heft 1, 1992, S. 76 77.

Vgl. zur aktuellen Problematik Hoffmann, J., Mobile Messe - Kreuz und quer, in: Wirtschaftswoche, Nr. 1/2, vom 4.1.1996, S. 72-74; 0.V., Klasse statt Masse, in: Focus, Nr. 42, 1995, S. 102; Koch, K.-W., Messemarkt kommt ins Rollen, in: Der Handel, Heft 11, 1995, S. 26; 0.V. Messe oder Event, in: asw, Heft 8, 1996, S. 108-111; o.V., Virtueller Rundgang, in: Markt und Mittelstand, Heft 4, 1996, S. 90-91. Einen Überblick über messeähnliche Veranstaltungskonzepte geben Selinski. H. Sperling, U. A., Marketinginstrument Messe: Arbeitsbuch für Studium und Praxis, Köln 1995, S. $15 \mathrm{ff}$. sowie S. $115 \mathrm{ff}$.; vgl. speziell zu Mischformen von Messen und Kongressen Selinski. H., Messe- und Kongressmarketing, Berlin 1983, S. $64 \mathrm{ff}$. 
Aufkommen dieser messeähnlichen Veranstaltungen, bei denen sich im Gegensatz zu herkömmlichen Messeveranstaltungen die ausstellenden Unternehmen zumeist allein oder aber in einem ausgewählten Konkurrenzumfeld präsentieren und sich damit dem Wettbewerbsdruck, der normalenweise auf Messen herrscht, entziehen, ist z. T. auch Ausdruck einer sich in letzter Zeit stärker zeigenden Kritik an dem Medium Messe. Diese beruht dabei zum einen auf der Feststellung steigender Messekosten für die Aussteller. ${ }^{29}$ Sie werden als Ursache dafür erachtet, daß Unternehmen, die sich bereits an Messen beteiligt haben, immer größere Messebudgets bereitstellen müssen, um ihre Beteiligungsintensität beibehalten zu können. Zum anderen fehlen vielen Ausstellern angesichts der gestiegenen Messekosten und des Wandels der Messen von Order- zu Informationsveranstaltungen überzeugende Nachweise des Nutzens von Messebeteiligungen. Die in diesem Zusammenhang seit den $80 \mathrm{er}$ Jahren geführte Diskussion um die „Messetransparenz“ zeigt die vermehrten Bestrebungen der Aussteller zur Steigerung der Effizienz bei Messebeteiligungen. ${ }^{30}$ Messen stehen daher vor der Herausforderung, ihre Rolle als Erfolgsfaktor im Marketing nachzuweisen. ${ }^{31}$

Die genannten Entwicklungen stellen potentielle Aussteller bei der Planung ihrer Messebeteiligungen zunehmend vor ein Spannungsfeld. Einerseits sind nicht zuletzt aufgrund der zunehmenden Bedeutung der direkten Kommunikation und Interaktion mit den Marktpartnern verstärkte Messeaktivitäten erforderlich. Ande-

29 Die Angaben zur Entwicklung der Messekosten schwanken je nach dem, welche Messeveranstaltungen einbezogen werden. Schätzungen gehen von einer Steigerung der gesamten Messekosten um 85 \% allein in den Jahren von 1973 bis 1988 aus. Vgl. Schober. P. M., Messen und Ausstellungen. Teilnehmen oder wegbleiben? - Eine kleine Entscheidungshilfe, in: Marketing Journal, 21. Jg., Heft 4, 1988, S. 400 ff. Betrachtet man nur die Standmiete, so werden Preissteigerungsraten von bis zu 59 \% innerhalb von 10 Jahren pro Quadratmeter Standfläche angegeben. Vgl. Rost. D., Die Rolle der Messen im Marketing, in: Schöttle. K. M. (Hrsg.), Jahrbuch des Marketing, 4. Ausgabe, Wiesbaden 1986, S. $137 \mathrm{ff}$. Einer aktuellen Untersuchung des AUMA zufolge liegt der Durchschnittspreis für einen Quadratmeter Standfläche auf Messen in Deutschland bei DM 1.138,--. Vgl. Ausstellungs- und Messe-Ausschuss der Deutschen Wirtschaft e. V. (AUMA) (Hrsg.), Die Messekosten der deutschen Aussteller, Bergisch Gladbach 1994, S. 15.

30 Vgl. Peters. M., Mehr Messetransparenz durch neue Ansätze der Messeerfolgskontrolle, in: $\mathrm{m}+\mathrm{a}$ report, Heft 2, 1980, S. 18-23; Rost. D., Ausstellen oder Aussteigen?, in: asw, Heft 1, 1982, S. 46-54; Schwickent. E., Transparenz der Besucher kommt zu kurz, in: HB, vom 24.11.1982, S. 16; Rost. D., Transparenz schafft Effizienz, in: asw, Heft 9, 1983, S. 68-72; Rost. D., Messetransparenz - ein Imperativ, in: asw, Heft 8, 1984, S. 30-32; Spryß. W. M., Mit Forschung Lücken füllen, in Verlagsbeilage der FAZ, Nr. 20, vom 25.01.1994, S. B2; Rominski. D., Neue Ausstellerwünsche, in: asw, Heft 6, 1995, S. 102-106. 
rerseits ergibt sich angesichts begrenzter Budgets ${ }^{32}$ und expandierendem Messeangebot die Notwendigkeit zu einem strategischen Management von Messebeteiligungen, das sich vor allem in einer zielorientierten Messeselektion und messespezifischen Positionierung i. S. einer bewußten Wettbewerbsdifferenzierung und messebesucherbezogenen Profilierung äußert. ${ }^{33}$

Erfahrungen im Bereich der Planung von Messebeteiligungen zeigen aber, daß Entscheidungen bezüglich der Beschickung von Messeveranstaltungen trotz ihrer z. T. erheblichen Kostenwirkungen vielfach nicht auf der Basis eines strategischen Entscheidungsprozesses, sondern häufig durch Wettbewerbsdruck begründet werden. ${ }^{34}$ In diesem Zusammenhang ist auch auf eine bislang unzureichende Berücksichtigung des Management von Messebeteiligungen in der wissenschaftlichen Auseinandersetzung im Marketing hinzuweisen. ${ }^{35}$ Zwar reichen älteste wissenschaftliche Veröffentlichungen zum Messewesen bis in das 19. Jahrhundert zurück $^{36}$, doch fehlte es bis in jüngster Zeit an umfassenderen Behandlungen des Managementprozesses von Messebeteiligungen.

32 Blieben in den 70er Jahren die Ausgaben der ausstellenden Unternehmen in der Bundesrepublik Deutschland mit geschätzten 3,3 Mrd. DM im Jahre 1970 und 3,5 Mrd. DM im Jahre 1978 noch relativ konstant, wurden die Ausgaben für Messen im Jahre 1980 auf 6 Mrd. DM geschätzt. Vgl. Gräbener, W., Die Messepolitik als Marketinginstrument dargestellt am Beispiel von Investitionsgüterproduzenten, Göttingen 1981, S. 18 f. Gegenwärtig geben deutsche Unternehmen für ihr Messe-Engagement mehr als geschätzte $12 \mathrm{Mrd}$. DM aus. Vgl. o.V., Klasse statt Masse, a.a.O., S. 102. Allerdings wird von zukünftig eher stagnierenden Messebudgets der Aussteller ausgegangen. Vgl. $\underline{0 . V}$., Stabile Großwetterlage für klassische Werbung, in: HB, Nr. 207, vom 26.10.1995, S. 12.

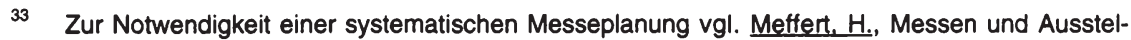
lungen als Marketinginstrument, a.a.O., S. 79; Strothmann, K.-H., Segmentorientierte Messepolitik, a.a.O., S. 102; Roth, G. D., Messen und Ausstellungen verkaufswirksam planen und durchführen, Landsberg/Lech 1981, S. 11.

34 Vgl. Mortsiefer. J., Die Intuition alleine reicht nicht - Messebeteiligungen auf der Basis eines Nutzungs-Konzeptes, in: Küffner. G.. Mortsiefer. J. (Hrsg.), Messeplatz Europa. Messen als Bestandteil des betrieblichen Marketings, Frankfurt a.M. 1990, S. 3 u. S. 11 f.; Huckemann, M. ter Weiler. D. S., Messen meßbar machen. Die 5 trojanischen Pferde des MesseMarketing, Neuwied, Kriftel, Berlin 1995, S. V ff. und S. 6.

Vgl. Wenge. H.-U. Müller. A., Das Management von Messe-Beteiligungen, in: Berndt. R. Hermanns, A. (Hrsg.), Handbuch Marketing-Kommunikation, Wiesbaden 1993, S. 73. Insbesondere die Unternehmenspraxis fordert die Wissenschaft zu Forschungsaktivitäten in diesem Bereich auf. Vgl. Berghäuser, B., Messen als Entscheidungsproblem, in: Deutsche Werbewissenschaftliche Gesellschaft e.V. (Hrsg.), Messen als Marketing-Instrument, Bonn 1983, S. 5 ff. 


\section{Management von Messebeteiligungen als Gegenstand der Untersuchung}

Erst seit Mitte der 70er Jahre werden in der wissenschaftlichen Forschung spezifische Fragestellungen des Management von Messebeteiligungen diskutiert. ${ }^{37}$ Der noch am Anfang stehenden wissenschaftlichen Auseinandersetzung mit betriebswirtschaftlichen Fragestellungen von Messebeteiligungen steht eine Vielzahl von Monographien gegenüber, die auf Erfahrungen bzw. subjektiven Eindrücken von Praktikern aus dem Messewesen oder Vertretern von Messe-Consulting-Unternehmen basiert. ${ }^{38}$ Inhalt dieser Beiträge sind meist in der Praxis bewährte Regeln und Handlungsvorschriften i. S. v. Heuristiken für die Ausgestaltung von Messebeteiligungen. Eine über die individuellen Einzelerfahrungen hinausgehende objektivierbare, wissenschaftliche Fundierung der Erkenntnisse ist im Rahmen dieser Beiträge allerdings nicht gegeben. ${ }^{39}$ Zur Erlangung wesentlicher Erkenntnisfortschritte ist es daher für die Wissenschaft um so mehr erforderlich, grundsätzliche Aussagen über die Konzeption des Management von Messebeteiligungen abzuleiten.

37 Vgl. Meffert. H., Einführungs- und Schlußworte anläßlich des 16. Münsteraner Führungsgesprächs am 13./14.03.1989, in: Meffert. H. Wagner. H. Backhaus. K. (Hrsg.), Messemarketing - Bestandsaufnahme und Perspektiven, Dokumentationspapier Nr. 52 der Wissenschaftlichen Gesellschaft für Marketing und Unternehmensführung e.V., Münster 1989, S. 1 ff. sowie S. $68 \mathrm{ff}$;; Meffert. H. Gass, C., Messen und Ausstellungen im System des Kommunikationsmix, a.a.O., S. 1.

Beispielhaft seien in der deutschsprachigen Literatur enwähnt Huckemann, M. ter Weiler. D. S.. Messen meßbar machen. Die 5 trojanischen Pferde des Messe-Marketing, a.a.O.; Naumann, C., Erfolgreich auf der Messe: Handbuch für die Beteiligung an Messen und Ausstellungen, Heidelberg 1993; Zotter. R., Erfolgreiches Messemarketing. Leitfaden für Analyse, Planung, Durchführung, Nachbearbeitung, Erfolgskontrolle bei Messebeteiligungen im In- und Ausland, Zürich 1993; Zerres. R., Ausstellen und Gewinnen. Der Kompass für die erfolgreiche Messepraxis, Woldert 1992; Földy. R., Die Messe als Markt. Beteiligungsstrategien für Aussteller, Wien 1990; Goschmann. K., Die erfolgreiche Beteiligung an Messen und Ausstellungen von A - Z, Landsberg/Lech 1988; Scheitlin. V., Messebeteiligung erfolgreich gestalten, St. Gallen, Stuttgart, Steyr 1984; Kustenaar. J., Messehandbuch - ein Leitfaden für Messebeteiligungen, Bern, Stuttgart 1983; Roth.G. D., Messen und Ausstellungen verkaufswirksam planen und durchführen, a.a.O.; Hoche. K., Handbuch für Aussteller, 111 Messetips, München 1974; Haeberle, K. E., Erfolg auf Messen und Ausstellungen: Handbuch für Teilnahme, Organisation, Gestaltung, Technik, Stuttgart 1967. Vgl. auch aus dem angloamerikanischen Sprachraum Siskind. B. The Successful Exhibitor's Handbook. Trade Show Techniques for Beginners and Pros, 2. Ed., Vancouver, Bellingham 1994; Christman. C., The Complete Handbook of Profitable Trade Show Exhibiting, Englewood Cliffs 1991; Dudley, J. W., Successful Exhibiting, Holbrook 1991; Weintraub. D. K., Trade Show Exhibiting: The Insider's Guide for Entrepreneurs, 0. O. 1991; Chapman, E. A., Exhibit Marketing: A Survival Guide for Managers, New York u. a. 1987.

39 Vgl. Funke, K., Messeentscheidungen: Handlungsalternativen und Informationsbedarf, Frankfurt a.M., Bern, New York 1986. S. 4. and Universität Münster - 978-3-631-75073-5 
In einer Gesamtschau der vorliegenden wissenschaftlichen Arbeiten wird deutlich, daß bislang eher fragmentarische Erkenntnisse zu diesem Themengebiet vorliegen. Dies ist zum einen in den Forschungsansätzen, die den Arbeiten zugrunde liegen, begründet und zum anderen auf die Beschränkung des Forschungsfokus hinsichtlich spezifischer Fragestellungen zurückzuführen. Während sich Selinski und Benad dem Management von Messebeteiligungen aus einem gestaltpsychologischen Ansatz ${ }^{40}$ nähern und vor allem Wahrnehmungswirkungen ${ }^{41}$ von Messeständen bei Messebesuchern untersuchen ${ }^{42}$, beschreibt die Mehrzahl der Forschungsansätze den Prozeß der Beteiligung an Messeveranstaltungen aus der managementbezogenen Perspektive des Ausstellers. ${ }^{43}$ Eine zusammenfassende Übersicht der bestehenden Forschungsansätze findet sich in Abb. 2. Dabei wurde zur Einordnung der Forschungsansätze auf die folgenden Beurteilungskriterien zurückgegriffen:

40 Unter der Gestaltpsychologie ist ein theoretischer Erklärungsansatz zur psychologischen Erklärung der Wahrnehmung von Gegenständen, die sich in ihrer Ausprägung bzw. "Prägnanz" unterscheiden, zu verstehen. Vgl. zu den theoretischen Grundlagen Stadler. M. Seeger. $F$. Raeithel. A., Psychologie der Wahrnehmung, 2. Aufl., München 1977; Behrens. G., Das Wahrnehmungsverhalten des Konsumenten, Frankfurt 1982.

41 Die Wahrnehmungswirkungen werden i. S. von momentanen Gedächtnisreaktionen auf optische Reize erfaßt, d. h. direkt nach Verlassen des Messestandes bzw. beim Verlassen des Messegeländes werden die Auskunftspersonen zu gestalterischen Eindrücken befragt. Inhaltlich stellen Akzeptanzmessungen Textverständlichkeits- und Erinnerungstests sowie Bekanntheitsanalysen den Schwerpunkt der Forschungsbemühungen dar. Vgl. zum verhaltenswissen-

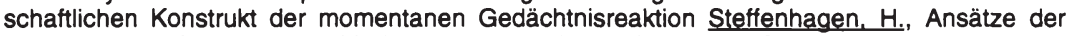
Werbewirkungsforschung, in: Marketing ZFP, 6. Jg., Heft 2, 1984, S. 77-88. Zu den verschiedenen Tests und Analyseformen vgl. Schenk. M., Wirkungen der Werbekommunikation, Köln u. a. 1990 .

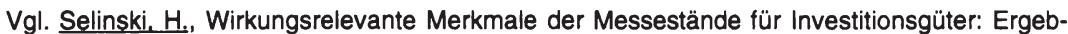
nisse einer empirischen Untersuchung auf der Hannover-Messe 1977, in: Strothmann, K.-H. (Hrsg.), Arbeitspapier des Instituts für Markt- und Verbrauchsforschung, Nr. 8, 1978; Benad. G.. Messestandanalyse - Ergebnisse von Befragungen auf 40 Messeständen bei 4800 Personen, Düsseldorf 1984.

43 Vgl. Gräbener. W., Die Messepolitik als Marketinginstrument, dargestellt am Beispiel von Investitionsgüterproduzenten, a.a.O., Täger, U. C. Ziegler. R., Die Bedeutung von Messen und Ausstellungen in der Bundesrepublik Deutschland für den Inlands- und Auslandsabsatz in ausgewählten Branchen, in: ifo-Institut für Wirtschaftsforschung e. V. (Hrsg.), ifo-Studien zu Handels- und Dienstleistungsfragen, Nr. 25, München 1984; Mortsiefer, J., Messen und Ausstellungen als Mittel der Absatzpolitik mittelständischer Herstellerbetriebe, Göttingen 1986; Rosson. Ph. Seringhaus. F. H. R., Reaching Export Objectives through International Trade Fairs, in: Trade Show Bureau (Ed.), Report No. IN 6, Denver 1991; Schmalen. H. Wagner, A., Empirische Untersuchung zur Messebeteiligung von Unternehmen, Arbeitspapier, o. Nr., Passau 1990; Head. J., Positioning, Objectives, and Selection Factors for Trade Shows, in: Trade Show Bureau (Ed.), Report No. MC24, Denver 1992; Gopalakrishna S. Williams J., Identifying Important Factors in Lead Efficiency at Trade Shows, in: Trade Show Bureau (Ed.), Report No. SM 23, Denver 1993; Spryß. W. M.. Nerb. G. Reuter, J., Messeziele - Messeeffizienz, Münster-Sarmsheim 1994; Gopalakrishna. S. Lilien. J. D.. Sequeira. I. K., Do Trade Shows Pay Off?, in: JoM, Vol. 59. July 1995, S. 75-83; Tanner. J. F. Chonko, L. B., Trade Show Objectives, Management, and Staffing Practices, in: IMM, Vol. 24, 1995, S. 257-264. 
- Abbildung des Managementprozesses von Messebeteiligungen ${ }^{44}$,

- Berücksichtigung verschiedener Messetypen ${ }^{45}$,

- Branchenzugehörigkeit ${ }^{46}$ der untersuchten Aussteller ${ }^{47}$ sowie

- unternehmensgrößenspezifische Unterschiede der untersuchten Aussteller. ${ }^{48}$

Die Bestandsaufnahme der angeführten Studien macht deutlich, daß bislang überwiegend lediglich ausgewählte Teilbereiche des Management von Messebeteiligungen untersucht wurden und zeigt insbesondere die Notwendigkeit, den Managementprozeß von Messebeteiligungen ganzheitlich zu erfassen.

Dabei wird zunächst die eindeutige Kennzeichnung des Begriffs „Messebeteiligung" wesentlich durch den Umstand erschwert, daß kein singuläres Merkmal besteht, das eine präzise Bestimmung und Abgrenzung des Begriffs

44 Um Aussagen zum Management von Messebeteiligungen machen zu können, ist zur Beachtung von Interdependenzen eine Berücksichtigung aller Managementphasen zu gewährleisten.

Zur Ananlyse des Einflusses von Messeveranstaltungen auf die Ausgestaltung von Messebeteiligungen sind möglichst viele verschiedene Messetypen in die Untersuchung einzubeziehen.

Im folgenden werden die Begriffe Branche, Wirtschaftssektor, Wirtschaftszweig, Industrie und Markt synonym verwendet. Vgl. hierzu Fronhoff, B., Die Gestaltung von Marketingstrategien, Köln 1986, S. 169 f.; Mauthe. K. D., Strategische Analyse, München 1984, S. 198.

47 Die Zugehörigkeit eines Ausstellers zu einem Wirtschaftszweig stellt vielfach eine Schlüsselvariable für die Auswahl der Messen und Ausgestaltung der Messebeteiligungen dar. Vgl. hierzu Strothmann, K.-H. Roloff. E., Charakterisierung und Arten von Messen, in: Berndt. R. Hermanns, A. (Hrsg.), Handbuch Marketing-Kommunikation, Wiesbaden 1993, S. 717 f.

48 Aufgrund unterschiedlicher Ressourcenausstattung sowie vermuteter Verschiedenheit der Beteiligungsmotive an Messeveranstaltungen sind auch die Auswirkungen unterschiedlicher Unternehmensgrößen, die i. d. R. an der Beschäftigtenzahl oder am Jahresumsatz festgemacht werden, zu untersuchen. Vgl. hierzu Mortsiefer. J., Messen und Ausstellungen als Mittel der Absatzpolitik mittelständischer Herstellerbetriebe, a.a.O. S. 21 f. 


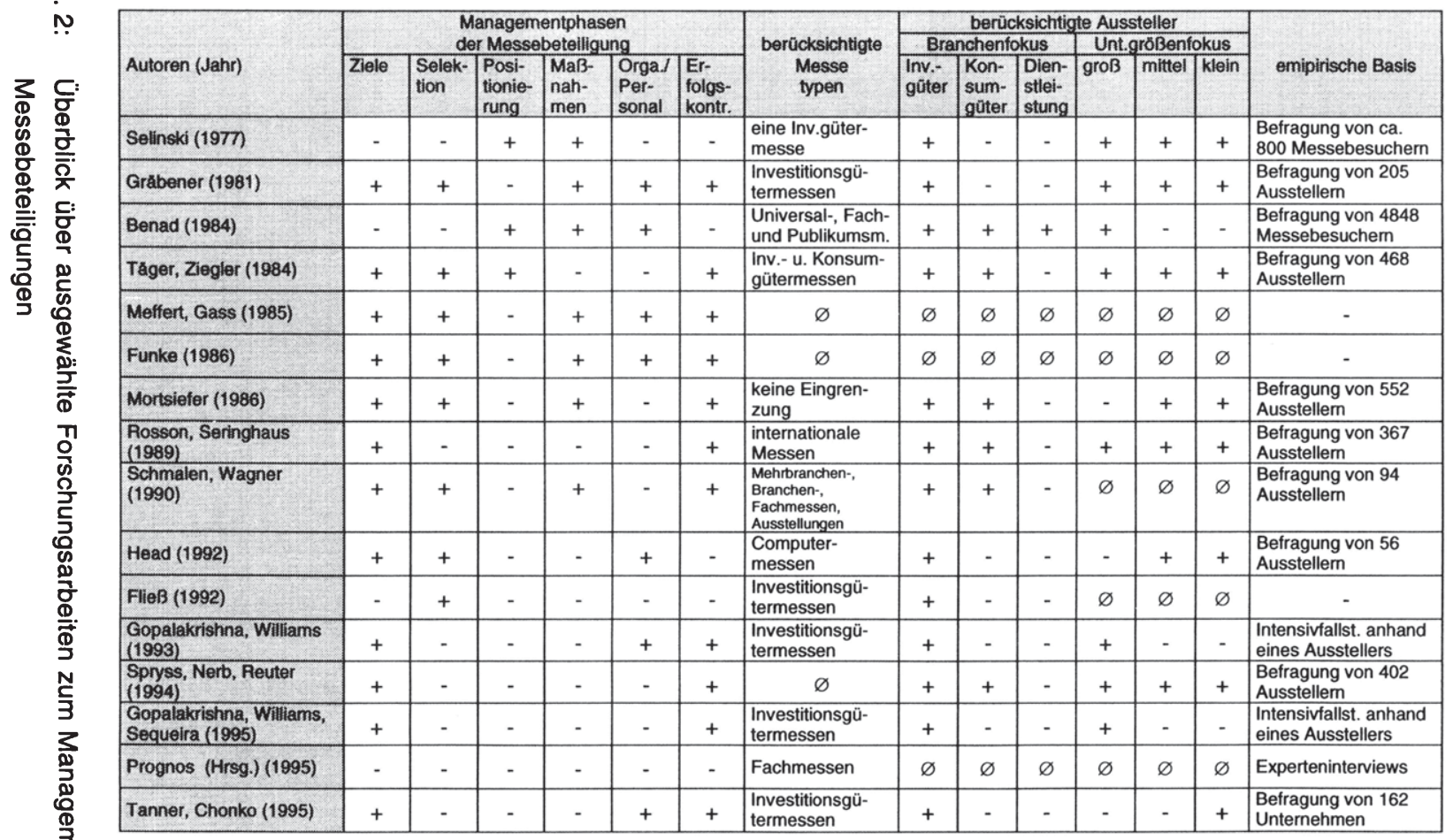

- = trifft nicht $2 \mathrm{u}$

ऽ $\varnothing=$ keine Aussage 
"Messe“49 ermöglicht. Diese Tatsache ist nicht zuletzt darauf zurückzuführen, daß sich in der Messepraxis eine Vielzahl verschiedener Messen entwickelt hat. Durch Typologisierungen ${ }^{50}$ wird auf der Grundlage ausgewählter Merkmale der Versuch unternommen, Grundformen von Messen zu charakterisieren, um sich damit auch den sich aus der Messevielfalt ergebenden Konsequenzen für das Management von Messebeteiligungen zu nähern. ${ }^{51}$ Eine von Funke in der Literatur vor-

49 Der Begriff der Messe wird in der Literatur mit unterschiedlichen Schwerpunktsetzungen belegt und je nach Autor anhand differenzierter Abgrenzungskriterien wie z. B. Repäsentativität des Ausstellungsprogramms für eine bestimmte Branche oder Anteil der Fachbesucher an der Gesamtbesucherzahl etc. definiert und von verwandten Begriffen wie Ausstellung, Salon, Schau, Börse u. a. abgegrenzt. Zwar existiert eine legaldefinitorische Unterscheidung der beiden zentralen Begrifflichkeiten "Messen" und "Ausstellungen" in der Gewerbeordnung, doch hat sich diese Unterscheidung in der Praxis als kaum haltbar erwiesen. Meffert plädiert aufgrund der vielfältigen Gemeinsamkeiten der beiden Veranstaltungsarten für eine synonyme Verwendung beider Begriffe. Vgl. Meffert, $\mathrm{H}_{\text {, }}$, Messen und Ausstellungen als Marketinginstrument, in: Verlag Wirtschaft und Finanzen (Hrsg.), Messen als Marketinginstrument, Düsseldorf 1988, S. 10. Der Argumentation wird insbesondere deshalb in dieser Arbeit gefolgt, da Veranstaltungsbezeichnungen mittlerweile kaum mehr in der Lage sind, über die Veranstaltungsart Auskunft zu geben. Hinsichtlich einer definitorischen Abgrenzung eines umfassenden Messebegriffs wird HASELOFF gefolgt, der unter Messen zeitlich und örtlich festgelegte Veranstaltungen mit Marktcharakter versteht, die ein umfassendes Angebot eines oder mehrerer Wirtschaftszweige bieten. Vgl. Haseloff, O.W., Die Reduktion der Komplexität: Messen heute Kommunikation, Information, Entscheider, Köln 1980, S. 14 f. Eine umfassende Darstellung der Diskussion um die Begriffsbestimmung von Messen und Ausstellungen findet sich bei Mortsiefer. J., Messen und Ausstellungen als Mittel der Absatzpolitik mittelständischer Herstellerbetriebe, a.a.O., S. $21 \mathrm{ff}$. Vgl. zur Legaldefinition von Messen und Ausstellungen Gewerbeordnung (GewO) in der Fassung vom 01.01.1987. Titel IV \$\$ 64,65. Zur Kritik an einer begrifflichen Trennung zwischen Messen und Ausstellungen vgl. auch Selinski. H. Sperling. $U$. A., Marketinginstrument Messe: Arbeitsbuch für Studium und Praxis, a.a.O., S. $13 \mathrm{ff}$.; Mortsiefer. J., Messen und Ausstellungen als Mittel der Absatzpolitik mittelständischer Herstellerbetriebe, a.a.O., S. 21. Messen und Ausstellungen werden hier unter dem Begriff "Marktveranstaltungen" subsumiert.

50 Vgl. zum Einsatzspektrum von Typologien Knoblich. H., Die typologische Methode in der Betriebswirtschaftslehre, in WiSt, Heft 4, April 1972, S. 141-147 und die dort angegebene Literatur.

51 In diesem Zusammenhang kann dabei Tietz hervorgehoben werden. Vgl. Tietz. B., Bildung und Verwendung von Typen in der Betriebswirtschaftslehre, dargestellt am Beispiel der Typologie der Messen und Ausstellungen, Köln, Opladen 1960, S. 149 ff. Eine grundlegende Klassifizierung von Konsumgüter- und Investitionsgütermessen legt dabei Strothmann vor. Vgl. Strothmann, K.-H., Die Messe- und Ausstellungswerbung im Überblick, in: Tietz. B. (Hrsg.), Die Werbung - Handbuch der Kommunikations- und Werbewirtschaft, Bd. 2, Landsberg/Lech 1982 , S. $1620 \mathrm{f}$. Eine spezielle Typenbeschreibung für Investitionsgütermessen liefert Merdian. Vgl. Merdian. M., Die Tragfähigkeit des Systemansatzes und der typologischen Definitionsform für die Darstellung von IG-Messen unter Berücksichtigung einer sozialwissenschaftlich verstandenen Kommunikationstheorie, Berlin 1983, S. 36 ff. Ein großer Teil der Beiträge aus der Unternehmenspraxis beinhaltet eine Auseinandersetzung mit (ausgewählten) Messetypen und deren Entwicklungen bzw. Entwicklungsperspektiven. Vgl. hierzu stellv. Groth. C., Das Leistungspotential von Fachmessen, a.a.O., S. $57 \mathrm{ff}$. sowie Berghäuser. B., So lassen sich Messen messen, in: Marketing Journal, Nr. 9, 1976, S. 61 ff. 
genommene Analyse zu den zentralen Typologisierungskriterien ergab die folgenden Differenzierungsmerkmale von Messen: ${ }^{.2}$

- Breite des Angebots (Universalmessen, Spezialmessen bzw. Solo- und Monomessen, Branchenmessen sowie Fach- bzw. Verbundmessen),

- Angebotsschwerpunkt (Konsum- und Investitionsgütermessen),

- Funktion einer Messe (Informations- und Ordermessen),

- geographische Aussteller- und Besucherreichweite (regionale, überregionale, nationale und internationale Messen),

- Zugehörigkeit der Aussteller bzw. Besucher zu Branchen bzw. Wirtschaftsstufen (Industriemessen, Handelsmessen, Landwirtschaftsmessen und Dienstleistungsmessen) sowie

- Hauptrichtung des Absatzes (Export- und Importmessen).

Eine solche Klassifizierung verfolgt im wesentlichen den Zweck, die veranstaltungsspezifischen Managementprobleme einer Messebeteiligung zu erhellen. Als problematisch ist hierbei jedoch zum einen die Tatsache einzuordnen, daß eine typologische Bestimmung von Messeveranstaltungen allenfalls tendenziell möglich ist, da die angeführten Messetypen i. d. R. nicht in Reinform existieren. So scheint bspw. eine Unterscheidung in Informations- und Ordermessen nur noch wenig zur Differenzierung von Planungsentscheidungen bei Messebeteiligungen beizutragen, da auf Informationsmessen stets auch Kaufabschlüsse getätigt, umgekehrt auf Ordermessen auch Informationen ausgetauscht werden. Damit kann zum anderen aus Managementsicht nicht hinsichtlich jedes Abgrenzungskriteriums von der gleichen Entscheidungsrelevanz für ausstellende Unternehmen ausgegangen werden. ${ }^{53}$ Neben der genannten funktionalen Unterscheidung von Messen dürfte auch eine Unterscheidung der Messen nach der Hauptrichtung des Absatzes nur von nachgeordneter Bedeutung sein, da sich Überschneidungen zum Aspekt der geographischen Aussteller- und Besucherreichweite ergeben. Einen vergleichsweise hohen Stellenwert wird dagegen das Kriterium "Breite des Angebots" einnehmen, da hier nicht nur die Branchenzugehörigkeit der Aussteller und Messebesucher, sondern auch das Kriterium „Angebotsschwerpunkt der Messen" subsumiert werden kann.

52 Vgl. Funke. K., Messeentscheidungen: Handlungsalternativen und Informationsbedarf, a.a.O., S. 4; ähnlich auch Hugger, P. F., Nutzen-Kosten-Analyse der regionalwirtschaftlichen Auswirkungen von Messen und Ausstellungen, München 1986, S. $24 \mathrm{ff}$.

53 Vgl. Wenge, H.-U., Planung von Messebeteiligungen, in: Strothmann, K.-H. Busche. M. (Hrsg.), Handbuch Messemarketing, Wiesbaden 1992, S. 293. 
Betrachtet man Messebeteiligungen des weiteren aus der instrumentellen Perspektive des Ausstellers, so ist zunächst die Position dieses Instruments im Marketingsystem zu bestimmen. Hier hat sich bis heute nicht zuletzt wegen der sog. Multifunktionalität von Messebeteiligungen keine einstimmig vertretene Sichtweise herauskristallisiert. Zahlreiche Autoren vertreten die Ansicht, daß Ausstellern mit Messebeteiligungen ein weitgehend eigenständiges Marketinginstrument zur Verfügung steht, das das "klassische Marketing-Mi““54 um einen zusätzlichen Instrumentalbereich erweitert. ${ }^{55}$

Unbestritten liegt das Potential von Messebeteiligungen auf mehreren Ebenen. Bei ihrem Einsatz spielen durchaus sortiments-, konditionen- und distributionspolitische Überlegungen der Aussteller eine wichtige Rolle. ${ }^{56}$ Meffert macht allerdings deutlich, „daß eine Messebeteiligung heute im wesentlichen durch kommunikationspolitische Aktivitäten bestimmt wird, die sich als Resultante aus den im Marketing des Unternehmens verfolgten produkt-, distributions- und preispolitischen Zielsetzungen ergeben“. Er plädiert daher dafür, Messebeteiligungen dem kommunikationspolitischen Instrumentarium zuzuordnen. ${ }^{57}$ Das Kommunikationsinstrument Messebeteiligung ist damit eigenständig neben die klassische Medienwerbung, den persönlichen Verkauf, die Verkaufsförderung sowie die Öffentlichkeitsarbeit und die neueren Kommunikationsinstrumente ${ }^{58}$ zu stellen. Dieser Argumentation soll im Rahmen der vorliegenden Arbeit gefolgt werden.

54 Unter dem "klassischen Marketing-Mix“ soll hier nach Meffert die Zuordnung der Marketinginstrumente zu den Mix-Bereichen "Produkt-Mix“, Kontrahierungs-Mix“, „Distributions-Mix" und „Kommunikations-Mix" verstanden werden, die sich im Marketing allgemein durchgesetzt hat. Vgl. Meffert. H., Marketing. Grundlagen der Absatzpolitik, 7. Aufl., Wiesbaden 1986, S. $114 \mathrm{ff}$.

55 Vgl. stellvertretend Jaspert. $F$, Das synergetische Potential ist groß - die Messe als Teil der betrieblichen Absatzpolitik, in: Küffner. G. Mortsiefer, J. (Hrsg.), Messeplatz Europa, Messe als Bestandteil des betrieblichen Marketings, Frankfurt a. M. 1990, Groth, C. Lentz. I., Die Messe als Dreh- und Angelpunkt: Multifunktionales Instrument für erfolgreiches Marketing, Landsberg/Lech 1993, S. 17 u. S. 36-50; Selinski. H.. Sperling. U. A., Marketinginstrument Messe: Arbeitsbuch für Studium und Praxis, a.a.O., S. $96 \mathrm{ff}$.

56 Vgl. Wenge. H.-U. Müller. A., Das Management von Messe-Beteiligungen, a.a.O., S. 730 f.

57 Vgl. zu dieser Argumentation Meffert. H., Messen und Ausstellungen als Marketinginstrument, a.a.O., S. 10.

58 Hierunter fallen Kommunikationsinstrumente wie z. B. Event-Marketing, Sponsoring, Product Placement, Multimedia-Marketing u. a. Vgl. hierzu Tomczak, T. Müller, F.. Müller. R., NichtKlassiker der Kommunikation: Erfolgsreserven im Erklärungswettbewerb, in: Tomczak. T. Müller. F. Müller. R. (Hrsg.), Die Nicht-Klassiker der Unternehmenskommunikation, St. Gallen 1995, S. $16 \mathrm{f}$. 
Es ist allerdings zu berücksichtigen, daß Messebeteiligungen einen spezifischen Instrumentalcharakter aufweisen. Einerseits sind sie zwar mehr als andere Kommunikationsinstrumente durch weitere Marketingmaßnahmen zu ergänzen, andererseits vermögen sie deren Wirkung aber auch vielseitiger und $z$. T. gezielter zu unterstützen als dies anderen Instrumenten möglich wäre ( $\mathrm{vgl}$. Abb. 3). So ist ein ausstellendes Unternehmen grundsätzlich in der Lage, auf dem Messestand gleichzeitig Beeinflussungswirkungen zu erzielen und von Kunden formulierte Erkenntnisse, Bedürnnisse sowie Probleme i. S. d. Informationsgewinnung aufzunehmen, um diese bspw. in die Produktpolitik einfließen zu lassen oder gezielt geeignete Handelspartner für Vertriebskooperationen zu suchen und damit die Distributionspolitik zu unterstützen.

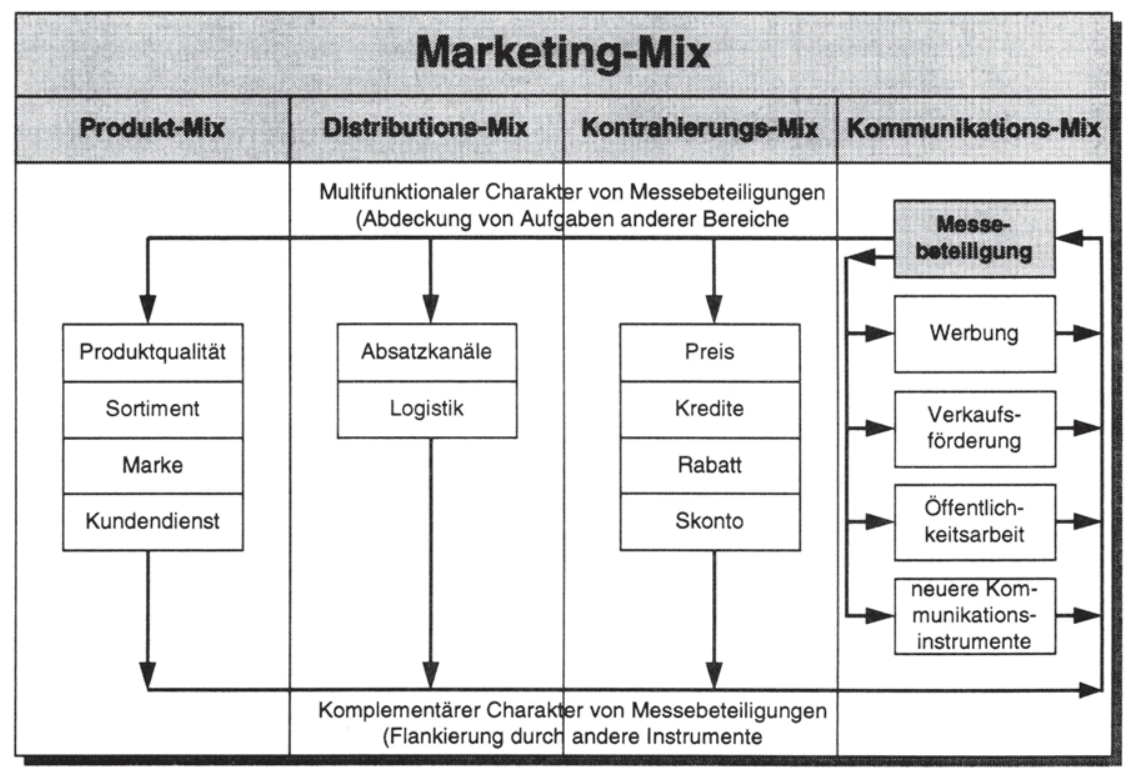

Abb. 3: Stellung von Messebeteiligungen im Marketing-Mix 
Greift man daher den Gedanken auf, daß Messebeteiligungen das Ergebnis einer Kombination verschiedener Einzelaktivitäten mit instrumentellem Charakter darstellen, so können sie als eigenständiger Submix-Bereich innerhalb der Kommunikationsinstrumente betrachtet werden. Die Notwendigkeit einer Integration der Messeaktivitäten in die Kommunikationsmaßnahmen ${ }^{59}$ einerseits und die Abstimmung des gesamten Messebeteiligungs-Mix andererseits deuten auf die Komplexität des Management von Messebeteiligungen hin. ${ }^{60}$ Letztere läßt eine Vielzahl von Planungs-, Kontroll- und Durchsetzungsproblemen sichtbar werden, die im folgenden behandelt werden sollen. Sie sind zunächst in einen systematischen Managementprozeß im Rahmen einer strategischen und operativen Messebeteiligungsplanung und -steuerung einzuordnen. ${ }^{61}$ Abb. 4 zeigt die wesentlichen Phasen eines solchen Managementprozesses im Überblick. ${ }^{62}$

59 Vgl. Raffée. H., Integrierte Kommunikation, in: Werbeforschung \& Praxis, Heft 3, 1991, S. 8790.

Vgl. Jaspert. F., Marketinginstrument Messe: Das synergetische Potential ist groß, in: BddW, Nr. 52, vom 14.03.1989, S. 7.

61 Die klassische Managementlehre baut ihr Grundverständnis des betrieblichen Steuerungsprozesses auf dem Prozeß-Paradigma auf. Hiernach wird Management als eine systematische Abfolge von Funktionen begriffen, an deren Anfang die Planung als geistiger Entwurf der zukünftig zu erreichenden Ziele und der hierzu zu ergreifenden Maßnahmen steht (Primat der Planung). Ihr folgen die Organisation als Strukturentwurf für den Aufgabenvollzug sowie die Führung als Veranlassung und Überwachung des Aufgabenvollzugs. Der Prozeß mündet in die Kontrolle, die schließlich Informationen über den Zielerreichungsgrad an die Planung zurückkoppelt, um bei weiteren Planungen Berücksichtigung zu finden. Vgl. zum Primat der Planung Adam. D., Planung und Entscheidung: Modelle - Ziele - Methoden; mit Fallstudien und Lösungen, 4. Aufl., Wiesbaden 1996, S. 12 ff.; Griffith. R. W., Management, 2. Aufl., Boston 1987, S. $10 \mathrm{ff}$. Zu einer gesamthaften Einordnung der Prozeßfunktionen vgl. Gutenberg. E., Grundlagen der Betriebswirtschaftslehre, Bd 1: Die Produktion, 24. Aufl., Berlin u. a. 1983, S 235 f.; Gutenberg. E., Die Unternehmung als Gegenstand betriebswirtschaftlicher Theoriebildung, Frankfurt a. M. 1967, S. 25 f.; Steinmann, H. Schrevögg. G., Management: Grundlagen der Unternehmensführung; Konzepte, Funktionen, Praxisfälle, Wiesbaden 1990, S. $103 \mathrm{ff}$.

62 Vgl. Meffert. H., Messen und Ausstellungen als Marketinginstrument, a.a.O., S. 14.

Ralf Ueding and Universität Münster - 978-3-631-75073-5 


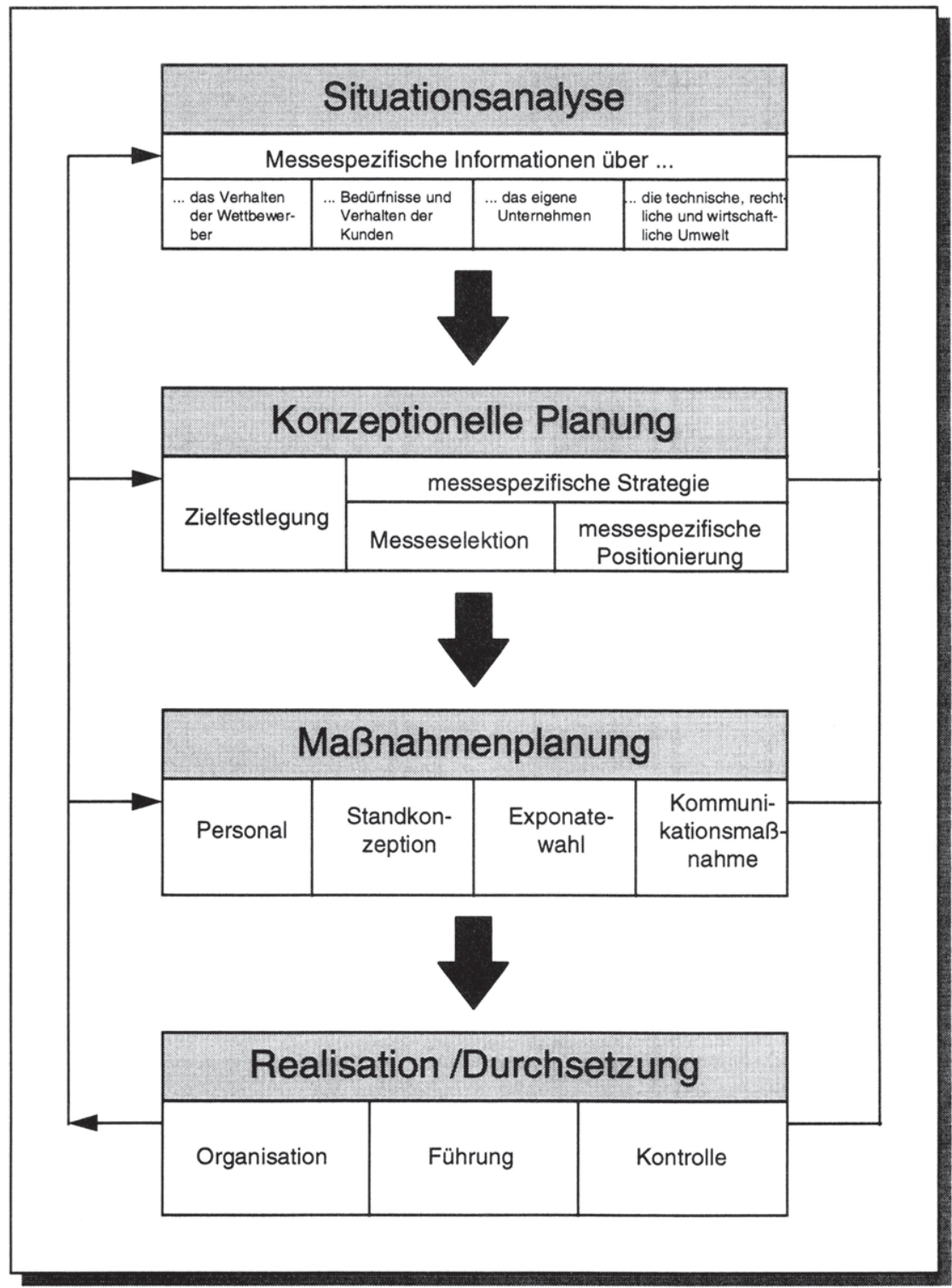

Abb. 4: Managementprozeß der Messebeteiligung 
Messebeteiligungen umfassen daher den Prozeß

- der Planung, Organisation, Führung sowie Kontrolle aller Aktivitäten,

- die mit der Teilnahme an einer zeitlich begrenzten und räumlich festgelegten Veranstaltung verbunden sind,

- um spezifische Marketing- bzw. Kommunikationsziele zu erreichen.

Die zentralen Fragestellungen des Messeengagements von Unternehmen lassen sich dabei anhand eines Paradigmas ${ }^{63}$ der Messebeteiligungsentscheidung ${ }^{64}$ darstellen:

- Was wird mit einer Beteiligung

- an welchen Messe(type)n

- wo

- wann

- wie

- zu welchen Kosten

- mit welchen Wirkungen erreicht?
(Messebeteiligungsziele)

(Messekonzeption)

(Messeort)

(Messezeitraum)

(Messebeteiligungsform)

(Messebeteiligungskosten)

(Messebeteiligungserfolg)

Das Paradigma macht deutlich, daß die einzelnen Entscheidungstatbestände insbesondere vor dem Hintergrund des jeweiligen situativen Kontextes eines Ausstellers in einen systematischen Managementprozeß von Messebeteiligungen zu überführen sind.

Die Planung der Messebeteiligung ist dabei in zwei zentrale Teilprozesse aufzuspalten. Während die konzeptionelle Planung im Anschluß an die Situationsanalyse die Festlegung von Messezielen und -strategien i. S. einer ziel- und budgetorientierten Messeselektion und einer messespezifischen Positionierung des Ausstellers umfaßt, sind der Maßnahmenplanung die stärker instrumentalen Bestandteile zuzuordnen. Die Realisation bzw. Durchsetzung beinhaltet die Organisations-, Führungs- und Kontrollprozesse der Messebeteiligung. Die

63 Vgl. Meffert. H., Marketing, in: Management-Enzyklopädie, Bd. 4, München 1971, S. 392; Meffert. H., Marketingforschung und Käuferverhalten, 2. Aufl., Wiesbaden 1992, S. 22.

64 Im Rahmen dieses Paradigmas wird die grundsätzliche Entscheidung, „ob überhaupt“ Messen als Kommunikationsinstrument einzusetzen sind als positiv entschieden vorausgesetzt. 
Erkenntnisse, die in dieser Phase gewonnen werden, gehen im Rahmen eines Feedbackprozesses in weitere Planungsentscheidungen ein.

Die verschiedenen Teilbereiche des damit grob gekennzeichneten Managementprozesses sind mit arteigenen Problemen und Fragestellungen behaftet, die im folgenden gekennzeichnet werden sollen.

Eine fundierte Situationsanalyse bildet generell den Ausgangspunkt jeder Marketingentscheidung. ${ }^{65}$ Aus der Gesamtheit möglicher Kontextvariablen sind dabei allerdings nur jene zu untersuchen, die im Rahmen der jeweiligen Planung Relevanz besitzen. Bezüglich der Messebeteiligungsentscheidungen weisen vor allem vier situativ bestimmte Bereiche grundsätzliche Planungsrelevanz auf: ${ }^{66}$

- unternehmens- und produktbezogene Faktoren

- marktbezogene Faktoren

- messebezogene Faktoren

- sonstige umweltbezogene Faktoren

Hinsichtlich der unternehmensbezogenen Faktoren ist zunächst die Unternehmensdemographie zu erfassen, die sich insbesondere in der Branchenzugehörigkeit und Unternehmensgröße darstellen läßt. In engem Zusammenhang mit der Größe des ausstellenden Unternehmens stehen auch die für Messebeteiligungen verfügbaren finanziellen und personellen Ressourcen. Sie beeinflussen die Entscheidung hinsichtlich einer Messebeteiligung maßgeblich. ${ }^{67}$ Mit Blick auf die produktbezogenen Faktoren sind vor allem die Merkmale des Produkts bzw. des Sortiments - hier insbesondere seine Mobilität, Ausstellungsfähigkeit und Erklärungsbedürftigkeit - entscheidungsrelevante Einflußfaktoren.

Als die wesentlichen marktbezogenen Einflußfaktoren können neben dem Verhalten und den Bedürfnissen der potentiellen Zielgruppen die Bedingungen im relevanten Markt herangezogen werden. Bezüglich der Marktbedingungen sind insbesondere das Verhalten der Wettbewerber, der Partner in den vor- und

65 Vgl. Meffert. H., Marketing. Grundlagen der Absatzpolitik, 7. Aufl., a.a.O., S. 57.

66 Vgl. Wenge. H.-U., Planung von Messebeteiligungen, a.a.O., S. 293.

67 Vgl. Meffert. H., Messen und Ausstellungen als Marketinginstrument, $a_{3}$ a 0. , S, $80 \mathrm{H}$.

Ralf Ueding and niversitat Menster - 978-3-631-75073-5 
nachgelagerten Marktstufen sowie die allgemeine Marktentwicklung zu berücksichtigen. $^{68}$

Der Messetyp bzw. die einzelne Messeveranstaltung selbst stellen einen Kontextfaktor dar, der nach verschiedenen Fragestellungen hin aufgespalten werden kann. So ist insbesondere zu klären, ob die vom Unternehmen ausgewählten Zielgruppen auf der Messe als Besucher erwartet werden und welche Informationsbedürfnisse bzw. welches Kaufverhalten diese aufweisen. Des weiteren sind die geographische Reichweite der Veranstaltung, die Serviceleistungen der Veranstalter sowie mitunter das Image des Messeplatzes und seine Umfeldqualität wesentliche Anhaltspunkte hinsichtlich einer Messebeteiligungsentscheidung. ${ }^{69}$

Im Rahmen der Beachtung sonstiger umweltbezogener Einflußfaktoren sind insbesondere ökonomische, rechtlich-politische, technologische und gesellschaftliche Umwelteinflüsse zu berücksichtigen. In diesem Zusammenhang sind bspw. Innovations- oder Konjunkturzyklen zu nennen, die ein Unternehmen in der Entscheidung für oder wider eine Messebeteiligung beeinflussen können.

Die situativen Einflußfaktoren gehen als Ausgangsparameter in die konzeptionelle Planung von Messebeteiligungen ein. In dieser Phase des Managementprozesses sind zunächst die Ziele, die mit der Messebeteiligung verfolgt werden sollen, festzulegen. Dabei bilden mit den Unternehmens- und Marketingzielen in Übereinstimmung gebrachte Messebeteiligungsziele die Voraussetzung für eine effiziente Messebeteiligung. Ein Messebeteiligungsziel läßt sich allgemein definieren als ein Zustand, der durch Beteiligung an Messen und damit verbundene Aktivitäten angestrebt wird. ${ }^{70}$ Dabei soll die Etfizienz von Messebeteiligungen als Verhältnis von eingesetzten Ressourcen und dem Grad der Zielerreichung und damit als $\mathrm{Maß}$ für den Erfolg des Messeengagements interpretiert werden. ${ }^{71}$ Die Formulierung der Messebeteiligungsziele gehört vor diesem Hintergrund zu den entscheidenden, da prädisponierenden, Planungsschritten des Messeengagements.

68 Vgl. Meffert. H., Messen und Ausstellungen als Marketinginstrument, a.a.O., S. 15.

69 Vgl. Wenge. H.-U. Müller. A., Das Management von Messe-Beteiligungen, a.a.O., S. 735.

70 Vgl. zur allgemeinen Definition von Handlungszielen Meffert. $H_{\text {. }}$, Marketing. Grundlagen der Absatzpolitik, 7. Aufl., a.a.O., S. $74 \mathrm{ff}$.

71 Vgl. Böcker. K., Marketing-Kontrolle, München 1987, S. 244 f. 
Auf der Grundlage der festgelegten Messebeteiligungsziele ist innerhalb der konzeptionellen Messeplanung die Messeselektionsentscheidung zu treffen. Geht man davon aus, daß die Grundsatzentscheidung einer Teilnahme an Messen gefallen ist, so sollte im Rahmen der Messeselektion die Intensität der Messebeteiligung festgelegt werden. Hier lassen sich zwei unterschiedliche Messeselektionsstrategien unterscheiden. Während sich ein Unternehmen im Zuge einer konzentrierten Selektionsstrategie auf einige wenige relevante Messen beschränkt, sieht eine diversifizierte Selektionsstrategie eine intensive Beteiligung an vielen z. T. unterschiedlichen Messen vor. Die Gründe einer diversifizierten Messeselektionsstrategie liegen insbesondere im Vorhandensein vieler verschiedener Kunden- bzw. Zielgruppen eines Unternehmens, während konzentrierte Selektionsstrategien vorwiegend von kleineren Unternehmen verfolgt werden. ${ }^{72}$ Die konkrete Zahl der Messen, an denen sich ein Unternehmen beteiligt, wird dabei zum einen durch die Bewertung jeder Messe sowie zum anderen durch vorhandene Ressourcen begrenzt.

Im Zentrum einer konzeptionellen Messeselektion steht daher zunächst das Bewertungsproblem von Messeveranstaltungen. Das Angebot an Messen ist deshalb einer Bewertung zu unterziehen, um festzustellen,

1. ob die zur Auswahl stehenden Messen generell in der Lage sind, die Erreichung der angestrebten Kommunikationsziele des Unternehmens zu unterstützen oder diese eher durch den Einsatz anderer Kommunikationsinstrumente erreicht werden können (inter-instrumentelle Substitutionskonkurrenz),

2. welche der zur Auswahl stehenden Messen den geforderten Beitrag zur Erreichung der formulierten Messebeteiligungsziele erbringen kann (intra-instrumentelle Substitutionskonkurrenz).

Gleichzeitig mit der generellen Bewertung der Messen erfolgt im Rahmen der Messebeteiligungsplanung die Budgetierung der Messebeteiligungen. Gegenstand der Budgetierung ist zum einen die Festlegung des Budgets für alle Beteiligungen eines Planungszeitraumes sowie zum anderen die Festlegung des Etats einer

Vgl. Täger. U. C. Ziegler, R., Die Bedeutung von Messen und Ausstellungen in der Bundesrepublik Deutschland für den Inlands- und Auslandsabsatz in ausgewählten Branchen, a.a.O., S. $54 \mathrm{ff}$. 
einzelnen Beteiligung und der mit ihr verbundenen Messeaktivitäten. ${ }^{73}$ Die zentralen Probleme der Budgetierung sind dabei in der Wahl eines Budgetierungsverfahrens, das eine zielorientierte Zuordnung der vorhandenen Mittel zu einzelnen Messebeteiligungen gewährleistet sowie in der theoretisch erforderlichen Simultaneität von Messeselektion und -budgetierung zu sehen. ${ }^{74}$ Ferner ist für die spätere Budgetkontrolle die Ausarbeitung von Planbudgets für einzelne Bereiche der Messebeteiligung notwendig. Hier sind nach Kostenbereichen ${ }^{75}$ aufgeteilte Budgetvorgaben zu erarbeiten. ${ }^{76}$

Im Rahmen der konzeptionellen Planung von Messebeteiligungen ist schließlich neben der Zielbildung und der Messeselektion auch der grundsätzliche Messeauftritt des Unternehmens festzulegen und i. S. einer messespezifischen Positionierung vorzunehmen. ${ }^{\pi}$

Die Positionierung von Unternehmen oder Produkten ist ein zentrales Konzept des Marketing. ${ }^{78}$ Wenngleich der Begriff Positionierung von vielen Autoren in der Marketingliteratur aufgegriffen wird, so hat sich bislang keine einheitliche Defini-

73 Raffée. H., Messen als Herausforderung für die Marketing-Theorie, in: Deutsche Werbewissenschaftliche Gesellschaft e.V. (Hrsg.), Messen als Marketing-Instrument, Bonn 1983, S. 80.

74 Einen Überblick über vorhandene Budgetierungsmethoden gibt Yang. $C$. Determining Advertising Budgets, in: Yang. C. (Ed.), Handbook of Marketing Research, New York u. a. 1974, S. 165-187. In der Messepraxis überwiegen Budgetierungsansätze, die sich an Richtwerten orientieren, wie z. B. am Umsatz oder den messespezifischen Ausgaben der Konkurrenz. Dabei werden Messen nach ihrer Bedeutung für das Unternehmen geordnet und erhalten mittels eines sukzessiven Verfahrens ihren Messeetat bis das für Messebeteiligungen zur Verfügung stehende Budget aufgezehrt ist. Vgl. Wenge. H.-U. Müller, A., Das Management von MesseBeteiligungen, a.a.O., S. 741.

75 Eine mögliche Aufspaltung der Gesamtkosten einer Messebeteiligung nach sechs allgemeinen Kostenarten schlägt der AUMA vor. Er teilt die messespezifischen Kosten auf in Grundkosten (Standmiete, Gebühren, Energie- und Wasserversorgung), Kosten für Standausstattung (Standbau, Mobiliar, technische Einrichtungen, Standgestaltung), Kosten für Standservice und Kommunikation (Bewirtung, Werbegeschenke, Informationsmaterial, Telefon, Fax etc.), Kosten für Transport und Entsorgung (Logistik, Abfallbeseitigung), Personalkosten (direkte und indirekte Personalkosten, Reise-, Übernachtungs- und Bewirtungskosten der Standmitarbeiter) und andere Kosten. Vgl. Ausstellungs- und Messe-Ausschuss der Deutschen Wirtschaft e.V. (AUMA) (Hrsg.), Die Messekosten der deutschen Aussteller, a.a.O., S. 7 f.

76 Vgl. Köhler, R., Kommunikations-Management im Unternehmen, in: Berndt. R. Hermanns, A. (Hrsg.), Handbuch Marketing-Kommunikation, Wiesbaden 1993, S. 101 f.

77 Vgl. zur Notwendigkeit der messespezifischen Positionierung Krone. K. Huber. B., Messen als Instrument der Unternehmenspolitik, in: Strothmann. K. -H. Busche. M. (Hrsg.), Handbuch Messemarketing, Wiesbaden 1992, S. 150 f.; Huckemann. M. ter Weiler. D. S., Messen meßbar machen. Die 5 trojanischen Pferde des Messe-Marketing, a.a.O., S. 16.

78 Vgl. Mühlbacher. H. Dreher, A. Gabriel-Ritter, A., Strategische Positionierung - Grundpfeiler des Marketings in komplexen und dynamischen Umwelten, in: DBW, 56. Jg, Heft 2, 1996, S. 203; Ries, A. Trout. J., Positioning: The Battle in Your Mind, New York 1986, S. $23 \mathrm{ff}$. 
tion ergeben. ${ }^{79}$ Gemein ist den verschiedenen Ansichten allerdings, daß mit der Positionierung aus Unternehmenssicht eine Dominanz beim Nachfrager und eine Differenzierung gegenüber Wettbewerbern erreicht werden soll. ${ }^{80} \mathrm{I}$. d. S. geht es letztlich darum, für das eigene Angebot bzw. Unternehmen eine „unique selling proposition" zu besetzen. ${ }^{81}$ Die Positionierung bezieht sich dabei stets auf die subjektive Wahrnehmung des Nachfragers und weniger auf die objektiven Gegebenheiten. ${ }^{82}$

Aus der entscheidungsorientierten Perspektive soll daher unter der Positionierung die aktive Planung, Gestaltung und Kontrolle der Außenwahrnehmung von Unternehmen bzw. Unternehmenseinheiten (z. B. Produkten) verstanden werden. ${ }^{83}$ Als Ziel der Positionierung kann damit der Aufbau von langfristigen Wettbewerbsvorteilen bzW. „komparativen Konkurrenzvorteilen ${ }^{44}$ bezeichnet werden ${ }^{85}$, wobei zur Erreichung der Positionierungsziele immer wieder die Notwendigkeit einer kommunikationspolitischen Profilierung ${ }^{86}$ genannt wird. ${ }^{87}$

Zweck der messespezifischen Positionierung ist die bewußte Profilierung des Ausstellers bei den Messezielgruppen einerseits und die Differenzierung gegenüber den Messe-Wettbewerbern andererseits. Insbesondere die konkurrenzorientierte Differenzierung des eigenen Messeauftritts stellt angesichts zunehmender Pro-

79 Vgl. Aaker. D. A. Shansby, J. G.; Positioning Your Product, in: Business Horizons, Vol. 25, NO. 3, 1982, S. 56-62.

80 Vgl. Rossiter. J. Percy, L., Advertising Theory and Practice, New York 1994, S. 14 f.

81 Vgl. Becker. J., Konzeptionelle Grundfragen der Positionierung, in: Tomczak. T. Rudolph. Th. Roosdore. A. (Hrsg.), Positionierung: Kernentscheidung des Marketing, St. Gallen 1996, S. 12.

82 Vgl. Esch. F.-R. Levermann, S., Positionierung als Grundlage des strategischen Kundenmanagements, in: Thexis, Heft 3, 1995, S. 8 f.

83 Vgl. Trommsdortf. V., Positionierung, in: Tietz. B. (Hrsg.), Handbuch des Marketing, 2. Aufl., Stuttgart 1995, Sp. 2056.

84 Vgl. hierzu Backhaus. K., Investitionsgütermarketing, 4. Aufl., München 1995, S. 14 ff.

85 Vgl. Becker. J., Konzeptionelle Grundfragen der Positionierung, a.a.O., S. 13.

86 Im Unterschied zur Positionierung ist mit der Profilierung hauptsächlich die Schaffung von Präferenzen bei potentiellen Abnehmern durch den Einsatz des absatzpolitischen Instrumentariums gemeint. Vgl. hinsichtlich der Herausarbeitung der zentralen Abgrenzungen Ceyp. M., Ökologieorientierte Profilierung im vertikalen Marketing - dargestellt am Beispiel der Elek-

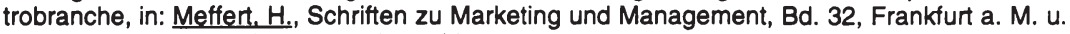
a. 1996, S. 4 und die dort angegebene Literatur.

87 Vgl. Ries. A. Trout, J, Positioning: The Battle in Your Mind, a.a.O., S. $19 \mathrm{ff}$.; Belz. Chr., Unternehmenspositionen mit integrierter Kommunikation besetzen, in: Tomczak. T. Rudolph. Th. Roosdore. A. (Hrsg.), Positionierung: Kernentscheidung des Marketing, St. Gallen 1996, S. 122 ff.; Feige. St., Handelsorientierte Markenführung - Strategien zur Profilierung von Konsumgüterherstellern beim Handel, Frankfurt a. M. U. a. 1996, S. $1 \mathrm{ff}$. 
dukthomogenität und -vielfalt ein Problem dar. Daher rücken vermehrt Aspekte der kommunikativen Alleinstellung des Ausstellers anläßlich einer Messeveranstaltung in den Vordergrund. Die Marktpositionierung des Unternehmens und seiner Produkte sollte den Ausgangspunkt der messespezifischen Positionierung bilden, um auf diese Weise ein konsistentes, mit anderen Kommunikationsmaßnahmen abgestimmtes Erscheinungsbild des Unternehmens i. S. einer Corporate Identity ${ }^{88}$ beim Messebesucher zu hinterlassen. ${ }^{89}$

Die bisherigen Ausführungen haben gezeigt, daß Messebeteiligungsziele, Messeselektion und messespezifische Positionierung die Basis für die strategische Ausrichtung einer Messebeteiligung bilden. $\mathrm{Da}$ in dieser Phase des Managementprozesses die grundsätzlichen Entscheidungen bezüglich der Messebeteiligung getroffen werden, soll das hier zum Ausdruck kommende Entscheidungsverhalten des Unternehmens als seine messespezifische Grundhaltung ${ }^{90}$ interpretiert werden.

Die auf der jeweils bestehenden messespezifischen Grundhaltung basierenden Maßnahmenplanung setzt sich im wesentlichen aus den vier Instrumenten des Messebeteiligungs-Mix „Standkonzeption“, „Exponate“ sowie „Standpersonal“ und "Kommunikationsmaßnahmen" zusammen. ${ }^{91}$

Zur gestalterischen Konzeption des Messestandes zählen Entscheidungen zur Plazierung des Standes, d. h. der Bestimmung seiner räumlichen Lage innerhalb des Messegeländes, der Standform und -gestaltung. ${ }^{92}$ Aufgrund vorhandener veranstalterspezifischer Messekonzepte (z. B. Belegung einzelner Hallen mit Vertretern einer bestimmten Branche) hat der einzelne Aussteller häufig nur wenig Einfluß auf die Wahl der Plazierung des Standes. Trotzdem wird er den Versuch unternehmen, die Standplazierung unter Berücksichtigung des Besucherverhal-

${ }_{88}$ Zu den Grundlagen der Corporate Identity zählt die Schaffung eines zeitlich stabilen und einheitlichen Erscheinungsbildes des Unternehmens und seiner Produkte. Vgl. Birkigt. K. Stadler. M., Corporate Identity - Grundlagen, in: Birkigt. K. Stadler, M. Funck. H.J. (Hrsg.), Corporate Identity. Grundlagen, Funktionen, Fallbeispiele, Landsberg/Lech, 5. Aufl., 1992, S. 11-59.

89 Vgl. Meffert. H., Strategische Unternehmensführung und Marketing: Beiträge zur marktorientierten Unternehmenspolitik, Wiesbaden 1988, S. 119.

90 Vgl. zu einem ähnlichen Vorgehen Kirchgeorg. M., Ökologieorientiertes Unternehmensverhalten: Typologien und Erklärungsansätze auf empirischer Grundlage, Wiesbaden 1990, S. $12 \mathrm{ff}$.

91 Vgl. Wenge. H.-U. Müller. A., Das Management von Messe-Beteiligungen, a.a.O., S. 741 f.

92 Vgl. Meffert. $H_{1}$, Messen und Ausstellungen als Marketinginstrument, a a, $0 .$, S, 21 
tens der Zielgruppen sowie der von der Messegesellschaft geplanten Plazierung der Konkurrenzstände zu optimieren. So ist eine Standplazierung zu erwirken, die thematisch dem eigenen Ausstellungsschwerpunkt entspricht und in dessen unmittelbarer Umgebung auch die wesentlichen Wettbewerber ausstellen. Darüber hinaus spielen bei der Plazierungsplanung auch infrastrukturelle Gegebenheiten auf dem Messegelände (z. B. Beleuchtung, Belüftung, Tragfähigkeit der Böden etc.) eine wesentliche Rolle. Spezifische Planungsprobleme ergeben sich des weiteren beim sog. „Standsplitting“, bei dem sich ein Aussteller entscheidet, auf mehreren Ständen an verschiedenen Orten des Messegeländes auszustellen. ${ }^{93}$ Die Wahl der geeigneten Standform sowie Entscheidungen hinsichtlich der Standgestaltung hängen neben den finanziellen Ressourcen des Ausstellers und den vorhandenen Messefaszilitäten wesentlich von den messebezogenen Zielen und der Positionierungsentscheidung des Ausstellers auf der Messeveranstaltung ab. $^{94}$

Für die Konzeption des Messestandes ist auch die Auswahl der Exponate ein wesentlicher Planungsschritt. Grundsätzlich ist die Zusammenstellung der Exponate abhängig von der Messethematik und somit von den Erwartungen und Interessen der Messe- oder präziser der Standbesucherzielgruppen Das ausgestellte Produktprogramm kann dabei zwischen der Präsentation der gesamten Produktpalette bis hin zur Ausstellung eines zielgruppenspezifisch tiefen Angebotsprogramms variieren. ${ }^{95}$ Eine relativ breit gefächerte Exponatepräsentation bietet sich vor allem bei einem eher heterogenen Messepublikum an, während fachlich hochqualifizierte Zielgruppen eher über ein bedarfsgerecht tiefes Ausstellungsprogramm angesprochen werden. Die Auswahl der Exponate wird darüber hinaus maßgeblich durch wettbewerbsperspektivische Überlegungen mitgeprägt. Fokussierte Ausstellungsprogramme stehen dabei meist für spezifische Kompetenz, während ein breit gefächertes Exponateangebot mit dem Leistungspotential eines Universalanbieters verbunden wird. $^{96}$

93 Vgl. Schwickert. E. Kindt. M. Stallmann, H., Messeauswahl und -gestaltung, a.a.O., S. $222 \mathrm{ff}$.

94 Vgl. Staeger. H., Auf dem Weg in das Jahr 2000 - Konsequenzen für Aussteller, Veranstalter und insbesondere Messebauer, in: Marketing Journal, Heft 1, 1989, S. 52-56.

95 Diese Feststellung gilt natürlich nur eingeschränkt für Anbieter von Anlagen- oder Systemtechnologien (z. B. Hersteller von Kraftwerken). Diese Unternehmen werden schwerpunktmäßig nur Modelle oder Teilmodule als Exponate ausstellen können. 
Der persönliche Kontakt am Messestand ist eines der hervorstechendsten Wesensmerkmale von Messen. Der Erfolg einer Messebeteiligung hängt daher nicht zuletzt vom Standpersonal als den kommunikativen Repräsentanten des Unternehmens ab. ${ }^{97}$ Aus diesem Grund ist personellen Aspekten ein besonderes Augenmerk zu schenken. Im Rahmen der Auswahl und des Einsatzes des Standpersonals ist vor allem auf fachliche und persönliche Qualifikation zu achten, aber auch der zeitliche und zahlenmäßige Einsatz festzulegen. ${ }^{98}$

Grundsätzlich sollte das Standpersonal vor dem Hintergrund des wachsenden Stellenwerts des Informations- und Interaktionsaspektes von Messen aus Mitgliedern der Geschäftsführung, des Verkaufs, Fachberatern sowie Fachleuten im Organisations-, Betreuungs- sowie Servicebereich und damit aus allen Hierarchieebenen des Unternehmens zusammengesetzt sein. ${ }^{99}$ Der Grund hierfür ist in der Tatsache zu sehen, daß jedem Gesprächspartner ein "gleichwertiges" Mitglied der Standbesatzung gegenübergestellt werden kann. Dabei wird bei der Auswahl des Standpersonals auf das Konzept des Buying-Center-Modells zurückgegriffen. ${ }^{100} \mathrm{Da}$ Besucheranalysen in der Bundesrepublik und auch in den USA ergeben haben, daß bis zu $50 \%$ der Besucher von Messen aus dem Middlemanagement und sogar bis zu $30 \%$ aus dem Topmanagement kommen ${ }^{101}$, geht man aufgrund der "Ähnlichkeitshypothese" von der Notwendigkeit aus, einzelnen Messestandbesuchern adäquate, hierarchisch auf der gleichen Ebene angesiedelte Gesprächspartner gegenüberzustellen. Dies hat zur Entwicklung des sog. "Selling-Center" als Counterpart des Buying Center geführt. ${ }^{102}$

Neben der fachlichen Qualifikation und dem Kompetenzbereich sind auch persönlichkeitsorientierte Merkmale des Standpersonals von Bedeutung, wie z. B. Kon-

97 Vgl. Mortsiefer, J., Die Intuition alleine reicht nicht - Messebeteiligungen auf der Basis eines Nutzungs-Konzeptes, a.a.O., S. 59.

98 Vgl. Meffert, H., Messen und Ausstellungen als Marketinginstrument, a.a.O., S. 88.

99 Vgl. Vogt. R. E., Worauf vor, während und nach der Messe zu achten ist, in: Verkauf + Marketing, Heft 2, 1987, S. 8-10; Goschmann, K., Die erfolgreiche Beteiligung an Messen und Ausstellungen von A-Z, a.a.O., S. 214.

100 Vgl. zur Bedeutung von Buying Center für Kaufentscheidungen bei Investitionsgütern Webster. F. E. Wind Y., Organizational Buying Behavior, Englewood Cliffs 1972; Backhaus. K., Investitionsgütermarketing, 4. Aufl., a.a.O., S. 60-73; Spiegel-Verlag (Hrsg.), Der Entscheidungsprozeß bei Investitionsgütern, Hamburg 1982, S. $15 \mathrm{ff}$.

$101 \mathrm{Vgl}$. Gesellschaft zur freiwilligen Kontrolle von Messe- und Ausstellungszahlen (FKM) (Hrsg.), Bericht 1995, Bergisch Gladbach 1996, S. 28 ff. sowie Trade Show Bureau (Ed.), The Power of Trade Shows, Denver 1992. 
taktfreudigkeit, Fremdsprachenkenntnisse, Gewandtheit in Ausdruck und Sprache sowie Kommunikationsflexibilität. ${ }^{103}$ Daher ist schon vor der Messebeteiligung Wert auf eine gezielte Vorbereitung des Standpersonals zu legen. Zur Realisierung eines möglichst großen Erfolgs der Messebeteiligung ist es nicht nur notwendig, die Standmitarbeiter über die Zielsetzungen, die mit der Messebeteiligung verbunden sind, zu informieren. Darüber hinaus sollten veranstaltungsbezogene Schulungsmaßnahmen durchgeführt werden, um zum einen auf die physische und psychische Belastung vorzubereiten und zum anderen Argumentationsinhalte und -schwerpunkte zu vermitteln sowie das Kommunikationsverhalten am Messestand zu trainieren. ${ }^{104}$

Der direkte Dialog des Standpersonals mit den Standbesuchern wird schließlich durch den Einsatz weiterer Kommunikationsmaßnahmen des Ausstellers unterstützt bzw. ergänzt. Unter den kommunikativen Maßnahmen eines Ausstellers sollen dabei alle Aktivitäten verstanden werden, die sich direkt auf eine konkrete Messebeteiligung beziehen. Demnach sind nicht nur die Standwerbung oder die persönliche Kommunikation des Standpersonals während einer Messeveranstaltung den kommunikativen Maßnahmen zuzuordnen. Vielmehr sind auch solche Aktivitäten der messebezogenen Kommunikation zu subsumieren, die auf dem Messegelände oder außerhalb des Messegeländes bzw. vor oder nach der Messe flankierend eingesetzt werden. So sind z. B. der Einsatz der Öffentlichkeitsarbeit vor, während und nach der Messe sowie Direktwerbemaßnahmen zum Hinweis auf die Messebeteiligung im Rahmen der Vor-Messe-Werbung zu den kommunikativen Maßnahmen im Rahmen einer Messebeteiligung zu zählen.

Die Vielzahl der Planungsebenen und -gegenstände sowie deren weitgehende Interdependenz hat deutlich werden lassen, daß Aspekte der Realisation bzw. Durchsetzung der geplanten Maßnahmen an Bedeutung gewinnen. Hierbei sind

103 Vgl. Roth. G. D., Messen und Ausstellungen verkaufswirksam planen und durchführen, a.a.O., S. $105 \mathrm{ff}$.

104 Vgl. Meffert. H., Messen und Ausstellungen als Marketinginstrument, a,a.O., S, 23

Ralf Ueding and Universitat Munster - 978-3-631-75073-5 
insbesondere die Bereiche der Organisation ${ }^{105}$, der Führung sowie der Kontrolle der Messebeteiligung näher zu beleuchten.

Im Rahmen der Organisation von Messebeteiligungen ist die Umsetzung der geplanten Maßnahmen sicherzustellen. Damit sind einerseits Fragen der Standorganisation angesprochen, andererseits aber auch Aspekte der Koordination ablauftechnischer Fragestellungen einer Messebeteiligung zu berücksichtigen. Im Rahmen der Standorganisation sind neben der Bestimmung eines Standleiters ebenfalls die Aufgaben des Standpersonals sowie die individuellen Präsenzzeiten der Standmitarbeiter festzulegen. Hinsichtlich der Koordination ablauftechnischer Prozesse sind eine detaillierte Terminplanung für den Standauf- und -abbau sowie eine entsprechende Prozeßkontrolle vorzunehmen. Sofern einzelne Aktivitäten, wie z. B. Standbau und Catering, an externe Dienstleister vergeben werden, bedarf es der Auswahl sowie der gegenseitigen Information und Koordination der Beteiligten. $^{106}$

Führung bzw. Führen als Prozeß der zielorientierten Verhaltensbeeinflussung ${ }^{107}$ gewinnen bei der Realisation einer Messebeteiligung insbesondere unter dem Aspekt der Steuerung des Verhaltens der Standmitarbeiter an Bedeutung. Führen heißt dabei einerseits, Einfluß auf die Mitarbeiter auszuüben, die zur Erreichung der gesteckten Ziele beitragen (Leistungsaspekt) und andererseits, Bedingungen zu schaffen, die eine Zielerreichung ermöglichen (Zufriedenheits- bzw. Motivationsaspekt). ${ }^{108}$ Leistungs- und Motivationsaspekte sind dabei eng miteinander verknüpft. Der Ereignischarakter von Messen sowie die bereits enwähnten Vorbereitungs- und Schulungsmaßnahmen können in diesem Zusammenhang als Bestand-

105 Der Begriff der Organisation als Managementfunktion wird in der Managementtheorie auf zwei unterschiedliche Ebenen bezogen. Einerseits ist Organisation Ausdruck genereller Regeln zur Strukturierung von Daueraufgaben (Vorgänge mit Wiederholungscharakter) und findet ihren Ausdruck in der "Aufbauorganisation", andererseits wird mit Organisation die gestaltende Tätigkeit des Organisierens selbst verbunden, die unter der Bezeichnung "Ablauforganisation" zusammengefaßt wird. Vgl. Steinmann. $H$. Schreyögg. G., Management: Grundlagen der Unternehmensführung; Konzepte, Funktionen, Praxisfälle, a.a.O., S. 358 ff.; Wild. J., Grundlagen der Unternehmensplanung 4. Aufl., Hamburg 1982, S. 34 ff. Hier soll der Fokus der Betrachtung auf den zuletzt genannten Aspekt gelegt werden.

Vgl. Mortsiefer. J., Die Vorbereitung entscheidet über den Erfolg - Planung und Vorbereitung einer Messebeteiligung, in: Küffner. G. Mortsiefer. J. (Hrsg.), Messeplatz Europa. Messen als Bestandteil des betrieblichen Marketings, Frankfurt a.M. 1990, S. 59 ff.

Vgl. Steinle. C., Führung, Grundlagen, Prozesse und Modelle der Führung in der Unternehmung, Stuttgart 1978, S. 13. 
teile der Motivation der Standmitarbeiter gesehen werden. Daneben existieren weitere monetäre bzw. nicht-monetäre Anreize (z. B. Gehaltszulagen, Sonderzahlungen, Auszeichnungen etc.), die den Mitarbeitern in Verbindung mit der Tätigkeit auf dem Messestand gewährt werden können.

Als letzter Schritt des Management von Messebeteiligungen wird im Rahmen der Kontrolle die Erfolgsmessung, die sich sowohl auf eine Betrachtung des Gesamtengagements als auch auf die Analyse einzelner messespezifischer Aktivitäten beziehen kann, durchgeführt. ${ }^{109}$ Das zentrale Problem der Erfolgskontrolle von Messebeteiligungen liegt in der Erfassung der relevanten Erfolgsdimensionen, die sich aus den Messebeteiligungszielen ableiten lassen. ${ }^{110}$ Die Intensität der Erfolgskontrolle ist daher nicht selten abhängig vom Budget, das hierfür im Rahmen des Messebeteiligungsetats zur Verfügung gestellt wird, von den unter Beachtung der verfolgten Ziele in Betracht zu ziehenden Methoden der Erfolgskontrolle sowie von organisatorischen Aspekten. Im Hinblick auf diese organisatorischen Aspekte ist insbesondere zwischen der internen und der extern an Dienstleister vergebenen Durchführung der Erfolgskontrolle zu unterscheiden. Für die Vergabe der Erfolgskontrolle an externe Dienstleister sprechen häufig das mangelnde Knowhow sowie fehlende Personalkapazitäten auf seiten des Ausstellers. ${ }^{111}$ Für die Durchführung von Erfolgskontrollen ist allerdings das erwartete Kosten-NutzenVerhältnis und ihr Beitrag für den Erfolg zukünftiger Messebeteiligungen, der in Abb. 4 durch einen Feedback-Prozeß gekennzeichnet ist, von maßgeblicher Bedeutung.

109 Vgl. Wenge. H.-U.. Müller, A., Das Management von Messe-Beteiligungen, a.a.O., S. 744.

110 Vgl. Winnen, R. Beuster. A., Kontrolle des Messeerfolgs, in: Strothmann, K.-H., Busche. M. (Hrsg.), Handbuch Messemarketing, Wiesbaden 1992, S. 378.

111 Vgl. Meffert. H. Ueding. R., Ziele und Nutzen von Messebeteiligungen, in: Ausstellungs- und Messe-Ausschuss der Deutschen Wirtschaft e. V. (AUMA) (Hrsg.), AUMA Edition Nr. 4, 1996, S. $69 \mathrm{f}$. 


\section{Zielsetzung und Gang der Untersuchung}

Die Diskussion der Arbeiten zum Management von Messebeteiligungen zu Beginn des Kapitels 2 hat als erstes Defizit das Fehlen einer umfassenden Untersuchung des gesamten Managementprozesses offengelegt. Die generelle Zielsetzung der nachfolgenden Untersuchung besteht daher in der Entwicklung eines Ansatzes zur Identifikation und Beschreibung bestimmter Grundhaltungen und der darauf aufbauenden empirischen Analyse der Ausgestaltung des Management von Messebeteiligungen am Beispiel von Ausstellern auf Messeveranstaltungen in Deutschland.

In diesem Zusammenhang existiert bislang keine leistungsfähige Typologie ${ }^{112}$ messespezifischer Grundhaltungen von Ausstellern. Diese Grundhaltungen sind aber, wie in den vorangegangenen Ausführungen bereits dargestellt, zentraler Ausgangspunkt des Management von Messebeteiligungen. Vor diesem Hintergrund besteht ein erstes, deskriptiv-taxonomisches Teilziel der vorliegenden Arbeit in der Ableitung einer Grundhaltungstypologie. Dieser Untersuchungsschritt erfolgt durch eine vergleichende Analyse der Ausgestaltungsformen von Messebeteiligungen bei empirisch ermittelten Grundhaltungstypen, die sich durch ähnliche Schwerpunktsetzungen bei den Basisdimensionen "messespezifische Zielkonzeption", "Messeselektion" und "messespezifische Positionierung" kennzeichnen lassen.

Ausgehend von dieser Typologie besteht ein zweites, explikatives Teilziel der Arbeit in der Klärung der Fragen, inwieweit die messespezifischen Grundhaltungen durch ausstellerinterne und -externe Kontingenzfaktoren beeinflußt werden und welche Einflüsse diese auf die Ausgestaltung von Messebeteiligungen auf deren Erfolg haben. Des weiteren ist auf der Basis der empirisch gewonnenen Erkenntnisse eine praktisch-normative Zielsetzung auf die Frage gerichtet, welche Gestaltungsempfehlungen zur Optimierung der Ausgestaltung von Messebeteiligungen für Aussteller formuliert werden können.

112 Die Typenbildung stellt eine wichtige Methode zur Komplexitätsreduktion dar. Es wird hierbei davon ausgegangen, daß die Vielzahl von real zu beobachtenden Phänomenen gewisse Gleichförmigkeiten bzw. Muster aufweist, so daß sich Eigenschaftsprofile von Einzelfällen zu Typen mit ähnlichen Eigenschaftsausprägungen zusammenfassen lassen. Vgl. Scholz. Chr., Strategisches Management, Berlin, New York 1987, S. $77 \mathrm{ff}$. 
Aus den übergeordneten Zielsetzungen der Untersuchung leiten sich die vier folgenden Forschungsschwerpunkte ab:

1. Ableitung und Systematisierung relevanter Dimensionen messespezifischer Grundhaltungen und Identifikation realer Grundhaltungstypen;

2. Ermittlung und Darstellung von Ausgestaltungsformen von Messebeteiligungen der identifizierten Grundhaltungstypen;

3. Systematisierung und Operationalisierung relevanter Einflußfaktoren des Management von Messebeteiligungen auf der Grundlage eines Bezugsrahmens;

4. empirische Überprüfung des Bezugsrahmens zur Ableitung von Aussagen über relevante Ausgestaltungsformen des Management von Messebeteiligungen und die Erfolgswirkung der untersuchten Einflußfaktoren;

Das theoretisch-methodische Vorgehen der Untersuchung ist den situativen bzw. Kontingenzansätzen zuzuordnen. ${ }^{113}$ Dabei darf jedoch nicht übersehen werden, daß der situative Ansatz die notwendigen Bedingungen für eine eigenständige Theorie nicht zu erfüllen vermag. ${ }^{114}$ Die zentrale Kritik situativer Erklärungsansätze richtet sich dabei auf den Determinismus zwischen Situation und Unternehmensverhalten bzw. Organisationsstruktur, der dem Handlungsspielraum der Entscheidungsträger nicht hinreichend Rechnung trägt und das Unternehmensverhalten als primär reaktiv kennzeichnet. ${ }^{115}$ Aus dieser Kritik heraus ist die Entwicklung unternehmenspolitisch-situativer Erklärungsansätze $e^{116}$, auf die auch in der vorliegenden Untersuchung Bezug genommen wird, entstanden. Hierbei wird das Unternehmensverhalten in Abhängigkeit der Ziel- und Strategieentscheidungen des Management und der situativen Einflüsse untersucht.

113 Der situative bzw. Kontingenzansatz der Marketingtheorie geht auf Kast und Rosenzweig zurück. Er basiert auf der Hypothese, daß die Effektivität und Effizienz bestimmter Ausprägungen des Unternehmensverhaltens von einem Fit mit externen und internen Situationsfaktoren abhängig ist. Der Begriff Situation oder Kontext wird dabei als "offenes Konstrukt" interpretiert, in das je nach der betrachteten Problemstellung unterschiedliche als relevant anzusehende Variablen Eingang finden. Vgl. Kast. F.. Rosenzweig. J., Organization and Management: A Contingency Approach, Tokio 1970.

Vgl. Lehnert. St., Die Bedeutung von Kontingenzansätzen für das strategische Management, Frankfurt a.M., Bern, New York 1983, S. 115.

115 Vgl. zur kritischen Einschätzung situativer Erklärungsansätze Lehnert, St., Die Bedeutung von Kontingenzansätzen für das strategische Management, a.a.O., S. $169 \mathrm{ff}$. sowie Schreyögg. G., Umwelt, Technologie und Organisationsstruktur, Bern, Stuttgart 1978, S. 235 ff. 
Als Begründung für die Auswahl eines unternehmenspolitisch-situativen Ansatzes zur Erklärung messespezifischer Grundhaltungen läßt sich anführen, daß die bereits in den 70er Jahren auf Fragestellungen des Marketing übertragene entscheidungsorientierte Sichtweise ${ }^{117}$ die Situationsanalyse an den Ausgangspunkt von Ziel-, Strategie- und Maßnahmenentscheidungen stellt. Unter der Annahme, daß sich reale Entscheidungsprozesse nach dieser Struktur vollziehen, kann von einer situativen Bedingtheit der Gestaltung von Messebeteiligungen ausgegangen werden.

Die Vorgehensweise dieser Arbeit ist als konzeptionell-literaturgestützt einzustufen, wobei die konzeptionellen Überlegungen mit einer empirischen Untersuchung unterlegt werden. Die ausgeprägte Komplexität des Untersuchungsgegenstandes Management von Messebeteiligungen läßt es notwendig erscheinen, die Untersuchung aus Gründen eines noch handhabbaren Abstraktionsgrades auf ausgewählte Fragestellungen auszurichten. Diese werden allerdings so gewählt, da $\beta$ der Managementproze $\beta$ von Messebeteiligungen in seinen wesentlichen Phasen sichtbar wird.

Abb. 5 faßt als kontingenztheoretischer Bezugsrahmen ${ }^{118}$ der vorliegenden Arbeit die relevanten Einflußfaktoren und Gestaltungsdimensionen des Management

117 Vgl. Meffert. H., Die Leistungsfähigkeit der entscheidungs- und systemorientierten Marketinglehre, in: von Kortzfleisch. G. (Hrsg.), Wissenschaftsprogramm und Ausbildungsziele der Betriebswirtschaftslehre, Berlin 1971, S. 167 ff.; Backhaus, K., Der entscheidungs- und verhaltensorientierte Ansatz in der Investitionsgüterwerbung, in: Rost. D. Strothmann, K.-H. (Hrsg.), Handbuch Werbung für Investitionsgüter, Wiesbaden 1983; Heinen. E., Einführung in die Betriebswirtschaftslehre, 9. Aufl., Wiesbaden 1985, S. $259 \mathrm{ff}$.

118 Bezugsrahmen stellen Aussagensysteme dar, "die von ihrer logischen Konsistenz und Operationalisierung her nicht den strengen Anforderungen an ein Hypothesensystem genügen". Vgl. Kubicek, $H$., Heuristische Bezugsrahmen und heuristisch angelegte Forschungsdesigns als Elemente einer Konstruktionsstrategie empirischer Forschung, in: Köhler, R. (Hrsg.), Empirische und handlungstheoretische Forschungskonzeptionen in der Betriebswirtschaftslehre, Stuttgart 1977, S. 17 f. Bezugsrahmen sind daher als provisorische Erklärungsmodelle zu interpretieren, die neben der Beeinflussung zukünftiger Forschungsvorhaben Orientierungshilfen für die Lösung praktischer Problemstellungen geben sollen. Vgl. Kirsch. W., Entscheidungsprozesse, Bd. 3: Entscheidungen in Organisationen, Wiesbaden 1971, S.241 $\mathrm{Hf}_{1}$ 


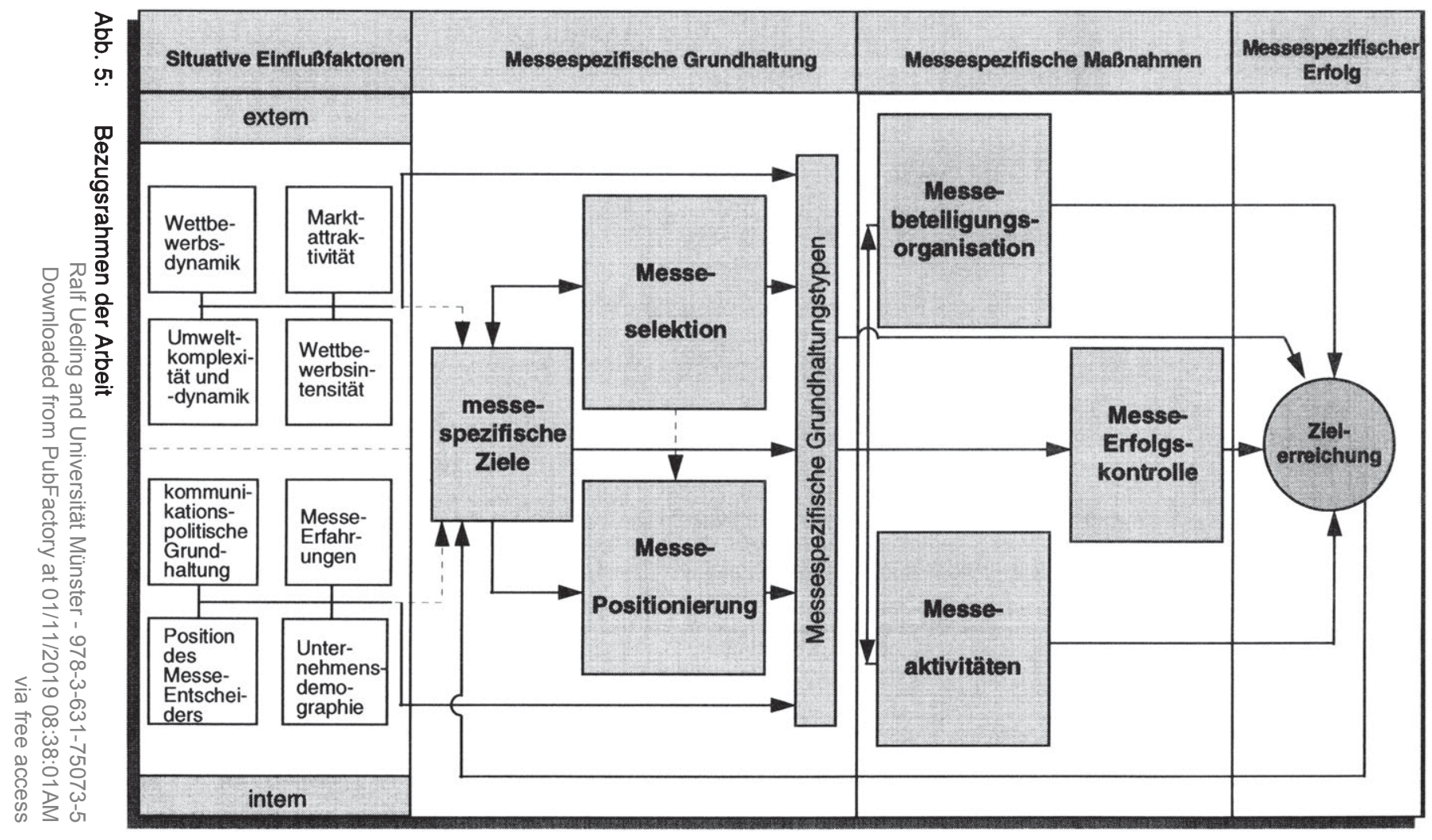


von Messebeteiligungen sowie ihre möglichen Wirkungsbeziehungen zusammen ${ }^{119}$, die in den einzelnen Analyseschritten eingehend diskutiert werden. Auf der Basis konzeptioneller Überlegungen zu den Ausgestaltungsformen und Einflußfaktoren des Management von Messebeteiligungen werden Untersuchungshypothesen abgeleitet und am Beispiel ausstellender Unternehmen bei Messeveranstaltungen in Deutschland empirisch überprüft.

Mit den beschriebenen Ziel- und Schwerpunktsetzungen der Arbeit ist bereits der Gang der Untersuchung vorgezeichnet. Im Kapitel B werden zunächst die konzeptionellen Grundlagen zur Analyse der Grundhaltungen im Management von Messebeteiligungen erörtert. Ausgehend von einer Systematisierung der Dimensionen messespezifischer Grundhaltungen, die das Spektrum verschiedener Verhaltensweisen ausstellender Unternehmen zur Begegnung der Herausforderungen von Messebeteiligungen abbilden, werden im Anschluß daran die aktuellen messespezifischen Grundhaltungen bei empirisch ermittelten messespezifischen Grundhaltungstypen dargestellt.

Im Mittelpunkt der Ausführungen im Kapitel C steht zunächst die Analyse der Möglichkeiten zur Ausgestaltung von Messebeteiligungen. Hierbei werden zunächst verschiedene Optionen der Messebeteiligungsorganisation, messebezogener Aktivitäten sowie Maßnahmen zur Erfolgskontrolle gekennzeichnet. Im Anschluß daran werden die beschriebenen Ausgestaltungsoptionen bezüglich ihrer Relevanz für die empirisch abgeleiteten Grundhaltungstypen gewürdigt und im Hinblick auf ihre Erfolgswirkungen untersucht.

Kapitel $D$ ist der Analyse situativer Einflußfaktoren auf die Ausgestaltung des Management von Messebeteiligungen gewidmet. Hierbei werden unternehmensexterne und -interne Einflußfaktoren einer getrennten Untersuchung unterzogen. Auf der Grundlage der ermittelten Ausgestaltungsformen von Messebetei-

119 Bei der Ausgestaltung von Bezugsrahmen sind die Anforderungen hinsichtlich Vollständigkeit und Operationalisierbarkeit zu erfüllen. Um dem Kriterium Vollständigkeit zu genügen, muß der Bezugsrahmen sämtliche relevanten Einflußfaktoren berücksichtigen bzw. adaptieren können. Die Adaptivität kennzeichnet in diesem Zusammenhang die Anpassungsfähigkeit an reale, sich verändernde Umweltsituationen. Vgl. hierzu Meffert. $H_{\text {. }}$ Steffenhagen. $H_{\text {., }}$ Marketingprognosemodelle - Quantitative Grundlagen des Marketing, Stuttgart 1977, S. $55 \mathrm{ff}$. Die Operationalisierbarkeit fordert darüber hinaus, daß die Formulierung des Bezugsrahmens so präzise erfolgt, daß er den Kriterien Einfachheit und Handhabbarkeit genügt. Vgl. zu den Anforderungen der Operationalisierbarkeit in der Betriebswirtschaftslehre $\mathrm{Ob} . \mathrm{K}$. D., Methodologie der Sozialwissenschaften, Reinbek 1970, S. $135 \mathrm{ff}$.; Andritzky, K., Die Operationalisierbarkeit von Theorien zum Konsumentenverhalten, Berlin 1977, S. 1 ff. 
ligungen sowie der ermittelten Wirkungsbeziehungen wird im Kapitel $E$ ein Ansatz zur systematischen Messebeteiligungskontrolle entwickelt. Des weiteren enthält dieses Kapitel abschließend Implikationen für die Ausgestaltung von Messebeteiligungen und Schlußfolgerungen für die Messewirtschaft sowie für weitere wissenschaftliche Untersuchungen.

\section{Design der empirischen Untersuchung}

Für die empirische Untersuchung wurde im Sommer 1995 in Zusammenarbeit mit dem Ausstellungs- und Messe-Ausschuss der Deutschen Wirtschaft e.V. (AUMA) ein Fragebogen zum Thema "Ziele und Nutzen von Messebeteiligungen" an deutsche Aussteller verschickt. ${ }^{120}$ Insgesamt konnten 2.320 Fragebogen an Entscheidungsträger für Messebeteiligungen in den Unternehmen versandt werden $^{121} .602$ zurückerhaltene Fragebögen wurden in die empirische Untersuchung einbezogen - dies entspricht einer Rücklaufquote von $25,9 \%$.

Die Zusammensetzung der Stichprobe ergibt sich aus Abb. 6, die die Strukturdarstellungen auf aggregierter Branchenebene sowie nach Umsatzgrößen und Beschäftigtenzahl umfaßt. Es zeigt sich nicht nur eine relativ ausgewogene branchenbezogene Verteilung der in die Stichprobe aufgenommenen Unternehmen, sondern darüber hinaus auch eine hinreichende Präsenz kleinerer Unternehmen.

Kernbestandteil der Befragung war ein 12-seitiger Fragebogen mit 23 Fragen und insgesamt 257 Variablen. Der Schwerpunkt der Befragung lag auf geschlossenen Fragestellungen, mit deren Hilfe die Einschätzungen der Messeverantwortlichen

120 Vgl. hierzu auch Meffert. H. Ueding. R., Ziele und Nutzen von Messebeteiligungen, Köln 1996, S. $18 \mathrm{ff}$.

121 Hierbei wurde aus den Ausstellerkatalogen von 30 Messeveranstaltungen unterschiedlicher Größe und Marktbedeutung eine Zufallsstichprobe gezogen. Bei den Messen handelte es sich sowohl um Investitionsgüter- als auch Konsumgütermessen und Verbraucherausstellungen, um damit das breite Spektrum der in Deutschland stattfindenden Messen zu berücksichtigen. Basis für die Ziehung der Stichprobe war eine 1993 durchgeführte Befragung des AUMA zu den Messekosten der Aussteller, bei der eine nach sechs verschiedenen Veranstaltungstypen und Ausstellergröße geschichtete Zufallsstichprobe von 4.140 Unternehmen aus 19.371 erfaßten Ausstellern gezogen wurde. Grundlage der vorliegenden Untersuchung war eine Zufallsauswahl von 2.400 Unternehmen aus den vorliegenden 4.140 Unternehmensadressen. Die in den 0 . g. Ausstellerkatalogen als Entscheidungsträger für Messebeteiligungen ausgewiesenen Unternehmensrepräsentanten wurden angeschrieben. Vgl. zur statistischen Basis der empirischen Untersuchung sowie zur Auswahl der Messeveranstaltungen Ausstellungsund Messe-Ausschuss der Deutschen Wirtschaft e. V. (AUMA) (Hrsg.), Die Messekosten der deutschen Aussteller, a.a.O. S. $8 \mathrm{ff}$.

Ralf Ueding and Universität Münster - 978-3-631-75073-5 


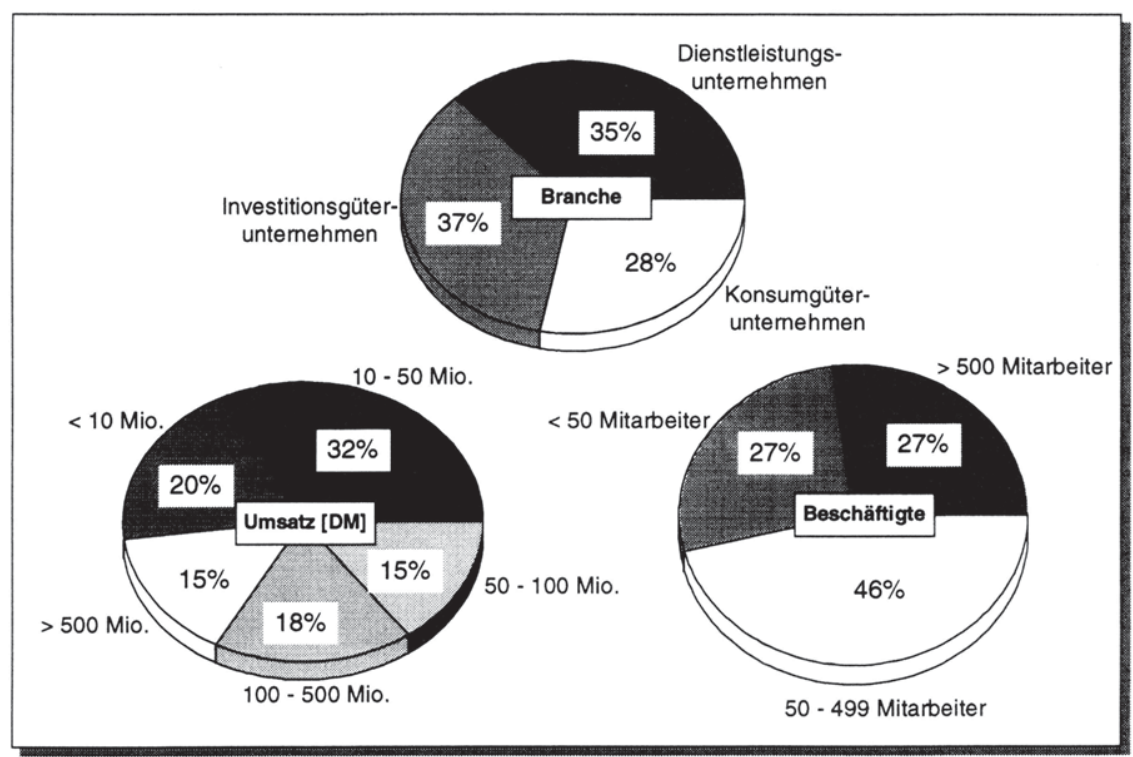

Abb. 6: Zusammensetzung der Stichprobe

erfaßt werden sollten. Die überwiegende Mehrzahl der Variablen wird auf 5-stufigen, bipolaren Ratingskalen erhoben, da diese eine problemlose Überführung in multivariate Verfahren der Datenanalyse ermöglichen ${ }^{122}$. Der detaillierte Fragebogen sowie die Fragenformulierungen wurden aufgrund von 16 in der Konzeptionsphase geführten Expertengesprächen ${ }^{123}$ und einem anschließenden Expertenworkshop beim AUMA konzipiert. Zur Überprüfung der Verständlichkeit wurden die endgültigen Fragebögen einem Pretest bei 45 zufällig ausgewählten Ausstellern unterzogen. Die Befragungsinhalte basieren auf den Situationsvariablen, Bestim-

122 Auf Ratingskalen erhobene Daten werden in der empirischen Forschung zumeist unter der Annahme einer vorliegenden Intervallskalierung verarbeitet. Solange aber die für intervallskalierte Daten notwendige Voraussetzung gleicher Skalenabstände unbestätigt ist, muß strenggenommen von lediglich ordinalskalierten Werten ausgegangen werden. In der vorliegenden Arbeit wird jedoch dem in der Literatur üblichen Vorgehen gefolgt und eine Intervallskalierung angenommen. Vgl. hierzu auch Meffert. H., Marketingforschung und Käuferverhalten, 2. Aufl., a.a.O., S. 185; Backhaus. K. et al., Multivariate Analysemethoden: Eine anwendungsorientierte Einführung, a.a.O., S. XV f.

Vgl. die Liste der Gesprächspartner im Anhang dieser Arbeit. 
mungsfaktoren und Erfolgsdimensionen des kontingenztheoretischen Bezugsrahmens. Im Schwerpunkt wurden folgende Fragenkomplexe erfaßt ${ }^{124}$ :

- Im ersten Teil wurden zunächst externe Situationsvariablen abgefragt. Hierzu zählen neben den Ausprägungen des Wettbewerbs auch das Kundenverhalten sowie die rechtlichen und politischen Rahmenbedingungen.

- Der zweite Fragenkomplex, der die internen Situationsvariablen beinhaltet, wird mit Fragen zur kommunikationspolitischen Grundhaltung eingeleitet und umfaßt neben Fragen zur aktuellen und zukünftigen Bedeutung von Messebeteiligungen im Kommunikations-Mix auch solche zum Integrationsgrad von Messebeteiligungen in übergeordnete Marketingkonzepte, zur Anzahl von Messebeteiligungen und Position von Messeentscheidern in den Unternehmen.

- Im dritten Teil werden Fragen zu Zielen von Messebeteiligungen gestellt. Hierbei werden Einschätzungen zur aktuellen und zukünftigen Wichtigkeit der Ziele, zur aktuellen Zielerreichung sowie zur Operationalisierung der Ziele abgefragt.

- Der vierte Teil beinhaltet Fragen zur Messeselektion und zur messespezifischen Positionierung der Aussteller.

- Ein fünfter Fragenkomplex bezieht sich auf die Aktivitäten der Aussteller vor, während und nach der Messe sowie die Nutzung des Dienstleistungsangebots der Messeveranstalter in der jeweiligen Phase.

- Der sechste Teil umfaßt Fragen zur Kontrolle des Erfolgs von Messebeteiligungen. Neben der Durchführungsintensität werden die eingesetzten Kontrollmethoden und Messebeteiligungsbudgetanteile für die Erfolgskontrolle sowie die organisatorische Ausgestaltung ermittelt.

- Schließlich werden abschließend die Demographika der in die Befragung einbezogenen Unternehmen festgehalten, die neben der Branchenzugehörigkeit auch Umsatzklassen und Beschäftigtenzahl enthalten.

124 Vgl. auch den Fragebogen im Anhang dieser Arbeit. 
Die statistische Datenauswertung erfolgt mit Hilfe des im lokalen Netzwerk der Wirtschaftswissenschaftlichen Fakultät der Universität Münster implementierten Softwarepaketes SPSS (Superior Performing Statistical Software) for Windows Version 6.01. Zur Analyse der fast ausschließlich metrisch skalierten Daten kommen verschiedene uni-, bi- und multivariate statistische Verfahren zur Anwendung. Zur Prüfung des Modells der messespezifischen Grundhaltung kommen auf der Ebene der Zielkonzeption das der Kausalanalyse zuzurechnende Verfahren der konfirmatorischen Faktorenanalyse sowie weiterhin die Clusteranalyse und die Diskriminanzanalyse zum Einsatz. ${ }^{125}$ Die Untersuchung von Mittelwertunterschieden verschiedener messespezifischer Grundhaltungstypen erfolgt auf der Basis des Scheffé-Tests. ${ }^{126}$

125 Vgl. ausführlich zu den einzelnen Prozeduren Schubö. W. et al., SPSS: Handbuch der Programmversionen 4.0 und SPSS-X 3.0, Stuttgart, New York 1991, S. 282 ff.; Brosius. G., SPSS/PC+ Advanced Statistics and Tables - Einführung und praktische Beispiele, Hamburg u. a. 1989, S. $97 \mathrm{ff}$.; Backhaus, K. et al., Multivariate Analysemethoden: Eine anwendungsorientierte Einführung, a.a.O., S. 90-165; allgemeine Verfahrensdarstellungen finden sich auch bei

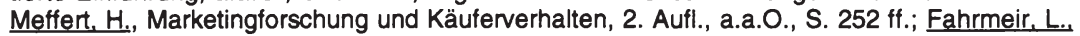
Häußler, W. Tutz, G., Diskriminanzanalyse, in: Fahrmeir. L.. Hamerle, A. (Hrsg.), Multivariate statistische Verfahren, Berlin, New York 1984, S. $301 \mathrm{ft}$.

Die Anwendung des als besonders konservativ geltenden Scheffé-Tests empfiehlt sich insbesondere bei ungleichen Gruppengrößen. Vgl. Bauer. F., Datenanalyse mit SPSS, Berlin u. a. 1984, S. 84 f. 


\section{B. Ableitung und Identifikation von messespezifischen Grundhal- tungstypen}

\section{Ableitung messespezifischer Grundhaltungen}

Während in der Literatur bereits erste Ansätze zur Erfassung spezifischer Verhaltensweisen bei Messebesuchern bestehen ${ }^{127}$, existiert bislang kein Ansatz zur Identifikation von Grundhaltungen hinsichtlich des beteiligungsbezogenen Messemanagement von Ausstellern. Im Rahmen der folgenden Ausführungen soll daher zunächst das Spektrum möglicher Grundhaltungformen erörtert werden, auf deren Basis Aussteller den Managementproze $\beta$ von Messebeteiligungen gestalten. Die sich anschließende empirische Überprüfung wird zeigen, inwieweit die zur Kennzeichnung der messespezifischen Grundhaltung herangezogenen Beschreibungsdimensionen in der Lage sind, konsistente Grundhaltungstypen zu identifizieren.

\subsection{Kennzeichnung der Dimensionen einer messespezifischen Grundhal- tung}

Die Erarbeitung eines Beschreibungsansatzes zur Ableitung messespezifischer Grundhaltungen verlangt zunächst die Präzisierung und Systematisierung der hierfür relevanten Managementbereiche sowie ihrer möglichen Ausprägungsformen und Beziehungen zueinander. Dabei zeichnet sich eine messespezifische Grundhaltung durch die drei Dimensionen "Messeziele“, „Messeselektion“ und „messespezifische Positionierung" aus. ${ }^{128}$

127 Strothmann hat in einer Untersuchung bei Bürokommunikations- bzw. EDV-Messen den Versuch einer Besuchertypologie unternommen und konnte vier unterschiedliche Verhaltenstypen der Messenutzung identifizieren: den intensiven Messenutzer, den punktuellen Messenutzer, den Messebummler und den praxisorientierten Messenutzer. Vgl. Spiegel Verlag (Hrsg.), Messen und Messebesucher in Deutschland, Hamburg 1992; Strothmann, K.-H., Im Verhalten unterscheiden sich Messe-Besucher beachtlich, in: Marketing Journal, Heft 2, 1992, S. 164169 sowie zu besuchertypenspezifischen Aussagen aus der Perspektive der Aussteller Strothmann, K.-H., Segmentorientierte Messepolitik, in: Strothmann, K.-H., Busche, M. (Hrsg.), Handbuch Messemarketing, Wiesbaden 1992, S. 112.

Vgl. die allgemeine Ableitung der Dimensionen messespezifischer Grundhaltungen in Kap. A.2 dieser Arbeit. 
Aus entscheidungsorientierter Sicht ist zunächst den Messebeteiligungszielen als zentralen Determinanten der Messebeteiligung besondere Bedeutung beizumessen. Grundsätzlich bringt der Zielbezug des Management von Messebeteiligungen zum Ausdruck, daß es sich nicht in "ad hoc" ergriffenen Einzelentscheidungen erschöpft, sondern aufbauend auf einer Analyse der relevanten unternehmensinternen und -externen Rahmenbedingungen als ein systematischer strategisch intendierter Planungsprozeß darstellt, der in das Zielsystem der Unternehmung integriert ist. ${ }^{129}$

Die strategische Dimension der Planung von Messebeteiligungen zeigt sich ebenfalls in der zieladäquaten Messeselektion. Vor dem Hintergrund der Leistungsfähigkeit und Vielfalt von Messen ${ }^{130}$ sowie einem weiter zunehmenden Messeangebot macht Strothmann darauf aufmerksam, daß Unternehmen u. U. dazu neigen, das Instrument Messebeteiligung zu überfordern, indem „alles nur Denkbare" zu erreichen versucht wird. ${ }^{131} \mathrm{Er}$ plädiert damit für einen Abstimmungsprozeß zwischen Messebeteiligungszielen und Selektion von Messen ${ }^{132}$, in dem auch die Zielkonzeption einer veranstaltungsspezifischen Anpassung unterliegen kann, um "durch ein derartiges zielgerichtetes Vorgehen Aussteller davor zu bewahren, mit dem Messeauftritt allzu hochgesteckte Erwartungen zu verbinden“"133.

Fuchs weist bezüglich der Festlegung der messebeteiligungsbezogenen Ziele auf den unmittelbaren Zusammenhang zur messespezifischen Positionierung hin. Mit der Entscheidung über die Festlegung der Messebeteiligungsziele geht für ihn die „Verabschiedung der Positionierung und der Kernbotschaft, die das Unternehmen von anderen differenzieren und den Hauptnutzen des Angebots für den Kunden

129 Heinen führt an, daß aufgrund der überwiegend entscheidungsorientierten Konzipierung der modernen Betriebswirtschaftslehre dem Zielsystem der Unternehmung als einer der Hauptentscheidungsdeterminanten besondere Aufmerksamkeit zu widmen ist. Vgl. Heinen, E., Grundlagen betriebswirtschaftlicher Entscheidungen - Das Zielsystem der Unternehmung, 3. Aufl., Wiesbaden 1976, S. 23 ff.; zur Notwendigkeit der Einbeziehung messespezifischer Zielsetzungen in das Zielsystem der Unternehmung vgl. Meffert. $H$., Messen und Ausstellungen als Marketinginstrument, a.a.O., S. 78 f.

Vgl. die hierzu gemachten Ausführungen in Kap. A.1. dieser Arbeit.

Vgl. Strothmann, K.-H., Segmentorientierte Messepolitik, in: Strothmann, K.-H., Busche. M. (Hrsg.), Handbuch Messemarketing, Wiesbaden 1992, S. 101.

Selinski und Sperling sprechen in diesem Zusammenhang davon, daß die Selektionsentscheidung gemeinsam durch die messespezifischen Ziele des Ausstellers und "den Charakter der Messeveranstaltung" determiniert wird. Vgl. Selinski, H., Sperling. U. A., Marketinginstrument Messe: Arbeitsbuch für Studium und Praxis, a.a.O., S. 108.

Vgl. Strothmann, K.-H., Segmentorientierte Messepolitik, in: Strothmann. K.-H., Busche, M. (Hrsg.), Handbuch Messemarketing, Wiesbaden 1992, S. 101. 
thematisieren" ${ }^{134}$ einher. Mit diesem Hinweis wird deutlich, daß auch die Positionierungsentscheidung bei Messebeteiligungen strategischen Charakter besitzt, da es hierbei zum einen um die Umsetzung der übergeordneten Positionierungsstrategie des ausstellenden Unternehmens und zum anderen um seine grundsätzliche Profilierung ggü. den Kunden bzw. Differenzierung ggü. den Wettbewerbern auf der Messe geht. ${ }^{135}$ Hierzu bedarf die messespezifische Positionierung der notwendigen Abstimmung mit den Messezielen sowie eine Anpassung an die ausgewählten Messen und die dort vertretenen Besucherzielgruppen.

Abb. 7 gibt einen ersten Überblick über die Dimensionen der messespezifischen Grundhaltung von Ausstellern und deren Beziehungen zueinander.

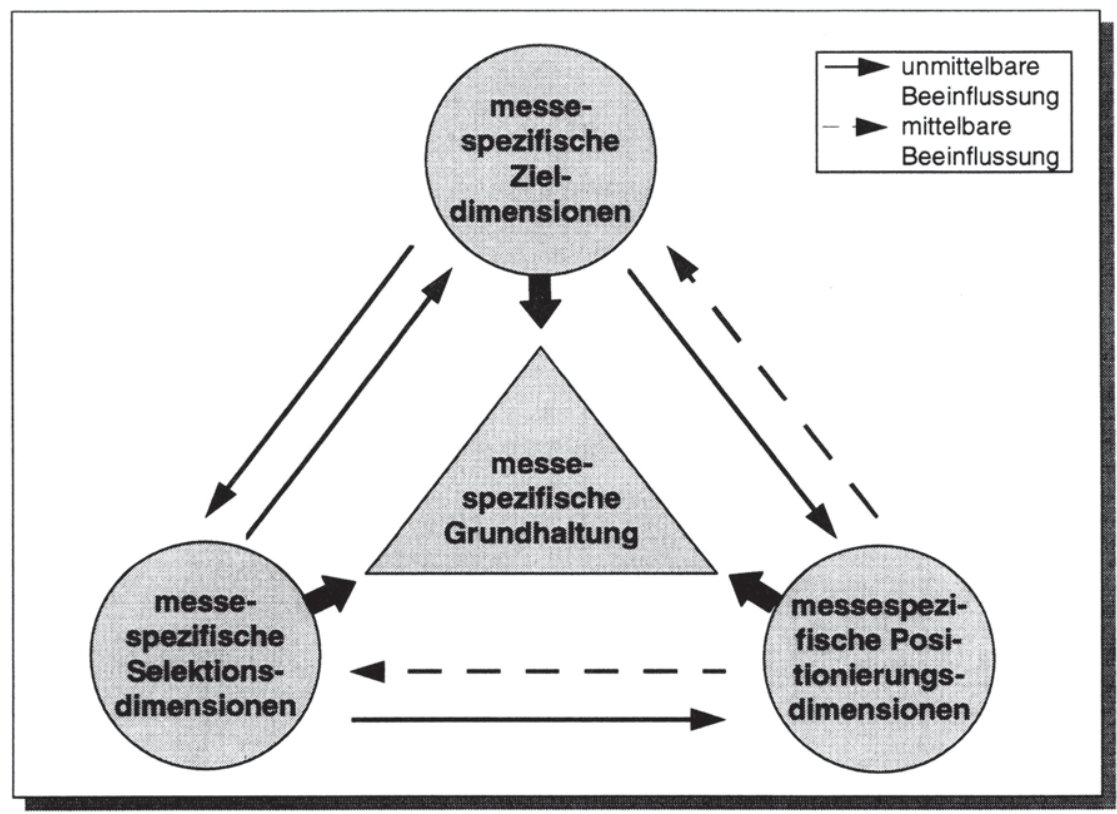

Abb. 7: Konzept messespezifischer Grundhaltungen von Ausstellern

134 Vgl. Fuchs, W. A., Die Messe als Kommunikationsprozeß, in: Werbeforschung \& Praxis, Heft 3, 1995, S. 99.

135 Vgl. auch Meffert. $H_{\text {. }}$, Messen und Ausstellungen als Marketinginstrument, a.a.O., S. 78. 
Es ist zu vermuten, daß sich auf der Basis dieser drei strategischen Grundhaltungsdimensionen unterschiedliche Grundhaltungstypen beschreiben lassen. Damit lautet die Basishypothese zur Ableitung von Grundhaltungstypen:

$\mathrm{H}_{\mathrm{GH}}$ : Es lassen sich auf der Grundlage der Messe-Zielkonzeption, MesseSelektionskonzeption und Messe-Positionierungskonzeption homogene Entscheidungsmuster und damit Typen von Grundhaltungen ableiten, die sich signifikant voneinander unterscheiden.

\subsection{Messe-Zielkonzeption}

Messebeteiligungsziele ${ }^{136}$ bilden den Ausgangspunkt des Managementprozesses und kennzeichnen jene zukünftigen Zustände, die aus Sicht des Ausstellers als erstrebenswert angesehen und durch entsprechende Handlungen erreicht werden sollen. ${ }^{137}$ Durch die Vorgabe anzustrebender Zustände werden die Messebeteiligungsziele zu konzeptionellen Orientierungs- bzw. Richtgrößen des Handelns hinsichtlich der Messebeteiligung. ${ }^{138}$ An innen richten sich alle weiteren Entscheidungen im Managementprozeß der Messebeteiligung aus. Eine in diesem Sinne verstandene Zielkonzeption für Messebeteiligungen übernimmt damit grundsätzlich mehrere Funktionen, insbesondere: ${ }^{139}$

136 Über das spezifische Untersuchungsobjekt "Messebeteiligungsziele“ liegen bislang im Vergleich $\mathrm{zu}$ anderen Bereichen der Unternehmenskommunikation, wie z. B. der Werbung, nur wenige konzeptionelle Beiträge vor, so daß zu einem gewissen Teil ein Transfer der Erkenntnisse der Kommunikations- bzw. der Werbeforschung auf die hier zu behandelnde Themenstellung

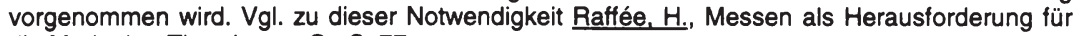
die Marketing-Theorie, a.a.O., S. 77.

137 Vgl. hierzu allgemeingültige Ausführungen bei Meffert, $H$., Unternehmensziele, in: Schöttle, K. M. (Hrsg.), Jahrbuch des Marketing, Essen, 1971, S. 22-34; Heinen, E., Grundlagen betriebswirtschaftlicher Entscheidungen - Das Zielsystem der Unternehmung, 3. Aufl., a.a.O., S. 49 ff.; Dinkelbach, W., Ziele, Zielvariablen und Zielfunktionen, in: DBW, Heft 1, 1978, S. 51-58.

Vgl. Amon, P., Messen + Ausstellungen: Messe-Ziele exakt formulieren und kontrollieren, in: Marketing Journal, Heft 1, 1991, S. 56; Cavanaugh. S., Setting Objectives and Evaluating the Effectiveness of Trade Show Exhibits, in Journal of Marketing, October 1976, S. 100-103.

Vgl. Steffenhagen, $H_{\text {. }}$ Funke, K., Messen und Ausstellungen: Formulieren Sie präzise MesseZiele, die später auch nachprüfbar sind, in: Marketing Journal, Heft 6, 1986, S. 546 sowie ausführlich Funke, K., Messeentscheidungen. Handlungsalternativen und Informationsbedarf, a.a.O., S. 12-15. 


\section{Entscheidungs- bzw. Steuerungsfunktion}

Der Einsatz des Marketing-Instrumentariums anläßlich einer Messe orientiert sich an den Messebeteiligungszielen. Die Instrumentekombination, die nach Meinung der Messe-Entscheider am meisten zur Zielerreichung beizutragen vermag, wird eingesetzt. Ziele stellen also ein Auswahl- bzw. Bewertungskriterium dar.

\section{Kontrollfunktion}

Der Erfolg einer Messebeteiligung wird jeweils am Grad der Zielerreichung gemessen. Ohne definierte Zielsetzungen können weder Ergebnisbewertungen erfolgen, noch die notwendigen Schlußfolgerungen gezogen werden.

\section{Koordinationsfunktion}

Ziele tragen zur Verhaltensabstimmung zwischen verschiedenen Bereichen und/oder Marketingaktionen des Ausstellers bei. Durch übergreifende Zielsetzungen werden messespezifische Aktivitäten verschiedener Unternehmensbereiche, Aktionen auf verschiedenen Messeveranstaltungen sowie Messe- und andere Marketingaktivitäten in eine bestimmte Richtung gelenkt, aufeinander abgestimmt und damit koordiniert.

\section{Motivationsfunktion}

Mitarbeiter auf Messeständen werden einerseits durch Zielvorgaben motiviert, bestimmte Leistungen zu erbringen. Andererseits haben sie bei der Erreichung von Zielsetzungen Erfolgserlebnisse, die Zufriedenheit auslösen und zusätzlicher Ansporn sein können.

Aus den angeführten Grundfunktionen von Messebeteiligungszielen ergeben sich spezifische Anforderungen an eine Messe-Zielkonzeption des Ausstellers.

Bei der Ableitung der Messebeteiligungsziele aus den Marketing- und Kommunikationszielen ist zu beachten, daß sie in einem sog. Mittel-Zweck-Verhältnis zu diesen stehen ${ }^{140}$. Folgerichtig stellen die hierarchisch übergeordneten Marketing- und Kommunikationsziele den Orientierungsrahmen für die Messebeteiligungsziele dar. Für die Messebeteiligungsziele bedeutet dies, daß sie Mittel zum Erreichen übergeordneter Marketing- bzw. Kommunikationsziele sind und damit keinen originären, sondern abgeleiteten (derivativen) Charakter haben. Als Handlungsanweisungen sind sie dann sinnvoll einsetzbar, wenn sie sich konsistent in das Zielsystem der Unternehmung, insbesondere in die Marketing- und Kommunikationsziele, einfügen lassen.

140 Zum Mittel-Zeck-Verhältnis von Zielen vgl. Hauschildt. J., Zielsysteme, in: Grochla. E. (Hrsg.), Handbuch der Organisation, 2. Aufl., Stuttgart, 1984, S. 2419-2430. 
Messebeteiligungsziele können - analog zu den übergeordneten Kommunikationsund Marketingzielen einer Unternehmung - zunächst grundsätzlich nach psychographischen und ökonomischen Zielsetzungen unterschieden werden. ${ }^{141}$ Unter psychographischen Messebeteiligungszielen sollen solche Ziele verstanden werden, die auf mentale Prozesse des Messe- bzw. Messestandbesuchers ausgerichtet sind und sowohl affektiv-gefühlshafte als auch kognitiv-rationale Ergebnisse zeitigen. Schließlich sollen beim Adressaten bestimmte beabsichtigte Verhaltensweisen prädisponiert werden. ${ }^{142} \mathrm{Zu}$ den ökonomischen Zielsetzungen von Messebeteiligungen zählen solche Zielinhalte, die im wesentlichen monetäre Größen wie Umsatz und Marktanteil beinhalten und die mit den psychographischen Zielsetzungen i. S. der dargelegten Mittel-Zweck-Beziehung korrespondieren. ${ }^{143}$

Sowohl die psychographischen als auch die ökonomischen Messebeteiligungsziele lassen sich weiter in direkte vs. indirekte Zielsetzungen unterteilen. ${ }^{144}$ Ein direktes ökonomisches Beteiligungsziel wäre bspw. eine bestimmte Umsatzhöhe, die während einer Messe erzielt und allein durch diese induziert wird. Wird dagegen beabsichtigt, den Nachmesse-Umsatz auf der Messe zu fördern, so wäre damit ein indirektes ökonomisches Messeziel formuliert. Mit dem Ziel der Erhöhung des Bekanntheitsgrades des Ausstellers bei den Messebesuchern wäre folglich ein direktes psychographisches Messebeteiligungsziel abgefaßt. Werden dagegen auch Marktforschungsziele (z. B. hinsichtlich von Meinungen und Einstellungen ggü. dem Unternehmen oder bestehenden Branchenproblemen) mit einer Messebeteiligung verfolgt, so sind diese als indirekte psychographische Zielsetzung einzustufen.

Darüber hinaus lassen sich Messebeteiligungsziele auch nach den angestrebten Verhaltenswirkungen bei den Messebesuchern in Zielsetzungen, die primär momentane Reaktionen, dauerhafte Gedächtnisreaktionen oder aber finale Verhal-

141 Vgl. Meffert. H., Marketing. Grundlagen der Absatzpolitik, 7. Aufl., a.a.O., S. 82. Einige Autoren benutzen zur Benennung psychographischer Zielsetzungen auch die synonym zu verwendenden Begriffe „außerökonomische“ bzw. "kommunikative“ Ziele. Vgl. stellv. Selinski, H. Sperling. U. A., Marketinginstrument Messe: Arbeitsbuch für Studium und Praxis, a.a.O., S. 107.

142 Vgl. Meffert. H., Das System des Kommunikations-Mix, Münster 1979, S. 156.

143 Vgl. Behrens, K. C., Absatzwerbung, Wiesbaden 1963, S. $50 \mathrm{ff}$.

144 Vgl. Mortsiefer, J., Die Vorbereitung entscheidet über den Erfolg - Planung und Vorbereitung einer Messebeteiligung, a.a.O., S. $52 \mathrm{ff}$. 
tensreaktionen auslösen sollen, unterscheiden. ${ }^{145}$ Den momentanen Reaktionen sind alle reizinduzierten Verhaltensweisen zu subsumieren, die sich unmittelbar bei oder direkt nach der Reizdarbietung bei den Messebesuchern ergeben. ${ }^{146}$ Mit dauerhaften Gedächtnisreaktionen sind jene psychischen bzw. nicht beobachtbaren Verhaltensweisen gemeint, die eine gewisse zeitliche Stabilität aufweisen und Einfluß auf das spätere Kaufverhalten haben. Damit sind bspw. Messebeteiligungsziele angesprochen, die auf die Kenntnisse der Messebesucher, ihr Interesse, ihre Einstellungen sowie ihre Zufriedenheit und Handlungsabsichten fokussiert sind. Hinsichtlich der finalen Verhaltensreaktionen sind solche Zielsetzungen zusammenzufassen, die am sichtbaren Kauf- bzw. Verwendungsoder Weiterempfehlungsverhalten ansetzen. ${ }^{147}$

Darüber hinaus besteht die Möglichkeit der Zielsystematisierung nach den Phasen des Kaufprozesses, auf die durch eine Messebeteiligung differenziert Einfluß genommen werden soll. Hiernach lassen sich Messebeteiligungsziele den Bereichen Absatzvorbereitung, -anbahnung, -durchführung und -erhaltung zuordnen. $^{148}$

Den bislang skizzierten Zielkategorien ist gemein, daß sie lediglich allgemeine Ansätze zur Zielsystematisierung im Marketing auf das Management von Messebeteiligungen übertragen. Zur Erfassung messespezifischer Zielkonzeptionen von Ausstellern schlägt Meffert daher einen Systematisierungsansatz vor, der sich an den Funktionen von Messen ${ }^{149}$ anlehnt und damit unmittelbar den Bezug zwischen der Funktionalebene einer Messeveranstaltung und der Zielebene eines einzelnen Ausstellers herstellt. Er unterscheidet die folgenden vier Zielkategorien, denen spezifische Zieldimensionen zugeordnet werden können: ${ }^{150}$

145 Vgl. Steffenhagen. H. Funke. K., Messen und Ausstellungen: Formulieren Sie präzise MesseZiele, die später auch nachprüfbar sind, a.a.O., S. $548 \mathrm{ff}$.

146 Hierunter wird das sog. Impulsverhalten ebenso zusammengefaßt wie die spontane Aktivierung von Messebesuchern. Vgl. Steffenhagen. H. Funke. K., Messen und Ausstellungen: Formulieren Sie präzise Messe-Ziele, die später auch nachprüfbar sind, a.a.O., S. 548.

147 Diese Austührungen basieren weitestgehend auf den Erkenntnissen der Werbewirkungsforschung, die von Steffenhagen auf das Messegeschehen übertragen werden. Vgl. Steffenhagen, H., Ansätze der Werbewirkungsforschung, a.a.O., S. 77-88.

$148 \mathrm{Vgl}$. Amon, $\mathrm{P}$., Messen + Ausstellungen: Messe-Ziele exakt formulieren und kontrollieren, a.a.O., S. 56; Schober. P. M., Messen und Ausstellungen, teilnehmen oder wegbleiben? - Eine kleine Entscheidungshilfe, a.a.O., S. 400-402.

149 Als zentrale Messefunktionen werden von Meffert die Informations-, Motivations-, Beeinflussungs- und Verkaufsfunktion identifiziert. Vgl. Meffert. $H$., Messen und Ausstellungen als Marketinginstrument, a.a.O., S. $10 \mathrm{f}$. 
- Informationsziele

- Ziele der Informationsweitergabe

- Ziel der Informationsbeschaffung

- Ziele der Markterkundung

- Motivationsziele

- mitarbeiterorientierte Ziele

- veranstalterorientierte Ziele

- besucherorientierte Ziele

- Beeinflussungsziele

- Einstellungsziele

- Bekanntheitsziele

- Imageziele

\section{- Verkaufsziele}

- Verkaufsvorbereitungsziele

- Verkaufsdurchführungsziele

- Verkaufserhaltungsziele

Dieser Systematisierungsansatz messespezifischer Ziele soll im Rahmen der vorliegenden Arbeit insofern eine weitere Spezifizierung erhalten, als insbesondere die Zielkategorien "Beeinflussungs-“ und "Verkaufsziele“ vor dem Hintergrund der aktuellen Entwicklungen im (Messe-)Marketing der Unternehmen differenzierter zu betrachten sind.

Das Anspruchsspektrum an das Kommunikationsmanagement von Unternehmen ist in jüngster Zeit durch die Integration von Aspekten des Beziehungsmarketing, die eine an Beeinflussungszielen orientierte Marktbearbeitung ergänzen, erweitert worden. ${ }^{151}$ Damit einhergehend gewinnen Aspekte der Kundenbindung und des

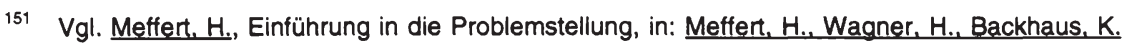
(Hrsg.), Beziehungsmarketing - neue Wege zur Kundenbindung, Dokumentation eines Workshops der Wissenschaftlichen Gesellschaft für Marketing und Unternehmensführung e.V., Nr. 90, Münster 1994, S. 1. 
Aufbaus langfristiger Geschäftsbeziehungen ${ }^{152}$, aber auch die Berücksichtigung der Anforderungen von Anspruchsgruppen ${ }^{153}$ für das Zielsystem von Messebeteiligungen, zunehmend an Bedeutung. Der persönliche Kontakt bzw. der Dialog mit Kunden und Anspruchsgruppen stellen damit Schlüsselgrößen im aktuellen Messemarketing von Ausstellern dar. ${ }^{154}$

Daher sind zunächst die von Meffert aufgeführten "Verkaufsziele“ zu differenzieren. Hinter den Zieldimensionen „Verkaufsvorbereitungs-“ und „Verkaufserhaltungsziele" stehen vermehrt eigenständige Zieldimensionen der Kundenbindung und des Kundenkontaktes, die mit der Zielkategorie "Kontaktziele“ wiedergegeben werden können ${ }^{155}$, während "Verkaufsdurchführungsziele" sich treffend mit der Zielkategorie „Verkaufsziele“ charakterisieren lassen.

Da Messen heute auch als Forum für den Dialog mit Anspruchsgruppen genutzt werden ${ }^{156}$, ist die übergreifende Zielkategorie "Beeinflussungsziele" in die eigenständigen Kategorien "markt(partner)bezogene Beeinflussungsziele" und „anspruchsgruppenbezogene Beeinflussungsziele“ ${ }^{157}$ aufzuspalten.

152 Geschäftsbeziehungen enthalten nach Diller die folgenden Definitionselemente: Sie sind von ökonomischen Zielen geleitet, stellen Interaktionsprozesse mit personalen Kontakten und langfristigen Geschäftsperspektiven sowie einer damit verbundenen investiven Komponente der beteiligten Partner dar. Vgl. Diller. H., Beziehungsmanagement und Marketing, in: Backhaus. K. Diller. H. (Hrsg.), Dokumentation des 1. Workshops der Arbeitsgruppe "Beziehungsmanagement" der wissenschaftlichen Kommission für Marketing im Verband der Hochschullehrer für Betriebswirtschaftslehre, Münster, Nürnberg 1994, S. 1 f.

153 Unter Anspruchsgruppen werden im Rahmen dieser Arbeit solche Personenmehrheiten verstanden, die ihre Interessen ggü. dem ausstellenden Unternehmen nicht aus der marktbezogenen Perspektive, sondern aus ökologischen, gesellschaftlichen oder politisch-rechtlichen Erwägungen begründen wie z. B. staatliche Institutionen, Umweltschutzorganisationen, Branchenverbände sowie Parteien, Medien und andere Interessenvertretungen. Vgl. Kirchgeorg. M., Ökologieorientiertes Unternehmensverhalten: Typologien und Erklärungsansätze auf empirischer Grundlage, a.a.O., S. 60 und die dort zitierte Literatur.

Vgl. Dudley. J. W., Successful Exhibiting, a.a.O., S. 32 ff.; Selinski, H., Sperling. U. A., Marketinginstrument Messe: Arbeitsbuch für Studium und Praxis, a.a.O., S. 108.

Selinski, H. Sperling. U. A., Marketinginstrument Messe: Arbeitsbuch für Studium und Praxis, a.a.O., S. 108

Vgl. Henkel. H. O., Neue Funktionen der Messen, in: Der Handel, Heft 11, 1995, S. 31; Strothmann, K.-H. Roloff. E., Charakterisierung und Arten von Messen, a.a.O., S. 713.

Krone und Huber verwenden stattdessen die Begriffe "Agenda Setting“ und "Lobbying". Vgl. Krone, K. Huber. B., Messen als Instrument der Unternehmenspolitik, a.a.O., S. 151 f.; vgl. auch McCombs. M. E. Shaw, D.L., The Agenda Setting Function of the Media, in: Public Opinion Quaterly, No. 36, 1972 , S. S. 176-187. 
Damit ergibt sich ein messezielbezogener Systematisierungsansatz, der die sechs Zielkategorien

- Informationsziele,

- Motivationsziele,

- marktbezogene Beeinflussungsziele,

- anspruchsgruppenbezogene Beeinflussungsziele,

- Verkaufsziele und

- Kontaktziele

enthält. Zu ihrer Operationalisierung kann den Zielkategorien die Vielzahl bestehender Einzelzielsetzungen, die in der Literatur in Verbindung mit Messebeteiligungen angeführt wird ${ }^{158}$, eindeutig zugeordnet werden (vgl. Abb. 8).

Im Rahmen der messespezifischen Zielkonzeption sind nun die zu verfolgenden Zielsetzungen festzulegen. Hierbei kann theoretisch zwischen „fokussierten" Zielkonzeptionen, die lediglich ein einzelnes oder aber wenige ausgewählte Zielsetzungen umfassen und breit gefächerten "Zielbündeln“ i. S. „mehrdimensionaler" Zielkonzeptionen unterschieden werden. Empirischen Untersuchungen zufolge, ist davon auszugehen, daß Aussteller i. d. R. mehrdimensionale Zielkonzeptionen mit ihren Messebeteiligungen verfolgen. ${ }^{159}$

158 Auf der Grundlage einer Analyse von in der Literatur aufgeführten Messebeteiligungszielen, soll aus Gründen der Überschaubarkeit eine Fokussierung auf dominante Messebeteiligungsziele erfolgen. Die vorliegende Auswahl wurde daher zunächst auf der Basis der Nennhäufigkeit jedes Einzelziels in der einschlägigen Literatur vorgenommen und des weiteren einem Expertengremium der ausstellenden Wirtschaft im Rahmen eines Workshops zur Diskussion sowie Relevanzprüfung vorgelegt und bestätigt. Vgl. im Hinblick auf eine umfassende Darstellung möglicher Messebeteiligungsziele in der Literatur Fließ.S., Messeselektion. Entscheidungskriterien für Investitionsgüteranbieter, Wiesbaden 1994, S. 12 f.; Selinski. H. Sperling. U. A., Marketinginstrument Messe: Arbeitsbuch für Studium und Praxis, a.a.O., S. 108 f.; Schober. P. M., Messen und Ausstellungen: Teilnehmen oder wegbleiben? - Eine kleine Entscheidungshilfe; a.a.O., S. 402; Chapman. E. A., Exhibit Marketing: A Survival Guide for Managers, a.a.O., S. 57 ff.; Raffée. $H_{\text {. }}$, Messen als Herausforderung für die MarketingTheorie, a.a.O., S. 75 f.; Gräbener. W., Die Messepolitik als Marketinginstrument dargestellt am Beispiel von Investitionsgüterproduzenten, Göttingen 1981, S. 159 f.; Weishäupl, G., Die Messe als Kommunikationsmedium - unter besonderer Berücksichtigung der Öffentlichkeitsarbeit und Werbung einer Messegesellschaft, München 1980, S. 64-76 und die an diesen Fundstellen jeweils zitierte Literatur. Vgl. zu den Teilnehmern des Workshops „Ziele und Nutzen von Messebeteiligungen" die Teilnehmerliste im Anhang 2. S. 274.

159 Täger und Ziegler weisen bspw. in inrer Studie darauf hin, daß Kontakt- bzw. marktbezogenen Beeinflussungsziele (Fachgespräche führen, Unternehmensbekanntheit erhöhen und Zahl der Geschäftsverbindungen erhöhen) von nahezu allen Ausstellern gleichzeitig verfolgt wurden. Vgl. Täger, U. C. Ziegler. R., Die Bedeutung von Messen und Ausstellungen in der Bundesrepublik Deutschland für den Inlands- und Auslandsabsatz in ausgewählten Branchen, a.a.O., S. 51 f. Auch SpryB, Nerb und Reuter kommen bei ihrer Untersuchung von Messebeteiligungszielen zu dem Ergebnis, daß bei der überwiegenden Zahl der Aussteller eine mehrdimensionale Zielorientierung vorliegt. Vgl. Spryß.W. M. Nerb. G.. Reuter. J., Messeziele Messeetfizienz, a.a.O., S. $10 \mathrm{f}$. 


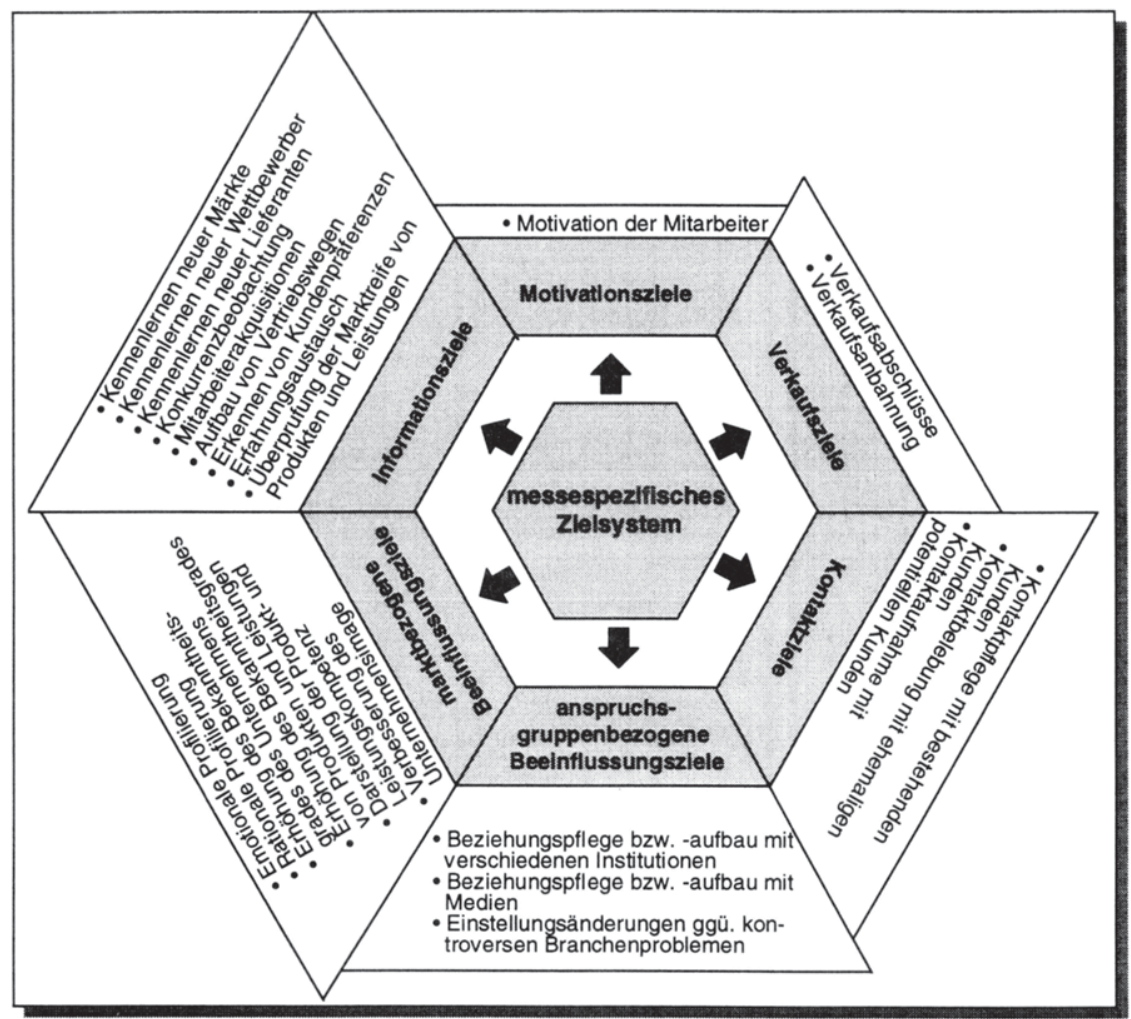

Abb. 8: Aufstellung von Messebeteiligungszielen nach Zielkategorien

Allerdings sind in diesen Zielkonzeptionen nicht alle gleichzeitig verfolgten Zielsetzungen von ähnlicher Bedeutung. Vielmehr wird eine Gewichtung der Messebeteiligungsziele vorgenommen. Diese kann in Form einer eindeutigen Prioritätenbildung (ein Hauptziel und andere Ziele als Nebenbedingungen) durchgeführt werden, oder aber auf der Grundlage einer internen Präferenzstruktur durch eine kombinierte und damit jeweils partiell angestrebte Zielverfolgung umgesetzt werden. ${ }^{160}$ Einen ersten Überblick über den aktuellen und zukünftigen Stellenwert einzelner Messebeteiligungsziele vermittelt Abb. 9.

\footnotetext{
160 Vgl. zur allgemeinen Darstellung von Zielgewichtungen Kupsch. P., Unternehmensziele, Stuttgart, New York 1979, S. 26 ff. Einen direkten Bezug zu Messebeteiligungszielen stellt Funke her. Vgl. Funke. K., Messeentscheidungen. Handlungsalternativen und Informationsbedarf, a.a.O., S. $18 \mathrm{ff}$.
} 


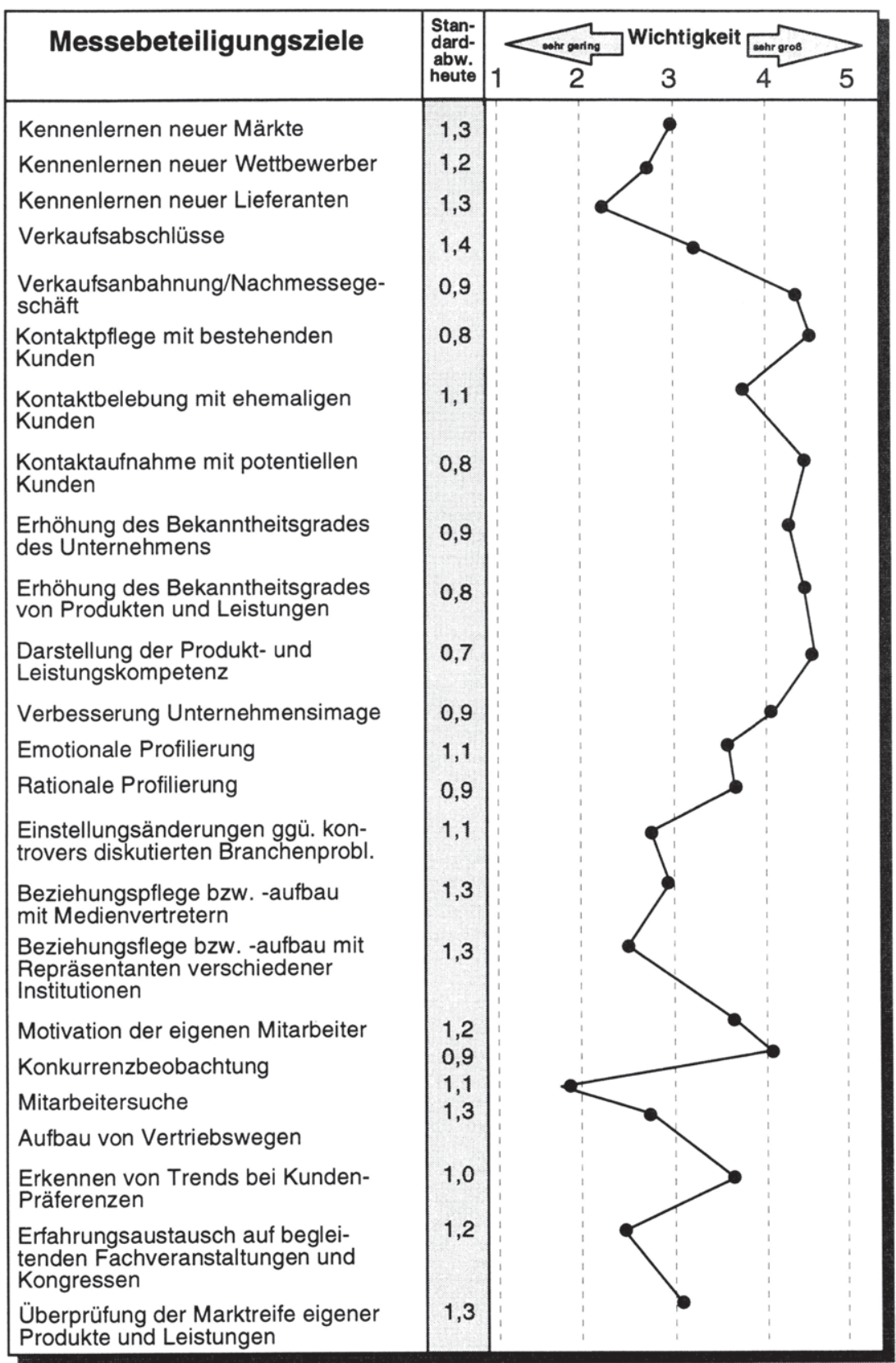

Abb. 9: Bedeutung von Messebeteiligungszielen 
Obwohl die untersuchten Aussteller eine Vielzahl von Messebeteiligungszielen verfolgen, existieren klar abgestufte Bedeutungsgewichte für die einzelnen Zielsetzungen, die einerseits zu eindeutig präferierten und andererseits weitgehend unbedeutenden Messebeteiligungszielen führen. Darüber hinaus kann davon ausgegangen werden, daß sich die Bedeutungsgewichte der verschiedenen Messebeteiligungsziele zukünftig in ihrer Struktur nicht wesentlich verschieben werden.

An erster Stelle der aktuellen Zielsetzungen von Messebeteiligungen steht die Kontaktaufnahme mit potentiellen Kunden, die mit einem Mittelwert von 4,7 den höchsten Wert erreicht. Den allgemein hohen Stellenwert der Kontaktziele auf Messen unterstreichen auch die Zielsetzungen der Kontaktpflege mit bestehenden Kunden, die insgesamt die zweithöchste Zielpriorität genießt, und die Kontaktbelebung mit ehemaligen Kunden, die ebenfalls einen hohen Stellenwert einnimmt.

Damit wird deutlich, daß eine Vielzahl ausstellender Unternehmen heute das Instrument der Messebeteiligung vornehmlich im Bereich des Beziehungsmarketing und der Kundenbindung einsetzen. Nicht die undifferenzierte Massenwirkung steht damit im Mittelpunkt der Messepolitik, sondern es wird eine zunehmende Individualisierung der Messekontakte auf der Seite der Aussteller angestrebt.

Vor diesem Hintergrund sind auch der hohe Stellenwert der Zielsetzungen Darstellung der Produkt- und Leistungskompetenz, Erhöhung des Bekanntheitsgrades von Produkten und Leistungen sowie Verkaufsanbahnung/Nachmessegeschäft einzuordnen. Die Messebeteiligung soll die Wahrnehmung der Messebesucher auf das aktuelle Angebot lenken und es als innovative und kompetente Kaufalternative ausweisen. Dieser Befund ist auch im Zusammenhang mit der Tatsache zu würdigen, daß vor allem auf Messen das Bedürfnis nach Informationen über neuartige Produkte häufig besonders groß ist und gerade die Bekanntmachung der Produkte bzw. der Leistungen einen wesentlichen Schritt zur Anbahnung von Verkäufen darstellt. Grundsätzlich bleibt anzumerken, daß der produktorientierte Kompetenznachweis i. S. eines Kundennutzens sowie das Bekanntheitsziel für auf der Messe angebotene Produkte und Leistungen unumgänglich erscheinen. Bei Kaufentscheidungen werden nur diejenigen Produkte und Leistungen beachtet, die zur Menge der wahrgenommenen und akzeptierten Alternativen gehören. Solange ein Angebot nicht als aktuelle Alternative im Bewußtsein des Kaufentscheiders i. S. eines "Top of mind" gesichert ist, kann eine positive Beurteilung oder ein positives Image picht wirksam werden. 
Gleichzeitig nutzen die Untemehmen Messeveranstaltungen auch zur Selbstdarstellung. Die Zielsetzungen Erhöhung des Bekanntheitsgrades des Unternehmens sowie Verbesserung des Unternehmensimage gehören zu den zehn wichtigsten Messebeteiligungszielen und machen deutlich, daß Messen gerade in Zeiten hoher Wettbewerbsintensität einen Beitrag zur Konkurrenzdifferenzierung leisten sollen.

Konkurrenzbeobachtung und das Erkennen von Trends bei Kundenpräferenzen bilden einen weiteren wesentlichen Zielschwerpunkt von Messebeteiligungen. Dies unterstreicht, daß Messen nicht nur als Medium der Informationsabgabe vom Unternehmen an den Kunden, sondern vielmehr auch als Instrument zur Aufnahme von Informationen über Kunden und Wettbewerber genutzt werden.

In der Gruppe der Messebeteiligungsziele mit einem mittleren Bedeutungsgewicht befinden sich Zielsetzungen wie Verkaufsabschlüsse, emotionale und rationale Profilierung, Beziehungspflege mit Medien, Einstellungsänderungen gegenüber kontrovers diskutierten Branchenproblemen, Kennenlernen neuer Märkte und Wettbewerber sowie Motivation der Mitarbeiter und Überprüfung der Marktreife von Produkten und Leistungen. Als weniger wichtig sind die Ziele Mitarbeitersuche, Kennenlernen neuer Lieferanten und Beziehungen zu Institutionen sowie Erfahrungsaustausch auf begleitenden Fachveranstaltungen und Kongressen einzustufen.

Ein Vergleich der Zielprofilverläufe bzgl. des heutigen und zukünftigen Stellenwertes von Messebeteiligungszielen in der Gesamtstichprobe zeigt, daß die Bedeutung der einzelnen Zielsetzungen im Vergleich zueinander relativ konstant bleibt. Allerdings ist eine allgemeine Bedeutungszunahme aller Messebeteiligungsziele zu konstatieren. Letzteres ist dabei als Ausdruck des gewachsenen Anspruchsspektrums der Aussteller gegenüber Messebeteiligungen zu bewerten sowie der Bemühungen, den Nutzen von Messebeteiligungen weiter zu steigern.

Die Festlegung von Messebeteiligungszielen erfordert nicht nur die Auswahl und Ordnung der zu verfolgenden Zielsetzungen. Vielmehr sind die Zielformulierungen zu präzisieren, damit sie die erwünschte Steuerungskraft bezüglich der zu ergreifenden Messeaktivitäten erhalten. Vollständige und damit operationale Zielfor- 
mulierungen liegen vor, wenn die Ziele in den folgenden Zieldimensionen festgeschrieben werden: ${ }^{161}$

\section{- Inhalt der Messebeteiligungsziele}

Bei der Frage nach den Inhalten von Messebeteiligungszielen steht die Formulierung der zu bewirkenden Reaktionen der Messestandbesucher im Vordergrund. Es geht somit um die Frage: "Was soll bei den Besuchern bewirkt werden?" Der konkrete Zielinhalt kann sich dabei bspw. zwischen den in Abb. 8 dargestellten Messebeteiligungszielen bewegen.

\section{- Ausmaß der Messebeteiligungsziele}

Mit dem Zielausmaß ist das Anspruchsniveau der Zielerreichung im Hinblick auf die jeweiligen Zielgrößen festzulegen. Grundsätzlich kann dabei zwischen unbegrenzten ("so viel wie möglich") und begrenzten ("5\% mehr als bisher) Anspruchsniveaus unterschieden werden. Der zuletzt genannten Alternative ist jedoch der Vorzug zu geben, da sie eine konkrete Überprüfung der Zielerreichung zuläßt. ${ }^{162}$

\section{- Zeitbezug der Messebeteiligungsziele}

Der Zeitbezug von Messebeteiligungszielen stellt auf einen bestimmten Zeitpunkt oder Zeitabschnitt ab, in dem das Ziel erreicht werden soll. Zeitliche Bezugsgrößen können bspw. sein:

- der Zeitraum mehrerer Messen (z. B. für best. Gruppen von Messen)

- der Veranstaltungszeitraum einer Messe

- der einzelne Veranstaltungstag einer Messe

\section{- Segmentbezug der Messebeteiligungsziele}

Im Rahmen der Festlegung des betreffenden Segmentes, in dem das jeweilige Messebeteiligungsziel verwirklicht werden soll, geht es um die Bestimmung und Auswahl von Zielgruppen, die mit der Messebeteiligung primär angesprochen werden sollen.

\section{- Objektbezug der Messebeteiligungsziele}

161 Vgl. Steffenhagen. H. Funke. K., Messen und Ausstellungen: Formulieren Sie präzise MesseZiele, die später auch nachprüfbar sind, a.a.O., S. 547.

162 Vgl. Heinen. E., Grundlagen betriebswirtschaftlicher Entscheidungen - Das Zielsystem der Unternehmung, 3. Aufl., a.a.O., S. 85. 
Der letzte Schritt der Zielkonkretisierung besteht in der Bestimmung der verschiedenen Produkt- bzw. Leistungsbereiche des Unternehmens, für die die Messebeteiligungsziele Gültigkeit besitzen sollen.

Auf diese Weise abgeleitete und exakt formulierte Messebeteiligungsziele bilden auf der einen Seite die Voraussetzung für eine effektive Planung und Durchführung des Messeengagements sowie andererseits die Grundlage einer effizienten Erfolgsbestimmung. ${ }^{163}$

Abb. 10 gibt einen zusammenfassenden Überblick über die wesentlichen Schritte zur Bildung einer messespezifischen Zielkonzeption von Ausstellern.

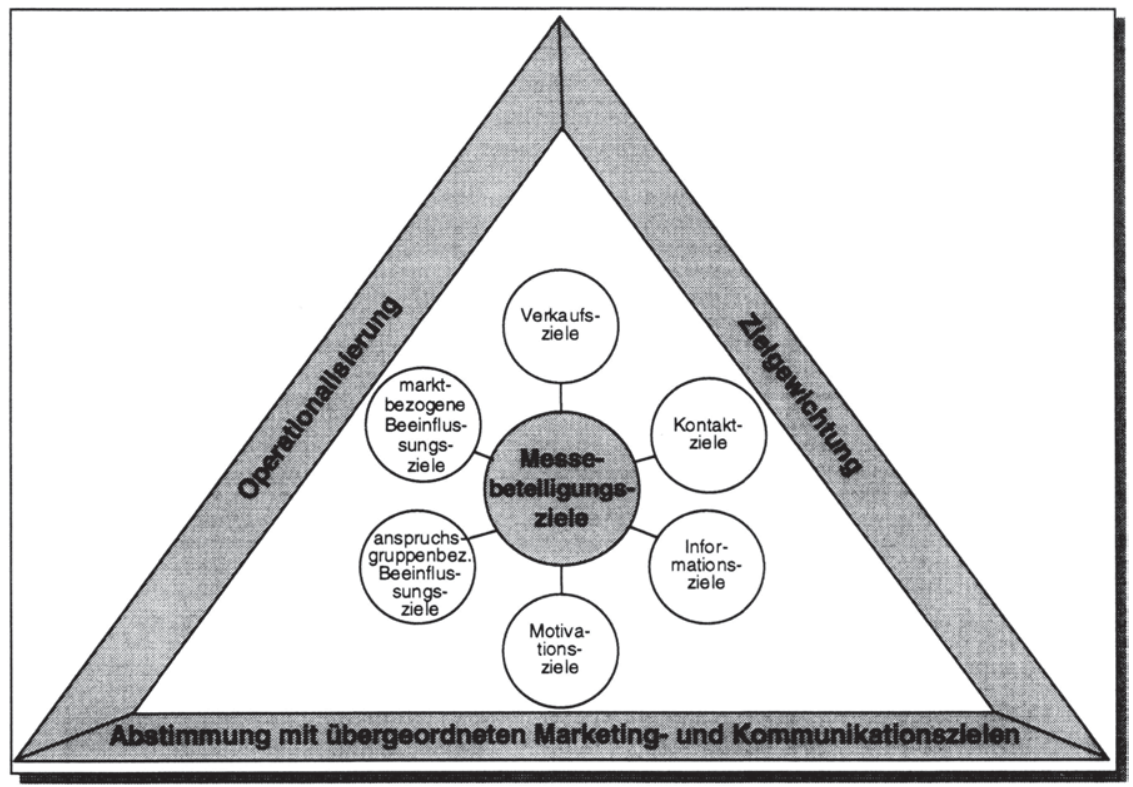

Abb. 10: Bestandteile der messespezifischen Zielkonzeption von Ausstellern

163 Vgl. bzgl. des Zusammenhangs operational formulierter Ziele und der Erfolgskontrolle die Ausführungen in Kap. C. 1.31 dieser Arbeit. 
Vor dem Hintergrund der in diesem Kapitel getroffenen theoretischen Aussagen und der vorliegenden empirischen Befunde lassen sich die folgenden Basishypothesen im Rahmen der weitergehenden empirischen Untersuchung einer Überprüfung unterziehen:

$\mathrm{H}_{\mathrm{zk} 1}$ : Ausstellende Unternehmen verfolgen auf Messen eine Vielzahl verschiedener Zielsetzungen, die zu eigenständigen und voneinander unabhängigen Hauptzielkategorien zusammengeführt werden können.

$\mathrm{H}_{\mathrm{zk} 2}$ : Ausstellende Unternehmen setzen bei der Festlegung der Messe-Zielkonzeption verschiedene Prioritäten. Es lassen sich aber generelle Zielmuster identifizieren, die sich signifikant voneinander unterscheiden und damit zur Beschreibung der messespezifischen Grundhaltungstypen einen wesentlichen Beitrag leisten. 


\subsection{Messe-Selektionskonzeption}

Die Selektion von Messen umfaßt einen mehrstufigen Entscheidungsproze ${ }^{164}$, der in Abb. 11 dargestellt ist. Die Erfassung verschiedener Veranstaltungsalternativen stellt die erste Stufe der Messeselektion dar. Dabei ist aufgrund der wachsenden Vielzahl von Messeveranstaltungen im In- und Ausland einerseits und begrenzter Informationsverarbeitungskapazitäten sowie des unvollkommenen Wissensstandes der Entscheider über das gesamte Messeangebot andererseits davon auszugehen, daß nicht alle, sondern nur eine begrenzte Zahl von Alternativen berücksichtigt werden kann. ${ }^{165}$ Bei der Zusammenstellung der relevanten Messeveranstaltungen vermögen insbesondere die Veröffentlichungen des AUMA zum Messeplatz Deutschland sowie zu ausgewählten Messeveranstaltungen im Ausland die Transparenz aus Ausstellersicht zu erhöhen. ${ }^{166}$ In diesem ersten Schritt der Messeselektion geht es zunächst um die Bestimmung der generellen thematischen bzw. inhaltlichen Affinität zwischen Messeveranstaltung und Aussteller. ${ }^{167}$

164 Vgl. Bamberg. G. Coenenberg. A. G., Betriebswirtschaftliche Entscheidungslehre, 3. Aufl., München 1991, S. 14 ff.; Saliger. E, Betriebswirtschaftliche Entscheidungstheorie. Eine Einführung in die Logik individueller und kollektiver Entscheidungen, 2. Aufl., München, Wien 1988, S. 2 ff. und die dort jeweils angegebene Literatur.

Vgl. Pfohl, H.-C.. Braun, G, E., Entscheidungstheorie. Normative und deskriptive Grundlagen des Entscheidens, Landsberg/Lech 1981, S. 90.

Vgl. die in Fußnote 24 in Kapitel A angeführte Literatur des AUMA zu den national und international stattfindenden Messeveranstaltungen.

167 Unter der thematischen bzw. inhaltlichen Affinität zwischen Messe und Aussteller soll bspw. die Abdeckung eines bestimmten Wirtschaftsbereiches, dem der Aussteller auch angehört, oder aber Problembereiche, die durch die betreffende Veranstaltung angesprochen werden, zu deren Lösung auch der Aussteller durch sein spezifisches Produktangebot beizutragen vermag, verstanden werden. Vgl. zu einer ähnlichen Sichtweise Funke. K., Messeentscheidungen. Handlungsalternativen und Informationsbedarf, a.a.O., S. $47 \mathrm{f}$. 


\section{Bestimmung der Veranstaltungsalternativen}

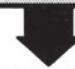

\section{Beschreibung der Veranstaltungsalternativen anhand ihrer zentralen Veranstaltungseigenschaften}

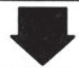

Bewertung der Veranstaltungsalternativen

Abb. 11: Entscheidungsprozeß der Messeselektion (in Anlehnung an Fließ, S., Messeselektion. Entscheidungskriterien für Investitionsgüteranbieter, Wiesbaden 1994, S. 18)

Im zweiten Schritt des Entscheidungsprozesses sind die Veranstaltungsalternativen anhand ihrer zentralen Eigenschaften ${ }^{168}$ einzuordnen bzw. zu charakterisieren, um auf diese Weise die Vielzahl unterschiedlich fokussierter Messeveranstaltungen unter spezifischen Gesichtspunkten, die im folgenden näher erörtert werden sollen, zu bewerten.

Zur Beschreibung der Eigenschaften von Messeveranstaltungen wurden bislang in der Literatur hauptsächlich die bereits in Kapitel A. 2. genannten Abgrenzungskriterien von Messen "geographische Reichweite“, „Breite des Angebots" bzw. „Angebotsschwerpunkt" herangezogen ${ }^{169}$, um auf der Basis von Veranstaltungstypologien eine Charakterisierung und Selektion von Messeveranstaltungen vorzunehmen. Abb. 12 enthält die zwei im deutschen Sprachraum wesentlichen Typologisierungsansätze. Zusätzlich existiert eine Kategorisierung von Messen, die die

168 Strothmann und Roloff verwenden statt dessen den Begriff "Charakter" von Messen. Vgl. Strothmann, K.-H. Roloff, E., Charakterisierung und Arten von Messen, a.a.O., S. $714 \mathrm{ff}$. 


\section{Typologie der Fachmessen}

\begin{tabular}{|c|c|c|c|}
\hline $\begin{array}{c}\text { Ver- Reichweite } \\
\text { anstatungs- } \\
\text { charakter }\end{array}$ & International & National & Regional \\
\hline $\begin{array}{c}\text { Solo- bzw. } \\
\text { Monomesse }\end{array}$ & & & \\
\hline $\begin{array}{c}\text { Verbund- } \\
\text { messe }\end{array}$ & & & \\
\hline $\begin{array}{c}\text { Universal- } \\
\text { messe }\end{array}$ & & & \\
\hline
\end{tabular}

Quelle: Meffert. $\mathrm{H}_{2}$, Messen und Ausstellungen als Marketinginstrument, in: Verlag Wirtschaft und Finanzen (Hrsg.), Messen als Marketinginstrument, Düsseldorf 1988, S. 11

\section{Messe-Typologie}

\begin{tabular}{|c|c|c|}
\hline $\begin{array}{c}\text { Breite } \\
\text { des Angebotes }\end{array}$ & $\begin{array}{c}\text { Investitions- } \\
\text { güter }\end{array}$ & \multirow{2}{*}{$\begin{array}{c}\text { Konsum- } \\
\text { güter }\end{array}$} \\
\hline Fachmesse & fachtechnisch & \\
\hline & anwendungstechnisch & \\
\hline $\begin{array}{c}\text { Branchen- } \\
\text { messe }\end{array}$ & & \\
\hline $\begin{array}{c}\text { Verbund- } \\
\text { messe }\end{array}$ & & \\
\hline
\end{tabular}

Quelle: Roloff, E., Messen und Medien, Ein sozialpsychologischer Ansatz zur Öffentlichkeitsarbeit, Wiesbaden 1992, S. 12

Abb. 12: Messetypologische Ansätze 
FKM nach den drei Hauptgruppen „Mehrbranchenmessen“, „Fachmessen“ und „Verbraucherausstellungen“ für ihre Berichterstattung unterscheidet. ${ }^{170}$

Die Wahl der Unterscheidungskriterien ist dabei allerdings nicht unproblematisch. Zum Teil sind die Kriterien bei Ausstellern und Besuchern von unterschiedlicher Bedeutung und oft von überhöhtem Anspruch. ${ }^{171}$ Darüber hinaus ist festzustellen, daß eine Vielzahl von Überschneidungsmöglichkeiten vorhanden ist, so daß die eindeutige Zuordnung realer Veranstaltungen zu den genannten Messetypen häufig nur tendenziell möglich erscheint. ${ }^{172}$ Damit ist deren Eignung, als Basis für die Einordnung und Selektion von Messeveranstaltungen zu dienen, als unzulänglich anzusehen.

In dieser Untersuchung wird daher in Anlehnung an eine Messe-Kategorisierungskonzeption von Strothmann und Roloff der Versuch unternommen, „zutreffende messecharakterisierende Merkmalspaare zu entwickeln und diese zur realitätsnahen Beschreibung von Messen heranzuziehen. Dabei wird davon ausgegangen, $\mathrm{da} ß$ keines der einzelnen im folgenden angeführten Merkmale vollständig auf eine Messe zutrifft. Es ist vieimehr zu sehen, daß es sich dabei um Eigenschaften (von Messeveranstaltungen, Anm. d. Verf.) handelt, die .... bei jeder Messe eine mehr oder weniger starke Ausprägung haben (aus Sicht des Ausstellers, Anm. d. Verf.). Aus diesem Grund ist zwischen den Merkmalen, die zu einem Merkmals-paar gehören, gedanklich eine Skala eingebracht. Diese Skala bietet die Möglich-keit,

170 Es werden darüber hinaus noch branchenbezogene Untergruppen von Messen gebildet, bspw. im Bereich der Fachmessen „Messen für Bau, Ausbau und deren Ausrüstungen“ bzw. "Transport, Verkehr und deren Ausrüstungen" etc. Vgl. FKM-Gesellschaft zur Freiwilligen Kontrolle von Messe- und Ausstellungszahlen e. V. (Hrsg.), Bericht 1990, Bergisch Gladbach 1991, S. 31.

171 Dies gilt in besonderem Maße für das Kriterium der Internationalität von Messen. Groth weist zu Recht darauf hin, daß sich viele Messen als "international“ bezeichnen, die bei der Analyse der Besucherstruktur aus Sicht der Aussteller bestenfalls nationalen Charakter für sich reklamieren können. Des weiteren macht er deutlich, daß dieses Selektionskriterium für Messen nicht geschützt ist. Vgl. Groth, C., Das Leistungspotential von Fachmessen, a.a.O., S. 59. Allein die Mitglieder der FKM - Gesellschaft zur Freiwilligen Kontrolle von Messe- und Ausstellungszahlen $\boldsymbol{e}$. V. - haben hierfür in ihren allgemeinen Regeln eine Übereinkunft getroffen: "Eine Veranstaltung darf die Bezeichnung „international“ nur führen, wenn mindestens 10 Prozent der Aussteller ausländische Aussteller sind“. Vgl. FKM-Gesellsschaft zur Freiwilligen Kontrolle von Messe- und Ausstellungszahlen e. V. (Hrsg.), Bericht 1990, a.a.O., S. 8. Diese Vereinbarung hat aber lediglich Gültigkeit für diejenigen Messen, die von einem Mitglied bei der FKM angemeldet worden sind. Eine Anmeldungspflicht besteht hingegen nicht.

172 Sog. Fachmessen sind meist - wenngleich auch nur an ausgewählten Messetagen - auch dem Privatpublikum geöffnet und umgekehrt.

Ralf Ueđing and Universität Münster - 978-3-631-75073-5 
durch die Wahl eines Skalenwertes die jeweilige Merkmalsausprägung für jede Messe zu bestimmen“. ${ }^{173}$

Den Ausgangspunkt bei der Entwicklung des Merkmalssystems bildet eine grundlegende Einteilung der Messen nach den hauptsächlich auf innen ausgestellten Güterarten. Danach werden Investitionsgüter-, Konsumgüter- und Dienstleistungsmessen unterschieden. ${ }^{174} \mathrm{Da}$ es diese Messen in "Reinkultur" nicht gibt, wird das folgende Merkmalspaar zur Beschreibung von Messen herangezogen:

\section{Homogenităt versus Heterogenität des Ausstellungsprogramms}

Die Differenzierungsmöglichkeit zwischen den Extrempunkten ermöglicht die Untersuchung des Spezialisierungsgrades bzw. der Spannweite des Güterangebots bzw. Ausstellungsprogramms der Messen, die von den Ausstellern für eine Beteiligung ausgewählt werden. ${ }^{175}$ Dabei soll für die empirische Analyse weniger die Frage im Vordergrund stehen, welche Güterarten im einzelnen auf der jeweiligen Messe vertreten waren, sondern vielmehr die subjektive Einschätzung der Breite der angebotenen Produktpalette insgesamt, um vor allem einen Eindruck von der Vielfalt bzw. Dichte des jeweiligen Messeangebots zu erhalten.

Ein weiteres Merkmalspaar soll Aufschluß über die gewünschten Wirkungen beim Besucher einer Messeveranstaltung geben. Hierbei wird zwischen den Prioritäten des Verkaufens und des Informierens unterschieden, so daß sich folgendes Merkmalspaar ergibt ${ }^{176}$ :

\section{Verkaufs- versus Informationsorientierung der Messe}

173 Damit machen die Autoren deutlich, daß mit den erwähnten Merkmalspaaren nicht dichotome Variablen, sondern jeweils Extremalausprägungen auf einem "Kontinuum" zu verbinden sind. Vgl. Strothmann, K.-H.. Roloff, E., Charakterisierung und Arten von Messen, a.a.O., S. 717.

174 Vgl. Strothmann, K.-H. Roloff, E., Charakterisierung und Arten von Messen, a.a.O., S. 717 f.

175 Vgl. Strothmann, K.-H. Roloff, E., Charakterisierung und Arten von Messen, a.a.O., S. 718.

176 Es sei an dieser Stelle nochmals darauf verwiesen, daß das angeführte Merkmalspaar zur Beschreibung der Orientierung einer Messeveranstaltung, nicht eines Ausstellers, herangezogen wird. Vgl. hierzu auch Meffert. $H_{\text {. }}$, Messen und Ausstellungen als Marketinginstrument, a.a.O., S. 77 . 
Anhand des nachstehenden Begriffspaares kann eine Messeveranstaltung danach eingeordnet werden, ob sie primär auf den Besuch von Fachpublikum ausgerichtet ist oder eher für Privatbesucher konzipiert wurde. Die stärkere Fachbesucher- bzw. Privatbesucherorientierung hat vor dem Hintergrund der mit einer Messebeteiligung verbundenen Zielgruppenansprache von Ausstellern erheblichen Einfluß auf die Messeselektion:

\section{Fachpublikum versus Privatpublikum}

Wie bereits dargestellt, nehmen zahlreiche Messen das Attribut der Internationalität für sich in Anspruch. ${ }^{177}$ Grundsätzlich kann zunächst die Analyse der Besucherschaft Indizien für die Internationalität der Messe liefern. Gleiches gilt aber auch für die Zusammensetzung der Ausstellerschaft. Dabei steht im Zusammenhang mit der vorliegenden Untersuchung nicht die Klärung der Frage im Vordergrund, ab welchem "prozentualen Anteil" ausländischer Besucher oder Aussteller die Bezeichnung "international" objektiv angebracht erscheint. Vielmehr soll analysiert werden, wie die Aussteller die von ihnen präferierten Messeveranstaltungen bezüglich des Attributes der "Internationalität" einschätzen und inwieweit dieses Relevanz für die Messeselektion besitzt. Damit ergeben sich die folgenden zu untersuchenden Merkmalspolaritäten:

\section{Nationalität versus Internationalität der Messebesucher}

und

\section{Nationalität versus Internationalität der Aussteller}

Mit einem weiteren Merkmalspaar ist dem Tatbestand Rechnung zu tragen, daß immer mehr Messeveranstalter Kongreß- und Tagungsräumlichkeiten einrichten und damit den Ausstellern die Möglichkeit bieten, anläßlich einer Messe eigene

177 Vgl. Groth, C., Determinanten der Veranstaltungspolitik von Messegesellschaften, in: Strothmann, K.-H., Busche, M. (Hrsg.), Handbuch Messemarketing, Wiesbaden 1992, S. 167 f.; o.V., Zuwachs im Internationalen, in: $m$ + a Report, März 1996, S. 23 f.; o.V., Impulsgeber für Warenströme, in: ZAW-service, Nr. 185, 1995, S. 18 f.; o.V., Das deutsche Messewachstum kommt heute aus dem Ausland, in: FAZ, Nr. 280, vom 1.12.1995, S. 30. 
Begleitveranstaltungen durchzuführen. ${ }^{178}$ Die Bedeutung, die derartige Begleitveranstaltungen haben, kann bei einzelnen Messen sehr unterschiedlich sein. So gibt es Messen, bei denen der Kongreß dominiert und die Ausstellung von Produkten und Dienstleistungen lediglich als ein Kongreßbestandteil anzusehen ist. ${ }^{179}$ Bei anderen Messeveranstaltungen genießt die eigentliche Messe absolute Priorität, die ausstellenden Unternehmen erhalten jedoch die Möglichkeit, Vortragsveranstaltungen, Symposien oder auch Empfänge in dafür bereitgestellten Gebäudefaszilitäten durchzuführen. Der zunehmende Stellenwert von Begleitveranstaltungen bei Messeveranstaltungen läßt es daher notwendig erscheinen, Messen auch unter dem Aspekt zu selektieren, welchen Raum Begleitveranstaltungen einnehmen:

\section{Viele versus keine Begleitveranstaltungen}

Anhand der aufgeführten Merkmalspaare kann ein Aussteller die zur Auswahl stehenden Messeveranstaltungen grob einordnen und zunächst diejenigen Messen auswählen, die den gewünschten Merkmalen (z. B. Zielgruppenbezug, angestrebte Präsenz im internationalen oder nationalen Wettbewerbsumfeld, Durchführung einer vom Messestand getrennten Diskussionsveranstaltung mit wichtigen Kunden und Meinungsführern etc.) in vollem Umfang bzw. weitestgehend entsprechen.

Daran anschließend ist in einem dritten Schritt eine Einzelbewertung der ausgewählten Veranstaltungsalternativen vorzunehmen. Die einzelnen Veranstaltungsalternativen sind anhand der aufgrund ihrer Veranstaltungsstruktur zu erwartenden Zielerreichungsbeiträge zu bewerten und in eine Rangfolge zu bringen. ${ }^{180}$

In der Literatur werden hierfür verschiedene Verfahren zur Messebewertung vorgeschlagen. ${ }^{181}$ Zum Teil handelt es sich um solche Modelle, die speziell für die

178 Vgl. Selinski, H., Begleitveranstaltungen von Messen, in: Strothmann, K.-H., Busche, M. (Hrsg.), Handbuch Messemarketing, Wiesbaden 1992, S. 486 ff.; Strothmann, K.-H., Verbundveranstaltungen des Messe- und Kongreßwesens im Investitionsgüter-Marketing, in: Rost, $D$. Strothmann, K.-H. (Hrsg.), Handbuch der Werbung für Investitionsgüter, Wiesbaden 1983, S. $391 \mathrm{ff}$.

179 Vgl. Winkler, K.-A., Kongreß mit Ausstellung, in: Strothmann, K.-H., Busche, M. (Hrsg.), Handbuch Messemarketing, Wiesbaden 1992, S. $502 \mathrm{ff}$.

180 Vgl. Fließ. S., Messeselektion. Entscheidungskriterien für Investitionsgüteranbieter, a.a.O., S. $19 \mathrm{f}$.

181 Auf eine eingehende Darstellung der folgenden Ansätze zur Messebewertung soll im Rahmen dieser Arbeit verzichtet werden. Hinsichtlich spezifischer Informationen zu den einzelnen Verfahren sei auf die jeweils angegebene Literatur verwiesen. 
Messebewertung entwickelt worden sind, des weiteren existieren auch Ansätze, bestehende Bewertungsmodelle angrenzender Bereiche auf das Messebewertungsproblem zu übertragen. Zur Gruppe der spezifischen Messebewertungsmodelle zählen Checklisten zur Messeauswahl ${ }^{182}$, Punktbewertungsmodelle zur Messeselektion ${ }^{183}$, Prognosemodelle des Messeerfolges ${ }^{184}$ sowie Portfoliomodelle ${ }^{185}$ und Korrespondenzanalysen. ${ }^{186} \mathrm{Zu}$ den Adaptionsmodellen zählen die Investitionsrechenverfahren ${ }^{187}$ und das Mediaselektionsmodell. ${ }^{188}$

Der Einsatz mathematischer Verfahren (Investitionsrechenverfahren, Prognosemodelle des Messeerfolgs, Mediaselektionsmodell) scheitert vielfach an der hierfür erforderlichen Datenbasis. So lassen sich bei der Anwendung von Investitionsrechenverfahren die messebeteiligungsspezifischen Ein- und Auszahlungen nur mit erheblichen Unschärfen bestimmen. ${ }^{189}$ Prognosemodelle beruhen i. d. R. auf Vergangenheitsdaten und entfalten häufig nur dann prognostischen Charakter, wenn keine wesentlichen Veränderungen der Umfeldbedingungen eintreten. ${ }^{190} \mathrm{Da}$ Messen aber teilweise in längeren Zyklen veranstaltet werden, besitzen Prognosemodelle für die Bewertung und Selektion von Messen nur einen geringen Aussagegehalt.

182 Vgl. Schober. P. M., Messen und Ausstellungen: Teilnehmen oder wegbleiben? - Eine kleine Entscheidungshilfe; a.a.O., S. 400-402; Hentschel. M., So können auch mittelständische Unternehmen auf Auslands-Messen gehen, in: Marketing Journal, 20. Jg., Heft 3, 1987, S. 248-253; Spryß, W. Einzelaspekte der Messe- und Ausstellungswerbung, in: Tietz. B. (Hrsg.), Die Werbung - Handbuch der Kommunikations- und Werbewirtschaft, Bd. 2, Landsberg/Lech 1982, S. 1639-1662; Rasche. H. O., Wie man Messe-Erfolge programmiert, Praktiker-Checkliste, Heiligenhaus 1980.

Vgl. Berghäuser, B., Messen als Entscheidungsproblem, a.a.O., S. 9 ff.; Detroy, E. N., So organisieren Sie eine verkaufsaktive Messe, in: asw, 15. Jg., Nr. 21/22, 1982, S. 120-125.

Vgl. Fritsch, S., Prognosemodell für Konsumgütermessen, Berlin 1979; Funke, K., Messeentscheidungen. Handlungsalternativen und Informationsbedarf, a.a.O., S. $354 \mathrm{ff}$.

Funke. K. Messeentscheidungen. Handlungsalternativen und Informationsbedarf, a.a.O., S. $261 \mathrm{ff}$.; Fließ. S., Messeselektion. Entscheidungskriterien für Investitionsgüteranbieter, a.a.O., S. $198 \mathrm{ff}$.

Fließ. S., Messeselektion. Entscheidungskriterien für Investitionsgüteranbieter, a.a.O., S. 206 $\mathrm{ff}$.

Vgl. Raffée. H., Messen als Herausforderung für die Marketing-Theorie, a.a.O., S. 81 f.

Vgl. Schwenzner, J. M., Messen und Ausstellungen im Kreuzfeuer der Begriffe Reichweitenanalyse und Messeerfolgskontrolle, in: GfK-Mitteilungen zur Markt- und Absatzforschung, 18. Jg., Heft 1, 1972, S. 10-20.

Vgl. Raffée. H., Messen als Herausforderung für die Marketing-Theorie, a.a.O., S. 81.

Vgl. Meffert. H. Steffenhagen, H., Marketingprognosemodelle - Quantitative Grundlagen des Marketing, a.a.O., S. $146 \mathrm{ff}$. 
Die im Rahmen von Mediaselektionsmodellen benötigten Daten - wie z. B. Reichweiten, Reichweitenüberschneidungen und Anzahl der Kontakte - sind bei Messeveranstaltungen meist nicht verfügbar. Der Turnus von Messen spricht darüber hinaus mit einer hohen Wahrscheinlichkeit für eine sich ändernde Besucherstruktur. ${ }^{191}$ Auch die Vergleichbarkeit der zur Verfügung stehenden Besucherdaten ist aufgrund unterschiedlicher Erfassungsmethoden häufig nicht gegeben ${ }^{192}$, so daß Mediaselektionsmodelle derzeit kaum zur Bewertung und Auswahl von Messen herangezogen werden können. ${ }^{193}$

Letztlich bleiben damit Checklisten bzw. Punktbewertungsmodelle für die praktische Anwendung zur Messebewertung relevant. Während Checklisten lediglich die Aufgabe haben, alle entscheidungsrelevanten Faktoren für eine Messebewertung aufzulisten und damit für eine entsprechende Informationssammlung zu sorgen, geben Punktbewertungsmodelle darüber hinausgehende Entscheidungshilfen. Üblich ist bei Punktbewertungsmodellen ein mehrstufiges Vorgehen. Die Analysekriterien sind dabei unternehmensindividuell festzulegen und $\mathrm{zu}$ gewichten. Zunächst werden diejenigen Messen aussortiert, die den unabdingbaren Kriterien nicht genügen. Diesen sog. „Musts" einer Messebeteiligung sind primär die situativen Bedingungen, wie z. B. Erfordernisse der Wettbewerbssituation (Imageschäden bei Nichtbeteiligung), Substitutionsmöglichkeiten der Messebeteiligung durch andere Marketinginstrumente sowie messespezifische Gegebenheiten (Verfügbarkeit von quantitativ und qualitativ hinreichenden Standflächen), zuzuordnen.

Die folgende Analyse enthält hauptsächlich Kriterien, die das Umfeld der potentiellen Messebeteiligung direkt betreffen. Ansetzend bei den Messebeteiligungszielen und -zielgruppen sind zusätzlich bspw. die Messekosten und damit Budgetrestriktionen zu bewerten, aber auch die Serviceleistungen sowie das Marketing der Messeveranstalter im Vorwege der Veranstaltung können als Bewertungskriterien hinzugezogen werden. Neben der Tatsache, daß keine theoretisch richtigen Bewertungsgewichte festgelegt werden können, ist des weiteren darauf hinzuweisen, daß auch über die Anzahl der zu berücksichtigenden Bewertungskriterien

191 Vgl. Rominski.D., Neue Besucherwünsche, in: Asw, Heft 6, 1995, S. 96-100.

192 Der FKM hat zwar einheitliche Erhebungsstandards entwickelt, aber die Messeveranstalter sind nicht gezwungen, die Messeveranstaltungen nach diesem Ansatz überprüfen zu lassen. Vgl. hierzu auch die Ausführungen in FN 171 der vorliegenden Arbeit.

193 Vgl. Mortsiefer, J., Die Intuition alleine reicht nicht - Messebeteiligungen auf der Basis eines Nutzungs-Konzeptes, a.a.O. S. 17 f. 
keine Aussagen gemacht werden können. Dennoch stellt das Punktbewertungsverfahren unter den gegebenen Umständen das flexibelste und praxisorientierteste Modell der Messebewertung dar. ${ }^{194}$

Innerhalb der gegebenen Budgetrestriktionen sind im letzten Schritt diejenigen Messeveranstaltungen auszuwählen, an denen sich das Unternehmen aufgrund eines positiven Bewertungsergebnisses als Aussteller beteiligen wird. ${ }^{195}$ Das Ergebnis der Messeselektion von Ausstellern soll im Rahmen dieser Arbeit anhand der vorgestellten messecharakterisierenden Merkmale festgehalten werden. Abb. 13 stellt die Merkmale, anhand derer die Messe-Selektionskonzeption der ausstellenden Unternehmen dargestellt werden soll, zusammenfassend dar.

\begin{tabular}{|c|lll|}
\hline $\begin{array}{c}\text { Ausstellungs- } \\
\text { programm } \\
\text { kriterien }\end{array}$ & homogen \\
\hline $\begin{array}{c}\text { Grundorientierung } \\
\text { der Messe }\end{array}$ & $\begin{array}{l}\text { verkaufs- } \\
\text { orientiert }\end{array}$ \\
\hline Messebesucher & $\begin{array}{l}\text { Fach- } \\
\text { publikum }\end{array}$ \\
\hline $\begin{array}{c}\text { Einzugsberelch bel } \\
\text { Messebesuchern }\end{array}$ & $\begin{array}{l}\text { national, } \\
\text { regional }\end{array}$ \\
\hline $\begin{array}{c}\text { Einzugsbereich } \\
\text { bei Ausstellern }\end{array}$ & $\begin{array}{l}\text { national, } \\
\text { regional }\end{array}$ \\
\hline $\begin{array}{c}\text { Durchführung } \\
\text { von Begleit- } \\
\text { veranstaltungen }\end{array}$ & viele & \\
\hline
\end{tabular}

Abb. 13: Operationalisierungsansatz zur Erfassung der Messe-Selektion

Einen ersten Überblick über die Struktur der von den befragten Ausstellern im Rahmen ihrer Messe-Selektion ausgewählten Messeveranstaltungen gibt Abb. 14. Wie aus dem Mittelwertprofil ersichtlich wird, nehmen die Ausstellerunternehmen in der Tendenz eine deutliche Fachbesucherorientierung bei den von innen selek-

\footnotetext{
194 Vgl. Berghäuser, B., Messen als Entscheidungsproblem, a.a.O., S. 9.

195 Vgl. Fließ. S., Messeselektion. Entscheidungskriterien für Investitionsgüteranbieter, a.a.O., S. $19 \mathrm{f}$.
} 
Messen in Deutschland wahr. Ferner sind die Messen eher informations- als verkaufsorientiert und stark international ausgerichtet - sowohl auf der Besucherals auch verstärkt auf der Ausstellerseite. Bezüglich der Breite des Ausstellungsprogramms besteht die Tendenz zur Auswahl von Messeveranstaltungen mit eher homogener Struktur. Weitgehende Indifferenz zeigt sich hingegen hinsichtlich der Intensität von Begleitveranstaltungen im Rahmenprogramm. Insgesamt zeigen die relativ hohen Standardabweichungen über alle genannten Variablen der Messeselektion, daß in das vorgestellte Mittelwertprofil Messekonzepte mit einer $\mathbf{z}$. $T$. deutlich unterschiedlichen Veranstaltungsstruktur eingehen.

Vor dem Hintergrund der theoriegeleiteten Ausführungen läßt sich folgende Basishypothese zur Messeselektion formulieren, die im Rahmen der weiteren empirischen Analyse einer Überprüfung unterzogen werden soll:

$\mathrm{H}_{\text {sk: }}$ : Ausstellende Unternehmen setzen im Rahmen ihrer Messeselektion in Abstimmung zwischen vorhandenem Messeangebot und ihren messespezifischen Zielsetzungen verschiedene Prioritäten. Es lassen sich allerdings generelle Selektionsmuster identifizieren, die sich signifikant voneinander unterscheiden und zur Beschreibung der messespezifischen Grundhaltungstypen einen Beitrag zu leisten vermögen.

\begin{tabular}{|c|c|c|c|c|}
\hline $\begin{array}{l}\text { Messetypbestimmende } \\
\text { Merkmale }\end{array}$ & \begin{tabular}{|l|} 
Standard- \\
abwol- \\
chung
\end{tabular} & & Mitteluerte & \\
\hline Ausstellungsprogramm & 1,28 & homogen & & heterogen \\
\hline Grundorientierung der Messe & 1,24 & $\begin{array}{l}\text { verkauts- } \\
\text { orientiert }\end{array}$ & & $\begin{array}{l}\text { Informa- } \\
\text { tionsorlen- } \\
\text { tlert }\end{array}$ \\
\hline Messebesucher & 1,17 & $\begin{array}{l}\text { Fachpub- } \\
\text { llikum }\end{array}$ & & $\begin{array}{l}\text { Prvat- } \\
\text { publikum }\end{array}$ \\
\hline $\begin{array}{l}\text { Einzugsbereich der } \\
\text { Messebesucher }\end{array}$ & 1,31 & $\begin{array}{l}\text { national, } \\
\text { regional }\end{array}$ & & $\begin{array}{l}\text { Inter: } \\
\text { national }\end{array}$ \\
\hline $\begin{array}{l}\text { Einzugsbereich der } \\
\text { Aussteller }\end{array}$ & 1,16 & $\begin{array}{l}\text { national, } \\
\text { regional }\end{array}$ & & $\begin{array}{l}\text { Inter } \\
\text { national }\end{array}$ \\
\hline $\begin{array}{l}\text { Durchführung von } \\
\text { Begleitveranstaltungen }\end{array}$ & 1,26 & viele & & keine \\
\hline
\end{tabular}

Abb. 14: Selektionsprofil von Messen in Deutschland 


\subsection{Messe-Positionierungskonzeption}

Messebeteiligungen weisen nicht nur die Möglichkeit einer Positionierung und kommunikativen Profilierung des Ausstellers oder seines Leistungsangebotes auf, sondern verlangen diese vielmehr als notwendige Voraussetzung für ein erfolgreiches Messeengagement. ${ }^{196}$ Aus der potentialorientierten Sichtweise bieten Messen für die Aussteller die Möglichkeit, an ihren Messeständen das Ergebnis inrer Produktpolitik und Forschungs- bzw. Innovationsaktivitäten zu demonstrieren. Des weiteren sind Aussteller in der Lage, die Botschaften der kontinuierlich betriebenen Kommunikationsaktivitäten in die Konzeption des Messestandes zu integrieren. Nicht zuletzt deshalb gehen Strothmann und Roloff davon aus, daß mit Messebeteiligungen die "Marketingbilanz" des Unternehmens präsentiert werden kann. Sie zeigen auf, daß „sämtliche im Marketing-Mix vereinigten Instrumente, die auch im Marketingalltag angewendet werden, in optimaler Abstimmung das Geschehen am Messestand prägen. Letztlich ist der Messestand die Plattorm, auf der Unternehmen die Inhalte ihrer Corporate Identity ausbreiten können". ${ }^{197}$

Wenngleich zahlreiche Unternehmen im Rahmen der Werbung und anderer Kommunikationsinstrumente den Versuch unternehmen, ihre Außenwahrnehmung und insbesondere die ihrer Produkte und Leistungen i. S. einer strategischen Positionierung bewußt und unternehmensindividuell zu steuern ${ }^{198}$, kann dieses Fazit für Messebeteiligungen überwiegend nicht gezogen werden. Vielmehr wird unter Hinweis auf Erfahrungen von Messe-Experten und Eindrücken von Messebesuchern immer wieder darauf aufmerksam gemacht, daß der Messeauftritt von ausstellenden Unternehmen vielfach ähnlich ist und damit kaum zur Differenzierung vom Wettbewerb und zur Profilierung bei Messebesuchern beizutragen vermag. ${ }^{199}$ Dies verwundert um so mehr, als Aussteller sich auf Messen

196 Vgl. Stibi. H. D., Messen und Ausstellungen - Auf dem Weg ins Kommunikations-Mix, in: Marketing Journal, Heft 1, 1983, S. 66.

197 Vgl. Strothmann, K.-H., Roloff, E., Charakterisierung und Arten von Messen, a.a.O., S. 712.

198 Vgl. die zahlreichen Beispiele bei Antonoff, R., Cl-Report '92: Das Jahrbuch vorbildlicher Cl, Darmstadt 1992; Raffeé. H. Wiedmann. K.-P., Corporate Identity als strategische Basis der Marketingkommunikation, in: Berndt. R.. Hermanns. A. (Hrsg.), Handbuch Kommunikation, Wiesbaden 1993, S. 43-67 sowie Tomczak. T. Reinecke, S., Die Rolle der Positionierung im strategischen Marketing, in: Thommen, J. P. (Hrsg.), Management-Kompetenz, Zürich 1995 , S. 499-517.

199 Vgl. Fröhder. St., Was passiert wirklich auf der Messe? - zweifeln Sie mal wieder, in: Marketing Journal, Heft 1, 1995, S. 47. Roth weist darauf hin, daß "es gilt, eine eigene Form zu finden; denn nichts ist werbend, was sich anpaßt, sondern nur das, was sein eigenes Profil behauptet". Vgl. Roth, G. D., Messen und Ausstellungen verkaufswirksam planen und durchführen, a.a.O., S. 105

Ralf Ueding and Universität Münster - 978-3-631-75073-5 
meist in einem außergewöhnlich dichten Wettbewerbsumfeld bewegen und die Messebesucher somit die Möglichkeit zu einem direkten Konkurrenzvergleich haben.

Allerdings macht auch eine Durchsicht der aktuellen, insbesondere der angloamerikanischen Literatur zum Management von Messebeteiligungen deutlich, daß dem Aspekt der Positionierung von Ausstellern bei Messeveranstaltungen bislang kaum Aufmerksamkeit geschenkt wurde. ${ }^{200}$ Daher erscheint es angebracht, in Anlehnung an die in der Literatur vorhandenen Ansätze zur Positionierung eine Messe-Positionierungskonzeption für Aussteller zu entwickeln, die verschiedene grundlegende Ansatzpunkte zur Positionierung und Profilierung auf Messen enthält.

Die Einordnung eines Ausstellers im Wahrnehmungsraum der Messebesucher hat eine erhebliche Auswirkung auf den Markterfolg eines Angebotes. ${ }^{201}$ Dabei stellt die Positionierung kein Planungsproblem dar, das "uno actu“ zu lösen ist, vielmehr handelt es sich um ein sequentielles Entscheidungsproblem, das sich in zwei Entscheidungsstufen unterscheiden läßt. ${ }^{202}$ Auf strategischer Ebene ist zunächst eine Entscheidung hinsichtlich der angestrebten Messeposition zu treffen. In diesem Zusammenhang ist die Frage des grundsätzlichen Messeauftritts zu beantworten. Hierbei sind insbesondere der generellen Unternehmens- und Produktpositionierung sowie der Übereinstimmung mit der anzustrebenden Messeposition ein besonderer Stellenwert einzuräumen. Auf der operativen Ebene ist schließlich der Schwerpunkt des Instumenteeinsatzes festzulegen, der sich aus dem Instrumente-Mix Standgestaltung, Exponate, Personaleinsatz und Kommunikationsmaßnahmen zusammensetzt. ${ }^{203}$

200 Nur wenige Autoren weisen auf die Notwendigkeit der messespezifischen Positionierung von Ausstellern als Bestandteil der strategischen Planung von Messebeteiligungen direkt hin. Vgl. Fuchs. W. A., Die Messe als KommunikationsprozeB, a.a.O., S. 99; Krone. K. Huber. B., Messen als Instrument der Unternehmenspolitik, a.a.O., S. $150 \mathrm{f}$. Mittelbare Hinweise finden sich bei Dudley. J. W., Successful Exhibiting, a.a.O., S. 42 f.; Weintraub. D. K., Trade Show Exhibiting: The Insider's Guide for Entrepreneurs, 0. O. 1991, S. 131 f.; Spryß.W. M., Messen und Ausstellungen - Mit dem "Messe-Diagramm" vorbereiten - von den Zielen bis zum Controlling, in: Marketing Journal, Heft 4, 1987, S. 369.

Dies ist um so eher der Fall, je stärker ein Unternehmen die Marktbearbeitung relevanter Zielgruppen im Rahmen von Messebeteiligungen vornimmt.

202 Vgl. hierzu die allgemeingültigen Ausführungen von Kühn. R., Angebotspositionierung als Ansatz zur Präzisierung von Wettbewerbsstrategien, in: Tomczak. T. Rudolph. Th. Roosdorp. A. (Hrsg.), Positionierung: Kernentscheidung des Marketing, St. Gallen 1996, S. 113, die auf den Bereich der Messebeteiligung übertragen werden. 
Die Aufgabe der strategischen Messepositionierung besteht darin, den Handlungsrahmen für die Ausgestaltung der Messeaktivitäten sowie das Verhalten gegenüber den Messeteilnehmern (Messebesucher, Wettbewerber, Anspruchsgruppen, Veranstalter etc.) vorzugeben. ${ }^{204}$ Sie weist damit konstitutiven Charakter auf. In diesem Zusammenhang ist die Bedeutung der folgenden Optionen für eine strategische Messepositionierung als besonders relevant zu erkennen: ${ }^{205}$

- Produkt- bzw. Sortimentsorientierung,

- Problemlösungs- bzw. Nutzenorientierung,

- Kommunikations- bzw. Erlebnisorientierung sowie

- Wettbewerbsorientierung.

Die produkt- bzw. sortimentsorientierte Messepositionierung eines Ausstellers ist als Ausdruck einer primär auf die technischen Ressourcen des Unternehmens und sein Leistungsspektrum ausgerichteten Messe-Positionierungskonzeption zu interpretieren. ${ }^{206} \mathrm{Im}$ Sinne einer Inside-Out-Orientierung ${ }^{207}$ werden aus Unternehmenssicht erfolgversprechende Produkte kreiert und im Rahmen einer Messeveranstaltung dem interessierten Publikum vorgestellt. ${ }^{208}$ Ansatzpunkt für eine produkt- bzw. sortimentsorientierte Messepositionierung sollten Produktinnovationen sein, um damit die spezifische Kompetenz des Unternehmens hervorzuheben. Einen Differenzierungs- und Profilierungsvorteil kann ein Aussteller allerdings nur dann erlangen, wenn die präsentierten Innovationen einen dauerhaften Kundennutzen erbringen und zudem auf eine besondere Fähigkeit im Unternehmen treffen. ${ }^{209}$ Folgende vier Voraussetzungen sollten daher

204 Vgl. allgemein Wind. Y., Positioning Analysis and Strategy, in: Day. G. S. Weitz, B. Wensley R. (Ed.), The Interface of Marketing and Strategy, Greenwich 1990, S. 387-412.

205 Die Relevanz leitet sich wesentlich aus den in der Literatur zum Management von Messebeteiligungen diskutierten Gestaltungsprinzipien von Messeständen ab, die aber strenggenommen als Folge der strategischen Messepositionierung anzusehen sind.

Vgl. zum ressourcenorientierten Ansatz im Rahmen der Positionierung Rasche, C. Wolfrum. B., Ressourcenorientierte Unternehmensführung, in: DBW, 54. Jg., Heft 4, 1994, S. 502.

Wesen der Inside-Out-Orientierung im Rahmen der Positionierung ist die Entwicklung von Produkten in einem ersten Schritt, für die in einem zweiten Schritt Nachfrager mit entsprechenden Bedürfnissen gesucht werden. Prägendes Merkmal einer solchen Positionierung ist damit die Bedarfsweckung. Vgl. Tomczak. T. Roosdorp. A., Positionierung - Neue Herausforderungen verlangen neue Ansätze, in: Tomczak. T. Rudolph. Th. Roosdorp. A. (Hrsg.), Positionierung: Kernentscheidung des Marketing, St. Gallen 1996, S. 29.

208 Vgl. Geschka, H. Eggert-Kipfstuhl, K., Innovationsbedarfserfassung, in: Tomczak. T., Reinecke. S. (Hrsg.), Marktforschung, St. Gallen 1994, S. 127. 
im Rahmen einer produkt- bzw. sortimentsorientierten Messepositionierung erfüllt sein: Nicht-Imitierbarkeit, Unternehmensspezifität, Nicht-Substituierbarkeit und Fähigkeit zur Nutzenstiftung der vorgestellten Produkte und Leistungen am Markt. ${ }^{210}$ Damit wird auch der primär rationale Charakter der Positionierung deutlich. Eine wesentliche Gefahr dieser im Messewesen als "klassisch“ zu bezeichnenden Positionierungskonzeption liegt in der Präsentation von "ScheinInnovationen", so daß Unternehmen ihre spezifisch rationale Produktkompetenz, die sie auf- bzw. auszubauen suchen, in der Wahrnehmung der Messebesucher zumindest teilweise verlieren können. Darüber hinaus werden häufig solche Produkteigenschaften in den Vordergrund gestellt, die stärker das Interesse und Engagement des Anbieters für sein Angebot widerspiegeln als die Interessen und Wünsche der Nachfrager. ${ }^{211}$

Als Ausdruck einer an den Kundenbedürfnissen und -problemen ausgerichteten Messe-Positionierungskonzeption stellt sich die Problemlösungs- bzw. Nutzenorientierung der Aussteller dar. Im Gegensatz zur zuvor genannten InsideOut-Orientierung liegt diesem Positionierungsansatz eine Outside-In-Orientierung $^{212}$ zugrunde. Ziele dieser Positionierung sind die Demonstration der kundenindividuellen Problemlösungskompetenz des Ausstellers auf der einen Seite und die Gewinnung von Informationen über zentrale Problemstellungen bei aktuellen und potentiellen Kunden auf der anderen Seite, um bereits latent vorhandenen Kundenbedürfnissen besser und schneller als die Wettbewerber mit maßgeschneiderten Lösungskonzepten zu begegnen. ${ }^{213}$ Im Fokus des Interesses steht für den Aussteller damit weniger die Darstellung vorhandener Produkte oder Leistungen, sondern die Deckung des Informationsbedarfs zur sachgerechten Leistungsgestaltung und -erstellung. Damit ist auch mit dieser Positionierungskonzeption eine stärkere Gewichtung rationaler Aspekte bei der Planung der Messebeteiligung verbunden.

210 Vgl. zur Beziehung von Ressourcenausstattung und Bildung von Differenzierungspotentialen Rasche. C.. Wolfrum, B., Ressourcenorientierte Unternehmensführung, a.a.O., S. $503 \mathrm{ff}$. und die dort zitierte Literatur.

211 Vgl. zu einem ähnlichen Phänomen in der Werbung Kroeber-Riel. W., Strategie und Technik der Werbung. Verhaltenswissenschaftliche Ansätze, a.a.O., S. 47 f.

212 Die Outside-In-Orientierung ist ein Ansatz der Positionierung, bei dem zunächst die latent vorhandenen Bedürfnisse einer bestimmten Kundengruppe identifiziert werden, um dann anschließend nach Problemlösungen zu suchen. Vgl. Tomczak. T. Roosdore. A., Positionierung - Neue Herausforderungen verlangen neue Ansätze, a.a.O., S. 29.

213 Vgl. Hamel, G. Prahalad, C. K., So spürten Unternehmen neue Märkte auf, in: Harvard Manager, Heft 2, 1992, S. 44 ff. 
Demgegenüber liegt der Kommunikations- bzw. Erlebnisorientierung eine stärker an der emotionalen Ansprache der Messebesucher ausgerichtete Positionierungskonzeption zugrunde, die vor allem auf den Ereignischarakter des Messestandes und seine kommunikationsfördernde Atmosphäre abstellt. Ziel dieser Positionierung ist damit nicht die sachlich-rationale Darstellung des Unternehmens und seines Leistungsprogramms, sondern das Unternehmen bzw. sein Angebot in der emotionalen Erfahrungs- und Erlebniswelt des Nachfragers zu verankern. ${ }^{214}$ Diese Positionierung ist vor allem dann angebracht, wenn die sachlichen Qualitätsunterschiede zwischen den angebotenen Produkten und Leistungen nur gering sind und der Nachfrager sich auf ein akzeptables Qualitätsniveau des Angebots verläßt. ${ }^{215}$ Die auf dem Messestand vermittelten emotionalen Anregungen und Erlebnisse weisen dabei i. S. eines Zusatznutzens eine grundsätzliche Bedeutung für die Attraktivität und Aktualität des Unternehmens und seines Angebots auf. ${ }^{216}$ Die Gefahr kommunikations- bzw. erlebnisbetonter Messepositionierung für Aussteller besteht vor allem in der Widersprüchlichkeit der vermittelten emotionalen Erlebnisse und Erfahrungen am Messestand mit den Erfahrungen, welche der Nachfrager außerhalb der Messe mit dem Unternehmen bzw. seinen Produkten und Leistungen macht. Damit sieht sich eine solche Positionierungskonzeption dem Risiko der Unglaubwürdigkeit ausgesetzt. Daher ist es für den Erfolg einer erlebnis- und kommunikationsorientierten Positionierung um so entscheidender, daß die Erlebnisvermittlung konsistent in das gesamte MarketingMix einbezogen wird. ${ }^{217}$

${ }^{214}$ Vgl. Kroeber-Riel, W., Strategie und Technik der Werbung. Verhaltenswissenschaftliche Ansätze, a.a.O.

215 Kroeber-Riel spricht in diesem Zusammenhang auch von der Selbstverständlichkeit der sachlichen Qualität von Gütern, die zunehmend zu einem „Hintergrundphänomen“ wird, über das sich Nachfrager kaum mehr Gedanken machen. Vgl. Kroeber-Riel, W., Strategie und Technik der Werbung. Verhaltenswissenschaftliche Ansätze, a.a.O., S. 68 f.

216 Zur aktuellen Bedeutung der Schaffung von Zusatznutzen zur Differenzierung im sich verschärfenden Wettbewerb vgl. Laakmann. K., Value-Added Services als Profilierungsinstrument im Wettbewerb: Analyse, Generierung und Bewertung, Frankfurt a. M. u. a. 1995, S. 3 ff.

217 Vgl. Kroeber-Riel. W. Strategie und Technik der Werbung. Verhaltenswissenschaftliche Ansätze, a.a.O., S. 73. 
Wie bereits dargestellt, soll die strategische Messepositionierung zu einer Abgrenzung des eigenen Messeauftritts gegenüber den Wettbewerbern und damit auch zu einer Heraushebung des eigenen Messestils aus der "Masse der Aussteller" beitragen. Hierzu dienen die bisher beschriebenen Positionierungen. Allerdings wurde im Rahmen dieser Arbeit bereits darauf hingewiesen, daß sich bei Ausstellern häufig der Eindruck der inhaltlichen und formalen Austauschbarkeit ${ }^{218}$ zeigt. $^{219}$ Eine solche Positionierungsstrategie, die vornehmlich das Ausstellungsverhalten der Konkurrenz kopiert und damit nicht eine Differenzierungs-, sondern primär eine Imitationsstrategie verfolgt, wird im Rahmen dieser Arbeit als Orientierung an Wettbewerbern bezeichnet. Sie ist damit - falls der eine solche Positionierungsstrategie verfolgende Aussteller vom Messebesucher wahrgenommen wird - lediglich geeignet, die Zugehörigkeit zu einem bestimmten Wettbewerbsumfeld zu demonstrieren. Dies kann ein Unternehmen allerdings auch mit weniger aufwendigen Kommunikationsmaßnahmen als mit einer Messebeteiligung erreichen.

Vor dem Hintergrund der skizzierten strategischen Positionierung sind Entscheidungen hinsichtlich des Stellenwertes und des Einsatzes der Instrumente des Messebeteiligungs-Mix auf der operationalen Ebene der Positionierung zu treffen. Nicht jedes der existierenden Instrumente weist für die Positionierungsstrategien dieselbe Relevanz auf. In diesem Zusammenhang kann auf das sog. DominanzStandard-Modell ${ }^{20}$ verwiesen werden. Kühn entwickelte eine Instrumentetypologie, die dominierende, komplementäre, standardisierte und marginale Marketing-

218 Unter der inhaltlichen Austauschbarkeit soll hier die Ähnlichkeit der Botschaftsinhalte und unter der formalen Austauschbarkeit die Ähnlichkeit der gestalterischen Elemente einer Messebeteiligung verstanden werden. Zu einer ähnlichen Abgrenzung im Bereich der Werbung vgl. Kroeber-Riel. W. Strategie und Technik der Werbung. Verhaltenswissenschaftliche Ansätze, a.a.O., S. 50.

Vgl. hierzu die auf Seite 69 in Fußnote 199 zitierte Literatur.

Das Dominanz-Standard-Modell wurde von Kühn entwickelt und geht von der Frage aus, ob nicht Ansatzpunkte existieren, die es erlauben, bestimmte Marketing-Instrumente in den Marketingüberlegungen in den Vordergrund zu stellen bzw. eine bestimmte Sequenz der Instrumente des Marketing-Mix festzulegen. Vgl. Kühn. R., Marketing - Analyse und Strategie, Zürich 1995, S. 44. Daneben wurden weitere Modelle zur Reduktion der Planungskomplexität auf der operationalen Ebene der Positionierung entwickelt, bspw. das Zonenmodell von Rudolph bzw. die instrumentellen Strategiemodelle von Haedrich und Tomczak. Bei beiden zuletzt genannten Modellen steht ebenfalls wie bei Kühn die Benennung von „Schwerpunktinstrumenten“ zur Umsetzung der Positionierungsentscheidung im Vordergrund. "Vgl. Rudolph. Th., Positionierungs- und Profilierungsstrategien im europäischen Einzelhandel, St. Gallen 1993; Haedrich, G.. Tomczak. T., Strategische Markenführung, Bern, Stuttgart 1990. 
instrumente unterscheidet. Die Kategorien können wie folgt charakterisiert werden. ${ }^{221}$

- Dominierende Instrumente

sind für den Differenzierungs- und Profilierungserfolg ggü. Wettbewerbern ausschlaggebend.

- Komplementäre Instrumente

erweisen sich für die Wirkungsunterstützung der dominierenden Instrumente als notwendig bzw. zweckmäßig.

\section{- Standardinstrumente}

führen bei Nichterreichen eines allgemein etablierten Standards mit hoher Wahrscheinlichkeit zu Mißerfolgen, während das Übertreffen des Standards entweder kaum realisierbar ist oder von den Nachfragern nicht honoriert wird.

\section{- Marginale Instrumente}

sind solche Instrumente, deren Einsatz aus Sicht des Marketing-Management und vor dem Hintergrund einer bestimmten Situation als bedeutungslos eingeschätzt wird.

Diese Kategorien lassen sich im Rahmen von Messebeteiligungen nicht definitiv einzelnen der genannten Instrumente zuordnen. ${ }^{222}$ Vielmehr ist eine Einordnung vor dem Hintergrund der gewählten Positionierungsstrategie vorzunehmen. Ohne bereits an dieser Stelle auf eine nähere Zuordnung des Messebeteiligungs-Mix zu den drei differenzierungs- und profilierungsfähigen Positionierungsstrategien eingehen zu wollen ${ }^{223}$, läßt sich aus Plausibilitätsüberlegungen ableiten, daß im Rahmen einer erlebnisorientierten Messe-Positionierungskonzeption weniger einzelne Exponate als vielmehr der Einsatz aktivierender Kommunikationsinstrumente dominierenden Charakter aufweisen werden. Steht dagegen die Produkt- bzw. Sortimentsorientierung im Vordergrund, so werden aus Sicht des MesseEntscheiders wesentlich die überzeugende Präsentation der Exponate sowie die Fachkompetenz des Standpersonals an Bedeutung gewinnen.

Abb. 15 gibt einen zusammenfassenden Überblick über die Entscheidungsfelder im Rahmen der Messe-Positionierungskonzeption, wobei deutlich wird, daß

221 Vgl. Kühn, R., Marketing-Mix, in: Poth, L. G. (Hrsg.), Marketing, Bd. 3, Neuwied 1979, S. 40 ff.

222 Vgl. Kapitel A.2. sowie Seite 70 dieser Arbeit.

223 Eine intensive Auseinandersetzung mit dem „Fit“ von Positionierung und eingesetztem Marketinginstrumentarium erfolgt in Kapitel C.1.2. 


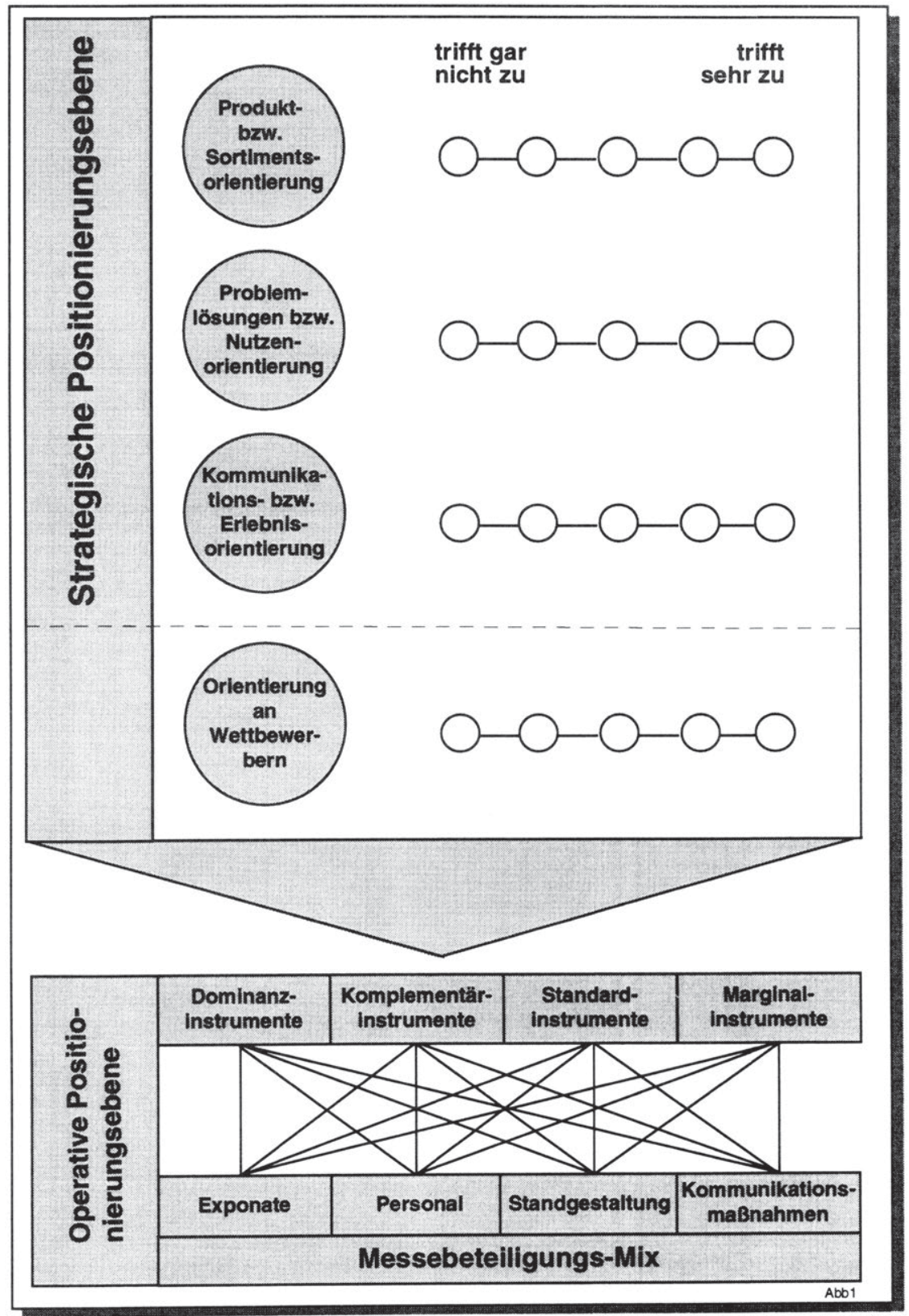

Abb. 15: Strategische und operative Entscheidungsfelder der Messe-Positionierungskonzeption

Ralf Ueding and Universität Münster - 978-3-631-75073-5

Downloaded from PubFactory at 01/11/2019 08:38:01AM 
hierbei die vorgestellten strategischen Positionierungsalternativen sich nicht gegenseitig ausschließen, sondern auch gleichzeitig in verschiedener Intensität verfolgt werden können. ${ }^{224}$

Die Ergebnisse der Befragung zur strategischen Positionierung, die für die Beschreibung der messespezifischen Grundhaltungen besondere Bedeutung besitzen, sind hinsichtlich einer ersten Einordnung ihres jeweiligen Stellenwertes für ausstellende Unternehmen Abb: 16 zu entnehmen.

Dabei fällt zunächst auf, daß die Produkt- bzw. Sortimentsorientierung der Messestandgestaltung den dominierenden Einfluß ausübt. Fast drei Viertel der befragten Unternehmen geben an, daß diese Positionierungskonzeption auf ihre Messebeteiligung zutrifft. Lediglich $11 \%$ der in der Stichprobe enthaltenen Unternehmen lehnen diese Positionierung für ihre Messebeteiligung ab. Allerdings machen ca. $60 \%$ der Unternehmen auch deutlich, daß eine problemlösungsbzw. nutzenorientierte Messepositionierung einen nahezu ebenso großen Stellenwert genießt. Ein lediglich durchschnittlicher Stellenwert kommt im Durchschnitt der Stichprobe der Kommunikations- bzw. Erlebnisorientierung von Messebeteiligungen zu. Dieser messen lediglich ca. $37 \%$ der Befragten eine höhere Bedeutung bei. Fast die gleiche Anzahl der Befragten sieht in dieser Grundorientierung allerdings keine relevante bzw. umsetzbare Messepositionierungsstrategie.

Den geringsten Stellenwert nimmt aber die Orientierung an Wettbewerbern ein. Über $75 \%$ der Befragten lehnen eine solche Positionierung bei Messebeteiligungen ab. Nicht einmal $7 \%$ der Befragten sehen in dieser Grundorientierung eine relevante messepolitische Gestaltungsperspektive.

224 Diese Tatsache ist nicht zuletzt darauf zurückzuführen, daß insbesondere Aussteller mit einer entsprechend großen Standfläche in der Lage sind, verschiedene Positionierungskonzepte zielgruppenspezifisch auf einem oder an verschiedenen Messeständen zu realisieren. Ein solches Vorgehen birgt allerdings die Gefahr der Verzettelung, so daß keinem der Standbesucher die jeweils intendierte Profilierungsdimension deutlich zu machen ist.

Ralf Ueding and Universität Münster - 978-3-631-75073-5 


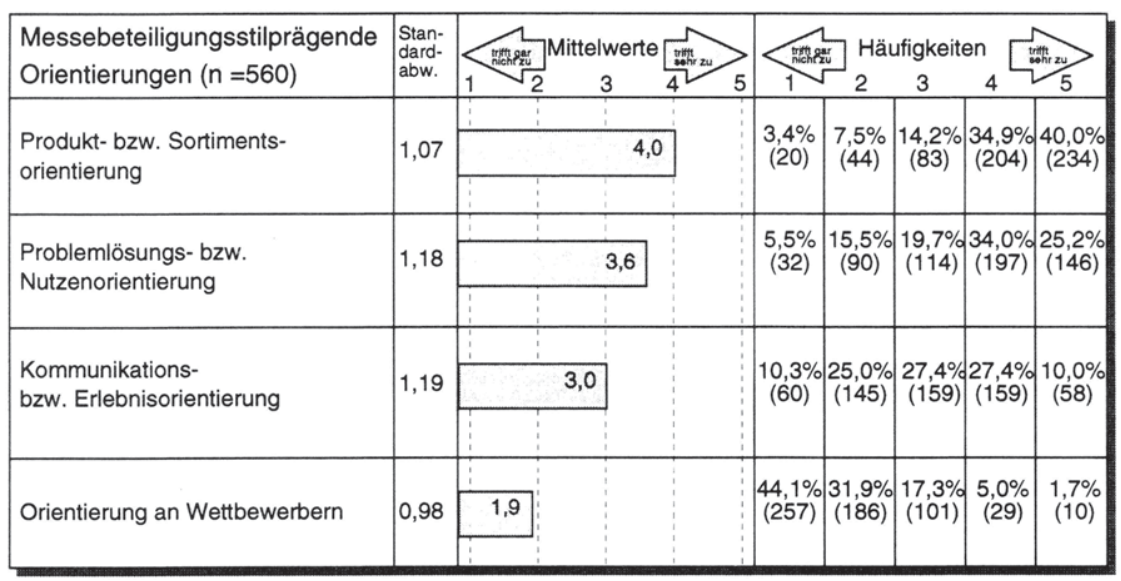

Abb: 16: Stellenwert der Positionierungskonzeptionen bei Messebeteiligungen

Angesichts der Ausführungen zur Messe-Positionierungskonzeption lassen sich die folgenden Untersuchungshypothesen anschließend einer empirischen Überprüfung unterziehen:

$H_{\text {Pk1: }}$ : Die Messepositionierung wird in unmittelbarer Abstimmung mit den Messezielen und dem Charakter der ausgewählten Messeveranstaltung(en) festgelegt, d. h. verschiedene Messezielkonzeptionen sowie Messeselektionskonzeptionen haben signifikant unterschiedliche Messepositionierungen zur Folge.

Diese Basishypothese soll durch folgende Tendenzhypothesen, die den Einfuß der messespezifischen Zielkonzeption auf die Messepositionierung beschreiben, ergänzt werden:

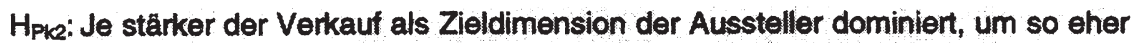
werden produkt- bzW. sortimentsorientierte Positionierungskonzepte verfolgt.

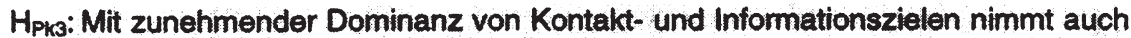
die Relevanz einer problemlösungs- bzw. nutzenorientierten Messepositionierung für die Aussteller zu.

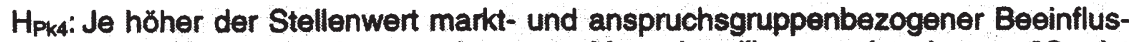
sungsziele für die Ausgestaltung von Messebeteiligungen ist, desto größer ist die Bedeutung kommunikations- bzW. enlebnisorientierter Messepositionierungskonzeptionen. 
$H_{P k 5}$ : Je weniger Zielorientierung eine Messebeteiligung aułweist, um so eher wird eine wettbewerbsorientierte Messepositionierung des Ausstellers an Relevanz gewinnen.

Unter Berücksichtigung des in der Theorie dargestellten Zusammenhangs zwischen Messe-Selektionskonzeption und messespezifischer Positionierung sind folgende Hypothesen für eine anschließende empirische Überprüfung ableitbar:

$H_{\text {Pk6: }}$ Je homogener das Ausstellungsprogramm einer Messeveranstaltung, um so weniger Relevanz besitzt eine produkt- bzw. sortimentsorientierte Positionierungskonzeption.

$H_{p k 7:}$ Mit zunehmender Informationsorientierung der Messeveranstaltung wird eine problemlösungs- bzw. nutzenorientierte Messepositionierung für die Aussteller an Wichtigkeit gewinnen.

$H_{\text {Pkg: }}$ Je stärker Fachpublikum auf einer Messeveranstaltung vertreten ist, um so stärker nimmt die Bedeutung problemlösungs- bzw. nutzenorientierter Messepositionierungskonzeptionen zu.

$H_{p k 9}$ : Je mehr Begleitveranstaltungen mit einer Messeveranstaltung verbunden sind, desto eher werden Aussteller eine kommunikations- bzw. erlebnisorientierter Messepositionierungskonzeption verfolgen. 


\section{Identifikation messespezifischer Grundhaltungstypen auf der Basis einer empirischen Analyse}

Im folgenden sollen aufbauend auf den in Kapitel B.1. dargestellten Dimensionen einer messespezifischen Grundhaltung homogene Grundhaltungstypen auf empirischer Basis abgeleitet werden. Hierzu werden in einem ersten Schritt die in Kapitel B.1.2 dargestellten Messebeteiligungsziele sowie das hinter den Einzelzielen vermutete Modell der Messe-Zielkonzeption einer konfirmatorischen Faktorenanalyse unterzogen. Anschließend werden zur Bildung der messespezifischen Grundhaltungstypen die ermittelten Zielfaktoren sowie die in den Kapiteln B.1.3 und B.1.4 vorgestellten Variablen der Messeselektion und der messespezifischen Positionierung in eine Clusteranalyse einbezogen. Nach der Beschreibung der gefundenen Cluster-Lösung erfolgt eine Überprüfung der Clusterstabilität sowie die Ermittlung der für die Typendifferenzierung zentralen Grundhaltungsdimensionen auf der Basis diskriminanzanalytischer Untersuchungen. Abschließend erfolgt eine zusammenfassende Würdigung der empirisch ermittelten Grundhaltungstypen.

\subsection{Konfirmatorische Faktorenanalyse für die messespezifische Zielkon- zeption von ausstellenden Unternehmen}

Die große Zahl der primärstatistisch erfaßten Variablen zur Kennzeichnung der verschiedenen Zielsetzungen, die Aussteller mit Messebeteiligungen verfolgen ${ }^{225}$, macht es in einem ersten Analyseschritt erforderlich, die Einzelvariablen zu höher aggregierten Konstrukten zu verdichten. Als statistisches Verfahren zur Erreichung dieser Datenreduktion kommt insbesondere die Faktorenanalyse in Betracht. ${ }^{226}$ Klassischerweise wird die Faktorenanalyse dabei als Instrument zur Entdeckung von Variablenbeziehungen verstanden, indem der Versuch unternommen wird, aus einer gegebenen Variablenstruktur hypothetische Konstrukte statistisch zu ermitteln. Neben dieser exploratorischen Ausrichtung der Faktorenanalyse existiert jedoch auch die Möglichkeit, eine Modell- bzw. Hypothesenprüfung zu verfolgen. Diese sog. konfirmatorische Faktorenanalyse kommt dabei als Bestandteil der

225 Es wurden 24 Einzelzielsetzungen erhoben. Vgl. Frage 8 auf den Seiten 4 und 5 des Fragebogens im Anhang dieser Arbeit.

226 Vgl. Backhaus. K. et al., Multivariate Analysemethoden: Eine anwendungsorientierte Einführung, a.a.O., S. 189-260 und insb. S. 190 f.; Bauer, F., Datenanalyse mit SPSS, a.a.O., S. 201 ff. 
Kausalanalyse und hier in erster Linie des LISREL-Modells (LISREL = Linear Structural Relationship) zum Einsatz. ${ }^{227}$

Für die Überprüfung des Strukturmodells der Messe-Zielkonzeption besitzt die konfirmatorische Faktorenanalyse als struktur-prüfendes Verfahren eine hohe Eignung. Das Verfahren ermöglicht die Prüfung auf Basis theoretischer Überlegungen abgeleiteter Hypothesen über die Anzahl von Faktoren (Zieldimensionen) eines komplexen Konstrukts (Messe-Zielkonzeption), die Beziehungen zwischen den Faktoren sowie die Beziehungen zwischen den Faktoren und ihren Indikatoren. 228

Zur Beurteilung der globalen Anpassungsgüte von konfirmatorischen Faktorenanalysen können drei, auch bei vollständigen LISREL-Modellen verwendete Fit-Maße verwendet werden. ${ }^{229}$ Der "goodness of fit-Index“ (GFI) sowie der „adjusted goodness of fit-Index" (AGFI), bei dem eine Anpassung des GFI um die Freiheitsgrade des Modells erfolgt, stellen Prüfgrößen für den durch eine Modellstruktur erklärten Anteil der Stichprobenvarianz dar. Beide Maße weisen einen Wertebereich zwischen 0 und 1 auf, wobei Werte nahe 1 eine hohe Anpassungsgüte des Modells anzeigen. Der „root mean square residual“-Index (RMR) gibt dagegen die durch ein Gesamtmodell nicht erklärte Restvarianz an. Hier deuten Werte nahe 0 auf einen sehr guten Fit des Modells hin. ${ }^{230}$

227 Zu den spezifischen Besonderheiten der Kausalanalyse bzw. des LISREL-Modells vgl. Backhaus. K. et al., Multivariate Analysemethoden: Eine anwendungsorientierte Einführung, a.a.O., S. 322-430; Jöreskog. K. G.. Sörbom. D., LISREL 7, A Guide to the Programme and Applications, 2. Aufl., Chicago 1989.

Die Anwendung konfirmatorischer Faktorenanalysen gehört heute zum Standardprogramm der empirischen Forschung. Daher soll im Zusammenhang mit der grundsätzlichen Anwendung dieser statistischen Analysemethode auf die einschlägige Literatur verwiesen werden. Vgl. für einen Überblick Backhaus, K. et al., Multivariate Analysemethoden: Eine anwendungsorientierte Einführung, a.a.O., S. 409 ff. sowie die dort angegebene Literatur.

229 Vgl. zu den einzelnen Fit-Maßen Backhaus, K. et al., Multivariate Analysemethoden: Eine anwendungsorientierte Einführung, a.a.O., S. $397 \mathrm{ff}$. Vor der Parameterberechnung muß ferner die Identifizierbarkeit eines Modells überprüft werden. Dies geschieht üblicherweise anhand der Freiheitsgrade des Modells, die einen Wert größer 0 aufweisen müssen. Damit ist allerdings nur eine notwendige Bedingung der Identifizierbarkeit erfüllt. Vgl. Jöreskog. K. G.. Sörbom, D. LISREL 7, A Guide to the Programme and Applications, 2. Aufl., a.a.O., S. $16 \mathrm{ff}$.

230 Ein viertes Beurteilungskriterium, der Chi Quadrat-Anpassungstest, wird im folgenden nicht verwendet, da in der Literatur Vorbehalte gegen dessen Eignung geäußert werden. Diese betreffen insbesondere Verzerrungseffekte, die in Abhängigkeit vom Stichprobenumfang und bei Abweichungen von der Normalverteilungsprämisse auftreten können. Vgl. Jöreskog. K. G. Sörbom, D., LISREL 7, A Guide to the Programme and Applications, 2. Aufl., a.a.O., S. 43; Backhaus. K. et al., Multivariate Analysemethoden: Eine anwendungsorientierte Einführung, a.a.O., S. 394 f. 
Zur Überprüfung der in Kapitel B.1.2 abgeleiteten Hypothesen werden die sechs Zieldimensionen und die insgesamt 24 zu deren Operationalisierung verwendeten Indikatorvariablen in die formale Modellstruktur einer konfirmatorischen Faktorenanalyse überführt. Die notwendige Bedingung für die Identifizierbarkeit des Modells ist bei 273 Freiheitsgraden erfüllt. Für die Parameterschätzung wird das iterative Schätzverfahren der ungewichteten kleinsten Quadrate (ULS) eingesetzt. ${ }^{231}$ Die Ergebnisse der konfirmatorischen Faktorenanalyse sind in Abb. 17 vollständig wiedergegeben.

231 LISREL stellt sowohl iterative als auch nicht iterative Schätzverfahren zur Verfügung. Von den grundsätzlich leistungsfähigeren iterativen Verfahren wird das ULS-Verfahren gewählt, da es keine spezielle Verteilung der zugrunde liegenden Datenbasis erfordert. Zu den Vorteilen der iterativen Schätzverfahren vgl. Backhaus, K. et al., Multivariate Analysemethoden: Eine anwendungsorientierte Einführung, a.a.O., S. $377 \mathrm{ff}$. 


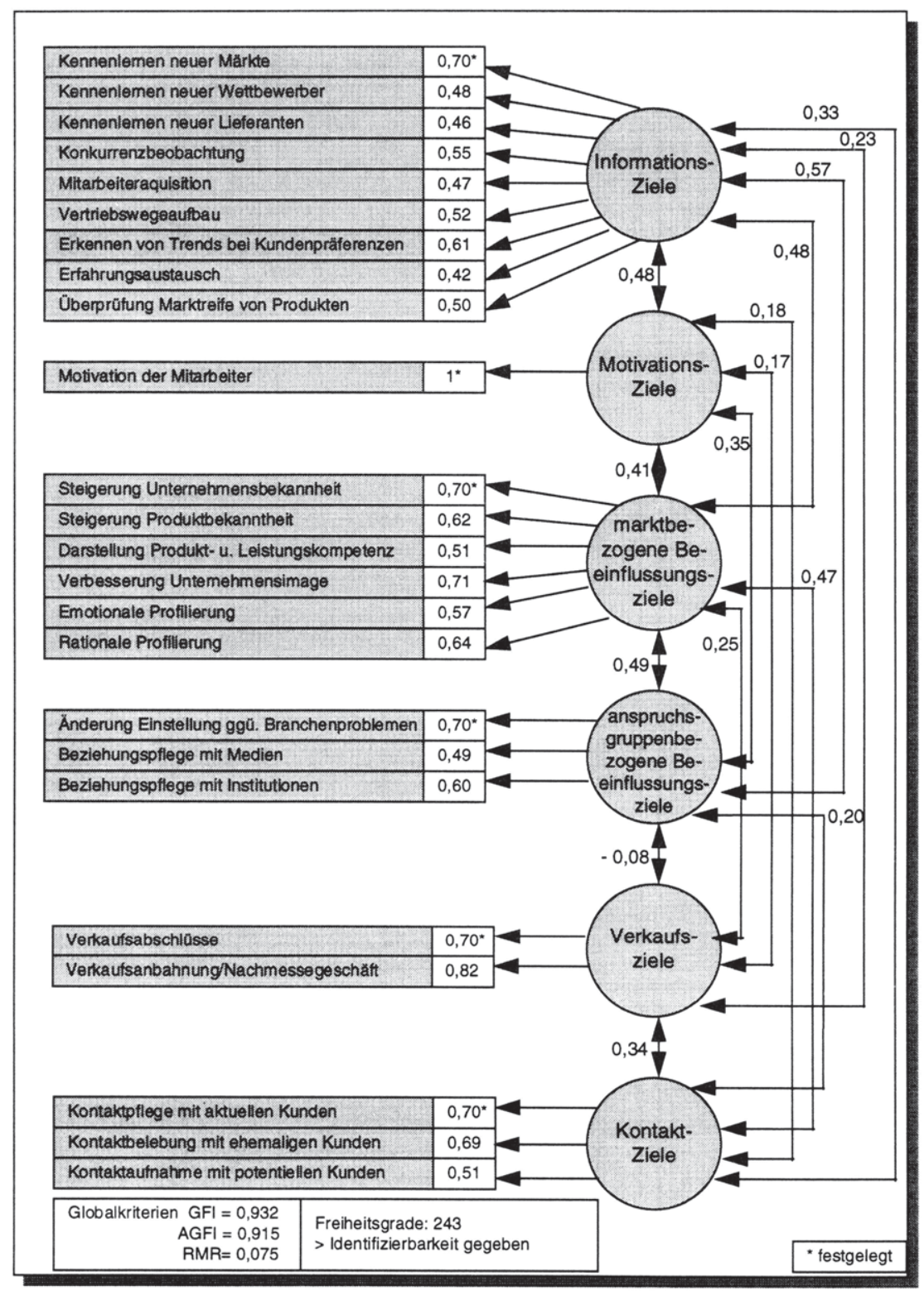

Abb. 17: Konfirmatorische Faktorenanalyse für das Modell der Messe-Zielkonzeption 
Die Fit-Maße zeigen mit Werten von 0.932 für den GFI-Index und 0,915 für den AGFI-Index zunächst eine sehr zufriedenstellende Anpassungsgüte der Modellstruktur an. Mit einem RMR-Index von 0,075 bleibt zudem lediglich ein Varianzund Kovarianzanteil von 7,5 Prozent der Stichprobe unerklärt. Die dem Modell der Messe-Zielkonzeption zugrunde gelegte Faktorenstruktur kann damit grundsätzlich bestätigt werden. Die mit der Hypothese $\mathrm{H}_{\mathrm{zk} 1}$ postulierte Eigenständigkeit der einzelnen Zieldimensionen soll anhand ihrer Diskriminanzvalidität ${ }^{232}$ beurteilt werden. Danach ist ein Faktor (Zieldimension) als eigenständig zu betrachten, wenn er sich von den übrigen Faktoren eindeutig unterscheidet, also keine hohen Korrelationen zu anderen Führungsdimensionen aufweist. ${ }^{233}$

Zur Beurteilung der Unterscheidbarkeit der Zieldimensionen als Bedingung der Eigenständigkeit sind die in Abb. 17 dargestellten Korrelationskoeffizienten der Faktoren zu analysieren. ${ }^{234}$ Hinsichtlich eines Grenzwertes, bei dessen Erreichen nicht mehr von unterscheidbaren Konstrukten bzw. Zieldimensionen ausgegangen werden kann, besteht in der Literatur keine eindeutige Meinung. Die Forschungspraxis sieht die Unterschiedlichkeit und damit Diskriminanzvalidität von Konstrukten bereits dann als gegeben an, wenn sie nicht vollständig miteinander korrelieren, d. h. einen Korrelationskoeffizienten kleiner als 1 aufweisen. ${ }^{235}$

232 Die Überprüfung der sog. Diskriminanzvalidität ist im Gegensatz zu den genannten Globalkriterien als Detailkriterium zu bezeichnen, anhand dessen die Anpassungsgüte von Teilstrukturen überprüft werden kann. Die Verwendung eines derartigen Kriteriums enweist sich als sinnvoll, da trotz einer zufriedenstellenden Globalanpassung von Modellstrukturen einzelne Modellkomponenten eine nur geringe Anpassungsgüte aufweisen können. Vgl. zu einer ausführlichen Darstellung der Detailkriterien für konfirmatorische Faktorenanalysen Fritz, W., Marketing-Management und Unternehmenserfolg: Grundlagen und Ergebnisse einer empirischen Untersuchung, 2. Aufl., Stuttgart 1995, S. $128 \mathrm{ff}$.

Die Unterscheidbarkeit hypothetischer Konstrukte bei konfirmatorischen Faktorenanalysen wird durch den Begriff der Diskriminanzvalidität erfaßt. Vgl. Fritz. W., Marketing-Management und Unternehmenserfolg: Grundlagen und Ergebnisse einer empirischen Untersuchung, 2. Aufl., a.a.O., S. $137 \mathrm{ff}$.

Im Gegensatz zur explorativen Faktorenanalyse, bei der orthogonale, also unabhängige Faktorenmuster extrahiert werden, läßt die konfirmatorische Faktorenanalyse Beziehungen zwischen den theoretisch abgeleiteten Konstrukten ausdrücklich zu. Hierin ist einer der wesentlichen Vorzüge dieses Verfahrens zu sehen.

Vgl. Bagozzi.R. P., Causal Modelling: A General Method for Developing and Testing Theories in Consumer Research, Monroe 1981, S. 197; Hildebrandt. L., Kausalanalytische Validierung in der Marketingforschung, in: Marketing ZFP, 6. Jg., 1984, S. 47. Balderjahn nennt dagegen einen Grenzwert von 0,6 für den Korrelationskoeffizienten, ab dem die Unabhängigkeit von Faktoren nicht uneingeschränkt angenommen werden kann. Vgl. Balderiahn, I., Das umweltbewußte Konsumentenverhalten. Eine empirische Studie, Berlin 1986, S. 171. 
Ein Blick in Abb. 17 macht indes deutlich, daß keine an den Wert von 1 nur annähernd heranreichenden Korrelationen zwischen den Zieldimensionen bestehen. Lediglich zwischen den Informationszielen und den anspruchsgruppenorientierten Beeinflussungszielen besteht ein Korrelationskoeffizient von $r=0,57$. Alle übrigen 14 Korrelationskoeffizienten weisen absolute Werte auf, die unterhalb von 0,5 liegen. ${ }^{236}$ Das spezifizierte Messe-Zielmodell kann daher, trotz der Nichtverfügbarkeit eindeutiger Beurteilungskriterien, als der Anforderung der Diskriminanzvalidität genügend angesehen werden.

Hinsichtlich der mit der Basishypothese $\mathbf{H}_{\mathbf{z k} 1}$ aufgestellten Eigenständigkeitsbehauptung für die 6 Zieldimensionen ist damit folgendes Ergebnis zu formulieren: Alle Zieldimensionen besitzen eine statistisch signifikante Eigenständigkeit. Mit Blick auf den RMR-Index von 0,075 kann weiterhin davon ausgegangen werden, $\mathrm{da} ß$ die real existierenden Zielsysteme der Aussteller nahezu vollständig durch die 6 Zieldimensionen beschrieben werden können. Angesichts der die Faktorenstruktur darüber hinaus insgesamt bestätigenden globalen Fit-Maße kann die zentrale Hypothese $\mathrm{H}_{2 \mathrm{k} 1}$ damit in vollem Umfang bestätigt werden.

Insgesamt zeigen die angelegten Kriterien, daß sich das Modell der übergeordneten Zielkategorien an der Realität bewährt. Mit der empirischen Bestätigung eines differenzierten Sets relevanter Zieldimensionen ist ein wichtiger Eckpfeiler für die nachfolgende Bildung von messespezifischen Grundhaltungstypen gelegt.

Zur weiteren Verwendung der auf der Grundlage der konfirmatorischen Faktorenanalyse identifizierten Zieldimensionen sind die entsprechenden Variablenausprägungen für jeden befragten Aussteller allerdings zunächst zu den einzelnen Zieldimensionen zu verdichten. Da die konfirmatorische Faktorenanalyse, wie sie über das LISREL-Programm zur Verfügung gestellt wird, nicht in der Lage ist, Faktorwerte ${ }^{237} \mathrm{zu}$ berechnen, als getrennte Variablen auszuweisen und somit für nachgelagerte statistische Analyseverfahren verfügbar zu machen, wird durch die

236 Damit erfült das Modell auch die von Balderjahn formulierten Anforderungen vollständig. Vgl. Fußnote 235.

237 Faktorwerte stellen die numerischen Ausprägungen der Faktoren bei den Untersuchungsobjekten dar. Die durch die i Faktoren ausgedrückten $n$ Variablenwerte je Untersuchungsobjekt $k$ werden also durch i Faktorwerte ersetzt, so daß eine Reduktion der Beobachtungswerte je Fall von $(n-i)$ erreicht werden kann. Vgl. hierzu auch Backhaus, $K$. et al., Multivariate Analysemethoden: Eine anwendungsorientierte Einführung, a.a.O., S. 233 f.; Bleymüller. J., Multivariate Analyse für Wirtschaftswissenschaftler. Manuskript, Münster 1989, S. 116 ff. 
Bildung von Index-Variablen ${ }^{238}$ die Struktur der konfimatorisch abgeleiteten Faktoren für die Stichprobenmitglieder modelliert. ${ }^{239}$ Diese 6 Index-Variablen gehen in die Ableitung der messespezifischen Grundhaltungstypen gemeinsam mit den 6 Variablen der Messeselektion und den 4 Variablen der Messe-Positionierungskonzeption ein.

\subsection{Ermittlung der Anzahl verschiedener Grundhaltungstypen}

Als Grundhaltungstypen werden in der vorliegenden Arbeit durchgängige Verhaltensmuster von Ausstellern bezeichnet, die sich in den strategischen Dimensionen „Messe-Zielkonzeption“, "Messe-Selektionskonzeption“ und "Messe-Positionierungskonzeption" einer Messebeteiligung manifestieren. ${ }^{240}$ Können bei ausstellenden Unternehmen identische Verhaltensmuster bzgl. der genannten Dimensionen nachgewiesen werden, so realisieren sie einen identischen Grundhaltungstyp. Für die Bildung derartiger, in sich homogener, aber untereinander möglichst heterogener Gruppen von Untersuchungsobjekten anhand zugehöriger Merkmalsausprägungen stellt die Clusteranalyse ein geeignetes statistisches Instrumentarium zur Verfügung. ${ }^{241}$

${ }^{238}$ Die Index-Variablen, die jeweils die einem Faktor (Zieldimension) zugehörigen Indikatorenwerte (Einzelzielsetzungen) enthalten, werden auf dem Wege der additiven Verknüpfung zusammengeführt und entsprechend der Anzahl der zu berücksichtigenden Indikatoren gewichtet. Der faktorenspezifischen Verdichtung der Ausgangsdaten für jeden befragten Aussteller in der Stichprobe liegt damit folgender Berechnungsalgorithmus zugrunde, der auf der Grundlage der vorliegenden Indikatorenstruktur von einer Gleichgewichtung der Ausgangsdaten ausgeht, was auch durch die relativ homogenen Faktorladungen naheliegend erscheint:

$$
F W_{i k}=1 / n \sum V_{j k}
$$

mit: $\mathrm{FW}_{\mathrm{ik}}=$ Faktorwert des Faktors i beim Aussteller $\mathrm{k}$

$\mathrm{V}_{\mathrm{jk}}=$ Wert des Indikators $\mathrm{j}$ beim Aussteller $\mathrm{k}$

$n=$ Anzahl der Indikatoren, die zu einem Faktor zusammengeführt werden

Zu einem ähnlichen Vorgehen vgl. Fritz. W., Marketing-Management und Unternehmenserfolg: Grundlagen und Ergebnisse einer empirischen Untersuchung, 2. Aufl., a.a.O., S. $223 \mathrm{ff}$. und die dort zitierte Literatur sowie Haedrich. G. Gussek. F.. Tomczak. T., Differenzierte Marktbearbeitung und Markterfolg im Reiseveranstaltermarkt der Bundesrepublik Deutschland, in Marketing ZFP, Nr. 1, 1989, S. 11-18. 
Aufgrund der vielfältigen, im Rahmen der Clusteranalyse verwendbaren Fusionierungs-Algorithmen ${ }^{242}$ soll nachfolgend ein dreistufiges Vorgehen gewählt werden: In einem ersten Schritt sind aus der Stichprobe von 602 befragten Unternehmen anhand des Single Linkage-Verfahrens ${ }^{243}$, einem zur Ausreißerselektion besonders geeigneten Fusionierungs-Algorithmus ${ }^{244}$, solche Aussteller zu identifizieren und auszusondern, die ein völlig atypisches Messebeteiligungsverhalten praktizieren, somit gewissermaßen Individualtypen der Messebeteiligung darstellen.

Auf dieser Basis kann dann nachfolgend das Ward-Verfahren eingesetzt werden, um in der um Individualtypen bereinigten Stichprobe übergreifende Grundhaltungstypen abzuleiten. Das hierarchische Ward-Verfahren nimmt eine schrittweise Zusammenfassung von Objekten vor, bis letztlich die Objektgesamtheit in einem gemeinsamen Cluster vereint ist. Die "richtige" Clusterzahl ist dann aus dem Verlauf des Fusionierungsprozesses zu ermitteln. ${ }^{245}$ Hierzu stellt das Ward-Verfahren

242 Die Fusionierungsalgorithmen können in zwei Hauptgruppen unterteilt werden. Hierarchische Verfahren weisen einen kontinuierlichen Fusionierungsproze $\beta$ auf, in dem entweder - ausgehend von der feinsten Partition, d. $h$. jedes Objekt bildet ein eigenes Cluster - die Objekte schrittweise zusammengefaßt werden (agglomerative Verfahren), oder - ausgehend von der gröbsten Partition, d. h. alle Objekte bilden ein einziges Cluster - die Gruppen schrittweise aufgeteilt werden (divisive Verfahren). Partionierende Verfahren gehen dagegen von einer bestimmten, vom Forscher vorgegeben, Gruppenstruktur aus und gruppieren die Objekte solange um, bis eine vorgegebene Zielfunktion ihr Optimum erreicht. Vgl. Steinhausen. D. Langer. K., Clusteranalyse: Einführung in Methoden und Verfahren der automatischen Klassifikation, Berlin, New York 1977, S. 69; Backhaus. K. et al., Multivariate Analysemethoden: Eine anwendungsorientierte Einführung, a.a.O., S. 281.

Das Single Linkage-Verfahren ist den hierarchisch-agglomerativen Clusterverfahren zuzurechnen. Es werden jeweils diejenigen Objekte zusammengefaßt, die die größte Ähnlichkeit bzw. die kleinste Distanz aufweisen. Vgl. Steinhausen, D. Langer. K., Clusteranalyse: Einführung in Methoden und Verfahren der automatischen Klassifikation, a.a.O., S. 77 f.

Bei Ausreißern handelt es sich um solche Objekte, die im Vergleich zu allen anderen zu klassifizierenden Objekten stark abweichende Merkmalsausprägungen aufweisen und daher keiner Gruppe sinnvoll zugewiesen werden können. Beläßt man diese Objekte in der Stichprobe, kann dies die Gruppenbildung stark beeinträchtigen. Das Single Linkage-Verfahren erlaubt aufgrund seines Fusionierungs-Algorithmus die Identifikation von Ausreißern, die erst am Ende des Fusionierungsprozesses den bis dahin gebildeten Gruppen zugeordnet werden. Vgl. Steinhausen. D.. Langer. K., Clusteranalyse: Einführung in Methoden und Verfahren der automatischen Klassifikation, a.a.O., S. 78.

Insofern entspricht das Ward-Verfahren in besonderer Weise der Problemstellung der vorliegenden Arbeit. Denn angesichts der Vielzahl relevanter Grundhaltungsdimensionen bei Messebeteiligungen und der für diese Dimensionen dargestellten Ausgestaltungsmöglichkeiten erscheint eine aus theoretischen Überlegungen begründete Ableitung der $\mathrm{zu}$ erwartenden Anzahl von Grundhaltungstypen nicht möglich. 
mit dem Elbow-Kriterium eine heuristische Entscheidungshilfe zur Verfügung. ${ }^{246}$ Vergleichende Untersuchungen von Bergs sowie Backhaus et. al. haben gezeigt, daß dieses Verfahren besonders geeignet ist, die „wahren“, d. h. in der Realität tatsächlich existierenden Gruppierungen zu identifizieren. ${ }^{247}$ Allerdings neigt das Ward-Verfahren dazu, möglichst gleich große Cluster zu bilden und ist nicht in der Lage, sehr große Gruppen bzw. solche mit kleiner Elementezahl zu erkennen. Zudem kann aufgrund des Fusionierungs-Algorithmus eine einmal vorgenommene Zuordnung von Objekten zu einem Cluster in den nächstfolgenden Fusionierungsstufen nicht mehr rückgängig gemacht werden, was mitunter suboptimale Zuordnungen einzelner Objekte zu den Objektgruppen zur Folge hat. Daher soll in einem dritten Schritt die gefundene Clusterlösung des Ward-Verfahrens durch die Anwendung eines iterativ-partionierenden Verfahrens ${ }^{248}$ der Clusteranalyse überprüft und ggf. verbessert werden. ${ }^{249}$

Die Anwendung des Single Linkage-Verfahrens für die Stichprobe von 602 Ausstellern führt zur Identifikation von lediglich 17 Ausreißern. Offensichtlich realisieren damit nur sehr wenige der befragten Aussteller Individualtypen der messespezifischen Grundhaltung, die ausschließlich für den speziellen Aussteller und

246 Demnach ergibt sich die "richtige" bzw. "wahre" Anzahl von Clustern bei derjenigen Fusionierungsstufe, bei der die Fehlerquadratsumme den stärksten Zuwachs verzeichnet. Die Gruppenbildung erfolgt beim Ward-Verfahren anhand der Analyse der Fehlerquadratsumme als Varianzkriterium i. S. eines Heterogenitätsmaßes. Dabei werden im Verlauf der Fusionierung jeweils diejenigen Objekte oder Gruppen zusammengefaßt, die die Fehlerquadratsumme und damit die Heterogenität innerhalb einer Gruppe am wenigsten erhöhen. Vgl. Bergs. S., Optimalität bei Clusteranalysen: Experimente zur Bewertung numerischer Klassifikationsverfahren, Münster 1980, S. 30 f.

Vgl. Bergs, S., Optimalität bei Clusteranalysen: Experimente zur Bewertung numerischer Klassifikationsverfahren, a.a.O., S. 96 f.; Backhaus. K. et al., Multivariate Analysemethoden: Eine anwendungsorientierte Einführung, a.a.O., S. 289.

Zur Anwendung kommt die "Quick-Cluster-Prozedur", die unter SPSS für die iterative Partionierung von Objektgesamtheiten zur Vertügung steht. Vgl. Norusis, M. J., SPSS Professional Statistics 6.1., Chicago 1994, S. 111.

Die partionierenden Verfahren verlangen eine genaue Vorstellung darüber, wie viele Gruppen von Objekten in der Stichprobe existieren, da andernfalls eine sinnvolle Startpartition nicht vorgegeben werden kann. Die Verbesserung einer Gruppenbildung wird im Rahmen eines Austauschprozesses von Objekten durch die Verringerung eines Varianzkriteriums angestrebt. Neben der Vorgabe der nach dem Ward-Verfahren ermittelten "wahren“ Clusterzahl kann weiterhin die Startpartition durch die Nutzung der Clustermittelwerte der "Ward-Lösung" näher spezifiziert werden. Vgl. Backhaus, K. et al., Multivariate Analysemethoden: Eine anwendungsorientierte Einführung, a.a.O., S. $282 \mathrm{ff}$. Diesem vorgeschlagenen Vorgehen wird im Rahmen der vorliegenden Arbeit gefolgt. 
nicht ausstellerübergreifend nachweisbar sind. ${ }^{250}$ In die nachfolgende Clusterbildung nach dem Ward-Verfahren können damit 585 Aussteller einbezogen werden. Abb. 18 ist die Entwicklung des Varianzkriteriums für die hier in Frage kommende Gruppenbildung anhand der Grundhaltungsdimensionen zu entnehmen.

Dabei wird deutlich, daß mit Ausnahme des letzten Fusionierungsschrittes, bei dem aber verfahrensbedingt stets ein starker Anstieg der Fehlerquadratsumme besteht, die absoluten und relativen Zuwächse nahezu stetig verlaufen. Allerdings liegen ab dem Übergang von der 5- zur 4-Cluster-Lösung die prozentualen Zuwächse der Fehlerquadratsumme jeweils im Bereich von 3,3 und 3,9 Prozent, während sie zuvor jeweils relativ konstant zwischen 2,6 und 2,8 Prozent festzustellen sind. Daher erscheint in dem relevanten Fusionierungsbereich die 5Cluster-Lösung sinnvoll.

Die Mittelwerte der clusterbildenden Grundhaltungsdimensionen, wie sie aus der Ward-Analyse hervorgehen, werden in einem weiteren Analyseschritt als Startpartition für die "Quick-Cluster"-Prozedur genutzt, und die Objekte, sprich Ausstellerunternehmen, werden den fünf vorgegebenen Gruppen neu zugewiesen. ${ }^{251} \mathrm{Tab}$. 1 zeigt das Ergebnis dieser abschließenden Gruppierung, wobei für jedes Cluster die jeweiligen Abweichungen der "Ziel-", "Selektions-" und „Positionierungsdimensionen" vom Gesamtmittelwert ausgewiesen werden.

250 Verbindliche Grenzwerte für das Vorliegen eines Ausreißers existieren nicht. Es werden daher alle Fälle herausgefiltert, deren Zuordnung zu den bestehenden Gruppen im Dendogramm des Single Linkage-Verfahrens bei Fehlerquadratsummen über 20 erfolgt. Dazu ist ergänzend anzumerken, daß in Dendogrammen eine Normierung der Fehlerquadratsumme auf einer Skala von 0 bis 25 erfolgt.

Die Kombination hierarchischer und partionierender Verfahren, wie sie in der vorliegenden Untersuchung angewendet wird, wird auch von Lingenfelder und Schneider vorgeschlagen. Sie verarbeiten die Ergebnisse einer nach dem Ward-Verfahren durchgeführten Clusteranalyse ebenfalls mit der "Quick-Cluster"-Prozedur von SPSS weiter. In der vorliegenden Analyse konnte beobachtet werden, daß nach 5 Iterationen keine Austauschprozesse von Objekten zwischen den 5 Gruppen mehr stattgefunden haben, so daß zu diesem Zeitpunkt die "optimale" Cluster-Struktur gegeben war. Zur Kombination hierarchischer und partionierender Verfahren vgl. Lingenfelder. M. Schneider. W., Die Kundenzufriedenheit - Bedeutung, Meßkonzept und empirische Befunde, in: Arbeitspapier des Instituts für Marketing der Universität Mannheim, Nr. 80, Mannheim 1990, S. 9 ff. Zur Anwendung der Cluster- und der QuickCluster-Prozedur in SPSS vgl. Schubö, W. et al., SPSS: Handbuch der Programmversionen 4.0 und SPSS-X 3.0, a.a.O. S. $282 \mathrm{ff}$. und $540 \mathrm{ff}$. 


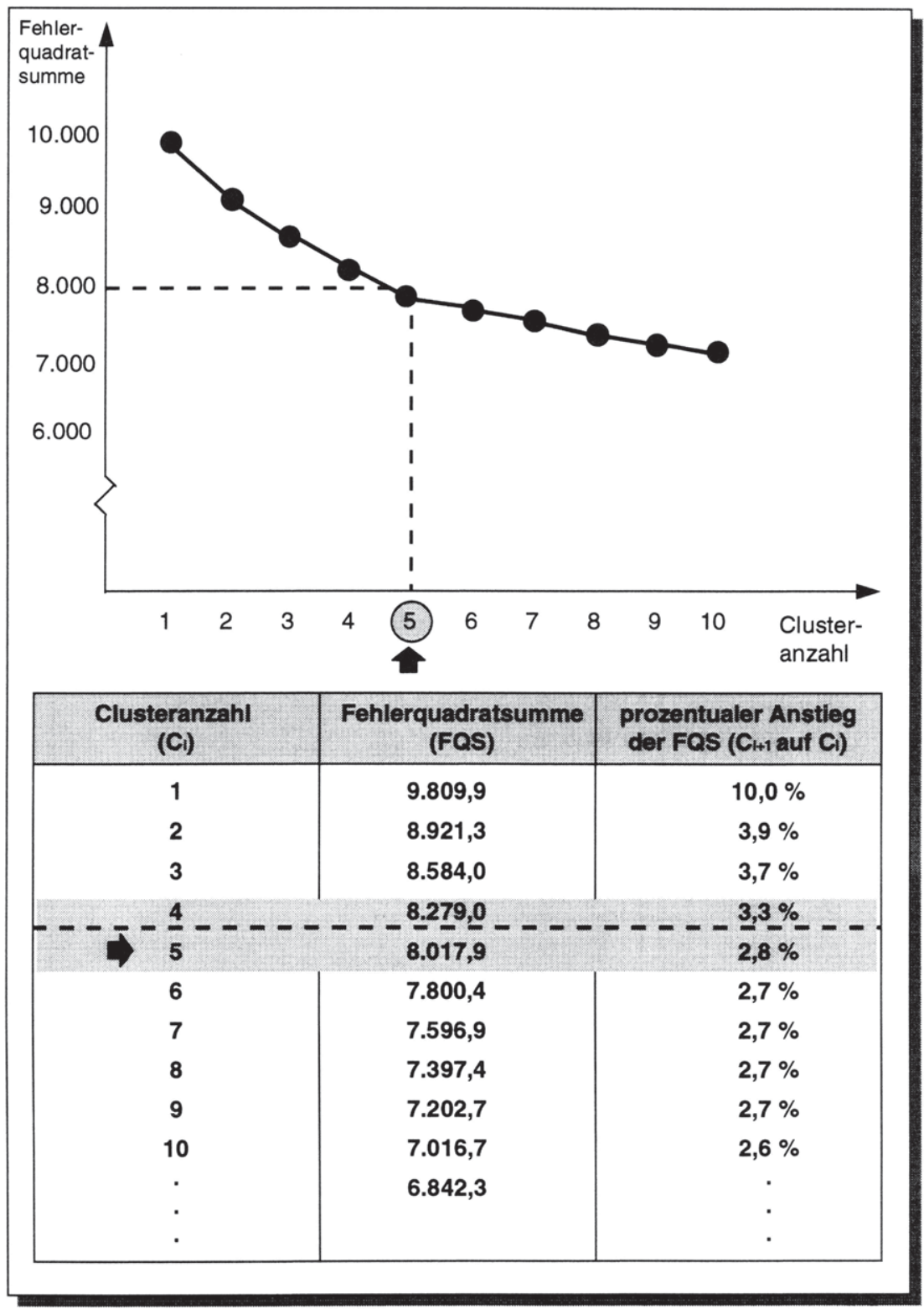

Abb. 18: Fusionierungsprozeß des Ward-Verfahrens und Varianzkriterium zur Bestimmung der Clusterlösung 


\begin{tabular}{|c|c|c|c|c|c|c|}
\hline \multirow{3}{*}{ 흘 홀흥 } & \multirow[b]{3}{*}{ Variablen } & \multicolumn{5}{|c|}{$\begin{array}{l}\text { Abweichungen der clusterbildenden } \\
\text { Variablen im jeweiligen Cluster vom } \\
\text { Gesamtmittelwert ( } n=541)\end{array}$} \\
\hline & & Cluster I & Cluster II & Cluster III & ICluster IV & Cluster V \\
\hline & & $\begin{array}{l}n=100 \\
18,5 \%\end{array}$ & $\begin{array}{l}n=155 \\
28,6 \%\end{array}$ & $\begin{array}{l}n=87 \\
16,1 \%\end{array}$ & $\begin{array}{l}n=101 \\
18,7 \%\end{array}$ & $\begin{array}{c}n=98 \\
18,1 \%\end{array}$ \\
\hline \multirow{6}{*}{ 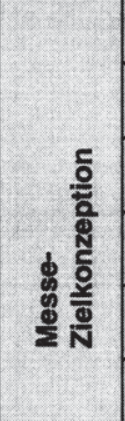 } & Informationszlele & $\mathbf{0}$ & 0 & - & - & + \\
\hline & Motivationsziele & - & 0 & -- & + & ++ \\
\hline & $\begin{array}{l}\text { marktbezogene } \\
\text { Beeinflussungsziele }\end{array}$ & $\mathbf{0}$ & 0 & - & $\mathbf{0}$ & + \\
\hline & $\begin{array}{l}\text { anspruchsgruppenbe- } \\
\text { zogene Beeinfl.-ziele }\end{array}$ & - & +++ & - & - & + \\
\hline & Verkaufszlele & +++ & -- & -- & - & + \\
\hline & Kontaktziele & $\mathbf{0}$ & 0 & - & $\mathbf{0}$ & + \\
\hline \multirow{6}{*}{ 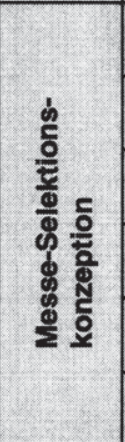 } & $\begin{array}{l}\text { Ausstellungs- } \\
\text { programm }\end{array}$ & -- & + & ++++ & -- & -- \\
\hline & $\begin{array}{l}\text { Grundorientierung } \\
\text { der Messe }\end{array}$ & --- & +++ & - & ++++ & - \\
\hline & $\begin{array}{l}\text { fachliche Orientierung } \\
\text { der Messe-Besucher }\end{array}$ & - & - & ++++ & -- & -- \\
\hline & $\begin{array}{l}\text { geogr. Einzugsbereich } \\
\text { der Besucher }\end{array}$ & +++ & ++++ & --- & ++ & --- \\
\hline & $\begin{array}{l}\text { geogr. Einzugsbereich } \\
\text { der Aussteller }\end{array}$ & ++ & +++ & -- & ++ & --- \\
\hline & $\begin{array}{l}\text { Durchführung von } \mathrm{Be}- \\
\text { gleitveranstaltungen }\end{array}$ & ++ & ---- & - & ++++ & + \\
\hline \multirow{4}{*}{ 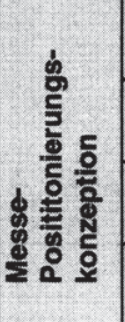 } & $\begin{array}{l}\text { Produkt- bzw. Sortl- } \\
\text { mentsorlentiertierung }\end{array}$ & ++ & - & -- & $\mathbf{0}$ & $\mathbf{0}$ \\
\hline & $\begin{array}{l}\text { Problemiösungs- bzw. } \\
\text { Nutzenorlentierung }\end{array}$ & - & ++ & -- & ++ & + \\
\hline & $\begin{array}{l}\text { Kommunikations- bzw } \\
\text { Erlebnisorientierung }\end{array}$ & - & ++ & - & $\mathbf{0}$ & $\mathbf{0}$ \\
\hline & $\begin{array}{l}\text { Wettbewerbs- } \\
\text { orlentierung }\end{array}$ & + & - & ++ & $\mathbf{0}$ & $\mathbf{0}$ \\
\hline \multicolumn{2}{|c|}{\begin{tabular}{l|l|} 
Abweichung der Cluster- & $\bar{X}$ \\
Mittelwerte vom & positiv \\
Gesamtmittelwert & negativ
\end{tabular} \mid} & $\begin{array}{l}-0,101 \\
0 \\
0\end{array}$ & $\begin{array}{c}11-0,251 \\
+ \\
-\end{array}$ & $\begin{array}{c}10,26-0,501 \\
++ \\
--\end{array}$ & $\begin{array}{r}10,51-0,81 \\
+++ \\
--\end{array}$ & $\begin{array}{r}>10,81 \\
++++ \\
---\end{array}$ \\
\hline
\end{tabular}

Tab. 1: Clusterbildende Merkmale der ermittelten Grundhaltungstypen 
Hervorzuheben sind mit Blick auf Tab. 1 zunächst die relativ homogenen Clustergrößen. ${ }^{252}$ Während das Cluster II als größtes Cluster 29 Prozent der Aussteller (absolut 155 Unternehmen) enthält, gehören dem kleinsten Cluster III immerhin noch 16 Prozent der befragten Aussteller (absolut 87 Unternehmen) an. Die Cluster I, IV und V vereinigen jeweils 100 bzw. 101 bzw. 98 Aussteller und damit jeweils ca. 18 Prozent aller befragten Unternehmen in sich. Darüber hinaus ist den deutlichen Mittelwertabweichungen, die sich bei der Mehrzahl der Grundhaltungsvariablen ergeben, zu entnehmen, daß offensichtlich wesentliche Unterschiede zwischen den identifizierten Grundhaltungstypen bestehen. Die Grundhaltungstypen sollen nachfolgend zunächst einzeln beschrieben werden. Da die Clusteranalyse eine rein auf statistischen, nicht aber auf inhaltlichen Kriterien beruhende Gruppenbildung vornimmt, ist zusätzlich eine Prüfung der inneren Konsistenz der gebildeten Typen vorzunehmen.

\subsection{Darstellung der Grundhaltungstypen}

Auf der Basis konkreter Mittelwertausprägungen der Grundhaltungsdimensionen, die in Tab. 2 wiedergegeben sind, lassen sich im folgenden die empirisch ermittelten, messespezifischen Grundhaltungstypen-Cluster differenzierter beschreiben und durch knappe, bewußt plakativ gehaltene Bezeichnungen charakterisieren: ${ }^{253}$

252 Diese ausgewogene Gruppengröße erweist sich insbesondere für die weitere statistische Analyse als vorteilhaft. Bei nur kleinen Fallzahlen in einzelnen Gruppen können statistisch gehaltvolle Aussagen über die entsprechenden Gruppen nicht mehr getroffen werden.

Zusätzlich zu den in Tab. 2 ausgewiesenen Mittelwerten der clusterbildenden Merkmale sind im Anhang 3 in den Tabellen A1 bis A5 die Häufigkeiten der clusterbildenden Merkmale für jede identifizierte Gruppe wiedergegeben. Für die vertiefende Analyse der Clusterspezifika sei auch auf diese Tabellen verwiesen. 


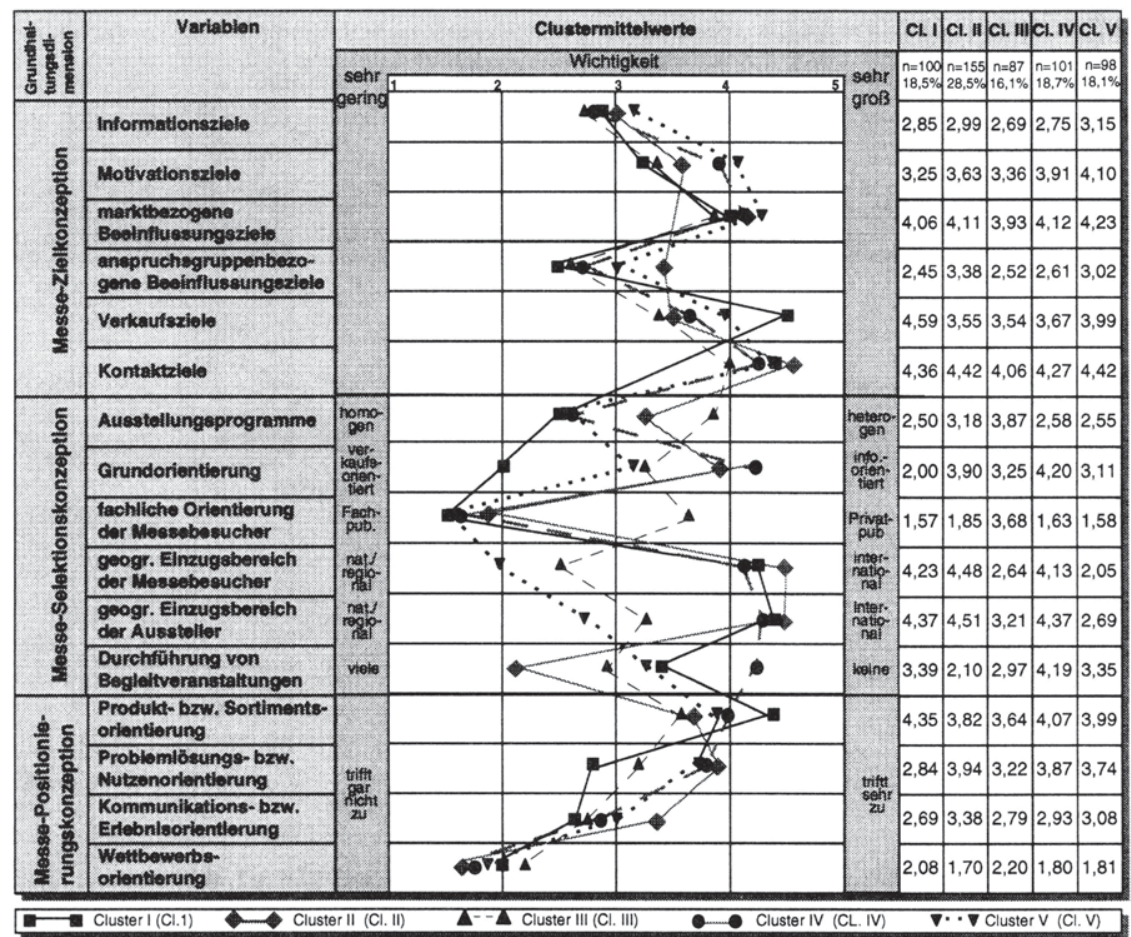

Tab. 2: Mittelwerte für die clusterbildenden Merkmale der ermittelten Grundhaltungstypen

\section{Cluster 1: fachbesucherorientierte Hardseller}

In diesem Cluster finden sich diejenigen Aussteller, deren Messe-Zielkonzeption durch eine verkaufsdominante Zielstruktur geprägt ist. Mit einem Mittelwert von 4,59 realisiert dieses Cluster den vergleichsweise höchsten Wert, wohingegen bei anderen Zieldimensionen höchstens eine durchschnittliche bzw. hinsichtlich der Motivations- und anspruchsgruppenbezogenen Beeinflussungsziele eine unterdurchschnittliche Zielwichtigkeit zu konstatieren ist.

Vor dem Hintergrund der vorliegenden Zielfokussierung wählen die Aussteller dieses Clusters schwerpunktmäßig solche Messeveranstaltungen aus, die eine eher homogene Struktur des Ausstellungsprogramms aufweisen - sei es aufgrund der Nomenklatur der Veranstaltung oder faktisch aufgrund ihrer thematischen Eingrenzung. Darüber hinaus sind die yon diesem Cluster selektierten Veranstaltungen mit 
Abstand am stärksten verkaufsorientiert und eindeutig auf das Fachpublikum ausgerichtet. Daneben zeichnen sich die Messen durch eine hohe Internationalität aus - sowohl auf der Besucher- als auch auf der Ausstellerseite. Aus Sicht der ausstellenden Unternehmen gehen von diesen Messeveranstaltungen eher wenige Impulse für die Durchführung von Begleitveranstaltungen zur Kommunikation mit verschiedenen Messezielgruppen aus.

Die durch die Ziel- und Selektionskonzeption deutlich werdende Verkaufsfokussierung bei Messebeteiligungen der Aussteller dieses Clusters findet auch ihre Entsprechung bei der messespezifischen Positionierungskonzeption. Hier konzentrieren sich die Aussteller im Vergleich zu allen anderen Ausstellern mit der höchsten Intensität auf das Produktprogramm, dessen Absatz anläßlich der Messe bzw. im direkten Nachmessegeschäft forciert werden soll.

In einer zusammenfassenden Würdigung kann für den ersten Grundhaltungstyp festgehalten werden, daß die Grundhaltungsdimensionen keine offensichtlichen Widersprüche aufweisen, so daß die Konsistenzbedingung als erfüllt angesehen werden kann. Für die weitere Untersuchung soll dieser Grundhaltungstyp als fachbesucherorientierter Hardseller gekennzeichnet werden.

\section{Cluster 2: kommunikationsorientierte Beziehungsmanager}

Dem zweiten Grundhaltungscluster gehören 28,6 Prozent der befragten Unternehmen an. Damit stellt dieses Cluster mit 155 Ausstellern den größten Anteil in der Stichprobe. Im Gegensatz zum Cluster I finden sich hier Ausstellerunternehmen, die den Verkaufszielen einen vergleichsweise nachrangigen Stellenwert einräumen. Für diese Unternehmen stehen vielmehr Kommunikationsziele, wie anspruchsgruppenbezogene Beeinflussungsziele, überdurchschnittlich stark im Vordergrund (vgl. auch Tab. 1). Wesentliche Zielgrößen stellen aber in diesem Cluster die Kontakt- und die marktbezogenen Beeinflussungsziele dar.

Diese messespezifische Zielkonzeption verbinden die Aussteller mit Messeveranstaltungen, die sich vor allem durch eine starke Informationsorientierung sowie eine hohe Internationalität und durch eine Vielzahl durchgeführter Begleitveranstaltungen auszeichnen. Schwerpunkt der Messezielgruppen bilden auch in diesem Cluster mehrheitlich die Fachbesucher. 
Im Rahmen der Positionierung stehen Aspekte der Profilierung einer Nutzen- bzw. Problemlösungskompetenz des Unternehmens im Mittelpunkt der Bemühungen. Daneben wird allerdings auch eine eher kommunikative bzw. erlebnisbetonte Positionierung angestrebt (vgl. auch Tab. 2). Allerdings liegt die Bedeutung einer produktorientierten Profilierung in diesem Cluster noch vor der Kommunikationsund Erlebnisorientierung.

Insgesamt kann aber auch hinsichtlich der Unternehmen dieses Grundhaltungstyps eine ebenfalls konsistente Messe-Grundhaltungskonzeption festgestellt werden. Im Mittelpunkt steht dabei vermehrt die Nutzung des Mediums Messe als Instrument des Beziehungsmarketing, bei dem vor allem kommunikative Potentiale von Messeveranstaltungen von diesem Cluster aufgegriffen werden. Im Ergebnis

soll dieser Grundhaltungstyp daher als kommunikationsorientierter Beziehungsmanager bezeichnet werden.

\section{Cluster 3: wettbewerbsorientierte Pflichtrepräsentanten}

Das dritte Cluster beinhaltet mit 87 Ausstellern lediglich 16,1 Prozent der Aussteller und stellt insgesamt damit die kleinste Gruppe von Ausstellern dar. Auch mit Blick auf Tab. 1 wird bereits aus der Gesamtsicht der Zieldimensionen eine im Vergleich zum Gesamtdurchschnitt geringere Zielorientierung deutlich. Nahezu jeder Zielorientierung wird ein niedrigerer Stellenwert zugeordnet als von den übrigen Ausstellerclustern. Den höchsten Stellenwert besitzen mit Abstand Kontaktziele ( $M W=4,06)$ sowie marktbezogene Beeinflussungsziele ( $M W=3,93$ ) für die Aussteller dieses Clusters.

Die im Rahmen der Messeselektion ausgewählten Messeveranstaltungen weisen eine hohe Heterogenität in bezug auf das Ausstellungsprogramm und eine ausgeprägte Privatbesucherorientierung auf. Des weiteren sind die ausgewählten Messeveranstaltungen hinsichtlich ihrer geographischen Reichweite bei Ausstellern und Messebesuchern deutlich auf nationales bzw. regionales Niveau eingeschränkt.

Obschon als Profilierungsrichtung hauptsächlich eine Produkt- bzw. Sortimentsorientierung angestrebt wird, weist dieser Positionierungstyp lediglich eine überdurchschnittliche Wettbewerbsorientierung auf. Damit wird deutlich, daß die Aussteller dieses Clusters sich am ehesten in ihrer Gestaltung der Messebeteiligung von den Aktivitäten der Wettbewerber leiten lassen, die vor dem Hintergrund der 
unterdurchschnittlichen Zielausprägung als eine „Pflichtrepräsentanz“ interpretiert werden kann.

Im Ergebnis läßt sich ein „eigenständig“ spezifisches Grundhaltungsmuster, über das die Ausgestaltung der Messebeteiligung erfolgt, nicht konstatieren. Vielmehr scheint eine zielorientierte Ausrichtung der Messeaktivitäten zu unterbleiben. Insofern erfüllt aber auch dieser Grundhaltungstyp die Konsistenzbedingung, denn es ergeben sich keine widersprüchlichen Ausgestaltungsformen der messespezifischen Grundhaltung, obwohl allerdings die gewählte Planungsform von Messebeteiligungen zunächst fraglich erscheint. Nachfolgend soll dieser Grundhaltungstyp als wettbewerbsorientierter Pflichtrepräsentant bezeichnet werden.

\section{Cluster 4: hybride Fachaussteller}

Das vierte Cluster, das $18,7 \%$ der befragten Aussteller enthält, umfaßt hinsichtlich der meisten Zieldimensionen eine eher durchschnittliche Ausgestaltung. Allerdings lassen diese Aussteller erkennen, daß sie mit Messebeteiligungen insbesondere die Möglichkeiten der Mitarbeitermotivation und damit auch innengerichtete Zielsetzungen verbinden (vgl. Tab. 1).

Die Aussteller dieses Clusters beteiligen sich darüber hinaus stark an internationalen Messeveranstaltungen, die primär der Informationsvermittlung eines ausgewählten Fachkreises dienen. Als Besonderheit dieser Veranstaltungen kann weiterhin die nahezu fehlende Durchführung von Begleitveranstaltungen festgehalten werden.

Die Informationsorientierung der Messeveranstaltungen sowie die auch bei diesem Grundhaltungstyp neben den Motivationszielen dominierenden Kontakt- und Marktbeeinflussungsziele schlagen sich in einer deutlichen Positionierung in Form der kundengerichteten Problemlösungs- bzw. Nutzenorientierung nieder. Insofern kann bei diesem Cluster von einer innen- und außengerichteten Ausgestaltung von Messebeteiligungen, die als hybride Grundhaltung interpretiert werden soll, ausgegangen werden.

Zusammenfassend liegt hier ein Grundhaltungstyp vor, bei dem vermutlich die Mitarbeiterorientierung nahezu gleichwertig neben unmittelbar kundenbezogenen Aspekten Beachtung findet. Dieser Grundhaltungstyp soll daher im weiteren Verlauf dieser Arbeit als hyprider Fachaussteller gekennzeichnet werden. 


\section{Cluster 5: ambitionierte Regionalaussteller}

Der fünfte Grundhaltungstyp, der von 98 befragten Unternehmen und damit 18,1 Prozent der Stichprobe praktiziert wird, umfaßt solche Aussteller, die insbesondere über eine überdurchschnittliche Zielorientierung verfügen - dies nicht nur hinsichtlich der Wichtigkeit einzelner Zieldimensionen, sondern auch bezogen auf die Bandbreite verfolgter Zielsetzungen. Mit Ausnahme der Verkaufsziele (die fachbesucherorientierten Hardseller in Cluster I verbinden mit dieser Zielkategorie einen höheren Stellenwert) wird jeder Zieldimension von diesem Cluster die vergleichsweise höchste Zielpriorität eingeräumt.

Interessanterweise sind die Aussteller des Clusters 5 insbesondere auf solchen Messeveranstaltungen vertreten, bei denen vor allem das nationale bzw. regionale Fachpublikum und das primär nationale Angebot zusammentreffen.

Hinsichtlich ihrer messespezifischen Positionierung dominiert die Produkt- bzw. Sortimentsorientierung, wenngleich diese Aussteller auch überdurchschnittlich stark eine Profilierung als Problemlöser bzw. Nutzenstifter aus Kundensicht anstreben.

Die Aussteller des fünften Grundhaltungstyps zeigen zusammenfassend eine ebenfalls konsistente Ausgestaltung des Messekonzepts, das sich vor allem durch eine Multioptionalität im Rahmen der Messezielkonzeption sowie die eindeutige Priorisierung von Messeveranstaltungen mit nationaler bzw. regionaler Reichweite auszeichnet. Insofern soll dieser Grundhaltungstyp daher als ambitionierter Regionalaussteller bezeichnet werden.

Die Clusteranalyse hat deutlich gemacht, daß sich hinsichtlich der messespezifischen Grundhaltung von Ausstellern verschiedene Typen identifizieren lassen. Bevor die Hypothese $H_{\mathrm{GH}}$ abschließend bestätigt werden kann, muß überprüft werden, ob es sich bei der gefundenen Clusterlösung um einen stabilen und signifikanten Befund handelt. 


\subsection{Diskriminanzanalytische Überprüfung der Clusterlösung und Ermittlung der zur Typendifferenzierung zentralen Grundhaltungsdimensionen}

Die zuvor durchgeführte Analyse der fünf identifizierten messespezifischen Grundhaltungstypen hat grundsätzliche Unterschiede in der Ausgestaltung der strategischen Ausrichtung des Messeengagements von Ausstellern gezeigt. Um die Unterschiede der Grundhaltungstypen auf der einen Seite statistisch abzusichern und auf der anderen Seite die für die Differenzierung der Typen wichtigsten Grundhaltungsvariablen und -dimensionen zu identifizieren, kann das Verfahren der Diskriminanzanalyse herangezogen werden. ${ }^{254}$

Die Güte der Diskriminanzanalyse und damit indirekt auch die Qualität der Clusterlösung lassen sich anhand des "Wilks'Lambda“ bzw. des „kanonischen Korrelationskoeffizienten" und des Anteiles der richtig reproduzierten Klassifikationen der Untersuchungsobjekte ermitteln. Dabei mißt der kanonische Korrelationskoeffizient das Verhältnis der durch die Diskriminanzfunktionen erklärten Streuung (zwischen den Gruppen) und Wilks'Lambda das der nicht erklärten Streuung (innerhalb der Gruppen) zur Gesamtstreuung (Streuung zwischen den Objekten bezogen auf die Variablen). Üblicherweise kommt bei der Prüfung der Diskriminanz Wilks'Lambda ${ }^{255}$ zur Anwendung. Es handelt sich hierbei um ein inverses Gütemaß, d. h. kleine Werte nahe Null deuten auf eine hohe Trennkraft der Diskriminanzfunktionen hin. ${ }^{256}$

Im vorliegenden Untersuchungsfall ergibt eine vorgeschaltete univariate Analyse der Trennfähigkeit der einzelnen Grundhaltungsdimensionen, daß alle Variablen für sich genommen signifikant (Irrtumswahrscheinlichkeit $<0,5$ Prozent) zwischen

254 Die Diskriminanzanalyse gehört zu den struktur-prüfenden Verfahren der multivariaten Datenanalyse. Wesentlicher Einsatzzweck ist die Analyse von Gruppenunterschieden. Das Verfahren bietet sich damit zur Validierung der Ergebnisse von Clusteranalysen an Vgl. Meffert. $H$., Marketingforschung und Käuferverhalten, a.a.O., S. 292 f.; Fahrmeir, L., Häußler, W. Tutz. G., Diskriminanzanalyse, a.a.O., S. 301 f., Backhaus, K. et al., Multivariate Analysemethoden: Eine anwendungsorientierte Einführung, a.a.O., S. 90-165.

Wilks Lambda bildet das Verhältnis von nicht erklärter Streuung zu Gesamtstreuung ab.

Die Diskriminanzanalyse stellt die abhängige (Diskriminanz-)Variable als eine Linearkombination der unabhängigen Variablen (=Prädiktoren) dar. Damit werden also nicht nur Mittelwertunterschiede auf ihre Signifikanz gegen Null getestet, sondern auch Diskriminanzfunktionen gebildet. Eine ausführliche Darstellung der Methodik der Diskriminanzanalyse sowie der zur Verügung stehenden Gütemaße findet sich bei Backhaus, K. et al., Multivariate Analysemethoden: Eine anwendungsorientierte Einführung, a.a.O., S. 90-165 und Brosius, G., SPSS/PC+ Advanced Statistics and Tables - Einführung und praktische Beispiele, a.a.O., S. $106 \mathrm{ff}$. 
den fünf Clustern trennen ${ }^{257}$ und somit in die weitere Diskriminanzanalyse einbezogen werden können. Die eigentliche Diskriminanzanalyse führt zur Bestimmung von vier signifikanten Diskriminanzfunktionen. Dabei erklären die ersten beiden Funktionen bereits 67,35 Prozent der Varianz zwischen den Grundhaltungstypen bei einem geringen Wilks'Lambda von nur 0,3455. Die Einbeziehung der beiden weiteren Funktionen erhöht die Trennfähigkeit auf 100 Prozent, verschlechtert allerdings das Wilks'Lambda trotz Wahrung der Signifikanz auf 0,631 bzw. den kanonischen Korrelationskoeffizienten auf einen Wert von $0,607 .{ }^{258}$ Dennoch ist insgesamt eine hohe Trennqualität der Analyse zu konstatieren. ${ }^{259}$

Mit Hilfe der Diskriminanzfunktion können im nächsten Schritt die Untersuchungsobjekte (Aussteller) klassifiziert, $\mathrm{d} h$. ihre Zugehörigkeit zu einer der vorgegebenen fünf Cluster geschätzt, und den „tatsächlichen“, d. h. der durch die Clusteranalyse erzeugten Gruppenzuordnung gegenübergestellt werden. Dies geschieht in Form einer Klassifikationsmatrix, wie sie für den vorliegenden Fall der Abb. 19 zu entnehmen ist. Mit einer "Trefferquote" von 92,79 Prozent ist ein sehr gutes Ergebnis erzielt worden. ${ }^{260}$ Lediglich zwischen Cluster II und Cluster IV ist eine nennenswerte Unschärfe zu beobachten, die sicherlich auf das in einigen Grundhaltungsdimensionen ähnlich verlaufende Grundhaltungstypenprofil zurückzuführen ist (vgl. Tab. 1 und Tab. 2). Bei den übrigen Grundhaltungstypen liegt der Anteil der Fehlzuordnungen dagegen zwischen 0 und 5,7 Prozent, so daß eine hohe Trennschärfe zwischen den Grundhaltungstypen zu konstatieren ist.

257 Vgl hierzu Backhaus, K. et al., Multivariate Analysemethoden: Eine anwendungsorientierte Einführung, a.a.O., S. 138.

$258 \mathrm{Vgl}$. dazu das SPSS-Listing der Diskriminanzanalyse im Anhnag der vorliegenden Arbeit.

259 Zur Beurteilung der Trennqualität von Diskriminanzanalysen im Mehrgruppenfall vgl. Backhaus, K. et al., Multivariate Analysemethoden: Eine anwendungsorientierte Einführung, a.a.O., S. 138 ff.; Brosius, G., SPSS/PC+ Advanced Statistics and Tables - Einführung und praktische Beispiele, a.a.O., S. $116 \mathrm{ff}$.

Die Güte der Klassifikation wird deutlich, wenn man sich vor Augen führt, daß sich in einem 5Gruppen-Fall bei Annahme annähernd gleich großer Cluster bei zufälliger Einordnung der Objekte eine Trefferquote von nur 20 Prozent ergeben würde. Allerdings ist zu berücksichtigen, daß die Trefferquote tendenziell überhöht ist, wenn sie auf Basis derselben Stichprobe berechnet wird, die gleichzeitig für die Ableitung der Diskriminanzfunktionen verwendet wird. Durch die mit 541 Objekten relativ große Stichprobe wird dieser Effekt jedoch deutlich vermindert. Vgl. hierzu Backhaus, K. et al., Multivariate Analysemethoden: Eine anwendungsorientierte Einführung, a.a.O., S. $115 \mathrm{f}$. 


\begin{tabular}{|c|c|c|c|c|c|c|}
\hline \multirow{2}{*}{\multicolumn{2}{|c|}{$\begin{array}{l}\text { Geschätzte Clusterzuge- } \\
\text { hórigkelt auf Basis der } \\
\text { Diskriminanz. } \\
\text { analyse }\end{array}$}} & \multirow{2}{*}{$\begin{array}{c}\begin{array}{c}\text { Cluster I } \\
\mathbf{n}=100\end{array} \\
\text { fachbesucher- } \\
\text { orientierte } \\
\text { Hardseller }\end{array}$} & \multirow{2}{*}{$\begin{array}{c}\begin{array}{c}\text { Cluster II } \\
n=155\end{array} \\
\begin{array}{c}\text { kommunikations- } \\
\text { attive Bezieh- } \\
\text { ungsmanager }\end{array}\end{array}$} & \multirow{2}{*}{\begin{tabular}{|c|}
$\begin{array}{c}\text { Cluster III } \\
\mathbf{n}=87\end{array}$ \\
$\begin{array}{c}\text { wettbewerbs- } \\
\text { orientierte Pllicht- } \\
\text { repräsentanten }\end{array}$ \\
\end{tabular}} & \multirow{2}{*}{$\begin{array}{c}\begin{array}{c}\text { Cluster IV } \\
\mathbf{n}=101\end{array} \\
\begin{array}{c}\text { hybride } \\
\text { Fachaussteller }\end{array}\end{array}$} & \multirow{2}{*}{$\begin{array}{c}\begin{array}{c}\text { Cluster V } \\
\mathrm{n}=98\end{array} \\
\begin{array}{c}\text { ambitionierte } \\
\text { Regional- } \\
\text { aussteller }\end{array}\end{array}$} \\
\hline & & & & & & \\
\hline $\begin{array}{l}\text { Cluster I } \\
n=100\end{array}$ & $\begin{array}{l}\text { fachbesucher- } \\
\text { orientierte } \\
\text { Hardseller }\end{array}$ & $93,0 \%$ & $2,0 \%$ & $3,0 \%$ & $0 \%$ & $2,0 \%$ \\
\hline $\begin{array}{l}\text { Cluster } 11 \\
n=155\end{array}$ & $\begin{array}{l}\text { kommunikations- } \\
\text { aktive Bezieh- } \\
\text { ungsmanager }\end{array}$ & $0,6 \%$ & $98,1 \%$ & $0,6 \%$ & $0,6 \%$ & $0 \%$ \\
\hline $\begin{array}{c}\text { Cluster in: } \\
n=87\end{array}$ & $\begin{array}{l}\text { wettbewerbs- } \\
\text { orientierte Pflicht- } \\
\text { repräsentanten }\end{array}$ & $0 \%$ & $5,7 \%$ & $89,7 \%$ & $1,1 \%$ & $3,4 \%$ \\
\hline $\begin{array}{l}\text { Cluster IV } \\
n=101\end{array}$ & $\begin{array}{c}\text { hybride } \\
\text { Fachaussteller }\end{array}$ & $1,0 \%$ & $9,9 \%$ & $0 \%$ & $86,1 \%$ & $3,0 \%$ \\
\hline $\begin{array}{l}\text { Cluster V } \\
n=98\end{array}$ & $\begin{array}{l}\text { ambitionierte } \\
\text { Regional- } \\
\text { aussteller }\end{array}$ & $0 \%$ & $2,0 \%$ & $3,1 \%$ & $1,0 \%$ & $93,9 \%$ \\
\hline
\end{tabular}

Abb. 19: Klassifikationsmatrix der diskriminanzanalytischen Untersuchung der messespezifischen Grundhaltungstypen

Insgesamt kann damit an dieser Stelle festgehalten werden, daß die Basishypothese $\mathrm{H}_{\mathrm{GH}}$ in vollem Umfang angenommen werden kann. Offensichtlich lassen sich im Rahmen von Messebeteiligungen differenzierte, durch signifikant abweichende Ausgestaltungsmuster der Grundhaltungsdimensionen gekennzeichnete MesseGrundhaltungstypen unterscheiden.

In der weiteren Analyse ist nunmehr der Frage nachzugehen, auf welche der grundhaltungstypenkonstituierenden Merkmale die Gruppenunterschiede in erster Linie zurückgeführt werden können. Zu diesem Zweck wird eine schrittweise Diskriminanzanalyse durchgeführt. ${ }^{261}$

261 Im Gegensatz zur klassischen Diskriminanzanalyse werden dabei nicht alle Variablen simultan zur Diskriminierung der Gruppen verwendet, sondern es erfolgt eine sukzessive Einbeziehung derjenigen Variablen, die zur Optimierung eines bestimmten Gütemaßes den höchsten Beitrag leisten und dabei signifikant zur Erhöhung der Diskriminanz zwischen den Gruppen beitragen. Als Gütemaß wird auch hier auf Wilks'Lambda zurückgegriffen. Als maximales Signifikanzniveau für die Aufnahme einer Grundhaltungsvariablen wurde der Wert $0,025(=2,5 \%)$, als minimales Signifikanzniveau für die abermalige Entfernung einer bereits aufgenommenen Grundhaltungsvariablen der Wert $0,05(=5,0 \%)$ vorgegeben. 
Wie Tab. 3 verdeutlicht, führt diese im vorliegenden Fall zu einer deutlichen Reduzierung der Grundhaltungsvariablen. Nur 12 von insgesamt 16 Variablen erweisen sich bei einem Signifikanzniveau von 2,5 Prozent als diskriminierungswirksam zwischen den Grundhaltungstypen. ${ }^{262}$ Die volle Diskriminanzwirkung einer Variablen, d. h. ihr Beitrag zur Trennung der Grundhaltungstypen, kann anhand des mittleren Diskriminanzkoeffizienten bestimmt werden. ${ }^{263}$ Die als Prozentwerte erfaßten Diskriminanzwirkungen der 12 Variablen und ihre Zuordnung zu den einzelnen Grundhaltungsdimensionen sind in Tab. 3 wiedergegeben.

Als wesentlichste Trennkriterien für Grundhaltungstypen unter ausstellenden Unternehmen erweisen sich damit die Unterscheidung der Messebesucher zwischen Fach- und Privatpublikum (Diskriminanzwirkung: 15,44 \%), die geographische Reichweite der Messeveranstaltung bei den Messebesuchern (Diskriminanzwirkung: 13, $85 \%$ ), die Grundorientierung der Messeveranstaltung (Diskriminanzwirkung: $12,71 \%$ ) sowie die Unterscheidung nach der Intensität durchgeführter Begleitveranstaltungen (Diskriminanzwirkung: 10,87 \%). Allein diese jeweils über 10 Prozent Diskriminanzwirkung besitzenden Variablen der messespezifischen Grundhaltung vermögen fast 53 Prozent der Unterschiede zwischen den Grundhaltungstypen zu erklären.

262 Zur Überprüfung der Diskriminierungskraft der in den Diskriminanzfunktionen verbleibenden 12 Variablen wurde wiederum eine Klassifikationsmatrix bestimmt. Dabei wurde mit 92,42 Prozent korrekt zugeordneter Aussteller ein fast identisches Klassifikationsergebnis erzielt. Dies bedeutet aber umgekehrt, daß mit den übrigen 4 Grundhaltungsvariablen lediglich eine marginale Verbesserung der Klassifikation um 0,37 Prozent möglich ist. Die Klassifikationsmatrix findet sich im Anhang 3 dieser Arbeit.

Der mittlere Diskriminanzkoeffizient errechnet sich durch die Multiplikation der von SPSS automatisch berechneten standardisierten Diskriminanzkoeffizienten mit den Eigenwerten der jeweiligen Diskriminanzfunktionen. Vgl. Backhaus, K. et al., Multivariate Analysemethoden: Eine anwendungsorientierte Einführung, a.a.O., S. 140 f. 


\begin{tabular}{|c|c|c|c|c|c|c|}
\hline $\begin{array}{l}\text { Grundhaltungs- } \\
\text { variablen 1) }\end{array}$ & $\begin{array}{l}\text { Wilks' } \\
\text { Lambda }\end{array}$ & $\begin{array}{l}\text { mittlerer } \\
\text { Diskrimi- } \\
\text { nlerungs- } \\
\text { koeffizient }\end{array}$ & $\begin{array}{l}\text { Diskrimi- } \\
\text { nlerungs- } \\
\text { wirkung } \\
\text { in } \% \text { 2) }\end{array}$ & $\begin{array}{l}\text { Variab- } \\
\text { len- } \\
\text { rang }\end{array}$ & $\begin{array}{l}\text { zugehörl- } \\
\text { ge Grund- } \\
\text { haltungs- } \\
\text { dimension }\end{array}$ & $\begin{array}{l}\text { Diskrimi- } \\
\text { nierungs- } \\
\text { wirkung } \\
\text { in \% }\end{array}$ \\
\hline Motivationsziele & 0,0691 & 0,4882 & $4,37 \%$ & 12 & \multirow[b]{2}{*}{$\begin{array}{l}\text { Messe- } \\
\text { zielkon- } \\
\text { zeption }\end{array}$} & \multirow[b]{2}{*}{$15,13 \%$} \\
\hline $\begin{array}{l}\text { anspruchsgruppenbe- } \\
\text { zogene Beeinfl.-ziele }\end{array}$ & 0,0651 & 0,5063 & $4,53 \%$ & 11 & & \\
\hline Verkaufsziele & 0,0747 & 0,6962 & $6,23 \%$ & 7 & \multirow{5}{*}{\begin{tabular}{|l|} 
\\
$\begin{array}{c}\text { Messe- } \\
\text { selek- } \\
\text { tions- } \\
\text { konzep- } \\
\text { tion }\end{array}$ \\
\end{tabular}} & \multirow{5}{*}{$69,65 \%$} \\
\hline $\begin{array}{l}\text { Ausstellungs- } \\
\text { programm }\end{array}$ & 0,1025 & 1,0038 & $8,98 \%$ & 5 & & \\
\hline $\begin{array}{l}\text { Grundorientierung } \\
\text { der Messe }\end{array}$ & 0,1748 & 1,4213 & $12,71 \%$ & 3 & & \\
\hline $\begin{array}{l}\text { fachliche Orientierung } \\
\text { der Messe-Besucher }\end{array}$ & 0,2798 & 1,7263 & $15,44 \%$ & 1 & & \\
\hline $\begin{array}{l}\text { geogr. Einzugsbereich } \\
\text { der Besucher }\end{array}$ & 0,4549 & 1,5480 & $13,85 \%$ & 2 & & \\
\hline $\begin{array}{l}\text { geogr. Einzugsbereich } \\
\text { der Aussteller }\end{array}$ & 0,0811 & 0,8714 & $7,79 \%$ & 6 & \multirow{5}{*}{\begin{tabular}{|c|}
$\begin{array}{c}\text { Messe- } \\
\text { positio- } \\
\text { hlerungs- } \\
\text { konzep- } \\
\text { tion }\end{array}$ \\
\end{tabular}} & \multirow[b]{4}{*}{$15,22 \%$} \\
\hline $\begin{array}{l}\text { Durchführung von Be- } \\
\text { gleltveranstaltungen }\end{array}$ & 0,1179 & 1,2157 & $10,87 \%$ & 4 & & \\
\hline $\begin{array}{l}\text { Problemlösungs- bzw. } \\
\text { Nutzenorlentlerung }\end{array}$ & 0,0903 & 0,6633 & $5,93 \%$ & 8 & & \\
\hline $\begin{array}{l}\text { Kommunikations-bzw. } \\
\text { Erlebnisorlentierung }\end{array}$ & 0,0602 & 0,5169 & $4,62 \%$ & 10 & & \\
\hline \multirow[t]{2}{*}{$\begin{array}{l}\text { Wettbewerbs- } \\
\text { orlentierung }\end{array}$} & 0,0624 & 0,5224 & $4,67 \%$ & 9 & & \\
\hline & & 11,1798 & $100 \%$ & & & \\
\hline \multicolumn{7}{|c|}{$\begin{array}{l}\text { 1) reduzierte Variablenstruktur nach schrittweiser Diskriminanzanalyse } \\
\text { 2) beschreibt den Anteil einer Grundhaltungsvariablen zur Klassifizierung der Fälle } \\
\text { 3) beschreibt den rechnerischen Gesamtanteil einer Grundhaltungsdimension zur } \\
\text { Klassifizierung der Fälle (nach schrittweiser Diskriminanzanalyse) }\end{array}$} \\
\hline
\end{tabular}

Tab. 3: Beiträge der Grundhaltungsdimensionen für die Diskriminierung der ermittelten Grundhaltungstypen 
Ordnet man die einzelnen Variablen den entsprechenden Führungsdimensionen zu, so zeigt sich die zentrale Bedeutung der Messe-Selektionskonzeption für die Diskriminierung der Grundhaltungstypen. Mit ca. 70 Prozent steuert diese Grundhaltungsdimension den absolut dominierenden Beitrag zur Diskriminanz der verschiedenen Gruppen von Ausstellern bei. Demgegenüber belaufen sich die Erklärungsbeiträge der Messe-Ziel- und der Messe-Positionierungskonzeption lediglich jeweils auf ca. 15 Prozent. Darüber hinaus gehen von drei Dimensionen der Messe-Zielkonzeption, den Kontakt-, Informations- und marktbezogenen Beeinflussungszielen. keine signifikanten Diskriminanzwirkungen aus. Offensichtlich weisen damit diese messespezifischen Zielsetzungen sowie der produkt- bzw. sortimentsorientierte Positionierungsansatz in allen fünf Grundhaltungstypen ähnliche Ausprägungen auf. ${ }^{264}$

Gleichzeitig unterstreicht die schrittweise Diskriminanzanalyse, daß die Streuungsbreite der Ausprägungsformen im Rahmen der Gestaltung der messespezifischen Grundhaltung deutlich variiert. Offensichtlich werden die in Kapitel B.1. abgeleiteten Gestaltungsfelder für die einzelnen Grundhaltungsdimensionen unterschiedlich stark von den Ausstellern genutzt. Damit erlangen vor dem Hintergrund dieser Ausgestaltungsunterschiede die hierzu in Kapitel B.1. abgeleiteten Hypothesen unmittelbar Relevanz, die abschließend im Rahmen einer inhaltichen Analyse der empirischen Grundhaltungstypen im folgenden Kapitel gewürdigt werden sollen.

\subsection{Zusammenfassende Würdigung der empirischen Grundhaltungstypen}

Die für die Grundhaltungstypen ermittelten Ausprägungsformen der in die Analyse einbezogenen Grundhaltungsdimensionen und inrer Indikatorvariablen sind vor dem Hintergrund der in Kapitel B.1. aufgestellten Hypothesen kritisch zu beleuchten.

Vor dem Hintergrund der geringen Unterschiede bei der Gewichtung einzelner Zieldimensionen durch die Aussteller ist mit Blick auf die Ausgestaltung der Zielkonzeption bei den fünf Grundhaltungstypen das Bedeutungsgewicht der Informations-, marktbezogenen Beeinflussungs- und Kontaktziele für die Bildung homogener Ausstellergruppen als nachrangig zu bezeichnen. Das heißt anderer-

$264 \mathrm{Vgl} \mathrm{hierzu} \mathrm{die} \mathrm{Tab.} \mathrm{2.} \mathrm{Diese} \mathrm{verdeutlicht,} \mathrm{daß} \mathrm{in} \mathrm{der} \mathrm{Tat} \mathrm{für} \mathrm{diese} \mathrm{Grundhaltungsindikatoren}$ geringere Mittelwertunterschiede zwischen den fünf Grundhaltungstypen bestehen.

Ralf Ueding and Universität Münster - 978-3-631-75073-5 
seits, daß sich die Gruppenunterschiede im Rahmen der Zielkonzeption vor allem durch die gruppenindividuelle Bedeutung von Motivations-, anspruchsgruppenbezogenen Beeinflussungs- und Verkaufszielen ableiten lassen. Dabei sind insbesondere hinsichtlich des Stellenwertes von Verkaufszielen signifikant unterschiedliche Gewichtungen in der Messe-Zielkonzeption der Grundhaltungstypen zu verzeichnen. Auf der Basis dieses Ergebnisses ist die Hypothese $\mathbf{H}_{\mathbf{z k} 2}$, wonach sich durchgängig unterschiedliche Zielmuster bei der Ableitung von messespezifischen Grundhaltungen feststellen lassen, die einen wesentlichen Beitrag zur Differenzierung der Grundhaltungstypen leisten, nur teilweise zu bestätigen.

Beleuchtet man des weiteren den für die Bildung der Grundhaltungstypen postulierten Zusammenhang zwischen Messe-Ziel- und -Selektionskonzeption, so hat die inhaltliche Konsistenzprüfung der fünf Cluster gezeigt, daß für die Beteiligung an Messen grundsätzlich von einem Abstimmungsprozeß zwischen Messezielen und Messeselektion auszugehen ist. Damit kann Hypothese $\boldsymbol{H}_{S k}$, nach der Aussteller jene Messen auswählen, die grundsätzlich zur Verfolgung der messespezifischen Zielsetzungen geeignet sind, bestätigt werden.

Neben der Überprüfung der Messe-Selektionskonzeption ist im weiteren auch die Richtigkeit der Hypothesen, die hinsichtlich der Ausgestaltung der Messe-Positionierungskonzeption formuliert worden sind, festzustellen.

Mit Blick auf Tab. 1 kann die Basishypothese $\mathbf{H}_{\mathbf{P k} 1}$, nach der verschiedene Messe-Zielkonzeptionen sowie Messe-Selektionskonzeptionen signifikant unterschiedliche Messepositionierungen zur Folge haben, voll bestätigt werden. Jedes der identifizierten Grundhaltungscluster legt einen deutlich von den anderen Gruppen verschiedenen Positionierungsschwerpunkt (vgl. Tab. 1).

Dabei kann hinsichtlich der Hypothesen, die den Zusammenhang zwischen der messespezifischen Zielkonzeption und der Messepositionierung zum Gegenstand haben folgendes festgehalten werden: Mit der Zunahme des Stellenwertes von Verkaufszielen gewinnen auch produkt- bzw. sortimentsorientierte Positionierungskonzepte an Bedeutung (vgl. Tab. 2). Insofern kann Hypothese $\mathrm{H}_{\mathrm{Pk} 2}$ im Rahmen der vorliegenden Untersuchung bestätigt werden. Tab. 2 läßt dagegen aber deutlich werden, daß von einem direkten Zusammenhang zwischen der Priorisierung von Kontakt- und Informationszielen und einer problemlösungs- bzw. nutzenorientierten Messepositionierung bei der Bildung von Grundhaltungstypen nicht ausgegangen werden kann. Daher ist Hypothese Hpro die diesen Zusam- 
menhang herstellt, aus Sicht der empirisch abgeleiteten Grundhaltungstypen abzulehnen. Allerdings steigt mit dem Stellenwert markt- und anspruchsgruppenbezogener Beeinflussungsziele die Bedeutung kommunikations- bzw. erlebnisorientierter Messepositionierungskonzeptionen, wie die Aussteller des Clusters II (kommunikationsorientierte Beziehungsmanager) nachweisen. Somit kann auch Hypothese $H_{P k 4}$ voll bestätigt werden. Zuletzt ist auch Hypothese $H_{P k 5}$ zuzustimmen, nach der mit vergleichsweise geringer Zielorientierung einer Messebeteiligung eine wettbewerbsorientierte Messepositionierung des Ausstellers an Relevanz gewinnt. Cluster III stellt einen solchen Fall dar, da diese Aussteller sich in nicht unerheblicher Weise an den Messekonzeptionen der Wettbewerber orientieren.

Stellt man die Beziehung zwischen der Messeselektion und der messespezifischen Positionierung in den Mittelpunkt der Betrachtung, so ist zunächst festzuhalten, daß Hypothese $\mathbf{H}_{\text {Pk6, }}$, wonach mit zunehmender Homogenität des Ausstellungsprogramms der Messeveranstaltung der Stellenwert produkt- bzw. sortimentsorientierter Positionierungskonzeptionen eher zurückgeht, abzulehnen ist. Wie insbesondere Tab. 2 deutlich macht, weisen im Gegenteil zu den Annahmen der angeführten Hypothese gerade Cluster I und IV trotz ihrer Präsenz auf den hinsichtlich ihres Ausstellungsprogramms am stärksten homogen wirkenden Messeveranstaltungen die mit Abstand höchste Produkt- bzw. Sortimentsorientierung auf.

Dagegen ist Hypothese $\mathbf{H}_{\mathrm{Pk} 7}$, die den Einfluß der Informationsorientierung von Messen auf eine problemlösungs- bzw. nutzenorientierte Profilierungskonzeption zum Inhalt hat, in vollem Umfang zuzustimmen. Mit Blick auf Tab. 1 wird ersichtlich, daß Cluster II und IV als diejenigen Gruppen von Ausstellern angesehen werden müssen, die hinsichtlich der Grundorientierung der Messeveranstaltungen am stärksten solche mit einer überdurchschnittlichen Informationsorientierung auswählen. Zugleich zeichnen sich die Positionierungskonzepte dieser Aussteller durch eine vergleichsweise höhere Problemlösungs- und Nutzenorientierung aus.

Der Einfluß der auf Messen anwesenden Zielgruppen auf die messespezifische Positionierung, der in Hypothese $\mathrm{H}_{\mathrm{Pk} 8}$ für die Beziehung zwischen Fachpublikum und problemorientierter bzw. nutzenorientierter Positionierungskonzeption angenommen wird, kann nicht bestätigt werden. So weist zwar Cluster I die höchste Fachbesucherorientierung bei den für die Messebeteiligung ausgewählten Messe- 
veranstaltungen auf, zugleich trifft aber eine problemlösungs- bzw. nutzenorientierte Positionierung auf diese Aussteller am wenigsten zu.

Auch hinsichtlich der Hypothese $\mathbf{H}_{\mathbf{P k} 9}$, nach der mit einer steigenden Anzahl von Begleitveranstaltungen auf Messen eine stärker kommunikations- bzw. erlebnisorientierte Positionierung der Aussteller einhergeht, kann nur z. T. bestätigt werden. Zwar zeigt Cluster II einen solchen Zusammenhang deutlich auf. Die Aussteller dieses Clusters wählen im Rahmen ihrer Messeselektion insbesondere solche Messen aus, auf denen eine hohe Zahl von Begleitveranstaltungen stattfindet. Allerdings zeigen die Ergebnisse der anderen Cluster, daß dieser Zusammenhang nicht durchgängig konstatiert werden kann und insofern zu relativieren ist.

Damit sind nunmehr sämtliche für die Überprüfung der inneren Konsistenz des Modells der messespezifischen Grundhaltung und die Bildung von Grundhaltungstypen empirischen Analyseschritte abgeschlossen. Im folgenden Hauptkapitel sollen i. S. des explikativen Teilziels der Arbeit Ausgestaltungsformen von Messebeteiligungen dargestellt und mit Blick auf das praktisch-normative Untersuchungsziel Erfolgswirkungen der grundhaltungsspezifischen Ausgestaltung von Messebeteiligungen untersucht werden. 


\section{Grundhaltungsspezifische Ausgestaltung und Erfolg von Messebeteiligungen}

Im Rahmen der folgenden Ausführungen werden zunächst relevante Maßnahmen zur Ausgestaltung von Messebeteiligungen konzeptionell abgeleitet und als Grundlage für die sich anschließende empirische Analyse anhand der im Kapitel B identifizierten Grundhaltungen dargestellt. Weiterhin sollen die Erfolgswirkungen der Grundhaltungen anhand von ausgewählten Erfolgsmaßen untersucht werden.

\section{Kennzeichnung der Maßnahmen zur Ausgestaltung von Messebeteiligun- gen}

Messebeteiligungen lassen sich als Kombination verschiedener Einzelaktivitäten charakterisieren. Zur Erreichung der gesteckten Messebeteiligungsziele ist die Vielzahl möglicher Maßnahmen im Rahmen eines integrierten Messebeteiligungskonzeptes - als Umsetzung der messespezifischen Grundhaltung - zu planen. ${ }^{265}$ Wie in Kapitel B dieser Arbeit gezeigt werden konnte, lassen sich fünf messespezifische Grundhaltungstypen von Ausstellern empirisch identifizieren, die signifikante Unterschiede in der Festlegung messespezifischer Zielsysteme, der Messeselektion und der messespezifischen Positionierung aufweisen. Aus entscheidungsorientierter Sicht ist daher davon auszugehen, daß der strategische Charakter der messespezifischen Grundhaltungen auf der Ebene der maßnahmenpolitischen Umsetzung unterschiedliche Akzentsetzungen zur Folge haben wird. ${ }^{266}$ Auf der Basis dieser Annahme soll folgende Basishypothese den weiteren Ausführungen vorangestellt werden:

$\mathrm{H}_{\mathrm{Ak}}$ : Messespezifische Grundhaltungen prägen die Maßnahmen zur Ausgestaltung von Messebeteiligungen wesentlich, d. $h$. verschiedene Grundhaltungstypen ergreifen signifikant unterschiedliche Aktivitäten zur Durchführung von Messebeteiligungen.

265 Vgl. Jaspert, F., Das synergetische Potential ist groß - die Messe als Teil der betrieblichen Absatzpolitik, a.a.O., S. 36 ff.; Fischer, A., Messen als Chance - Erfahrungen einer Unternehmung, in: Thexis, Heft 2, 1989, S. 21.

266 Vgl. auch Selinski. H. Sperling. U. A., Marketinginstrument Messe: Arbeitsbuch für Studium und Praxis, a.a.O., S. 118, die einen Zusammenhang zwischen dem strategischen und operativen Management von Messebeteiligungen postulieren. 
Als zentrale Entscheidungsfelder kristallisieren sich dabei die Ausgestaltung der messebezogenen Aktivitäten, die Durchführung von Maßnahmen zur Erfolgskontrolle sowie die Festlegung organisatorischer Strukturen der Messebeteiligung heraus, die im folgenden zunächst systematisiert und im Überblick dargestellt werden sollen. ${ }^{267}$

\subsection{Messebezogene Aktivitäten}

Eine Messebeteiligung kann im Rahmen einer chronologisch orientierten Betrachtung in ein "Phasen-Modell" aufgespalten werden ${ }^{268}$, wobei zweckmäßigerweise die Vor-Messe-Phase, die Messe-Phase und die Nach-Messe-Phase zu trennen sind. ${ }^{269}$ Innerhalb jeder dieser drei Phasen fallen spezifische Aktivitäten an. Im folgenden sollen diejenigen Aktivitäten näher dargestellt werden, die eine primär messebesucherorientierte Fokussierung aufweisen. ${ }^{270}$

267 Vgl. Fuchs, W. A., Die Messe als Kommunikationsprozeß, a.a.O., S. 99 f.

268 Wesen chronologischer Ansätze sind die Einordnung und Beschreibung von messebezogenen Aktivitäten nach ihrem zeitlichen Anfall. Eine nach der Zeitdimension erfolgende Aufspaltung messespezifischer Maßnahmen geht dabei auf eine lange Tradition zurück. Vgl. hierzu Reuther. K. H., Die betriebswirtschaftliche Bedeutung der Messen und Ausstellungen für die Konsumgüterindustrie, München 1956, S. 75 ff. sowie S. 168 ff., Blom, H., Messen und Ausstellungen als Mittel der Absatzförderung in der Schweizerischen Maschinenindustrie, Freiburg, Winterthur 1960, S. 76 ff., Lohmaier. P., Die Messe - Bedeutung und Problematik aus der Sicht der Aussteller, in: Arbeitspapier des Instituts für Absatzwirtschaft der Universität Augsburg Nr. 1/85, Augsburg 1985, S. 28 ff. Die zentrale Kritik an dieser Sichtweise richtet sich vor allem auf Abgrenzungsprobleme, die einzelne Aktivitäten verursachen, die in mehreren Phasen gleichermaßen Relevanz besitzen, so daß mitunter Zuordnungsprobleme auftreten können. Zur Kritik an chronologischen Ansätzen vgl. Funke, K., Ausstelleraktivitäten auf Messen - Eine kritische Analyse einschlägiger Darstellungen und Systematisierungsansätze in der Literatur, in: Arbeitsbericht Nr. 03/86 des Instituts für Wirtschaftswissenschaften der RWTH Aachen, Aachen 1986, S. $14 \mathrm{f}$. Diese Kritik muß allerdings insofern relativiert werden, als chronologische Ansätze hinsichtlich der Planung von Messeaktivitäten zumindest in der Lage sind, das Planungsproblem nach der Zeitdimension zu strukturieren und somit ein geeignetes Instrument zur Auswahl und zeitgerechten Umsetzung der Aktivität darstellen.

269 Vgl. zur Verwendung eines Drei-Phasen-Modells Roth, G. D., Messen und Ausstellungen verkaufswirksam planen und durchführen, a.a.O., S. $123 \mathrm{ff}$.; Müller, K., Die Messepolitik im Marketing der handwerklichen Zulieferer, Göttingen 1985, S. 213 ff.; Roloff. E., Messen und Medien. Ein sozialpsychologischer Ansatz zur Öffentlichkeitsarbeit, Wiesbaden 1992, S. 215 ff.

Zur Notwendigkeit einer besucherorientierten Maßnahmengestaltung bei Messebeteiligungen vgl. Funke, K., Ausstelleraktivitäten auf Messen - Eine kritische Analyse einschlägiger Darstellungen und Systematisierungsansätze in der Literatur, a.a.O., S. 4 ff., der insbesondere eine Abgrenzung zu solchen Aktivitäten vornimmt, die primär an organisations- und ablauftechnischen Fragestellungen orientiert sind. 


\subsection{Aktivitäten in der Vor-Messe-Phase}

In der Vor-Messe-Phase besteht die zentrale Aufgabe des Messemarketing neben der Überwachung der vorbereitenden Arbeiten hinsichtlich Standgestaltung ${ }^{271}$ und Exponateauswahl ${ }^{272}$ vor allem in der Bekanntmachung der eigenen Messebeteiligung bei den potentiellen Zielgruppen sowie der Auswahl und Qualifizierung des Standpersonals. ${ }^{273}$ Um auf die Messebeteiligung aufmerksam zu machen, werden in dieser Phase schwerpunktmäßig Messeinformations- bzw. -einladungsaktionen durchgeführt. ${ }^{274}$ Dabei werden in Abhängigkeit der Zielgruppenstruktur persönliche und/oder massenmediale Kommunikationsinstrumente eingesetzt.

$\mathrm{Zu}$ den Instrumenten der unpersönlichen Kommunikation mit Messe-Zielgruppen gehören die Vor-Messe-Werbung ${ }^{275}$ in Massenmedien, wie z. B. Zeitschriften, Messevorberichte in der Presse sowie Anzeigen und Einträge im Messekatalog. ${ }^{276}$ Die wesentlichen Formen der persönlichen Einladung zum Besuch von Messeständen sind die direkten Terminabsprachen mit aktuellen bzw. potentiellen Kunden durch den Außendienst des Unternehmens, Direct Mail-Aktionen sowie Einladungen an ausgewählte Pressevertreter. ${ }^{277}$

271 Vgl. Funke, K., Messeentscheidungen. Handlungsalternativen und Informationsbedarf, a.a.O., S. $189 \mathrm{ft}$.

272 Vgl. Funke, K., Messeentscheidungen. Handlungsalternativen und Informationsbedarf, a.a.O., S. $101 \mathrm{ff}$.

273 Vgl. Selinski, H. Sperling, U. A., Marketinginstrument Messe: Arbeitsbuch für Studium und Praxis, a.a.O., S. 118 f.

274 Vgl. Küng. P., Der Einsatz des Verkaufs an Messen, a.a.O., S. 47.

275 Die Vor-Messe-Werbung zielt dabei auf eine Steuerung des Verhaltens aktueller und potentieller Kunden ab. Ihre Hauptaufgabe besteht darin, für die Messebeteiligung einen möglichst hohen Bekanntheitsgrad aufzubauen. Vgl. Becker, J., Marketing-Konzeption - Grundlagen des strategischen Marketing-Managements, 4. Aufl., München 1992, S. 467.

276 Vgl. Mortsiefer, J., Messen und Ausstellungen als Mittel der Absatzpolitik mittelständischer Herstellerbetriebe, a.a.O., S. 401 f.; Selinski, H. Sperling, U. A., Marketinginstrument Messe: Arbeitsbuch für Studium und Praxis, a.a.O., S $179 \mathrm{ff}$. sowie $185 \mathrm{f}$.

277 Vgl. Schmitz, U., Messepolitik eines Unternehmens der Investitionsgüterindustrie, in: Strothmann, K.-H. Busche, M. (Hrsg.), Handbuch Messemarketing, Wiesbaden 1992, S. 393; Zahner, W., Directmail - Sein Einsatz im Marketing, Bern, 1991, S. 18 ff.; Kohle, G. U., Von der "Messe-Einladung" zur Einladung mit Konzept, in: Marketing Journal, Heft 5, 1991, S. 460-466; Pidun. R., Messen und Ausstellungen: Messe-Promotion - einfach faszinierend und glaubwürdig, in: Marketing-Journal, Heft 1, 1990, S. 70-71. 
Da Messebeteiligungen lediglich zeitlich begrenzte Möglichkeiten der direkten Kommunikation mit Messebesuchern darstellen, kommt der Qualifizierung des Standpersonals in der Vor-Messe-Phase eine entsprechend große Bedeutung $z u .{ }^{278} \mathrm{Um}$ eine möglichst optimale Zielerreichung des Messeengagements sicherzustellen, ist das Standpersonal in dieser Phase nicht nur genau über die Ziele und Zielgruppen der Messebeteiligung zu informieren, sondern darüber hinaus im Hinblick auf Verhaltens ${ }^{279}$ und inhaltliche Argumentationsaspekte ${ }^{280}$ auf dem Messestand vorzubereiten.

\subsection{Aktivitäten in der Messe-Phase}

Ausgangspunkt einer zielorientierten Messebeteiligung ist der Kontakt zu den anvisierten Zielgruppen auf dem eigenen Messestand. ${ }^{281}$ Zur Anbahnung dieser Kontakte stehen nicht nur die bereits angeführten vorbereitenden Aktivitäten in der Vor-Messe-Phase zur Verfügung, sondern es existieren insbesondere auch während der Messe Möglichkeiten, gezielte Maßnahmen zu ergreifen, die im wesentlichen drei Bereichen zuzuordnen sind:

- Akquisition der Messebesucher i. S. der Wahrnehmung des eigenen Messestandes durch das Messepublikum als Voraussetzung für die

- Intensivierung des Dialogs mit ausgewählten Zielgruppen auf dem Messestand sowie die

- Aktivierung des anonymen Messestandpublikums.

278 Vgl. Mortsiefer, J., Die Vorbereitung entscheidet über den Erfolg - Planung und Vorbereitung einer Messebeteiligung, a.a.O., S. 59 ff., o.V., Mitarbeiterschulung - Hymne in der Halle: Unternehmen bereiten sich mit ungewöhnlichen Methoden auf den Messeauftritt vor, in: Wirtschaftswoche, Heft 1/2 vom 5.1.1995, S. $70 \mathrm{ff}$.

Vgl. Aries, L. A., Bereiten Sie ihr Standpersonal intensiv auf das Messe-Verkaufsgespräch vor, in: Marketing-Journal, Heft Nr. 4, 1986, S. 365-368; Strothmann, K.-H., Suchen Sie das Gespräch mit dem Standbesucher, in: Marketing-Journal, Heft Nr. 4, 1989, S. 388 f.; Spryß, W.

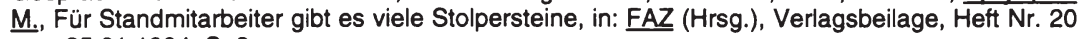
vom 25.01.1994, S. 8.

Vgl. Ausstellungs- und Messe-Ausschuss der Deutschen Wirtschaft e. V. (Hrsg.), Erfolgreiche Messebeteiligung Made in Germany, Bonn 1995, S. 73 ff.; Wagner, G., Innovatives MesseTeam-Training, in: FAZ (Hrsg.), Verlagsbeilage, Heft Nr. 20 vom 25.01.1994, S. 5; Mortsiefer. $\underline{J}$., Informationen sammeln und auswerten - Die Abwicklung einer Messebeteiligung, in: Küffner. G. Mortsiefer, J. (Hrsg.), Messeplatz Europa. Messen als Bestandteil des betrieblichen Marketings, Frankfurt a.M. 1990, S. 62 f.

281 Vgl. hierzu auch die Ergebnisse der Arbeiten von Täger, U. C. Ziegler, R., Die Bedeutung von Messen und Ausstellungen in der Bundesrepublik Deutschland für den Inlands- und Auslandsabsatz in ausgewählten Branchen, a.a.O., S. 30 und 52 f.; Mortsiefer, J., Messen und Ausstellungen als Mittel der Absatzpolitik mittelständischer Herstellerbetriebe, a.a.O. S. $322 \mathrm{ff}$.

Ralf Ueding and Universitat Munster - 978-3-631-75073-5 
Während die Kommunikationsarbeit der Messeveranstalter darauf gerichtet ist, Ausstellern und Messebesuchern ein Forum der Begegnung zur Verfügung zu stellen $^{282}$, müssen die Aktivitäten der Aussteller während der Messe zunächst darauf abzielen, die Messebesucher zum eigenen Messestand zu leiten. Deshalb kommt der Besucherakquisition eine große Bedeutung zu. Die Überbrückung der Wahrnehmungshürde bei den Messebesuchern sowie die Lenkung ihrer Aufmerksamkeit und ihres Interesses auf den eigenen Messestand stellen sich dabei als wesentliche Herausforderungen dar. $^{283}$

Als Hilfestellung für Messebesucher stellen die meisten Messegesellschaften computergestützte Messe-Informationssysteme zur Verfügung. ${ }^{284}$ Diese InfoTerminals, die vor allem im Eingangsbereich der Messen zu finden sind, sollen dem Besucher die Orientierung auf dem Messegelände erleichtern und einen Überblick über das Ausstellungsprogramm vermitteln. Für Aussteller bietet sich die Möglichkeit, ihren Stand werblich in diesem Informationssystem herauszustellen und so ein gezieltes Auffinden des Messestandes zu ermöglichen. Auch Außenwerbeflächen, die z. T. im Umfeld und auf dem Gelände der Messeveranstaltung selbst zur Verfügung stehen, können vom Aussteller genutzt werden, um im Rahmen der Außenwerbung auf die eigene Messebeteiligung hinzuweisen. ${ }^{285}$ Darüber hinaus läßt sich insbesondere mittels Werbeaktionen an Standorten in der Nähe des eigenen Messestandes eine erhöhte Aufmerksamkeit hinsichtlich der eigenen Messebeteiligung erzielen und Interesse an einem Besuch des Messestandes wecken (z. B. Verteilung von Werbegeschenken, Handzetteln mit Wegbeschreibung zum Stand, Karten für ein Preisausschreiben, die am Messestand abgegeben werden können etc.). ${ }^{286}$

War die Besucherakquisition in der Vor-Messe- und der Messe-Phase erfolgreich, werden sich interessierte Messebesucher auf dem Messestand einfinden. Doch

282 Vgl. Roloff. E., Messen und Medien. Ein sozialpsychologischer Ansatz zur Öffentlichkeitsarbeit, a.a.O., S. 46 f.; Selinski, H., Messe- und Kongreßmarketing, a.a.O., S. 60 f.

283 Vgl. Selinski, H. Sperling, U. A., Marketinginstrument Messe: Arbeitsbuch für Studium und Praxis, a.a.O., S. 185.

284 Vgl. Tauberer. A. Wartenberg. W., Serviceleistungen von Messegesellschaften, in: Strothmann, K.-H.. Busche, M. (Hrsg.), Handbuch Messemarketing, Wiesbaden 1992, S. 245 f.

Unter Außenwerbung ist die großflächige Belegung von (standexternen) Werbeflächen zu verstehen, die meist in den Messe(innen)städten bzw. an Verkehrsknotenpunkten in der Nähe des Messegeländes zu finden sind. Vgl. Selinski, H. Sperling, U. A., Marketinginstrument Messe: Arbeitsbuch für Studium und Praxis, a.a.O., S. 187.

286 Vgl. Selinski, H. Sperling, U. A., Marketinginstrument Messe: Arbeitsbuch für Studium und Praxis, a.a.O., S. 187. 
wird es i. d. R. nicht mit allen Besuchern zu persönlichen Gesprächen kommen. Der persönliche Dialog wird hauptsächlich zwischen den Vertretern des Unternehmens und den auf der Messe erwarteten Zielgruppen forciert. Unterstützend kann dabei die Präsenz der Unternehmensleitung auf dem Messestand wirken. Die Anwesenheit des oberen Management und seine Bereitschaft zum Dialog mit Kunden und Anspruchsgruppen, wie z. B. Pressevertretern und Repräsentanten von Interessengruppen, signalisiert nicht nur die Offenheit des Unternehmens zum Austausch mit Dritten, sondern sie erhöht auch den Stellenwert und die Kompetenzanmutung der Kommunikation aus Sicht der Gesprächspartner. ${ }^{287}$

Zur Initiierung und Intensivierung eines fachlich orientierten Dialogs mit relevanten Zielgruppen können darüber hinaus Begleitveranstaltungen wie z. B. Podiumsdiskussionen, Fachvorträge, festliche Rahmenveranstaltungen oder Pressekonferenzen beitragen. Diese Veranstaltungen dienen nicht nur der konzentrierten Informationsvermittlung, sondern weisen i. d. R. auch einen besonderen Ereignischarakter auf, der bei den Vertretern der verschiedenen Zielgruppen meist zu einer Verstärkung der positiven Eindrücke am Messestand führt. ${ }^{288}$

Um die Wirkung der Messepräsentation darüber hinaus beim sonstigen Standpublikum zu erhöhen, sind Maßnahmen zur Aktivierung der Standbesucher unerläßlich. Nur aktivierte Besucher bringen den Messe-Botschaften des Ausstellers die notwendige Aufmerksamkeit entgegen, um diese bewußt wahrzunehmen und gedanklich zu verarbeiten. Den Standbesuchern sollen der Messestand und seine zentralen Produkt- bzw. Unternehmensbotschaften im Wettbewerb der Messekommunikation möglichst lange im Gedächtnis verhaftet bleiben. Neben der notwendigen Informationsvermittlung ist daher auch ein emotional geprägtes Messestand-Erlebnis i. S. eines "Event" ${ }^{289}$ für die Messebesucher zu erzeugen. ${ }^{290}$

287 Vgl. Roth, G. D., Messen und Ausstellungen verkaufswirksam planen und durchführen, a.a.O., S. $139 \mathrm{ff}$.

288 Vgl. zum Facettenreichtum von Begleitveranstaltungen bei Messen Selinski, $H_{\text {. }}$, Begleitveranstaltungen von Messen, a.a.O., S. $485 \mathrm{ff}$. Zu den Einsatzbereichen und Integrationsmöglichkeiten vgl. insbesondere Strothmann, K.-H., Verbundveranstaltungen des Messe- und Kongreßwesens im Investitionsgüter-Marketing, a.a.O., S. 74 ff.; Strothmann. K.-H., Die Instrument-Integration im Messe- und Kongreßwesen - Messestand-Konzeption und Begleitveranstaltung, in: Rost, D. Strothmann, K. -H. (Hrsg.), Handbuch der Werbung für Investitionsgüter, Wiesbaden 1983, S. 219 ff.

Zu den Grundlagen des Event-Marketing vgl. Ueding. R., Event-Marketing, in: Meffert, $H$., Lexikon aktueller Marketing-Stichwörter, Wien 1994, S. $59 \mathrm{ff}$.

290 Vgl. Selinski. H. Sperling, U. A., Marketinginstrument Messe: Arbeitsbuch für Studium und Praxis, a.a.O., S. 193 f. 
Für die Umsetzung sind Produktshows (Produkt in Aktion) ebenso geeignet wie z. B. Video- oder Filmvorführungen, der Einsatz von Musikgruppen oder Auftritte, sog. Testimonials (z. B. Kino- bzw. TV-Stars, Sportler) als Sympathie- und Informationsträger. Statt eines einfachen Kommunikationstransfers werden vom Aussteller damit Information und Unterhaltung gemeinsam i. S. eines "Infotainment" angeboten. Dabei ist aufgrund der multisensorischen Anspracheform der Standbesucher von einer erhöhten Aktivierung der Anwesenden auszugehen.

\subsection{Aktivitäten in der Nach-Messe-Phase}

Neben die operativ notwendigen Aktivitäten (z. B. Rückführung der Exponate) treten i. S. einer "strategischen Nachbereitung der Messebeteiligung" in der Nach-Messe-Phase vor allem Maßnahmen zur Aufarbeitung und Aufbereitung des gesamten Messegeschehens.

Diese nachbereitenden Aktivitäten der Messebeteiligung sind insbesondere auf die unterschiedlichen Anforderungen folgender Zielgruppen auszurichten: 291

- aktuelle und potentielle Kunden, zu denen auf der Messe persönliche Kontakte geknüpft werden konnten,

- zur Messe eingeladene aktuelle und potentielle Kunden, die den Messestand nicht besucht haben,

- an allgemeinen Unternehmensinformationen interessierte Standbesucher, die nicht zu den unmittelbar anvisierten Zielgruppen des Unternehmens gehören ( $z$. B. Wissenschaftler, Lehrer, Berufseinsteiger $u$. a.),

- Vertreter der Presse (vor allem Fach-, Wirtschafts- und Tagespresse) sowie Anspruchsgruppen, die insbesondere bereits in der Vor-Messe-Phase in die Maßnahmen der Vorabinformation einbezogen waren sowie

- die eigenen Mitarbeiter.

291 Vgl. Naumann, C., Die Arbeit nach der Arbeit - Messe-Nachbereitung und Erfolgskontrolle, in: Küffner, G. Mortsiefer, J. (Hrsg.), Messeplatz Europa, Messe als Bestandteil des betrieblichen Marketings, Frankfurt a.M. 1990 , S. 75 f.

Ralf Ueding and Universität Münster - 978-3-631-75073-5 
Von zentraler Bedeutung ist dabei die systematische Weiterverfolgung der auf der Messe geknüpften Kontakte mit aktuellen und potentiellen Kunden. ${ }^{292}$ Im Mittelpunkt stehen hier die Bezugnahme auf konkrete Gesprächsinhalte am Messestand sowie die Weiterführung des Dialogs mit dem Gesprächspartner zum Aufbau langfristiger Geschäftsbeziehungen. Auch den Informationsinteressen der Standbesucher, die für das Unternehmen keine unmittelbaren Zielgruppen der Messebeteiligung darstellen, ist in dieser Phase i. S. einer individualisierten MessePR systematisch nachzugehen.

Die Nachbereitung darf allerdings nicht auf die Weiterverfolgung der direkten Messekontakte beschränkt bleiben. Auch diejenigen Kunden, die zum Besuch des Messestandes eingeladen wurden, diesen aber nicht besucht haben, sind jetzt ebenfalls mit Blick auf die eigenen Messeergebnisse anzusprechen. Ein werblich orientierter Messebericht des Ausstellers kann sowohl zur Profilierung des eigenen Unternehmens beitragen als auch zu direkten Geschäftskontakten nach der Messeveranstaltung führen. ${ }^{293}$

Möglichkeiten zur Nach-Messe-Werbung mit den Ergebnissen der Messebeteiligung ergeben sich des weiteren durch die Abfassung von Messeberichten im Rahmen der Pressearbeit des Unternehmens sowie durch den Einsatz klassischer Werbemittel, wie z. B. Anzeigen. Durch gezielte Ansprache der Pressevertreter und Bereithaltung geeigneter Informationen bzw. Werbemittel können diese NachMesse-Aktivitäten eine hohe Wirkung bei den Zielgruppen des Unternehmens erzeugen, da in dieser Phase das allgemeine Interesse an der gerade beendeten Messeveranstaltung noch vergleichsweise groß ist. ${ }^{294}$

Nicht zuletzt sind auch die eigenen Mitarbeiter im Rahmen eines internen Messeberichts über die Ergebnisse der Messebeteiligung zu informieren. ${ }^{295}$ Für die an der Messeveranstaltung beteiligten Mitarbeiter bedeutet dieser Bericht eine

292 Vgl. Naumann, C., Die Arbeit nach der Arbeit - Messe-Nachbereitung und Erfolgskontrolle, a.a.O., S. 81.

293 Vgl. Naumann, C., Die Arbeit nach der Arbeit - Messe-Nachbereitung und Erfolgskontrolle, a.a.O., S. 81.

294 Vgl. Wenge, H.-U. Müller, A., Das Management von Messe-Beteiligungen, a.a.O., S. 742 f. sowie Mortsiefer, J., Informationen sammeln und auswerten - Die Abwicklung einer Messebeteiligung, a.a.O., S. 67.

295 Vgl. Spryß, W. M., Messen und Ausstellungen: Mit dem "Messe-Diagramm" vorbereiten - von den Zielen bis zum Controlling, a.a.O., S. 370; Dostal. G., Messen und Ausstellungen: So decken Sie die Stärken und Schwächen Ihrer Messe-Beteiligung auf, in: Marketing-Journal, Heft 2, 1987, S. $140 \mathrm{ff}$. 
geeignete Feedback-Möglichkeit, und für die nicht auf der Messe anwesenden Mitarbeiter stellt er bei entsprechender Ausgestaltung eine relevante Informationsquelle dar. Neben der Informationsfunktion weisen interne Messeberichte, die über den Verlauf und das Ergebnis der eigenen Messebeteiligung Auskunft geben, aber auch bedeutende Möglichkeiten der Motivation der gesamten Mitarbeiterschaft eines Unternehmens auf. Wesentliche Voraussetzung hierfür ist natürlich eine hinreichende Diffusion des Messeberichts im Unternehmen. ${ }^{296}$

Die nachfolgende Abbildung zeigt die angeführten Aktivitäten, getrennt nach der Vor-Messe-, Messe- und Nach-Messe-Phase, in einem zusammenfassenden Überblick.

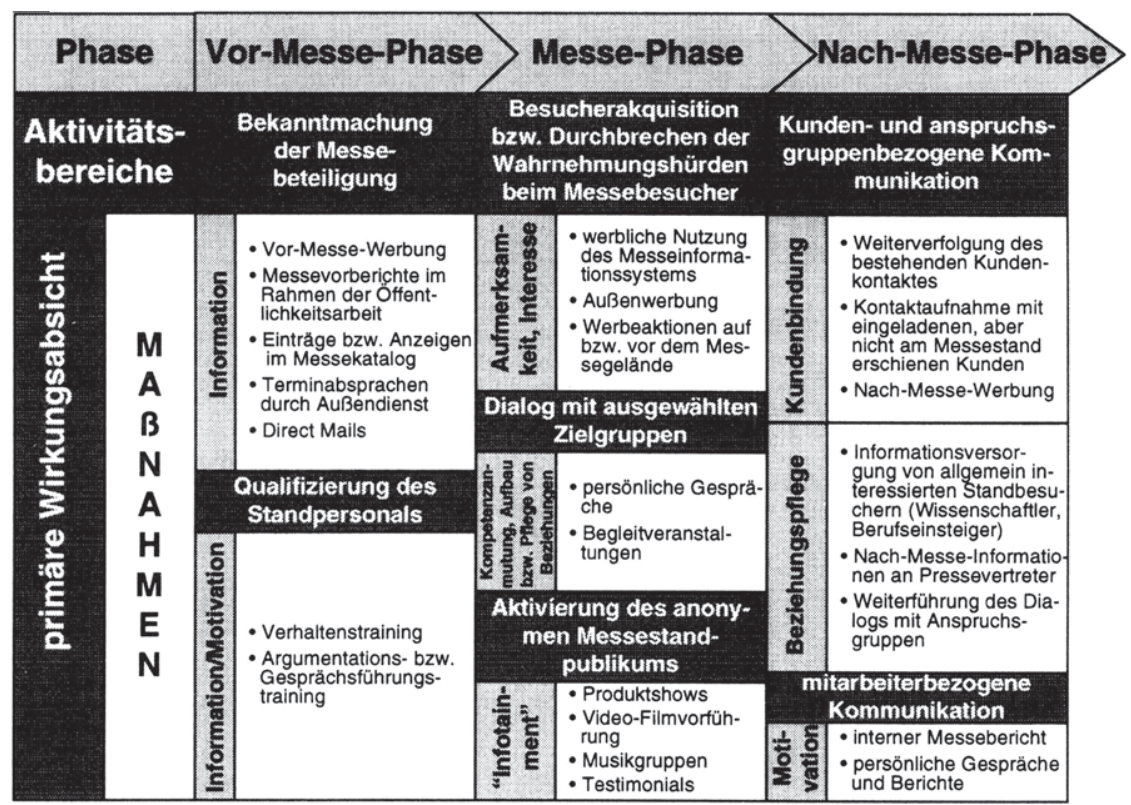

Abb. 20: Synopse phasenspezifischer Messe-Aktivitäten

296 Vgl. hierzu die Ausführungen in Kap. C.1.2.4 dieser Arbeit. 


\subsection{Erfolgskontrolle von Messebeteiligungen}

Messebeteiligungen stellen für ausstellende Unternehmen i. d. R. eine hohe Kostenbelastung dar. ${ }^{297}$ Nicht zuletzt aufgrund eines generell wachsenden Kostenbewußtseins und einer zunehmend systematischen Planungsorientierung in Unternehmen nimmt die Betrachtung von Kosten-Nutzen-Relationen eingesetzter Kommunikationsinstrumente einen bedeutender werdenden Stellenwert ein. ${ }^{298}$ In Verbindung mit Messebeteiligungen wird zwar seit geraumer Zeit der Versuch unternommen, die insgesamt angefallenen Kosten festzustellen, der Erfolg des Messeengagements - als Ausdruck seines Nutzens - wird hingegen nur selten analysiert. $^{299}$

Der Begriff der Messeerfolgskontrolle soll dabei aus Sicht der Aussteller als die systematische Gewinnung und Auswertung von spezifischen Daten einer Messeveranstaltung zur Kontrolle des ökonomischen und psychographischen Erfolges einer Messebeteiligung i. S. eines "Soll-Ist-Vergleichs" interpretiert werden. ${ }^{300}$ Gegenstand der Messeerfolgskontrolle ist die Feststellung des Zielerreichungsgrades der verfolgten Messebeteiligungsziele.

297 Vgl. hierzu die Ausführungen in Kap. A. 1 dieser Arbeit.

Vgl. Schoop, K. Sandt, B., Die Messeerfolgskontrolle, in: Tietz, B., Die Werbung - Handbuch der Kommunikations- und Werbewirtschaft, Band 2, Landsberg/Lech 1982, S. 1666.

Mortsiefer sieht hiertür u. a. folgende Gründe: Die Vielzahl von Erfolgsgrößen, die mit einer Messebeteiligung verbunden sein können (z. B. ökonomischer vs. psychographischer und direkter vs. indirekter Erfolg), der Wandel der meisten Messen von Order- zu Informationsmessen, der eine Erfolgsermittlung auf der Basis ökonomischer Größen erschwert, Probleme der Kausalität und Zurechenbarkeit von Erfolgswirkungen bei Messebeteiligungen (da meist mehrere Messen beschickt und/oder auch andere Kommunikationsinstrumente vor und nach einer Messebeteiligung eingesetzt werden) sowie die Wahl des Kontrollzeitpunktes bzw. -zeitraumes (Problem der Bestimmung der über eine Messebeteiligung hinausgehenden Erfolgswirkungen). Vgl. Mortsiefer, J., Messen und Ausstellungen als Mittel der Absatzpolitik mittelständischer Herstellerbetriebe, a.a.O., S. 439 ff.; Stiege, J., Messebeteiligungen sollen gründlich bewertet werden, in: BddW vom 24.04.95, S. 1. Bestätigende empirische Befunde finden sich darüber hinaus bei Täger, U. C. Ziegler, R., Die Bedeutung von Messen und Ausstellungen in der Bundesrepublik Deutschland für den Inlands- und Auslandsabsatz in ausgewählten Branchen, a.a.O., S. $63 \mathrm{ff}$.

Vgl. auch Winnen, R., Beuster, A., Kontrolle des Messeerfolgs, a.a.O., S. $368 \mathrm{ff.}$ 
Die Basis der Erfolgskontrolle bilden somit die Zielsetzungen, die in Verbindung mit der Messebeteiligung verfolgt worden sind. ${ }^{301}$ Im Rahmen eines systematischen Vorgehens sind dabei aus den Messebeteiligungszielen operationale Erfolgskriterien abzuleiten ${ }^{302}$, da erst die Festlegung der Erfolgskriterien eine exakte Analyse des Zielerreichungsgrades ermöglicht. ${ }^{303}$ Eng mit der Auswahl der Erfolgskriterien verbunden ist die Wahl der Methoden der Erfolgskontrolle. ${ }^{304}$ Dabei lassen sich einige Methoden zur Messung mehrerer Erfolgskriterien heranziehen, während andere Verfahren lediglich für bestimmte Fragestellungen eine besondere Eignung aufweisen.

Eine Übersicht über mögliche aus den Messebeteiligungszielen ableitbare Erfolgskriterien sowie Methoden zu deren Messung vermitteln die folgenden Tabellen. ${ }^{305}$

301 Die Messeerfolgskontrolle wird indes von vielen Unternehmen nur i. S. ökonomischer Kenngrößen interpretiert. Das Ergebnis einer Messebeteiligung wird bspw. ausschließlich in Form des in dieser Zeit erzielten Auftragsvolumens bzw. Umsatzes dargestellt. Wie bei allen anderen Marketinginstrumenten besteht in einer solch vereinfachenden Sichtweise zum einen das Problem, daß das Umsatzergebnis nicht Resultat der Messebeteiligung allein ist. Vielmehr existieren Wechselwirkungen mit anderen Marketingmaßnahmen, die berücksichtigt werden müssen. So kann bspw. der angestrebte Umsatzzuwachs auf der Messe ausbleiben, weil in der Preispolitik des Unternehmens falsche Annahmen über die Preisbereitschaft der Kunden enthalten sind - und dies trotz einer gelungenen Präsentation des Produkt- bzw. Leistungsprogramms am Messestand. Zum anderen ist mit der Präsenz auf Messen und Ausstellungen in wachsendem Maße die Verfolgung psychographischer Zielsetzungen verbunden. Deren Erreichung kann mit Kontrollmethoden, die lediglich quantifizierbare Wirkungen von Messebeteiligungen überprüfen, nicht unmittelbar wiedergegeben werden. Vielmehr sind für die Überprüfung dieser Messebeteiligungsziele qualitative Verfahren der Erfolgskontrolle einzusetzen.

Vgl. zur Notwendigkeit der Operationalisierung von Messebeteiligungszielen die Ausführungen in Kap. B. 1.2 dieser Arbeit.

303 Vgl. allg. Heinen, E., Grundlagen betriebswirtschaftlicher Entscheidungen - Das Zielsystem der Unternehmung, 3. Aufl., a.a.O., S. 86 f.

304 Im folgenden sollen die Begriffe "Methode“ und „Verfahren“ der Erfolgskontrolle synonym verwendet werden.

Die Aufstellung der Erfolgskriterien sowie der Methoden zur Erfolgsmessung in den Tabellen soll dabei beispielhafte Möglichkeiten zur Erfolgskriterienbildung und Kontrolle der Ziele eines Ausstellers aufzeigen und erhebt daher keinen Anspruch auf Vollständigkeit. Vgl. zur Entwicklung von Erfolgskriterien Meffert. $\mathrm{H}$., Messen und Ausstellungen als Marketinginstrument, a.a.O., S. 25; Amon, P., Messen + Ausstellungen: Messe-Ziele exakt formulieren und kontrollieren, a.a.O., S. 56 f. Ein erster Ansatz zur Verbindung der Erfolgskriterien mit Methoden der Erfolgskontrolle findet sich bei Winnen, R.. Beuster, A., Kontrolle des Messeerfolgs, a.a.O., S. 375 f. 


\begin{tabular}{|c|c|c|}
\hline Informationsziele & Erfolgskriterien & MeBmethoden \\
\hline Kennenlernen neuer Märkte & $\begin{array}{l}\text { - Zahl der neuen Märkte } \\
\text { - Marktvolumen und } \\
\text { Wettbewerbsstruktur }\end{array}$ & $\begin{array}{l}\text { Messebesucherstruk- } \\
\text { turanalyse }\end{array}$ \\
\hline $\begin{array}{l}\text { Kennenlernen neuer Wettbe- } \\
\text { werber }\end{array}$ & $\begin{array}{l}\text { - Anzahl und Größe der } \\
\text { Wettbewerber } \\
\text { - Art und Umfang des Leistungs- } \\
\text { programms }\end{array}$ & $\begin{array}{l}\text { Ausstellerstruktur- } \\
\text { analyse }\end{array}$ \\
\hline $\begin{array}{l}\text { Kennenlernen neuer } \\
\text { Lieferanten }\end{array}$ & - Zahl und Qualität neuer Lieferanten & $\begin{array}{l}\text { Ausstellerstrukturanalyse, } \\
\text { Gesprächsprotokoll }\end{array}$ \\
\hline $\begin{array}{l}\text { Anbahnung von Gemein- } \\
\text { schaftsunternehmen }\end{array}$ & $\begin{array}{l}\text { - Zahl und Qualität potentieller } \\
\text { Kooperationspartner }\end{array}$ & Gesprächsprotokoll \\
\hline Konkurrenzbeobachtung & $\begin{array}{l}\text { - Merkmale und Verhalten der } \\
\text { Konkurrenten }\end{array}$ & Ausstellerbeobachtung \\
\hline Mitarbeiterakquisition & $\begin{array}{l}\text { Zahl der erfolgreichen } \\
\text { Bewerbungen, die zu Einstellungen } \\
\text { führen }\end{array}$ & Kontaktzählung \\
\hline Aufbau von Vertriebswegen & $\begin{array}{l}\text { - Anzahl und Struktur neuer } \\
\text { Vertriebswege }\end{array}$ & $\begin{array}{l}\text { Ausstellerstrukturanalyse, } \\
\text { Gesprächsprotokoll }\end{array}$ \\
\hline $\begin{array}{l}\text { Erkennen von Trends bei } \\
\text { Kundenpräferenzen }\end{array}$ & $\begin{array}{l}\text { - Merkmale, Verhalten und Urteile } \\
\text { der Abnehmer }\end{array}$ & $\begin{array}{l}\text { Gesprächsprotokoll, } \\
\text { Besucherbefragung, } \\
\text { Besucherbeobachtung, } \\
\text { Wegeverlaufsanalyse }\end{array}$ \\
\hline Erfahrungsaustausch & $\begin{array}{l}\text { - Zahl der geführten Gespräche } \\
\text { - Gesprächsinhalte }\end{array}$ & $\begin{array}{l}\text { Zählung pers. Kontakte } \\
\text { Gesprächsprotokoll }\end{array}$ \\
\hline $\begin{array}{l}\text { Überprüfung der Marktreife } \\
\text { von Produkten und } \\
\text { Leistungen }\end{array}$ & $\begin{array}{l}\text { - Akzeptanz bei Kunden } \\
\text { - Verhalten der Kunden }\end{array}$ & $\begin{array}{l}\text { Besucherbefragung } \\
\text { Gesprächsprotokoll } \\
\text { Standpersonalbefragung }\end{array}$ \\
\hline
\end{tabular}

Tab. 4: Erfolgskriterien und Methoden für die Kontrolle von Informationszielen 


\begin{tabular}{|c|c|c|}
\hline Motivationsziele & Erfolgskriterien & Meßmethoden \\
\hline Motivation der Mitarbeiter & $\begin{array}{l}\text { - Zufriedenheit des } \\
\text { Standpersonals }\end{array}$ & Standpersonalbefragung \\
\hline $\begin{array}{c}\text { marktbezogene } \\
\text { Beeinflussungsziele }\end{array}$ & Erfolgskriterien & MeBmethoden \\
\hline $\begin{array}{l}\text { Erhöhung des Bekanntheits- } \\
\text { grades des Unternehmens }\end{array}$ & 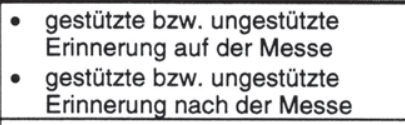 & $\begin{array}{l}\text { Besucherbefragung, } \\
\text { Nachmessebefragung }\end{array}$ \\
\hline $\begin{array}{l}\text { Erhöhung des Bekanntheits- } \\
\text { grades von Produkten und } \\
\text { Leistungen }\end{array}$ & $\begin{array}{l}\text { - gestützte bzw. ungestützte } \\
\text { Erinnerung auf und nach der } \\
\text { Messe } \\
\text { - Zahl der Messestandbesucher } \\
\text { - Beachtung Exponate }\end{array}$ & $\begin{array}{l}\text { Standbesucherzählung, } \\
\text { Besucherbefragung, } \\
\text { Nachmessebefragung, } \\
\text { Wegeverlaufsanalyse }\end{array}$ \\
\hline $\begin{array}{l}\text { Darstellung der Produkt- } \\
\text { und Leistungskompetenz }\end{array}$ & $\begin{array}{l}\text { - Urteile und Meinungen der } \\
\text { Standbesucher } \\
\text { - Anzahl der ausgegebenen } \\
\text { Informationsprospekte }\end{array}$ & $\begin{array}{l}\text { Besucherbefragung, } \\
\text { Zählung der abgegebe-nen } \\
\text { Prospekte }\end{array}$ \\
\hline $\begin{array}{l}\text { Verbesserung } \\
\text { Unternehmensimage }\end{array}$ & $\begin{array}{l}\text { - Urteile, Goodwill, Einstellungen } \\
\text { der Standbesucher }\end{array}$ & $\begin{array}{l}\text { Besucherbefragung, } \\
\text { Nachmessebefragung }\end{array}$ \\
\hline Emotionale Profilierung & $\begin{array}{l}\text { - Aktivierungs- bzw. Aufmerk- } \\
\text { samkeitsgrad der Stand- } \\
\text { besucher } \\
\text { - Emotionen der Standbesucher }\end{array}$ & Standbesucherbeobachtung \\
\hline Rationale Profilierung & $\begin{array}{l}\text { - Urteile und Meinungen der } \\
\text { Standbesucher }\end{array}$ & $\begin{array}{l}\text { Besucherbefragung, } \\
\text { Gesprächsprotokoll }\end{array}$ \\
\hline $\begin{array}{l}\text { anspruchsgruppen- } \\
\text { bezogene Beeinflus- } \\
\text { sungsziele }\end{array}$ & Erfolgskriterien & Meßmethoden \\
\hline $\begin{array}{l}\text { Einstellungsänderungen } \\
\text { ggü. kontroversen } \\
\text { Branchenproblemen }\end{array}$ & $\begin{array}{l}\text { - Einstellungen bei Presse, } \\
\text { Multiplikatoren, Messepublikum } \\
\text { - }\end{array}$ & $\begin{array}{l}\text { Focusgruppenbefragung, } \\
\text { Besucherbefragung, } \\
\text { Ausstellerbefragung } \\
\end{array}$ \\
\hline $\begin{array}{l}\text { Beziehungspflege bzw. -auf- } \\
\text { bau mit Medien }\end{array}$ & $\begin{array}{l}\text { - Anzahl und Qualität der } \\
\text { Kontakte zu Medienvertretern } \\
\text { - Anzahl abgegebener Presse- } \\
\text { Infos } \\
\end{array}$ & $\begin{array}{l}\text { Kontaktzählung, } \\
\text { Kontaktbewertung, } \\
\text { Zählung verbreiteter } \\
\text { Presse-Infos } \\
\end{array}$ \\
\hline $\begin{array}{l}\text { Beziehungspflege bzw. -auf- } \\
\text { bau mit versch. Institutionen }\end{array}$ & $\begin{array}{l}\text { - Anzahl und Qualität der } \\
\text { Kontakte zu Vertretern der } \\
\text { Institutionen }\end{array}$ & $\begin{array}{l}\text { Kontaktzählung, } \\
\text { Kontaktbewertung }\end{array}$ \\
\hline
\end{tabular}

Tab. 5: Erfolgskriterien und Methoden für die Kontrolle von Motivations- sowie markt- und anspruchsgruppenbezogenen Beeinflussungszielen 


\begin{tabular}{|c|c|c|}
\hline Verkaufsziele & Erfolgskriterien & Meßmethoden \\
\hline Verkaufsabschlüsse & $\begin{array}{l}\text { - Zahl der Aufträge } \\
\text { - Anzahl verkaufter Produkte } \\
\text { - Höhe des direkten Messe-Umsatzes }\end{array}$ & Umsatzkontrolle \\
\hline $\begin{array}{l}\text { Verkaufsanbahnung / } \\
\text { Nachmessegeschäft }\end{array}$ & $\begin{array}{l}\text { - Zahl der in Aussicht stehenden } \\
\text { Aufträge } \\
\text { - Zahl der abgegebenen Angebote } \\
\text { - Anzahl der ausgegebenen } \\
\text { Informationsprospekte } \\
\end{array}$ & $\begin{array}{l}\text { Gesprächsprotokoll, } \\
\text { Zählung der } \\
\text { abgegebenen Prospekte }\end{array}$ \\
\hline Kontaktziele & \begin{tabular}{|c|} 
Erfolgskriterien \\
\end{tabular} & MeBmethoden \\
\hline $\begin{array}{l}\text { Kontaktpflege mit } \\
\text { bestehenden Kunden }\end{array}$ & $\begin{array}{l}\text { - Anzahl und Bedeutung der Kunden } \\
\text { - Besuchsquote der eingeladenen } \\
\text { Kunden }\end{array}$ & $\begin{array}{l}\text { Zählung der Kontakte, } \\
\text { Gesprächsprotokoll, } \\
\text { Kontaktbewertung, } \\
\text { Resonanzanalyse }\end{array}$ \\
\hline $\begin{array}{l}\text { Kontaktbelebung mit } \\
\text { ehemaligen Kunden }\end{array}$ & $\begin{array}{l}\text { - Anzahl und Bedeutung der Kunden } \\
\text { - Besuchsquote der eingeladenen } \\
\text { Kunden }\end{array}$ & $\begin{array}{l}\text { Zählung der Kontakte, } \\
\text { Gesprächsprotokoll, } \\
\text { Kontaktbewertung, } \\
\text { Resonanzanalyse }\end{array}$ \\
\hline $\begin{array}{l}\text { Kontaktaufnahme mit poten- } \\
\text { tiellen Kunden }\end{array}$ & $\begin{array}{l}\text { - Anzahl und Bedeutung der Kunden } \\
\text { - Anzahl Messestandbesucher } \\
\text { - Besuchsquote der eingeladenen } \\
\text { Kunden }\end{array}$ & $\begin{array}{l}\text { Zählung der Kontakte, } \\
\text { Zählung der Standbe- } \\
\text { sucher, Gesprächspro- } \\
\text { tokoll, } \\
\text { Kontaktbewertung, } \\
\text { Resonanzanalyse }\end{array}$ \\
\hline
\end{tabular}

Tab. 6: Erfolgskriterien und Methoden für die Kontrolle von Verkaufs- und Kontaktzielen

Die verschiedenen Methoden, die den Ausstellern im Rahmen ihrer Messeerfolgskontrolle zur Verfügung stehen, werden in Abb. 21 in systematisierter Form dargestellt. ${ }^{306}$ Dabei wird deutlich, daß hierbei, analog zur Trennung der Messebeteiligungsziele in ökonomische und psychographische Zielsetzungen (vgl. Kap. B. 1.2), eine ebensolche Unterscheidung auch für die aufgeführten Verfahren vorgenommen werden kann. ${ }^{307}$

306 Im Rahmen dieser Arbeit werden aus Gründen der Komplexität und Verständlichkeit des Fragebogens lediglich solche Methoden in die Untersuchung aufgenommen, die in der Messepraxis eine ${ }_{n}$ gewisse" Verbreitung gefunden haben. Insofern liegt hier eine aus praxeologischen Überlegungen heraus vorgenommene Einschränkung der Auswahl von Methoden zur Erfolgskontrolle vor, die allerdings auf der Basis eines Expertenworkshops erfolgte. Vgl. hierzu die Teilnehmerliste der AUMA-Workshops im Anhang 2 dieser Arbeit.

307 Die aufgeführten Methoden sind in der überwiegenden Mehrzahl den primärstatistischen Erhebungen zuzuordnen, da es sich bei messerelevanten Sekundärdaten meist um allgemeine Rahmendaten handelt, die nur wenig Aussagekraft zur Beurteilung des Messebeteiligungserfolges eines einzelnen Ausstellers entwickeln. Vgl. hierzu auch Funke, K., Messeentscheidungen. Handlungsalternativen und Informationsbedarf, a.a.O., S. $290 \mathrm{ff}$. 


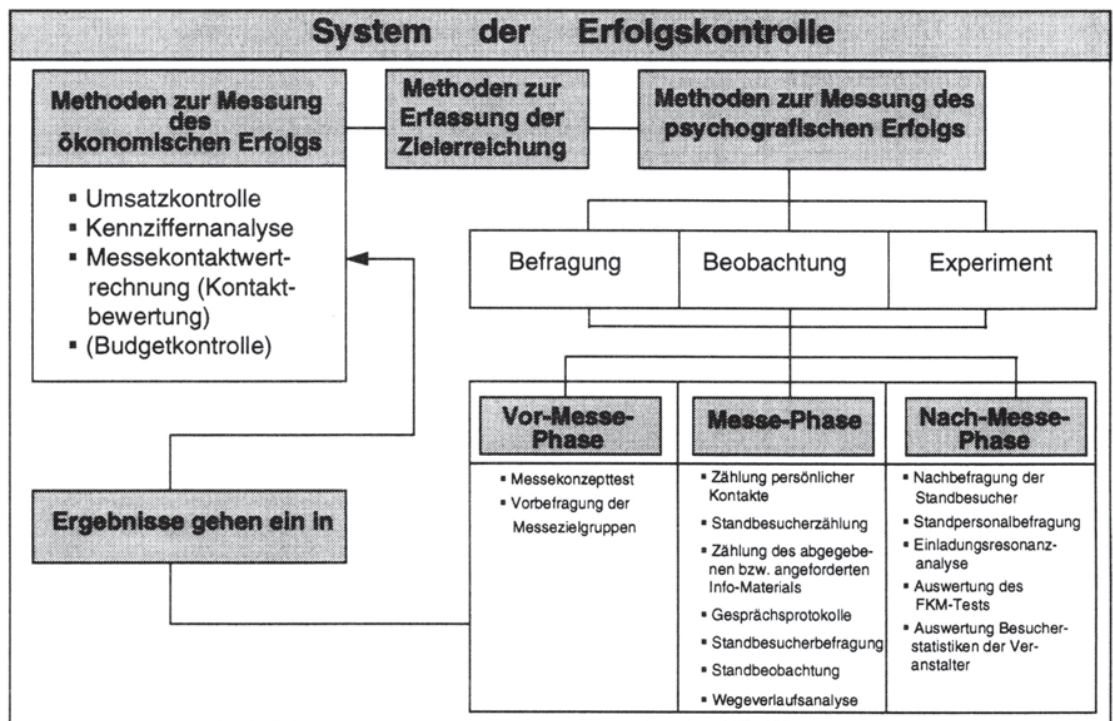

für die Erfassung der Zielerreichung einer einzelnen Messebeteiligung

Abb. 21: Systematisierung der Methoden zur Messeerfolgskontrolle

Zur Kontrolle des psychographischen Erfolgs stehen grundsätzlich die Erhebungsformen "Befragung", "Beobachtung" und "Experiment" zur Verfügung. ${ }^{308}$ Die in Abb. 21 aufgeführten Methoden können jeweils einer der genannten Erhebungsformen zugeordnet werden. Somit fällt die Wahl der Erhebungsform mit der Entscheidung über den Einsatz einer Methode zusammen. Dies besitzt wichtige Implikationen für den Kontrollierenden, da die Erhebungsformen unterschiedliche Ansprüche an die Vorbereitung und Durchführung der Erfolgskontrolle stellen. ${ }^{309}$

Die Methoden der Erfolgskontrolle sind darüber hinaus hinsichtlich ihrer zeitlichen Zielung des Einsatzes strukturierbar. Hierbei kann wiederum auf das "PhasenKonzept" einer Messebeteiligung zurückgegriffen werden. ${ }^{310}$ Damit wird deutlich, daß die Methoden der Erfolgskontrolle entweder in der Vor-Messe-, der Messe-

308 Vgl. Meffert. H., Marketingforschung und Käuferverhalten, a.a.O., S. 182 ff.; Berekoven, L. Eckert, W. Ellenrieder. P., Marktforschung: methodische Grundlagen und praktische Anwendung. 6. Aufl., Wiesbaden 1993, S. 88 ff.

309 Während bspw. die Durchführung einer einfachen Zählung nur wenige Vorbereitungs- und Personalressourcen erfordert, bedart eine Befragung intensiver inhaltlicher Vorbereitung und personeller Betreuung durch den Kontrollierenden. 
oder der Nach-Messe-Phase greifen müssen. Der Vor-Messe-Phase kommt in diesem Kontext eine besondere Bedeutung zu, da die Erfolgskontrolle in dieser Phase vor allem der Erprobung des Messebeteiligungskonzepts sowie der Überprüfung der Zielgruppenerreichung dient. Die Erfolgskontrolle in der Messe-Phase dient dagegen vor allem der gezielten Erfassung des Geschehens auf dem Messestand.

In der Nach-Messe-Phase finden schwerpunktmäßig die Evaluierung des ökonomischen Erfolges der Messebeteiligung sowie die Überprüfung des Zielerreichungsgrades derjenigen psychographischen Messeziele statt, die gesamthaft erst nach Beendigung der Messeveranstaltung beurteilt werden können. Im Hinblick auf die Überprüfung der ökonomischen Zielsetzungen einer Messebeteiligung ist darauf hinzuweisen, daß einige der aufgeführten Verfahren explizit Ergebnisse der Kontrolle des psychographischen Erfolgs verwenden. Insofern sind beide Bereiche nicht losgelöst voneinander zu betrachten, sondern prinzipiell miteinander verbunden.

Im folgenden werden die wichtigsten Methoden der Erfolgskontrolle von Messebeteiligungen vorgestellt. Dabei werden jeweils die Ziele, die Erhebungsform, die zeitliche Zielung sowie die Vor- und Nachteile der Methoden kurz gekennzeichnet. Das Phasen-Konzept der Messebeteiligung bildet dabei den Ausgangspunkt der Betrachtung.

\subsection{Methoden zur Erfolgsprognose in der Vor-Messe-Phase}

In der Vor-Messe-Phase stehen dem Aussteller grundsätzlich der Messekonzepttest sowie die Vorbefragung der Messezielgruppen als Methoden der Erfolgskontrolle zur Verfügung. Dabei weisen diese Verfahren i. S. einer "ex ante-Analyse" stark prognostischen Charakter auf und dienen damit eher der Erfolgsprognose. ${ }^{311}$

311 Prognosen stellen Zukunftsaussagen mit einem gewissen Objektivitätsgrad dar. Sie beinhalten Vorhersagen wahrscheinlicher oder möglicher Ereignisse bzw. Entwicklungen. Vgl. hierzu Meffert, H. Steffenhagen. $H_{\text {. }}$, Marketingprognosemodelle - Quantitative Modelle des Marketing, a.a.O., S. 5. Insofern sollen Erfolgsprognosen hier als Instrument der Potentialabschätzung einzelner Gestaltungselemente bzw. Aktivitäten im Zusammenhang mit Messebeteiligungen verstanden werden. 
- Messekonzepttest

Ziel des Messekonzepttests ${ }^{312}$ ist die Prognose der Wirkungen einzelner Bestandteile des Messekonzeptes bzw. des gesamten Messestandes eines Ausstellers. Durch systematische Variation der einzelnen Bestandteile des Messestandes im Rahmen eines experimentellen Designs sollen die jeweils wirkungsstärksten Gestaltungsalternativen für einzelne Messestandelemente, wie z. B. Exponate, Plakate, Standbeschriftungen und -farben etc., ermittelt werden. ${ }^{313}$ Gleichzeitig können Handlungsanweisungen und Gestaltungsempfehlungen für einzelne Instrumente des Messemarketing abgeleitet werden. Im Vordergrund dieses Verfahrens stehen die momentanen Reaktionen der Zielpersonen (wie z. B. Aufmerksamkeit und spontanes Interesse) als unmittelbare Konsequenz der Kommunikationsinstrumente am Messestand und der äußeren Gestaltung des Standes selbst sowie seiner Exponate. ${ }^{314}$ Damit dient der Messekonzepttest primär der Kontrolle bzw. Prognose der Erreichung von marktbezogenen Beeinflussungszielen.

Der wesentliche Vorteil dieses Verfahrens ist vor allem in der gezielten Aufdekkung von Ursache-Wirkungs-Zusammenhängen zu sehen, ohne dabei - im Gegensatz zu einer Untersuchung während des Messegeschehens - den Ablauf der Messebeteiligung zu stören. ${ }^{315}$ Allerdings stellt die Repräsentativität des Untersuchungssamples ein entscheidendes Problem des Messekonzepttests dar. Oft kann nur mit „Kleinst"-Stichproben gearbeitet werden, die sich zudem häufig aus Messe(bau)-Experten zusammensetzen und damit nicht als repräsentativ für die Messebesucher angesehen werden können. ${ }^{316}$ Dennoch ist davon auszugehen, daß durch den Einsatz dieses Verfahrens Anhaltspunkte über die Wirksamkeit bestimmter Messekonzeptelemente des Ausstellers zu erhalten sind.

312 Diese Methode wurde in Anlehnung an sog. Labortestmärkte in der Werbewirkungsforschung entwickelt und weist daher vielfach Ähnlichkeiten dazu auf. Vgl. Rehorn. J., Markttests, Neuwied 1977, S. $45 \mathrm{ff}$. sowie Rehorn. J., Labortestmarkt und Minitestmarkt - Alternativen zum Prägen von Marktchancen, in: asw, Heft 9, 1977, S. 51-60; Hossinger. H.-P., Pretests in der Marktforschung - Die Validität von Pretestverfahren unter besonderer Berücksichtigung der Tachistoskopie, Würzburg, Wien 1982, S. 6 ff.

313 Vgl. Gräbener. W. Die Messepolitik als Marketinginstrument dargestellt am Beispiel von Investitionsgüterproduzenten, a.a.O., S. $325 \mathrm{ff}$.

$314 \mathrm{Vgl}$. Funke, $\mathrm{K}$, Messeentscheidungen. Handlungsalternativen und Informationsbedarf, a.a.O., S. 315 .

$315 \mathrm{Vgl}$. Doyle, P. Gidengil, B. Z., A Review of In-Store Experiments, in: Journal of Retailing, No. 2, 1977 , S. 47-62.

316 Vgl. Gluth, H. Keine schwarzen Messe - Erfolgskontrolle mit den Methoden der empirischen Sozialforschung, in: Verlagsbeilage zur FAZ vom 23.01.1996, S. B7. 


\section{- Vorbefragung der Messezielgrupppen}

Hauptzweck einer Befragung von Messezielgruppen im Vorwege einer Messebeteiligung ist die Beschaffung von Informationen über ihre Messepräsenz sowie Vorstellungen und Erwartungen bezüglich der in Kürze stattfindenden Veranstaltung. ${ }^{317}$ Damit dient der Einsatz dieser Form der direkten Befragung, die i. d. R. telefonisch durchgeführt wird, dem Aussteller als Entscheidungshilfe im Rahmen der Messebeteiligungsentscheidung, denn er ist nach Durchführung der Befragung in der Lage einzuschätzen, ob die von inm auf der Messe enwarteten Zielgruppen dort anzutreffen sind oder nicht.

Darüber hinaus ermöglicht eine Vorbefragung potentieller Messebesucher die Erhebung vielfältiger Zusatzinformationen für den Aussteller. Mögliche Erhebungsthemen können neben der Bedeutung der Messe für die Informationsbeschaffung des Befragten auch sein Informationsverhalten sowie seine Informationsbedürnisse und Wünsche an die Aussteller auf dieser Messeveranstaltung sein. Damit eignet sich dieses Verfahren insbesondere zur Einschätzung der Erreichung von Informations- und Kontaktzielen. ${ }^{318}$

Ein wesentlicher Vorteil dieser Methode zur Kontrolle des Messeerfolges liegt in seiner "Frühwarn-Funktion". ${ }^{319}$ Zum einen wird eine zielgruppenorientierte Messeselektion unterstützt und zum anderen können Messebeteiligungsziele und geplante Präsentationsinhalte einer Überprüfung unterzogen werden. Als nachteilig wirkt sich insbesondere die Notwendigkeit eines relativ umfangreichen Adressenbestandes aus, der die Zielgruppenstruktur hinreichend widerspiegelt. Darüber hinaus lassen sich Befragungen solcher Art mit vertretbarem Aufwand im allgemeinen nur bei inländischen Zielgruppen durchführen.

Vgl. o.V., Wirkungskontrollen für Messebeteiligungen, in: Fachverband Messe- und Ausstellungsbau e.V. (FAMAB) (Hrsg.), Jahresbericht '95, S. 21.

Vgl. Gelszus Messe-Marktforschung GmbH (Hrsg.), Mehr Erfolg auf Messen durch Messewirkungskontrolle, Dortmund 1994, S. 19.

Vgl. zur Notwendigkeit des Einsatzes von geeigneten Instrumenten mit Frühwarn-Funktion; Liebl, F., Strategische Frühaufklärung, München 1996, S. 5 f. Zurlino, F., Zukunftsorientierung von Industrieunternehmen durch strategische Früherkennung, München, Wien 1995; Steger. U. Winter. $M_{\text {. }}$, Strategische Früherkennung zur Antizipation ökologisch motivierter Marktveränderungen, in: DBW, 56. Jg., Heft 5, 1996, S. 607-629. 


\subsection{Methoden zur Erfolgskontrolle in der Messe-Phase}

Erhebungen der Aussteller während einer Messeveranstaltung können auf dem Messegelände oder außerhalb dieses Areals stattfinden. Allerdings bilden die nicht auf dem Messegelände durchgeführten Erhebungen eher die Ausnahme. Die Aussteller konzentrieren sich in dieser Phase auf Erhebungsmethoden, die auf dem eigenen Messestand bzw. in dessen unmittelbarer Nähe Anwendung finden. Dies nicht zuletzt auch deshalb, weil i. d. R. ausstellerseitige Erhebungen auf dem Messegelände nach geltenden Veranstaltungsbestimmungen nicht gestattet sind. ${ }^{320}$

Dabei werden die Methoden der Erfolgskontrolle von Messebeteiligungen im folgenden nach dem mit innen verbundenen Erhebungsaufwand und der Aussagekraft der Methoden im Hinblick auf ihre Ergebnisse in aufsteigender Reihenfolge behandelt.

Die einfachsten Methoden der Erfolgskontrolle stellen die Zählungen dar. ${ }^{321}$ Ihre Erscheinungsformen sollen zunächst skizziert werden.

\section{- Zählung des abgegebenen bzw. angeforderten Informationsmaterials}

Mit dem Einsatz dieser Erhebungsform verfolgen Aussteller die Zielsetzung, Angaben über das Informationsverhalten und das konkrete Produktinteresse der Messestandbesucher $\mathrm{zu}$ erhalten. Festgehalten werden die Anfangs- und Endbestände aller Informationsmaterialien, die am Messestand für die Besucher zur Verfügung stehen sowie die entsprechende Zahl der entgegengenommenen Anforderungen. Die Auswertung der Daten ermöglicht nicht nur die Feststellung des Schwerpunktes des Besucherinteresses, sondern gibt auch in begrenztem Umfang Auskunft über Wahrnehmung und Akzeptanz des Produkt- bzw.

320 Vgl. Funke, K., Messeentscheidungen. Handlungsalternativen und Informationsbedarf, a.a.O., S. $298 \mathrm{f}$. Aus diesem Grund werden im folgenden ausschließlich solche Methoden der Erfolgskontrolle von Messebeteiligungen dargestellt, die auf dem Messestand bzw. seiner unmittelbaren Nähe eingesetzt werden können.

321 Zählungen sind beobachtende bzw. registrierende Verfahren, bei denen Häufigkeiten einzelner, genau gekennzeichneter Individualverhaltensweisen ermittelt werden. Durch Zählungen ermittelte Daten sind damit Reaktionsdaten, die in einem Vorgang erhoben und aggregiert werden. Vgl. Cunningham, M. T. White, J. G., The Role of Exhibitions in Industrial Marketing An Evaluation of the International Machine Tool Exhibition, in: Industrial Marketing Management, No. 3, 1974, S. 238 f. 
Leistungsangebots am Messestand und dient damit vor allem der Überprüfung bestimmter Informations- und Beeinflussungsziele. ${ }^{322}$

Ohne Zweifel ist die unproblematische Umsetzung dieser Kontrollmethode am Messestand ihr größter Vorteil. Andererseits kann nicht eindeutig nachvollzogen werden, ob die Mitnahme von Informationsmaterial bei Messestandbesuchern in jedem Fall auf ein hohes Produktinteresse schließen läßt. ${ }^{323}$ Insofern kann dieses Instrument der Erfolgskontrolle immer nur ein flankierend eingesetztes Verfahren darstellen, das um weitere Analysemethoden zu ergänzen ist, wenn valide Erkenntnisse über den Erfolg der Messebeteiligung ermittelt werden sollen.

\section{- Standbesucherzählung}

Die Standbesucherzählung ist ein beobachtendes bzw. registrierendes Verfahren zur quantitativen Bestimmung der Besucherfrequenz auf dem Messestand während einzelner Messetage bzw. der gesamten Veranstaltungsdauer. Nach den Verhältnissen des Messestandes werden Zählpunkte festgelegt, die mit entsprechendem Personal besetzt werden. Gezählt werden alle Besucher, die den Stand betreten. Die Zählung erfolgt an allen Tagen über die gesamte Öffnungszeit der Messeveranstaltung. ${ }^{324}$ Eine Alternative zum Einsatz von Personal stellt seit längerem die Verwendung von Kameras dar. Seit kurzem existiert auch die Möglichkeit, Standbesucher mit magnetischen Chipkarten auszustatten, die beim Betreten des Messestandes einen Zählimpuls an einem "Computer-Counter" auslösen. ${ }^{325}$

Ziel der Standbesucherzählung ist die Ermittlung von Daten zur Bestimmung des Besucherinteresses. ${ }^{326}$ Ein Vergleich der Gesamtbesucherzahl der Veranstaltung mit der Standbesucherzahl gibt des weiteren Auskunft darüber, ob ein angemessener Anteil Messebesucher den eigenen Stand besucht und das

322 Vgl. Hagen, R., Erfolgskontrolle auf Verbrauchermessen - dargestellt am Beispiel der Analyse des Informationsstandes des Deutschen Bundestages, in: planung und analyse, Heft 7, 1983, S. 292-296.

Vgl. Schönemann, K., Messen und Kataloge: Die Sache mit den Katalogen, in: Der Fremdenverkehr, Heft 2, 1989, S. 16.

Vgl. Gelszus, R. H., Messen und Ausstellungen: Vergessen Sie die Messe-Wirkungskontrolle nicht, in: Marketing Journal, Heft 2, 1985, S. 176.

Vgl. o.V., Erfolgskontrolle - Umstrittene Methode, in: FAMA intern, Heft 2, 1985, S. 12.

Vgl. Gelszus Messe-Marktforschung GmbH (Hrsg.), Mehr Ertolg auf Messen - Wie Sie Ihren Erfolg messen und neue Potentiale entdecken, 0.0., o. J., S. 6. 
enwartete Besucheraufkommen erreicht wurde. In begrenztem Umfang läßt die Anwendung dieser einfachen Kontrollmethode damit also Aussagen über den Aktivierungsgrad des Messestandes (Auslösen von Interesse) sowie den Bekanntheitsgrad der ausgestellten Produkte und Leistungen bei den Messebesuchern zu.

Die entscheidenden Vorteile dieser Methode zur Erfolgskontrolle von Messebeteiligungen liegen in ihrer vergleichsweise einfachen und wenig kostenintensiven Handhabung. Zudem lassen sich einige Aussagen über den Standbesuch hinaus machen (z. B. Geschlecht und ungefähres Alter der Standbesucher). Nachteilig ist aber, daß die ermittelte Gesamtbesucherzahl des Messestandes i. d. R. deutlich höher ist als das tatsächliche Standbesucheraufkommen, da einige Standbesucher (z. B. Fachbesucher, die den Stand mehr als einmal aufsuchen, um bspw. einen bestimmten Gesprächspartner zu sprechen) mehrfach gezählt werden. Zudem weisen die rein quantitativen Informationen über die Standbesucherzahl nur wenige Implikationen für die Erfolgsbeurteilung der Messebeteiligung auf.

\section{- Zählung persönlicher Kontakte}

Eine Zählung persönlicher Kontakte als Methode der Erfolgskontrolle weist im Gegensatz zur Standbesucherzählung wesentliche Unterschiede bei der Auswahl der gezählten Standbesucher und den die Zählung vornehmenden Personen auf. Es werden nämlich nur diejenigen Standbesucher in die Statistik aufgenommen, mit denen ein persönliches Gespräch geführt wird. Zudem wird i. d. R. auch die zeitliche Verteilung der Kontakte über den einzelnen Messetag festgehalten. ${ }^{327}$ Damit ist die Aufzeichnung der Kontakte Aufgabe des Fachpersonals am Messestand, das diese Erhebungen neben seinen Verkaufs- und Informationsaufgaben übernehmen muß.

Die methodische Zielsetzung dieser Methode liegt somit darin, quantitative Aussagen über Zahl und Zeitpunkte der Kontakte zwischen Standpersonal und -besuchern zu ermöglichen. Damit bildet die Anwendung dieser Methode eine Basis für die Personaleinsatzplanung am Messestand sowie die standpersonalbezogene Bewertung von quantitativen Kontaktzielen. ${ }^{328}$

327 Vgl. Krueger, J., Messen und Ausstellungen: Die Kontakt-Kosten sichtbar machen, in: Marketing Journal, Heft 5, 1984, S. 484-489.

328 Vgl. Josef. F., Effizienzsteigerung durch Messekontrolle: Wie man aus Fehlern lernen kann, in: MesseMarkt, O. Nr., Februar 1986, S. 56-57. 
Dem Vorteil der einfachen Handhabung stehen allerdings einige Nachteile entgegen. An hochfrequentierten Messeständen können dem Fachpersonal aufgrund der hohen Kontakthäufigkeit Fehler in der Aufzeichnung der Kontaktanzahl unterlaufen. Darüber hinaus sagen quantitative Kontaktzahlen nichts über die Dauer und den Inhalt der zustande gekommenen Kontakte sowie über die "Qualität" der Standbesucher - z. B. hinsichtlich ihrer Zielgruppenzugehörigkeit aus. Es erscheint daher wenig sinnvoll, Standmitarbeiter bzw. den Erfolg einer Messebeteiligung ausschließlich an der Anzahl der Kontakte zu Standbesuchern zu messen. Damit kann auch dieses Verfahren lediglich der Ausgangspunkt für weitere, die qualitativen Aspekte von Messekontakten berücksichtigende Untersuchungen sein (vgl. insbesondere den Punkt "Gesprächsprotokolle" in diesem Kapitel).

\section{- Wegeverlaufsanalyse}

Die Wegeverlaufsanalyse ${ }^{329}$ dient der Messung von Besucherströmen sowie der Darstellung des Besucherverhaltens in räumlicher und zeitlicher Sicht auf dem Messestand. Sie wird bei einer möglichst großen Zahl von Standbesuchern durchgeführt, die keine Kenntnis von der Beobachtungssituation haben. Nur eine lückenlose Aufzeichnung der Besucherbewegungen auf dem Messestand ist in der Lage, Informationen über den Hauptwegeverlauf der Standbesucher, bevorzugte Haltepunkte sowie weniger frequentierte Bereiche des Standes zu liefern. Häufig werden technische Hilfsmittel wie Film- oder Videokameras für die Beobachtung der Besucherströme hinzugezogen, die die durch Rechercheure vorgenommenen Wegeverlaufsanalysen ergänzen. ${ }^{330}$

Ziel des Einsatzes dieses Verfahrens ist die Sammlung von Informationen im Hinblick auf die Ausgestaltung des Messestandes. Insbesondere interessieren in diesem Zusammenhang Angaben über die Hauptbesucherströme, die Intensität der Benutzung einzelner Ein- und Ausgänge sowie die Attraktivität einzelner Exponate. Damit ist die Wegeverlaufsanalyse vor allem zur Überprü-

329 Synonym wird auch der Begriff "Wegeanalyse“ verwendet. Vgl. Groth. C., Die Wegeanalyse Ein Instrument zur Verhaltensforschung bei Messeveranstaltungen und ähnlichen speziellen Märkten, in: GfK Mitteilungen zur Markt- und Absatzforschung, Heft 3, 1968, S. 87-90. Messebeteiligung auf, a.a.O., S. 140-144. 
fung von Präsentations- bzw. Bekanntheitszielen hinsichtlich bestimmter Produkte (Exponate) geeignet. ${ }^{331}$

Da Besucher bei der Anwendung dieser Methode während ihres Messestandbesuches keine methodenbedingte Beeinflussung erfahren, kann der Beobachter den Einfluß von Standarchitektur und Gestaltungselementen des Messestandes auf das sichtbare Verhalten der Standbesucher messen. Die ausgewählten Standbesucher sind allerdings hinsichtlich ihrer demographischen Strukturen nicht identifizierbar, so daß eine Zuordnung zu spezifischen Messezielgruppen des Ausstellers nicht möglich ist.

\section{- Standbesucherbefragung}

Gegenstand von Befragungen während einer Messeveranstaltung sind die Ermittlung von Standbesucherstrukturen und die Beurteilung des Messestandes im Hinblick auf das wahrgenommene Informationsangebot, die Verständlichkeit der Informationen, die Auswahl der Exponate, die Qualität der Kontakte mit dem Standpersonal, seine Freundlichkeit, die Attraktivität des Messestandes insgesamt etc. ${ }^{332}$

Mit dem Einsatz von Standbesucherbefragungen steht den Ausstellern ein höchst flexibles Verfahren zur Kontrolle des Messeerfolges zur Verfügung, das sowohl isoliert, aber auch in Kombination mit den oben genannten beobachtenden Verfahren eingesetzt werden kann. Diese Flexibilität erlangt die Befragung für ausstellende Unternehmen deshalb, weil sowohl der Kreis der Befragten als auch die Befragungsinhalte individuellen Zielsetzungen des Ausstellers angepaßt werden können. ${ }^{333}$

Steht die Erfassung der Standbesucherstruktur im Vordergrund, wird in diesem Zusammenhang auch von Besucherstrukturbefragung gesprochen. Dagegen

331 Vgl. Bachmeir, H. M., Marktforschung auf Messen, in: Meyer, P. W. (Hrsg.), Arbeitspapiere zur Schriftenreihe Schwerpunkt Marketing der Universität Augsburg, Bd. 23, Augsburg 1989, S. 25 f.

Vgl. Gelszus, R. H., Messen und Ausstellungen: Vergessen Sie die Messe-Wirkungskontrolle nicht, a.a.O., S. 174.

Rost. D., Maßstäbe zur Beurteilung der Effizienz von Messen, in: VDI-Nachrichten, Nr. 115, 1973, S. 333-338. 
liegt bei Besucherintensivbefragungen der Schwerpunkt auf der Behandlung komplexer Themen. ${ }^{334}$

Im Rahmen der Besucherstrukturbefragung erfolgt die Auswahl der Auskunftspersonen nach dem Zufallsprinzip aus den Besuchern, die den Stand verlassen. Bei der Besucherintensivbefragung effolgt eine Selektion der Auskunftspersonen nach Zielgruppen. Nur diejenigen Standbesucher, die sich nach einigen Fragen zur Person sowie ggf. zum durch sie vertretenen Unternehmen als Mitglied der gewünschten Zielgruppe erweisen, werden um ein weiterührendes Interview gebeten.

Der wesentliche Vorteil der Anwendung von Befragungen liegt in der Möglichkeit, nicht beobachtbare Reaktionen der Standbesucher auf die Aktivitäten der Aussteller näher ergründen zu können. Es ist dem Aussteller daher bspw. möglich, Informationen über die Wirkung seiner messebezogenen Maßnahmen hinsichtlich der Einstellungen und Meinungen der Standbesucher zu erlangen. Ferner können Aktivierungs- sowie Wahrnehmungs- und Verarbeitungswirkungen, die spontan beim Besuch des Messestandes entstanden sind, aufgenommen werden. Explizit können Standbesucher z. B. über Gelegenheiten zu einem persönlichen Gespräch mit dem Standpersonal befragt werden, um so die Wirkungen persönlicher und unpersönlicher Kommunikation am Messestand zu untersuchen. ${ }^{335}$

Diesen Vorteilen stehen die generell für Befragungen geltenden Risiken gegenüber. ${ }^{336} \mathrm{Um}$ weitgehend repräsentative Ergebnisse einer Stichprobenbefragung zu erhalten, müßte zudem die Grundgesamtheit der Messestandbesucher streng genommen schon vor Messebeginn bekannt sein. Die zahlenmäßig exakte Erfassung der Messebesucher kann aber erst nach Beendi-

Vgl. zu den wesentlichen Unterschieden der beiden Befragungsarten Gelszus Messe-Marktforschung $\mathrm{GmbH}$ (Hrsg.), Mehr Erfolg auf Messen durch Messewirkungskontrolle, a.a.O., S. 9 $\mathrm{ff}$.

Vgl. Funke, K., Messeentscheidungen. Handlungsalternativen und Informationsbedarf, a.a.O., S. $303 \mathrm{f}$.

So können die Informationen, die der Aussteller mittels einer Befragung von den Standbesuchern erhält, sog. systematischen Fehlern unterliegen. Sind die eingesetzten Fragen interpretationsfähig und die Interviewer nehmen unbewußt Einfluß auf die Beantwortung der Fragen (soziale Erwünschtheit von Antworten), oder die Struktur der auskunftswilligen stimmt nicht mit derjenigen der nicht-auskunftswilligen Standbesucher überein, kommt es zu befragungsbedingten Fehlinterpretationen der Daten. Zu den allgemeinen Risiken von Befragungen vgl. Böhler. H., Marktforschung, Stuttgart u. a. 1985, S. 121 ft.; Hammann, P. Erichson, B., Marktforschung, Stuttgart, New York 1978, S. $30 \mathrm{ff}$. 
gung der Messe ermittelt werden. Trotz dieser zu bedenkenden Fehlerquellen stellen die verschiedenen Formen der Standbesucherbefragung eine zentrale Informationsquelle zur Überprüfung der Zielerreichung insbesondere im Hinblick auf die psychographischen Zielsetzungen dar.

\section{- Gesprächsprotokoll}

Die systematische Erfassung aller am Messestand geführten Kundengespräche gehört zu den unabdingbaren Elementen der Messeerfolgskontrolle. Gesprächsprotokolle, die auch als Berichtsbogen, Messekontaktbogen oder Kontakterfassungsbogen bezeichnet werden, haben die Aufgabe, die auf dem Messestand geführten Gespräche schriftlich festzuhalten. Durch den Einsatz dieses Verfahrens können somit Informationen über den Standbesucher sowie den Gesprächsverlauf bzw. -inhalt jeweils nach Tag und Uhrzeit dokumentiert werden. $^{337}$

Grundsätzlich steht dem Aussteller der Einsatz von einerseits weitgehend formalisierten sowie andererseits nicht formalisierten Gesprächsprotokollen ${ }^{338}$ zur Verfügung. Während formalisierte Gesprächsprotokolle auf Basis eines streng strukturierten Vorgehens durchgeführt werden und auf die Aufzeichnung des einzelnen Gesprächs ausgerichtet $\operatorname{sind}^{339}$, weisen nicht formalisierte Gesprächsberichte i. d. R. keine spezifische Struktur auf und haben häufig einen eher zusammenfassenden Charakter, so daß letztere häufig nach Ablauf eines Messetages zum Einsatz kommen. Damit erhält der Standmitarbeiter Gelegenheit, die Inhalte der geführten Gespräche zu reflektieren und Besonderheiten festzuhalten. Jedoch muß hierbei davon ausgegangen werden, daß relevante Informationen möglichenweise vergessen und nicht aufgezeichnet werden. Dagegen besteht der Vorteil eines formalisierten Protokollbogens darin,

337 Vgl. Selinski, H., Messe- und Kongreßforschung (Teil II), in: planung und analyse, Heft 5, 1984, S. 214 f.

338 Gelszus spricht in diesem Zusammenhang von Gesprächsnotizen. Vgl. Gelszus MesseMarktforschung GmbH (Hrsg.), Mehr Erfolg auf Messen durch Messewirkungskontrolle, a.a.O., S. 21.

339 Zum grundsätzlichen Aufbau von formalisierten Gesprächsprotokollen vgl. Jaspersen. $H$., Mehr Messe-Transparenz: Hüls, in: Marketing Journal, Heft 2, S. 169-173 sowie in erweiterter Form Meffert, $H$. Ueding. R., Ziele und Nutzen von Messebeteiligungen, a.a.O., S. 58 f. 
daß er die Aufzeichnung aller relevanten Gesprächspunkte weitgehend unterstützt. ${ }^{340}$

Der wesentliche Nachteil des Einsatzes von Gesprächsprotokollen besteht in ihrer Selektivität, da diejenigen Standbesucher, die kein Gespräch mit dem Standpersonal führen, sondern sich nur auf dem Messestand umsehen und anhand des Prospektmaterials sowie der Exponate informieren, nicht erfaßt werden.

Nachdem die wichtigsten Methoden der Erfolgskontrolle, die einem Aussteller in der Messe-Phase zur Verfügung stehen, vorgestellt worden sind, sollen im Folgenden die Verfahren zur Überprüfung der Zielerreichung gekennzeichnet werden, die nach Beendigung der Messebeteiligung ergriffen werden können.

\subsection{Methoden zur Erfolgskontrolle in der Nach-Messe-Phase}

Der Grad der Zielerreichung kann vielfach erst nach Beendigung einer Messebeteiligung vollständig erhoben werden. Dies trifft insbesondere für die Kontrolle des ökonomischen Erfolgs zu. Des weiteren kann es für einen Aussteller von großer Wichtigkeit sein, Informationen über die Fristigkeit der psychographischen Wirkungen der Messebeteiligung bei den Standbesuchem zu erhalten. Nicht zuletzt sollte auch das Standpersonal bei Abschluß der Messebeteiligung "Manöverkritik" üben können. Zum einen, um seine Erfahrungen als Bindeglied zwischen Unternehmen und Messebesucher über das Geschehen am Messestand wiederzugeben und zum anderen, um die gewonnenen Informationen für die Organisation und Durchführung der nächsten Messebeteiligung zu verwenden. Damit ergibt sich auch nach der Messe der Bedarf für weitere Erhebungen.

\section{- Nach-Messebefragungen}

Die vielfältigen optischen und akustischen Reize auf einer Messeveranstaltung sowie die große Anzahl der geführten Gespräche bewirken bei Messebesuchern, daß die auf der Messe aufgenommenen Informationen nur kurzfristig

340 Durch den Einsatz von Personal Computern bereits bei der Erstellung der Gesprächsprotokolle (z. B. während des Gesprächs) kann eine Effektivitätssteigerung dieses Instruments der Erfolgskontrolle erreicht werden, da es möglich wird, die erfaßten Protokolle "über Nacht" auszuwerten und an das Unternehmen weiterzuleiten, um dort die ggf. notwendig werdenden Maßnahmen (z. B. Versendung von Informationsunterlagen) ohne zeitliche Verzögerungen zu ergreifen. 
präsent bleiben. Erst einige Zeit nach dem Messebesuch konsolidiert sich der Gesamteindruck der Veranstaltung. Als wichtig empfundene Informationen bleiben im Gedächtnis verhaftet, eher unwichtige Eindrücke gehen schnell verloren. Deshalb gibt eine Besucherbefragung nach der Messe ein individuell reflektierendes Gesamtbild der Messeveranstaltung wieder und ist besonders geeignet, Aussagen über die mittel- und längerfristige Wirksamkeit des Messeengagements eines Ausstellers bei der Zielgruppe zu treffen. ${ }^{341}$ Dabei ist insbesondere die Kontrolle der Erreichung von Beeinflussungs- und Verkaufszielen Gegenstand der meist telefonisch durchgeführten Befragung. Die Aussagen der Auskunftspersonen können auch durch Fragen im Hinblick auf die Wirkungen der Messeaktivitäten von Wettbewerbern relativiert werden. ${ }^{342}$

Der wesentliche Vorteil einer Nachbefragung von Standbesuchern ist in der Tatsache zu sehen, daß die Befragung zu einem Zeitpunkt durchgeführt wird, zu dem kurzwirkende Eindrücke i. d. R. bereits vergessen worden sind. Die für den Standbesucher wichtigen Dinge treten damit klarer in den Vordergrund. Der Aussteller kann vor allem solche Aspekte seiner Messeaktivitäten abfragen, mit denen längerfristige Verhaltenswirkungen beim Messebesucher erzielt werden soliten. ${ }^{343}$

Nachbefragungen können schließlich - nicht nur i. S. einer Kontrollgruppe auch jene Mitglieder der Zielgruppe erfassen, die die Veranstaltung bzw. den Messestand nicht besucht haben. Hierdurch kann der Aussteller vor allem die Motive des Nicht-Besuchs einer Messeveranstaltung oder des Messestandes erfahren. Diese Informationen können zu einer besseren Einschätzung der Messeveranstaltung bezüglich des Präsenzanteils der anvisierten Zielgruppe an der Gesamtbesucherzahl sowie damit auch zur Fundierung zukünftiger Messebeteiligungsentscheidungen beitragen. ${ }^{344}$

${ }^{441}$ Vgl. Funke, K., Messeentscheidungen. Handlungsalternativen und Informationsbedarf, a.a.O., S. $308 \mathrm{ft}$.

342 Vgl. Gelszus Messe-Marktforschung GmbH (Hrsg.), Mehr Erfolg auf Messen durch Messewirkungskontrolle, a.a.O., S. 20.

343 Funke, K., Messeentscheidungen. Handlungsalternativen und Informationsbedarf, a.a.O., S. 308.

344 Gelszus Messe-Marktforschung GmbH (Hrsg.), Mehr Erfolg auf Messen durch Messewirkungskontrolle, Dortmund 1994, S. 20.

Ralf Uéding and Universität Münster - 978-3-631-75073-5 
Vorteilen und Funktionen von Nach-Messebefragungen stehen indes auch Nachteile gegenüber. In erster Linie sind dies die zusätzlich entstehenden Kosten, die vor allem aus dem organisatorischen Doppelaufwand (Erfassung der erforderlichen Adressen während und Durchführung der Befragung nach der Messe) und den für die Durchführung benötigten Personalressourcen resultieren.

\section{- Standpersonalbefragung}

Befragungen des Standpersonals am Ende einer Messeveranstaltung bieten die Möglichkeit, Informationen über die Qualität der Messevorbereitung, den Veranstaltungsverlauf aus Sicht der Standbesatzung sowie Anregungen aus dem Standbesucherkreis $\mathrm{zu}$ erhalten. Standpersonalbefragungen dienen darüber hinaus auch der Kontrolle von Motivationszielen, die bezüglich der Mitarbeiter formuliert wurden. ${ }^{345}$

Die Durchführung der Standpersonalbefragung geschieht i. d. R. durch kurze Einzelinterviews oder Gruppengespräche ${ }^{346} \mathrm{Da}$ dabei nur subjektive Eindrücke der Mitarbeiter wiedergegeben werden können, ist eine konkrete Überprüfung der Erreichung von kunden- bzw. anspruchsgruppenorientierten Messebeteiligungszielen mit Hilfe dieser Besprechungen nicht möglich. Somit darf diese Methode streng genommen nur insofern zu den Methoden der Erfolgskontrolle von Messebeteiligungen gezählt werden, als die Überprüfung von mitarbeitergerichteten Zielsetzungen vorgenommen werden soll. Die Ergebnisse dieser Befragungen können zudem i. S. einer Tätigkeitskontrolle genutzt werden, um korrigierend in den Ablauf zukünftiger Messebeteiligungen einzugreifen. Ferner können sie Anhaltspunkte für eine gezielte Messenacharbeit liefern. ${ }^{347}$

345 Vgl. Berghäuser. B., Messen und Ausstellungen: Entscheidungshilfen für die „richtige" Beteiligung, in: Marketing Journal, Heft 2, 1983, S. 166-168; Leicher. R., Die Manöverkritik. Unerläßlicher Bestandteil der Messenacharbeit, in: Ausstellungs- und Messe-Ausschuss der Deutschen Wirtschaft e. V. (Hrsg.), Literatursammlung des AUMA, Köln O. J., S. 79.

Selinski und Sperling schlagen dagegen anonyme schriftliche Befragungen der Standmitarbeiter vor, um den Informationsnutzen der Erhebung für das ausstellende Unternehmen zu erhöhen. Vgl. Selinski, H., Sperling, U. A., Marketinginstrument Messe: Arbeitsbuch für Studium und Praxis, a.a.O., S. 224.

347 Vgl. Berghäuser, B., Messen und Ausstellungen: Entscheidungshilfen für die „richtige" Beteiligung, a.a.O., S. 168. 


\section{- Einladungsresonanzanalyse}

$\mathrm{Zu}$ den wichtigsten Zielsetzungen einer Messebeteiligung zählen die Kontaktanbahnung zu potentiellen Kunden sowie die Kontaktpflege mit aktuellen Geschäftspartnern. ${ }^{348}$ Daher unternehmen die meisten Aussteller besondere Anstrengungen, diese Messezielgruppen zum Besuch des Messestandes einzuladen. Die Resonanz auf die versandten Einladungen ist damit mitentscheidend für den Erfolg der Messebeteiligung. Zur Gesamtbewertung des Messebeteiligungserfolges zählt darum zwingend die Überprüfung der Einladungsresonanz - auch um die Einladungsaktion auf mögliche Defizite hin zu analysieren. ${ }^{349}$ Voraussetzung hierfür ist aber, daß sowohl die ausgesandten Einladungen als auch die dieser Einladung gefolgten Besucher individuell festgehalten worden sind.

Die Ergebnisse der Resonanzanalyse sind zur gezielten Messenacharbeit bei den eingeladenen Kunden einzusetzen. Diejenigen Kunden, die auf dem Messestand erschienen, sind aufbauend auf den Ergebnissen der Gespräche am Messestand zu kontaktieren. Bei Kunden, die trotz Einladung nicht auf dem Messestand erschienen sind, können die Gründe für ihr Fernbleiben erfragt werden. Darüber hinaus bieten sich durch die Abgabe eines Messeberichts aus Sicht des Ausstellers interessante Anknüpfungspunkte für die Initiierung bzw. Weiterführung des Dialogs mit den Kunden.

\section{- Auswertung der Besucherstatistiken des Veranstalters}

Auch die Messegesellschaften bemühen sich häufig um die Erhebung von Besucherdaten ihrer Veranstaltung. Im Rahmen von Besucheranalysen der Messegesellschaften werden je Veranstaltungstag Messebesucher zu ihren Messeeindrücken befragt. ${ }^{350}$ Welche Strukturmerkmale der Besucher erhoben werden, bleibt dabei den Messegesellschaften überlassen. I. d. R. werden aber zumindest die regionale Herkunft, das Geschlecht, Alter und der Beruf sowie die Besuchsmotive der Auskunftspersonen erfaßt. Da dem Veranstalter auch die Gesamtzahl der Messebesucher bekannt ist, können durch Hoch-

Vgl. hierzu die empirischen Ergebnisse in Kap. B.1.2 dieser Arbeit.

Vgl. Selinski, H., Sperling, U. A., Marketinginstrument Messe: Arbeitsbuch für Studium und Praxis, a.a.O., S. 236.

Darüber hinaus besteht vereinzelt das Angebot einer Befragungsbeteiligung für Aussteller, die es ihnen ermöglicht, eigene Frageninhalte im Fragenkatalog der Messegesellschaft zu plazieren. Eine solche kombinierte Befragung wird vielfach auch als "Messebus" bezeichnet. Vgl. Gelszus Messe-Marktforschung GmbH (Hrsg.), Mehr Erfolg auf Messen - Wie Sie Ihren Erfolg messen und neue Potentiale entdecken, o.O., o. J., S. 5. 
rechnungen der Stichprobe sowohl quantitative als auch qualitative "Strukturdaten" der Messeveranstaltung ermittelt werden. ${ }^{351}$

Die Informationen über die Besucherstruktur der Messeveranstaltung geben dem Aussteller die Möglichkeit zur Überprüfung des Anteils seiner Messezielgruppen an der Gesamtbesucherzahl einer Messeveranstaltung. Damit erhält er einen groben Anhaltspunkt zur Beurteilung der segmentspezifischen Reichweite $^{352}$ der Messe. Diese Reichweitendaten sind aber wenig aussagekräftig, wenn ein Aussteller sie allein als Erfolgsmaßstab für die Zielgruppenerreichung heranzieht. Hingegen kann der Vergleich der Standbesucherstruktur mit der Struktur der Gesamtbesucherschaft interessante Erkenntnisse im Hinblick auf die Zielgruppenerreichung liefern. Dafür müssen aber nicht nur die Messegesellschaft und der Aussteller diese Besucherstrukturen erhoben haben. Vielmehr ist es zwingend notwendig, daß die erhobenen Daten vergleichbar sind. Hierzu bedarf es der Abstimmung zwischen Messegesellschaften und Ausstellern. ${ }^{353}$

Der Vergleich der beiden Strukturprofile ermöglicht dem Aussteller die Überprüfung der Effektivität seiner Zielgruppenansprache vor und während der Messe. Die Zahlen beider Auswertungen haben dann auch prognostischen Charakter, wenn davon ausgegangen werden kann, daß sich die Besucherstruktur bei der nächsten Messeveranstaltung nicht maßgeblich verändern wird.

Hier seien beispielhaft die Erhebungen der Deutschen Messe AG, Hannover, enwähnt, die in regelmäßigen Abständen die Ergebnisse der Besucherbefragungen ausgewählter Veranstaltungen in den Messe-Nachrichten Hannover sowie in gebundenen Publikationen veröffentlicht. Vgl. bspw. Deutsche Messe AG (Hrsg.), Das transparente Medium - Aussteller- und BesucherAnalysen Hannover Messe ‘94, Hannover 1994.

Der Begriff der Reichweite stellt die Messeveranstaltung in eine Analogie zum Bereich der Printund elektronischen (Radio- und TV-)Medien. Mit dem Reichweitenbegriff sind in Zusammenhang mit diesen Medien normative Festlegungen hinsichtlich der Ermittlung des Nutzeranteils eines bestimmten Mediums in einer fest umrissenen Grundgesamtheit verbunden, die im Rahmen von Messeveranstaltungen noch nicht entwickelt wurden. Darüber hinaus fällt auch die Bestimmung der Besuchergrundgesamtheiten von Messen - u. a. wegen der Probleme einer geographischen Abgrenzung des Einzugsbereichs - schwer. Vgl. hierzu Strothmann, K.-H., Messeforschung, in: Deutsche Werbewissenschaftliche Gesellschaft e.V. (Hrsg.), Messen als Marketing-Instrument, Bonn 1983, S. 104.

Auch hier existieren keine allgemeingültigen Erhebungsstandards, wenngleich diese seit langem auf seiten der Aussteller gefordert werden. Nicht zuletzt auch deshalb, um miteinander konkurrierende Messeveranstaltungen in ihrem Leistungsprofil vergleichen zu können. Vgl. hierzu Merbold, C., Strukturerhebungen noch die Ausnahme - Transparenz läßt weiter sehr zu wünschen übrig, in: HB, Nr. 235, vom 07.12.1983, S. 17; Schwenzner, J. M., Messen und Ausstellungen im Kreuzfeuer der Begriffe Reichweitenanalyse und Messeerfolgskontrolle, a.a.O., S. 10-20. 
Kritisch bleibt bezüglich der Besucherstatistiken der Veranstalter anzumerken, $\mathrm{da} ß$ sie nicht für jede Veranstaltung vorliegen. Sind sie indes vorhanden, werden sie nicht immer den Ausstellern zur Verfügung gestellt. Darüber hinaus kann jeder Veranstalter die Befragungsinhalte individuell festlegen. Damit wird nicht nur die Vergleichbarkeit der Leistungsdaten verschiedener Messeveranstaltungen erschwert, sondern es bedarf auch für den einzelnen Aussteller eines erheblichen Aufwands, seine eigenen Erhebungen an wechselnde Erhebungsstandards der Messegesellschaften anzupassen, um jeweils die Vergleichbarkeit der individuellen mit den Gesamtergebnissen herzustellen.

Insbesondere die Kritik im Hinblick auf die Datenvergleichbarkeit hat zu einem standardisierten Verfahren der Besucherstrukturermittlung beigetragen, das im folgenden kurz skizziert wird.

\section{- Auswertung des FKM-Besucherstrukturtests}

Die Gesellschaft zur freiwilligen Kontrolle von Messe- und Ausstellungszahlen (FKM) hat ein standardisiertes Konzept zur Ermittlung von Messebesucherstrukturdaten entwickelt, das auf allen Messeveranstaltungen zur Anwendung kommen kann und dessen korrekte Durchführung durch einen Wirtschaftsprüfer kontrolliert wird. ${ }^{354}$

Der AUMA veröffentlicht die von der FKM vorgeschriebenen Besucherstrukturdaten der untersuchten Messen in seinen jährlich erscheinenden Handbüchern "Messeplatz Deutschland" und "Regional", ebenso die FKM in ihrem Jahresbericht. $^{355}$

Aussteller können diese Daten als Grundlage bei Entscheidungen über Messebeteiligungen, aber auch zu Vergleichen der Besucherschaft mit der erreichten (ex-post) bzw. anvisierten (ex-ante) Zielgruppe heranziehen. Insgesamt

354 Zu den genauen Durchführungsvorschriften vgl. Gesellschaft zur freiwilligen Kontrolle von Messe- und Ausstellungszahlen (FKM) (Hrsg.), Satzung und Regeln, Köln 1994, S. 20-22. Ziel des FKM-Besucherstrukturtests ist die Vergleichbarkeit der auf verschiedenen Messeveranstaltungen ermittelten Daten zu Merkmalen und Verhalten der Besucher. Die FKM schreibt hierfür die zu erhebenden Strukturmerkmale vor. So sind im Rahmen von Fachbesucherstrukturtests die folgenden Informationen abzufragen: Regionale Herkunft (Inland: Bundesland; Ausland: Staatszugehörigkeit) Wirtschaftszweig, Betriebsgröße Berufliche Stellung, Entscheidungskompetenz, Aufgabenbereich im Unternehmen, Häufigkeit des Messebesuchs sowie Aufenthaltsdauer.

Vgl. Ausstellungs- und Messe-Ausschuss der Deutschen Wirtschaft e. V. (AUMA), (Hrsg.), Handbuch Messeplatz Deutschland '96, Köln 1995, S. 7 ff.; Gesellschaft zur freiwilligen Kontrolle von Messe- und Ausstellungszahlen (FKM) (Hrsg.). Bericht 1995, a.a.O., S. $8 \mathrm{ff}$. 
erlauben die Ergebnisse der FKM-geprüften Besucheranalysen den Vergleich verschiedener Messeveranstaltungen im Hinblick auf ihre Besucherschaft und häufig auch deren Verhalten und Motive. Auf Grundlage dieser Informationen kann ein Aussteller Schlüsse für die zukünftige Entwicklung der Messe ziehen. Schließlich ist es möglich, auf der Basis der Kenntnisse der Besucherstruktur (ex ante) Anhaltspunkte für die Ausgestaltung der Messebeteiligung, insb. die Festlegung der Messebeteiligungsziele und -aktivitäten zu gewinnen.

Dem steht allerdings entgegen, daß zahlreiche Messeveranstaltungen existieren, deren Besucherstruktur nicht nach diesem standardisierten Verfahren überprüft wird. ${ }^{356}$ Andererseits darf die Prognosekraft der Besucherstrukturdaten nicht überschätzt werden. Angesichts der Dynamik des Messemarktes, auf dem ständig neue Messeveranstaltungen entstehen, vorhandene Messekonzepte verändert oder bislang integrierte Bereiche von Messeveranstaltungen zu eigenständigen Messen weiterentwickelt werden, erscheint die Relevanz vergangenheitsorientierter Daten für zukunftsorientierte Messebeteiligungsentscheidungen in vielen Fällen zunehmend fraglich. ${ }^{357}$

\section{- Umsatzkontrolle}

Für viele Aussteller besitzen Verkaufsziele eine hohe Priorität im Zielsystem ihrer Messebeteiligungen - und dies gilt nicht nur für diejenigen Branchen, in denen heute die Orderfunktion von Messen ein großes Bedeutungsgewicht hat. ${ }^{358}$ Daher liegt es nahe, eine Analyse der Messeumsätze vorzunehmen und deren Soll-lst-Abweichungen als Indikator für den Erfolg der Messebeteiligung heranzuziehen. Dabei umfaßt der Messeumsatz i. d. R. nicht nur alle unmittelbar auf der Messeveranstaltung abgeschlossenen Verkäufe und Aufträge, sondern auch die mittelbaren Messeaufträge, die maßgeblich auf der Messe initiiert wurden und erst später entgegengenommen werden können. ${ }^{359}$

356 Vgl. Rost. D., Messetransparenz - ein Imperativ, a.a.O., S. 30-32.

Eine umfassende Kritik von Besucherstrukturtests aus Sicht der Aussteller findet sich bei Funke. K., Messeentscheidungen. Handlungsalternativen und Informationsbedarf, a.a.O., S. $326 \mathrm{ff}$. Messebeteiligungen, die in Kap. B.1.2 dieser Arbeit wiedergegeben werden. 
Doch ergibt sich hierbei ein doppeltes Zurechnungsproblem. Zum einen ist fraglich, ob die Verkäufe auf der Messe sowie die dort erhaltenen Aufträge tatsächlich den Messeaktivitäten zurechenbar oder ursächlich eher auf zeitlich vor der Messe liegende Verkaufsaktivitäten des Unternehmens zurückzuführen sind. Zum anderen stellt sich die Frage der Festlegung des Zeitintervalls in der Nach-Messe-Phase, in dem die eingehenden Aufträge noch den Auswirkungen der Messebeteiligung zugeschrieben werden können. Die ungefähre Zurechnung der Käufe zum Messeumsatz ist nur durch eine Befragung der Kunden hinsichtlich ihrer Kaufentscheidungsprozesse möglich. Überschneidungsfreie Zurechnung wird allerdings um so schwieriger, je weiter die Kaufhandlung zeitlich der Messeveranstaltung nachgelagert ist. ${ }^{360}$

Die Tatsache, daß das Kriterium "Messeumsatz" als alleiniger Erfolgsindikator einer Messebeteiligung nur einen geringen Anspruch auf Repräsentativität hat, wurde schon früh aufgrund des Wandels der Messen zu informations- und kommunikationsorientierten Veranstaltungen erkannt und führt daher zu einer eher kritischen Beurteilung dieses Erfolgskriteriums. ${ }^{361}$ Der Umsatz einer Messebeteiligung sollte daher immer nur eines von mehreren Kriterien zur Messung des Erfolgs sein, und er ist gänzlich ungeeignet zur Beurteilung des außerökonomischen Erfolgs von Messebeteiligungen.

\section{- Kennziffernanalyse}

Die Kennziffernanalyse, auch Kennzahlenanalyse genannt, dient zum Vergleich von Beteiligungen an verschiedenen Messeveranstaltungen und alternativer Marketinginstrumente hinsichtlich ihrer Kosten-Nutzen-Relation. ${ }^{362}$ Sie stellt insofern eine Erweiterung der Umsatzanalyse dar, als sie neben dem Umsatz weitere Kriterien zur Erfolgsmessung heranzieht. Neben den ökonomischen Größen können auch psychographische Erfolgskriterien in die Analyse eingehen.

Eine umfassende Möglichkeit zur Berechnung des Messe- und besonders des messeinduzierten Nachmesseumsatzes stellt Funke mit dem "modellgestützten Verfahren zur Ermittlung auftragsbezogener Messewirkungen“ vor. Allerdings besitzt dieses Modell nur wenig praktische Relevanz, da die getroffenen Annahmen des Modells hinsichtlich des Kaufentscheidungsprozesses von Fachbesuchern sowie die vorausgesetzten Informationen in der Messepraxis nicht vorzufinden sind. Vgl. Funke, K., Messeentscheidungen. Handlungsalternativen und Informationsbedarf, a.a.O., S. $354 \mathrm{ff}$.

Schwenzner, J. M. Andersen, E., Der Messe-Test, in: Wagner, K. (Hrsg.), Allgemeines Statistisches Archiv, Bd. 39, München 1955, S. 25-30.

362 Vgl. Haeberle, K. E., Erfolg auf Messen und Ausstellungen - Handbuch für Teilnahme, Organisation, Gestaltung, Technik, a.a.O., S. 271; Wedel, P. Graf von, Messen - vom Markt zum Marketing, Frankfurt 1977 S 47 . 
Kennziffern werden dabei zunächst eingesetzt, um die Wirtschaftlichkeit einer Messebeteiligung nachzuweisen. So sind bspw. die sog. Messekoeffizienten ein $\mathrm{Ma}$ der Wirtschaftlichkeit von Verkaufsbemühungen am Messestand. Die Koeffizienten werden aus dem Verhältnis des Messe- und Nachmesseumsatzes zu den Messekosten gebildet. Sie können damit auf eine einzelne Veranstaltung (I) oder auf eine bestimmte Zeitspanne (II) (Z. B. ein Jahr) bezogen werden. ${ }^{363}$ Vornehmlich unter dem Stichwort Effizienzanalysen werden in Analogie zu dem aus der klassischen Werbung stammenden "Tausenderpreis" Kennzahlen gebildet. ${ }^{364}$

Der zentrale Nachteil der bisher genannten Kennziffern liegt darin, daß sie unterschiedliche Besucherqualitäten nicht zu berücksichtigen vermögen. Weiterhin schließen sie die Möglichkeit der ausschließlichen oder fukussierten Bearbeitung einer bestimmten sowie der differenzierten Bearbeitung mehrerer Zielgruppen aus. Eine weitere Gruppe der Kennzahlen stellt mithin den Zielgruppenbezug her. ${ }^{365}$

Aussteller bedienen sich z. T. weiterer Kennzahlen. Den Messekosten werden bspw. die Anzahl der Gesprächskontakte, die Quadratmeterzahl des Messestandes oder die Zahl der Standmitarbeiter gegenübergestellt.

Wesentliche Voraussetzung einer effektiven Kennziffernanalyse ist neben der korrekten Ermittlung der relevanten Größen (z. B. Umsatz, Messekosten, Kontakte etc.) eine über mehrere Messen und Veranstaltungstermine hinweg konstante und konsequente Anwendung einheitlicher Methoden der Datener-

363 Z. B. Messekoeffizient I= Wert der Abschlüsse auf einer Messe/Kosten der Messebeteiligung oder Messekoeffizient II= Jahresumsatz des Unternehmens/jährliche Kosten der Messebeteiligungen. Vgl. Blohm, H., Messen und Ausstellungen in der Schweizer Maschinenindustrie, a.a.O., S. 135. Haeberle schlägt zwei weitere Berechnungsmöglichkeiten der Wirtschaftlichkeit vor, die Vergleichskennzahlen mit anderen Marketinginstrumenten darstellen: $z$. B. den sales efficiency quotient, der die Kosten der Auftragserlangung auf der Messe in ein Verhältnis zu den Kosten der üblichen Auftragserlangung setzt. Oder den project efficiency quotient, der die Kosten für den Standbesuch von Interessenten mit den Kosten für den üblichen Besuch vergleicht.

Z. B. Standkontaktpreis $=$ Messekosten / Anzahl Kontakte am Messestand oder Messebesucherpreis $=$ Messekosten $/$ Messebesucher $\times 1000$. Vgl. Cunningham, M. T., White, J. G., The Role of Exhibitions in Industrial Marketing - An Evaluation of the International Machine Tool Exhibition, a.a.O., S. 239.

Als Beispiele können daher angeführt werden: Fachbesucherpreis=Messekosten/Anzahl Fachbesucher auf der Messe oder Zielgruppenpreis = Zielgruppenspezifische Messekosten / Anzahl Zielgruppenmitglieder am Messestand. Vgl. Naumann, C., Ist es eine Messe wert? Den Messeerfolg organisieren und kontrollieren, in: BddW, Nr. 170 vom 06.09.1982, S. 7. 
mittlung, um auf diese Weise eine Vergleichbarkeit der Ergebnisse der Kennziffernanalyse zu gewährleisten.

Als zentrale Kritik an Kennziffernanalysen ist allerdings anzuführen, daß sie vielfach den Nutzen von Messebeteiligungen an Größen festmachen, die lediglich als Wirkungsvoraussetzungen und nicht als Erfolgswirkungen i. S. v. Zielerreichungsgraden zu bezeichnen sind und somit nur begrenzt zur Beurteilung des Erfolgs einer Messebeteiligung herangezogen werden können. Darüber hinaus erfolgt bei der Interpretation der Kennzahlen auch keine Berücksichtigung qualitativer Aspekte der Begegnungen zwischen Ausstellern und Nachfragern. ${ }^{366}$ Diese zuletzt genannte Kritik hat zur Entwicklung der Messekontaktwertrechnung geführt.

\section{- Messekontaktwertrechnung}

Die Messekontaktwertrechnung verfolgt das Ziel einer quantitativen und qualitativen Erfassung aller Besucherkontakte auf dem Messestand. Neben einer zahlenmäßigen Erfassung der Standbesucherkontakte beinhaltet diese Methode der Erfolgskontrolle von Messebeteiligungen eine qualitative Unterscheidung der Standbesucher nach vier Gesichtspunkten: ${ }^{367}$

- Dauer der geschäftlichen Beziehung (Neu- oder Altkunde)

- Herkunft (Inland oder Ausland)

- Kompetenz (Geschäftsleitung oder Mitarbeiter)

- Orderverhalten (mit Auftrag oder ohne Auftrag)

Gemäß ihrer Bedeutung für den Aussteller werden den einzelnen Besuchern oder Besuchergruppen sog. Kontaktwertpunkte zugeteilt. Die Gewichtung der einzelnen Parameter erfolgt im Vorfeld einer Messebeteiligung unter Berücksichtigung der jeweils durch das Unternehmen anvisierten Zielgruppen. Zur Erfassung der Gesprächspartner werden i. d. R. Gesprächsprotokolle verwendet. Mittels der vorab festgelegten Kontaktwertpunkte wird nun die Bewertung der Gespräche vorgenommen. Ein Vergleich der gewonnenen Daten mit den Soll-Vorstellungen sowie die Betrachtung von Vergleichsdaten mehrerer Messeveranstaltungen erlauben Aussagen über die Rentabilität der Messebeteili- 
gungen, wenn den Kontaktwerten z. B. die Messekosten gegenübergestellt werden. ${ }^{368}$ Denkbar sind auch Vergleiche mit den von der Gesellschaft zur freiwilligen Kontrolle von Messe- und Ausstellungszahlen (FKM) erhobenen Daten zur Besucherstrukturanalyse (vgl. "Auswertung des FKM-Besucherstrukturtests" in diesem Kapitel). Dadurch läßt sich $u$. a. feststellen, ob es gelungen ist, die angestrebte Zielgruppe auf der Messe effizient zu erreichen.

Kritisch bleibt bei diesem Verfahren anzumerken, daß unterschiedliche Auftragsvolumina sowie die Ergebnisse des Nachmessegeschäfts unberücksichtigt bleiben. Dennoch ist es eine sehr flexible Methode zur Überprüfung der Erreichung von Kontaktzielen der Aussteller, die darüber hinaus einen sehr einfachen und nachvollziehbaren Aufbau aufweist. Allerdings kommt dieses Verfahren nicht ohne die Kombination mit anderen Methoden der Erfolgskontrolle aus (Kontaktzählung, Gesprächsprotokoll).

Im Rahmen der Erläuterungen zu den letztgenannten Methoden der Kontrolle des Erfolgs von Messebeteiligungen wurde deutlich, daß den Messekosten als Bezugsgröße für die Ergebnisse der Zielerreichung ein wesentlicher Stellenwert zukommt. Die Bewertung von Messebeteiligungen erfolgt damit stets auch unter Budget-Aspekten und umfaßt somit die Betrachtung der Kosten von Messebeteiligungen.

Die konsequente und kontinuierliche Feststellung der Messekosten mit einem einheitlichen Kostenschema ermöglicht im Rahmen der Budgetkontrolle nicht nur die Kostenbewertung einer einzelnen Messebeteiligung i. S. eines Soll-IstVergleichs. Vielmehr schafft sie die Voraussetzung für einen Kostenvergleich der Beteiligungen verschiedener Jahre sowie verschiedener Veranstaltungen. ${ }^{369}$

Es muß allerdings betont werden, daß die Budget- bzw. die dahinter stehende Kostenkontrolle i. e. S. keine Methoden der Erfolgskontrolle darstellen, sondern lediglich eine notwendige Voraussetzung für die Durchführung von Kosten-NutzenAnalysen bilden und damit eine Grundlage für Effizienz- und Wirtschaftlichkeitsüberlegungen bei Messebeteiligungen legen.

368 Vgl. Naumann, C., Was war die letzte Messe wert? - Kontaktwertmessung nach Punkten, a.a.O., S. 17.

369 Auf eine ausführliche Darstellung der Durchführung einer Budgetkontrolle soll an dieser Stelle verzichtet werden, da die wesentlichen Aspekte der Erfassung der Messekosten bereits in Kapitel A.2 dieser Arbeit gewürdigt wurden. 


\subsection{Verwendung der Ergebnisse der Erfolgskontrolle}

Eine Steigerung der Messebeteiligungseffizienz ist nur dann möglich, wenn aus den ermittelten Kontrollergebnissen auch konkrete Handlungskonsequenzen abgeleitet werden können. ${ }^{370}$ Diesem Aspekt wird allerdings in der Literatur zum Management von Messebeteiligungen bislang keine Aufmerksamkeit geschenkt.

Die Untersuchung der Auswirkungen der Erfolgskontrolle auf Planung und Durchführung von Messebeteiligungen verfolgt im Rahmen dieser Arbeit daher zwei grundsätzlich unterschiedliche Analyseziele. Zum einen sind zunächst die Adressaten der Kontrollergebnisse im Unternehmen zu betrachten. Zum anderen sind auch diejenigen Entscheidungsbereiche bzw. -felder zu skizzieren, innerhalb derer die Aussteller Konsequenzen aus den Ergebnissen der Messeerfolgskontrolle ziehen.

Hierbei sollen hinsichtlich der potentiellen Adressaten die Bereiche

- Unternehmensleitung und

- Absatzorganisation sowie

- die am Messestand beteiligten Mitarbeiter und darüber hinaus

- Mitarbeiter, die nicht aktiv an der Messebeteiligung mitgewirkt haben, aber dennoch von ihren Ergebnissen tangiert werden,

als potentielle Zielgruppen für die Präsentation der Messebeteiligungsergebnisse unterschieden werden. Diese Differenzierung erscheint sinnvoll, um auf der einen Seite den Stellenwert der Messebeteiligung und ihrer Ergebnisse im hierarchischen System des Ausstellers festzustellen sowie auf der anderen Seite die Diffusion der Messeergebnisse im ausstellenden Unternehmen zu analysieren.

Mit Blick auf die Entscheidungsfelder sind als relevante Handlungsoptionen hier

- die Anpassung des gesamten Messeengagements,

- die Entscheidung über die Weiterführung der jeweiligen Messebeteiligung,

- die Diskussion mit den Messegesellschaften über das Veranstaltungskonzept,

370 Hiermit sind vor allem die Führungs- und Steuerungsaufgaben des Management angesprochen, dessen Aufgabe es ist, die Informationen der Messeerfolgskontrolle in Korrekturmaßnahmen zu überführen. Vgl. Staehle, W. H., Management: eine verhaltenswissenschaftliche Perspektive, a.a.O., S. 510 f. 
- die Überarbeitung der Messestandkonzeption sowie

- die Bewertung und Auswahl der Standmitarbeiter

zu nennen. Durch diese Aufspaltung in die genannten Entscheidungsfelder sind einerseits zumindest die Richtungen der Handlungskonsequenzen ansatzweise zu erfassen. Andererseits bedarf es aber darüber hinaus auch der Ermittlung der Verwendungsintensität der Ergebnisse der Messeerfolgskontrolle in jedem dieser Entscheidungsfelder, um die Handlungsschwerpunkte zu erfassen und damit Aussagen über die konkrete Reichweite der Informationen der Erfolgskontrolle ableiten zu können.

Nachdem bereits in den Ausführungen dieses Kapitels sowie durch die Vielfalt der in Kapitel C. 1.1 aufgeführten messebezogenen Aktivitätsbereiche die Evidenz organisatorischer Fragestellungen im Rahmen des Management von Messebeteiligungen deutlich wurde, wird im folgenden diese Anforderung aufgenommen und einer Analyse möglicher Ausgestaltungsoptionen unterzogen.

\subsection{Messebeteiligungsorganisation}

In der Literatur wird in vielfältiger Weise auf die Notwendigkeit der Abstimmung zwischen strategischen und organisatorischen Managementkonzepten hingewiesen. ${ }^{371}$ Dabei wird insbesondere im Marketing der Erfolg von Strategiekonzepten eng mit der Effizienz ihrer organisationalen Umsetzung verbunden. ${ }^{372}$ I. d. S. läßt sich der Grundgedanke „Structure follows Strategy ${ }^{\text {‘373 }}$ auch auf die Beteiligung an Messeveranstaltungen übertragen, da mit den messespezifischen Grundhaltungen strategische Verhaltenspläne vorgegeben werden, die im Rahmen organisatorischer Umsetzungskonzepte zu verwirklichen sind. In Anlehnung an Hofer und

371 Vgl. Frese, E., Grundlagen der Organisation: Konzept, Prinzipien, Strukturen, 5. Aufl., Wiesbaden 1993, S. 295 ff.; Kieser, A. Kubicek. H., Organisation, 3. Aufl., Berlin, New York 1992, S. 225 ff.; Meffert. H., Marketing-Management: Analyse - Strategie - Implementierung, a.a.O., S. $287 \mathrm{ff}$.

Vgl. Hilker, J., Marketingimplementierung: Grundlagen und Umsetzung am Beispiel ostdeutscher Unternehmen, Wiesbaden 1993, S. 47 ff.

373 Diese von Chandler erstmalig 1962 formulierte These bezog sich ursprünglich auf den Zusammenhang zwischen Diversifikationsstrategien und dem Aufkommen divisionalisierter Organisationsformen in Unternehmen. Vgl. Chandler. A. D., Strategy and Structure. Chapters in the History of Industrial Enterprise, Cambridge 1962. Mittlerweile liegen verschiedene Ansätze vor, die den Zusammenhang zwischen der organisationalen Struktur eines Unternehmens bzw. einer Unternehmenseinheit und verschiedenen Strategiedimensionen untersuchen. Vgl. hierzu Bühner, R., Strategie und Organisation, Wiesbaden 1985, insb. S. $14 \mathrm{ff}$. 
Schendel äußert sich die Verfolgung einer Strategie u. a. im grundlegenden Muster des Ressourceneinsatzes. ${ }^{374} \mathrm{Um}$ zu analysieren, ob und in welcher Weise die messespezifischen Grundhaltungstypen auf unterschiedliche Ressourcen zurückgreifen, sollen diesbezüglich zwei zentrale Aspekte der Durchführung von Messebeteiligungen untersucht werden: Zum einen der Grad der Integration von externen Dienstleistungsspezialisten im Rahmen der Vorbereitung und Durchführung der Messebeteiligung (hier bezeichnet als der "Externalisierungsgrad“) und zum anderen der Grad der Entscheidungskompetenz der mit dem Management von Messebeteiligungen betrauten Institution in der Organisation des ausstellenden Unternehmens (hier bezeichnet als "Autonomiegrad“). ${ }^{375} \mathrm{Im}$ folgenden sollen die für die empirische Untersuchung relevanten Aspekte kurz erläutert werden.

\subsection{Externalisierungsgrad}

Im Rahmen ihres Messemarketing stehen Aussteller bezüglich der organisatorischen Ausgestaltung von Messebeteiligungen vielfach vor einer "Make or Buy"Entscheidung. ${ }^{376}$ Es existiert eine Vielzahl von Dienstleistungsspezialisten, die Ausstellern die Planung und/oder organisatorische Abwicklung der Messebeteiligung ganz oder teilweise abnehmen. Häufig externalisierte Bereiche bilden heute

374 Vgl. Hofer, C. W. Schendel, D., Strategy Formulation: Analytical Concepts, St. Paul u.a. 1978, S. $45 \mathrm{ft}$.

375 Frese verwendet die Begriffe Entscheidungskompetenz und -autonomie nicht synonym, sondern weist darauf hin, daß erstere stark durch inhaltliche Kompetenzen und letztere vor allem durch den vorhandenen Kompetenzspielraum gekennzeichnet sind. Vgl. Frese, E., Grundlagen der Organisation: Konzept, Prinzipien, Strukturen, 5. Aufl., a.a.O., S. 43 ff. Da beide Einflüsse in die Betrachtung dieser Arbeit eingehen, soll der allgemeingültigere Begriff der Entscheidungsautonomie zur Systematisierung der organisatorischen Gestaltung einer Messebeteiligung herangezogen werden.

376 Unter dem Begriff "Make-or-Buy" ist die Entscheidung über Eigenerstellung oder Fremdvergabe von Produkten und Leistungen zu verstehen. Zumeist wird die Frage nach "Make-or-Buy" nur mit dem Fertigungsbereich in Verbindung gebracht. Vgl. hierzu bspw. Ramser, H.-J., Eigenerstellung oder Fremdbezug von Leistungen, in: Kern, W. (Hrsg.), Handwörterbuch der Produktion, Stuttgart 1979, Sp. 435-450. Aber auch im Marketing existiert ein breites Spektrum möglicher Make-or-Buy-Fragestellungen. Vgl. Fischer, M., Make-or-Buy-Entscheidungen im Marketing, Wiesbaden 1993, S. 3 ff.; Nieschlag. R., Dichtl, E., Hörschgen. H., Marketing, 15. Aufl., Berlin 1988, S. 211 ff. sowie die dort jeweils zitierte bzw. zusammengestellte Literatur. Die Fragestellungen hinsichtlich der organisatorischen Ausgestaltung von Messebeteiligungen sowie der Durchführung bzw. Übernahme einzelner Teilleistungen zur Realisation des angestrebten Messeauftritts des Ausstellers können diesem Entscheidungsspektrum explizit zugeordnet werden. 
bereits Standbau, Logistik, Catering sowie Standservices. ${ }^{377}$ Insbesondere mit Blick auf die Konzeption und Durchführung von Maßnahmen zur Erfolgskontrolle von Messebeteiligungen sei auf die wachsende Bedeutung von Dienstleistungsunternehmen hingewiesen. ${ }^{378}$

Auch die Messegesellschaften weisen ein breites ausstellergerichtetes Dienstleistungsspektrum auf. Das Service-Angebot der Messeveranstalter umfaßt neben der Schaffung der notwendigen insfrastrukturellen Voraussetzungen für die Durchführung einer Messe u. a. Mietmessestände, Standreinigungs- und -bewachungsdienste, Dekorations- und Pressedienste sowie die Erstellung von Werbemitteln und die Bereiche Dolmetscher- sowie Fotoservice bis hin zur Organisation von Begleitveranstaltungen, wie z. B. Pressekonferenzen oder Fachkongresse. $^{379}$

Aus Sicht der Aussteller weist die Nutzung externer Dienstleister im Gegensatz zur eigenverantwortlichen Organisation der Messebeteiligung sowohl Vor- als auch Nachteile auf, die gegeneinander abzuwägen sind. Wesentliche Vorteile sind in der Nutzung des spezifischen Know-how eines Spezialisten sowie in der Möglichkeit zur Konzentration auf die Kernbereiche der Messebeteiligung durch den Aussteller selbst zu sehen. Als nachteilig können sich bei der Inanspruchnahme externer

377 Vgl. Roth, G. D., Messen und Ausstellungen verkaufswirksam planen und durchführen, a.a.O.,

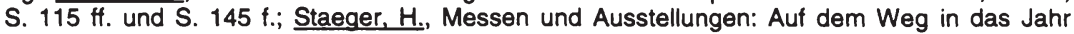
2000 - Konsequenzen für Aussteller, Veranstalter und insbesondere Messebauer, in: Marketing-Journal, Heft 1, 1989, S. 56; Winnen, R., Entscheidungshilfen für den Mittelstand Die Bedeutung der Messeberatung nimmt zu, in: BddW, Nr. 65, vom 04.04.1989, S. 7.

Winnen sieht hier einen der wichtigsten Einsatzbereiche für Messe-Marktforschungsgesellschaften bzw. Messe-Consulting-Unternehmen. Vgl. Winnen, R., Entscheidungshilfen für den Mittelstand - Die Bedeutung der Messeberatung nimmt zu, in: Küffner, G. Mortsiefer, J. (Hrsg.), Messeplatz Europa, Messe als Bestandteil des betrieblichen Marketings, Frankfurt a. M. 1990, S. $146 \mathrm{ff}$.

Taeger unterscheidet in diesem Zusammenhang in unmittelbare und mittelbare Leistungen der Messegesellschaften, wobei die unmittelbaren Leistungen sich direkt auf Aussteller und Besucher beziehen, während mittelbare Leistungen auf die Schaffung der notwendigen Rahmenbedingungen (z. B. Parkraum, Anbindung an öffentlichen Personen-Nahverkehr etc.) ausgerichtet sind. Vgl. Taeger, M., Messemarketing - Marketing-Mix von Messegesellschaften unter Berücksichtigung wettbewerbspolitischer Rahmenbedingungen, Göttingen 1993, S. $139 \mathrm{ff}$. Allg. zum Service-Angebot ausgewählter Messegesellschaften vgl. die Beitragsreihe "Services von Messegesellschaften", die im Marketing Journal seit Heft 3, 1986 in unregelmäßiger Abfolge erschienen ist. Z. B. o.V., Services der Messegesellschaften, in: Marketing Journal, Heft 1, 1991, S. 61. 
Dienstleister aber der erhöhte Koordinationsaufwand für den Aussteller sowie insgesamt höhere Messebeteiligungskosten ${ }^{380}$ auswirken. $^{381}$

\subsection{Autonomiegrad}

Hinsichtlich der Planung und Durchführung von Messebeteiligungen in ausstellenden Unternehmen sind grundsätzlich drei Formen der organisatorischen Umsetzung zu berücksichtigen, die sich hauptsächlich durch die Breite ihres Aufgabengebietes und den Spielraum ihrer Entscheidungskompetenzen unterscheiden.

Dabei ist in einer ersten Option davon auszugehen, daß die Messebeteiligung innerhalb einer Organisationsstruktur geplant und umgesetzt wird, die keine besondere messespezifische Stelle bzw. Abteilung aufweist. Es ist anzunehmen, daß sich in diesem Fall projektähnliche interdisziplinäre Teams zusammenfinden, die bereichsübergreifend die Ausgestaltung der Messebeteiligung vornehmen. Als Teammitglieder sind Mitglieder der Unternehmensleitung, Mitarbeiter aus der Absatzorganisation sowie - sofern vorhanden - aus dem Forschungs- und Entwicklungsbereich denkbar. $^{382}$

Im Gegensatz dazu besteht die Möglichkeit der Einrichtung einer zentralen Messeabteilung, die als in der Unternehmensorganisation etablierter Spezialbereich die Aufgaben des Vorschlags sowie der Festlegung des Messeprogramms und der -aktivitäten für alle messerelevanten Unternehmensbereiche übernimmt. Von zentraler Bedeutung hierbei ist, daß der Messeabteilung i. d. R. sowohl die Budgetals auch die Ergebnisverantwortung für jede Messebeteiligung übertragen wird. ${ }^{383}$

\footnotetext{
380 Kostenerhöhungen durch die Inanspruchnahme von externen Dienstleistungen können insbesondere aus dem angeführten höheren Koordinations- und Informationsaufwand sowie der Entstehung bzw. Erhöhung von Suchkosten resultieren. Vgl. hierzu allg. Buzzell, R. D., Is Vertical Integration Profitable?, in: HBR, 61. Jg., January-February 1983, S. 92-102.

Vgl. Fischer, M., Make-or-Buy-Entscheidungen im Marketing, a.a.O., S. $23 \mathrm{ff}$.

Vgl. hierzu die Ergebnisse einer empirischen Untersuchung von Tanner und Chonko, die diese Form der Organisation von Messebeteiligungen aus Ausstellersicht als die weitaus häufigste erkennen. Vgl. Tanner, J. F. Chonko, L. B., Trade Show Objectives, Management, and Staffing Practices, a.a.O., S. 260 f.; Williams, J. D. Gopalakrishna, S. Cox, J. M., Trade Show Guidelines for Smaller Firms, in: IMM, Vol. 22, 1993, S. 316 f.
}

383 Vgl. Kijewski, V., Yoon, E., Young, G., How Exhibitors Select Trade Shows, in: IMM, Vol. 22, 1993, S. 296 f. 
Als dritte Organisationsform soll die Einrichtung einer unternehmensinternen (Messe-) Serviceorganisation in die Betrachtung einbezogen werden. Diese plant auf Anweisung der Unternehmensleitung bzw. verschiedener messerelevanter Unternehmensbereiche die operativen Tätigkeiten im Zusammenhang mit den Messebeteiligungen und ist in der Hauptsache mit Fragen der Umsetzung einer vorgegebenen Messebeteiligungskonzeption betraut. Wesentlich hierbei ist, daß die Budget- und Ergebnisverantwortung der Messebeteiligungen nicht bei der Messe-Serviceorganisation liegen, sondern bei den Unternehmensbereichen, die sie beauftragen. ${ }^{384}$

Nachdem die relevanten Maßnahmen zur Ausgestaltung einer Messebeteiligung theoretisch dargestellt worden sind, soll im folgenden Kapitel ihre empirische Relevanz für die Grundhaltungstypen einer Überprüfung unterzogen werden.

384 Vgl. Mortsiefer, J., Messen und Ausstellungen als Mittel der Absatzpolitik mittelständischer Herstellerbetriebe, a.a.O., S. $154 \mathrm{ff}$. und $167 \mathrm{ff}$. 


\section{Empirische Analyse der Maßnahmen zur Ausgestaltung von Messebetei- ligungen}

Im Rahmen der nachfolgenden empirischen Untersuchung der Maßnahmen zur Ausgestaltung von Messebeteiligungen werden analog zur theoretisch abgeleiteten Systematik die grundhaltungstypenspezifischen Besonderheiten dargestellt. Darüber hinaus wird auch der jeweils erreichte Messebeteiligungserfolg analysiert. $^{385}$

Die Analyse der Unterschiede hinsichtlich der Ausgestaltung und Erfolgswirkungen von Messebeteiligungen zwischen den fünf empirisch ermittelten Grundhaltungstypen erfolgt auf der Basis von Mittelwertvergleichen. ${ }^{386}$ Als Testverfahren zur Überprüfung der Signifikanz von Mittelwertunterschieden wird die einfaktorielle Varianzanalyse eingesetzt, die mit dem F-Wert ein Kriterium für die Globalbeurteilung von Mittelwertunterschieden zur Verfügung stellt. ${ }^{387} \mathrm{Um}$ zusätzliche Aussagen darüber zu treffen, zwischen welchen Grundhaltungstypen signifikante Unterschiede hinsichtlich der Ausgestaltungsvariablen bestehen, sind multiple Mittelwertvergleichstests notwendig. Hierzu wird auf das Testverfahren von Scheffé zurückgegriffen. ${ }^{388}$ Es wird in der Literatur vor allem deshalb vorgeschlagen, da es auch bei ungleichen Gruppenumfängen zu verläßlichen Ergebnissen führt.

385 Hinsichtlich der den Untersuchungen zugrunde liegenden Daten ist darauf hinzuweisen, daß zur Beschreibung der Ausgestaltungsformen und Erfolgswirkungen von Messebeteiligungen ausschließlich subjektive Einschätzungen der "Messebeteiligungs-Entscheider" erhoben worden sind. Um Meßfehler hier so weit wie möglich zu reduzieren und eine Vergleichbarkeit der Antworten zu gewährleisten, wurden bei der Variablenoperationalisierung vielfach beispielhafte Umschreibungen abstrakter Sachverhalte zu deren Erläuterung mit angegeben. Vgl. hierzu den Fragebogen im Anhang dieser Arbeit.

386 Signifikante Mittelwertunterschiede können dabei als Indikator dafür angesehen werden, daß die messespezifischen Grundhaltungen einen Erklärungsbeitrag für Ausgestaltungs- und Erfolgsunterschiede bei Messebeteiligungen leisten. Dabei ist allerdings zu berücksichtigen, daß varianzanalytische Verfahren strenggenommen nur Korrelationsbeziehungen zwischen Variablen offenlegen, ohne allerdings direkt Auskunft über die Einfluß- und Wirkungsrichtung, $d$. h. die Kausalität der Beziehung, zu geben. Vgl. Diehl, J. M., Varianzanalyse, Frankfurt a. M. 1977 , S. $30 \mathrm{ff}$.

Der F-Test gibt bei mehreren Mittelwerten Auskunft darüber, ob sich die Mittelwerte in ihrer Gesamtheit signifikant unterscheiden, nicht aber darüber, zwischen welchen einzelnen Mittelwerten signifikante Unterschiede bestehen. Vgl. Sachs, L., Angewandte Statistik: Planung und Auswertung, Methoden und Modelle, 4. Aufl., Berlin, Heidelberg, New York 1974, S. 205 ff.; Diehl, J. M., Varianzanalyse, a.a.O., S. 39 f.

Vgl. Bosch, K., Statistik-Taschenbuch, München, Wien 1992, S. 507 f.; Schubö, W. et al., SPSS: Handbuch der Programmversionen 4.0 und SPSS-X 3.0, a.a.O., S. 503 ff.; Norusis, M. J., SPSS/PC + Statistics 4.0, Chicago 1990, S. B-27 f. 


\subsection{Analyse der messebezogenen Aktivitäten}

\subsection{Aktivitäten in der Vor-Messe-Phase}

Die Erfassung der Aktivitätsschwerpunkte in der Vor-Messe-Phase wurde mit zehn Variablen operationalisiert. ${ }^{389}$ Tab. 7 enthält den Mittelwertvergleichstest für die fünf messespezifischen Grundhaltungstypen. Mit Blick auf die Werte des F-Tests wird zunächst deutlich, daß bei einem Signifikanzniveau von $\alpha=0,1$ mit der Ausnahme einer Variablen von signifikanten Mittelwertunterschiede zwischen den fünf Grundhaltungstypen ausgegangen werden kann.

In der oberen Hälfte der Tabelle sind die Abweichungen der Grundhaltungstypen vom Gesamtmittelwert der Stichprobe hinsichtlich der Vor-Messe-Aktivitäten ausgewiesen. Mit Blick auf das vorliegende Mittelwertprofil der Gesamtstichprobe zeigt sich zunächst der allgemein hohe Stellenwert der Formen der persönlichen Ansprache von potentiellen Messestandbesuchern ${ }^{390}$, während die unpersönliche, auf eine größere Breitenwirkung abzielende Kommunikation deutlich in den Hintergrund tritt. Als Ausnahme ist hierbei der Eintrag im Messekatalog zu bewerten, der heute allerdings als nahezu obligatorisch anzusehen ist, da dieser Eintrag i. d. R. gleichzeitig mit der Anmeldung des Ausstellers zur Messe erfolgt - sofern der Veranstalter einen Messekatalog erstellt. ${ }^{391}$

Erwähnenswert erscheinen ebenfalls die für alle befragten Aussteller gleichermaßen geltende geringe Bedeutung der Standpersonalschulung durch externe Trainer ( $M W=1,74)$ sowie das deutlich höhere Aktivitätsniveau bei Schulungsmaßnahmen durch ausstellerinterne Führungskräfte $(M W=3,46) .{ }^{392}$

389 Vgl. zur Operationalisierung der Variablen die Frage 19 (Vor-Messe-Phase) des Fragebogens im Anhang der Arbeit.

390 Hierbei sind insbesondere die Mittelwerte der Maßnahmen "Direct Mails an aktuelle Kunden" $(4,27)$, "Direct Mails an potentielle Kunden" $(3,98)$ und "Terminabsprachen durch den Außendienst" $(3,91)$ zu nennen, die deutlich machen, daß diese Instrumente häufig bzw. regelmäßig eingesetzt werden.

Das Instrument „Eintrag im Messekatalog" weist mit einem Mittelwert von 4,85 die insgesamt höchste Einsatzintensität aller Instrumente auf. Für alle anderen - nicht obligatorischen Maßnahmen der unpersönlichen Kommunikation ergeben sich allerdings Mittelwerte, die zwischen 2,57 (Anzeigen im Messekatalog) und 3,25 (Messevorberichte für die Presse) liegen, so daß von eher geringen Aktivitätsniveaus auszugehen ist. 


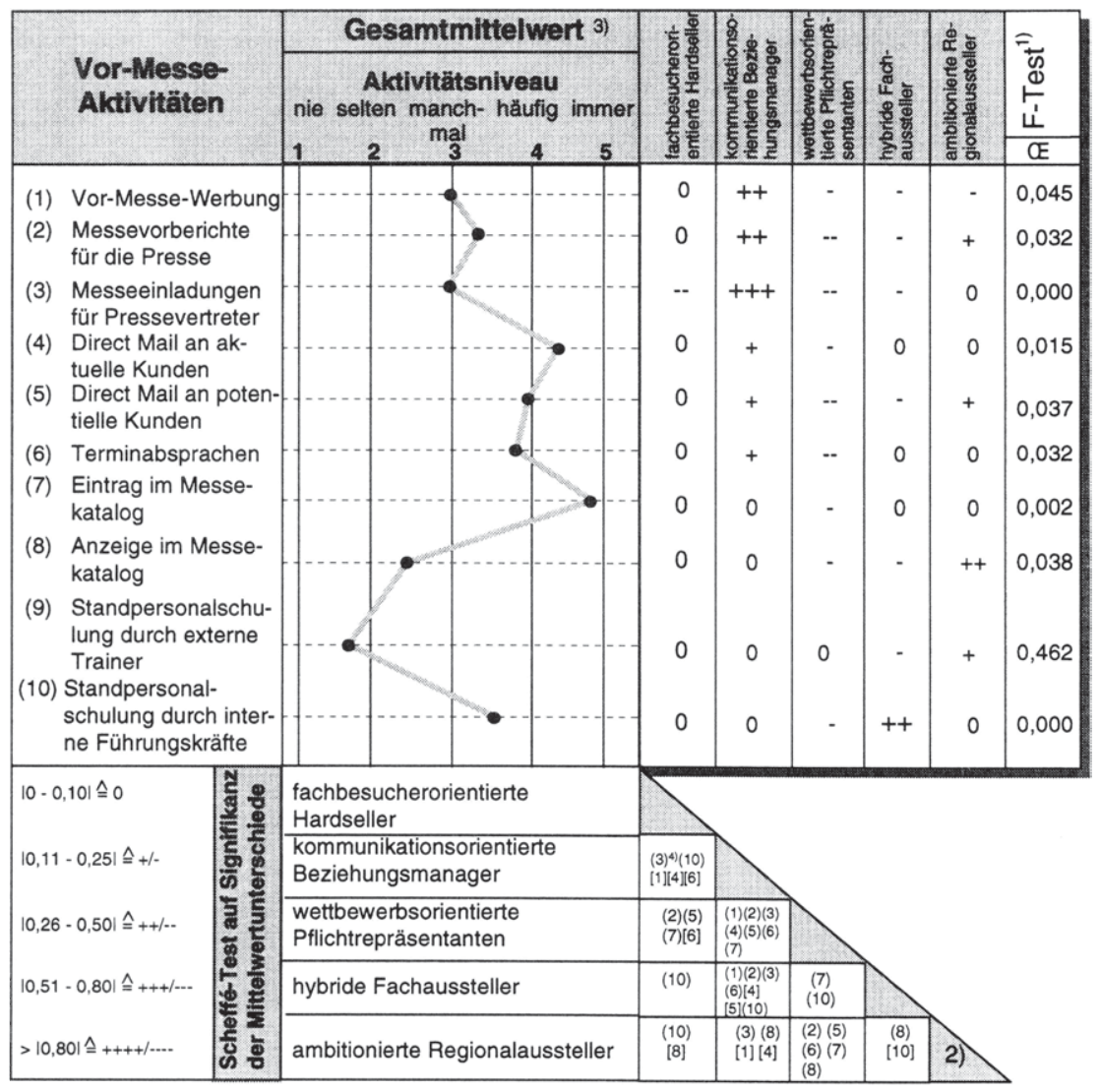

1) One-way-Analyse zur Globalbeurteilung der Mittelwertunterschiede

2) Angabe der Signifikanzniveaus: (i) $\hat{=} \alpha=0,05 /$ [i] $\hat{=} \alpha=0,10$

3) Standardabweichung vom Gesamtmittelwert zwischen 0,57 und 1,50

4) Ziffern beziehen sich auf die jeweiligen Items in der Tabelle

Tab. 7: Varianzanalytische Prüfung auf Unterschiede der Vor-Messe-Aktivitäten zwischen den messespezifischen Grundhaltungstypen 
Mit Blick auf die typenspezifischen Mittelwertabweichungen fällt zunächst auf, daß besonders die Aussteller im Cluster der kommunikationsorientierten Beziehungsmanager ein vergleichsweise überdurchschnittliches Vor-Messe-Engagement aufweisen. Sie unternehmen in besonderer Weise den Versuch, im Vorfeld der Messe auf die eigene Messebeteiligung durch die Nutzung persönlicher und massenmedialer Kommunikationsformen hinzuweisen. Im Vergleich zu den übrigen Grundhaltungstypen ist zudem das besondere Interesse dieser Aussteller an Pressekontakten zu konstatieren. ${ }^{393}$ Dagegen werden die meisten anderen Maßnahmen der Vor-Messe-Phase - insbesondere die Mitarbeiterqualifizierung durch externe Dienstleister oder aber interne Führungskräfte - nur durchschnittlich eingesetzt.

Ihr vergleichsweise hohes Interesse an der Bekanntmachung ihrer Messebeteiligung machen auch die ambitionierten Regionalaussteller deutlich, da sie mit Messevorberichten für die Presse, Direct Mails an potentielle Kunden und Anzeigen im Messekatalog insbesondere solche Instrumente überdurchschnittlich einsetzen, die direkt oder indirekt zur Schaffung von Aktualität der Messebeteiligung bei potentiellen Messebesuchern beizutragen vermögen.

Ein insgesamt unterdurchschnittliches Aktivitätsniveau weisen die drei verbleibenden Cluster auf, wobei die Gruppe der wettbewerbsorientierten Pflichtrepräsentanten die schwächste Nutzungsintensität der untersuchten Instrumente aufweist. Offensichtlich scheint sich der vermutete Zusammenhang zwischen der messespezifischen Grundhaltung und der Ausgestaltung der Messebeteiligung hinsichtlich dieses Grundhaltungstyps zumindest für die Vor-Messe-Phase zu bestätigen, da eine in diesem Fall vergleichsweise schwach ausgeprägte Zielorientierung unterdurchschnittliche Vor-Messe-Aktivitäten zur Folge hat. Erwähnenswert erscheint des weiteren das Aktivitätsprofil des Clusters IV, das die Gruppe der hybriden Fachaussteller beinhaltet. Hier sei vor dem Hintergrund der festgestellten Mitarbeiter- bzw. Motivationszielorientierung des Clusters auf die für diese Aussteller hohe Bedeutung der Standpersonalschulung durch interne Führungskräfte hingewiesen. Damit ist davon auszugehen, daß für diese Unternehmen die Motivationsziele nicht zuletzt auch durch die intensive Vorbereitung der Standmitarbeiter auf die bevorstehenden Messeaufgaben erreicht werden sollen.

393 Dieser Befund unterstützt die hohe Konsistenz dieses Grundhaltungstyps, denn ihre stark anspruchsgruppen- und marktgerichteten Zielorientierung findet ihre Entsprechung in der Intensität der eingesetzten Kommunikationsinstrumente. 
Die ermittelten Unterschiede zwischen den fünf messespezifischen Grundhaltungstypen werden durch die Ergebnisse des Scheffè-Tests, die im unteren Teil der Tab. 7 wiedergegeben sind, bestätigt. Die Resultate sind wie folgt zu interpretieren:

Ausgehend von der Kopfspalte der Tabelle, welche die messespezifischen Grundhaltungstypen enthält, ergibt sich eine untere Dreiecksmatrix, die alle paarweisen Kombinationen von Grundhaltungstypen enthält. Die Ziffern innerhalb der Zellen der Matrix verweisen auf die untersuchten Vor-Messe-Aktivitätsvariablen. Anhand der Klammern, in welche die Ziffern gesetzt worden sind, lassen sich schließlich die beiden alternativen Signifikanzniveaus ablesen (Irrtumswahrscheinlichkeit von 5 bzw. 10 Prozent), mit denen der Scheffé-Test durchgeführt wurde. Beispielsweise unterscheiden sich kommunikationsorientierte Beziehungsmanager und fachbesucherorientierte Hardseller auf einem Signifikanzniveau von $\alpha=0,05$ hinsichtlich des Einsatzes von Messeeinladungen für Pressevertreter (Variable 3) und der Standpersonalschulung durch interne Führungskräfte (Variable 10), sowie auf einem Signifikanzniveau $\alpha=0,1$ hinsichtlich des Einsatzes der Vor-Messe-Werbung (Variable 1), der Nutzung von Direct Mails an aktuelle Kunden (Variable 4) und der Terminabsprachen durch den Außendienst des ausstellenden Unternehmens (Variable 6). ${ }^{394}$

Zusammenfassend kann eine Polarisierung der Grundhaltungstypen in zwei Gruppen konstatiert werden. Während insgesamt ein hohes Aktivitätsniveau hinsichtlich der Vor-Messe-Aktivitäten für die Gesamtstichprobe festgestellt werden kann, weisen die kommunikationsorientierten Beziehungsmanager und die ambitionierten Regionalaussteller in ausgewählten Aktivitätsbereichen ein überdurchschnittliches Engagement auf, während sich bei den anderen drei Grundhaltungstypen in dieser Phase ein als unterdurchschnittlich zu bezeichnendes Aktivitätsniveau einstellt. Darüber hinaus soll als wichtiges Zwischenergebnis festgehalten werden, daß entgegen des in der Literatur konstatierten hohen Stellenwertes einer adäquaten Vorbereitung der Standmitarbeiter auf ihre messebezogenen Aufgaben, clusterübergreifend die Standpersonalschulung durch externe Trainer den geringsten Stellenwert für die Aussteller aufweist. Wie bereits beschrieben, ist nur

\footnotetext{
394 Nach dem Scheffé-Test erweisen sich 39 der insgesamt 100 einzelnen Mittelwertpaare als signifikant unterschiedlich voneinander. Damit ergeben sich für die Maßnahmen der VorMesse-Phase die stärksten Unterschiede im Vergleich aller beschreibenden Clustermerkmale, wie sie in Kapitel $\mathrm{C}$ diskutieft werden.
} 
die Gruppe der hybriden Fachaussteller hinsichtlich der Standpersonalschulung durch interne Führungskräfte überdurchschnittlich aktiv. Damit ist aber zu vermuten, daß eine Vielzahl von Ausstellern durch mangelnde Vorbereitung ihrer Standmitarbeiter, letztere dadurch nicht bzw. kaum in die Lage versetzen, die sich auf der Messe bietenden Kontaktpotentiale in vollem Umfang für das Unternehmen auszuschöpfen.

Im folgenden wird daher zu analysieren sein, ob sich insbesondere die grundhaltungstypenspezifichen Tendenzen auch in den weiteren Phasen identifizieren lassen.

\subsection{Aktivitäten in der Messe-Phase}

Die Aktivitätsschwerpunkte von Ausstellern in der Messe-Phase wurden im Rahmen der Befragung durch elf Variablen repräsentiert. ${ }^{395}$ Zur Überprüfung der Ausprägungsunterschiede soll wiederum ein Mittelwertvergleichstest für die messespezifischen Grundhaltungstypen durchgeführt werden, dessen Ergebnisse in Tab. 8 dokumentiert sind.

Hinsichtlich des Gesamtprofils ergeben sich für die Mehrzahl der Instrumentevariablen geringe Intensitätsniveaus. Eine Ausnahme bildet lediglich die Präsenz der Unternehmensleitung auf dem Messestand mit einem Gesamtmittelwert von 4,21 .

395 Vgl. zur Operationalisierung der Variablen die Frage 19 (Messe-Phase) des Fragebogens im Anhang der Arbeit. 


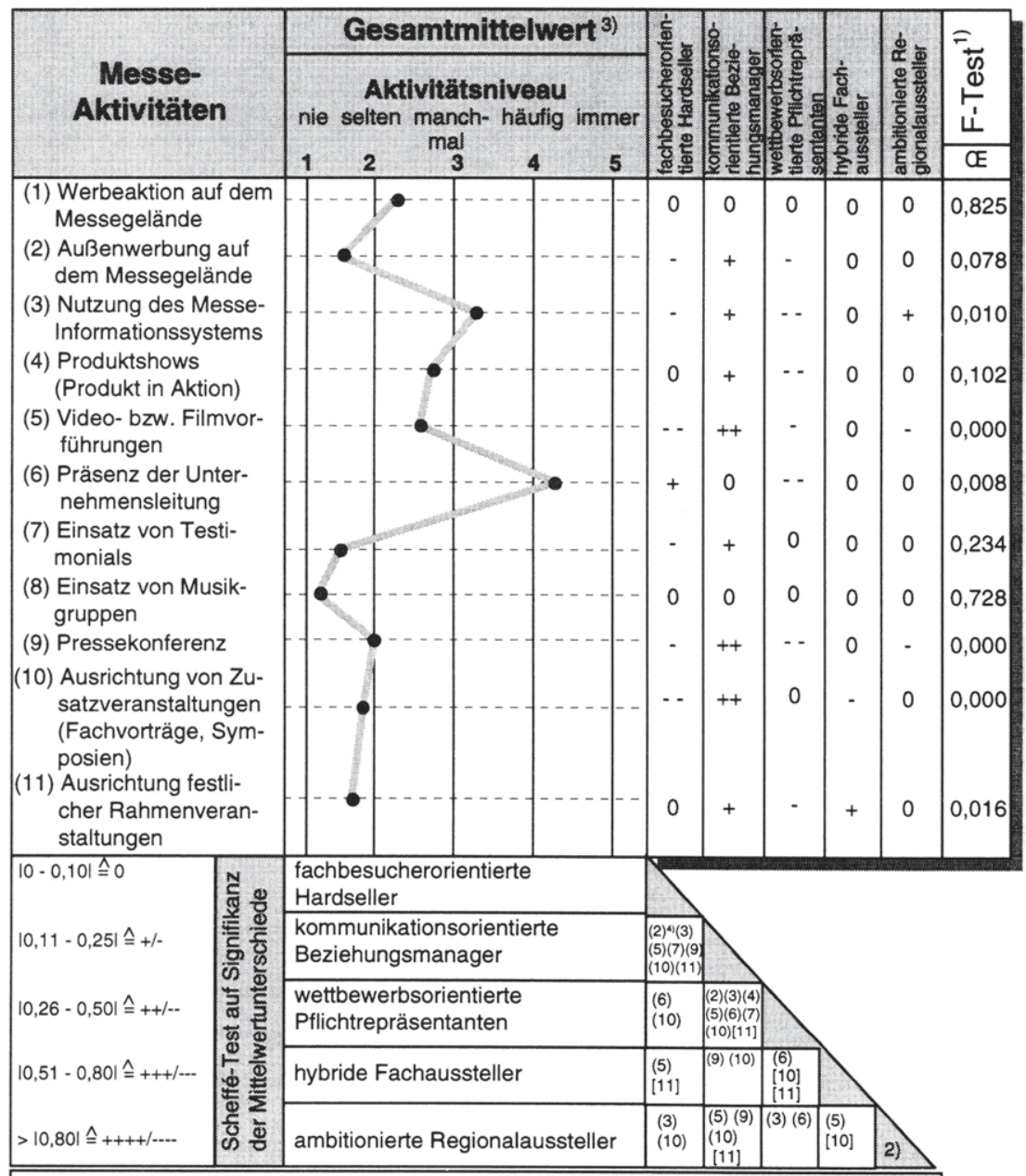

1) One-way-Analyse zur Globalbeurteilung der Mittelwertunterschiede

2) Angabe der Signifikanzniveaus: (i) $\hat{=} \alpha=0,05 /[i] \hat{=} \alpha=0,10$

3) Standardabweichung vom Gesamtmittelwert zwischen 0,69 und 1,51

4) Ziffern beziehen sich auf die jeweiligen Items in der Tabelle

Tab. 8: Varianzanalytische Prüfung auf Unterschiede der Messe-Aktivitäten zwischen den messespezifischen Grundhaltungstypen 
Betrachtet man die typenspezifischen Mittelwertabweichungen im einzelnen, dann zeigt sich auch hinsichtlich der meisten Messe-Aktivitäten für das Cluster der kommunikationsorientierten Beziehungsmanager ein überdurchschnittliches Aktivitätsniveau. ${ }^{396}$ So werden im Vergleich zum Gesamtmittelwertprofil aller befragten Aussteller die Instrumente "Außenwerbung auf dem Messegelände", „Nutzung des Messe-Informationssystems für werbliche Hinweise“, „Produkt Shows (Produkt in Aktion)“, „Einsatz von Testimonials" sowie „Ausrichtung von festlichen Rahmenveranstaltungen" überdurchschnittlich und die Instrumente "Video- bzw. Filmvorführungen“, "Pressekonferenz" sowie "Ausrichtung von fachlichen Zusatzveranstaltungen" stark überdurchschnittlich eingesetzt. ${ }^{397}$ Bemerkenswert erscheinen in dieser Betrachtung vor allem die empirischen Befunde für die Gruppe der ambitionierten Regionalaussteller, die sich zwar in der Vor-MessePhase überdurchschnittlich stark engagieren, in der Messe-Phase indes mit Ausnahme der Nutzung des Messeinformationssystems hinsichtlich der abgefragten Variablen höchstens durchschnittliche Einsatzintensitäten realisieren. Trotz ihres sehr anspruchsvollen Zielsystems nutzen diese Aussteller die Möglichkeiten der Besucherakquisition auf der Messe (Werbeaktion und Außenwerbung auf dem Messegelände) und die Maßnahmen der Aktivierung der Standbesucher (ProduktShows, Video- bzw. Filmvorführungen, Einsatz von Testimonials, Musikgruppen und Zusatzveranstaltungen) nur unter- bzw. durchschnittlich. ${ }^{398}$ Es ist daher trotz der hochgesteckten Ziele dieses Grundhaltungstyps davon auszugehen, daß kaum Anstrengungen unternommen werden, um sich im Kommunikationswettbewerb auf der Messe durch außergewöhnliche Aktivitäten zu profilieren.

Ein weiterer interessanter Befund ergibt sich für die hybriden Fachaussteller. Zeichnete sich dieser Grundhaltungstyp in der Vor-Messe-Phase im Vergleich zum Gesamtprofil der Aussteller durch überdurchschnittliche Aktivitäten zur Mitarbeiterschulung aus, so steht zu vermuten, daß auch seine einzig überdurch-

396 Der Scheffé-Test weist für dieses Cluster die mit Abstand meisten signifikanten Mittelwertunterschiede zu den übrigen Clustern aus. Im Paarvergleich sind 21 von 44 Mittelwerten signifikant verschieden.

Das im Vergleich deutlich höhere Aktivitätsniveau in den Bereichen „Produkt Show“, "Videobzw. Filmvorführungen“, "Einsatz von Testimonials“, "Ausrichtung festlicher Rahmenveranstaltungen" entspricht dabei der am stärksten ausgeprägten Erlebnisorientierung des MessePositionierungskonzepts dieses Clusters, während die Ausrichtung von "Pressekonferenzen" und "fachlichen Zusatzveranstaltungen“ den Kommunikationscharakter der Messebeteiligungskonzeption dieser Aussteller besonders widerspiegelt.

Der Nutzen akquisitionsfördernder und aktivierender Instrumente wird offensichtlich aufgrund der hohen Fachbesucherorientierung der von diesem Cluster ausgewählten Messeveranstaltungen nicht hoch eingeschätzt. 
schnittlichen Aktivitäten hinsichtlich der Ausrichtung festlicher Rahmenveranstaltungen eine den mitarbeitergerichteten Motivationszielen entsprechende Orientierung aufweisen.

Die hohe Fachbesucher- und Verkaufsorientierung der Aussteller in Cluster I dokumentiert sich dagegen in einer überdurchschnittlich hoch ausgeprägten Präsenz der Unternehmensleitung während der Messebeteiligung. Dieser Befund deckt sich mit den Ergebnissen amerikanischer Studien, die ebenfalls einhergehend mit der Anwesenheit des Top-Managements von Unternehmen eine Zunahme der Verkaufs- bzw. Ordertätigkeit am Messestand konstatieren. ${ }^{399}$ Darüber hinaus weist dieser Grundhaltungstyp jedoch hinsichtlich jeder weiteren Messe-Aktivität, die abgefragt worden ist, eine unterdurchschnittliche Ausprägung auf. Das geringste Aktivitätsniveau realisieren allerdings - wie auch schon in der Vor-Messe-Phase - die wettbewerbsorientierten Pflichtaussteller, die hinsichtlich sieben der elf abgefragten Aktivitätsvariablen ein unterdurchschnittliches sowie $z$. T. stark unterdurchschnittliches Engagement dokumentieren.

Mit Blick auf die Ergebnisse des Scheffé-Tests wird deutlich, daß alle Grundhaltungstypen sich zumindest bei jeweils zwei Variablen signifikant unterscheiden. Dabei ist zu bedenken, daß der F-Test die Zahl signifikant unterschiedlicher Variablenausprägungen für die Variablen "Werbeaktion auf dem Messegelände“, „Einsatz von Testimonials“ und „Einsatz von Musikgruppen“ als nicht gegeben ausweist (Signifikanzniveau $\alpha=0,1$ ) und sich damit die relevante Zahl der Aktivitätsvariablen auf acht verringert. Zusammenfassend kann insbesondere für das Cluster II von einem signifikant unterschiedlichen Aktivitätsniveau gegenüber allen anderen Ausstellergruppen ausgegangen werden. ${ }^{400}$

Insgesamt bleibt festzuhalten, daß sich zwar signifikante Unterschiede zwischen den Clustern hinsichtlich der Aktivitäten in der Messe-Phase ergeben. Im Vergleich zu den Vor-Messe-Aktivitäten ist allerdings ein für alle Aussteller als gering zu bezeichnendes Aktivitätsniveau zu konstatieren.

399 Vgl. Williams, J. D. Gopalakrishna, S., Cox, J. M., Trade Show Guidelines for Smaller Firms, a.a.O., S. 322 f.; Tanner, J. F., Chonko. L. B., Trade Show Objectives, Management, and Staffing Practices, a.a.O., S. 271.

400 Immerhin weist dieses Cluster 21 von 32 signifikanten Unterschieden gegenüber den anderen Clustern auf, wobei lediglich zwei Signifikanzniveaus bei nur $10 \%$ liegen. 


\subsection{Aktivitäten in der Nach-Messe-Phase}

Zur Analyse der Aktivitätsschwerpunkte der verschiedenen Grundhaltungstypen in der Nach-Messe-Phase wird wiederum ein Mittelwertvergleichstest durchgeführt, dessen Ergebnisse in Tab. 9 wiedergegeben sind.

Auch hier zeigen sich hinsichtlich des Gesamtprofils für drei der vier Instrumentevariablen geringe Intensitätsniveaus. Eine Ausnahme bildet lediglich die systematische Weiterverfolgung der Messekontakte mit einem Gesamtmittelwert von 4,36. ${ }^{401}$ Offensichtlich findet nahezu unabhängig vom verfolgten Grundhaltungstyp eine dominante Orientierung der Aussteller an der Weiterführung der am Messestand erzielten Gesprächskontakte statt.

Mit Blick auf die verschiedenen Grundhaltungstypen verdeutlicht der F-Test, daß hinsichtlich der systematischen Weiterverfolgung der Messekontakte, der Werbung mit Messeergebnissen und dem Verfassen eines Messeberichtes für die Presse signifikante Unterschiede zwischen den Grundhaltungstypen bestehen. Allerdings macht der Scheffé-Test deutlich, daß zwischen den fachbesucherorientierten Hardsellern und sowohl den hybriden Fachausstellern als auch den ambitionierten Regionalausstellern keine signifikanten Unterschiede in bezug auf unterschiedliche Aktivitätsniveaus feststellbar sind. Gleiches gilt für die kommunikationsorientierten Beziehungsmanager und die hybriden Fachaussteller. Für alle anderen Typenvergleiche ergibt sich jedoch wenigstens ein signifikanter Mittelwertunterschied.

Bei Betrachtung der typenspezifischen Mittelwertunterschiede zeigen sich folgerichtig nur wenige grundhaltungsspezifische Besonderheiten. Festzuhalten bleibt zunächst auch hier, daß wiederum die wettbewerbsorientierten Pflichtaussteller die geringste Aktivitätsintensität aufweisen und sich vor allem aufgrund der stark unterdurchschnittlich ausgeprägten Weiterverfolgung der Messekontakte in der Nach-Messe-Phase von allen anderen Grundhaltungstypen signifikant unter

401 Die Variablen "Werbung mit Messeergebnissen“ $(2,08)$, „Verfassen eines internen Messeberichtes" (3.37) "sowie "Verfassen eines Messeberichtes für die Presse" $(2,25)$ weisen deutlich geringere Mittelwerte auf. 


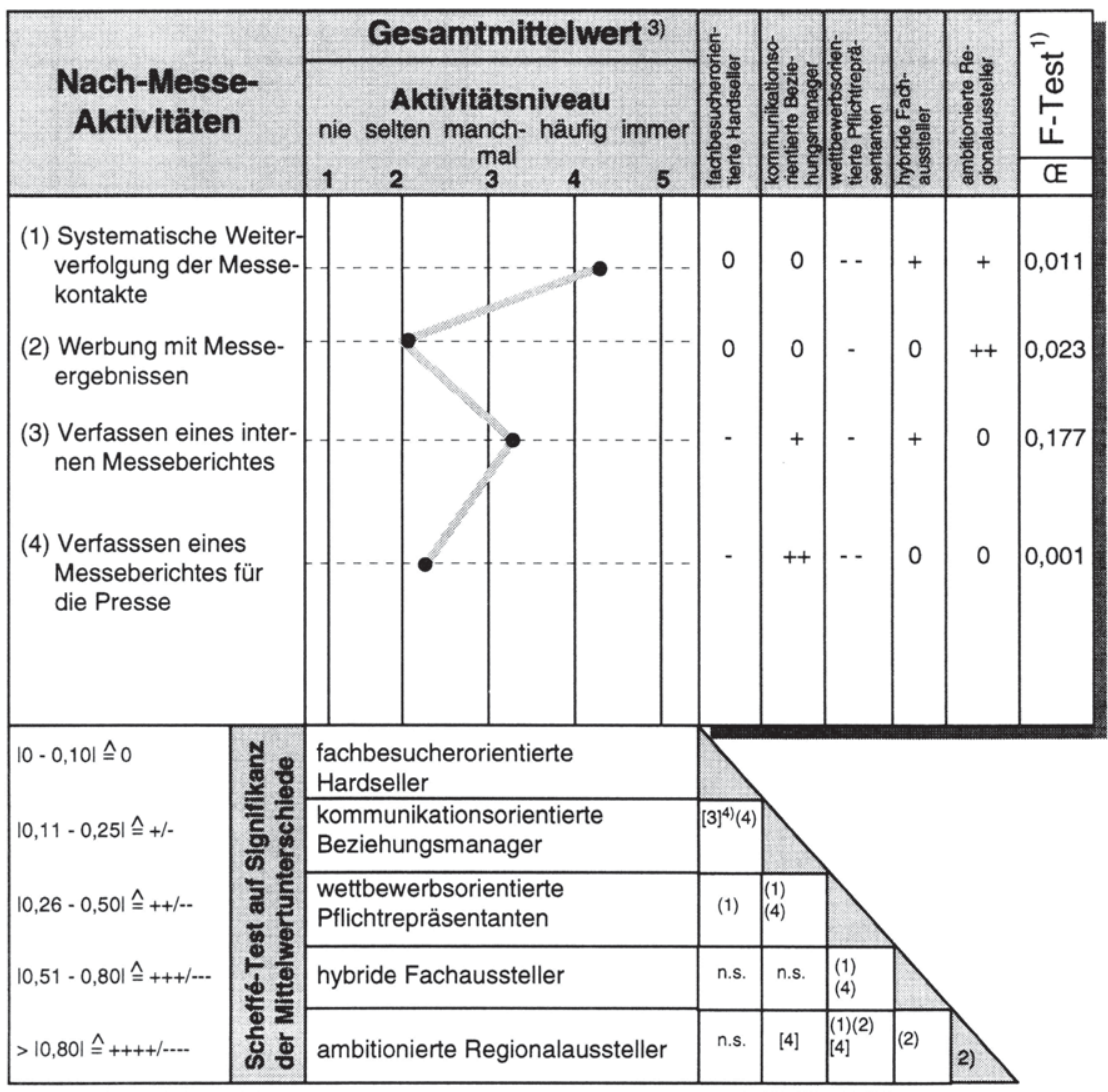

1) One-way-Analyse zur Globalbeurteilung der Mittelwertunterschiede

2) Angabe der Signifikanzniveaus: (i) $\hat{=} \alpha=0,05 /[i] \hat{=} \alpha=0,10$

3) Standardabweichung vom Gesamtmittelwert zwischen 0,91 und 1,44

4) Ziffern beziehen sich auf die jeweiligen Items in der Tabelle

Tab. 9: Varianzanalytische Prüfung auf Unterschiede der Nach-Messe-Aktivitäten zwischen den messespezifischen Grundhaltungstypen

scheiden. ${ }^{402}$ Darüber hinaus macht auch Cluster II (kommunikationsaktive Beziehungsmanager) deutlich, daß es in signifikant stärkerem Maße den Kontakt zur

402 Diese Tatsache läßt sich vor allem dadurch erklären, daß die wettbewerbsorientierten Pflichtaussteller ihren Messebeteiligungsschwerpunkt im Bereich der Publikumsmessen haben, in deren Rahmen i. d. R. die Anonymität der Messebesucher größer ist, die direkten persönlichen Messekontakte sporadischer sind und damit die Nachverfolgung dieser Messekontakte weniger stark im Vordergrund stehen.

Ralf Ueding and Universität Münster - 978-3-631-75073-5 
Presse durch das Abfassen von Nach-Messe-Presseberichten sucht, als die übrigen Ausstellercluster. ${ }^{403}$

Zusammenfassend bleibt daher festzuhalten, daß das Engagement aller Ausstellergruppen primär auf die Weiterverfolgung der Messekontakte fokussiert ist, während die anderen Aktivitäten eine deutlich geringere Bedeutung einnehmen. Unterschiede zwischen den einzelnen Ausstellergruppen sind als lediglich marginal zu konstatieren.

Hinsichtlich der Aktivitäten der fünf messespezifischen Grundhaltungstypen kann bislang vor allem von einem unterschiedlichen Verhalten in der Vor-Messe-Phase ausgegangen werden. In der Messe- und Nach-Messe-Phase nehmen die Unterschiede zwischen den Ausstellergruppen dagegen zunehmend ab. Es wird daher im folgenden interessant sein zu prüfen, ob diese Konversion der Aktivitätsniveaus auch hinsichtlich der Ausgestaltung der Erfolgskontrolle von Messebeteiligungen festzustellen ist.

\subsection{Analyse der Erfolgskontrolle von Messebeteiligungen}

Wesentlicher Bestandteil der Erfolgskontrolle von Messebeteiligungen und zugleich zentrale Voraussetzung für inre zielorientierte Durchführung ist die Operationalisierung der Messebeteiligungsziele. Im folgenden soll daher zunächst analysiert werden, in welcher Weise die Grundhaltungstypen ihre Zielformulierungen jeweils operationalisieren.

\subsection{Zieloperationalisierung}

Hinsichtlich der Messung des Grades der Operationalisierung von messespezifischen Zielsetzungen wurden in der Befragung sieben Variablen eingesetzt. ${ }^{404} \mathrm{Tab}$.

403 Allerdings beträgt der Mittelwert dieses Clusters für die Verfassung von Messeberichten für die Presse lediglich 2,85, was insgesamt dennoch nur auf eine insgesamt geringe Anwendungsintensität dieses Instrumentes schließen läßt.

404 Hierdurch werden der Zeit-, Objekt- und Segmentbezug sowie die Festlegung des Ausmaßes der Messebeteiligungsziele erfaßt. Die Erfassung der Zielinhalte wurde hiervon getrennt in einer anderen Frage abgefragt und ist bereits in Kap. B 1.2 dargestellt worden. Dem Aspekt der schwierigen Bestimmung des Zeitbezugs von Messebeteiligungszielen wurde in Form von drei Items mit unterschiedlicher zeitlicher Dimensionierung in besonderer Weise Rechnung getragen. 
10 gibt die Ergebnisse des Mittelwertvergleichstests, der hier ebenfalls zur Herausarbeitung der Unterschiede zwischen den Grundhaltungstypen angewendet wird, wieder.

Der die Mittelwerte der Gesamtstichprobe wiedergebende Gesamtprofilverlauf zeigt zunächst eine wichtige Besonderheit: Bei den Ausstellern besteht mit einem Mittelwert von 2,63 hinsichtlich des Items „Messeziele werden nicht weiter operationalisiert" das allgemeine Bewußtsein, ihre Messebeteiligungsziele weniger häufig zu operationalisieren, als dies notwendig ist. Analysiert man das Mittelwertprofil weiter, so ist festzustellen, daß die Aussteller die inhaltlichen Zielfestlegungen hinsichtlich der zeitlichen Dimensionierung zum überwiegenden Teil auf einzelne Messeveranstaltungen ( $M W=4,20)$ beziehen.

Ebensowenig wie ein Herunterbrechen der Messebeteiligungsziele auf einzelne Messetage erfolgt, wird der zeitliche Horizont auf mehrere Messen und damit auf eine längerfristige Perspektive ausgedehnt. Dies unterstreicht, daß ausstellende Unternehmen Messeveranstaltungen als singuläre Veranstaltungen bewerten. ${ }^{405}$

Des weiteren wird deutlich, daß sowohl der Segment- als auch der Objektbezug der Messebeteiligungsziele weitgehend nur sporadisch hergestellt wird. Besonders auffällig ist in diesem Zusammenhang die geringe Bedeutung der Festlegung des anzustrebenden Ausmaßes der Messebeteiligungsziele. Mit einem Mittelwert von nur 2,80 wird es im Durchschnitt der Aussteller nur manchmal festgelegt.

Mit Blick auf die Mittelwertunterschiede der messespezifischen Grundhaltungstypen, lassen sich überdies kaum signifikante Abweichungen vom Gesamtprofilverlauf feststellen. Der F-Test macht vielmehr deutlich, daß bei einem Signifikanzniveau von $\alpha=0,1$ lediglich zwei der sieben Variablen signifikante Unterschiede zwischen den Ausstellergruppen erwarten lassen. Auch der durchgeführte Scheffé-Test bestätigt diesen Befund.

\footnotetext{
405 Hierzu mögen verschiedene Faktoren beitragen. Zum einen ist davon auszugehen, daß Messeveranstaltungen, die thematisch und gleichzeitig hinsichtlich ihrer Besucher- und Ausstellerreichweite hohe Überschneidungen aufweisen relativ selten vorzufinden sein werden. Zum anderen sind in einem solchen Fall die Veranstaltungstermine von den Messegesellschaften i. d. R. „entzerrt", so daß ein zielfokussierte Zusammenfassung dieser Messen für Aussteller aus zeitlicher Perspektive erschwert wird. Darüber hinaus spricht auch der häufig postulierte "Eventcharakter" von Messen aus Sicht der Aussteller für eine einzelmessespezifische Betrachtung von Messezielsetzungen.
} 


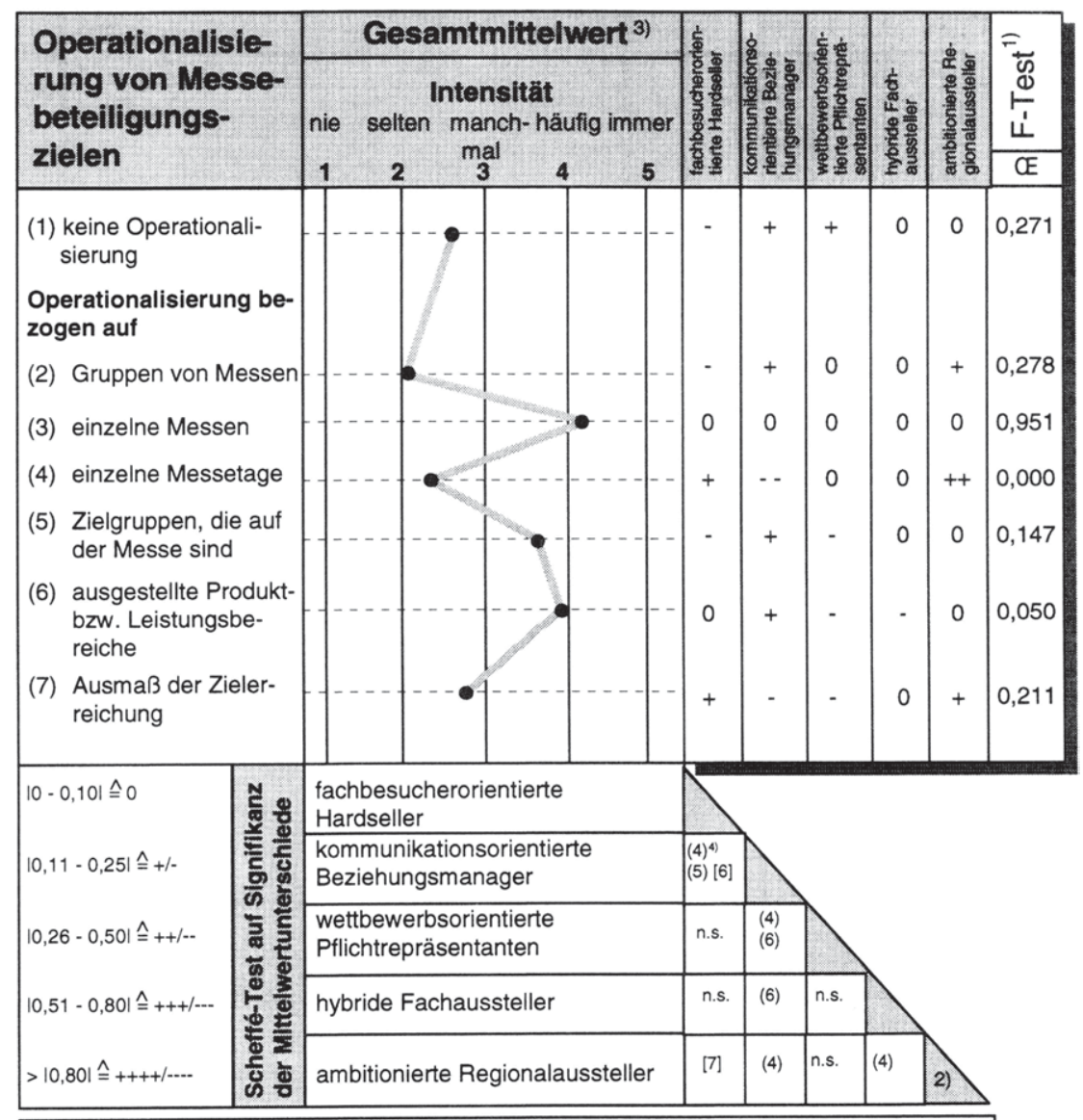

1) One-way-Analyse zur Globalbeurteilung der Mittelwertunterschiede

2) Angabe der Signifikanzniveaus: (i) $\hat{=} \alpha=0,05 /$ [i] $\hat{=} \alpha=0,10$

3) Standardabweichung vom Gesamtmittelwert zwischen 0,99 und 1,37

4) Ziffern beziehen sich auf die jeweiligen Items in der Tabelle

Tab. 10: Varianzanalytische Prüfung auf Unterschiede der Zieloperationalisierung zwischen den messespezifischen Grundhaltungstypen 
Als Fazit ist daher zum einen zu konstatieren, daß die Zieloperationalisierung offensichtlich unabhängig von der verfolgten Grundorientierung auf Messen vorgenommen wird. Zum anderen bleibt festzuhalten, daß die Operationalisierung der Messebeteiligungsziele vor dem Hintergrund der vorliegenden Befragungsergebnisse als insgesamt nicht hinreichend zu bezeichnen ist. Interessantenweise wird der für die Kontrolle der Zielerreichung so wesentliche Aspekt der Formulierung des angestebten Zielerreichungsgrades gerade von den kommunikationsorientierten Beziehungsmanagern, die sich durch eine überdurchschnittliche Zielorientierung und ein vergleichsweise hohes Aktivitätsniveau über alle MessePhasen hinweg auszeichnen, mit einem Mittelwert von 2,63 im geringsten Maße umgesetzt.

Aufgrund dieser Teilergebnisse der empirischen Analyse ist zu folgern, daß die Erfolgskontrolle der Aussteller insgesamt nur unpräzise Ergebnisse hinsichtlich der Erreichung der inhaltlich formulierten Messebeteiligungsziele zu Tage bringen kann.

\subsection{Stellenwert der Erfolgskontrolle}

Vor dem Hintergrund der mangelnden Operationalisierung der Messebeteiligungsziele der Aussteller stellt sich unmittelbar die Frage nach dem Stellenwert der Erfolgskontrolle von Messebeteiligungen in Abhängigkeit der messespezifischen Grundhaltungstypen. Zur Einschätzung dieser Problematik wird im Rahmen dieser Arbeit zunächst grundsätzlich untersucht, ob die Aussteller Untersuchungen zur Kontrolle des Erfolgs durchführen. ${ }^{406}$ Als Stufen der Kontrollintensität werden die drei Optionen „immer" (d. h. bei jeder Messebeteiligung), „nur bei ausgewählten Veranstaltungen" und "nie" (d. h. bei keiner Messebeteiligung) unterschieden. ${ }^{407}$ Diejenigen Aussteller, die immer Erfolgskontrollen bei ihren Messebeteiligungen vornehmen, sollen im Rahmen dieser Arbeit als die "Kontrollaktiven“ bezeichnet werden. Diejenigen Aussteller, die lediglich bei „ausgewählten Veranstaltungen“ Erfolgskontrollen durchführen, sollen im weiteren als die "Kontrollselektiven" und

406 Vgl. Frage 10 des Fragebogens im Anhang 5 dieser Arbeit.

407 Im Hinblick auf die Gesamtstichprobe ergibt sich das folgende Ergebnis: Lediglich $27,4 \%$ der Aussteller stellen bei jeder Messebeteiligung Untersuchungen zur Erfolgskontrolle ihrer messebezogenen Aktivitäten an, 35,7\% der Aussteller machen indes deutlich, daß sie nie solche Erfolgskontrollen durchführen. Immerhin $36,9 \%$ der befragten Aussteller untersuchen bei ausgewählten Veranstaltungen den Erfolg ihrer eigenen Messebeteiligung. Vgl. Meffert, $H_{\text {. }}$ Ueding. R., Ziele und Nutzen von Messebeteiligungen, a.a.O., S. 67.

Ralf Ueding and Universität Münster - 978-3-631-75073-5 
solche, die „nie" Erfolgskontrollen durchführen als die „Kontrollaversen“ bezeichnet werden.

Dabei ist nachfolgend zu untersuchen, ob zwischen den einzelnen Grundhaltungstypen und den drei Intensitätsstufen der Erfolgskontrolle ein Zusammenhang besteht. Zur Überprüfung dieser Fragestellung werden die messespezifischen Grundhaltungstypen und die Intensitätstypen der Erfolgskontrolle im Rahmen einer Kreuztabellierung einer empirischen Analyse unterzogen. Mittels dieses Verfahrens kann untersucht werden, ob die identifizierten Grundhaltungstypen hinsichtlich der Intensität der Erfolgskontrolle signifikante Unterschiede aufweisen. Ist dies der Fall, kann von eindeutigen Beziehungen zwischen den messespezifischen Grundhaltungen und dem für sie geltenden Stellenwert der Erfolgskontrolle von Messebeteiligungen ausgegangen werden. ${ }^{408}$

Die Ergebnisse der Kreuztabellierung sind in Tab. 11 vollständig dokumentiert. Diese offenbart, daß sich hinsichtlich der messespezifischen Grundhaltungstypen sehr wohl Unterschiede in der Intensität der Erfolgskontrolle ihrer Messebeteiligungen ergeben, denn es zeigen sich z. T. deutliche Abweichungen von den statistisch zu erwartenden Zellenhäufigkeiten.

So weist das Cluster der kommunikationsorientierten Beziehungsmanager ein überdurchschnittlich starkes Ablehnungsverhalten gegenüber Erfolgskontrollen auf. Hinsichtlich der Gruppe der Kontrollaktiven sind diese Aussteller deutlich unterrepräsentiert $\left(x_{i}=31 \text { ggü. }\left(x_{i}\right)=41,8\right)^{409}$ und bezogen auf die Gruppe der Kontrollaversen deutlich stärker vertreten als statistisch zu erwarten gewesen wäre $\left(x_{i}=65\right.$ ggü. $\left.\left(x_{i}\right)=55,2\right)$.

408 Im Rahmen der Kreuztabellierung wird entsprechend des Umfangs der einzelnen Grundhaltungstypen sowie der Intensitätstypen der Erfolgskontrolle eine zu erwartende Häufigkeit für jede Zelle der Kreuztabelle berechnet. Diesen Erwartungswerten wird jeweils die tatsächliche Häufigkeit, wie sie sich aus dem Datensatz ergibt, gegenübergestellt. Abweichungen der tatsächlichen von der statistisch zu enwartenden Häufigkeit deuten sodann auf Beziehungen zwischen den Grundhaltungs- und Intensitätstypen der Erfolgskontrolle hin. 


\begin{tabular}{|c|c|c|c|c|c|c|}
\hline \multicolumn{2}{|c|}{$\begin{array}{l}\text { messespexiflecher } \\
\text { Grundhaltungstyp }\end{array}$} & Cluster I & Cluster II & Cluster IfI & Cluster IV & Cluster V \\
\hline 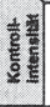 & \begin{tabular}{|l|}
$x_{1}:=$ tatsăch- \\
licher Zeilen- \\
wert \\
$\left(x_{1}\right):=$ erwarteter \\
Zeilenwert \\
\end{tabular} & $\begin{array}{c}\text { fachbesucherorien- } \\
\text { tierte Hardseller }\end{array}$ & $\begin{array}{l}\text { kommunikations- } \\
\text { orientierte Bezie- } \\
\text { hungsmanager }\end{array}$ & $\begin{array}{c}\text { wettbewerbsorien- } \\
\text { tierte Pflichtaus- } \\
\text { steller }\end{array}$ & $\begin{array}{c}\text { hybride } \\
\text { Fachaussteller }\end{array}$ & $\begin{array}{c}\text { ambitionierte } \\
\text { Regionalaussteller }\end{array}$ \\
\hline \multirow{2}{*}{\multicolumn{2}{|c|}{ Kontrollaktive }} & $\begin{array}{c}27 \\
(26,7)\end{array}$ & $\begin{array}{c}31 \\
(41,8)\end{array}$ & $\begin{array}{c}24 \\
(23,7)\end{array}$ & $\begin{array}{c}29 \\
(26,9)\end{array}$ & $\begin{array}{c}35 \\
(26,5)\end{array}$ \\
\hline & & 0 & $\cdots$ & 0 & + & ++ \\
\hline \multirow{2}{*}{\multicolumn{2}{|c|}{ Kontrollselektive }} & $\begin{array}{c}36 \\
(35,9)\end{array}$ & $\begin{array}{c}57 \\
(56,1)\end{array}$ & $\begin{array}{c}32 \\
(31,9)\end{array}$ & $\begin{array}{c}33 \\
(36,9)\end{array}$ & $\begin{array}{c}38 \\
(35,5)\end{array}$ \\
\hline & & 0 & 0 & 0 & - & + \\
\hline \multirow{2}{*}{\multicolumn{2}{|c|}{ Kontrollaverse }} & $\begin{array}{c}35 \\
(35,4)\end{array}$ & $\begin{array}{c}65 \\
(55,2)\end{array}$ & $\begin{array}{c}31 \\
(31,4)\end{array}$ & $\begin{array}{c}39 \\
(36,2)\end{array}$ & $\begin{array}{c}24 \\
(35,0)\end{array}$ \\
\hline & & 0 & 4 & 0 & + & $\cdots$ \\
\hline
\end{tabular}

\begin{tabular}{|c|c|c|c|}
\hline Chi Quadrat-Test gemas & Slgniflikanzniveau & $x_{1}-\left(x_{1}\right)$ & $0-2,0 \quad 2,01-5,0 \quad>5,0$ \\
\hline Person & 0,17573 & positiv & 0 \\
Likelihood Ratio & 0,15824 & negativ & 0 \\
\hline
\end{tabular}

1) Zuorchung von 535 Befragten in 65 Fällen mit Missing Values und vorheriger Selektion von 6 Ausreißem.

Tab. 11: Zusammenhang zwischen Intensität der Erfolgskontrolle und den messespezifischen Grundhaltungstypen

Genau umgekehrt verhalten sich die ambitionierten Regionalaussteller. Sie sind deutlich stärker kontrollaktiv $\left(x_{i}=35\right.$ ggü. $\left.\left(x_{i}\right)=26,5\right)$ und weisen eine weniger große Anzahl Aussteller auf, die sich ablehnend ggü. der Messeerfolgskontrolle verhalten als dies statistisch zu enwarten ist $\left(x_{i}=24\right.$ ggü. $\left.\left(x_{i}\right)=35,0\right)$. In der Gruppe der hybriden Aussteller sind sowohl die kontrollaktiven $\left(x_{i}=29\right.$ ggü. $\left.\left(x_{i}\right)=26,9\right)$ als auch die kontrollaversen $\left(x_{i}=39\right.$ ggü. $\left.\left(x_{i}\right)=36,2\right)$ Aussteller stärker vertreten als dies statistisch zu erwarten ist. Die beiden anderen Grundhaltungscluster weisen dagegen keine Abweichungen von den statistisch erwarteten Werten auf.

Aus den Ergebnissen der Analyse läßt sich folgendes Fazit ziehen: Die überwiegende Mehrheit der Aussteller ist als kontollavers bzw. -selektiv einzustufen. ${ }^{410}$ Lediglich das Cluster der ambitionierten Regionalaussteller weist eine überdurchschnittlich kontrollaktive Haltung ggü. seinen Messebeteiligungen auf. In Zusammenhang mit den Ausführungen zu den empirischen Untersuchungen in Kapitel C

\footnotetext{
410 Als zentrale Gründe für dieses Verhalten wird von den Ausstellern vor allem das als ungünstig empfundene Kosten-/Nutzenverhältnis der Erfolgskontrolle von Messebeteiligungen sowie fehlende Personalkapazitäten und die unabhängig vom eigenen Messebeteiligungserfolg geltende Unverzichtbarkeit von Messebeteiligungen für den Aussteller betont. Vgl. Meffert. $H$. Ueding, R., Ziele und Nutzen von Messebeteiligungen, a.a.O., S. 69 f.
} 
2.21 (Operationalisierung der Messebeteiligungsziele) läßt sich damit erwartungsgemäß festhalten, daß diejenigen Aussteller mit der weitestgehenden Operationalisierung ihrer Messebeteiligungsziele auch diejenigen sind, die am ehesten eine Erfolgskontrolle durchführen. Insbesondere am Beispiel der kommunikationsorientierte Beziehungsmanager wird deutlich, daß eine mangelnde Zieloperationalisierung folgerichtig bei der Mehrzahl der Aussteller mit einer deutlichen Vernachlässigung der Erfolgskontrolle einhergeht. Dadurch muß ein erheblicher Teil der durch das überdurchschnittliche Aktivitätsniveau vor während und nach der Messe hervorgerufenen Wirkungen als weitgehend "unkontrollierter Aktionismus" zumindest derjenigen Aussteller dieses Clusters bezeichnet werden, die als kontollavers bzw. -selektiv zu bezeichnen sind.

Schließlich zeigt der Chi Quadrat-Wert von 0,175, daß de facto nur ein Teil der Aussteller in den Grundhaltungstypen von dem Gesamtergebnis abweichende Kontrollaktivitäten vornimmt. Nachdem die Kontrollintensität Gegenstand der Untersuchung war, soll im folgenden untersucht werden, inwiefern Unterschiede hinsichtlich qualitativer Gesichtspunkte der Messeerfolgskontrolle existieren, die sich im Einsatz der Methoden der Erfolgskontrolle äußern.

\subsection{Einsatz der Methoden zur Kontrolle des Messebeteiligungserfolgs}

Um die Bedeutung der in Kapitel C 1.3 beschriebenen Methoden zur Erfolgskontrolle für die Aussteller der Grundhaltungstypen ermitteln zu können, wird die Intensität der Nutzung dieser Methoden herangezogen. Des weiteren sind die von den Ausstellern eingesetzten Methoden der Erfolgskontrolle von Messebeteiligungen hinsichtlich ihrer prinzipiellen Kongruenz mit den verfolgten Messebeteiligungszielen zu überprüfen.

Im folgenden wird aus Gründen der Übersicht, die Analyse des Einsatzes der Methoden der Erfolgskontrolle in zwei Schritten vollzogen. Zunächst werden die relevanten Methoden, die vornehmlich in der Vor-Messe- und Messe-Phase einer Messebeteiligung zum Einsatz kommen, analysiert. Danach soll der Einsatz derjenigen Methoden, die erst in der Nach-Messe-Phase anwendbar sind, detailliert untersucht werden. Um sicherzustellen daß die Urteile der Befragten bezüglich der eingesetzten Methoden der Erfolgskontrolle auf konkreten Durchführungs- bzw. Anwendungserfahrungen beruhen, werden in Verbindung mit diesem Untersuchungsgegenstand lediglich diejenigen Aussteller berücksichtigt, die entweder 
zu den im zuvor behandelten Abschnitt der Arbeit als „Kontrollselektive“ oder „Kontrollaktive" bezeichneten Ausstellern gehören. ${ }^{411}$

Die Ergebnisse eines Mittelwertvergleichstests hinsichtlich des Einsatzes der Methoden der Erfolgskontrolle, die in der Vor-Messe- und Messe-Phase eingesetzt werden, zeigt Tab. 12. Der die Mittelwerte der Gesamtstichprobe wiedergebende Gesamtprofilverlauf zeigt insgesamt eine eher sporadische Nutzung der Methoden der Erfolgskontrolle im Zusammenhang mit der Vor-Messe- und MessePhase. Mit einem Mittelwert von 4,11 wird lediglich die Methode der Zählung persönlicher Kontakte am Messestand häufig bzw. regelmäßig durchgeführt. Alle anderen der insgesamt zehn Methoden werden höchstens manchmal bzw. selten eingesetzt. Darüber hinaus zeigen die Mittelwerte, daß vor allem die einfachen "Zählmethoden“ auf eine breitere Akzeptanz bei den Ausstellern stoßen, denn auch die Standbesucherzählung, die Analyse der Kontakte im Zeitablauf der Messe sowie die Zählung des abgegebenen Informationsmaterials werden in der Messe-Phase deutlich häufiger eingesetzt, als die komplexeren Methoden mit mehr Erhebungsaufwand wie z. B. Besucherbefragungen und -beobachtungen.

Hierin spiegelt sich offensichtlich die Schwierigkeit der Umsetzung von Erfolgskontrollen bzw. ihre mangelnde Akzeptanz während des laufenden Messegeschäfts wider, und dies obwohl die weitere Analyse zeigt, daß die Störung des Ablaufs der Messeaktivitäten am Stand von den meisten Befragten kaum als Grund für die fehlende Erfolgskontrolle genannt wird. ${ }^{412}$

411 Damit verringert sich zwar die Zahl der auswertbaren Datensätze, aber die Validität der aus der statistischen Analyse zu gewinnenden Erkenntnisse wird deutlich verbessert. Insofern wird der Verlust eines Teils der Stichprobenbasis in Kauf genommen. 


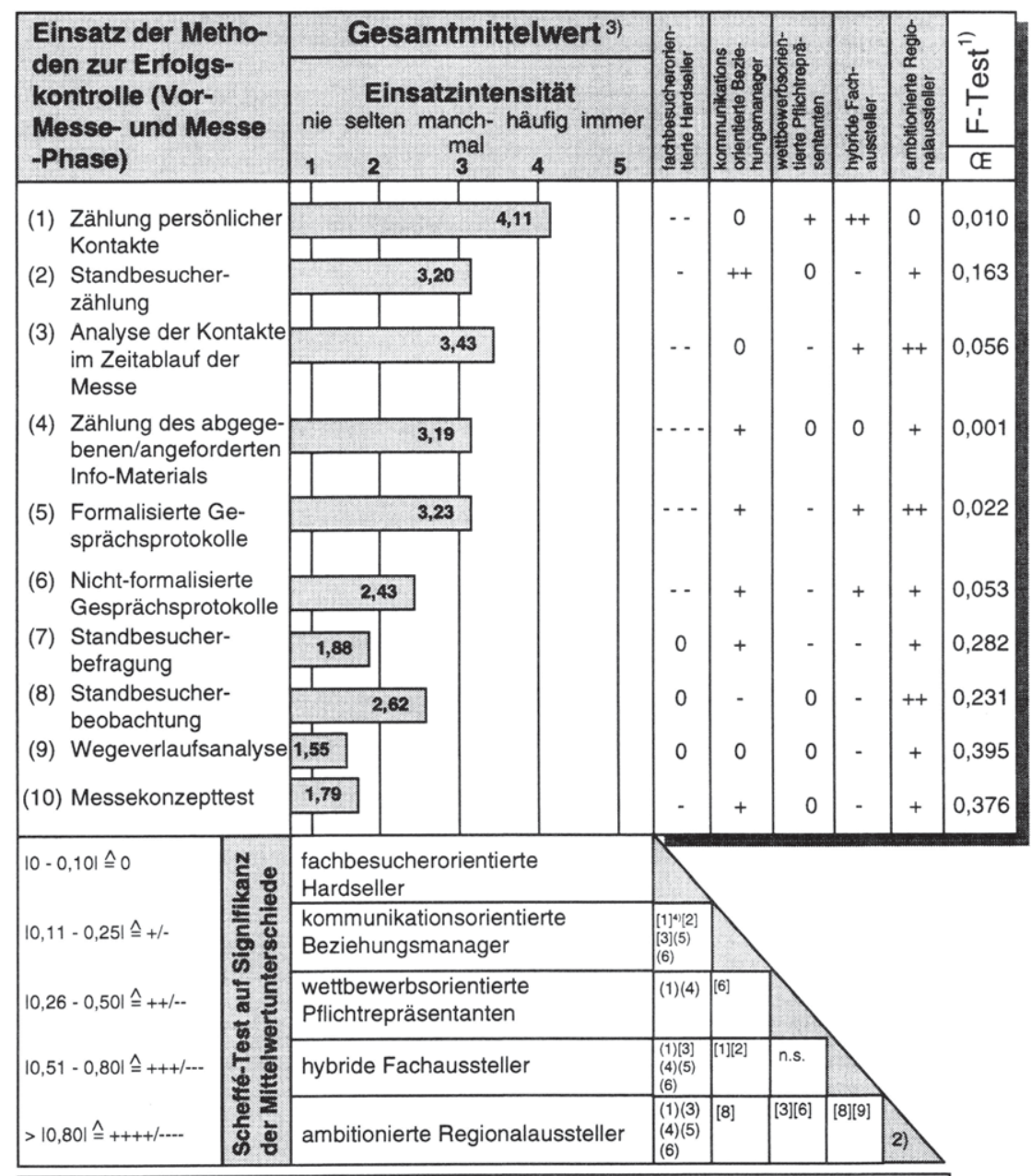

1) One-way-Analyse zur Globalbeurteilung der Mittelwertunterschiede

2) Angabe der Signifikanzniveaus: (i) $\hat{=} \alpha=0,05 /[i] \hat{=} \alpha=0,10$

3) Standardabweichung vom Gesamtmittelwert zwischen 1,18 und 1,83

4) Ziffern beziehen sich auf die jeweiligen Items in der Tabelle

Tab. 12: Varianzanalytische Prüfung auf Unterschiede beim Einsatz der Methoden der Erfolgskontrolle in der Vor-Messe- und Messe-Phase zwischen den messespezifischen Grundhaltungstypen 
Mit Blick auf die einzelnen Grundhaltungstypen lassen sich mit Hinweis auf den Scheffé-Test einige signifikante Unterschiede zwischen den Ausstellergruppen feststellen. Als am stärksten kontrollavers kristallisiert sich in der Vor-Messe- und Messe-Phase das Cluster der fachbesucherorientierten Hardseller heraus. Hier zeigt sich deutlich, daß für diese Aussteller Erfolgskontrollen in dieser Phase ihrer Messebeteiligung kaum eine relevante Rolle spielen, was nicht zuletzt auf ihre überdurchschnittliche Fokussierung auf den Verkauf während der Messe zurückführbar sein dürfte. Erstaunlich ist aber in diesem Zusammenhang dennoch, daß die Aussteller dieses Clusters auch deutlich unterdurchschittliche Anstrengungen unternehmen, die Gespräche mit den Fachbesuchern für Zwecke des Nachmessegeschäfts zu protokollieren. Ähnlich inaktiv verhalten sich ebenfalls die wettbewerbsorientierten Pflichtrepräsentanten. ${ }^{413}$

Kontrollaktiv verhalten sich dagegen die ambitionierten Regionalaussteller, die nahezu jede der genannten Methoden vergleichsweise am intensivsten zur Kontrolle des eigenen Messebeteiligungserfolges einsetzen. Neben den Zählungen persönlicher Kontakte, die auch bei diesen Ausstellern die höchste Priorität genießen ( $M W=4,14)$, sind es vor allem der Einsatz von formalisierten Gesprächsprotokollen ( $M W=3,71$ ) und die Beobachtung der Standbesucher (MW $=2,94$ ), durch die sich dieses Cluster besonders in dieser Phase der Messebeteiligung auszeichnet. Eine stark selektive Auswahl von Methoden zur Kontrolle ihres Messebeteiligungserfolges nehmen dagegen die hybriden Fachaussteller vor. Im Vergleich zum Durchschnitt der Aussteller sind diese überduchschnittlich auf die Auswertung der persönlichen Kontakte mit den Messestandbesuchern fokussiert, was durch den vergleichsweise starken Einsatz der Zählung persönlicher Kontakte zwischen Besucher und Personal, der Analyse dieser Kontakte im Zeitablauf der Messe sowie auf die Protokollierung der Gesprächsinhalte determiniert wird.

Weniger die Feststellung qualitativer Komponenten der individuellen Kontakte zwischen Standpersonal und -besuchern, als vielmehr die Breitenwirkung der Messebeteiligung steht im Vordergrund der Erfolgskontrolle im Cluster der kommunikationsorientierten Beziehungsmanager. Diese Aussteller setzten als einzige überdurchschnittlich stark das Instrument der Standbesucherzählung ein

413 Insbesondere hinsichtlich dieser Aussteller scheint sich ihre mangeinde Orientierung an operational formulierten Messebeteiligungszielen auch in einer geringen Nutzungsintensität der Messeerfolgskontrolle niederzuschlagen.

Ralf Ueding and Universität Münster - 978-3-631-75073-5 
( $M W=3,50)$ und zählen das abgegebene bzW. angeforderte Informationsmaterial $(M W=3,40)$. Sie sind zwar hinsichtlich der Erfolgskontrolle im Vergleich zu den anderen Ausstellerclustern aktiver, das absolute Nutzungsniveau deutet dabei aber auf eine eher gelegentliche Anwendung der Methoden hin.

Um zu einem umfassenden Überblick über den Stellenwert der Messeerfolgskontrolle für die Grundhaltungstypen zu gelangen, ist weiterhin die Nutzungsintensität der Kontrollmethoden, die schwerpunktmäßig in der Nach-Messe-Phase greifen, einer Analyse zu unterziehen. Tab. 13 gibt dabei die Ergebnisse der empirischen Untersuchung wieder. Der Verlauf des Gesamtprofils macht dabei zunächst deutlich, daß die Intensität der Nutzung von Methoden zur Erfolgskontrolle sich auch in der Nach-Messe-Phase nicht wesentlich verändert. Kaum ein Instrument wird im Durchschnitt häufiger als im Rahmen von Maßnahmen zur Kontrolle des Messebeteiligungserfolges genutzt. ${ }^{414}$ Eine Ausnahme bildet zum einen die Budgetkontrolle, die allerdings eher als Ausdruck einer strikten Kostenkontrolle denn als Erfolgskontrolle i. S. einer Analyse des Nutzens von Messebeteiligungen zu werten ist. Interessant erscheint zum anderen, daß eine Vielzahl von Ausstellern die Messeerfolgsergebnisse mehrerer Jahre im Rahmen einer Längsschnittanalyse miteinander vergleichen.

Mit Bezug zu den einzelnen Grundhaltungstypen weist der F-Test auf einem Signifikanzniveau von $\alpha=0,1$ für zehn der zwölf Variablen signifikante Mittelwertunterschiede aus. Der differenzierte Scheffé-Test zeigt weiterhin, daß insgesamt 54 Mittelwertpaare mit Irrtumswahrscheinlichkeiten von maximal zehn Prozent signifikant unterschiedlich voneinander sind. Hinsichtlich der Kennziffernanalyse und der Einladungsresonanzanalyse ergeben sich nach dem F-Test keine signifikanten Mittelwertunterschiede zwischen den Grundhaltungstypen.

414 Allerdings macht die relativ hohe Standardabweichung zwischen 1,28 und 1,74 für die angeführten Kontrollmethoden deutlich, daß sich hier erhebliche Unterschiede in der Stichprobe ergeben. 


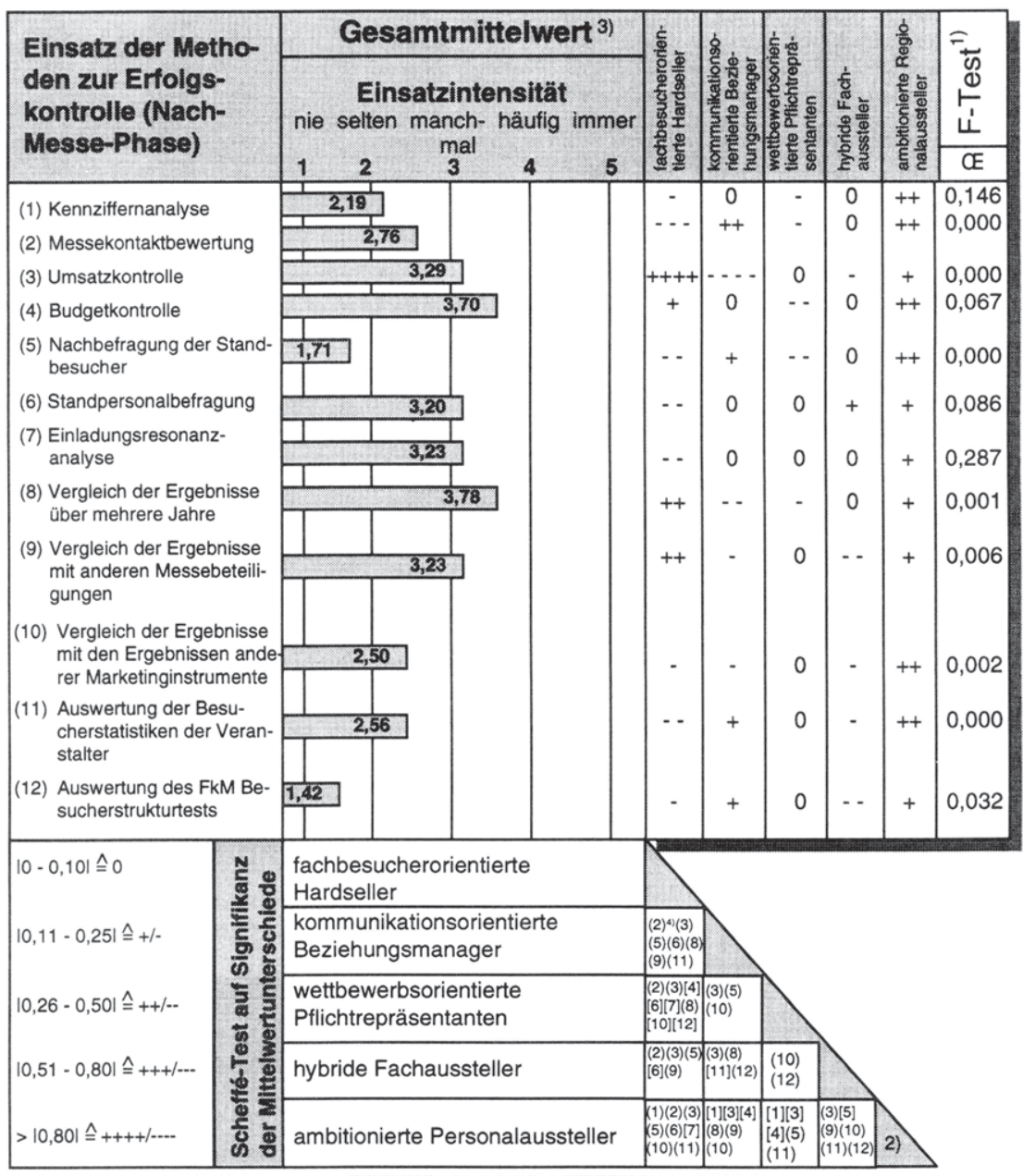

1) One-way-Analyse zur Globalbeurteilung der Mittelwertunterschiede

2) Angabe der Signifikanzniveaus: (i) $\hat{=} \alpha=0,05 /[i] \hat{=} \alpha=0,10$

3) Standardabweichung vom Gesamtmittelwert zwischen 1,28 und 1,74

4) Ziffern beziehen sich auf die jeweiligen Items in der Tabelle

Tab. 13: Varianzanalytische Prüfung auf Unterschiede beim Einsatz der Methoden der Erfolgskontrolle in der Nach-Messe-Phase zwischen den messespezifischen Grundhaltungstypen 
Im Hinblick auf die Besonderheiten der einzelnen Grundhaltungstypen bei der Erfolgskontrolle in der Nach-Messe-Phase ist zunächst auf das Cluster der ambitionierten Regionalaussteller hinzuweisen. Auch in dieser Phase der Messebeteiligung zeigen sie vergleichsweise das mit Abstand umfangreichste Kontrollengagement, wogegen die wettbewerbsorientierten Pflichtaussteller und die hybriden Fachaussteller das entgegengesetzte Kontrollverhalten an den Tag legen. Interessanterweise nutzen die letztgenannten Aussteller einzig die Befragung des eigenen Standpersonals stärker als alle anderen Aussteller. ${ }^{415}$ Das Cluster der fachbesucherorientierten Hardseller fokussiert sich dagegen auf die Kontrolle der Messebeteiligungsumsätze, die auch im Vergleich zu den Vorjahren und mit Beteiligungen an anderen Messen verglichen werden. Die kommunikationsorientierten Beziehungsmanager legen besonderen Wert auf eine Bewertung der Messekontakte, stellen aber in vergleichsweise geringerem Umfang Erfolgsvergleiche mit anderen Messebeteiligungen an.

In einem ersten grundhaltungstypenspezifischen Zwischenfazit hinsichtlich des Einsatzes der Methoden zur Erfolgskontrolle ist folgendes festzuhalten:

Cluster 1: Die fachbesucherorientierten Hardseller konzentrieren sich überdurchschnittlich auf die Kontrolle der Umsätze sowie der Einhaltung des Messebudgets der einzelnen Messebeteiligung und den Erfolgsvergleich i. S. einer Zeitreihenanalyse sowie eines direkten Beteiligungsvergleichs verschiedener Messeveranstaltungen.

Cluster 2: Die kommunikationsorientierten Beziehungsmanager sind stark überdurchschnittlich an Besucherstrukturdaten interessiert. Hierfür nutzen sie insbesondere verschiedene besucherbezogene Zählungen sowie Gesprächsprotokolle und Sekundärstatistiken der Messeveranstalter und unabhängiger Organisationen (FKM) über die Zusammensetzung der Messebesucher.

Cluster 3: Den wettbewerbsorientierten Pflichtausstellern genügt eine quantitative Erfassung der Besucherfrequenz, daher konzentrieren sie sich mehr als der Durchschnitt der Aussteller auf die Zählung von Kontakten bzw. Besuchern am Messestand.

415 Dieses Kontrollverhalten kann als Bezugnahme auf die im Zusammenhang mit dem Zielsystem dieses Clusters diskutiente Mitarbeiterorientierung gewertet werden. 
Cluster 4: Die hybriden Fachaussteller sind ebenfalls an primär kontaktorientierten Informationen interessiert und nutzen mit Gesprächsprotokollen und Standpersonalbefragungen vergleichsweise intensiv ihre Mitarbeiter als Informationsquellen während und nach einer Messeveranstaltung.

Cluster 5: Die ambitionierten Regionalaussteller weisen ein besonders umfangreiches Engagement im Rahmen der Erfolgskontrolle ihrer Messebeteiligungen auf. Zudem vergleichen sie das Ergebnis der einzelnen Messebeteiligung deutlich mehr als andere Aussteller mit den Ergebnissen anderer Messebeteiligungen und Marketinginstrumente. Dies zeigt, daß die Aussteller dieses Clusters den Versuch unternehmen, die Effizienz des gesamten Messeengagements im Wettbewerb der Marketinginstrumente zu erfassen.

Neben der Darstellung der Kontrollintensität der einzelnen Grundhaltungstypen, soll im folgenden der "Fit" zwischen verfolgten Messebeteiligungszielen und dem Einsatz der Methoden der Erfolgskontrolle durchgeführt werden. Hierzu ist auf die Zielkonzeption der Grundhaltungscluster zurückzugreifen. Ausgehend von der Annahme, daß wichtigere Zielsetzungen eher einer Kontrolle hinsichtlich ihrer Zielerreichung unterzogen werden als die nicht priorisierten Ziele, soll die folgende Analyse auf die Messebeteiligungsziele mit den jeweils höchsten Bedeutungsgewichten begrenzt werden. ${ }^{416}$ Diesen Zielsetzungen sollen im Rahmen einer "Fit-Analyse" die Methoden der Erfolgskontrolle gegenübergestellt werden, die in den jeweiligen Clustern im Durchschnitt der Befragten mindestens manchmal eingesetzt werden. ${ }^{417}$

416 Dabei wird die Betrachtung aus praxeologischen Gründen auf die Analyse derjenigen Ziele jedes Clusters eingegrenzt, die eine überdurchschnittliche Wichtigkeit aufweisen. Daher werden nur solche Zielsetzungen berücksichtigt, die auf der im Fragebogen gewählten 5-poligen Skala im Durchschnitt der Cluster mindestens einen Wert von 4 aufweisen (wobei 1 = sehr gering(e) und $\mathbf{5}=$ sehr groß(e) Zielwichtigkeit bedeuten).

417 Die Fokussierung der relevanten Methoden zur Erfolgskontrolle auf diejenigen mit zumindest regelmäßiger Einsatzintensität erscheint deshalb sinnvoll, da es sich bei den Messebeteiligungszielen um generelle Zielsetzungen handelt, die nicht messebeteiligungsspezifisch, sondern -übergreifend verfolgt werden. Regelmäßig eingesetzte Methoden der Erfolgskontrolle weisen in diesem Zusammenhang die höchste Wahrscheinlichkeit auf, messeübergreifend eingesetzt zu werden. 
Die Ergebnisse dieser Gegenüberstellung sind in Tab.14 wiedergegeben. Mit Blick auf die Messebeteiligungsziele zeigen sich zunächst drei deutliche Phänomene: Zum einen beschränken sich die mit hoher Priorität von den Grundhaltungsclustern verfolgten Zielsetzungen auf eine geringe Anzahl von Zieldimensionen. In diesem Zusammenhang wird deutlich, daß Kontakt- bzw. Kundenbindungs- und produkt- bzw. unternehmensbezogene Bekanntheits- und Kompetenzziele das Zielsystem der Aussteller sowohl insgesamt als auch in den Grundhaltungsclustern wesentlich bestimmen. Zum anderen wird aber auch deutlich, daß sich nicht nur die Zielgewichte hinsichtlich identischer Zielsetzungen z. T. deutlich in den Grundhaltungsclustern unterscheiden, sondern sich auch deutliche Unterschiede in der Anzahl der prioritär verfolgten Zielsetzungen ergeben.

Hinsichtlich des Einsatzes der Methoden zur Erfolgskontrolle zeigt sich ebenfalls, daß nicht nur die absolute Anzahl der mindestens manchmal eingesetzten Methoden der Erfolgskontrolle erheblich zwischen den Clustern divergiert, sondern auch ihre jeweilige Anwendungshäufigkeit. Während fachbesucherorientierte Hardseller lediglich drei Methoden regelmäßig einsetzen, nutzen die ambitionierten Regionalaussteller dagegen elf Verfahren mindestens manchmal. In diesem Zusammenhang ist auf die oben festgestellten Unterschiede in der Anzahl der gleichzeitig mit hoher Priorität verfolgten Messebeteiligungsziele sowie auf die Unterschiede in den Zielgewichtungen beider Cluster hinzuweisen, die maßgeblich für den deutlich unterschiedlichen Methodeneinsatz verantwortlich sein dürften.

Eine grundhaltungstypenbezogene Analyse läßt zunächst für die fachbesucherorientierten Hardseller deutlich werden, daß diese die Erreichung der stark in den Vordergrund gestellten Verkaufsziele durch den häufigen Einsatz von Umsatzkontrollen prüfen. Die Erreichung von Kontaktzielen wird lediglich hinsichtlich ihrer quantitativen Dimension durch den sporadischen Einsatz von Zählungen persönlicher Kontakte festgehalten. Befragungsverfahren von Standbesuchern oder Standmitarbeitern zur Feststellung der von den Standbesuchern wahrgenommenen Produktkompetenz bzw. Veränderungen der Produktbekanntheit werden von diesen Ausstellern selten bzw. nie durchgeführt. Über den Grad der diesbezüglichen Zielerreichung liegen den Ausstellern damit i. d. R. keine relevanten Informationen vor. 


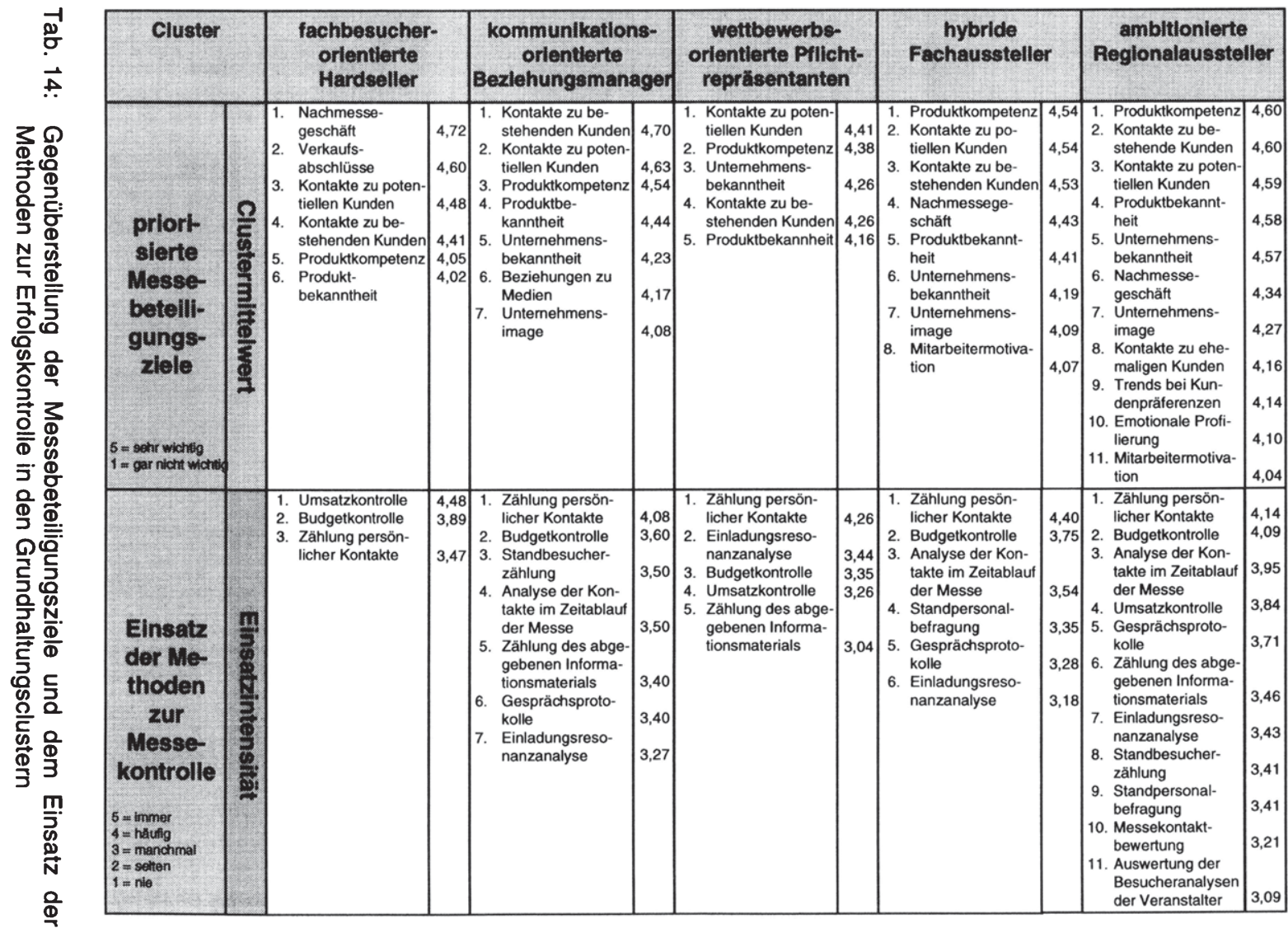


Die kommunikationsorientierten Beziehungsmanager verfolgen neben den stark priorisierten Kontakt- bzw. Beziehungszielen auch produkt- und unternehmensbezogene Bekanntheits- und Imageziele. Während erstere sich hinsichtlich ihrer quantitativen Erreichung durch den Einsatz von Zählverfahren (Zählung der persönlichen Kontakte, der Standbesucher, des abgegebenen Informationsmaterials, Einladungsresonanzanalyse) und hinsichtlich qualitativer Kontaktkriterien durch den Einsatz von Gesprächsprotokollen überprüfen lassen, erfolgt kaum ein Einsatz adäquater Methoden zur Kontrolle der Bekanntheits- und Imageziele. Allenfalls durch eine qualitative Interpretation der Einladungsresonanz sowie durch entsprechende Anmerkungen in den Gesprächsprotokollen der Standmitarbeiter ließen sich schwache Indikatoren hinsichtlich des Image des Ausstellers bzw. seiner Produkte bei denjenigen Standbesuchern ableiten, mit denen eine persönliche Kontaktaufnahme möglich war. Das anonyme Stand- bzw. Messepublikum wird dabei nicht berücksichtigt.

Bei Betrachtung der zentralen Zielsetzungen der wettbewerbsorientierten Pflichtaussteller fallen die produktbezogenen Zielaspekte "Darstellung der Produktkompetenz" und "Erhöhung der Unternehmensbekanntheit" als Besonderheiten dieses Clusters auf. Ebenso wichtig sind allerdings Kontaktziele hinsichtlich potentieller und bestehender Kundenbeziehungen. ${ }^{418}$ Eine Analyse des Einsatzes der Methoden zur Erfolgskontrolle der Messebeteiligungen zeigt aber, daß lediglich die Zählung der persönlichen Kontakte am Messestand mit hoher Intensität betrieben wird. Die anderen Verfahren werden dagegen eher sporadisch eingesetzt. Auch hier gilt, was bereits für das zuvor behandelte Cluster bemerkt wurde, daß die eingesetzten Methoden zwar die quantitative Erfassung von Kontakten unterstützen, allerdings über die Erreichung der Kompetenzdarstellungs- und Bekanntheitsziele nur wenig Aussagen getroffen werden können. Interessanter Weise stellen die Aussteller dieses Clusters mit durchaus höherer Intensität Umsatzkontrollen an, obwohl Verkaufsziele einen eher geringen Stellenwert in ihrem Gesamtzielsystem darstellen. ${ }^{419}$ Mit dieser Vorgehensweise bei der Erfolgskontrolle kann allerdings die Erreichung der Mehrzahl der prioritären Messebeteiligungsziele dieses Clusters nur ansatzweise überprüft werden.

418 Insgesamt ergeben sich aber die im Vergleich aller Grundhaltungstypen niedrigsten Zielgewichte, was die bereits an anderer Stelle dieser Arbeit festgestellte geringe Zielorientierung dieser Aussteller unterstreicht.

419 Verkaufsziele gehen mit einem Mittelwert von 3,06 in das Zielsystem der Aussteller dieses Clusters ein und sind damit von eher nachrangiger Bedeutung. 
Die hybriden Fachaussteller sind dagegen durch ihr "Methoden-Mix" der Erfolgskontrolle weitgehend in der Lage, Aussagen über die Erreichung ihrer wesentlichen Messeziele zu machen. Die auch hier angestrebten Kontaktziele können hinsichtlich ihrer Erreichung durch den Einsatz der Zählung der persönlichen Kontakte, der Analyse der Kontakte im Verlauf der Messe sowie der Einladungsresonanzanalyse bereits quantitativ kontrolliert werden. Darüber hinaus geben Gesprächsprotokolle sowie Standpersonalbefragungen die "indirekte" Möglichkeit, Eindrücke hinsichtlich der beim Standbesucher wahrgenommenen Kompetenzanmutung bzw. Produkt- und Unternehmensbekanntheit zu sammeln. Eine direkte Messung dieser Wirkungen auf die Messebesucher erfolgt jedoch selten. Mit dem Instrument der Standpersonalbefragung setzen diese Aussteller darüber hinaus ein Verfahren ein, daß zur direkten Überprüfung von Motivationswirkungen bei Mitarbeitern geeignet erscheint.

Wie bereits zuvor angedeutet, verfolgen die ambitionierten Regionalaussteller die vergleichsweise hochgestecktesten Ziele und wenden das breiteste Spektrum an Maßnahmen zur Kontrolle des Messebeteiligungserfolges ein. ${ }^{420}$ Eine Analyse der regelmäßig eingesetzten Methoden der Erfolgskontrolle zeigt, daß vor allem die Erreichung der Kontaktziele in quantitativer und qualitativer Hinsicht umfassend überprüft und bewertet (Messekontaktbewertung) wird. Für die Bewertung der Zielerreichung hinsichtlich der Kompetenz- und Bekanntheitsziele, werden auch in diesem Cluster schwerpunktmäßig solche Methoden genutzt, die lediglich indirekte Schlüsse bzgl. eines angestrebten Zielerreichungsgrades zulassen. Bemerkenswert erscheint die relative Gleichgewichtung der eingesetzten Verfahren, die sich - ähnlich wie die messespezifischen Ziele - auf relativ homogenen Niveau befinden, was für eine methodenübergreifende Kontrollintensität dieser Aussteller spricht.

Der über alle Cluster hinweg hohe Stellenwert der Budgetkontrolle ${ }^{421}$ zeigt schließlich, daß die Einhaltung von Kostenzielgrößen für Messebeteiligungen bei den hier untersuchten Aussteller nahezu selbstverständlich geworden ist.

420 Setzt man dem bspw. die weniger zielorientierten Aussteller der Gruppe der wettbewerbsorientierten Pflichtaussteller entgegen, so erscheint es sinnvoll anzunehmen, daß mit zunehmender Zielorientierung der Aussteller das Niveau der Erfolgskontrolle zunimmt.

421 Dies ist jeweils in Relation zu den anderen Methoden innerhalb eines Clusters zu interpretieren. 
Insgesamt bleibt festzuhalten, daß die Erfolgskontrolle der ausstellenden Unternehmen in den meisten Fällen nicht hinreichend ausgestaltet ist, um über die Erreichung aller wesentlich erscheinenden Messebeteiligungsziele valide Erkenntnisse zu gewinnen. Dabei ist festzustellen, daß die Aussteller vornehmlich solche Methoden der Erfolgskontrolle nutzen, die nur zur quantitativen, nicht aber zur qualitativen Auswertung der Messebeteiligung in der Lage sind. Eine Korrelationsanalyse zur Aufdeckung der Zusammenhänge zwischen dem Einsatz der verschiedenen Verfahren ergab zudem keine signifikanten Beziehungen. Dies zeigt, daß die Methoden der Erfolgskontrolle von den ausstellenden Unternehmen unabhängig voneinander angewendet werden, obwohl inhaltliche Bezüge zwischen einzelnen Methoden bestehen und genutzt werden könnten. ${ }^{422} \mathrm{Da}$ das zur Verfügung stehende Methodenspektrum durch die Aussteller nur sehr selektiv genutzt wird, ist insgesamt davon auszugehen, daß die Vielfalt der Ziele, die mit Messebeteiligungen verfolgt wird, von den meisten Ausstellern nicht hinreichend auf ihre Erreichung hin überprüft wird.

Vor diesem Hintergrund sind die Implikationen, die Aussteller aus den Ergebnissen der Messeerfolgskontrolle ziehen, differenziert zu betrachten. Einerseits ist anzunehmen, daß von den Informationen der Messeerfolgskontrolle aufgrund ihrer Selektivität und Subjektivität ${ }^{423}$ kaum normative Veränderungsempfehlungen ausgehen können. Andererseits eröffnet die Analyse der Verwendung der Ergebnisse der Erfolgskontrolle beim Aussteller, Ansatzpunkte für eine auf die jeweils erforderlichen Informationsbedürfnisse ausgerichteten Anpassung der Erfolgskontrolle von Messebeteiligungen. Darüber hinaus ist nur auf diese Weise eine Einschätzung der Bedeutung von Messeerfolgskontrollen und ihrer Ergebnisse für den Planungsprozeß von Messebeteiligungen möglich.

\subsection{Verwendung der Ergebnisse der Erfolgskontrolle}

Um einen Eindruck über die Verwendung der Ergebnisse der Erfolgskontrolle in den Unternehmen zu erhalten lassen sich im folgenden sowohl die Adressaten für die Kontrollergebnisse im ausstellenden Unternehmen als auch die Entschei-

422 Vgl. hierzu die Ausführungen in Kap. C 1.2 dieser Arbeit.

${ }^{423}$ Diese Subjektivität ist insbesondere hinsichtlich qualitativer Kontrollgrößen zu interpretieren, da die Aussteller hier hauptsächlich auf das spezifisch interessengesteuerte Urteil ihrer Standmitarbeiter vertrauen. 
dungsfelder, für die die Informationen der Erfolgskontrolle genutzt werden, analysieren.

Die Ergebnisse der empirischen Untersuchung sind in Tab. 15 dokumentiert. Bei Betrachtung des Gesamtprofilverlaufs zeigt sich, daß die Ergebnisse der Erfolgskontrolle von Messebeteiligungen - im Falle ihrer Erhebung - überwiegend auch in den ausstellenden Unternehmen diskutiert wird. Der geringe Mittelwert von 2,06 zeigt, daß ein Unterlasssen der Diskussion der Messeergebnisse für die große Mehrzahl der Aussteller nicht zutrifft. ${ }^{424}$ Dies bedeutet jedoch, daß im Kreis der befragten Aussteller auch solche zu finden sind, die zwar Erfolgskontrollen durchführen, die gewonnenen Daten aber nicht weiter verwenden. ${ }^{425}$ Hauptadressat für die Präsentation und Diskussion der Messebeteiligungsergebnisse ist die Unternehmensleitung (mit einem MW von 3,87 die absolut höchste Ausprägung). Mit einer nahezu ähnlichen Intensität werden die Messebeteiligungsergebnisse auch an die Verkaufsorganisation weitergegeben, die damit nicht nur über aktuelle Veränderungen im Wettbewerbsumfeld des Ausstellers informiert werden kann, sondern auch in die Lage versetzt wird, eine gezielte Nacharbeitung der Messekontakte durchzuführen.

Ein wesentlicher Adressat ist auch das am Messestand anwesende Personal, das damit in eine aktive Nachbereitung der Messebeteiligung einbezogen wird. Eine untergeordnete Rolle spielt in diesem Zusammenhang die Verdichtung der Messeergebnisse zu einem internen Bericht, der auch den Mitarbeitern zur Verfügung gestellt wird, die nicht am Messestand beteiligt waren.

Die Ergebnisse der Erfolgskontrolle werden in den ausstellenden Unternehmen meist zur Beurteilung des weiteren Engagements bei der zu bewertenden Veranstaltung oder zur Überarbeitung der Messestandkonzeption genutzt. Überdurchschnittlich oft werden die Ergebnisse einzelner Messebeteiligungen auch zum Anlaß genommen, das gesamte Messeengagement zu hinterfragen. Nur eine unterdurchschnittliche Zahl von Ausstellern nutzt die Informationen der Erfolgskontrolle nicht bzw. selten als Basis zukünftiger Messeentscheidungen. ${ }^{426}$

${ }_{424}$ Hier sei darauf hingewiesen, daß im Gegensatz zu den anderen Items der Batterie, ein geringer Wert aufgrund der negativen Antwortvorgabe eher positiv zu werten ist. Erfolgskontrolle aufgrund der Tatsache, daß sie nicht das Informationsbedürfnis der Messeverantwortlichen befriedigen, vermehrt ungenutzt bleiben. 


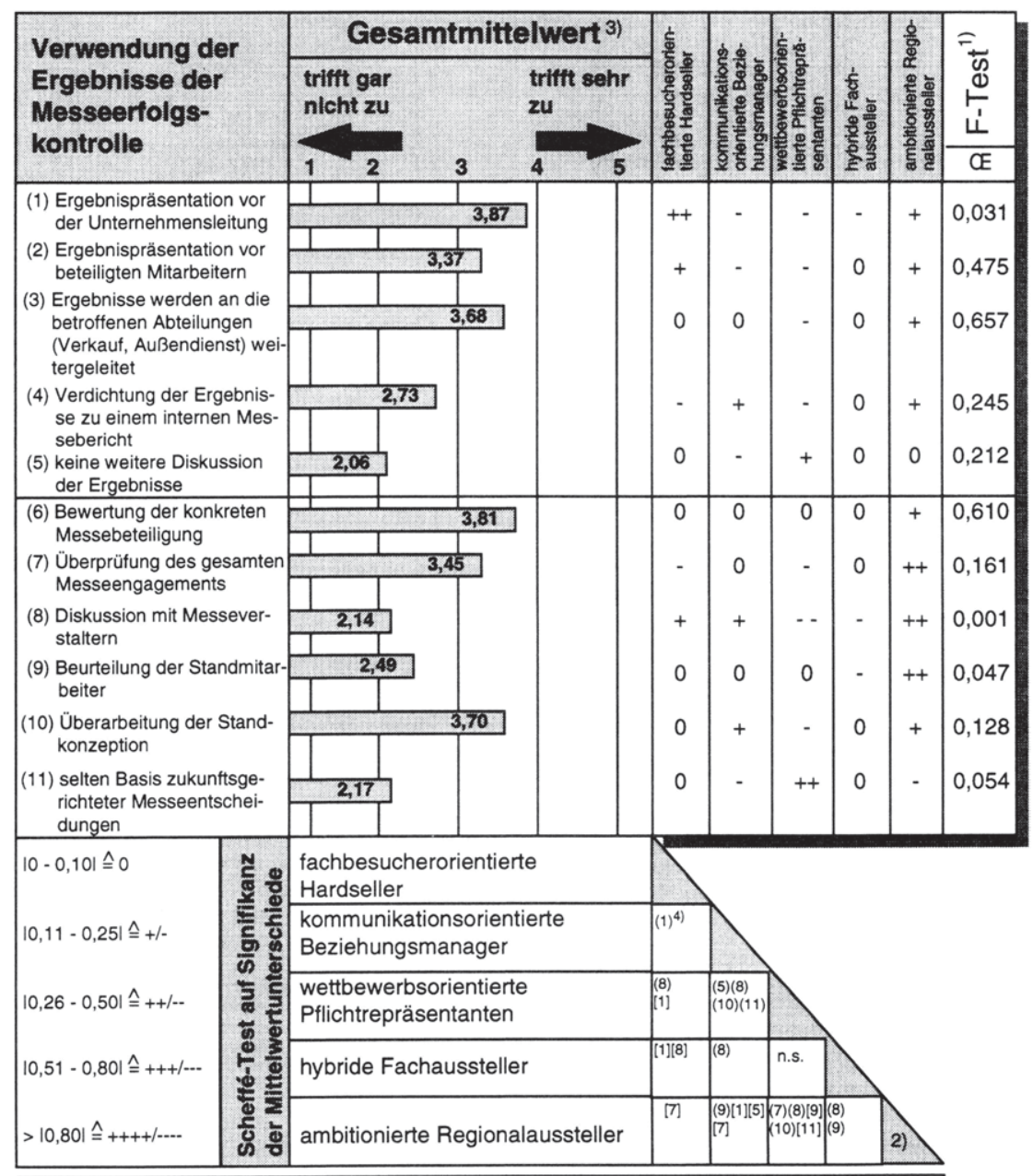

1) One-way-Analyse zur Globalbeurteilung der Mittelwertunterschiede

2) Angabe der Signifikanzniveaus: (i) $\hat{=} \alpha=0,05 /[i] \hat{=} \alpha=0,10$

3) Standardabweichung vom Gesamtmittelwert zwischen 1,14 und 1,53

4) Ziffern beziehen sich auf die jeweiligen Items in der Tabelle

Tab. 15: Varianzanalytische Prüfung auf Unterschiede bei der Verwendung der Ergebnisse der Erfolgskontrolle zwischen den messespezifischen Grundhaltungstypen 
Hinsichtlich der Unterschiede in den fünf Grundhaltungsclustern zeigt der F-Test bei einem Signifikanzniveau von $\alpha=0,1$ lediglich für vier Dimensionen der Ergebnisverwendung von Kontrollinformationen globale Mittelwertunterschiede an. Im Individualbereich der Grundhaltungstypen werden nach dem Scheffé-Test darüber hinaus 22 Mittelwertpaare als signifikant unterschiedlich voneinander ausgewiesen. Die Mittelwertunterschiede beziehen sich hier auf insgesamt sieben Variablen, so daß das Ergebnis der Globalanalyse anhand des F-Tests in seinem Aussagegehalt zu relativieren ist. ${ }^{427}$

Mit Blick auf die einzelnen Grundhaltungstypen zeigt sich zunächst, daß konsequenterweise auch hier die ambitionierten Regionalaussteller das mit Abstand größte Engagement aufweisen und sich insbesondere von den wettbewerbsorientierten Pflichtrepräsentanten deutlich unterscheiden, die hinsichtlich nahezu jeder Verwendungsrichtung der Erfolgsergebnisse eine unterdurchschnittliche Ausprägung aufweisen. Nicht zuletzt deshalb ist zu erklären, daß diese Aussteller kaum zur Diskussion der Messebeteiligungsergebnisse im Unternehmen neigen und diese vergleichsweise seltener als Basis ihrer künftigen Messeentscheidungen nutzen. $^{428}$

Die Art und Weise des Umgangs mit den Ergebnissen der Erfolgskontrolle und ihrer Nutzung für zukünftige Entscheidungen zur Erfolgssteigerung von Messebeteiligungen sind insbesondere auch vor dem Hintergrund organisationsbedingter Besonderheiten zu diskutieren. Es ist davon auszugehen, daß die Handhabung der Erfolgskontrolle insgesamt im Zusammenhang mit dem Autonomiegrad der Messeentscheider und dem Grad der Integration externer Dienstleistungsunternehmen in den Prozeß der Messebeteiligungsgestaltung (insbesondere im Rahmen der Erfolgskontrolle) gesehen werden muß. Zur Erhellung dieses hypothetischen Zusammenhangs soll im folgenden die Messebeteiligungsorganisation und insbesondere die Integration externer Dienstleister bei der Erfolgskontrolle untersucht werden.

427 Diskrepanzen zwischen beiden Testverfahren ergeben sich vor allem dann, wenn nur zwei Mittelwerte signifikant unterschiedlich voneinander sind und die übrigen eng um den Gesamtmittelwert streuen. In diesem Fall weist der F-Test regelmäßig relativ hohe $\alpha$-Werte aus.

428 Hinsichtlich beider Fragen bestehen überdurchschnittliche Ausprägungen, die aufgrund der Struktur der dazugehörigen Fragen als ein vergleichsweise zurückhaltenderes Verhalten der Aussteller zu werten ist. 


\subsection{Analyse der Messebeteiligungsorganisation}

Zur Erhellung des Zusammenhangs zwischen den messespezifischen Grundhaltungen und der Organisation der Messebeteiligungen wurden im Fragebogen hinsichtlich der intraorganisationalen Verankerung der Messebeteiligungsverantwortlichen und zur Prüfung der Frage, inwieweit externe Dienstleistungen im Rahmen von Messebeteiligungen genutzt werden, insgesamt acht Variablen operationalisiert.

Tab. 13 zeigt die von den Ausstellern ergriffenen organisatorischen Optionen im Gesamtprofil über alle Befragten sowie die typenspezifischen Mittelwertabweichungen. Hinsichtlich des Gesamtprofils ergeben sich für die Organisationsvariablen mittlere bis geringe Anwendungsniveaus. Bei drei der acht Variablen weisen die Grundhaltungstypen nach dem globalen F-Test bei einem Signifikanzniveau von $\alpha=0,1$ keine signifikanten Unterschiede auf. Mit Blick auf die typenspezifischen Paarvergleiche wird deutlich, daß sich lediglich die wettbewerbsorientierten Pflichtrepräsentanten und die hybriden Fachaussteller sowie die wettbewerbsorientierten Pflichtrepräsentanten und die fachbesucherorientierten Hardseller nicht signifikant unterscheiden. Alle anderen acht Typvergleiche weisen mindestens hinsichtlich einer Variablen auf einem Signifikanzniveau von $\alpha=0,05$ signifikante Gruppenunterschiede aus.

Eine typenspezifische Analyse der Besonderheiten einzelner Grundhaltungen zeigt, daß vor allem das Cluster der ambitionierten Regionalaussteller eine unterdurchschnittliche Integration externer Dienstleister hinsichtlich operativer Tätigkeiten im Prozeß der Ausgestaltung von Messebeteiligungen vornimmt. Die Aussteller dieser Gruppe wickeln in deutlich höherem Maße die Planung und Durchführung allein ab. Dabei nutzen sie am ehesten von allen Ausstellem eine zentrale Messeabteilung, die eigenverantwortlich die Planung und Abwicklung der Messebeteiligung übernimmt. Hinsichtlich der Nutzung der Dienstleistungen von Messegesellschaften in der Vor-Messe-, Messe- und Nach-Messe-Phase zeigen sie allerdings interessanterweise das insgesamt breiteste Nutzungsniveau. Eine ähnliche Wahrnehmung haben die kommunikationsorientierten Beziehungsmanager, die nicht nur die Services der Veranstalter zumindest in der Vor-Messeund Messe-Phase überdurchschnittlich nutzen, sondern insgesamt die deutlichste Orientierung zu Dienstleistungsanbietern im Rahmen ihrer Messeplanung und durchführung aufweisen. 
Die übrigen Aussteller weisen ein dem Profilverlauf des Gesamtmittelwertes ähnliches Verhalten auf, daß sich vor allem durch ein gleichgewichtiges Nebeneinander von absolut selbständiger Planung und Durchführung von Messebeteiligungen einerseits und der Integration externer Dienstleistungsunternehmen andererseits auszeichnet. Beide Organisationsformen sind damit in den betrachteten Clustern gleichzeitig anzutreffen. Messeabteilungen bzw. eine MesseService-Organisation sind i. d. R. nicht vorhanden, so daß davon auszugehen ist, daß bei den Unternehmen dieser Cluster die Planung der Messebeteiligung durch Entscheider bzw. Teams vorgenommen wird, die neben dieser noch weitere Verantwortlichkeiten aufweisen. ${ }^{429}$ Die oben angeführte Integration externer Dienstleistungsunternehmen in den Prozeß der Planung und Durchführung von Messebeteiligungen erstreckt sich dabei allerdings in nur geringem Maße auf die Serviceangebote der Messeveranstalter sowie auf die Nutzung des Dienstleistungsangebotes von Messemarktforschungsinstituten, die Unterstützungsleistungen bei der Durchführung der Erfolgskontrolle anbieten.

Insgesamt kann damit hinsichtlich des organisatorischen Aspekts von Messebeteiligungen aus Sicht der Aussteller zusammenfassend festgehalten werden: Messebeteiligungen werden heute maßgeblich von den ausstellenden Unternehmen selbst geplant und lediglich bei der operativen Umsetzung wird der Service und das Know how von Dienstleistungsspezialisten fallweise hinzugezogen. ${ }^{430}$ Hinsichtlich der Erfolgskontrolle wird - sofern sie überhaupt durchgeführt wird ohne erkennbare Unterschiede zwischen allen betrachteten Grundhaltungstypen, nahezu gänzlich auf die Unterstützung und das Expertenwissen spezialisierter Anbieter verzichtet.

Nachdem umfassend die messespezifischen Aktivitäten, die Durchführung der Erfolgskontrolle und deren beider Umsetzung in den organisatorischen Konzepten der Aussteller dargestellt wurde, soll im folgenden die Erfolgswahrnehmung des Messeengagements der verschiedenen Grundhaltungstypen einer Analyse unterzogen werden. 429 Z. B. die Unternehmensleitung, die Marketingabteilung oder die Verkaufsabteilung. Vgl. zur
Position des Messeentscheiders in den befragten Unternehmen Kap. D. 4.24 dieser Arbeit.

430 Dies bestätigt damit auch die geringe Relevanz externer Trainer für die Schulung des Standpersonals. Vgl. hierzu nochmals die Ausführungen in Kap. C. 2.21

Ralf Ueding and Universität Münster - 978-3-631-75073-5 


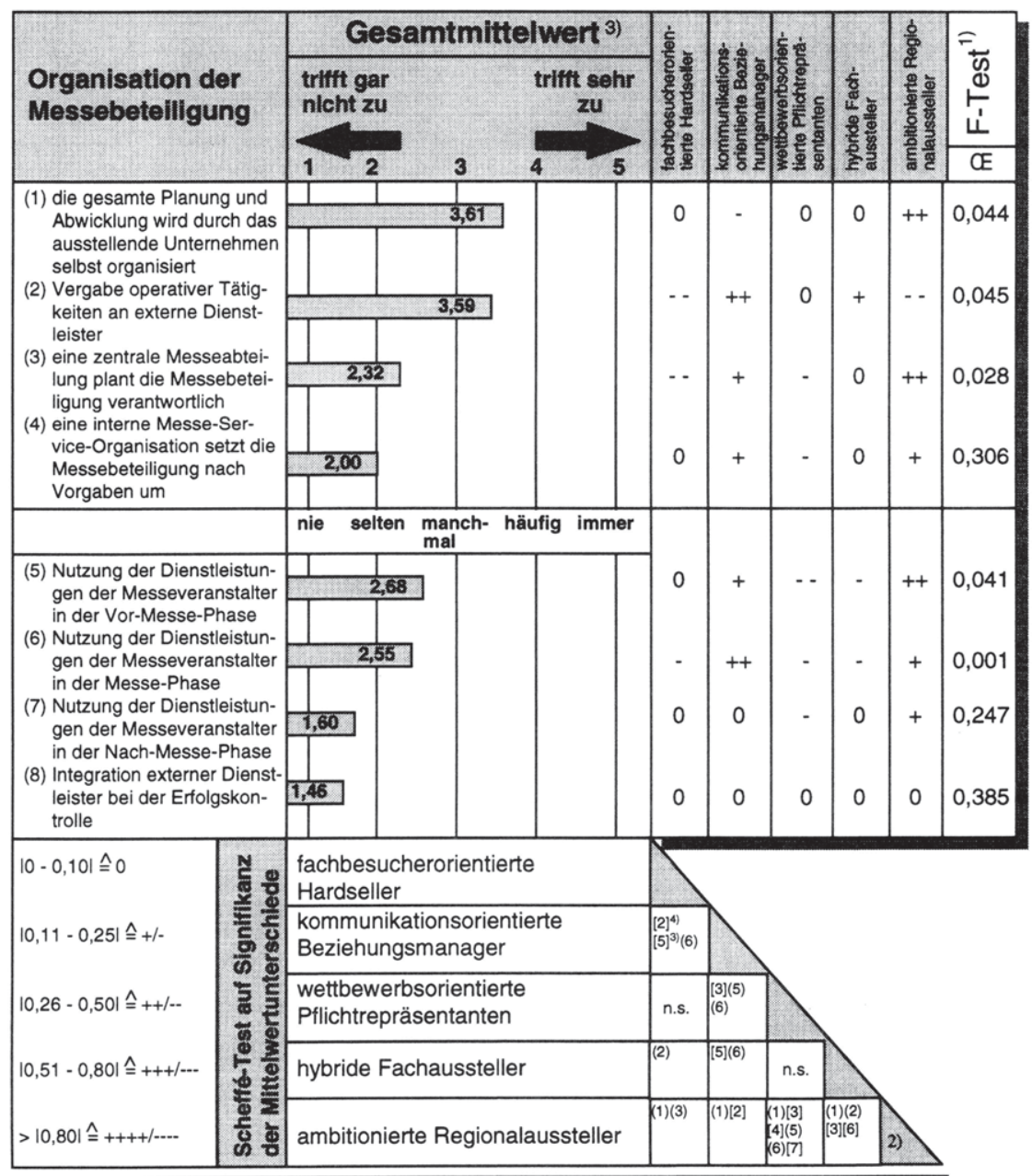

1) One-way-Analyse zur Globalbeurteilung der Mittelwertunterschiede

2) Angabe der Signifikanzniveaus: (i) $\hat{=} \alpha=0,05 /[i] \hat{=} \alpha=0,10$

3) Standardabweichung vom Gesamtmittelwert zwischen 0,95 und 1,60

4) Ziffern beziehen sich auf die jeweiligen Items in der Tabelle

Tab. 16: Varianzanalytische Prüfung auf Unterschiede bei der Organisation der Messebeteiligung zwischen den messespezifischen Grundhaltungstypen 


\subsection{Analyse des Erfolgs von Messebeteiligungen}

Nachdem insbesondere in Kap. C. 2.2 deutlich geworden ist, daß nur die wenigsten Aussteller inre Messeziele stets operational formulieren, nur eine Minderheit der Aussteller regelmäßige Untersuchungen zur Erfolgskontrolle anstellt und darüber hinaus nicht in jedem Fall die Adäquanz der eingesetzten Methoden gewährleistet ist, muß die nachfolgende empirische Analyse des Erfolgs von Messebeteiligungen differenziert betrachtet werden. Zum einen kann aufgrund des dargestellten Zusammenhangs in vielen Fällen nicht von einer objektiven Einschätzung des Erfolgs der Messebeteiligung ausgegangen werden. Zum anderen bleibt aber festzuhalten, daß vor allem der Erreichungsgrad der wichtigsten Messebeteiligungsziele von vielen Ausstellern - wenn auch ohne die theoretisch erforderliche Exaktheit - einer Analyse unterzogen werden muß. Darüber hinaus verfügen die Befragten durch die Beteiligung an durchschnittlich neun Messeveranstaltungen pro Jahr über geeignete Vergleichsmaßstäbe, an denen sie einzelne Messebeteiligungen und ihren relativen Erfolg zumindest abzuschätzen vermögen. ${ }^{431}$

Um die Messung der Erfolgswahrnehmung der ausstellenden Unternehmen zu ermöglichen, wurden diese zunächst gebeten, eine generelle Erfolgseinschätzung inrer Messebeteiligungen abzugeben. Hierzu stand eine 5-polige Skala zur Verfügung die mit "gar nicht erfolgreich“ bei dem Wert 1 und "sehr erfolgreich" für den Wert 5 ausgewiesen war. ${ }^{432} \mathrm{Um}$ die Aussteller zu einer vergleichenden Einschätzung verschiedener Messebeteiligungen und deren generellem Erfolg zu bewegen, wurden diese des weiteren gebeten, die drei Messebeteiligungen, die zusammen den Schwerpunkt ihres Messeengagements darstellen, jeweils hinsichtlich ihres jeweiligen Erfolges einzuschätzen. ${ }^{433}$ Daneben wurde für 24 ausgewählte

431 Damit kann die Kritik an der mangelhaften Validierung des Messeerfolges natürlich nicht gänzlich entkräftet werden, andererseits sollte dies kein Grund für eine Vernachlässigung der häufig subjektiven Erfolgswahrnehmung durch die Verantwortlichen von Messebeteiligungen sein. Es ist anzunehmen, daß die durchschnittlich vorhandenen Messeerfahrungen der befragten Aussteller - wenn zwar nicht zu einer objektiven - zumindest in Ansätzen zu einer rationalen Beurteilung ihres Messeengagements führen. Vgl. zur Entwicklung der Zahl der Messebeteiligungen pro Jahr Meffert, H. Ueding. R., Ziele und Nutzen von Messebeteiligungen, a.a.O., S. 23.

$432 \mathrm{Vgl}$. Frage 6 des sich in Anhang 5 befindenden Fragebogens.

433 Durch die Einschränkung auf die drei wesentlichen Messebeteiligungen wurden zwei Zwecke verfolgt: Zum einen wurde auf diese Weise erreicht, daß die Aussteller nicht ausschließlich "die" wichtigste Messebeteiligung, bei der die Gefahr eines vergleichsweise atypischen Messeverhaltens zu unterstellen wäre, zur Beurteilung ihres generellen Messeerfolges herangezogen haben. Zum anderen ist zu erwarten, daß durch den hohen Stellenwert der ausgewählten Messebeteiligungen insgesamt, die zur Beantwortung der Fragestellung notwendige Präsenz der Messeergebnisse bei den Befragten gegeben war. 
Zieldimensionen ${ }^{434}$ der durch die zuvor abgefragten Messebeteiligungen bewirkte Grad der Zielerreichung abgefragt. ${ }^{435}$ Der auf diese Weise mögliche Vergleich von zwei z. T. subjektiven Erfolgsmaßen für Messebeteiligungen soll dabei zur Objektivierung der Einschätzung der befragten Aussteller beitragen. ${ }^{436}$

Tab. 17 gibt das Ergebnis der grundhaltungsspezifischen Analyse des Erfolgs von Messebeteiligungen wieder. Hinsichtlich des Gesamtprofils ergeben sich für die Mehrzahl der Messebeteiligungsziele eher durchschnittliche Zielerreichungsgrade.

Betrachtet man die typenspezifischen Mittelwertabweichungen im einzelnen, so zeigt sich bezogen auf die Mehrzahl der Zieldimensionen ein überdurchschnittliches Zielerreichungsniveau lediglich für das Cluster der kommunikationsorientierten Beziehungsmanager. Dieser Grundhaltungstyp geht davon aus, die Ziele "Beziehungspflege bzw. -aufbau mit Vertretern der Medien“ und „Erfahrungsaustausch“ stark überdurchschnittlich erreicht zu haben ${ }^{437}$ und vermag aus seiner Perspektive auch hinsichtlich nahezu aller anderen Zielsetzungen

434 Vgl. zur Begründung der Auswahl die entsprechenden Ausführungen in Kap. B. 2 dieser Arbeit.

$435 \mathrm{Vgl}$. hierzu Frage 8 des Fragebogens in Anhang 5 dieser Arbeit.

436 Hinsichtlich des Fragebogendesigns wurden diese beiden Erfolgseinschätzungen getrennt voneinander abgefragt. Zwischen der Beantwortung der beiden Fragen war eine dritte davon unabhängige Frage geschaltet. Zudem wurde zunächst das generelle Erfolgsmaß erfragt, um eine weitestgehend unbeeinflußte Einschätzung beider Frageninhalte zu erreichen.

437 Diese Ziele werden von diesem Grundhaltungstyp auch überdurchschnittlich stark verfolgt. Offensichtlich verfügen diese Aussteller über eine insgesamt höhere Kompetenz beim Umgang mit Pressevertretern und es eröffnen sich informelle Wege des Erfahrungsaustauschs. 


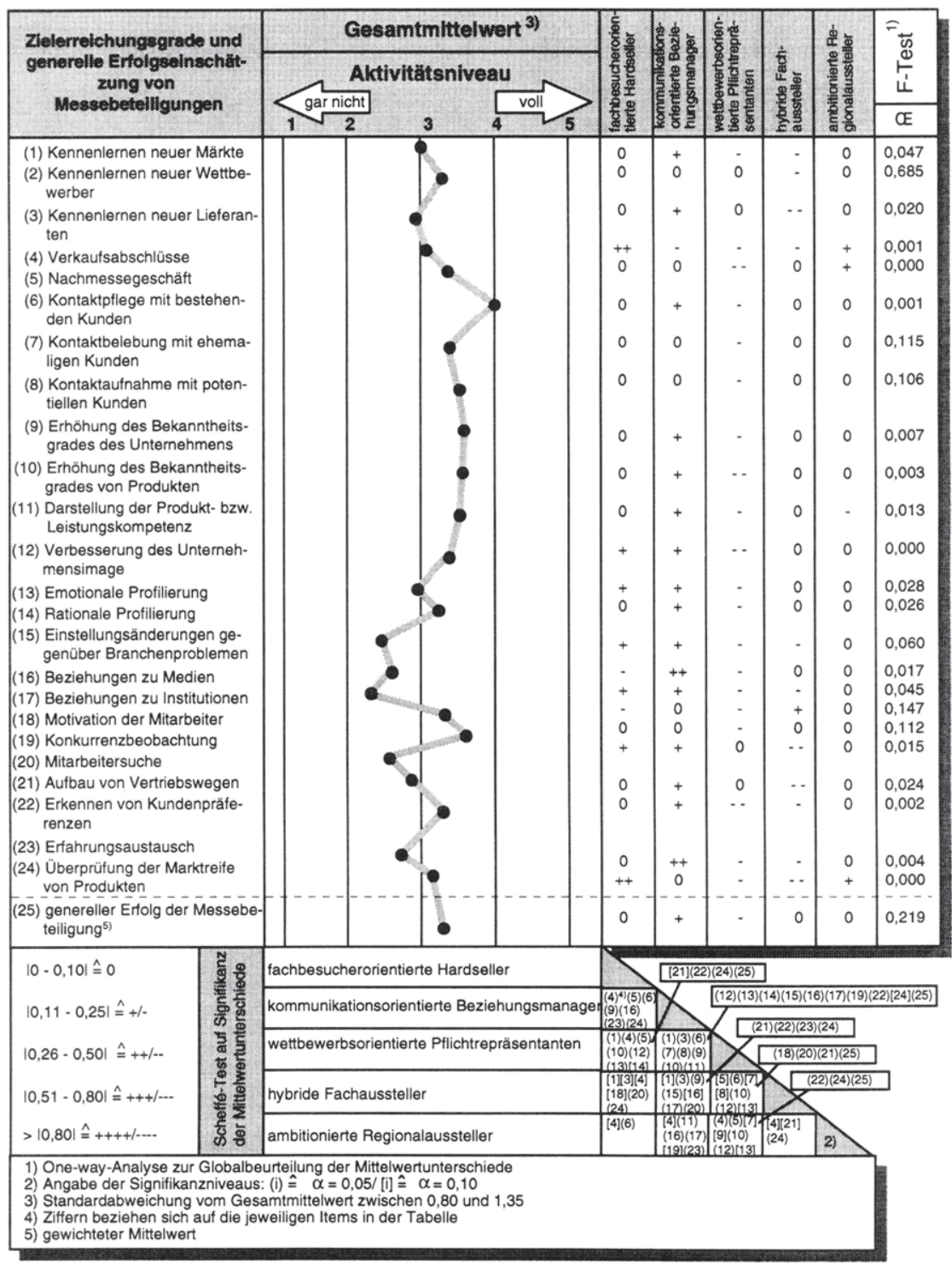

Tab. 17: Varianzanalytische Prüfung auf Unterschiede bei der Zielerreichung und der generellen Erfolgseinschätzung bei Messebeteiligungen zwischen den messespezifischen Grundhaltungstypen 
einen höheren Zielerreichungsgrad als der Durchschnitt der Aussteller umzusetzen. Es kann daher nicht verwundern, daß diese Aussteller insgesamt diejenigen sind, die mit Blick auf das generelle Erfolgsmaß einen überdurchschnittlichen Erfolg ihrer Messebeteiligungen ausweisen. Bemerkenswert sind auch die sich für die wettbewerbsorientierten Pflichtaussteller ergebenden empirischen Befunde. Der Mittelwertvergleichstest weist für nahezu sämtliche Zielvariablen und die generelle Erfolgseinschätzung signifikante, unterdurchschnittliche Ausprägungen auf. ${ }^{438}$

Ein weiterer interessanter Befund ergibt sich für die verkaufsorientierten Hardseller. Zeichnet sich dieser Grundhaltungstyp durch eine extrem starke Fokussierung auf Verkaufsziele aus ${ }^{439}$, so wird aufgrund des vorliegenden Befundes deutlich, daß diese Aussteller nicht nur ihre Verkaufsziele überdurchschnittlich erreichen, sondern auch Ziele wie z. B. "Überprüfung der Marktreife von Produkten" sowie "Verbesserung des Unternehmensimage“. Dennoch gehen die Aussteller lediglich von einem durchschnittlichen Erfolg ihrer Messebeteiligungen aus. Offensichtlich scheinen die überdurchschnittlichen Zielerreichungen in einigen weniger prioritär verfolgten Zielbereichen die nur durchschnittlichen Zielerreichungsbeiträge der Messebeteiligungen in wichtigeren Bereichen nicht kompensieren zu können. ${ }^{440}$

Der Mittelwertvergleichstest weist dagegen kaum signifikante Unterschiede zwischen den hybriden Fachausstellern und den ambitionierten Regionalausstellern aus. Beide Ausstellergruppen realisieren überwiegend durchschnittliche Zielerreichungsgrade und bestätigen diesen Eindruck auch durch eine durchschnittliche Gesamterfolgseinschätzung ihrer Messebeteiligungen. Dieser empirische Befund ist insbesondere mit Bezug auf die ambitionierten Regionalaussteller interessant, da sie die Ausstellergruppe mit dem breitesten mit Messebeteiligungen verfolgten Zielspektrum, das nahezu alle Einzelzielsetzungen mit einem hohen Stellenwert versieht, darstellen. Offensichtlich zeigt sich hier die Gefahr des "Verzettelns" bei zu breit und undifferenziert formulierten Zielsystemen, die im Rahmen dieser Arbeit

438 Diese Aussteller erreichen lediglich hinsichtlich des Ziels "Kontaktpflege mit bestehenden Kunden" einen Mittelwert von 3,76. Die Mehrzahl der anderen Zielerreichungsgrade bewegen sich zwischen 2,50 und 3,00.

Die empirischen Analysen in Kap. B 2.1 haben gezeigt, daß dieser Grundhaltungstyp ein insgesamt weniger breites, auf die Ziele des direkten Verkaufens und des Nachmessegeschäfts ausgerichtetes Zielsystem verfolgt.

440 Dieses Ergebnis stützt damit auch das Vorgehen in Kap C 2.23, bei dem zur Überprüfung des Einsatzes der Verfahren der Erfolgskontrolle und des inhaltlichen Bezugs zu den Messebeteiligungszielen eine Beschränkung auf die wichtigsten Ziele vorgenommen wurde. 
bereits als potentielles Risiko für das Marketinginstrument „Messebeteiligung“ identifiziert und diskutiert worden ist. ${ }^{441}$

Als wesentliches Ergebnis dieser Untersuchung kann zum einen festgehalten werden, daß der Eindruck der generellen Erfolgseinschätzung der Aussteller durch die Festlegung der Zielerreichungsgrade bestätigt wird, so daß von weitgehend homogenen Erfolgs- bzw. Zielerreichungseinschätzungen auf der Individualebene der Aussteller ausgegangen werden kann. Insgesamt ist allerdings für die Grundhaltungstypen ein eher zurückhaltendes Fazit hinsichtlich des Erfolgs der Messebeteiligungen zu ziehen, wobei dieser Befund letztlich nur im Zusammenhang mit den anderen empirischen Ergebnissen zu interpretieren ist. Hierzu soll der Versuch im folgenden zusammenfassenden Kapitel unternommen werden.

\subsection{Zusammenfassende Würdigung der empirischen Ergebnisse}

Die differenzierten Erörterungen im Rahmen der empirischen Analyse der Ausgestaltung von Messebeteiligungen und des Erfolgs verschiedener messespezifischer Grundhaltungstypen haben gezeigt, daß Aussteller im Rahmen ihres Messeengagements verschiedene Aktivitäten ergreifen, die als Ausdruck der jeweils verfolgten Grundhaltung differenziert werden können.

So wurde deutlich, daß insbesondere die kommunikationsorientierten Beziehungsmanager und die ambitionierten Regionalaussteller gemäß ihren ehrgeizig gesteckten kommunikativen Messebeteiligungszielen überdurchschnittlich stark Aktivitäten ergreifen, um im Vorfeld der Veranstaltung auf ihre Messebeteiligung aufmerksam zu machen. Des weiteren wurde aber auch deutlich, daß das Engagement der Aussteller allgemein mit Beginn der Messeveranstaltung nachläßt. Aber auch hier war für die kommunikationsorientierten Beziehungsmanager das vergleichsweise stärkste Aktivitätsniveau zu konstatieren. Damit kann dieses Cluster hinsichtlich der betrachteten Aktivitäten in der Vor-Messe-, der Messe- und der Nach-Messe-Phase als ein Extremtyp bezeichnet werden, dessen Pendant im wettbewerbsorientierten Pflichtrepräsentanten zu sehen ist. Dieser Grundhaltungstyp ist entsprechend seiner geringen Zielorientierung in allen genannten Phasen weit unterdurchschnittlich aktiv. Im Gegensatz zu den beiden aktiven Clustern und den eher als inaktiv zu bezeichnenden wettbewerbsorien-

441 Vgl. die Ausführungen in Kap. B. 1.1 dieser Arbeit und die dort zitierte Literatur.

Ralf Ueding and Universität Münster - 978-3-631-75073-5 
tierten Pflichtrepräsentanten stellen die fachbesucherorientierten Hardseller und die hybriden Fachaussteller Selektivtypen dar, die gemäß ihrer jeweiligen Grundhaltung lediglich ausgewählte Instrumente zur Unterstützung ihrer Messebeteiligung durchführen. Vor diesem Hintergrund ist damit die Hypothese $H_{A k}$, nach der die messebezogenen Aktivitäten der Aussteller signifikant auf ihre verfolgte messespezifische Grundhaltung zurückzuführen sind, anzunehmen.

Als wesentliches Problemfeld für alle Aussteller - unabhängig von ihrer Zugehörigkeit zu bestimmten Grundhaltungstypen - erweist sich dagegen die Operationalisierung der Messebeteiligungsziele. Hier sind kaum typenspezifische Unterschiede auszumachen. Allerdings erwiesen sich insbesondere die fachbesucherorientierten Hardseller und die ambitionierten Regionalaussteller als diejenigen mit der deutlichsten Operationalisierung der Messebeteiligungsziele. Die zuletzt genannten Aussteller sind es auch, die zu den kontrollaktiven Unternehmen zählen, während z. B. die kommunikationsorientierten Beziehungsmanager nicht zuletzt aufgrund der Tatsache, daß sie kaum eine wesentliche Operationalisierung ihrer Zielsetzungen vornehmen, zur Gruppe der kontrollaversen Ausstellern zählen. Dieser Befund bestätigt die Tatsache, daß eine fundierte Erfolgskontrolle nur dann erfolgen kann, wenn im Vorwege die Ziele operational formuliert worden sind. Es kann daher nicht verwundern, daß sich im sich anschließenden Analyseschritt ein signifikant unterschiedlicher Aussagewert der eingesetzten Methoden der Erfolgskontrolle für die einzelnen Grundhaltungstypen ergibt. Während diejenigen Aussteller mit einer relativ weitgehenden Operationalisierung der Zielsetzungen solche Methoden der Erfolgskontrolle einsetzen, mit denen sie z. T. auch tiefergehende Erkenntnisse über den Grad der Zielerreichung ihrer Messebeteiligungen erhalten, ist mit Blick auf diejenigen Aussteller, die ihre Messebeteiligungsziele kaum bzw. gar nicht operationalisieren, festzuhalten, daß der Aussagegehalt der Ergebnisse der eingesetzten Methoden i. S. der Erreichung der verfolgten Ziele nur wenig Informationen zu geben vermögen. Es kann daher kaum verwundern, daß die Mehrzahl der Aussteller hinsichtlich der Zielerreichung bzw. einer generellen Erfolgseinschätzung der Messebeteiligungen weitgehend indifferent ist. Beide Maße weisen allerdings eine bezüglich aller Aussteller hohe Übereinstimmung aus. 
Aufgrund der gewonnenen Erkenntnisse ist erwartungsgemäß festzustellen, daß die Mehrzahl der Aussteller kaum Konsequenzen aus den Ergebnissen der Erfolgskontrolle zieht. Lediglich die ambitionierten Regionalaussteller zeigen ein überdurchschnittliches Interesse, diese Informationen zur konzeptionellen Verbesserung ihres eigenen Messemarketing zu nutzen.

Ein Grund für die fehlende Stringenz bei der Erfolgskontrolle ist neben der weitgehend vernachlässigten Zieloperationalisierung mitunter auch in der geringen Integration externer Dienstleistungsunternehmen in diesem Bereich zu sehen, die ihr spezifisches Know how auf diese Weise kaum in das Marketing ausstellender Unternehmen einzubringen vermögen. In diesem Zusammenhang ist anzumerken, daß insgesamt der Eindruck entsteht, Messebeteiligungen sind allein Sache des ausstellenden Unternehmens. Dienstleistungen von Dritten werden - obwohl, wie gezeigt, vielfach kompetente Kommunikationsspezialisten im Rahmen der Planung und Durchführung der Messebeteiligung hinzuzuziehen wären - nicht in Anspruch genommen.

Insgesamt bleibt damit festzuhalten, daß die Aussteller die kommunikativen und absatzgerichteten Potentiale von Messebeteiligungen nicht zuletzt aufgrund ihrer $\mathbf{z}$. T. ehrgeizigen Zielsetzungen erkannt haben. Allerdings äußern sich grundhaltungsspezifische und auch ausstellerübergreifende Besonderheiten und auch Defizite im Management der Messebeteiligungen, die sich nicht zuletzt nur vor dem jeweiligen situativen Hintergrund der Grundhaltungstypen erklären lassen. Daher sollen die wesentlichen Rahmenbedingungen, innerhalb derer die Aussteller ihre Messebeteiligungen als Marketinginstrument einsetzen, im folgenden zur Erklärung der Unterschiede und Gemeinsamkeiten zwischen den verschiedenen Grundhaltungstypen herangezogen werden. 


\section{Grundhaltungsspezifische Analyse der situativen Einfluß- faktoren}

Aufbauend auf den in Kapitel B abgeleiteten messespezifischen Grundhaltungen und der in Kapitel $C$ vorgenommenen Untersuchung der grundhaltungsspezifischen Ausgestaltung und des Erfolgs von Messebeteiligungen erfolgt nachfolgend eine Analyse situativer Einflußfaktoren. In Analogie zum vorausgegangenen Kapitel werden dabei zunächst die relevanten situativen Einflußgrößen für die messespezifischen Grundhaltungen auf der Basis konzeptioneller Überlegungen abgeleitet. Die im Rahmen dieser Darstellungen formulierten Hypothesen dienen dabei als Grundlage der nachfolgenden empirischen Analyse.

\section{Identifikation von Einflußfaktoren des Management von Messebeteili- gungen}

In der Management- und Organisationstheorie besteht ein breiter Konsens darüber, daß eine Erklärung von Managementphänomenen nicht ohne die Berücksichtigung situativer Faktoren erfolgen kann. ${ }^{442}$ Wenngleich damit zwar der grundsätzliche Einfluß des situativen Kontextes auch auf das Management von Messebeteiligungen als gesichert angesehen werden kann, herrscht dennoch auf der konzeptionellen Ebene hinsichtlich der Art und Anzahl relevanter situativer Faktoren Uneinigkeit. ${ }^{443}$

Grundsätzlich kann in Anlehnung an Kieser und Kubicek zunächst zwischen monovariaten und multivariaten Situationskonzepten unterschieden werden. ${ }^{444}$ Während im Rahmen der monovariaten Ansätze jeweils nur ein als wesentlich erscheinender Situationsfaktor als zentrale Einflußgröße der Managementent-

442 Vgl. Steinmann, H. Schrevögg. G., Management: Grundlagen der Unternehmensführung: Konzepte, Funktionen, Praxisfälle, a.a.O., S. 507 f.; Staehle. W. H., Management: Eine verhaltenswissenschaftliche Perspektive, 4. Aufl., München 1989, S. 47; Brose. P., Konzeption, Varianten und Perspektiven der Kontingenztheorie, in: Journal für Betriebswirtschaft, $34 . \mathrm{Jg}$, Heft 5, 1984, S. 230 f.

443 Vgl. Lehnert. St., Die Bedeutung von Kontingenzansätzen für das Strategische Management, a.a.O., S. $176 \mathrm{ff}$. Lehnert nimmt dabei einen Zusammenhang zwischen dem Detaillierungsgrad der Situationsbestimmung und dem praktischen Nutzen von Kontingenzaussagen in Form eines umgekehrt U-förmigen Verlaufs an.

444 Vgl. hierzu und im folgenden Kieser, A., Kubicek, H., Organisation, 3. Aufl., a.a.O., S. 191 ff. 
scheidungen herausgestellt wird, ${ }^{445}$ verstehen multivariate Konzepte die Situation dagegen als ein mehrdimensionales Konstrukt. Die Wahl einer bestimmten Managementgrundhaltung ist i. d. S. nur durch die Berücksichtigung mehrerer, sich in der Wirkung ergänzender oder auch überlagernder, situativer Einflußfaktoren zu erklären. ${ }^{446}$

Mit Blick auf die Komplexität der im B-Kapitel dieser Arbeit identifizierten messespezifischen Grundhaltungstypen erscheint es wenig realistisch, deren Existenz durch die isolierte Betrachtung eines als dominant erscheinenden Einflußfaktors erklären zu wollen. Der vorliegenden Arbeit ist daher ein mehrdimensionales Situations- und Einflußfaktorenkonzept zugrunde zu legen. Ausgehend von dieser Überlegung soll mit Blick auf die weiteren Untersuchungen zur Erklärung der messespezifischen Grundhaltungstypen, und unter Rückgriff auf die zentralen Aussagen des situativen Ansatzes, der folgenden Basishypothese nachgegangen werden:

H sitt: Die Ausprägungen verschiedener situativer Rahmenbedingungen bzw. Einflußfaktoren eines ausstellenden Unternehmens beeinflussen seine messespezifische Grundhaltung, d. h. die Grundhaltungstypen unterscheiden sich signifikant hinsichtlich ihrer jeweiligen situativen Kontexte.

Die relevanten situativen Einflußfaktoren sind entsprechend der bei Kontingenzansätzen üblichen Differenzierung in Merkmale der unternehmensexternen Situation, welche von dem ausstellenden Unternehmen nicht - oder nur mittelbar beeinflußbar sind, und solche der unternehmensinternen Situation, die dem Entscheidungsbereich der Unternehmung selbst zugerechnet werden können, zu trennen. ${ }^{447}$ Dabei ist die unternehmensexterne Situation zusätzlich in Faktoren der

445 Die wichtigsten Ansätze priorisieren dabei entweder die Unternehmensgröße, die Organisationstechnologie, die Unternehmensumwelt oder die Bedürnnisstruktur der Organisationsmitglieder.

446 Kieser und Kubicek argumentieren entsprechend, wenn sie die Situation als ein offenes Konzept bezeichnen, das in Abhängigkeit vom jeweiligen Forschungsziel zu konkretisieren ist. Vgl. Kieser, A., Kubicek, H., Organisation, 3. Aufl., a.a.O., S. 205.

447 Vgl. hierzu stellvertretend für zahlreiche andere Quellen Kieser, A., Kubicek, H., Organisation, 3. Aufl., a.a.O., S. 208 f.; Meffert, H., Zur Bedeutung von Konkurrenzstrategien im Marketing, in: Marketing ZFP, 7. Jg., Heft 1, 1985, S. 17; Achrol, R. S., Reve, T., Stern, L. W., The Environment of Marketing Channel Dyads: A Framework for Comparative Analysis, in: JoM, Vol. 47 , No. 3,1983, S. 57. 
globalen und der aufgabenspezifischen Umwelt aufzuspalten. ${ }^{448}$ Unter den Einflußfaktoren der globalen Umwelt werden Subumwelten, wie die ökonomische, gesellschaftliche, technische, politisch-rechtliche und natürlich-ökologische Umwelt, subsumiert. ${ }^{449}$ Diesen Einflußfaktoren ist allerdings gemein, daß sie i. d. R. für alle ausstellenden Unternehmen die gleichen Ausprägungen aufweisen, sie insofern also nicht ge日ignet sind, Unterschiede zwischen Ausstellern zu erklären. ${ }^{450}$ Daher sollen sie auch im Rahmen dieser Untersuchung nicht explizit erfaßt werden. ${ }^{451}$

Dagegen weisen die Einflußfaktoren der aufgabenspezifischen Umwelt ausstellerindividuelle Unterschiede auf und sind daher von größerer Erklärungsrelevanz. ${ }^{452}$ Mit Blick auf die ausstellenden Unternehmen sollen ihre Markt- und Wettbewerbssituation betrachtet werden. ${ }^{453}$ Zusätzlich erscheint es angesichts der vorliegenden Problemstruktur der Arbeit unabdingbar, interne Einflußfaktoren hinsichtlich ihrer Wirkung auf die messespezifische Grundhaltung hin zu analysieren. $^{454}$ Zweckmäßigerweise sind in einem nächsten Analyseschritt die situativen Einflußfaktoren zu präzisieren.

448 Becker unterscheidet in inhaltlich weitgehend analoger Weise zwischen der Makro-Umwelt (= globale Umwelt) und der Mikro-Umwelt (= Aufgaben-Umwelt). Vgl. Becker. J., MarketingKonzeption, Grundlagen des strategischen Marketing-Managements, 4. Aufl., a.a.O., S. 76.

Zu einer ähnlichen Systematik vgl. Meffert. H., Marketing, Grundlagen der Absatzpolitik, 7. Aufl., a.a.O., S. $56 \mathrm{ff}$. und hier insbesondere Abb. 7 auf S. 58 .

Dieser Schluß triftt insbesondere deshalb zu, da in diese Untersuchung nur solche Aussteller einbezogen werden, die ihren Firmensitz in Deutschland haben und sich an Messen in Deutschland beteiligen. Vgl. hierzu auch die Ausführungen in Kap. A 4. dieser Arbeit.

Zu einer ähnlichen Argumentation vgl. Meurer, J., Führung von Franchisesystemen - Erklärungsansätze, Verhaltens- und Erfolgswirkungen auf der Grundlage einer empirischen Führungstypologie, Wiesbaden 1997 (im Druck), S. $138 \mathrm{ff}$. und die dort zitierte Literatur.

Mortsiefer beschäftigt sich bspw. insbesondere mit der Wettbewerbssituation von Ausstellern als einem wichtigen Einflußfaktor der Ausstellerheterogenität. Vgl. Mortsiefer, J., Messen und Ausstellungen als Mittel der Absatzpolitik mittelständischer Herstellerbetriebe, a.a.O., S. 531.

Zum zentralen Stellenwert der absatzmarktbezogenen Merkmale "Markt- und Wettbewerbssituation" vgl. Wöllenstein. St., Betriebstypenprofilierung in vertraglichen Vertriebssystemen: eine Analyse von Einflußfaktoren und Erfolgswirkungen auf der Grundlage eines Vertragshändlersystems im Automobilhandel, in: Meffert, $H$. (Hrsg.), Schriften zu Marketing und Management, Bd. 28, Frankfurt a.M., Berlin u. a. 1996, S. $98 \mathrm{ff}$.

454 Zur Relevanz unternehmensinterner Einflußfaktoren vgl. Khandwalla, $\mathrm{P}$., The Design of Organizations, New York u. a. 1977, S. $271 \mathrm{ff}$. 


\section{Markt- und Wettbewerbsbedingungen als zentrale Einflußfaktoren der Aufgabenumwelt}

Bei Betrachtung zentraler kontextbezogener Konzepte, wie sie während der 70er und $80 \mathrm{er}$ Jahre im Bereich der Organisationstheorie erarbeitet wurden, ist insbesondere eine immer stärkere Komprimierung der zur Kennzeichnung der Markt- und Wettbewerbssituation herangezogenen Dimensionen augenscheinlich. Werden von Khandwalla noch fünf relevante Umweltdimensionen diskutiert, verdichtet Mintzberg diese weiter zu vier Dimensionen. ${ }^{455}$ Miller und Friesen erfassen schließlich die Markt- und Wettbewerbssituation nur noch anhand von drei Dimensionen: Umweltdynamik, Heterogenität bzw. Komplexität und Feindlichkeit der Umwelt. ${ }^{456} \mathrm{Da}$ dieses dreidimensionale Umweltkonzept durch seine generalisierende Struktur unmittelbar auch für die Situationserfassung von messespezifischen Grundhaltungstypen geeignet erscheint, wird es den folgenden Ausführungen zugrunde gelegt.

Durch die Dimension der Umweltdynamik wird das Ausmaß der Veränderlichkeit der Markt- und Wettbewerbsbedingungen im Zeitablauf abgebildet. ${ }^{457}$ Eine dynamische Umwelt liegt demzufolge immer dann vor, wenn sich häufige Veränderungen der relevanten Umweltfaktoren ergeben, diese eine starke Ausprägung aufweisen und zudem unregelmäßig auftreten. ${ }^{458}$ Bei jeweils entgegengesetzten Ausprägungen wird von einer statischen Umwelt gesprochen. Die Heterogenitäts- und Komplexitätsdimension erfaßt hingegen die Verschiedenartigkeit und Anzahl der vom Management zu berücksichtigenden Umweltbereiche. Eine

455 Khandwalla differenziert dabei zwischen den Dimensionen: Turbulenz, Feindlichkeit, Diversität, technische Komplexität und Restriktivität der Umwelt. Mintzberg dagegen verdichtet diese auf die folgenden vier Dimensionen: Komplexität, Diversität, Stabilität und Feindlichkeit der Umwelt. Vgl. Khandwalla. P., The Design of Organizations, a.a.O., S. $333 \mathrm{ff}$.; Mintzberg. H., The Structuring of Organizations. A Synthesis of the Research, Englewood Cliffs 1979, S. $268 \mathrm{ff}$.

456 Vgl. Miller. D. Friesen, P. H., Momentum and Revolution in Organizational Adaption, in: Academy of Management Journal, Vol. 23, No. 4, 1980, S. 591 H.; Miller. D. Friesen, P. H., Innovation in Conservative and Entrepreneurial Firms: Two Models of Strategic Momentum, in: SMJ, Vol. 3, No. 1, 1982, S. 1 ff. Die Umweltdynamik wird von den Autoren zuweilen auch als Umweltunsicherheit definiert. Auch Kieser und Kubicek gehen in Anlehnung an Jurkovich von einem dreidimensionalen Ansatz aus, wobei sie anstatt der Feindlichkeit der Umwelt die verwandte Dimension der Abhängigkeit verwenden. Vgl. Kieser, A. Kubicek, $H_{\text {., Organisation, }}$ 3. Aufl., a.a.O., S. 371; Jurkovich, R., A Core Typology of Organizational Environments, in: Administrative Science Quarterly, Vol. 19, o. Nr., 1974, S. $380 \mathrm{ff}$. 
komplexe Markt- und Wettbewerbssituation ist z. B. durch eine Vielzahl unterschiedlicher Kundengruppen, verschiedenartige strategische Verhaltensweisen der Wettbewerber oder aber eine hohe Internationalität der Märkte mit jeweils zu berücksichtigenden länderspezifischen Marktbedingungen gekennzeichnet. ${ }^{459}$

Die Feindlichkeit der Aufgabenumwelt soll im Rahmen der vorliegenden Arbeit insbesondere in Gestalt der absatzmarktbezogenen Teildimensionen der Wettbewerbsintensität und der Marktattraktivität Eingang finden. ${ }^{460}$ Dabei ist gleichermaßen bei einer hohen Wettbewerbsintensität oder einer geringen Marktattraktivität - etwa infolge eines nur geringen Wachstums oder eines niedrigen durchschnittlichen Preisniveaus - von einer feindlichen Unternehmensumwelt auszugehen. Der Grad der Umweltfeindlichkeit steigt um so mehr, je stärker die Intensität beider Ausprägungen kombiniert auftritt.

Nimmt man die Basishypothese $\mathrm{H}_{\text {sitt }}$, wonach sich ein Einfluß der situativen Faktoren auf die messespezifische Grundhaltung von Ausstellern zeigt, zunächst als gegeben an, dann stellt sich, ausgehend von den obigen Überlegungen, unmittelbar die weiterführende Frage, welche Konsequenzen von den möglichen Ausprägungen der drei Umweltdimensionen auf die messespezifische Grundhaltung und damit die Wahl eines bestimmten Grundhaltungstyps ausgehen können. Dieser Frage soll im folgenden nachgegangen werden.

Eine dynamische Umwelt erhöht die Unsicherheit, der sich ein Aussteller bei seinen marktgerichteten Aktivitäten gegenübergestellt sieht. ${ }^{461}$ Die Beschaffung von Informationen und die Prognose zukünftiger Entwicklungen der Markt- und Wettbewerbssituation werden entsprechend erschwert. Messeveranstaltungen vermögen aufgrund ihres Charakters als "Markt für Informationen“462 und ihrer damit einhergehenden Informationsfunktion über Wettbewerber und Kunden den Ausstellern wesentliche Anhaltspunkte hinsichtlich des zu erwartenden Ausmaßes der Wettbewerbsbedingungen und der Marktdynamik zu geben. Es erscheint daher wahrscheinlich, daß in dynamischen Markt- und Wettbewerbsumfeldern verstärkt

459 Vgl. Niemeier, J., Wettbewerbsumwelt und interne Konfiguration: Theoretische Ansätze und empirische Prüfung, Frankfurt a. M., Bern, New York 1986, S. 156. und „Marktattraktivität" Niemeier, J., Wettbewerbsumwelt und interne Konfiguration: Theoretische Ansätze und empirische Prüfung, a.a.O., S. $156 \mathrm{f}$. 
solche messespezifischen Grundhaltungen im Vordergrund stehen, die hinsichtlich der Messebeteiligungsziele eine überdurchschnittliche Informationsorientierung aufweisen.

Hinsichtlich der Auswirkungen der Komplexitätsdimension auf die messespezifische Grundhaltung ist insbesondere aufgrund der mit der Marktkomplexität verbundenen heterogenen Kundenstruktur und dem häufig kaum vorhersehbaren Verhalten der Wettbewerber und Anspruchsgruppen davon auszugehen, daß die ausstellenden Unternehmen vor allem von der Multifunktionalität ${ }^{463}$ der Messeveranstaltungen Gebrauch machen werden, indem sie messespezifische Grundhaltungen entwickeln, die sich durch differenzierte bzw. mehrdimensionale Messezielkonzepte auszeichnen. Ist ein wesentlicher Bestimmungsfaktor dieser Umweltkomplexität die Internationalität des Wettbewerbs bzw. der Kunden, dann liegt es nahe zu vermuten, daß Aussteller mit einer solchen Konstellation der Situationsfaktoren messespezifische Grundhaltungen aufweisen, die insbesondere im Rahmen der Messeselektion auf die Internationalität der Messeveranstaltungen rekurrieren. $^{464}$

Der Einfluß der über die Dimensionen der Wettbewerbsintensität und der Marktattraktivität $^{465}$ erfaßten Feindlichkeit der Aufgabenumwelt auf die Entwicklung messespezifischer Grundhaltungen ist differenziert zu betrachten. Einerseits ist davon auszugehen, daß Messebeteiligungen gerade in solchen Marktsituationen einen hohen Stellenwert besitzen, in denen eine hohe Wettbewerbsintensität - also eine hohe Umweltfeindlichkeit - festzustellen ist. Im Sinne einer Nutzung der Messebeteiligung als Instrument zur Profilierung im Wettbewerb kann dabei eine positive Korrelation zwischen der Wettbewerbsintensität und der Zielfokussierung der messespezifischen Grundhaltung der Aussteller angenommen werden. Andererseits zeigen zahlreiche Beispiele, daß bei geringer Attraktivität der Märkte -

463 Vgl. zum Begriff der Multifunktionalität von Messen Groth, C. Lentz, I., Die Messe als Dreh- und Angelpunkt - Multifunktionales Instrument für ein erfolgreiches Marketing, a.a.O., S. 16 f.

$464 \mathrm{Vgl}$. Ebert. D., Weltweite Entwicklungstendenzen im Messewesen, in: Strothmann, K. $-\mathrm{H}$. Busche, M. (Hrsg.), Handbuch Messemarketing, Wiesbaden 1992, S. 41 f., der Messen zunehmend als Forum für Informationen mit weltweiter Ausstrahlung und "mehr denn je als multilaterale Drehscheiben, auf denen die Aussteller alle wichtigen Entscheider aus allen wichtigen Märkten treffen", bezeichnet.

Im Zusammenhang mit der Marktattraktivität werden vielfach vornehmlich Einflüsse des Marktwachstums und des damit einhergehenden Marktpotentials untersucht. Vgl. dazu Meffert.

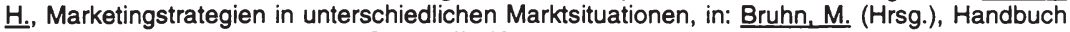
des Marketing, München 1989, S. 277 ff.; Kotler, Ph., Bliemel, F., Marketing-Management, 7. Aufl., Stuttgart 1992, S. $543 \mathrm{ff}$. 
also bei einer ebenfalls weitgehend als feindlich zu bezeichnenden Umweltsituation - auch die entsprechenden Messen wenig attraktiv für ausstellende Unternehmen erscheinen. Somit ist mit abnehmender Marktattraktivität auch der weitgehende Verlust einer klaren Zielstruktur gegenüber Messebeteiligungen zu enwarten.

Zusammenfassend sollen die Einflüsse der drei Dimensionen der Aufgabenumwelt auf die messespezifische Grundhaltung von Ausstellern in Gestalt verschiedener Hypothesen den empirischen Untersuchungen zugrunde gelegt werden:

Hsı: Je höher die Dynamik und Unsicherheit des Markt- und Wettbewerbsumfeldes ausgeprägt ist, desto häufiger werden die stark informationsorientierten Messegrundhaltungstypen wie der ambitionierte Regionalaussteller oder der kommunikationsorientierte Beziehungsmanager dominieren.

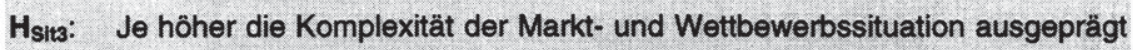
ist, um so häufiger wird eine differenzierte Zielkonzeption aufweisende Grundhaltung wie die der kommunikationsorientierten Beziehungsmanager bei Messebeteiligungen realisiert.

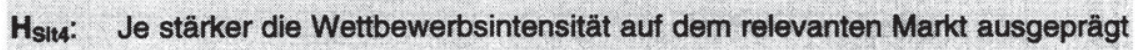
ist, desto häufiger wird der durch eine hohe Zielfokussierung geprägte Messegrundhaltungstyp des fachbesucherorientierten Hardsellers umgesetzt.

Hsits: Je geringer die Attraktivität des relevanten Marktes ausgeprägt ist, desto häufiger wird der kaum eine klare Zielstruktur aufweisende Grundhaltungstyp des wettbewerbsorientierten Pflichtausstellers realisiert. 


\section{Interne Einflußfaktoren der messespezifischen Grundhaltung}

Während zu den Einflußfaktoren der Aufgabenumwelt ausschließlich jene Merkmale gezählt wurden, die aus dem Verhalten vieler, nicht zum Einflußkreis des ausstellenden Unternehmens gehörenden Akteure resultieren, umfaßt die interne Situation diejenigen Faktoren, die auf das Unternehmen selbst zurückgehen. Gleichwohl bedeutet dieser Umstand nicht unmittelbar, daß diese Faktoren in jedem Fall kurzfristig disponierbare Aktionsparameter darstellen. Vielmehr sind die meisten internen Situationsvariablen nur längerfristig beeinflußbar. ${ }^{466} \mathrm{Im}$ folgenden sollen die für diese Untersuchung relevanten internen Einflußfaktoren näher skizziert werden.

\subsection{Stellenwert und Integration im Kommunikations-Mix}

Die Beteiligung an Messeveranstaltungen erfordert i. d. R. von den Unternehmen ein erhebliches Kommunikationsbudget und eine entsprechende Bindung qualifizierter Mitarbeiter für einen längeren Zeitraum. ${ }^{467}$ Nicht zuletzt aus diesen Gründen wird der Entscheidungsprozeß, sich an einer Messe als Aussteller zu beteiligen, häufig auch von der Überlegung beeinflußt, den hierfür notwendigen Budgetanteil innerhalb alternativer Instrumenteoptionen im Kommunikations-Mix zu verwenden. $^{468}$

Die Bedeutung von Messebeteiligungen für das Marketing und insbesondere für die Unternehmenskommunikation läßt sich daher an ihrem Stellenwert im Vergleich zu den anderen Kommunikationsinstrumenten festmachen. Die Zusammensetzung des Kommunikations-Mix ${ }^{469}$ sowie die relative Bedeutung der darin

466 Vgl. Patt, P.-J., Strategische Erfolgsfaktoren im Einzelhandel: Eine empirische Analyse am Beispiel des Bekleidungsfachhandels, in: Meffert, $H$. (Hrsg.), Schriften zu Marketing und Management, Bd. 16, Frankfurt a. M., Berlin u. a. 1988, S. 47.

467 Vgl. Täger, U. C. Ziegler, R., Die Bedeutung von Messen und Ausstellungen in der Bundesrepublik Deutschland für den Inlands- und Auslandsabsatz in ausgewählten Branchen, a.a.O., S. 144.

468 Vgl. Jaspersen, $H_{\text {. }}$, Messen beschicken oder Anzeigen schalten? Eine Antwort mit Zahlen, in: Marketing Journal, Heft 5, 1978, S. 456; Rost, D., Ausstellen oder Aussteigen?, a.a.O., S. 47.

469 Vgl. zur umfassenden Abgrenzung relevanterer Kommunikationsinstrumente Tietz, B., Das Konzept des integrierten Kommunikations-Mix, in: Tietz, B. (Hrsg.), Die Werbung - Handbuch der Kommunikations- und Werbewirtschaft, Bd. 3, Landsberg/Lech 1982, S. 2265-2297, insb. S. 2272; Meffert. H., Integrierte Marktkommunikation, Münster 0.J., S. 2 ff. 
enthaltenen Instrumente sind dabei als Ausdruck einer instrumentestrategischen Ausrichtung ${ }^{470}$ von Unternehmen zu interpretieren, die insbesondere die hierarchischen Beziehungen i. S. einer Rangordnung zwischen den einzelnen Kommunikationsinstrumenten betont.

Mit der Komplementarität von Kommunikationsinstrumenten ${ }^{471}$ sowie ihrem jeweiligen Anteil an strategischen und taktischen Komponenten ${ }^{472}$ werden in diesem Zusammenhang in der Literatur zwei wesentliche Komponenten zur Bildung einer solchen Rangordnung herangezogen. ${ }^{473}$

Betrachtet man den Grad an Komplementarität von Messebeteiligungen, dann wird dieser durch die Frage bestimmt, ob Messebeteiligungen einen eher konstruktiven oder ergänzenden Charakter für das Kommunikationskonzept von ausstellenden Unternehmen aufweisen. Eine strategische Bedeutung von Messebeteiligungen liegt dann vor, wenn diese einen „strukturellen“ bzw. "mittel- bis langfristigen Charakter besitzen". ${ }^{474}$ In diesem Zusammenhang ist im allgemeinen davon auszugehen, daß mit steigender Bedeutung von Kommunikationsinstrumenten auch ihre strategische Verankerung im Kommunikationskonzept wächst. Nicht zuletzt deshalb erscheint es sinnvoll, die strategische Bedeutung von Kommunikationsinstrumenten an ihrer Fähigkeit zu messen, die strategischen Marketing- und Kommunikationsziele des Unternehmens (z. B. Marktanteil, Umsatz, Gewinn) bei den Nachfragern bzw. im Markt durchzusetzen. Hier ergibt sich insofern ein direkter Zusammenhang zur Diskussion über die Komplementarität und Bestimmung von konstitutiven Kommunikationsinstrumenten. Konstitutive Instrumente sind zwingend notwendig, um die strategischen Marketing- bzw. Kommuni-

470 Vgl. Schürmann, U., Erfolgsfaktoren der Werbung im Produktlebenszyklus - Ein Beitrag zur Werbewirkungsforschung, in: Meffert, $H$. (Hrsg.), Schriften zu Marketing und Management, Bd. 19, Frankfurt a. M., Berlin u. a. 1993, S. 71.

471 Der Begriff der Komplementarität beschreibt dabei ergänzende bzw. sich stützende Beziehungen zwischen verschiedenen Instrumenten der Kommunikation. Vgl. hierzu und darüber

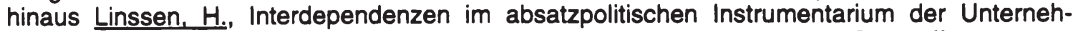
mung. Ein Beitrag zur optimalen Kombination der Absatzmittel, Berlin 1975, S. $130 \mathrm{ff}$.

472 Vgl. Meffert, H., Marketing, Grundlagen der Absatzpolitik, 7. Aufl., a.a.O., S. 115 f.

473 Vgl. Bruhn, M., Integrierte Unternehmenskommunikation, 2. Aufl., a.a.O., S. 74 f.; Becker, J., Marketing-Konzeption, Grundlagen des strategischen Marketing-Managements, 5. Aufl., München 1993, S. 474 f.

474 Becker, J., Marketing-Konzeption, Grundlagen des strategischen Marketing-Managements, 5. Aufl., a.a.O., S. 475. 
kationsziele zu erreichen. In der Literatur wird Messebeteiligungen teilweise eine strategische bzw. taktische Bedeutung zugeordnet. ${ }^{475}$

Mit Blick auf die messespezifischen Grundhaltungen ausstellender Unternehmen ist anzunehmen, daß sich in innen der generelle Stellenwert von Messebeteiligungen im Kommunikations-Mix widerspiegelt. Eine hohe Bedeutung von Messebeteiligungen dürtte dabei mit der Verfolgung strategischer Marketing- bzw. Kommunikationsziele einhergehen, während ein relativ niedriger Stellenwert von Messebeteiligungen keine ausgeprägt zielorientierte messespezifische Grundhaltung erwarten läßt. Diesbezüglich läßt sich folgende Basishypothese formulieren, die im Rahmen der empirischen Analyse überprüft werden soll:

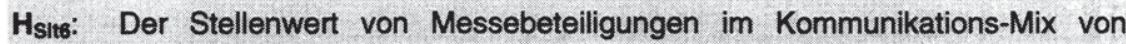 Unternehmen liefert einen signifikanten Erklärungsbeitrag für die Existenz der identifizierten messespezifischen Grundhaltungen.}

Eng verknüpft mit dem Stellenwert der Messebeteiligungen im KommunikationsMix ist die Frage nach ihrer Integration in ein übergeordnetes Kommunikationskonzept. Die Notwendigkeit für integrierte Kommunikationskonzepte wird in vielfältigen Entwicklungen und der Dynamik des kommunikativen Umfeldes von Unternehmen gesehen ${ }^{476}$ und gleichermaßen in Wissenschaft und Praxis erkannt. ${ }^{477}$ Ziel der integrierten Marktkommunikation ist die Bündelung differenzierter

475 Vgl. Bruhn, M. Zimmermann, A., Integrierte Kommunikationsarbeit in deutschen Unternehmen - Ergebnisse einer Unternehmensbefragung, in: Bruhn, M. Dahlhoff. D. (Hrsg.), Effizientes Kommunikationsmanagement. Konzepte, Beispiele und Erfahrungen aus der integrierten Unternehmenskommunikation, Stuttgart 1993, S. 168.

476 Diese Entwicklungen sind vor allem auf Veränderungen in den Medienmärkten zurückzuführen, die sowohl auf der Angebotsseite (Verdreifachung des Werbevolumens zwischen 1980 und 1995, steigende Zahl und Atomisierung von Medien etc.) als auch auf der Nachfrageseite (Informationsüberlastung, Werbereaktanzen, oberflächliche Informationsverarbeitung etc.) zu beobachten sind. Vgl. zu den Herausforderungen an die marktgerichtete Unternehmenskommunikation Kroeber-Riel, W., Kommunikation im Zeitalter der Informationsüberlastung, in: Marketing ZFP, 10. Jg., Nr. 3, 1988, S. 182 ff.; Bruhn, M., Neuere Entwicklungen in der Integrierten Kommunikation, in: Thexis, Heft 3, 1996, S. 12 f.

477 Vgl. stellv. für die Wissenschaft Bruhn, M., Integrierte Unternehmenskommunikation, 2. Aufl., a.a.O., S. 17 f.; Kroeber-Riel, W., Integrierte Kommunikation, in: BddW, vom 03.06.1991, S. 7; Bednarczuk. P., Strategische Kommunikationspolitik für Markenartikel in der Konsumgüterindustrie - Gestaltung und organisatorische Umsetzung, Offenbach 1990, S. 56 f.; stellv. für die Sicht der Praxis Berger. M., Energieverschwendung in der Kommunikation, in: Marketing Journal, Heft 6, 1988, S. 638 f.; Scherrer, M., Integrierte Kommunikation - das große Schlagwort, in: Horizont, Nr. 48, vom 30.11.1990, S. 46; Walter. P., Glaubwürdigkeit und Kontinuität sind das oberste Gebot, in: BddW, vom 07.09.1990, S. 4 
Kommunikationsmaßnahmen und die Nutzung synergetischer Kommunikationswirkungen zur Steigerung des Erfolges im Kommunikationswettbewerb. ${ }^{478}$

Mit Blick auf das Management von Messebeteiligungen stellt sich daher unmittelbar die Frage nach der Integrationsfähigkeit dieses Kommunikationsinstrumentes. Nicht zuletzt aufgrund der vielfältigen Kommunikationsaktivitäten, die mit Messebeteiligungen in der Vor-Messe-, Messe- und Nach-Messe-Phase i. d. R. verbunden sind, ist davon auszugehen, daß Messebeteiligungen nicht nur ein hohes Integrationspotential besitzen, sondern vielmehr für ihre erfolgreiche Durchführung zwingend in ein integriertes Kommunikationskonzept eingebettet sein müssen. ${ }^{479}$ Dabei sind verschiedene Integrationsrichtungen zu berücksichtigen, die nach inhaltlichen, formalen und zeitlichen Dimensionen zu differenzieren sind. ${ }^{480}$

Die inhaltliche Integration dient dazu, Messebeteiligungen mit anderen Kommunikationsinstrumenten thematisch zu verbinden und aufeinander abzustimmen. Als Verbindungslinien dienen dabei Slogans, Kernbotschaften, Schlüsselbilder u. a. Die formale Integration weiterer Kommunikationsinstrumente mit Messebeteiligungen wird durch die einheitliche Venwendung eines Corporate Design ${ }^{481}$ realisiert, das als Hauptkomponente die Einhaltung formaler Gestaltungsprinzipien wie Logos, Farben, Schriften und Schriftgrößen beinhaltet. Im Rahmen der zeitlichen Integration von Messebeteiligungen mit anderen Kommunikationsinstrumenten wird die Absicht verfolgt, sowohl den Einsatz der Kommunikationsinstrumente terminlich aufeinander abzustimmen, als auch ihre zeitliche Kontinuität zu gewährleisten.

Während empirische Untersuchungen einerseits die integrierte Marktkommunikation als einen wesentlichen Bestimmungsfaktor des Markterfolgs identifizieren ${ }^{482}$, zeigen andere empirische Arbeiten, daß die Integration von Messebeteiligungen in ein übergeordnetes Kommunikationskonzept in der Praxis auf zahlreiche Barrieren

478 Vgl. Raffée, H., Integrierte Kommunikation, a.a.O., S. 87.

479 Vgl. Jaspert, F., Das synergetische Potential ist groß - die Messe als Teil der betrieblichen Absatzpolitik, a.a.O., S. 36 f.

$480 \mathrm{Vgl}$. auch im folgenden Bruhn, M., Integrierte Unternehmenskommunikation, a.a.O., S. $40 \mathrm{ff}$.

481 Zum Begriff des Corporate Design vgl. Kroeber-Riel, W., Bildkommunikation - Imagerystrategien für die Werbung, München 1993, S. 301 f.; Demuth, A., Corporate Communications, in: Bruhn, M. (Hrsg.), Handbuch des Marketing, München 1989, S. 438 f.

482

Vgl. Gesamtverband Werbeagenturen (GWA) (Hrsg.), Werbung und Markterfolg. Eine empirische Untersuchung auf der Grundlage von Expertenschätzungen im Markenartikelbereich, Frankfurt a. M. 1992, S. $91 \mathrm{ff}$. 
stößt und somit nur selten umgesetzt wird. ${ }^{483}$ Bruhn und Zimmermann identifizieren als eine zentrale Barriere eines integrierten Kommunikationskonzeptes den generellen Mangel an Professionalität bzw. Kompetenz im Umgang mit den Instrumenten der Marktkommunikation ${ }^{484}$. Somit stellt der Grad der Integration von Messebeteiligungen in ein übergeordnetes Kommunikationskonzept eine spezifische Herausforderung an Messebeteiligungen dar, die es mit Blick auf die Grundhaltungstypen näher zu analysieren gilt.

Aus entscheidungsorientierter Sicht ist mit der Integration verschiedener Kommunikationsinstrumente zumeist auch eine zunehmende Differenzierung des Zielsystems verbunden. ${ }^{485}$ Dies gilt gleichermaßen für das übergeordnete Kommunikationskonzept als auch für die Zielsetzungen einzelner Kommunikationsinstrumente. ${ }^{486} \mathrm{Im}$ Hinblick auf das Zielsystem von Messebeteiligungen ist daher anzunehmen, daß mit einer zunehmend integrativen Ausrichtung dieses Kommunikationsinstrumentes ein differenzierteres Anspruchsspektrum i. S. mehrdimensionaler Zielsysteme einhergeht.

Auf Basis obiger Überlegungen läßt sich der empirischen Analyse folgende Basishypothese zugrunde legen:

\section{Hsirt Der Grad der Integration von Messebeteiligungen im Kommunikations-Mix von Unternehmen liefert einen signifikanten Erklärungsbeitrag für die Existenz der identifizierten messespezifischen Grundhaltungen.}

Weiterhin gilt es, auf dieser Grundlage folgende Tendenzhypothese im Rahmen der empirischen Analyse zu überprüfen:

\section{Hsitza: Je stärker Messebeteiligungen in ein übergeordnetes Kommunikations- konzept integriert werden, desto eher werden differenzierte bzw. mehr- dimensionale Zielkonzepte verfolgt, wie z. B. beim kommunikationsorien- tierten Beziehungsmanager bzw. ambitionierten Regionalaussteller.}

483 Vgl. Bruhn, M. Zimmermann, A., Integrierte Kommunikationsarbeit in deutschen Unternehmen - Ergebnisse einer Unternehmensbefragung, a.a.O., S. $166 \mathrm{ff}$.

484 Vgl. ebenda S. $177 \mathrm{ff}$.

485 Vgl. Bednarczuk, P., Strategische Kommunikationspolitik für Markenartikel in der Konsumgüterindustrie - Gestaltung und organisatorische Umsetzung, a.a.O., S. 58.

486 Vgl. Bruhn, M., Integrierte Unternehmenskommunikation, a.a.O., S. 7. 


\subsection{Messeerfahrungen}

Messebeteiligungen stellen nicht zuletzt aufgrund ihrer Vielfältigkeit und Multifunktionalität ein komplexes Planungsproblem dar. Für die Ausgestaltung des Messeengagements ist daher spezifisches Know-how zwingend notwendig. Dieses Wissen der Aussteller um die optimale Durchführung von Messebeteiligungen beruht zu einem großen Teil auf den eigenen Messeerfahrungen, die sich auf den gesamten Planungsprozeß beziehen.

Nach Raffée ist eine Messebeteiligung „ein Kalkül- und Kreativproblem“, bei dem bereits "die Auflistung von Messe-Zielen ein aus Erfahrung und Kreativität geborener Prozeß $B$ ist, der unternehmensindividuell in einen Abstimmungs- und Ableitungszusammenhang nicht nur mit Kommunikations-, sondern auch mit übergeordneten Marketing-Zielen zu bringen ist. ${ }^{487}$ Der Autor verdeutlicht damit neben der Komplexität des Planungsvorgangs nicht zuletzt auch, daß Messebeteiligungen ein Planungsproblem darstellen, bei dem Aussteller kaum von den Erfahrungen anderer Aussteller zu profitieren vermögen, da hierbei das Gewicht unternehmensspezifischer Ziel- und Ausgestaltungsoptionen deutlich gegenüber allgemeinen bzw. ausstellerübergreifenden Erkenntnissen überwiegt. Nach Rost führen Erfahrungsdefizite insbesondere zur Schwierigkeit der Gewinnung von Maßstäben für die Messebeteiligungsentscheidungen ${ }^{488}$, was in der Konsequenz dazu führt, daß unerfahrene Ausstellerunternehmen die Potentiale, die innen Messebeteiligungen bieten, oftmals nicht ausschöpfen. ${ }^{489}$

Messeerfahrungen können durch die insgesamt bestehende Dauer des bislang bestehenden Messeengagements (historische Messeerfahrungen) sowie durch eine vergleichsweise hohe Intensität des Einsatzes von Messebeteiligungen (intensitätsbedingte Messeerfahrungen) aufgebaut werden. Im Rahmen dieser Arbeit sollen die Messeerfahrungen nicht über die eher unternehmenshistorische Dimension der Dauer des insgesamt bestehenden Messeengagements eines Ausstellers, sondern über seine aktuelle Messebeteiligungsintensität operationalisiert werden. Da sich diese Dimension für Aussteller auf einen überschaubaren Zeitraum in der jüngsten Vergangenheit bzw. die Gegenwart bezieht, scheint die

487 Raffée, $H_{1}$, Messen als Herausforderung für die Marketing-Theorie, a.a.O., S. 73 f.

488 Vgl. Rost. D., Messen als Kommunikations-Problem, a.a.O., S. 44.

489 Vgl. Prinz Wittgenstein, P., Vorwort, in: Ausstellungs- und Messe-Ausschuss der Deutschen Wirtschaft e. V. (Hrsg.), Erfolgreiche Messebeteiligung Made in Germany, Bonn 1995, S. 3.

Ralf Ueding and Universital Munster - 978-3-631-75073-5 
Messebeteiligungsintensität eher geeignet zu sein, über die aktuell im ausstellenden Unternehmen vorhandenen Messeerfahrungen valide Informationen zu liefern.

Vor dem skizzierten Hintergrund können Messeerfahrungen als zentrale Einflußfaktoren des Aufbaus und der Umsetzung von messespezifischen Grundhaltungen bezeichnet werden. Dabei erscheint es offensichtlich, daß mit steigenden Messeerfahrungen Erfahrungskurveneffekte ${ }^{490}$ realisiert werden können. ${ }^{491}$ Die sich daraus ergebende Zunahme der Professionalität des Ausstellers bei der Planung und Durchführung von Messebeteiligungen erzeugt nahezu zwangsläufig ein wachsendes Anspruchsspektrum ggü. diesem Kommunikationsinstrument und führt somit zu einer Verfolgung differenzierter Zielsetzungen.

Auf Basis der obigen Überlegungen läßt sich die folgende Basishypothese als Grundlage der weiteren empirischen Analyse formulieren:

\section{Hsirs: Die Messebeteiligungsintensität liefert einen signifikanten Erklärungsbei- trag für die Existenz der identifizierten messespezifischen Grundhaltungs- typen.}

In Ergänzung dieser Basishypothese lassen sich des weiteren folgende Tendenzhypothesen einer empirischen Überprüfung unterziehen:

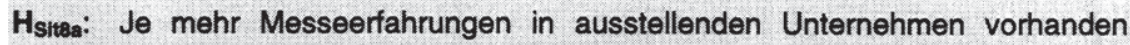
sind, desto eher werden differenzierte Grundhaltungen bei Messebeteiligungen verfolgt.

490 Der Begriff der Erfahrungskurve wurde in den 60er Jahren entwickelt und war ursprünglich auf den Produktionsbereich eines Unternehmens bezogen. Der Erfahrungskurveneffekt bringt zum Ausdruck, daß mit wachsender Produktionsmenge und damit zunehmenden Erfahrungen die Kosten der Leistungserstellung gesenkt werden können. Vgl. Ehrmann. H., MarketingControlling, 2. Aufl., Ludwigshafen 1995, S. 175 f. Übertragen auf den hier zu betrachtenden Untersuchungsgegenstand ist demgemäß eine höhere Effizienz i. S. eines besseren Kosten/Nutzen-Verhältnisses von Messebeteiligungen mit steigenden Messeerfahrungen zu enwarten.

Während über die Dimension der "Dauer des Messeengagements" eine Zeitreihenbetrachtung des Messeengagements i. S. der Messebeteiligungshistorie eines ausstellenden Unternehmens vorgenommen wird, stellt das Kriterium der "Messebeteiligungsintensität" das aktuelle Engagement von Ausstellern in den Vordergrund der Betrachtung. 


\subsection{Position des Messeentscheiders}

In der Unternehmenspraxis werden Entscheidungen bzgl. des für und wider von Beteiligungen an Messeveranstaltungen i. d. R. mit der Frage nach den hierfür zuständigen Messeentscheidern verknüpft. Dabei kommt der Position ${ }^{492}$ von Messeentscheidern im Unternehmen eine Schlüsselrolle im Rahmen der Auswahl zu beschickender Messeveranstaltungen sowie der Festlegung von Größenordnung und Ausgestaltung einzelner Beteiligungen zu. ${ }^{493}$ Mortsiefer stellt bspw. aufgrund empirischer Untersuchungen fest, daß Entscheidungen über Messebeteiligungen in ausstellenden Unternehmen häufig zur "Chefsache" erklärt werden. ${ }^{494} \mathrm{Die}$ in diesem Zusammenhang integrierten Entscheidungsfelder können dabei sehr weitreichend sein und schließen keineswegs nur strategische, sondern vielfach auch operative Tatbestände ein. ${ }^{495}$

Die Ursachen für dieses Unternehmensverhalten sind nicht zuletzt in der Struktur des Entscheidungsobjektes "Messebeteiligung" zu identifizieren. Zum einen stellen Messebeteiligungen aus Sicht des ausstellenden Unternehmens nicht nur komplexe mit zahlreichen Aktivitäten verbundene, sondern auch personal- und insbesondere kostenintensive Marketing- bzw. Kommunikationsmaßnahmen dar. Zum anderen ist in diesem Zusammenhang auch von einer weitgehenden Irreversibilität bei den wesentlichen einmal getroffenen Entscheidungen auszugehen. ${ }^{496}$ In Anlehnung an Gutenberg kann daher davon ausgegangen werden, daß

492 Der Begriff der Position wird in der Organisationstheorie als Stelle innerhalb einer Organisation wiedergegeben, der ein bestimmter Platz in der Organisationshierarchie zugewiesen wird. Dieser Platz wird wesentlich durch den organisatorischen Status sowie die Statussymbole der

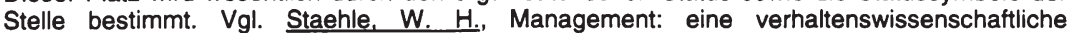
Perspektive, 4. Aufl., a.a.O., S. $246 \mathrm{ff}$.

Vgl. Mortsiefer, J., Die Vorbereitung entscheidet über den Erfolg - Planung und Vorbereitung einer Messebeteiligung, a.a.O., S. 51.

Vgl. Mortsiefer, J., Messen und Ausstellungen als Mittel der Absatzpolitik mittelständischer Herstellerbetriebe, a.a.O., S. $331 \mathrm{ff}$.

Spryß führt bspw. Fälle an, bei denen das oberste Management neben Entscheidungen über die Ziele von Messebeteiligungen auch Festlegungen hinsichtlich der farblichen Abstimmung der Kleidung der Hostessen und des Teppichbodens etc. vornehmen. Vgl. Spryß, W. M., Messen und Ausstellungen: Mit dem "Messe-Diagramm" vorbereiten - von den Zielen bis zum Controlling, a.a.O., S. 368.

Dies trifft insbesondere auf die Entscheidung zu, die einen Rückzug von einer Messebeteiligung zum Gegenstand hat. Hier ist hinsichtlich eines Umdenkens zu einem späteren Zeitpunkt aufgrund der bei vielen Messeveranstaltungen von einer Warteliste nachrückenden Aussteller, häufig nicht mit einer sofortigen Wiederaufnahme in den Kreis der ausstellenden Unternehmen zu rechnen. Aber auch viele Entscheidungen hinsichtlich der Standarchitektur und -gestaltung sowie weiterer konzeptioneller und operativer Elemente einer Messebeteiligung (Exponatewahl, Standmotto etc.) sind i. d. R. nur langfristig veränderbar. 
es sich bei Messebeteiligungsentscheidungen um „echte Führungsentscheidungen" handelt. ${ }^{497}$

Auch wenn davon auszugehen ist, daß Messebeteiligungsentscheidungen zumeist mit einer maßgeblichen Beteiligung von Führungskräften eines Unternehmens durchgeführt werden, so bestehen unternehmensindividuell durchaus Unterschiede im Hinblick auf deren hierarchische Stellung im Unternehmen. Die Ergebnisse der Untersuchung von Mortsiefer lassen den Schluß zu, daß mit der Beteiligungsentscheidung an Messeveranstaltungen sowohl die Unternehmensleitung als auch Führungskräfte in den für Messebeteiligungen zuständigen Bereichen betraut sein können. ${ }^{498}$ Geht man somit davon aus, daß unterschiedliche Konstellationen hinsichtlich der Position des Messeentscheiders in den ausstellenden Unternehmen existieren, dann ist der Frage nachzugehen, wie sich dies auf die Ausgestaltung der messespezifischen Grundhaltung auswirkt.

Es entspricht organisationstheoretischen Anforderungen, aber auch Erkenntnissen der Unternehmenspraxis, daß Führungskräfte im obersten Management einer Unternehmung sich im Zuge der organisationalen Arbeitsteilung im Schwerpunkt mit den unternehmensstrategischen Aspekten der Unternehmenssicherung und entwicklung auseinandersetzen, während das mittlere und untere Management sich auf die Optimierung der einzelnen Unternehmensbereiche i. S. einer Umsetzung der strategischen Vorgaben fokussiert. ${ }^{499}$ Mit Bezug auf die messespezifische Grundhaltung ausstellender Unternehmen ist daher davon auszugehen, daß diese bei maßgeblicher Entscheidungsbeteiligung durch die Unternehmensleitung eine stärker differenzierte und an übergeordneten Unternehmensinteressen ausgerichtete Prägung erhält, während bei zentraler Entscheidungsbefugnis auf der Bereichsebene eine eher konkrete Orientierung an spezifischen Bereichszielen zu erwarten ist.

497 Gutenberg knüpft „echte Führungsentscheidungen“ an die folgenden Bedingungen: "Fehlschläge und Mißerfolge nach dem Abschluß dieser Geschäfte führen unter Umständen zu finanziellen Verlusten und zu Prestigeeinbußen, die das Unternehmen schädigen, unter Umständen sogar seine Zukunft bedrohen.“. Gutenberg. E., Grundlagen der Betriebswirtschaftslehre, Bd. I, Die Produktion, 22. Aufl., Berlin, Heidelberg, New York 1976, S. $138 \mathrm{ff}$.

Vgl. Mortsiefer, J., Messen und Ausstellungen als Mittel der Absatzpolitik mittelständischer Herstellerbetriebe, a.a.O., S. 333, insb. Tabelle 47. Letztlich ist die hierarchische Position des Messeentscheiders sicherlich in Verbindung mit unternehmensdemographischen Gesichtspunkten zu beurteilen. Vgl. dazu die Ausführungen in Kap. D 3.4 dieser Arbeit.

499 Vgl. Steinmann, H. Schreyögg. G., Management: Grundlagen der Unternehmensführung; Konzepte, Funktionen, Praxisfälle, a.a.O., S. $116 \mathrm{ff}$. 
Auf Grundlage der vorangegangenen Ausführungen läßt sich im Rahmen der empirischen Analyse die folgende Basishypothese überprüfen:

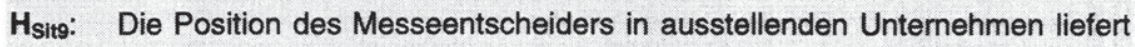
einen signifikanten Erklärungsbeitrag für die Existenz der identifizierten messespezifischen Grundhaltungstypen.

In Ergänzung dieser Basishypothese soll des weiteren folgende Tendenzhypothese einer empirischen Überprüfung unterzogen werden:

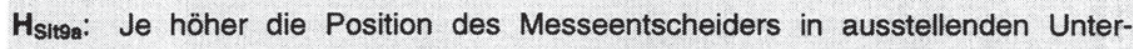
nehmen, desto eher werden differenzierte Grundhaltungen bei Messebeteiligungen verfolgt.

\subsection{Unternehmensdemographie}

Als letzte Gruppe von Einflußfaktoren sollen schließlich ausgewählte Merkmale der Unternehmensdemographie hinsichtlich ihres Einflusses auf die messespezifische Grundhaltung untersucht werden. Im Mittelpunkt stehen dabei Unternehmensgröße und Branchenzugehörigkeit. Über die Beziehungen dieser beiden unternehmensdemographischen Dimensionen zur Ausgestaltung von Messebeteiligungen liegen zwar zahlreiche Forschungsergebnisse vor, allerdings lassen diese nur z. T. Einblicke in messespezifische Grundhaltungen von ausstellenden Unternehmen zu.

So wird in den bislang vorliegenden Arbeiten eine grundsätzlich positive Korrelation zwischen der Unternehmensgröße und dem Umfang des Messeengagements angenommen. ${ }^{500}$ Mortsiefer sieht in dem Aspekt der Unternehmensgröße einen zentralen Einflußfaktor, der auf weitere größenabhängige Einflußfaktoren der Messebeteiligungsintensität ausstrahlt. Diesbezüglich nennt er u. a. das Messebudget, die Belastung der Organisationsstruktur durch Messebeteiligungen, die Personal- und Bildungsstruktur sowie die spezifischen Marketing-Kenntnisse im

500 Vgl. stellv. Spryß, W. M., Nerb, G. Reuter, J., Messeziele - Messeeffizienz, a.a.O., S. 4; Täger. U. C. Ziegler. R., Die Bedeutung von Messen und Ausstellungen in der Bundesrepublik Deutschland für den Inlands- und Auslandsabsatz in ausgewählten Branchen, a.a.O., S. 114 f.; Mortsiefer. J., Messen und Ausstellungen als Mittel der Absatzpolitik mittelständischer Herstellerbetriebe, a.a.Q.ą. S. 91 fing 
Unternehmen. ${ }^{501}$ Hinsichtlich eines Vergleichs zwischen Großindustrie und mittelständischen Unternehmen weist er nach, daß hinsichtlich jedes der zuletzt genannten Aspekte kleinere Unternehmen systematische Nachteile aufweisen. ${ }^{502}$. Die dokumentierte größere generelle Messebeteiligungszurückhaltung bei kleineren Unternehmen läßt insbesondere auf ein geringeres $M a ß$ an spezifischem Messebeteiligungs-Know how schließen. ${ }^{503}$

Bei Übertragung dieser Erkenntnisse auf die identifizierten messespezifischen Grundhaltungen erscheint in großen Unternehmen der Typ des kommunikationsorientierten Beziehungsmanagers besonders wahrscheinlich. Große Unternehmen weisen i. d. R. eine Vielzahl relevanter Märkte sowie Ziel- bzw. Anspruchsgruppen auf, die auch im Rahmen von Messekonzepten differenziert Berücksichtigung finden. Bei diesem Grundhaltungstyp besteht folgerichtig einerseits eine Kombination aus differenzierten Messebeteiligungszielen und andererseits eine hohe Internationalität der ausgewählten Messeveranstaltungen sowie eine signifikant höhere Kommunikations- und Erlebnisorientierung im Bereich der messespezifischen Positionierung. Demgegenüber dürfte der wettbewerbsorientierte Pflichtrepräsentant eher in kleinen Unternehmen anzutreffen sein, da dieser Grundhaltungstyp über eine vergleichsweise geringere Zielorientierung verfügt und seine Messeselektion und -positionierung am Durchschnitt der Aussteller ausrichtet. In diesem Verhalten kann vor dem Hintergrund der oben skizzierten Erkenntnisse ein durchaus unternehmensgrößenbezogenes Verhalten vermutet werden. Bemerkenswert erscheint angesichts der skizzierten Einflüsse der Unternehmensgröße der messespezifische Grundhaltungstyp des ambitionierten Regionalausstellers: Bei diesem liegt ein Ausprägungsmuster der Grundhal-

501 Vgl. Mortsiefer, J., Messen und Ausstellungen als Mittel der Absatzpolitik mittelständischer Herstellerbetriebe, a.a.O., S. 148.

502 Mortsiefer. J., Messen und Ausstellungen als Mittel der Absatzpolitik mittelständischer Herstellerbetriebe, a.a.O., S. 149 ff., insb. S. 176. Zu ähnlichen Erkenntnissen kommen auch andere Studien. Vgl. Tanner, J. F. Chonko, L. B., Trade Show Objectives, Management, and Staffing Practices, a.a.O., S. 257 ff.; Head, J., Positioning, Objectives, and Selection Factors for Trade Shows, a.a.O., S. $1 \mathrm{ff}$., die insbesondere unternehmensstrukturelle Nachteile von kleineren Unternehmen gegenüber großen Unternehmen hinsichtlich der Ausgestaltung von Messebeteiligungen ausmachen.

503 Vgl. Rosson, Ph. Seringhaus, F. H. R., Reaching Export Objectives through International Trade Fairs, in: Trade Show Bureau (Ed.), Report No. IN 6, Denver 1991, S. 2, die im Rahmen ihrer Studie in der Gruppe der "Erst-Nutzer" internationaler Messeveranstaltungen überproportional viele kleinere Unternehmen entdecken, während die größeren Unternehmen zum überwiegenden Teil der Gruppe der "regelmäßigen Aussteller" zugeordnet werden.

Ralf Ueding and Universität Münster - 978-3-631-75073-5 
tungsdimensionen vor, das potentielle Merkmale sowohl großer als auch kleiner Ausstellerunternehmen in sich vereinigt. ${ }^{504}$

Eine eindeutige Erklärung der komplexen messespezifischen Grundhaltungstypen über die Branchenzugehörigkeit als einem weiteren Merkmal der Unternehmensdemographie dürtte kaum möglich sein. ${ }^{505}$ Allerdings lassen sich Brancheneinflüsse auf einzelne Grundhaltungsdimensionen kennzeichnen. So erscheint es in den Branchen, in denen Messen heute überwiegend noch zu Orderzwecken genutzt werden (z. B. Textil (Mode), Möbel, Porzellan etc.), wahrscheinlich, daß bei den ausstellenden Unternehmen aufgrund dieser Prädisposition auf der Ebene der Zieldimension überwiegend der Grundhaltungstyp des fachbesucherorientierten Hardsellers realisiert wird. Des weiteren ist kaum zu erwarten, daß in Branchen, deren Vertreter international orientiert sind ( $z$. B. Maschinenbau, Chemische Industrie), Messeveranstaltungen mit überwiegend regionaler bzw. nationaler Reichweite Relevanz besitzen. Daher wären in diesen Branchen ambitionierte Regionalaussteller nicht zu erwarten. Da allgemein davon auszugehen ist, daß im Konsumgüter- und Dienstleistungsbereich Messen im Rahmen der Kommunikationspolitik insgesamt weniger intensiv genutzt werden als von Unternehmen der Investitionsgüterindustrie, ist davon auszugehen, daß der Grundhaltungstyp des wettbewerbsorientierten Pflichtrepräsentanten eher in den Branchen der Konsumgüterindustrie sowie im Dienstleistungsbereich realisiert wird.

504 So sind einerseits die Messebeteiligungsziele sehr stark ausdifferenziert und vergleichsweise stark ausgeprägt, was auf mittlere bis große Unternehmen hindeutet. Andererseits läßt die primäre Orientierung an Messeveranstaltungen mit nationalem bzw. regionalem Charakter eher kleine Ausstellerunternehmen erwarten.

Zwar erfolgen nahezu alle bislang vorliegenden Arbeiten zum Management von Messebeteiligungen $u$. a. oder ausschließlich im Zusammenhang mit einem spezifischen Branchenkontext. Allerdings sind keine konsistenten Verhaltensweisen von Branchen bei Messebeteiligungen i. S. komplexer Grundhaltungen zu identifizieren. Vgl. zum Branchenbezug der Untersuchungen den Überblick in Kap. A 2. dieser Arbeit und stellv. Täger, U. C. Ziegler, R., Die Bedeutung von Messen und Ausstellungen in der Bundesrepublik Deutschland für den Inlands- und Auslandsabsatz in ausgewählten Branchen, a.a.O., S. 114, die ihre umfassende Untersuchung anhand von 4 Wirtschaftszweigen (Industrie, Handel, Handwerk, Dienstleistungen) und innerhalb der Industrie nach 15 Industriebranchen (z. B. Chemische Industrie, Maschinenbau, Spielwaren etc.) differenzieren. 
Auf der Basis der Erkenntnisse der vorstehenden Ausführungen läßt sich die folgende Basishypothese formulieren:

Hsit10: Die Größe ausstellender Unternehmen liefert einen signifikanten Erklärungsbeitrag für die Existenz der messespezifischen Grundhaltungstypen.

In Ergänzung dieser Basishypothese sollen darüber hinaus die folgenden Hypothesen einer empirischen Überprüfung unterzogen werden:

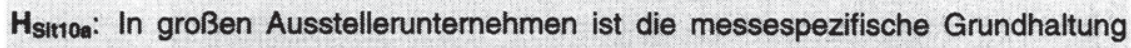
des kommunikationsorientierten Beziehungsmanagers, bei kleinen Unternehmen dagegen der wettbewerbsorientierte Pflichtrepräsentant signifikant häufiger anzutreffen als statistisch zu enwarten.

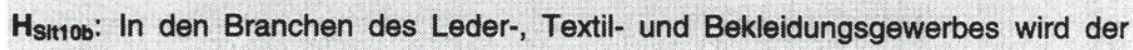
Grundhaltungstyp des fachbesucherorientierter Hardsellers signifikant häufiger realisiert als statistisch zu erwarten.

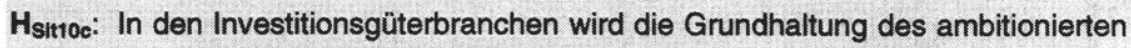
Regionalausstellers signifikant seltener realisiert als statistisch zu erwarten.

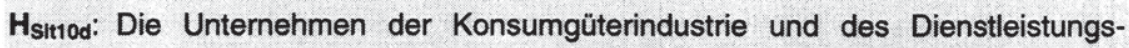
bereichs realisieren die Grundhaltung des wettbewerbsorientierten Pflichtausstellers häufiger als statistisch zu enwarten.

Mit den Dimensionen der Unternehmensdemographie ist die letzte Gruppe relevanter Einflußfaktoren messespezifischer Grundhaltungen skizziert worden. 


\section{Empirische Analyse von Einflußfaktoren auf die messespezifische Grundhaltung}

Die im konzeptionellen Teil dieses Kapitels aufgestellten Hypothesen sollen im folgenden einer empirischen Analyse unterzogen werden.

\subsection{Analyse des Einflusses der Markt- und Wettbewerbssituation}

Zur Erfassung des Markt- und Wettbewerbskontextes von ausstellenden Unternehmen stehen im Rahmen der empirischen Erhebung elf Variablen zur Verfügung. ${ }^{506}$ Zur Verdichtung dieser Variablen wird zunächst eine explorative Faktorenanalyse durchgeführt. ${ }^{507}$ Bei einer 4 Faktor-Lösung können die im Theorieteil abgeleiteten Dimensionen der Markt- und Wettbewerbssituation weitgehend identifiziert werden, was zunächst als deutlicher Hinweis auf die Güte des von Miller und Friesen entwickelten Umweltkonzeptes zu bewerten ist. Die Markt- und Wettbewerbssituation läßt sich damit im folgenden über die Dimensionen der Wettbewerbsintensität und Marktattraktivität ${ }^{508}$, die Umweltkomplexität bzw. dynamik sowie die Wettbewerbsdynamik abbilden.

Nach Zuordnung der elf Einzelvariablen zu den faktoranalytisch ermittelten Dimensionen der Markt- und Wettbewerbssituation zeigt Tab. 18 den Mittelwertvergleichstest für die fünf messespezifischen Grundhaltungstypen.

506 Vgl. hierzu die Operationalisierung der Variablen zur Markt- und Wettbewerbssituation in Frage 1 des Fragebogens im Anhang der Arbeit.

Das Verfahren der explorativen Faktorenanalyse wird gewählt, da im Theorieteil die Feindlichkeit der Aufgabenumwelt ausstellender Unternehmen über die Dimensionen der Wettbewerbsintensität und der Marktattraktivität erfaßt worden sind. Es ist allerdings keineswegs sicher, ob diese beiden Dimensionen im Rahmen des vorliegenden Untersuchungsgegenstandes differenziert oder kombiniert zu erfassen sind, so daß die Anwendungsvoraussetzung der konfirmatorischen Faktorenanalyse, nämlich die Existenz genauer Vorstellungen über die Anzahl relevanter Konstrukte, hier nicht als erfült angesehen werden muß. Die explorative Faktorenanalyse wird nach dem Verfahren der Hauptkomponentenanalyse mit anschließender Faktorrotation durchgeführt. Die Ergebnisse der Faktorenanalyse sind im Anhang der Arbeit dokumentiert.

508 Die explorative Faktorenanalyse führt damit zu einer Separation dieser beiden, die Feindlichkeit der Unternehmensumwelt abbildenden Teildimensionen. Insgesamt konnten von den gebildeten 4 Faktoren $55,8 \%$ der Varianz erklärt werden. 


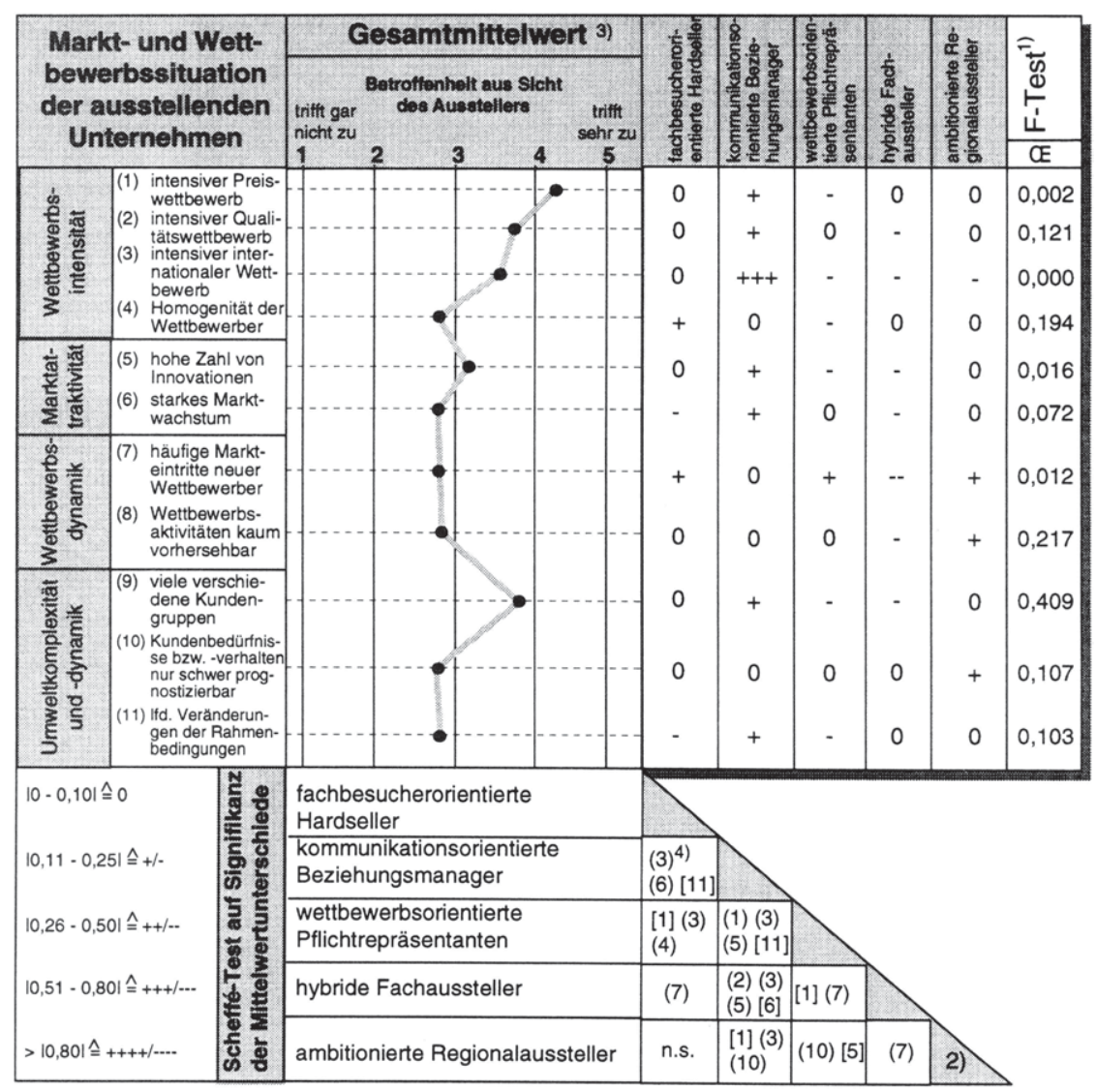

1) One-way-Analyse zur Globalbeurteilung der Mittelwertunterschiede

2) Angabe der Signifikanzniveaus: (i) $\hat{=} \alpha=0,05 /[i] \hat{=} \alpha=0,10$

3) Standardabweichung vom Gesamtmittelwert zwischen 0,86 und 1,31

4) Ziffern beziehen sich auf die jeweiligen Items in der Tabelle

Tab. 18: Einfluß der Markt- und Wettbewerbssituation auf die messespezifische Grundhaltung

Bei Betrachtung der F-Werte fällt zunächst auf, daß bei einem Signifikanzniveau von $\alpha<0,1$ offensichtlich nur für fünf der elf abgefragten Variablen signifikante Mittelwertunterschiede bestehen. Auch der paarweise Scheffée-Test deutet auf eine eher geringe Signifikanz der identifizierten Unterschiede hin. Lediglich das Cluster der kommunikationsorientierten Beziehungsmanager weist hinsichtlich 
mehrerer Variablen signifikante Unterschiede im Vergleich $\mathrm{zu}$ den anderen Ausstellergruppen aus. Dieses Ergebnis scheint zunächst darauf hinzudeuten, daß zwischen der Markt- und Umweltsituation von ausstellenden Unternehmen und der Ausgestaltung von Messebeteiligungen nur sehr schwache Beziehungen bestehen, so $\mathrm{da} B$ die Entstehung messespezifischer Grundhaltungen auf andere Einflußfaktoren zurückgeführt werden muß. Damit wären aber die im Theorieteil abgeleiteten Hypothesen, die relevante Einflüsse des situativen Kontextes postulieren, in ihrer Gesamtheit abzulehnen.

Eine weitergehende Analyse der Ergebnisse des Mittelwertvergleichstests offenbart jedoch einen Erklärungsansatz für diese zunächst ablehnende Haltung, der auf dem methodischen Vorgehen des Mittelwertvergleichstests beruht. Da die messespezifischen Grundhaltungstypen jeweils nur hinsichtlich einzelner Situationsvariablen verglichen werden, kann der situative Gesamtkontext, der durch die spezifische Kombination der Ausprägungen der vier Dimensionen der Markt- und Wettbewerbssituation gebildet wird, in seiner Wirkung auf die messespezifische Grundhaltung von Ausstellern nicht erfaßt werden. ${ }^{509} \mathrm{Da}$ aber davon auszugehen ist, daß ausstellende Unternehmen ihre messespezifische Grundhaltung auf eben diesen Gesamtkontext hin ausrichten, bedarf es einer weitergehenden Analyse, welche die messespezifischen Grundhaltungen in Beziehung zu derartigen situativen Gesamtkontexten bringt.

Um den Erklärungsgehalt situativer Gesamtkontexte auf die Bildung und Ausgestaltung von messespezifischen Grundhaltungen zu untersuchen, sind somit Kontexttypen zu bilden. Hierzu wird eine Clusteranalyse für die elf Situationsvariablen durchgeführt. ${ }^{510}$ Nach einer Ausreißerselektion über das Single-LinkageVerfahren sowie der Bestimmung der optimalen Clusterzahl nach dem WardAlgorithmus können mithilfe der partitionierenden Clusteranalyse drei Kontexttypen ermittelt werden, deren Clustermittelwerte und jeweiligen Abweichungen vom Gesamtmittelwert in der Tabelle 19 zusammenfassend dargestellt sind.

509 Es ergibt sich zwar bei Mittelwertvergleichen für jeden untersuchten Grundhaltungstyp ein eigenständiger Profilverlauf, allerdings kann dieser im vorliegenden Fall nicht als typenspezifischer situativer Kontext aufgefaßt werden. Es zeigte sich nämlich bei der Datenanalyse, daß die Standardabweichungen für die meisten Situationsvariablen bei typenbezogener Betrachtung höher ausgeprägt sind als in der Gesamtstichprobe. Diese Tatsache deutet darauf hin, daß die messespezifischen Grundhaltungstypen offensichtlich in unterschiedlichen situativen Kontexten realisiert werden.

Da durch die vier extrahierten Faktoren lediglich 55,8 Prozent der Varianz der Einzelvariablen erklärt werden kann, empfiehlt sich eine Clusterlösung auf der Grundlage der Einzelvariablen anstatt die Faktorwerte hierzu heranzuziehen. Dies erleichtert im übrigen auch die spätere Clusterinterpretation. 


\begin{tabular}{|c|c|c|c|c|c|c|c|c|c|}
\hline \multicolumn{4}{|c|}{ Variablen Ausprägungen } & \multicolumn{6}{|c|}{$\begin{array}{l}\text { Mittelwerte und Abweichungen der clusterbildenden } \\
\text { Variablen vom Gesamtmittelwert }\end{array}$} \\
\hline \multirow{3}{*}{ 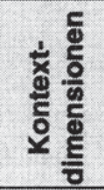 } & \multirow{3}{*}{\multicolumn{3}{|c|}{$\begin{array}{l}\text { EinfluBfaktoren der } \\
\text { Markt- und Wett- } \\
\text { bewerbssituation }\end{array}$}} & \multicolumn{2}{|c|}{ Cluster I } & \multicolumn{2}{|c|}{ Cluster II } & \multicolumn{2}{|c|}{ Cluster III } \\
\hline & & & & \multicolumn{2}{|c|}{$36,0 \% \quad n=201$} & \multicolumn{2}{|c|}{$40,3 \% \quad n=225$} & \multicolumn{2}{|c|}{$23,7 \% \quad n=133$} \\
\hline & & & & $\begin{array}{c}\text { Cluster- } \\
\text { Mittelwerte }\end{array}$ & $\begin{array}{l}\text { Mittelwert- } \\
\text { abwelchung }\end{array}$ & \begin{tabular}{|c|} 
Cluster- \\
Maltelwerte \\
\end{tabular} & $\begin{array}{l}\text { Mittalwert- } \\
\text { abwoichung }\end{array}$ & $\begin{array}{l}\text { Cluster- } \\
\text { Mittelwerte }\end{array}$ & Mittelwert- \\
\hline \multirow{4}{*}{ 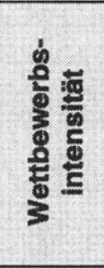 } & \multirow{4}{*}{\multicolumn{3}{|c|}{$\begin{array}{ll}\text { (1) intensiver Preis- } \\
\text { wettbewerb } \\
\text { (2) intensiver Quali- } \\
\text { tätswettbewerb } \\
\text { (3) intensiver interna- } \\
\text { tionaler Wettbewerb } \\
\text { (4) Homogenität der } \\
\text { Wettbewerber } \\
\end{array}$}} & 4,33 & 0 & 4,47 & + & 4,03 & -- \\
\hline & & & & 4,15 & ++ & 3,89 & 0 & 3,39 & -- \\
\hline & & & & 4,26 & +++ & 4,09 & ++ & 1,93 & --- \\
\hline & & & & 2,92 & + & 2,80 & 0 & 2,44 & - \\
\hline \multirow{2}{*}{ 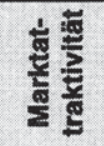 } & \multirow{2}{*}{\multicolumn{3}{|c|}{$\begin{array}{l}\text { (5) hohe Zahl von } \\
\text { Innovationen } \\
\text { (6) starkes Markt- } \\
\text { wachstum }\end{array}$}} & 3,82 & +++ & 3,19 & 0 & 2,63 & --- \\
\hline & & & & 3,49 & +++ & 2,44 & - & 2,41 & - \\
\hline \multirow{2}{*}{ 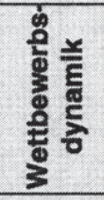 } & \multirow{2}{*}{\multicolumn{3}{|c|}{ 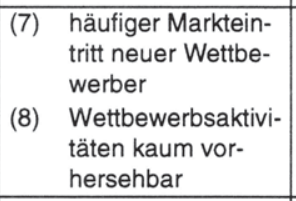 }} & 3,44 & ++++ & 2,36 & -- & 2,48 & -- \\
\hline & & & & 3,30 & + & 2,42 & -- & 2,95 & 0 \\
\hline \multirow{3}{*}{ 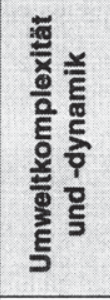 } & \multirow{3}{*}{\multicolumn{3}{|c|}{ 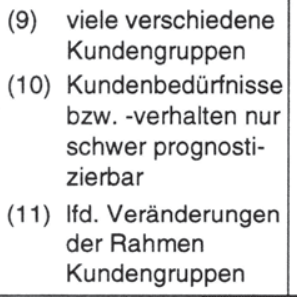 }} & 4,30 & +++ & 3,07 & -- & 3,83 & + \\
\hline & & & & 3,42 & +++ & 2,31 & --- & 3,10 & + \\
\hline & & & & 3,54 & +++ & 2,53 & -- & 2,47 & -- \\
\hline \multirow{3}{*}{\multicolumn{2}{|c|}{$\begin{array}{l}\text { Abweichung der } \\
\text { Cluster-Mittel- } \\
\text { werte vom } \\
\text { Gesamtmittelwert }\end{array}$}} & $\overline{\mathrm{X}}_{\mathrm{i}}$ & $10-0$ & $, 10|| 0,11$ & $1-0,25||$ & $0,26-0,5$ & 50110,51 - & $-0,801=$ & $>|0,80|$ \\
\hline & & positiv & 0 & & + & ++ & & & ++++ \\
\hline & & negativ & 0 & & - & -- & -.. & & -.-. \\
\hline
\end{tabular}

Tab. 19: Clusterbildende Merkmale der ermittelten Kontexttypen

Die Kontexttypen können dabei wie folgt interpretiert werden:

- Für den ersten Kontexttyp läßt sich für nahezu alle Bestimmungsgrößen der Markt- und Wettbe-werbssituation eine stark überdurchschnittliche Ausprägung 
konstatieren. Die im Clustervergleich deutlich überdurchschnittliche Wettbewerbsintensität resultiert dabei insbesondere aus der hohen Internationalität des Wettbewerbs. Gleichzeitig agieren die Unternehmen dieses Clusters in Märkten mit einer überdurchschnittlichen Zahl von Innovationen und einem insgesamt starken Marktwachstum. Auch hinsichtlich der Wettbewerbsdynamik und der Umweltkomplexität- und dynamik weist dieser Kontexttyp stark überdurchschnittliche Ausprägungen auf. Zusammenfassend kennzeichnet dieser erste Situationstyp damit komplexe internationale Wachstumsmärkte mit hoher Wettbewerbsintensität. In diesem situativen Kontext müssen sich 36 Prozent der untersuchten Aussteller (201 Unternehmen) behaupten.

- Das zweite Situationscluster, das für 40,3 Prozent der befragten Aussteller Relevanz besitzt und damit das größte Cluster der Untersuchung darstellt, zeichnet sich vorwiegend durch eine überdurchschnittliche Wettbewerbsintensität aus. Diese ist insbesondere auf einen intensiven internationalen Preis/Leistungswettbewerb zurückzuführen. Hinsichtlich der übrigen Dimensionen der Markt- und Wettbewerbssituation weist dieser Kontexttyp mehr oder wenig deutlich unterdurchschnittliche Ausprägungen auf. Sowohl die geringe Wettbewerbsdynamik als auch die unterdurchschnittliche Marktattraktivität lassen sich als deutliche Indizien starker Sättigungstendenzen interpretieren. Die ebenfalls deutlich unterdurchschnittliche Umweltkomplexität macht darüber hinaus auf die relative Transparenz der Markt- und Wettbewerbsbedingungen aufmerksam. Zusammenfassend charakterisiert dieser Kontexttyp international gesättigte Märkte mit hohem Preis-/Leistungswettbewerb.

- Der dritte Kontexttyp umfasst schließlich Märkte mit einer im Clustervergleich deutlich unterdurchschnittlichen Wettbewerbsintensität. Wie in den anderen Clustern dominiert auch hier eindeutig der Preis- vor dem Qualitätswettbewerb. Auffällig erscheint in diesem Zusammenhang der stark nationale Wettbewerbsbezug der Unternehmen. Die geringe Marktattraktivität wird durch ein unterdurchschnittliches Marktwachstum und eine im Clustervergleich deutlich geringere Innovationshäufigkeit dokumentiert. Die auf die Komplexität und Dynamik bezogenen Situationsvariablen deuten überdies auf insgesamt schwierige Marktbedingungen hin. Die Unternehmen dieses Clusters sind mit einer Vielzahl verschiedener Kundengruppen konfrontiert. Zudem ist die Prognostizierbarkeit der Kundenbedürfnisse im Vergleich zu Cluster II mit größeren Problemen verbunden. Gleichzeitig ist die Zahl der Neueintritte potentieller Wettbewerber in

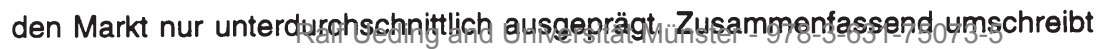


dieser Kontexttyp, der mit einem Anteil von 23,7 Prozent in der Stichprobe die geringste Bedeutung einnimmt, damit national gesättigte bzw. schrumpfende Märkte mit hoher Fragmentierung.

Mit dem empirischen Nachweis von drei eindeutig differenzierbaren Kontexttypen kann zunächst der erste Erklärungsansatz für das Ergebnis des Mittelwertvergleichstests, der von einem singulären situativen Kontext ausgeht, verworfen werden. ${ }^{511}$ Es ist daher nachfolgend zu untersuchen, ob - im Sinne des zweiten Erklärungsansatzes - über die Kontexttypen ein höherer Erklärungsbeitrag für die messespezifischen Grundhaltungstypen geleistet wird als über die einzelnen Situationsvariablen.

Dazu werden die Kontexttypen und messespezifischen Gundhaltungen in einem nächsten Untersuchungsschritt in eine Kontingenzanalyse überführt. Durch den Einsatz dieses Verfahrens kann untersucht werden, ob die identifizierten Grundhaltungstypen in einzelnen Kontexttypen signifikant häufiger auftreten als in anderen. Ist dies der Fall, kann von einem situativen Einfluß auf die messespezifische Grundhaltung von ausstellenden Unternehmen ausgegangen werden. Die Ergebnisse der Kontingenzanalyse sind in der Tab.20 dokumentiert. Aus dieser wird deutlich, daß entgegen der Ergebnisse des Mittelwertvergleichstests über die einzelnen Einflußgrößen von den Kontexttypen wesentliche Einflüsse auf die Wahl des messespezifischen Grundhaltungstyps ausgehen, da sich z. T. deutliche Abweichungen von den statistisch zu erwartenden Zellenhäufigkeiten ergeben. ${ }^{512}$

511 In diesem Zusammenhang ist darauf hinzuweisen, daß im Rahmen einer Diskriminanzanalyse die Güte der Clusterlösung überprüft wurde. Mit einem Anteil von 95,35 Prozent korrekt klassifizierter Fälle kann von einer trennscharfen Bildung der Situationscluster ausgegangen werden. Die Klassifikationsmatrix findet sich in Anhang 5 der Arbeit.

512 Die Kontingenzanalyse berechnet entsprechend des Umfangs der einzelnen Situations- und messespezifischen Grundhaltungstypen die tatsächliche Häufigkeit für jede Zelle der Kontingenztabelle und stellt dieser jeweils eine zu enwartende Häufigkeit gegenüber. Abweichungen der tatsächlichen von der statistisch zu enwartenden Häufigkeit sind methodisch zunächst lediglich als Beziehungen zwischen Kontext- und messespezifischem Grundhaltungstyp zu deuten. Da aber ein Einfluß der messespezifischen Grundhaltung auf die Situation der ausstellenden Unternehmen per definitionem ausgeschlossen werden kann, sind diese Beziehungen eindeutig i. S. situativer Einflüsse auf das Führungsverhalten zu interpretieren.

Ralf Ueding and Universität Münster - 978-3-631-75073-5 


\begin{tabular}{|c|c|c|c|c|c|c|}
\hline \multicolumn{2}{|c|}{$\begin{array}{l}\text { messespeaillecher } \\
\text { Grundhattungstyp' }\end{array}$} & Cluster I & Cluster H & Cluster HII & Cluster IV & Cluster V \\
\hline 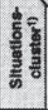 & \begin{tabular}{|l|}
$x_{1}:=$ tatsăch- \\
licher Zeilen- \\
wert \\
$(x):=$ erwarteter \\
Zeilenwert
\end{tabular} & $\begin{array}{c}\text { fachbesucherorien- } \\
\text { tierte Hardseller }\end{array}$ & $\begin{array}{l}\text { kommunikations- } \\
\text { orientierte Bezie- } \\
\text { hungsmanager }\end{array}$ & $\begin{array}{l}\text { wettbewerbsorien- } \\
\text { tierte Pflichtre- } \\
\text { präsentanten }\end{array}$ & $\begin{array}{c}\text { hybride } \\
\text { Fachaussteller }\end{array}$ & $\begin{array}{c}\text { ambitionierte } \\
\text { Regionalaussteller }\end{array}$ \\
\hline \multirow{2}{*}{ 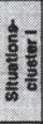 } & \multirow{2}{*}{$\begin{array}{c}\text { komplexe intema- } \\
\text { tionale Wachs- } \\
\text { tumsmärkte mit } \\
\text { hoher Wettbe- } \\
\text { werbsintensităt }\end{array}$} & $\begin{array}{c}32 \\
(33,2)\end{array}$ & $\begin{array}{c}61 \\
(52,7)\end{array}$ & $\begin{array}{c}25 \\
(28,6)\end{array}$ & $\begin{array}{c}27 \\
(35,0)\end{array}$ & $\begin{array}{c}36 \\
(31,5)\end{array}$ \\
\hline & & 0 & ++ & 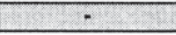 & $\cdots$ & + \\
\hline \multirow[t]{2}{*}{ 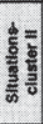 } & \multirow{2}{*}{$\begin{array}{c}\text { international } \\
\text { gesăttigte Märkte } \\
\text { mit hohem } \\
\text { Preis-/Leistungs- } \\
\text { wettbewerb }\end{array}$} & $\begin{array}{c}42 \\
(38,4)\end{array}$ & $\begin{array}{c}67 \\
(60,8)\end{array}$ & $\begin{array}{c}28 \\
(33,1)\end{array}$ & $\begin{array}{c}46 \\
(40,4)\end{array}$ & $\begin{array}{c}26 \\
(36,3)\end{array}$ \\
\hline & & + & ++ & - & + & $\cdots$ \\
\hline \multirow[t]{2}{*}{ 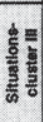 } & \multirow{2}{*}{$\begin{array}{c}\text { national gesättigte } \\
\text { Märkte mit } \\
\text { hoher } \\
\text { Fragmentierung }\end{array}$} & $\begin{array}{c}20 \\
(22,4)\end{array}$ & $\begin{array}{c}21 \\
(35,5)\end{array}$ & $\begin{array}{c}28 \\
(19,3)\end{array}$ & $\begin{array}{c}26 \\
(23,6)\end{array}$ & $\begin{array}{c}27 \\
(21,2)\end{array}$ \\
\hline & & 0 & $\cdots$ & ++ & 0 & + \\
\hline
\end{tabular}

\begin{tabular}{|c|c|c|c|c|c|}
\hline Chi Quadrat-Test gemäB & Signiflikanzniveau & $x_{1}-\left(x_{1}\right)$ & $0-3,0$ & $3,01-6,0$ & $>6,0$ \\
\hline $\begin{array}{l}\text { Pearson } \\
\text { Likelihood Ratio }\end{array}$ & $\begin{array}{l}0,0055 \\
0,0040\end{array}$ & $\begin{array}{l}\text { positiv } \\
\text { negatlv }\end{array}$ & $\begin{array}{l}0 \\
0\end{array}$ & + & $\begin{array}{l}++ \\
-\end{array}$ \\
\hline
\end{tabular}

1) Zuordnung von 512 Ausstellem bei 78 Fallen mit Missing Values und vorheriger Selektion von 10 Ausreißem

Tab.20: Zusammenhang zwischen Kontext- und messespezifischen Grundhaltungstypen

Der Grundhaltungstyp des fachbesucherorientierten Hardsellers wird eher in international gesättigten Märkten mit hohem Preis-/Leistungswettbewerb realisiert. $\left(x_{i}=42 \text { ggü. }\left(x_{i}\right)=38,4\right)^{513}$. Auch der hybride Fachaussteller ist in diesem Situationscluster überdurchschnittlich oft vertreten $\left(x_{i}=46\right.$ ggü. $\left.\left(x_{i}\right)=40,4\right)$. Dagegen ist dieser Grundhaltungstyp in komplexen internationalen Wachtumsmärkten mit hoher Wettbewerbsintensität deutlich unterrepräsentiert. Bei diesem Kontexttyp realisieren ausstellende Unternehmen häufiger als statistisch zu erwarten die Grundhaltungen des kommunikationsorientierten Beziehungsmangers $\left(x_{i}=61\right.$ ggü. $\left.\left(x_{i}\right)=52,7\right)$ bzw. des ambitionierten Regionalausstellers $\left(x_{i}=36\right.$ ggü. $\left(x_{i}\right)=$ 31,5). Wettbewerbsorientierte Pflichtrepräsentanten sind dagegen überdurchschnittlich oft vor dem Hintergrund national gesättigter Märkte mit hoher Fragmentierung anzutreffen $\left(x_{i}=28\right.$ ggü. $\left.\left(x_{i}\right)=19,3\right)$, während sie in den beiden anderen Kontexttypen seltener als statistisch zu erwarten realisiert werden. Während sich damit für den ersten, dritten und vierten messespezifischen Grundhaltungstyp deutliche Tendenzen hinsichtlich des jeweiligen situativen Hintergrunds

513 Dabei gibt $x_{i}$ die tatsächliche und $\left(x_{i}\right)$ die statistisch zu enwartende Häufigkeit eines messespezifischen Grundhaltungstyps bezogen auf ein einzelnes Situationscluster an.

Ralf Ueding and Universität Münster - 978-3-631-75073-5 
herausbilden, weist das Ergebnis der Kontingenzanalyse für den kommunikationsorientierten Beziehungsmanager und den ambitionierten Regionalaussteller weniger eindeutige Beziehungen aus. Während der kommunikationsorientierte Beziehungsmanager auch im Kontext international gesättigter Märkte mit hohem Preis-/Leistungswettbewerb in stärkerem Umfang als statistisch zu enwarten vorzufinden ist $\left(x_{i}=67\right.$ ggü. $\left.\left(x_{i}\right)=60,8\right)$, wird der Grundhaltungstyp des ambitionierten Regionalausstellers neben dem Einsatz in komplexen internationalen Wachstumsmärkten mit hoher Wettbewerbsintensität auch in den national gesättigten Märkten mit hoher Fragmentierung realisiert $\left(x_{i}=27\right.$ ggü. $\left.\left(x_{i}\right)=21,2\right)$.

Aus den Ergebnissen der Kontingenzanalyse lassen sich die folgenden Schlußfolgerungen für die im Theorieteil formulierten Hypothesen ziehen: Der mit der Hypothese $\mathbf{H}_{\mathbf{S I} 2}$ angenommene Einfluß der Dynamik der Markt- und Wettbewerbsbedingungen auf die messespezifische Grundhaltung, wonach eine hohe Dynamik und Unsicherheit des Markt- und Wettbewerbsumfeldes zu einer signifikant höheren Realisierung der u. a. stark an der Aufnahme von Marktinformationen interessierten Grundhaltungstypen der kommunikationsorientierten Beziehungsmanager und der ambitionierten Regionalaussteller führt, kann voll bestätigt werden. Sowohl der Typ des kommunikationsorientierten Beziehungsmanagers, als auch die ambitionierten Regionalaussteller sind im Situationscluster I, das als einziges durch eine außergewöhnlich hohe Markt- und Wettbewerbsdynamik gekennzeichnet ist, überdurchschnittlich stark vertreten. Alle anderen Grundhaltungstypen werden in diesem situativen Umfeld unterdurchschnittlich realisiert. Hypothese $\mathrm{H}_{\text {sit3 }}$ ist dagegen abzulehnen. In ihr wird der Zusammenhang zwischen der zunehmenden Komplexität der Umwelt- und Wettbewerbsbedingungen und der damit einhergehenden Differenzierung von messespezifischen Zielsystemen wie sie beim kommunikationsorientierten Beziehungsmanager konstatiert werden können, postuliert. Mit Blick auf Tab.20 wird deutlich, daß dieser Grundhaltungstyp stärker als statistisch zu erwarten in den Situationsclustern I und || realisiert wird. Beide Situationscluster weisen allerdings hinsichtlich der Wettbewerbsdynamik und der Umweltkomplexität und dynamik entgegengesetzte Ausprägungen auf. Damit kann der vermutete Zusammenhang zwischen dem kommunikationsorientierten Beziehungsmanager und der Komplexität der Marktbedingungen nicht bestätigt werden.

In diesem Zusammenhang kann auch Hypothese $\mathbf{H}_{\text {Sit4 }}$ teilweise bestätigt werden, wonach bei Zunahme der Wettbewerbsintensität der Grundhaltungstyp des an direkten Verkäufen interessierten fachbesucherprientierten 8 Hardselllers 3 verstärkt 
beobachtbar ist. Wie Tab.20 zu entnehmen ist, wird dieser Grundhaltungstyp gerade im Situationscluster II verstärkt umgesetzt, in dem der Preiswettbewerb vergleichsweise am höchsten ausgeprägt ist. Allerdings findet er in Situationscluster I, in dem ein stärkerer Qualitätswettbewerb vorherrscht, keine höhere Realisierung als statistisch zu erwarten. Insofern kann aus diesem Ergebnis die Erkenntnis gezogen werden, daß die Grundhaltung der fachbesucherorientierten Hardseller insbesondere dann realisiert wird, wenn die Wettbewerbsintensität sich vor allem über einen ausgesprochenen Preiswettbewerb definiert.

Zu bestätigen ist ebenfalls die den Einfluß der Marktattraktivität spezifizierende Hypothese $\mathbf{H}_{\text {Sits. }}$. In Übereinstimmung mit den theoretischen Überlegungen verfolgen ausstellende Unternehmen in den durch eine insgesamt geringe Attraktivität gekennzeichneten Märkten des Situationsclusters III besonders häufig die messespezifische Grundhaltung des wettbewerbsorientierten Pflichtrepräsentanten, der nicht nur eine vergleichsweise unterdurchschnittliche Zielorientierung aufweist, sondern auch insgesamt eher zurückhaltende messebezogene Aktivitäten entfaltet. Unter den Bedingungen internationaler Wachstumsmärkte des Situationsclusters I und international gesättigter Märkte mit hohem Preis/Leistungswettbewerb des Situationsclusters II ist dieser Grundhaltungstyp daher wohl auch signifikant seltener als statistisch zu erwarten, vertreten.

Interessanterweise scheinen wettbewerbsintensive Marktsituationen neben dem beschriebenen messebezogenen Grundhaltungsdimensionen auch eine entsprechend hohe Mitarbeiterorientierung zu erfordern, denn der Grundhaltungstyp des stark mitarbeiterfokussierten hybriden Fachausstellers wird in besonderem $\mathrm{Maße}$ in der hoch preiskompetitiven Marktsituation des Situationsclusters II realisiert. Offensichtlich steht hinter dieser Grundhaltung einerseits die Erkenntnis der Aussteller, sich mit dem Einsatz motivierter Mitarbeiter insgesamt besser in diesem Wettbewerbskontext behaupten zu können und andererseits die Einsicht Messebeteiligungen auch als Anreiz für Mitarbeiter nutzbar zu machen.

Zusammenfassend kann der Einfluß der Markt- und Wettbewerbssituation auf die Wahl der messespezifischen Grundhaltung und damit auch Hypothese $\mathbf{H}_{\text {str1 }}$ bestätigt werden. Inwiefern auch interne Einflußfaktoren diese Grundhaltungstypen bestimmen, soll im folgenden untersucht werden. 


\subsection{Analyse des Einflusses unternehmensinterner Faktoren}

Im Kapitel 3 dieses Teils der Arbeit sind mit dem Stellenwert und der Integration von Messebeteiligungen im Kommunikations-Mix, den im Unternehmen vorhandenen Messeerfahrungen, der Position des Messeentscheiders sowie den beiden Dimensionen der Unternehmensgröße und der Branchenzugehörigkeit sechs unternehmensinterne Einflußkategorien diskutiert und hinsichtlich möglicher Einflüsse auf die Bildung von messespezifischen Grundhaltungen analysiert worden. Zur empirischen Erfassung der Einflußwirkungen wird im folgenden auf Mittelwertvergleichstests zurückgegriffen.

\subsection{Einfluß des Stellenwerts und der Integration von Messebeteiligungen im Kommunikations-Mix}

Zur empirischen Erfassung des Stellenwertes von Messebeteiligungen standen in der Befragung fünfzehn Instrumentevariablen zur Verfügung. Um darüber hinaus die Integration von Messebeteiligungen in ein übergeordnetes Kommunikationskonzept zu untersuchen, wurden weiterhin vier Integrationsvariablen in den Fragebogen aufgenommen. ${ }^{514}$ Der die Mittelwerte der Gesamtstichprobe wiedergebende Gesamtprofilverlauf in Tab. 21 zeigt zunächst, daß Messebeteiligungen einen hohen Stellenwert in der Hierarchie der Kommunikationsinstrumente genießen (MW: 3,9). Hinter persönlichem Verkauf/Beratung/Außendienst (MW: 4,6) sowie Kundenservice nach ( $M W: 4,1)$ und vor dem Kauf ( $M W: 4,0$ ) belegen Messebeteiligungen einen Spitzenplatz in der Bedeutungseinschätzung der befragten Unternehmen. Ein Blick auf diese vier Instrumente macht deutlich, daß die befragten Unternehmen damit dem individuellen Kundendialog absolute Priorität einräumen.

514 Vgl. hierzu die Fragen 2 und 3 im Fragebogen.

Ralf Ueding and Universität Münster - 978-3-631-75073-5 

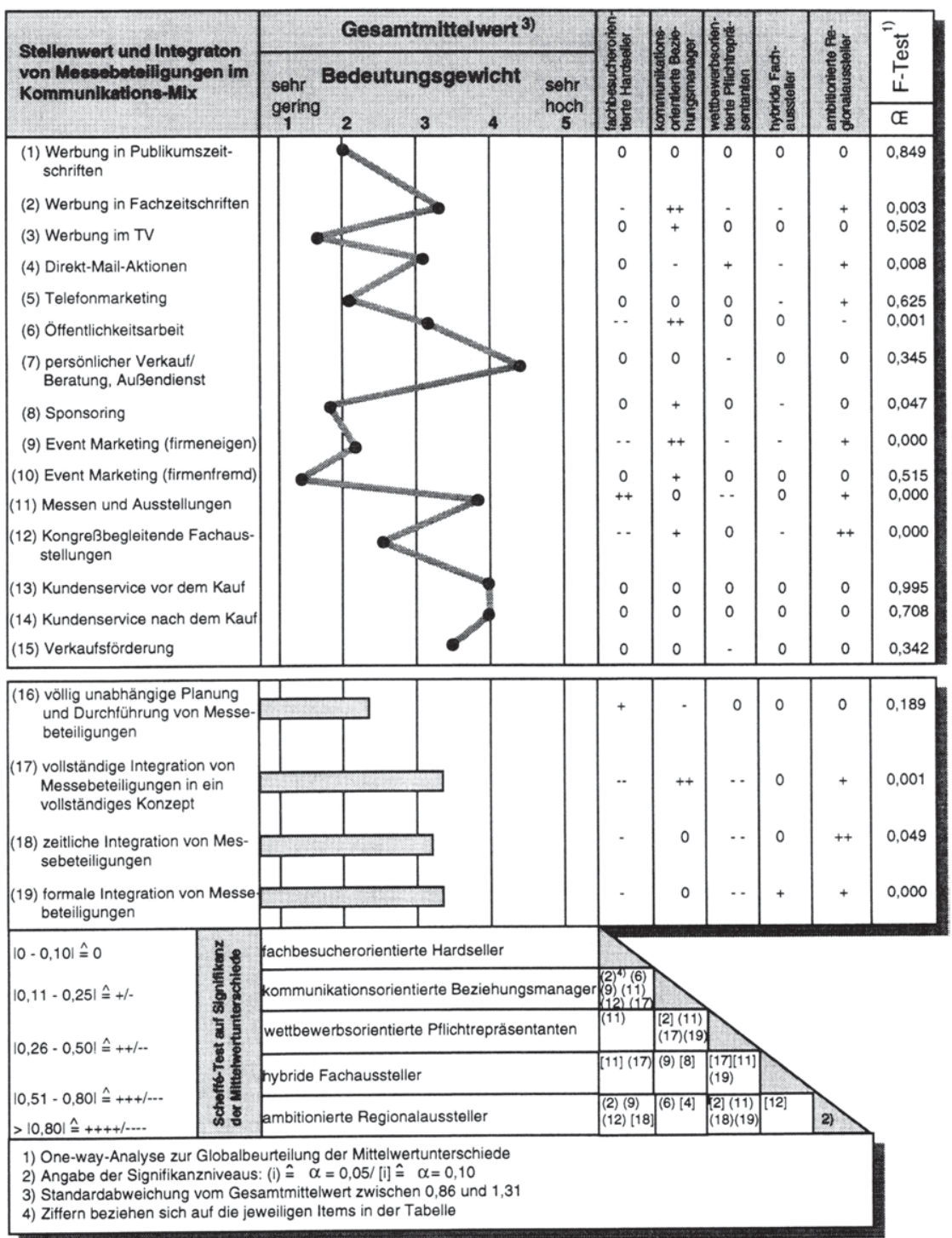

Tab. 21: Einfluß des Stellenwerts und der Integration von Messebeteiligungen auf die messespezifische Grundhaltung 
Mit Bezug zu den einzelnen messespezifischen Grundhaltungstypen weist der FTest auf einem Signifikanzniveau von $\alpha<0,10$ nur für sieben der fünfzehn Instrumente Mittelwertunterschiede aus ${ }^{515}$, wobei in der skizzierten Gruppe der wichtigsten vier Kommunikationsinstrumente lediglich für Messebeteiligungen signifikante Mittelwertunterschiede angegeben werden. Diese Tatsache deutet darauf hin, daß der persönliche Verkauf, die Beratung sowie der Außendienst, und der individuelle Kundenservice vor und nach dem Kauf für die befragten Unternehmen nahezu obligatorischen Charakter besitzen. ${ }^{516}$ Messebeteiligungen werden dagegen in Abhängigkeit der messespezifischen Grundhaltungstypen unterschiedlich beurteilt. Diesen Befund bestätigt auch der paarweise SchefféTest, der im Hinblick auf den Stellenwert von Messebeteiligungen für sechs von zehn durchgeführten Paarvergleichen zwischen den Grundhaltungsclustern signifikante Unterschiede bei einem Signifikanzniveau von mindestens zehn Prozent $(\alpha=0,10)$ ausweist.

Den vergleichsweise höchsten Stellenwert besitzen Messebeteiligungen für fachbesucherorientierte Hardseller, die lediglich dieses Instrument des Kommunikations-Mix überdurchschnittlich nutzen, während alle anderen nur durchschnittlich bzw. unterdurchschnittlich genutzt werden. Den vergleichsweise geringsten Stellenwert ordnen die wettbewerbsorientierten Pflichtrepräsentanten den Messebeteiligungen zu. Dies wird umso deutlicher, als daß in diesem Grundhaltungscluster alle anderen Mittelwerte nur unwesentlich um den Gesamtmittelwert schwanken ${ }^{517}$. Einen etwas erhöhten Stellenwert lassen die ambitionierten Regionalaussteller den Messebeteiligungen zukommen, wobei diese das verwandte Instrument der kongreßbegleitenden Fachausstellungen zusätzlich überdurchschnittlich stark nutzen. Eine im Vergleich eher durchschnitt-

515 Dieses Ergebnis muß insofern erstaunen, als erstens damit deutlich wird, daß das Kommunikations-Mix aller befragten Unternehmen sich insgesamt eher ähnelt und zweitens insbesondere Kommunikationsinstrumente, die in anderen Untersuchungen als zentrale Kommunikationsinstrumente bezeichnet werden - wie z. B. die TV-Werbung - stichprobenübergreifend eine eher geringe Bedeutung aufweisen (MW: 1,6). Vgl. stellv. Schürmann, U., Erfolgsfaktoren der Werbung im Produktlebenszyklus - Ein Beitrag zur Werbewirkungsforschung, a.a.O., S. 4 f. Als Begründung ist hier sicherlich die in diesen Studien meist fokussierte Betrachtung von Konsumgüter- bzw. Markenartikelunternehmen. In der dieser Arbeit zugrundeliegenden Stichprobe sind allerdings auch kleinere Unternehmen repräsentiert, die erfahrungsgemäß kaum Gebrauch von diesen Massenmedien machen.

516 Die obligatorische Bedeutung leitet sich dabei aus dem insgesamt hohen Stellenwert der Instrumente sowie der generellen Gültigkeit für alle Grundhaltungstypen ab.

517 Dieser Tatbestand trägt nachdrücklich zur Bezeichnung und Charakterisierung dieses Messegrundhaltungstyps bei, der Messen tatsächlich primär als Pflichtinstrument und damit "so wenig wie möglich" nutzt. 
liche Bedeutung besitzen Messebeteiligungen für die kommunikationsorientierten Beziehungsmanager und die hybriden Fachaussteller. Mit diesem Befund ist der

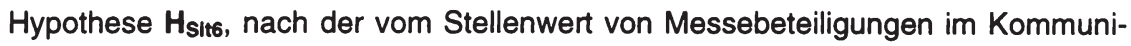
kations-Mix ein signifikanter Einfluß auf die Wahl der messespezifischen Grundhaltungen ausgeht, voll zuzustimmen.

Mit Blick auf die Integration von Messebeteiligungen in ein übergeordnetes Kommunikationskonzept zeigt sich interessanterweise bei Betrachtung der Mittelwerte der Gesamtstichprobe, daß die völlig unabhängige Planung und Durchführung von Messebeteiligungen insgesamt einen deutlich geringeren Stellenwert besitzt (MW: 2,5), als die verschiedenen Integrationsformen. Allerdings zeigt der Mittelwert von lediglich 3,3 für die vollständige Integration von Messebeteiligungen in übergeordnete Kommunikationskonzepte, daß eine Vielzahl ausstellender Unternehmen Messebeteiligungen allenfalls partiell in kommunikative Gesamtkonzepte einbindet.

Mit Blick auf die Grundhaltungstypen sind deutliche Unterschiede bei der Schwerpunktsetzung im Rahmen der jeweiligen Integrationsbemühungen zu konstatieren, die durch den globalen F-Test mit einem Signifikanzniveau von $\alpha<$ 0,1 bestätigt werden. Den vergleichsweise geringsten Integrationsgrad weisen die fachbesucherorientierten Hardseller und die wettbewerbsorientierten Pflichtaussteller auf, wobei hinsichtlich des erstgenannten Grundhaltungstyps die völlig unabhängige Planung und Durchführung von Messebeteiligungen überdurchschnittlich stark ausgeprägt ist. Die im Vergleich vollständigste Integration von Messebeteiligungen in ein übergeordnetes Kommunikationskonzept nehmen die kommunikationsorientierten Beziehungsmanager vor, während die hybriden Fachaussteller den Schwerpunkt ihrer Integrationsbemühungen auf formale Aspekte legen und die ambitionierten Regionalaussteller vergleichsweise überdurchschnittliche Anstrengungen zur vollständigen sowie insbesondere zeitlichen Integration von Messebeteiligungen unternehmen. Aufgrund der sich damit in der empirischen Analyse ergebenden deutlichen Unterschiede hinsichtlich der Integration von Messebeteiligungen in ein übergeordnetes Kommunikationskonzept kann die Hypothese $\mathbf{H}_{\mathbf{s i t}}$, die diesen Zusammenhang postuliert, angenom-

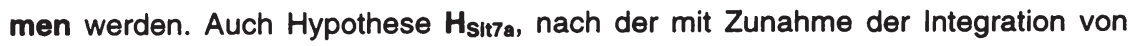
Messebeteiligungen eher die Grundhaltungen der kommunikationsorientierten Beziehungsmanager bzw. ambitionierten Regionalaussteller realisiert werden, ist vor dem Hintergrund der empirischen Erkenntnisse zuzustimmen. 


\subsection{Einfluß der Messeerfahrungen}

Zur Analyse des Einflusses der im ausstellenden Unternehmen vorhandenen Messeerfahrungen auf die jeweils verfolgte messespezifische Grundhaltung wird im folgenden, wie in Kapitel 3.2 dieses Teils der Arbeit herausgearbeitet, auf die durchschnittliche jährliche Messebeteiligungsintensität der „letzten Jahre“518 zurückgegriffen. Im oberen Teil der Abb. 22 ist zunächst die durchschnittliche Zahl der Messebeteiligungen differenziert nach messespezifischen Grundhaltungstypen ausgewiesen. ${ }^{519}$

Hinsichtlich der Zahl der durchschnittlichen jährlichen Messebeteiligungsintensität ergeben sich z. T. ausgeprägte Unterschiede zwischen den Grundhaltungstypen. Die geringste Anzahl mit im Jahresdurchschnitt sechs durchgeführten Messebeteiligungen weisen die kommunikationsorientierten Beziehungsmanager auf. Offensichtlich fokussieren sie ihre vergleichsweise ausgedehnten messebezogenen Aktivitäten auf ausgewählte Messeveranstaltungen. Hierfür spricht auch ihre ausgesprochen hohe Orientierung an internationalen Messeveranstaltungen, die damit der Messebeteiligungsintensität gewissen Schranken unterwirft. Auf fast dreimal so vielen Messeveranstaltungen engagiert sich die Gruppe der ambitionierten Regionalaussteller (MW: 16) und weist damit i. S. dieser Arbeit die meisten, aktuell im Unternehmen vorhandenen, Messeerfahrungen auf. Auch hier liegt die Vermutung nahe, daß vor allem die Festlegungen im Rahmen einer nationalen bzw. regionalen Charakter aufweisenden Messeselektionsstrategie für diese vergleichsweise hohe Messebeteiligungsintensität mitverantwortlich ist. Interessanterweise engagieren sich nämlich auch die wettbewerbsorientierten Pflichtrepräsentanten mit einer durchschnittlichen Intensität von elf Beteiligungen pro Jahr an derartigen Messeveranstaltungen. Dieses insgesamt zweithöchste Beteiligungsengagement verwundert umso mehr, als daß dieser Grundhaltungs-typ Messebeteiligungen im Vergleich den geringsten Stellenwert und insgesamt den geringsten Erfolg einräumt. In diesem Zusammenhang wird es im weiteren

518 Dabei wurde bewußt die offene Formulierung der Zeitdimension mit dem Begriff "letzte Jahre gewählt, um die messeveranstaltungsindividuellen Turni bei der Berechnung der durchschnittlichen jährlichen Messebeteiligungsintensität berücksichtigen zu können. Vgl. zur genauen Formulierung die Frage 4 im Fragebogen im Anhang $X$ dieser Arbeit.

519 Berücksichtigt sind hier explizit lediglich Beteiligungen an Messeveranstaltungen in Deutschland. Messebeteiligungsaktivitäten im Ausland werden - obwohl erhoben (s. Fragebogen) bewußt ausgeschlossen, um etwaige Verzerrungseffekte zu vermeiden. 


\section{Messespezifischer}

Grundhaltungstyp

(1) fachbesucherorien-

tierte Hardseller

(II) kommunikationsorien-

tierte Beziehungsmanager

(III) wettbewerbsorientierte

Pflichtrepräsentanten

(IV) hybride Fachaussteller

(V) ambitionierte Regional-

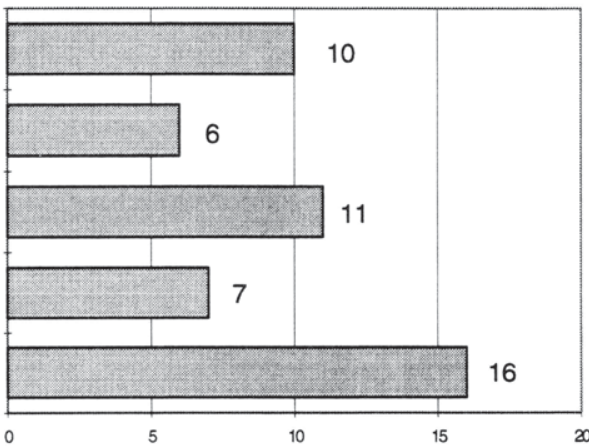

F-Test 0,0937

\section{Paarweiser Mittelwert- verglelchstest (Schefée)}

ambitionierte Regionalaussteller

hybride Fachausteller

wettbewerbsorientierte
Pflichtrepräsentanten

kommunikationsorien-

tierte Beziehungsmanager

fachbesucherorien-

tierte Hardseller

\section{Abb. 22: Messebeteiligungsintensität differenziert nach messespezifischen Grundhaltungstypen}


Verlauf der Arbeit interessant sein, mögliche Erklärungen für diese Tatsache aus den verbleibenden Einflußfaktoren abzuleiten.

Mit Blick auf die Signifikanz der Mittelwertunterschiede weist der globale F-Test zwar ein befriedigendes Signifikanzniveau von $\alpha=0,0937$ aus, doch der paarweise Scheffée-Test deutet aufgrund der kaum vorhandenen signifikanten Unterschiede zwischen den Grundhaltungstypen auf eine starke Streuung des Messeengagements und der damit jeweils einhergehenden Messeerfahrungen hin. Mit Blick auf die formulierten Hypothesen ist aufgrund der vorliegenden empirischen Ergebnisse folgendes festzuhalten: Hypothese $\boldsymbol{H}_{\text {sitz, }}$ nach der die Messebeteiligungsintensität einen signifikanten Erklärungsbeitrag für die Existenz der messespezifischen Grundhaltungstypen zu liefern vermag, kann nicht bestätigt werden. Ebenso ist auch Hypothese $\mathbf{H}_{\text {sitza, wonach mit steigenden }}$ Messeerfahrungen bzw. steigender Messebeteiligungsintensität zunehmend differenzierte Grundhaltungen realisiert werden, nur bedingt anzunehmen. Zwar verfolgen die ambitionierten Regionalaussteller als Cluster mit der höchsten durchschnittlichen Messebeteiligungsintensität eine sehr differenzierte Grundhaltung i. S. einer breit angelegten und ambitionierten Messebeteiligungsziel- sowie einer vergleichsweise anspruchsvollen Positionierungskonzeption. Doch mit den kommunikationsorientierten Beziehungsmanagern steht diesen Ausstellern eine Gruppe gegenüber, die eine auf der Ziel- und Positionierungsebene ähnliche Grundhaltung verfolgt, allerdings bei der insgesamt geringsten Messebeteiligungsintensität. Im Ergebnis kann damit in den Messeerfahrungen eines Ausstellers allenfalls ein peripherer Einflußfaktor der messespezifischen Grundhaltung gesehen werden. ${ }^{520}$

520 Eine ebenfalls für verschiedene Klassen von Messebeteiligungsintensitäten und messespezifischen Grundhaltungstypen durchgeführte Kontingenzanalyse erbrachte keine signifikanten Ergebnisse. Die Abweichungen der von der jeweils erwarteten Verteilung der Grundhaltungstypen in den "Intensitätsklassen" erwiesen sich insgesamt als relativ gering und zudem z. T. unsystematisch. 


\subsection{Einfluß der Position des Messeentscheiders}

Zur Operationalisierung des Einfusses der Position des Messeentscheiders im Unternehmen sollen vier verschiedene Unternehmensbereiche, in denen verantwortlich über die Beteiligung bzw. Nichtbeteiligung an einer Messeveranstaltung entschieden wird, unterschieden werden. Dies sind: Unternehmens- bzw. Geschäftsleitung, Vertriebsleitung, Verkaufsleitung und Marketingleitung. ${ }^{521}$ Tab. 22 stellt die Ergebnisse einer Kontingenzanalyse unter Zugrundelegung der genannten Unternehmensbereiche dar. ${ }^{522}$

Mit Blick auf die Häufigkeiten der einzelnen Unternehmensbereiche, in denen über die Teilnahme an einer Messeveranstaltung letztendlich entschieden wird, ist zunächst auf die deutliche Dominanz der Unternehmensführungsebene hinzuweisen. In mehr als der Hälfte aller in die Analyse einbezogenen Unternehmen entscheidet die Geschäftsleitung über das Messeengagement (54, 7 Prozent). In immerhin knapp einem Viertel der ausstellenden Unternehmen ist die Messebeteiligungsentscheidung eine Angelegenheit des Marketing (23,4 Prozent), während ca. 22 Prozent der Unternehmen die Vertriebs- bzw. die Verkaufsleitung mit dieser Entscheidung betrauen. Dieses zunächst nur auf die Position des Entscheiders bezogene Ergebnis bestätigt die empirischen Befunde von Mortsiefer nachhaltig. ${ }^{523}$

521 Im Rahmen der Befragung war nach der Position des Messeentscheiders in Form einer offenen Frage gefragt worden. 95,5 Prozent aller Antworten entfielen dabei auf die oben genannten vier Unternehmensbereiche. 1,4 Prozent gaben die Position des Werbeleiters, 0,2 Prozent die des Leiters der Presse- und Öffentlichkeitsarbeit und 2,9 Prozent sonstige Positionen, z. B. die des Messebeauftragten, an. Da hinter diesen Anteilswerten allerdings jeweils weniger als 15 Fälle in der Stichprobe stehen, soll der Einfluß dieser Messeentscheidungspositionen auf die messespezifischen Grundhaltungstypen in der empirischen Analyse nicht weiter verfolgt werden.

Mit Blick auf den Chi Quadrat-Test ist darauf hinzuweisen, daß der Zusammenhang zwischen der Position des Messeentscheiders und der messespezifischen Grundhaltung als signifikant zu bezeichnen ist (Signifikanzniveau $\alpha=0,0725$ ).

Vgl. Mortsiefer, J., Messen und Ausstellungen als Mittel der Absatzpolitik mittelständischer Herstellerbetriebe, a.a.O., S. $331 \mathrm{ff}$. 


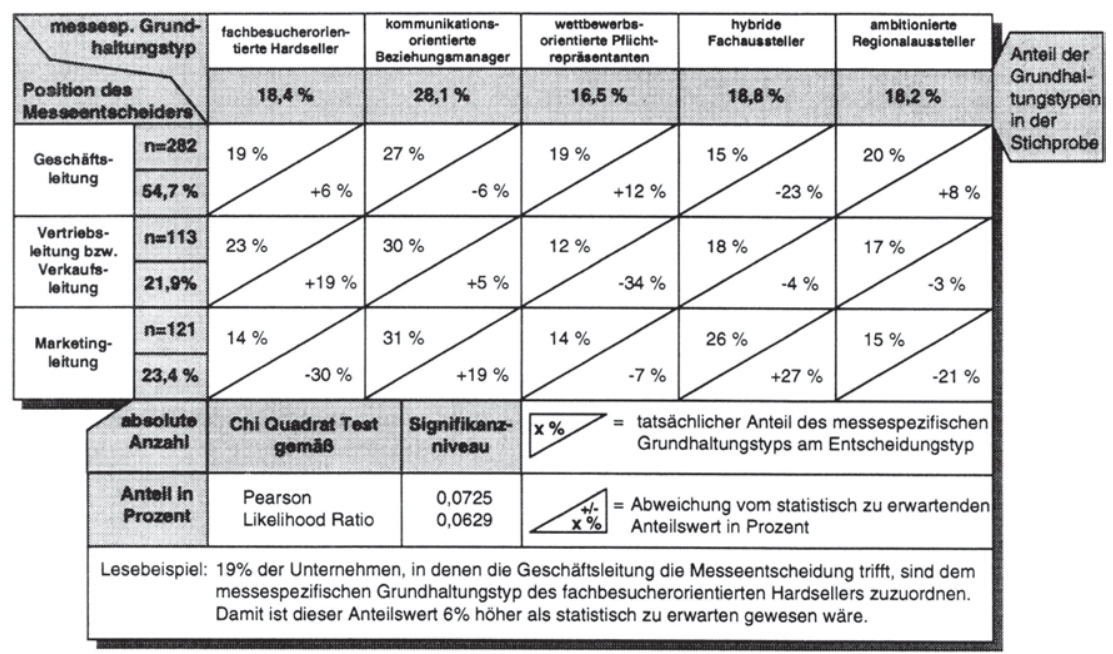

Tab. 22: Kontingenzanalytische Untersuchung des Zusammenhangs zwischen der Position des Messeentscheiders und der messespezifischen Grundhaltung

Auf die sich ergebenden Unterschiede hinsichtlich der messespezifischen Grundhaltungstypen soll im folgenden eingegangen werden: Es zeigt sich, daß i. S. der Hypothese $\mathbf{H}_{\mathbf{s i t g}}$, nach der ein signifikanter Einfluß der Position des Messeentscheiders auf die Wahl der messespezifischen Grundhaltung ausgeht, jeder Grundhaltungstyp spezifische Entscheiderstrukturen aufweist. So wird über Messebeteiligungen bei den fachbesucherorientierten Hardsellern insbesondere häufiger als statistisch zu erwarten auf den Ebenen der Vertriebs- bzw. Verkaufsleitung sowie der Geschäftsleitung entschieden, wobei erstere mit einer positiven Abweichung von 19 Prozent über dem statistisch zu erwartenden Anteilswert die Typencharakterisierung klar dominieren. Damit läßt sich die inhaltliche Schwerpunktsetzung dieses Grundhaltungstyps in konsistenter Weise auch in der Entscheiderposition wiederfinden.

Mit Blick auf die kommunikationsorientierten Beziehungsmanager fällt die insgesamt starke Orientierung an der Marketingleitung auf. Hier liegt der tatsächliche Wert um 19 Prozent über dem statistischen Erwartungswert. Während bei diesem Grundhaltungstyp auch die Verkaufs- und Vertriebsleitung etwas stärker als 
erwartet die maßgeblichen Messeentscheidungen trifft, ist dagegen die Beteiligung der Geschäftsleitung in diesem Bereich unterrepräsentiert. Dieses Ergebnis ist insofern etwas überraschend, als das durchaus hätte angenommen werden können, das das diesen Ausstellertyp charakterisierende breite und differenzierte Zielkonzept, auf eine im Typenvergleich überdurchschnittliche Entscheidungsrolle der Geschäftsleitung zurückgeht.

Eine deutliche Orientierung an den Messeentscheidungen der Geschäftsleitung findet sich allerdings bei den wettbewerbsorientierten Pflichtausstellern. Während die übrigen potentiellen Messeentscheider deutlich seltener als statistisch zu erwarten die relevanten Entscheidungen treffen, nimmt die Geschäftsleitung mit einer positiven Abweichung vom erwarteten Wert von 12 Prozent die offenkundig stärkere Entscheidungs-Präsenz in diesem Grundhaltungscluster ein. Somit stellt dieses Cluster auch die Gruppe der Aussteller dar, die mit Abstand die stärkste Entscheidungszentralisierung in der Führungsspitze des Unternehmens aufzuweisen haben. Zusammenfassend wird deutlich, daß Hypothese $\mathbf{H}_{\mathbf{s i t} 9 a}$, die mit steigender Position des Messeentscheiders in der Unternehmenshierarchie eine zunehmend anspruchsvollere bzw. differenziertere Messegrundhaltung einhergehen sieht, für diesen Grundhaltungstyp nicht angenommen werden kann. Die Ursache hierfür liegt in der Tatsache begründet, daß die wettbewerbsorientierten Pflichtaussteller sich nicht nur durch eine stark unterdurchschnittliche Konzeptionierung ihrer Messebeteiligungsgrundhaltung, sondern zudem durch eine insgesamt eher zurückhaltende Aktivitätsbereitschaft bei Messen kennzeichnen lassen. Vor dem Hintergrund der grundhaltungsspezifischen Analyse dieses Clusters ist sogar eher von einer Umkehrung der Aussage der Hypothese auszugehen.

Der vergleichsweise geringste Stellenwert der Geschäftsleitung für messebezogene Entscheidungen findet sich im Grundhaltungscluster der hybriden Fachaussteller (Abweichung vom statistisch zu erwartenden Anteilswert: - 23 Prozent). Gleichzeitig findet hier die deutlichste Fokussierung auf Unternehmen, in denen die Marketingleitung die relevanten Messeentscheidungen trifft (Abweichung vom statistisch zu erwartenden Anteilswert: +27 Prozent). Offensichtlich geht die überdurchschnittliche Mitarbeiterorientiertung in der Messezielkonzeption dieses Grundhaltungstyps auf die intensive Verankerung des Marketing im Unternehmen und der Erkenntnis um die Notwendigkeit eines internen Marketing zur Realisierung von Motivationszielen einher. Insofern erlangen bei diesem Grundhaltungstyp, im Gegensatz zu den o. g. kommunikationsorientierten Beziehungs- 
managern, die Marketingleiter ein besonderes Gewicht, als sie sowohl für eine Markt- als auch für eine Innenorientierung der Messebeteiligung Sorge tragen.

Ein interessantes Ergebnis zeigt sich auch im Zusammenhang mit den ambitionierten Regionalausstellern, die - wie bereits im Teil B dieser Arbeit gezeigt - über das breiteste und engagierteste Messezielkonzept im Rahmen der Ableitung von messespezifischen Grundhaltungen verfügen. In diesem Cluster zeigt sich, daß insbesondere die Geschäftsleitung stärker als statistisch zu erwarten die wesentlichen Messeentscheidungen trifft (Abweichung vom statistisch zu enwartenden Anteilswert: +8 Prozent). Vertriebs-, Verkaufs- und Marketingleitungen werden dagegen weniger oft als erwartet mit dieser Aufgabe konfrontiert. Damit existiert auch in diesem Grundhaltungscluster eine Gruppe von Ausstellern, in der ähnlich wie bei den fachbesucherorientierten Hardsellern und den wettbewerbsorientierten Pflichtausstellern vornehmlich die Geschäftsleitung selbst die letztliche Messebeteiligungsentscheidung fällt. Dabei wird gerade mit Blick auf die insgesamt eher differenziert angelegte Messezielkonzeption dieses Grundhaltungstyps und vor dem Hintergrund der andersartigen Grundhaltungen der beiden zuvor genannten Cluster aber deutlich, daß die Gruppe der Entscheider aus der Geschäftsleitung nicht homogene, sondern vielmehr heterogene Einflüsse auf die messespezifische Grundhaltung aufweist. Insofern kann die Hypothese $\mathbf{H}_{\text {sitga }}$ nicht angenommen werden. Dagegen ist die Basishypothese $\mathbf{H}_{\text {sitg }}$ sehr wohl anzunehmen, da die Grundhaltungstypen sich jeweils durch spezifische Entscheidungskonstellationen erklären lassen.

\subsection{Einfluß der Unternehmensdemographie}

Bei der Analyse unternehmensdemographischer Kontextfaktoren soll in einem ersten Untersuchungsschritt die Unternehmensgröße in ihrem Einfluß auf die messespezifische Grundhaltung analysiert werden. Mit der Zahl der Mitarbeiter und dem Gesamtumsatz des Unternehmens bzw. des in die Befragung einbezogenen Geschäftsfeldes werden dabei zwei klassische Kennziffern zur Operationalisierung der Unternehmensgröße herangezogen. In Abb. 23 sind die durchschnittliche Mitarbeiterzahl und der durchschnittliche Umsatz differenziert nach Grundhaltungstypen ausgewiesen. 


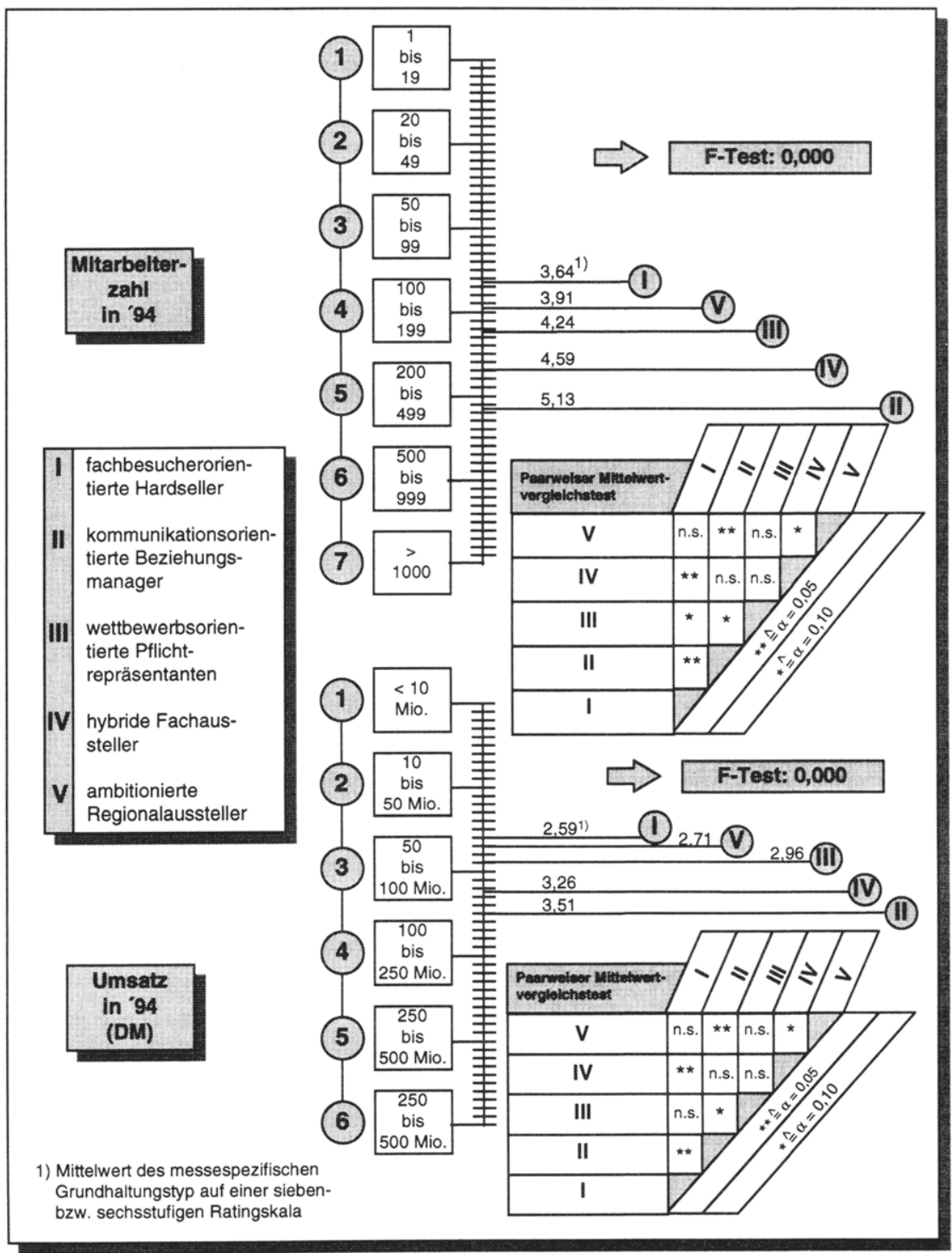

Abb. 23: Mitarbeiterzahl und Umatz differenziert nach messespezifischen Grundhaltungstypen 
Wie der globale F-Test zeigt, ist hinsichtlich beider Kennziffern von signifikanten Unterschieden zwischen den verschiedenen Grundhaltungstypen auszugehen. Auch die paarweisen Mittelwertvergleiche weisen für elf der 20 Mittelwertvergleiche signifikante Unterschiede mit einem Mindestsignifikanzniveau von $\alpha=0,10$ aus. Damit ist insgesamt von einem signifikanten Einfluß der Unternehmensgröße auf die Wahl der messespezifischen Grundhaltung auszugehen und Hypothese $\mathbf{H}_{\text {sit10, }}$, die diesen Zusammenhang postuliert, aufgrund dieses Ergebnisses anzunehmen.

Der Vergleich der grundhaltungsspezifischen Ergebnisse macht überdies deutlich, daß sich sowohl nach Umsatz als auch nach Mitarbeiterzahl die gleiche Rangfolge der Grundhaltungstypen ergibt. Der Grundhaltungstyp des fachbesucherorientierten Hardsellers wird demnach eher von Kleinunternehmen repräsentiert, während die kommunikationsorientierten Beziehungsmanager im wesentlichen die größeren Unternehmen darstellen. ${ }^{524}$ Die Grundhaltungstypen der ambitionierten Regionalaussteller, wettbewerbsorientierten Pflichtrepräsentanten und der hybriden Fachaussteller sind damit nach Mitarbeiterzahl und Umsatz in der Tendenz eher den mittelgroßen Unternehmen zuzuordnen. Mit diesem Ergebnis

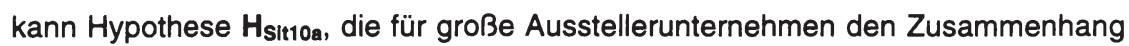
zum kommunikationsorientierten Beziehungsmanager behauptet und die wettbewerbsorientierten Pflichtaussteller durch kleine Unternehmen repräsentiert sieht, nur teilweise angenommen werden.

Auch hinsichtlich der Branchenzugehörigkeit als zweitem unternehmensdemographischen Merkmal sind im Theorieteil mögliche messespezifische Grundhaltungseinflüsse herausgearbeitet worden. Die Überprüfung der drei diesbezüglich formulierten Hypothesen kann anhand der in Tab. 23 dokumentierten Kontingenztabelle erfolgen. ${ }^{525}$

524 Dieses Ergebnis bestätigt auch eine durchgeführte Kontingenzanalyse für die fünf messespezifischen Grundhaltungstypen und die neun im Fragebogen aufgeführten Beschäftigtenklassen. Die fachbesucherorientierten Hardseller sind demnach häufiger als statistisch zu enwarten in den Unternehmen mit weniger als 100 Mitarbeitern vertreten, wobei mit abnehmender Unternehmensgröße die positive Abweichung des tatsächlichen vom enwarteten Anteilswert zunimmt. Genau gegenläufig ist das Ergebnis für die kommunikationsorientierten Beziehungsmanager, die überdurchschnittlich stark in Unternehmen mit mehr als 1000 Mitarbeitern realisiert werden. Vgl. zu den Beschäftigtenklassen im Fragebogen Frage $23 \mathrm{c}$ ). 


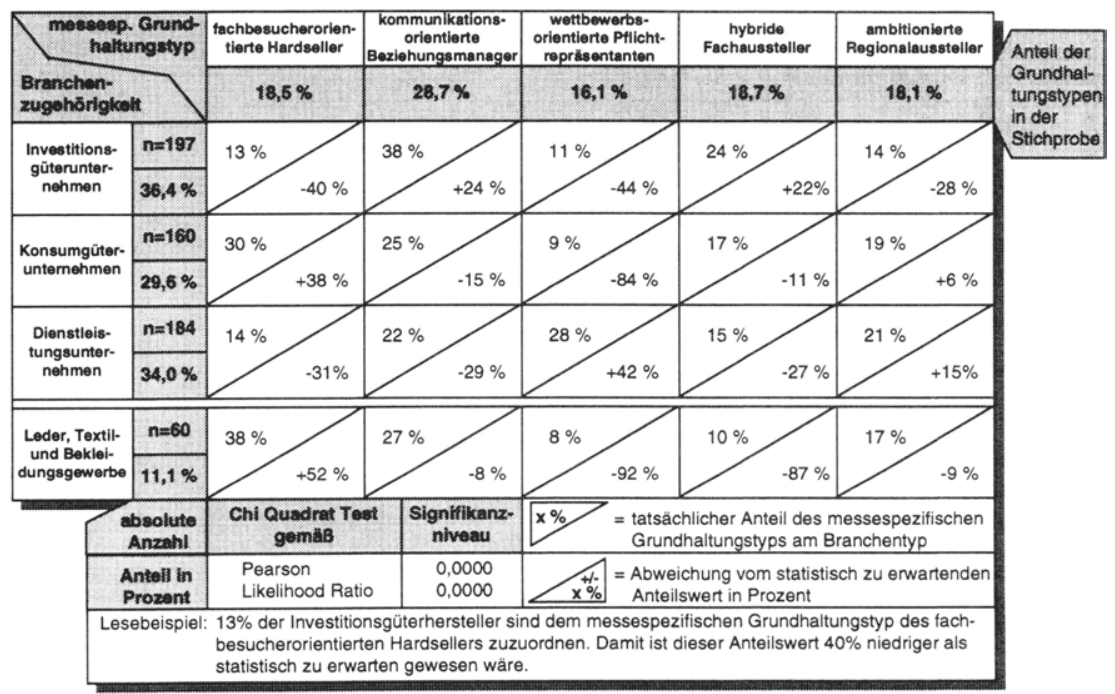

Tab. 23: Zusammenhang zwischen Branchenzugehörigkeit und messespezifischer Grundhaltung

Die für Unternehmen des Leder-, Textil- und Bekleidungsgewerbes als dominierend angenommene Verkaufsorientierung findet auch empirisch ihre Bestätigung. So sind in dieser Teilstichprobe von absolut sechzig Unternehmen gemäß der Hypothese $\mathbf{H}_{\text {sit10b }}$ nur fünf $z u$ finden (8 Prozent), für die die messespezifische Grundhaltung der wettbewerbsorientierten Pflichtrepräsentanten - diese weisen im Vergleich die geringste Verkaufsorientierung auf - eine wesentliche Relevanz besitzt. Dagegen beträgt die positive Abweichung vom statistisch zu erwartenden Anteilswert bei den fachbesucherorientierten Hardsellern 52 Prozent. Diesem Grundhaltungstyp gehören zudem die meisten Unternehmen dieses Gewerbes an. Die Hypothese $\mathbf{H}_{\text {sit } 10 b}$ ist demnach anzunehmen.

Auch der durch die Hypothese $\mathbf{H}_{\mathbf{s i t 1 0}}$ behauptete Zusammenhang zwischen der internationalen Marktbearbeitung der Investitionsgüterbranchen und des damit einhergehenden niedrigen Stellenwertes der messespezifischen Grundhaltung ambitionierter Regionalaussteller für diesen Industriesektor kann empirisch verifiziert werden. Die tatsächliche Zahl der Unternehmen, die diesen Grundhaltungstyp realisieren, bleibt deutlich hinter den erwarteten Anteilswerten zurück (28 Prozent) und stellt damit im Branchenvergleich die höchste Abweichung bei diesem Grundhaltungstyp dar. Hypothese $\mathbf{H}_{\mathbf{S t r 1} 0 \mathrm{c}}$ wird daher voll bestätigt. 
Dagegen kann der in Hypothese $\mathbf{H}_{\text {sit10d }}$ behauptete Zusammenhang nur partiell gestützt werden. In ihr wird die Erwartung geäußert, daß die Unternehmen der Konsumgüter- und Dienstleistungsbranchen, aufgrund eines eher geringen Stellenwertes von Messebeteiligungen für die Unternehmens- bzw. Marktkommunikation signifikant häufiger die messespezifische Grundhaltung der wettbewerbsorientierten Pflichtrepräsentanten realisieren als dies anzunehmen wäre. Mit Blick auf Tab. 23 zeigt sich, daß diese Annahme nur für die Dienstleistungsunternehmen voll zutrifft. Die wettbewerbsorientierten Pflichtrepräsentanten stellen hier nicht nur den größten Anteil der Aussteller (28 Prozent), sondern die positive Abweichung vom statistisch zu erwartenden Anteilswert ist mit 42 Prozent vergleichsweise am größten. Ein gegenteiliges Ergebnis stellt sich hingegen bei den Unternehmen der Konsumgüterindustrie ein. Während die Grundhaltung der wettbewerbsorientierten Pflichtrepräsentanten nahezu keine Bedeutung für diese Branche hat (nur 9 Prozent der Unternehmen realisieren diesen Grundhaltungstyp, dessen Anteil um 84 Prozent hinter dem statistisch zu erwartenden liegt), ist die Mehrzahl der Aussteller den fachbesucherorientierten Hardsellern zuzuordnen. Hypothese $\mathbf{H}_{\text {sit10d }}$ wird daher nur für die Dienstleistungsunternehmen bestätigt.

Eine Erklärung für die empirischen Ergebnisse der Konsumgüterindustrie kann nur an den Grundlagen der generierten Hypothese ansetzen. Die Vermutung, daß die Bedeutung von Messen für das Marketing von Konsumgüterherstellern generell als gering zu bezeichnen ist, kann auf der Basis der vorliegenden Erkenntnisse nicht bestätigt werden. Die fachbesucherorientierten Hardseller zeichnen sich im Gegenteil durch einen überdurchschnittlichen Stellenwert von Messebeteiligungen im Kommunikations-Mix aus. Diese hohe Bedeutung läßt sich vor allem aus der zunehmend stärker gewordenen Stellung des Handels aus Sicht der Konsumgüterhersteller ableiten ${ }^{526}$. Der sich daraus für die Herstellerseite ergebende Handlungsbedarf spiegelt sich in der Fachbesucherorientierung der von diesem Grundhaltungstyp überwiegend ausgewählten Messeveranstaltungen wider. Damit sind Messebeteiligungen als ein wesentliches Instrument des handelsgerichteten Marketing von Konsumgüterunternehmen zu interpretieren,

526 Vgl. Horn, H.-R., Category Management aus Sicht der Unternehmenspraxis (Zusammenfassung eines Vortrages), in: Meffert. H. Wagner, H. Backhaus, K (Hrsg.) Category Management - neue Herausforderung im vertikalen Marketing? - Dokumentationspapier der Wissenschaftlichen Gesellschaft für Marketing und Unternehmensführung e. V. Nr. 97, Münster 1995, S, 44. 
bei denen die Verkaufs- bzw. Orderorientierung der Aussteller i. S. eines „PushMarketing" durchaus plausibel erscheint. ${ }^{527}$

\subsection{Integrierte Analyse der Einflußfaktoren auf die messespezifische Grundhaltung}

Die Analyse der Einflußfaktoren auf die messespezifische Grundhaltung von ausstellenden Unternehmen erfolgte auf der Basis von Mittelwertvergleichstest und Kontingenzanalysen. Dabei wurde nicht untersucht, welche relative Bedeutung den als signifikant identifizierten Einflußfaktoren bei der Wahl einer messespezifischen Grundhaltung zukommt. Zur Beantwortung dieser Fragestellung lassen sich die untersuchten Einflußfaktoren im Rahmen einer Diskriminanzanalyse auf ihren Erklärungsbeitrag zur Trennung der fünf Grundhaltungstypen hin untersucht. ${ }^{528}$ Die hierbei ermittelten Diskriminanzwirkungen ${ }^{529}$ sind in Tab. 24 dargestellt.

Für die nach den verschiedenen untersuchten Kontextdimensionen systematisierten Einflußfaktoren zeigt sich, daß den umweltbezogenen Bestimmungsfaktoren mit einem erklärten Varianzanteil von zusammen 48,26 Prozent im Vergleich zu den unternehmensbezogenen Bestimmungsfaktoren (51,74 Prozent) eine vergleichbare Bedeutung zur Erklärung der Wahl von messespezifischen Grundhaltungstypen zukommt. Innerhalb der Gruppe der umweltbezogenen Bestimmungsfaktoren nehmen die Kontextfaktoren mit eine Anteil von 16,65 Prozent der erklärten Varianz die größte Bedeutung ein. Interessanterweise hat von den erhobenen Umweltfaktoren die Attraktivität von Märkten den geringsten Einfluß

527 Vgl. zur Notwendigkeit eines solchen Marketingkonzeptes zur Marktdurchsetzung von Produkten Szeliga, M., Push und Pull in der Markenpolitik - Ein Beitrag zur modellgestützten Marketingplanung am Beispiel des Reifenmarktes, in: Meffert. H. (Hrsg.), Schriften zum Marketing und Management, Bd. 29, Frankfurt a. M., Berlin u. a. 1996, S. $14 \mathrm{ff}$.

Zur Vermeidung von Multikollinearitäten wurden die Variablen im Rahmen einer Korrelationsanalyse auf Unabhängigkeit überprüft. Aufgrund der erwartungsgemäß hohen Korrelation $(0,925)$ der Einflußfaktoren "Beschäftigtenzahl" und "Gesamtumsatz" wurde letztere Variable aus der Diskriminanzanalyse herausgenommen. Alle anderen Variablen wiesen Korrelationen < 0,5 auf und wurden daher in der Analyse belassen.

Insgesamt beträgt der Anteil der korrekten Klassifikationen auf Basis der in Tab. 24 zusammengefaßten Variablen 87,63 Prozent. Unter Berücksichtigung des durch Wilk's Lambda generierten Chi Quadrat-Tests, der für die einzelnen Diskriminanzfunktionen ein Signifikanzniveau von 0,0086 ausgibt, weist das vorliegende Ergebnis auf eine zufriedenstellende Trennschärfe der Einflußfaktoren hin. Vgl. auch die im Anhang abgebildete Klassifikationsmatrix und die Diskriminanzfunktionen. 


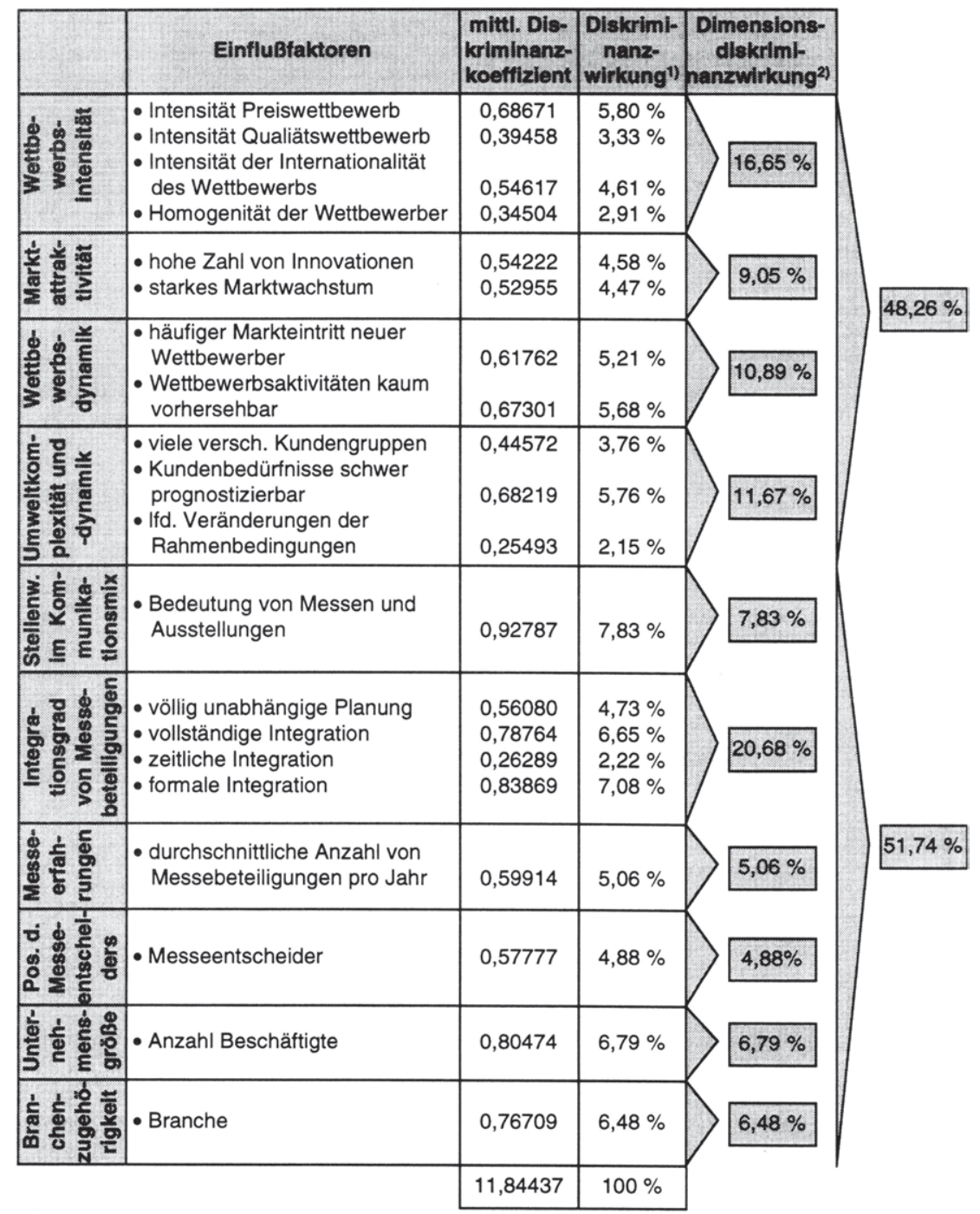

1) beschreibt den Anteil eines Einflußfaktors zur Klassifizierung der Fälle

2) beschreibt den rechn. Gesamtanteil einer Einstellungsdimension zur Klassifizierung der Fälle

Tab. 24: Diskriminatorische Bedeutung der Einflußfaktoren für die Bildung messespezifischer Grundhaltungen 
auf die Wahl einer messespezifischen Grundhaltung (9,05 Prozent). Diese Tatsache deutet, vor dem Hintergrund der von den Befragten überwiegend als gesättigt bezeichneten Märkte, darauf hin, daß Aussteller in Messebeteiligungen weitgehend unabhängig von der vorhandenen Marktsituation - überwiegend ein Kommunikationsinstrument zur proaktiven Gestaltung von Märkten und zur Entdeckung neuer Marktpotentiale sehen.

Bei den unternehmensbezogenen Einflußfaktoren tragen auf der Ebene der Einzelvariablen insbesondere die Stellung von Messebeteiligungen im Kommunikations-Mix (7,83 Prozent), die Beschäftigtenzahl als Ausdruck der Unternehmensgröße (6,79 Prozent) sowie sowohl die formale (7,08 Prozent) als auch die vollständige Integration von Messebeteiligungen in ein übergeordnetes Kommunikationskonzept (6,65 Prozent) und die Branchenzugehörigkeit (6,48 Prozent) zur Erklärung der Wahl einer messespezifischen Grundhaltung bei. Insgesamt können damit die direkt instrumentebezogenen Einflußfaktoren vergleichsweise stärker zur Diskriminierung zwischen den fünf Grundhaltungstypen beisteuern, als die restlichen unternehmensbezogenen Kontextfaktoren. Dies wiederum macht aber deutlich, daß messespezifische Grundhaltungen und ihr Stellenwert für das Marketing von Unternehmen sich weniger aus einer gemeinschaftlichbranchengerichteten, sondern vielmehr aus einer unternehmensindividuellkommunikationspolitischen Grundsatzperspektive heraus erklären läßt.

Insgesamt wird auch deutlich, daß die Analyse der Einflußfaktoren der messespezifischen Grundhaltung nicht auf einzelne Hauptvariablen zu reduzieren ist. ${ }^{530}$ Es zeigt sich vielmehr, daß eine Vielzahl umwelt- und unternehmensbezogener Bestimmungsfaktoren die jeweilige messespezifische Grundhaltung beeinflußt. Die in der Literatur teilweise anzutreffende isolierte Diskussion einzelner spezifischer Einflußfaktoren ist für die Analyse der Bildung und Ausgestaltung messespezifischer Grundhaltungen daher nicht geeignet. Es wird somit deutlich, daß die Beschränkung eines Forschungsdesigns auf wenige ausgewählte Aspekte eines Forschungs- bzw. Erklärungsansatzes nur begrenzt zu differenzierten Ergebnissen der Bestimmungsfaktoren von messespezifischen Grundhaltungen führt.

530 Die in der Diskriminanzanalyse berücksichtigten Einflußfaktoren sind als weitgehend unabhängig zu bezeichen, so daß eine Reduzierung der Einflußfaktoren auf wenige Hauptvariablen durch eine Faktorenanalyse somit zu keinem befriedigenden Ergebnis führen würde.

Ralf Ueding and Universität Münster - 978-3-631-75073-5 


\section{E. Ansatzpunkte für eine systematische Messebeteiligungskontrolle und Implikationen der Untersuchung}

\section{Zusammenfassende Würdigung der Untersuchungsergebnisse}

Die Fragestellung der Ausgestaltung und Einflußfaktoren des Messeengagements von Unternehmen nimmt in der Literatur bislang kaum einen nennenswerten Raum ein. In den wenigen existierenden Arbeiten sind teilweise übereinstimmende, häufiger jedoch divergierende Erkenntnisse über die zentralen Einflußfaktoren messestrategischer Entscheidungen von Unternehmen festzustellen. In diesem Zusammenhang war es das Ziel der Arbeit, auf theoretisch-konzeptioneller wie auch auf empirischer Basis einen Erklärungsansatz für messespezifische Grundhaltungen zu erarbeiten und gleichzeitig ihre Gestaltungs- und Erfolgswirkungen aufzuzeigen. Vor diesem Hintergrund wurde in der vorliegenden Untersuchung unter Verwendung des unternehmenspolitisch-situativen Erklärungsansatzes der Versuch unternommen, auf der Grundlage einer konzeptionellen Aufarbeitung und anschließenden empirischen Überprüfung die zentralen Ausgestaltungsparameter, den Messebeteiligungserfolg und die zentralen Kontextfaktoren messespezifischer Grundhaltungstypen zu analysieren.

Der zentrale, die vorliegende Arbeit insbesondere gegenüber dem traditionellen Vorgehen der in diesem Forschungsfeld vorliegenden empirischen Untersuchungen abgrenzende methodisch-konzeptionelle Ansatzpunkt bestand dabei in der Ableitung einer messespezifischen Grundhaltungstypologie als Basis für die nachfolgenden Untersuchungsschritte. Damit konnte die Untersuchung, im Gegensatz zu den bislang bestehenden branchen- bzw. unternehmensgrößenbezogenen Arbeiten, in weiten Teilen auf der Ebene homogener Ausstellergruppen geführt werden.

Zur Bildung messespezifischer Grundhaltungstypen wurden die Messebeteiligungs-Zielkonzeption, die Messe-Selektion und die messespezifische Positionierung als konstitutive Gestaltungsdimensionen herausgearbeitet und empirisch zu messespezifischen Grundhaltungen verdichtet. Die ermittelten Grundhaltungstypen gehen deutlich im Detaillierungsgrad ihrer Erfassung sowie in ihrem Aussagegehalt über den derzeitigen Forschungsstand im Bereich der Messeforschung hinaus (vgl. Tab. 25). So besteht in der Identifikation des wettbewerbsorientierten Pflichtrepräsentanten, bei dem eine grundsätzliche Messebeteiligungsorien- 
tierung weitgehend unterbleibt, ein bemerkenswerter Befund: Offensichtlich fehlt jedem sechsten Aussteller auf Messeveranstaltungen in Deutschland eine hinreichende strategische Orientierung und inhaltliche Begründung für sein Messeengagement.

Diesem Grundhaltungstyp diametral entgegengesetzte messespezifische Grundhaltungen stellen die kommunikationsorientierten Beziehungsmanager und die ambitionierten Regionalaussteller dar, die zusammen nahezu die Hälfte aller ausstellenden Unternehmen in Deutschland repräsentieren. Wenngleich für diese Grundhaltungstypen ähnlich anspruchsvolle Messe-Zielkonzeptionen und eine weitgehende Übereinstimmung in Fragen der ausstellerbezogenen MessePositionierung zu attestieren sind, so weisen sie doch in der Wahl der Messeveranstaltungen grundsätzliche Unterschiede auf: Während die kommunikationsorientierten Beziehungsmanager, die insgesamt mit fast 30 Prozent aller befragten Aussteller die größte Gruppe darstellen, primär auf internationalen Fachmessen mit Verbundveranstaltungscharakter ausstellen, finden sich die ambitionierten Regionalaussteller eher auf solche Fachmessen ein, die auf der Aussteller- wie auch auf der Besucherseite eine tendenziell regionale bzw. nationale Reichweite besitzen. Die Verbindung stark differenzierter und anspruchsvoller Zielvorstellungen mit den beschickten Fachmessen kann damit nicht zuletzt auch als Beleg für die Vielseitigkeit und von den Ausstellern wahrgenommene Leistungsfähigkeit des in Deutschland vorherrschenden Veranstaltungstyps verstanden werden. ${ }^{531}$

531 Zur Dominanz sowie zur breiten Akzeptanz von Fachmessen in Deutschland vgl. $\underline{0 .}$ V., Messen bleiben wichtiges Instrument, in: Handelsblatt, vom 16.01,1997, S. 24. 


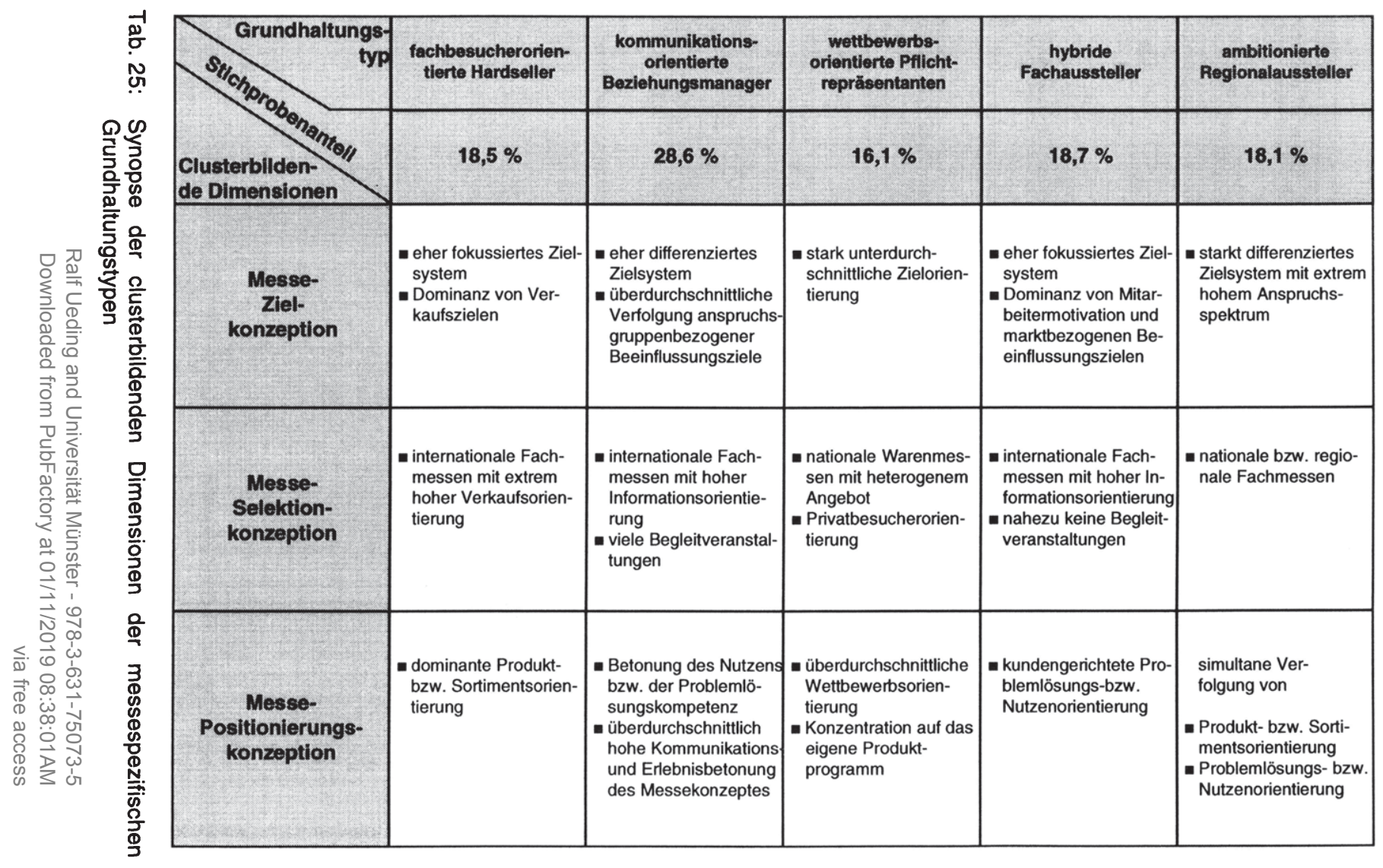


Als Aussteller mit eher fokussierten Messe-Zielsystemen wurden die fachbesucherorientierten Hardseller und die hybriden Fachaussteller herausgearbeitet, die beide jeweils nahezu ein Fünftel der Aussteller auf deutschen Messen repräsentieren. Während erstere primär den Versuch unternehmen, ihre dominanten Verkaufsziele auf internationalen Fachmessen mit starker Orderorientierung und über eine stark an den Produkten ausgerichtete Messepositionierung zu verfolgen, sind letztere vornehmlich auf internationalen Fachmessen mit überdurchschnittlichem Informationscharakter präsent, um dort ihre absatzmarktbezogenen und mitarbeiterorientierten Beziehungs- und Motivationsziele zu verfolgen.

Aufbauend auf der empirisch ermittelten Grundhaltungstypologie wurden i. S. des deskriptiven Teilziels der Arbeit die Fragestellungen der Umsetzung und des Erfolgs unter besonderer Berücksichtigung der messebezogenen Aktivitäten, der Messebeteiligungsorganisation und der Erfolgskontrollen von Messebeteiligungen diskutiert. Tab. 26 und Tab. 27 enthalten die zentralen Ergebnisse der im Kapitel C erfolgten Analyse.

Mit Blick auf den wettbewerbsorientierten Pflichtaussteller fällt auf, daß die unterdurchschnittliche Zielorientierung in eine aktivitätsbezogene Passivität mündet, die sich in ausgeprägter Form durch alle untersuchten Gestaltungsdimensionen verfolgen läßt. Es kann daher nicht verwundern, wenn dieser Grundhaltungstyp bezüglich der Erfolgsbeurteilung der Messebeteiligungen zu unterdurchschnittlichen Bewertungen gelangt. ${ }^{532}$ Insbesondere die weitgehend fehlende Zieloperationalisierung dürfte eine valide Einschätzung von Zielerreichungsgraden allerdings nahezu unmöglich machen. Zudem werden im Rahmen der Erfolgskontrolle Methoden eingesetzt, die kaum in der Lage sind, verläßliche Informationen über die angestrebten Erfolgsbeiträge der Messebeteiligungen zu geben.

532 An dieser Stelle sei nochmals darauf hingewiesen, daß es sich hierbei um Selbsteinschätzungen der Aussteller handelt, die vom Autor der Arbeit nur durch den Vergleich mit den abgefragten Zielerreichungsgraden der verfolgten Zielsetzungen validiert werden können. 


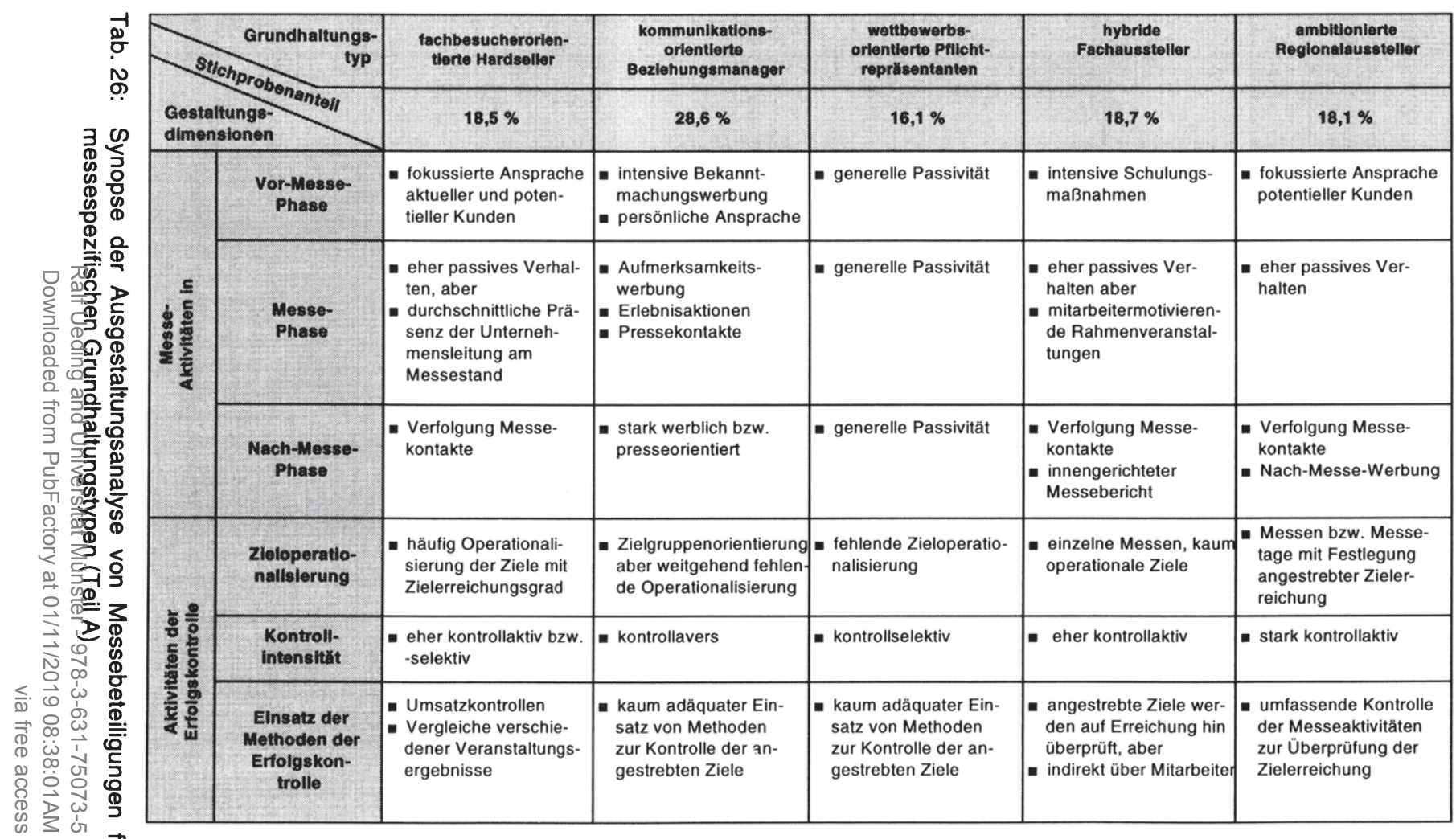




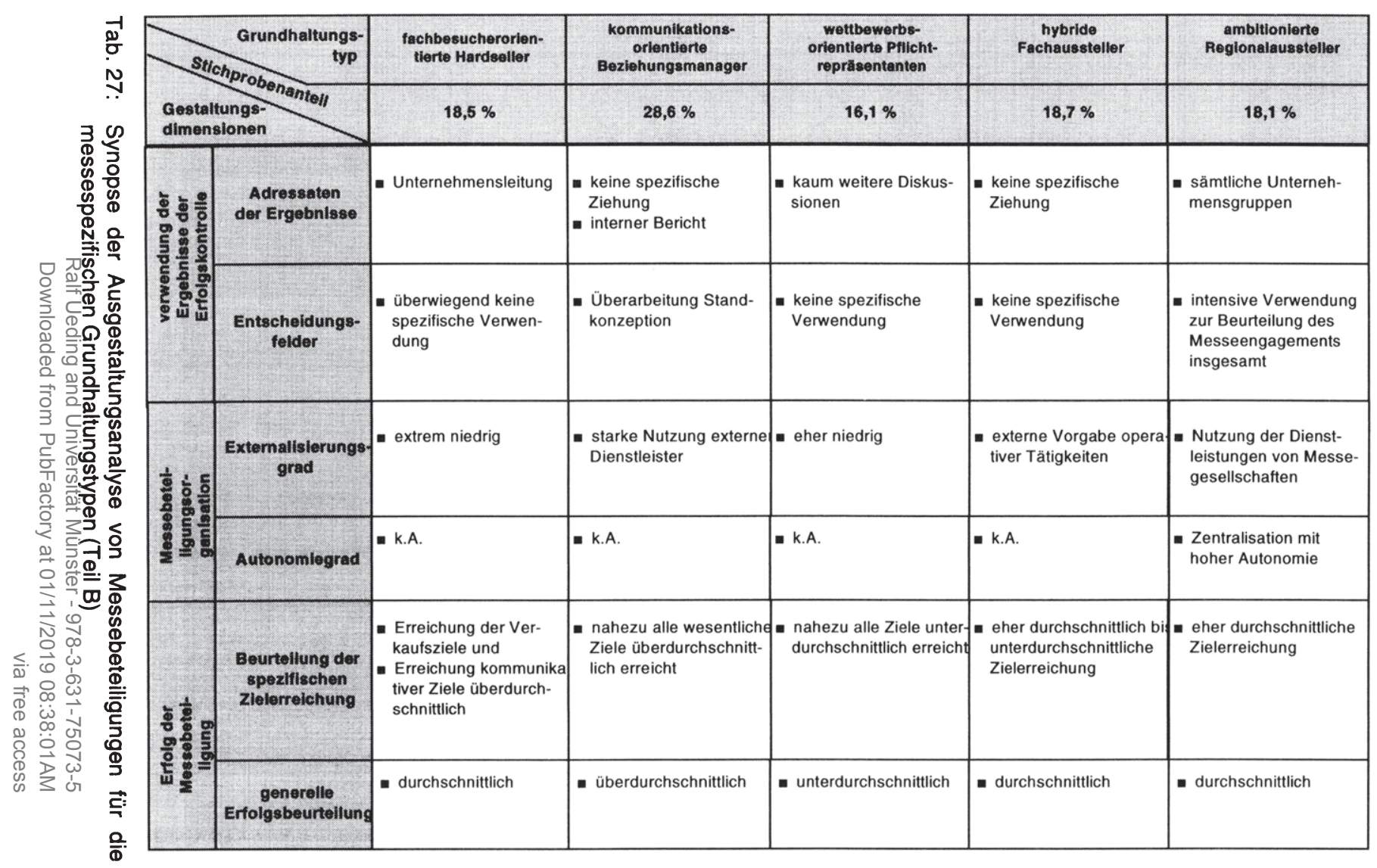


Als stark überdurchschnittlich kommunikationsaktiv können dagegen die kommunikationsorientierten Beziehungsmanager gelten, die sowohl in der VorMesse-, Messe- und Nach-Messe-Phase die vergleichsweise stärksten Anstrengungen unternehmen, um von potentiellen und aktuellen Messebesuchern wahrgenommen zu werden und Kontakte zu schaffen. Um so mehr muß es verwundern, daß diese Aussteller trotz des hohen Aktivitätsniveaus kaum Anstrengungen unternehmen, die Erreichung der hochgesteckten Ziele zu kontrollieren. Die Kontrollaversion dieses Grundhaltungstyps äußert sich folgerichtig in einer weitgehend fehlenden Bestimmung des angestrebten Ausmaßes der Zielerreichung und in einer nicht zieladäquaten Auswahl der Methoden zur Kontrolle des Messebeteiligungserfolges. Es kann darüber hinaus kaum verwundern, daß die Ergebnisse der sporadisch durchgeführten Messeerfolgskontrollen keine spezifischen Adressaten im Unternehmen finden. Es ist bei diesen Ausstellern davon auszugehen, daß sie den Versuch unternehmen vielfältige messebezogene Aktivitäten zu rechtfertigen. Wie der empirischen Analyse der Unternehmensdemographie in diesem Teil der Arbeit zu entnehmen ist, sind es vor allem die großen Unternehmen, die diesem Grundhaltungstyp angehören. Damit wird das zentrale Problem dieser Aussteller im Zusammenhang mit ihren Messebeteiligungen deutlich: Sie übernehmen zum einen vielfach Schrittmacher- bzw. Trendsetterfunktion bei Messen und stehen damit z. T. unter hohem politischen und gesellschaftlichen Druck. Erfolgsmeldungen werden daher als notwendige Marktsignale fast obligatorisch. Einer kritischen Analyse dürften diese Messebeteiligungserfolge allerdings kaum standhalten.

Wenngleich die ambitionierten Regionalaussteller hinsichtlich ihrer messebezogenen Aktivitäten weniger aktiv sind (dies trifft insbesondere auf die MessePhase zu, wo sie ein eher passives Messebeteiligungsverhalten zeigen) als die soeben beschriebenen Aussteller, so zeigen sie die höchste Kontrollintensität im Vergleich zu allen anderen Ausstellergruppen. Sie stellen der breiten Zielorientierung ihrer Messebeteiligungen ein ebensolches Kontrollinstrumentarium gegenüber, das sie in die Lage versetzt, die Erfolgsbeiträge der Messebeteiligungen kritisch und methodenadäquat zu hinterfragen. Für diese Aussteller kann davon ausgegangen werden, $d a ß \operatorname{der}$ im Teil $A$ dieser Arbeit beschriebene Managementprozeß konsequent verfolgt wird.

Auffällig an den empirischen Ergebnissen zur Ausgestaltung und zum Erfolg von Messebeteiligungen ist weiterhin, daß es stark auf die Verfolgung ökonomischer Zielgrößen fokussierten Ausstellern wie den fachbesucherorientierten Hard- 
sellern - offensichtlich besser gelingt, ihre spezifischen Erfolgsbeiträge zu analysieren. So operationalisieren sie ihre Zielsetzungen präziser und überprüfen die Zielerreichungsbeiträge einzelner Messebeteiligungen auch im Vergleich. Allerdings leiten diese Unternehmen aus den gewonnenen Erkenntnissen kaum Maßnahmen zur Verbesserung ihrer Messearbeit ab, wodurch zweifelsohne Entwicklungspotentiale dieses Kommunikationsinstruments für jene Aussteller ungenutzt bleiben werden.

Im Zusammenhang mit den hybriden Fachausstellern ist bezüglich des messespezifischen Verhaltens und der Ausgestaltung der Messebeteiligungen eine dominante Berücksichtigung der Belange der Mitarbeiter unverkennbar. Konfrontiert mit einem dynamischen und wettbewerbsintensiven Umfeld, scheint damit eine hohe Mitarbeiterorientierung und die hiermit einhergehende Kompetenzsicherung auch im Rahmen von Messebeteiligungen konsequent und zielführend zur Sicherung der Wettbewerbsfähigkeit dieses Grundhaltungstyps beizutragen. Neben der Tatsache, daß dieser Grundhaltungstyp die insgesamt intensivste Vorbereitung der Mitarbeiter auf das Messegeschehen vornimmt, fällt die ansonsten eher passive Haltung gegenüber den messebezogenen Aktivitäten auf. Die Mitarbeiterorientierung dokumentiert sich auch im Hinblick auf die Anwendung der Methoden zur Erfolgskontrolle. Es werden schwerpunktmäßig diejenigen verwandt, bei denen die Mitarbeiter als Auskunftspersonen über den Messebeteiligungserfolg befragt werden, wobei auch für diesen Grundhaltungstyp eine eher sporadische Messung der Zielerreichung zu konstatieren ist. Interessanterweise erwachsen in der Folge aus dem Erfolgsbeitrag der Messebeteiligungen auch hier keinerlei Implikationen für die zukünftige Messearbeit.

Im Sinne des explikativen Teilziels der Arbeit wurden in einem nächsten Schritt gemäß dem zugrunde gelegten Ansatz fünf Kontextdimensionen hinsichtlich ihrer Erklärungsbeiträge für die Herausbildung der identifizierten und näher beschriebenen Grundhaltungstypen untersucht und im Rahmen einer integrierten Analyse auf ihre relative Bedeutung hin überprüft. Zusammenfassend konnten dabei die folgenden, in der Tab. 28 synoptisch erfaßten, typenspezifischen Einflüsse ermittelt werden:

(1) Die fachbesucherorientierten Hardseller können auf der einen Seite als Reaktion auf schwierige Marktbedingungen begriffen werden. So sind diese Aussteller überdurchschnittlich stark mit einem Wettbewerbsumfeld konfrontiert, das sich insbesondere durch einen intensiven Preiswettbewerb aus- 
zeichnet. Auf der anderen Seite bedingen offensichtlich die geringe Unternehmensgröße und die Zugehörigkeit zu den Branchen der Konsumgüterindustrie die Realisierung einer hohen Verkaufsorientierung, da Messen in diesen Branchen auf der Besucherseite vielfach zu Orderzwecken genutzt werden. Gleichzeitig ist dieser messespezifische Grundhaltungstyp als Ausdruck einer isolierten absatzmarktgerichteten Profilierung zu interpretieren, da die Nutzung von Messebeteiligungen weitgehend unabhängig von den anderen Instrumenten des Kommunikations-Mix erfolgt.

(2) Eine überdurchschnittliche Präsenz in schwierigen situativen Kontexten konnte auch als erklärender Faktor für die kommunikationsorientierten Beziehungsmanager nachgewiesen werden, wobei sich die Komplexität einerseits aus der Internationalität der Märkte und andererseits aus den intensiven Wettbewerbsbedingungen ableiten läßt. Gleichzeitig ist dieses Grundhaltungsmuster insbesondere durch die Zugehörigkeit der Aussteller zu den großen Unternehmen der Investitionsgüterindustrie zu erklären. Die Notwendigkeit $\mathrm{zu}$ einem breiten, auf mehrere verschiedene Abnehmer- und Anspruchsgruppen gerichteten Beziehungsmarketing läßt diese Aussteller ein umfangreiches Spektrum von Aktivitäten im Umfeld der Messebeteiligung entfalten.

(3) Die Grundhaltung der wettbewerbsorientierten Pflichtaussteller mit der offensichtlichen Passivität bei der Ausgestaltung der Messebeteiligung sind hinsichtlich des situativen Kontextes vor allem durch nationale Märkte gekennzeichnet, die eine hohe Fragmentierung aufweisen und in denen die Bedürfnisse und das Verhalten der Kunden nur schwer prognostizierbar sind. Zudem handelt es sich bei diesen Ausstellern um Unternehmen mittlerer Größe, die nahezu gesamthaft dem Dienstleistungsbereich zuzuordnen sind. Bei dem Versuch, das Verhalten dieser Aussteller aus den genannten Faktoren zu erklären ist auf zwei sich überlagernde Effekte zu verweisen. Dienstleistungen lassen sich i. d. R. nur schwer visualisieren, so daß Messebesuchern kaum „tangible Produkte“ auf der Messe präsentiert werden können. Dies führt sicher auch zu dem insgesamt niedrigen Stellenwert von Messebeteiligungen für dieses Ausstellerunternehmen. Auf der anderen Seite existieren bislang kaum echte Dienstleistungsmessen, so da $\beta$ ausstellende Unternehmen der Dienstleistungsbranche aufgrund von Zielgruppenüberlegungen auch hier zu dem Schluß kommen müssen, Messebeteiligungen 


\begin{tabular}{|c|c|c|c|c|c|c|c|c|}
\hline \multirow[b]{2}{*}{$\begin{array}{l}\text { Einfluß- } \\
\text { faktoren }\end{array}$} & \multirow[b]{2}{*}{$\begin{array}{l}\text { Hypo- } \\
\text { thesen }\end{array}$} & \multirow[b]{2}{*}{$\begin{array}{l}\text { Empirisch unter- } \\
\text { suchte } \\
\text { Beziehungen }\end{array}$} & \multirow[b]{2}{*}{$\begin{array}{l}\text { Hypo- } \\
\text { thesen- } \\
\text { test }\end{array}$} & \multicolumn{5}{|c|}{ Empirlsch ermittelter ElnfluB messesp. GH 1): } \\
\hline & & & & \begin{tabular}{|c|} 
fachbesu- \\
cherorien- \\
tierte \\
Hardseller
\end{tabular} & \begin{tabular}{|c|} 
komm. Bo- \\
ziehungs- \\
manager
\end{tabular} & $\begin{array}{c}\text { wettb. } \\
\text { Palichtre- } \\
\text { präsen- } \\
\text { tanten }\end{array}$ & $\begin{array}{c}\text { hybride } \\
\text { Fachaus- } \\
\text { steller }\end{array}$ & $\begin{array}{l}\text { ambition. } \\
\text { Regionat- } \\
\text { aussteller }\end{array}$ \\
\hline $\begin{array}{l}\text { extern } \\
\text { und } \\
\text { intern }\end{array}$ & $\mathrm{H}_{\text {Sit1 }}$ & $\begin{array}{l}\text { Situation erklärt } \\
\text { messesp. GH-Typen }\end{array}$ & ๑ & & & & & \\
\hline \multirow{4}{*}{ 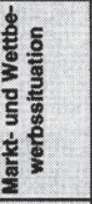 } & $\mathrm{H}_{\mathrm{Sit}}$ & $\begin{array}{l}\text { hohe } \\
\text { Wettbewerbsdynamik }\end{array}$ & ๑) & 4 & $\boldsymbol{t}$ & 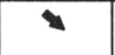 & 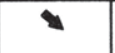 & 1 \\
\hline & $\mathrm{H}_{\mathrm{sit3}}$ & $\begin{array}{l}\text { hohe } \\
\text { Umweltkomplexität }\end{array}$ & $\odot$ & $\not$ & $\Rightarrow$ & 7 & $\Rightarrow$ & $\Rightarrow$ \\
\hline & $\mathrm{H}_{\mathrm{sit} 4}$ & $\begin{array}{l}\text { hohe } \\
\text { Wettbewerbsintensität }\end{array}$ & $\oplus / \odot$ & 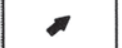 & 个 & 4 & 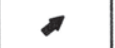 & $\Rightarrow$ \\
\hline & $\mathrm{H}_{\mathrm{Sit5}}$ & $\begin{array}{l}\text { geringe } \\
\text { Marktattraktivität }\end{array}$ & (๑) & $\Rightarrow$ & $t$ & $\hat{\mathbf{1}}$ & $\Rightarrow$ & $\Rightarrow$ \\
\hline \multirow{3}{*}{ 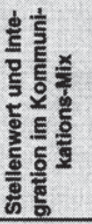 } & $\mathrm{H}_{\text {Sit6 }}$ & $\begin{array}{l}\text { Stellenwert erklärt } \\
\text { messesp. GH-Typen }\end{array}$ & ๑) & & & & & \\
\hline & $\mathrm{H}_{\text {Sit7 }}$ & $\begin{array}{l}\text { Integrationsgrad } \\
\text { erklärt messesp. } \\
\text { GH-Typen }\end{array}$ & ๑ & $\checkmark$ & ث & $\downarrow$ & $\Rightarrow$ & 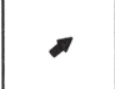 \\
\hline & $\mathrm{H}_{\text {Sit7a }}$ & Integrationsintensität & ๑) & $\downarrow$ & 步 & $\checkmark$ & $\Rightarrow$ & 1ै \\
\hline \multirow{2}{*}{ 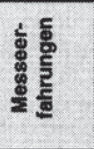 } & $\mathrm{H}_{\mathrm{sit8}}$ & $\begin{array}{l}\text { Messebeteiligungsin- } \\
\text { tensität erklärt } \\
\text { messesp.GH-Typen }\end{array}$ & $\odot$ & & & & & \\
\hline & $\mathrm{H}_{\mathrm{Sit} \text { a }}$ & $\begin{array}{l}\text { Umfang Messe- } \\
\text { erfahrung }\end{array}$ & ๑'○ & $\Rightarrow$ & $\mathbf{y}$ & & 4 & 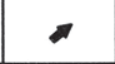 \\
\hline \multirow{2}{*}{ 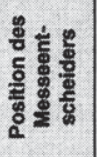 } & $\mathrm{H}_{\text {Sit9 }}$ & $\begin{array}{l}\text { Position des Messe- } \\
\text { entscheiders erklärt } \\
\text { messesp. GH-Typen }\end{array}$ & ๑ & & & & & \\
\hline & $\mathrm{H}_{\mathrm{sit9a}}$ & $\begin{array}{l}\text { Zugehörigkeit zu Füh- } \\
\text { rungsbereich }\end{array}$ & $\odot$ & 4 & 4 & $\downarrow$ & 4 & 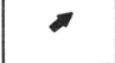 \\
\hline \multirow{5}{*}{ 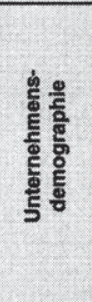 } & $\mathrm{H}_{\text {sit10 }}$ & $\begin{array}{l}\text { Unternehmensgröße } \\
\text { erklärt messesp. } \\
\text { GH-Typen }\end{array}$ & $\oplus$ & $\begin{array}{l}\text { eher kleine } \\
\text { Unterneh- } \\
\text { men }\end{array}$ & $\begin{array}{l}\text { ener grolie } \\
\text { Unterneh- } \\
\text { men }\end{array}$ & $\begin{array}{l}\text { ener mittere } \\
\text { Unterneh- } \\
\text { men }\end{array}$ & $\begin{array}{l}\text { ener mittlere } \\
\text { Unterneh- } \\
\text { men }\end{array}$ & $\begin{array}{l}\text { ener kleine bis } \\
\text { mittlere Unter- } \\
\text { nehmen }\end{array}$ \\
\hline & $\mathrm{H}_{\text {Sit10a }}$ & Großunternehmen & ๑) & $\Rightarrow$ & & & $\Rightarrow$ & $\Rightarrow$ \\
\hline & $\mathrm{H}_{\text {Sit10b }}$ & $\begin{array}{l}\text { Leder-, Textil- und Be- } \\
\text { kleidungsgewerbe }\end{array}$ & ๑) & ث & 4 & 1 & $\downarrow$ & 4 \\
\hline & $\mathrm{H}_{\text {Sit10c }}$ & $\begin{array}{l}\text { Investitionsgüterin- } \\
\text { dustrie }\end{array}$ & ๑) & $\downarrow$ & $\boldsymbol{\uparrow}$ & $\checkmark$ & $\uparrow$ & $\downarrow$ \\
\hline & $\mathrm{H}_{\text {Sit10d }}$ & $\begin{array}{l}\text { Konsumgüter- und } \\
\text { Dienstleistungsunter- } \\
\text { nehmen }\end{array}$ & $\oplus$ & \begin{tabular}{|l|} 
Kons. $^{2)}$ \\
$\mathrm{DL}^{3}{ }^{3)}$ \\
\end{tabular} & $\begin{array}{|ll|}\text { Kons. } & \checkmark \\
\text { DL. } & \checkmark \\
\end{array}$ & $\begin{array}{ll}\text { Kons. } & 1 \\
\text { DL. } & \mathbf{A} \\
\end{array}$ & $\begin{array}{ll}\text { Kons. } \\
\text { DL. }\end{array}$ & $\begin{array}{l}\text { Kons. } \\
\text { DL. }\end{array}$ \\
\hline \multicolumn{3}{|c|}{$\begin{array}{c}\text { 1) } \text { messesp. } \mathrm{GH}=\text { messespezifische } \\
\text { Grundhaltung }\end{array}$} & \multicolumn{6}{|c|}{ I = (stark) positiver Einflu 1} \\
\hline $\begin{array}{l}\text { 2) Kons. } \\
\text { 3) DL. }\end{array}$ & \multicolumn{2}{|c|}{$\begin{aligned} & \text { Grundhaltung } \\
= & \text { Konsumgüterunternehmen } \\
= & \text { Dienstleistungsunter- } \\
& \text { nehmen }\end{aligned}$} & $\Rightarrow$ & $\begin{array}{l}=\mathrm{kein} / \mathrm{gerin} \\
=(\mathrm{stark}) \mathrm{neg}\end{array}$ & $\begin{array}{l}\text { ger Einfluß } \\
\text { gativer Einfluß }\end{array}$ & & \multicolumn{2}{|c|}{$\begin{array}{l}\text {...auf die Wahl einer } \\
\text { messespezifischen } \\
\text { Grundhaltung }\end{array}$} \\
\hline \multicolumn{2}{|c|}{ Hypothesentest: } & ( $=$ Bestătigung & & = Ablehnu & & & & \\
\hline
\end{tabular}

Tab. 28: Synopse der Einflußfaktoren für die ermittelten messespezifischen Grundhaltungstypen 
allenfalls als flankierendes Instrument im Rahmen der Marketingbemühungen einzusetzen, da die Messezielgruppen i. d. R. nicht primär die Messe besuchen, um Kontakt zu den dort vertretenen Dienstleistern aufzunehmen. Es ist daher kaum verwunderlich, daß diese Aussteller Messebeteiligungen kaum in ihr Kommunikations-Mix integrieren.

(4) Für die Entstehung der Grundhaltung hybrider Fachaussteller konnte aus kontextspezifischer Sicht ein dem der fachbesucherorientierten Hardseller ähnliches Umfeld ausgemacht werden. Dem hohen Wettbewerbsdruck, der sich insbesondere in einem erhöhten internationalen Preiswettbewerb zeigt, versuchen diese Aussteller der Investitonsgüterindustrie mit überwiegend mittlerer Größe, durch eine intensive Mitarbeiterorientierung zu begegnen. Zunehmend stellt für diese Aussteller die synergetische Nutzung von Messebeteiligungen und anderen Kommunikationsinstrumenten eine vergleichsweise hohe Bedeutung dar.

(5) Der ambitionierte Regionalaussteller läßt sich dagegen kaum durch sein Markt- und Wettbewerbsumfeld erklären, da dieser Grundhaltungstyp sowohl in internationalen Wachstumsmärkten als auch in national gesättigten Märkten agiert. Dagegen zeichnen sich diese Aussteller durch eine, gemessen an den anderen Grundhaltungsclustern, hohe Messebeteiligungsintensität und damit zahlreichen Messeerfahrungen aus, die nicht zuletzt auch als Erklärung für das im Vergleich breiteste Anspruchsspektrum und die umfangreiche Kontrolle des Messebeteiligungserfolges dienen. Interessant ist in diesem Zusammenhang, daß es sich bei dieser Gruppe von Ausstellern vornehmlich um eher kleinere Unternehmen handelt. Dies wiederum verdeutlicht, daß kleinere Unternehmen Messebeteiligungen intensiver im Rahmen ihres Marketingkonzeptes einsetzen, als dies zu vermuten gewesen wäre. ${ }^{533}$

Betrachtet man die Ergebnisse der Einflußfaktorenanalyse zusammenfassend, dann konnte eine zentrale Kategorie von Einflußfaktoren über die - i. S. e. monokausalen Begründung - eine hauptsächliche oder gar ausschließliche Erklärung der messespezifischen Grundhaltungstypen möglich ist, nicht identifiziert werden. Damit erweist sich ex post die Wahl eines breiteren kontingenztheoretischen Bezugsrahmens als gerechtfertigt.

533 Vgl. hierzu auch die Ausführungen zu den fachbesucherorientierten Hardsellern in diesem Kapitel. 
Über die inhaltlichen Dimensionen der vorliegenden Arbeit hinaus erfordert eine kritische Würdigung der Untersuchungsergebnisse schließlich eine Auseinandersetzung mit der gewählten methodischen Vorgehensweise der Untersuchung. Folgende Aspekte sind dabei hervorzuheben:

(1) Vorbehalte lassen sich gegen die nahezu ausschließliche Verwendung qualitativer Daten vorbringen, die lediglich subjektive Einschätzungen der in den ausstellenden Unternehmen befragten Führungskräfte widerspiegein und Verzerrungseffekte verursachen können. Dem ist aber entgegenzuhalten, daß die Breite der abgefragten Themen sowie eine als gering einzuschätzende Bereitschaft der Messeentscheider in den Unternehmen zur Teilnahme an umfangreichen Felderhebungen eine zeiteffiziente Datenerhebung, wie sie im vorliegenden Fall durch die Verwendung geschlossener Fragen von Ratingskalen für die Antworten weitgehend gewährleistet wird, zwingend erforderlich erscheinen ließ. Außerdem wurde über die vorab geführten Expertengespräche ${ }^{534}$ sowie die Workshops ${ }^{535}$ und ausführlichen Pretests des Fragebogens versucht, zur Erhöhung der Validität der Messungen beizutragen.

(2) Hinsichtlich der Methodenwahl ist schließlich anzumerken, daß die eingesetzten varianz- und kontingenzanalytischen Verfahren streng genommen nicht zur Aufdeckung von Kausalbeziehungen geeignet sind, da sie lediglich Beziehungszusammenhänge aufdecken, nicht aber die jeweilige Wirkungsrichtung spezifizieren. Diesem Einwand wurde zu begegnen versucht, indem die inhaltlichen Aspekte der Untersuchung aus entscheidungstheoretisch bestätigten Erwägungen des Managementansatzes von Messebeteiligungen geschlossen wurden. Zusätzlich wurde an besonders sensiblen Stellen im Rahmen der Expertengespräche der Versuch unternommen, die tatsächlichen Wirkungsbeziehungen aufzudecken.

Im Verlauf der vorliegenden Arbeit wurde der Managementprozeß von Messebeteiligungen in seinen einzelnen Bestandteilen theoretisch abgeleitet und empirisch analysiert. Vor dem Hintergrund unterschiedlicher situativer Kontexte und organisationaler Voraussetzungen ließ sich sowohl eine Analyse der grundhaltungsspezifischen Ausgestaltung messebezogener Aktivitäten als auch deren

534 Vgl. die Liste der geführten Expertengespräche im Anhang dieser Arbeit.

535 Vgl. die Teilnehmerlisten der AUMA-Workshops zum Thema „Ziele und Nutzen von Messebeteiligungen" im Anhang dieser Arbeit. 
Erfolgskontrolle vornehmen. Inbesondere die hinsichtlich der Kontrolle von Messeaktivitäten erzielten Ergebnisse der Arbeit geben begründeten Anlaß, die Ausgestaltung dieses zentralen Parts der Messebeteiligungen kritisch zu hinterfragen $^{536}$.

Im folgenden soll deshalb ein Ansatz zur systematischen Kontrolle von Messebeteiligungen entwickelt werden, der nicht nur eine Kontrolle im Sinne einer Ergebnisvalidierung leistet, sondern darüber hinaus auch in der Lage ist, Kontrollinformationen über die Planung und Ausgestaltung des gesamten Managementprozesses zu liefern.

\section{Ableitung eines Ansatzes zur Kontrolle von Messebeteiligungen}

Grundsätzlich besitzt die Messebeteiligungskontrolle drei wesentliche Funktionen: Die im Rahmen der Kontrolle gewonnenen Daten dienen einerseits der Beurteilung bereits durchgeführter Messebeteiligungen und andererseits der Beurteilung aktueller Messeengagements, um ggf. auch kurzfristig korrigierend einwirken zu können. Darüber hinaus können sie - im Sinne eines Rückkoppelungseffektes - die Basis für zukünftige Beteiligungsentscheidungen darstellen. Die Messebeteiligungskontrolle besitzt somit in Anlehnung an allgemeine Kontrollsystematiken eine Diagnose- und eine Prognosefunktion. Gerade der prognostischen Kontrollfunktion kommt große Bedeutung zu, denn aufgrund der zumeist unzureichenden Datenbasis sind Entscheidungen hinsichtlich zukünftiger Messebeteiligungen durch ein hohes Maß an Unsicherheit gekennzeichnet.

Aus der Notwendigkeit einer Messebeteiligungskontrolle sowie deren drei wesentlichen Funktionen lassen sich die zentralen Elemente eines umfassenden Konzeptes ableiten. Ein solches Konzept der Messebeteiligungskontrolle ${ }^{537}$ hat aus zwei grundlegenden Teilbereichen zu bestehen: Dem der Prozeßkontrolle

536 Die unzureichenden Kontrollen von Messebeteiligungen zeigen sich zum einen in den offenkundigen Defiziten im Bereich der Messeerfolgskontrolle, aber auch in den z. T. deutlichen Inkonsistenzen zwischen der strategischen Ausrichtung der Messebeteiligungsplanung und der sich anschließenden Ausgestaltung von Messebeteiligungen bei einzelnen Grundhaltungstypen. Vgl. hierzu die Ausführungen in Kap. C 2. Messebeteiligungsmanagement vor, die das Thema der Messebeteiligungskontrolle in der hier vorliegenden Weise bearbeiten. Insofern ist auf die allgemeineren Erkenntnisse der MarketingKontrolle zurückzugreifen, auf die im folgenden schwerpunktmäßig rekurriert wird. 
dienenden Messebeteiligungs-Audit sowie der Ergebniskontrolle. ${ }^{538}$ In beiden Bereichen ist hinsichtlich des Kontrollablaufs ein identisches, aus vier Stufen bestehendes Vorgehen zu durchlaufen: ${ }^{539}$

- Festlegung von Standards bzw. Soll-Werten

- Ermittlung der Ist-Werte

- Standard- bzw. Soll - Ist - Vergleich sowie

- Auswertung der Vergleichsergebnisse

Grundlegende Unterschiede bestehen hingegen hinsichtlich der jeweils zu untersuchenden Kontrollobjekte. Während die Ergebniskontrolle sich mit der Messung und Beurteilung der erzielten Handlungserfolge beschäftigt ${ }^{540}$, ist es Aufgabe des Messebeteiligungs-Audits, eine Überprüfung der Konzeptionierung und des Ablaufs von Messebeteiligungen vorzunehmen. Es wird daher auch als Prozeßkontrolle bezeichnet. ${ }^{541}$

Ziel eines Messebeteiligungs-Audits ist es, möglichst frühzeitig planungs- und systembedingte Fehlentwicklungen zu identifizieren, um damit eine optimale Anpassung an dynamische Umweltentwicklungen zu gewährleisten. ${ }^{542}$ Unter dem Messebeteiligungs-Audit sind dabei fünf Teilbereiche zu subsumieren, die die wesentlichen Komponenten des Managementprozesses von Messebeteiligungen $^{543}$ umfassen: ${ }^{544}$

538 Vgl. allg. zur Prozeß- und Ergebniskontrolle, Nieschlag. R. Dichtl. E.. Hörschgen. H., Marketing, 15. Aufl., Berlin 1988, S. $913 \mathrm{ff}$.

539 Vgl. Meffert, H., Marketing. Grundlagen der Absatzpolitik, 7. Aufl., Wiesbaden 1986, S. 558 f.

$540 \mathrm{Da}$ die Ergebniskontrolle in Form von Soll-Ist-Vergleichen vorgenommen wird, bildet die verbindliche Formulierung von Sollvorgaben eine wesentliche Voraussetzung. Als Sollvorgaben fungieren zumeist die fixierten Messebeteiligungsziele, denen damit innerhalb der Ergebniskontrolle eine zentrale Bedeutung beizumessen ist. Die Grundlagen dieses Aspekts wurden im Rahmen der konzeptionellen Ausführungen zur Erfolgskontrolle von Messebeteiligungen dieser Arbeit bereits umfassend gewürdigt. Vgl. Kap. C 1.2.

541 Vgl. McDonald, M. H. B., The Marketing Audit - Translating Marketing Theory into Practice, Oxford, London u. a. 1993, S. 11.

542 Vgl. allg. Meffert, H., Marketing. Grundlagen der Absatzpolitik, 7. Aufl., Wiesbaden 1986, S. 571.

543 Vgl. zum Manangement-Prozeß von Messebeteiligungen Kap A. 2. dieser Arbeit.

544 Vgl. allg. Nieschlag, R. Dichtl. E. Hörschgen, H., Marketing, 17. Aufl. Berlin 1994, S. 932 ff.

Rall U eding and Universitat Mǘnster - 978-3-631-75073-5 
- das Prämissen-Audit

- das Ziele- und Strategien-Audit

- das Organisations-Audit

- das Maßnahmen-Audit

- das Audit der Erfolgskontrolle

Diese verschiedenen Elemente eines Gesamt-Audits setzen an unterschiedlichen Phasen der Messebeteiligung an. In Abb. 24 ist eine solche Zuordung vom inhaltlichen Fokus des Messebeteiligungs-Audits und den Messephasen vorgenommen.

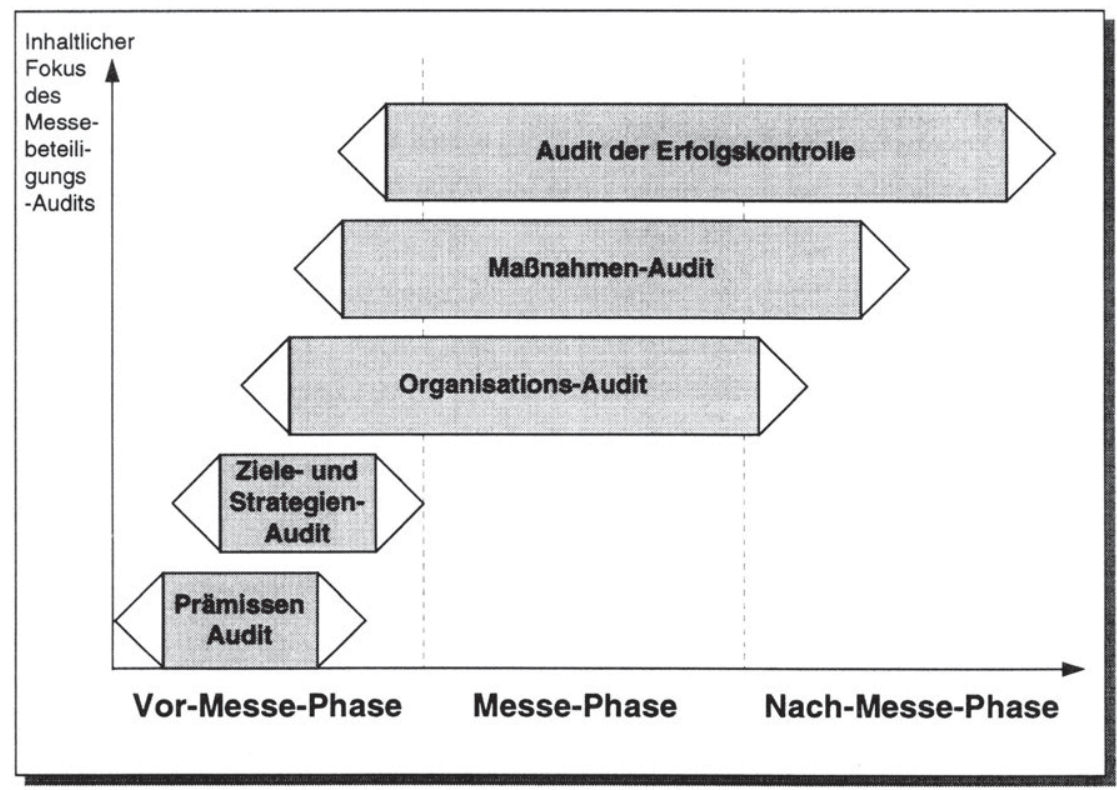

Abb. 24: Das Messebeteiligungs-Audit im Phasenüberblick

Aufgabe des Prämissen-Audits ist es zunächst, sämtliche der Planung von Messebeteiligungen zugrunde liegenden Annahmen festzustellen sowie deren Sinnhaftigkeit und Berechtigung zu überprüfen. Werden bspw. nicht sämtliche bedeutsamen Aspekte berücksichtigt oder ist die tatsächliche Umweltsituation des Unternehmens anders als angenommen, so können die gefällten Messebeteili- 
gungsentscheidungen nicht situationsgerecht sein. Es ist in diesem Zusammenhang zu fragen: ${ }^{545}$

- Sind die entscheidungsrelevanten Prämissen erkannt und bei der Planung der Messebeteiligung berücksichtigt worden?

- Wie aktuell, zuverlässig und präzise waren die Informationen über die Umweltsituation?

- Existieren systematische Ansätze einer Verdichtung und Interpretation der vorhandenen Informationen zu den Kontextbedingungen?

Wie im Teil B dieser Arbeit gezeigt, haben die Ziel- und Strategie-Entscheidungen weitreichende Bedeutung für die Planung und Ausgestaltung von Messebeteiligungen - sie legen die Grundhaltung für die Umsetzung der messespezifischen Aktivitäten fest. Dem Ziel- und Strategie-Audit kommt daher die Aufgabe zu, durch Kontrollen inhaltliche Fehlinterpretationen der messespezifischen Ziele und Strategien ${ }^{546} \mathrm{zu}$ identifizieren und möglichst auszuschalten. ${ }^{547}$ Dabei erhalten vor allem die folgenden Fragen Relevanz:

- Sind die messespezifischen Ziele mit den Kommunikations- und Marketingzielen des Unternehmens abgestimmt?

- Waren alle relevanten Unternehmensbereiche am Zielbildungsprozeß beteiligt?

- Welche Zielprioritäten wurden festgelegt?

- Sind sowohl das messebezogene Zielsystem als auch die daraus abgeleitete messespezifische Strategie operational und inhaltlich konsistent?

- Harmonieren die messespezifischen Strategien mit dem Selbstverständnis des Unternehmens und den übrigen kommunikationspolitischen Aktionsfeldern?

$545 \mathrm{Vgl}$. allg. Hildenbrand, W., Informationsmarketing in der Kommunikation zwischen Hersteller und Handelsvertreter, Frankfurt a. M. 1983, S. 162 ff.; Grotz-Martin, S., Informations-Qualität und Informationsakzeptanz in Entscheidungsprozessen, Saarbrücken 1976, S. 26 ff.

546 Die strategische Ebene von Messebeteiligungen wird dabei maßgeblich durch die Messeselektion und die messespezifische Positionierung bestimmt. Vgl. hierzu insbesondere die Ausführungen in Kap B 1. dieser Arbeit.

547 Hierbei ist zunächst der Ist-Zustand zu erfassen, d. h. es ist zu ermittlen, welche Ziele bzw. strategischen Ausrichtungen geplant sind, um sie auf ihre Operationalität, Konsistenz, Transparenz, Vollständigkeit, Aktualität und Realisierbarketi hin zu untersuchen. Vgl. Heinen, E., Grundlagen betriebswirtschaftlicher Entscheidungen. Das Zielsystem der Unternehmung, 3. Aufl., Wiesbaden 1976, S. 49 ff.; Szyperski. N.. Winand, U., Grundbegriffe der Unternehmensplanung, Stuttgart 1980, S. 50 f.; Korndörfer, W., Unternehmensführungslehre, 5. Aufl., Wiesbaden 1986, S. $38 \mathrm{ff}$. 
Ausgangspunkt des Maßnahmen-Audit ist die Bestandsaufnahme der Vor-MesseMesse- und Nach-Messeaktivitäten. In diesem Zusammenhang ist vor allem die inhaltliche Zusammensetzung der einzelnen Aktivitäten sowie die Höhe des jeweils benötigten Budgetanteils und dessen Aufteilung $\mathrm{zu}$ überprüfen. ${ }^{548}$ Dabei interessieren die folgenden Fragestellungen:

- Sind die geplanten bzw. realisierten Maßnahmen der Unternehmenssituation angemessen?

- Sind sie mit den messespezifischen Zielen und Strategien vereinbar?

- Erreichen die Aktivitäten der Vor-Messe-Phase die gewünschten Zielgruppen?

- Schaffen sie hinreichend Anreize zum Besuch des Messestandes?

- Bringen die Messe-Aktivitäten die gewünschten Zielgruppen zum Messestand?

- Dienen die Aktivitäten der Nach-Messe-Phase der konsequenten Weiterverfolgung der Messekontakte?

- Ist die Weiterleitung der erhaltenen Kundeninformationen an die relevanten Unternehmensbereiche sichergestellt?

Das Ziel des Organisations-Audit ist die Entdeckung von spezifischen Schwachstellen in der Ablaufplanung und Durchführung der Messebeteiligung. ${ }^{549}$ Dabei sind die Messe-Organisation und ihre Beziehungen zu anderen Funktionsbereichen der Unternehmung ${ }^{550}$ sowie zu externen Dienstleistungsanbietern, die in die Abwicklung der Messebeteiligung einbezogen werden, zu überprüfen. Zudem kann das Organisations-Audit bei Messebeteiligungen in zwei interdependente Teilbereiche aufgespalten werden: Das Koordinations- und das Auswahl-Audit. Zu fragen ist demnach: ${ }^{551}$

- Sind die mit der Messebeteiligung betrauten Unternehmensbereiche informell miteinander vernetzt (Koordinations-Audit)?

- Stimmen die Qualifikationsprofile der Mitarbeiter im Zusammenhang mit der Messebeteiligung mit den Anforderungsprofilen überein (Auswahl-Audit)?

\footnotetext{
548 Vgl. allg. Nieschlag, R. Dichtl, E., Hörschgen, H., Marketing, 17. Aufl., Berlin 1994, S. 933.

549 Vgl. allg. Adamowsky, S., Prüfung der Organisation, in: Grochla. E. (Hrsg.), HWO Handwörterbuch der Organisation, Stuttgart 1973, Sp. 1371-1377; Peemöller. V., Organisationsprüfung, in: Management-Enzyklopädie, Bd. 7, München 1975, S. 2556-2565.

550 Vgl. allg. Kotler, Ph., Gregor, W. Rodgers, W., The Marketing Audit Comes of Age, in: SMR, Vol. 19, Winter 1977, S. 25-45.

551 Vgl. allg. Kotler, Ph. Bliemel, Marketing-Management, 6. Aufl, Stuttgart 1994, S, 685 
- Wie wurde die Auswahl der externen Dienstleister vorgenommen (AuswahlAudit)?

- Sind Ausstellerunternehmen und externer Dienstleister in ihren Leistungsanforderungen und -profilen sowie terminlich aufeinander abgestimmt (Koordinations-Audit)?

Das Audit der Erfolgskontrolle hat schließlich zum Ziel, den Prozeß der Durchführung zur Messung und Bestimmung des Messebeteiligungserfolgs zu analysieren. Hierbei ist vor allem die Zweckmäßigkeit des Einsatzes der Methoden der Erfolgskontrolle mit Blick auf die verfolgten Messebeteiligungsziele zu untersuchen. In diesem Zusammenhang ist daher zu fragen:

- Wird der Erfolg einer Messebeteiligung überhaupt ermittelt?

- Wird die Erreichung der verfolgten Messebeteiligungsziele mit den richtigen Methoden der Erfolgskontrolle gemessen?

- Wird hinsichtlich der ermittelten Ergebnisse der Erfolgskontrolle eine „unternehmensinterne Messetransparenz" hergestellt?

- Werden Konsequenzen aus den Ergebnissen der Erfolgskontrolle gezogen, die in konkreten Handlungen münden?

- Werden die richtigen Konsequenzen gezogen und die richtigen Handlungsoptionen ergriffen?

Das aufgezeigte Messebeteiligungs-Audit trägt in zweierlei Hinsicht zu einer fundierten Analyse der Messebeteiligungsplanung und -durchführung sowie der Erfolgskontrolle bei. Zum einen vermag dieses Kontrollinstrument i. S. e. Ursachenanalyse Schwachstellen in Planung und Durchführung von Messebeteiligungen offenzulegen, indem der gesamte Messebeteiligungsprozeß einer systematischen und umfassenden Analyse unterzogen wird. Zum anderen wird insbesondere mit dem Audit der Erfolgskontrolle geprüft, ob und inwieweit tatsächlich eine zieladäquate Messung und Beurteilung des Messeerfolgs vorgenommen wird. Nur eine valide Erfassung der erreichten Zielausprägung gewährleistet schließlich, daß die Interpretation der gewonnen Ergebnisse zu den richtigen Schlußfolgerungen und Konsequenzen führt.

Ein Versuch dieses notwendige Messebeteiligungs-Audit auf die vorliegende Untersuchung zu übertragen, führt zu interessanten Erkenntnisse im Hinblick auf 
den Prozeß der Planung und Durchführung von Messebeteiligungen, für die identifizierten Grundhaltungstypen, die in Abb. 25 dargestellt sind. ${ }^{552}$

\begin{tabular}{|c|c|c|c|c|c|}
\hline $\begin{array}{l}\text { mesesep. Grund- } \\
\text { haltungstyp } \\
\text { Phasen } \\
\text { des Moses- } \\
\text { betelligungsaudits }\end{array}$ & $\begin{array}{l}\text { feohbeaucherorien- } \\
\text { terte Hardestler }\end{array}$ & \begin{tabular}{|c|}
$\begin{array}{c}\text { kommunikatione- } \\
\text { orlentlerto }\end{array}$ \\
Bexiohungemaneger
\end{tabular} & 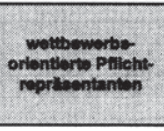 & $\begin{array}{l}\text { hybride } \\
\text { Fachausetellor }\end{array}$ & Regititionilarte \\
\hline Primiecenaudit & k.A. & k.A. & k.A. & k.A. & k.A. \\
\hline $\begin{array}{l}\text { zele und } \\
\text { Strateglen-Audit }\end{array}$ & 0 & - & $\cdot$ & 0 & + \\
\hline $\begin{array}{l}\text { Orgeniaations- } \\
\text { Audit }\end{array}$ & 0 & 0 & - & - & 0 \\
\hline $\begin{array}{c}\text { Maennahmen- } \\
\text { Audit }\end{array}$ & k.A. & k.A. & k.A. & k.A. & k.A. \\
\hline $\begin{array}{l}\text { Audit der } \\
\text { Entolgakontrolie }\end{array}$ & + & - & - & 0 & + \\
\hline - = viele Schw & n erkennbar & $0=$ weni & in erkennbar & um Schwä & erkennbar \\
\hline
\end{tabular}

Abb. 25: Synoptische Darstellung des Messebeteiligungs-Audits am Beispiel der messespezifischen Grundhaltungstypen

Es lassen sich zunächt zwei Typen identifizieren, die ein Messebeteiligungs-Audit nur mit geringen Schwächen durchlaufen würden. ${ }^{553}$ Dabei handelt es sich um die fachbesucherorientierten Hardseller und die ambitionierten Regionalaussteller. Solche Schwachpunkte sind bei den fachbesucherorientierten Hardsellern hinsichtlich des Ziel- und Strategien-Audit bei der Integration von Messebeteiligungen in eine übergeordnete Kommunikationskonzeption zu konstatieren.

552 Dabei ist anzumerken, daß im Rahmen dieser Untersuchung aufgrund des empirischen Designs eine dezidierte Analyse des Prämissen- und Organisations-Audits nur ansatzweise erfolgen kann. Aus diesem Grund wird hier auf eine spezifische Bewertung der Grundhaltungstypen verzichtet. Eine derartige Bewertung ließe sich ohnenhin nur mit Hilfe der Durchführung von Intensivfallstudien vornehmen. Dies erscheint vor dem Hintergrund der Untersuchungsergebnisse allerdings dringend erforderlich.

553 In diesem Zusammenhang wird bewußt eine präskriptive Perspektive eingenommen, die auf Plausibilitätsüberlegungen basiert, die schwerpunktmäßig auf einer Gesamtbetrachtung der grundhaltungsspezifischen Messekonzepte beruht. Dem Autor ist sehr wohl bewußt, daß wahrscheinlich ein Messebeteiligungs-Audit von den betrachteten Ausstellern nicht oder nur ansatzweise durchgeführt wurde. 
Dagegen zeichnen sich beide Ausstellertypen durch eine hohe Operationalisierung der verfolgten Messebeteiligungsziele aus. ${ }^{554}$ Im Rahmen des Maßnahmen-Audits sind - wie eigentlich für alle messespezifischen Grundhaltungstypen - auch für die fachbesucherorientierten Hardseller und die ambitionierten Regionalaussteller insbesondere bezüglich der Ziel-Strategie-Maßnahmen-Kongruenz leichte Inkonsistenzen unverkennbar. ${ }^{555}$ Bei beiden Ausstellertypen ist eine Übereinstimmung von Zielen und zugehörigen Maßnahmen lediglich anzatzweise feststellbar. Das Audit der Erfolgskontrolle kann indes als bestanden angesehen werden, da beide Grundhaltungstypen zieladäquate Methoden der Erfolgskontrolle einsetzen. ${ }^{556}$

Ein Blick auf die drei weiteren Grundhaltungstypen offenbart ein differenziertes Bild. Sowohl für den wettbewerbsorientierten Pflichtrepräsentanten als auch den hybriden Fachausteller ergeben sich durchaus erwartete Audit-Resultate. Während die, hinsichtlich des gesamten Messeengagements durch Passivität gekennzeichneten Pflichtaussteller folgerichtig auch nahezu in keiner Phase des AuditProzesses einer kritischen Überprüfung standhalten, läßt sich für den hybriden Fachausteller zumindest im Rahmen des Ziel-Strategien-Audits sowie des Audits der Erfolgskontrolle von einer hinreichenden Erfüllung der Auditanforderungen sprechen. Eine derartige Einschätzung resultiert aus der Tatsache, daß diese Aussteller ihre Messebeteiligungsziele zumindest formal und zeitlich in ein übergeordnetes Kommunikationskonzept integrieren ${ }^{557}$ und darüber hinaus auch im Rahmen der Erfolgskontrolle Instrumente eingesetzt werden, die in wesentlichen Teilbereichen zur Überprüfung der Zielerreichung beitragen.

Dagegen ist zu vermuten, daß ein Messebeteiligungs-Audit im Cluster der kommunikationsorientierten Beziehungsmanager zu ernüchternden Ergebnissen führen würde. Dieser, durch ein differenziertes Zielsystem und vielfältige messebezogene Aktivitäten gekennzeichnete Grundhaltungstyp, läßt nicht nur eine konsequente Operationalisierung seiner Messebeteiligungs-Ziele vermissen, sondern ist zudem - nahezu in logischer Konsequenz - in hohem Maße kontrollavers und

\footnotetext{
554 Vgl. die Ausführungen in Kap C dieser Arbeit.

Eine Korrelationaanalyse von Zielen und Maßnahmen, ergab für keinen der ermittelten Grundhaltungstypen nennenswerte Zusammenhänge. Dabei konnte mximal eine Korrelation von $r=0,4$ ermittelt werden, so daß die Ergebnisse dieser Analyse nicht näher ausgewiesen werden.

Vgl. dazu die Ausführungen in Kap. C 2.23 dieser Arbeit

Vg. dazu die Ausführungen in Kap. D 4.21
} 
läßt somit auch kaum eine geeignete Überprüfung von Zielerreichungsbeiträgen zu. Gerade weil dieser Aussteller durch eine hohe Integration des Instruments von Messebeiteiligungen in das übergeordnete Kommunikationskonzept gekennzeichnet ist, wäre eine Ermittlung des Zielerreichungsbeitrages des Messeinstrumentariums allerdings zwingend erforderlich, um eine optimale Allokation des Kommunikationsbudgets zu gewährleisten. Auf Basis der Audit-Ergebnisse ist daher zu befürchten, daß die im Rahmen der Ergebniskontrolle ermittelten Erfolgsbeurteilungen als wenig valide einzustufen sind.

Die Ergebniskontrolle stellt neben dem Messebeteiligungs-Audit den zweiten Bereich der Kontrolle von Messebeteiligungen dar. Im Rahmen der Ergebniskontrolle ist insbesondere die Bewertung der Effizienz von Messebeteiligungsaktivitäten in den Vordergrund zu stellen ${ }^{558}$.

Die Effizienzbewertung von Messebeteiligungsaktivitäten stellt somit einen zentralen Teilbereich der Ergebniskontrolle dar. Dabei gilt eine Messebeteiligung als effizient, wenn der erzielte Nutzen die entstandenen Kosten übersteigt und aufgrund einer alternativen Mittelverwendung kein höherer Nutzen hätte erzielt werden können ${ }^{559}$. Im Rahmen der Effizienzbewertung wird damit eine Beurteilung der Wirtschaftlichkeit von Messebeteiligungsaktivitäten und zudem die Sicherstellung einer optimalen Budgetallokation innerhalb des Kommunikationsmix angestrebt ${ }^{560}$. In diesem Zusammenhang kann eine Messebeteiligung als effizient interpretiert werden, wenn

- der erzielte Nutzen die entstandenen Kosten übersteigt (notwendige Bedingung) und

558 Neben der Effizienzbestimmung kann der Ergebniskontrolle auch der Bereich der Effektivität subsumiert werden. Diese wurde jedoch bereits im Rahmen des Messebeteiligungs-Audits thematisiert. Im Gegensatz zum Begriff der Effektivität, der die Eignung einer Maßnahme zur Erreichung angestrebter Ziele beschreibt, ist der Effizienzbegriff durch ein relationales Moment gekennzeichnet, das sich allgemein in der Verknüpfung von Input- und Output-Größen manifestiert. Diese Input- und Outputgrößen erfahren bei der Effizienzbewertung von Messebeteiligungsaktivitäten eine Konkretisierung in Form von Kosten- und Nutzendimensionen. Vgl. zur allgemeinen Erläuterung des Effizienzbegriffes: Welge, M. K., Unternehmensführung, Bd 1, Stuttgart 1985, S. 585.

559 In diesem Zusammenhang ist nochmals darauf hinzuweisen, daß in der vorliegenden Arbeit keine Erfassung der Messebeteiligungskosten vorgenommen wurde.

560 Die Einbeziehung des Opportunitätsansatzes in die Effizienzbewertung verdeutlicht, daß die Suche nach geeigneten Vergleichsobjekten eine wesentliche Aufgabe im Rahmen der Effizienzbewertung darstellt. 
- aufgrund einer alternativen Mittelverwendung kein höherer Nutzen hätte erzielt werden können (hinreichende Bedingung).

Für die Messeeffizienzbewertung bietet dabei damit sich ein zweistufiges Vorgehen an:

(1) Überprüfung der notwendigen Bedingung durch eine isolierte Wirtschaftlichkeitsanalyse einer Messebeteiligung; die Effizienz wird dabei als dichotomes Merkmal i. S. e. Ja-Nein-Aussage interpretiert.

(2) Effizienzvergleiche mit geeigneten Vergleichsobjekten bei Erfüllung der notwendigen Bedingung; Ziel ist die Sicherstellung einer optimalen Budgetallokation auf Basis des Opportunitätsansatzes.

Eine isolierte Wirtschaftlichkeitsanalyse zur Überprüfung der notwendigen Bedingung ist aber nur möglich, wenn Kosten und Nutzen einer Messebeteiligung die gleiche Maßeinheit aufweisen. Weil die Kosten stets einer monetären Bewertung unterliegen, können isolierte Aussagen über die Effizienz einer zu bewertenden Messebeteiligung somit nur dann getroffen werden, wenn die Nutzendimensionen sowohl quantifizierbar als auch unmittelbar monetär bewertbar sind. Diese Voraussetzungen erfüllen allerdings nur die ökonomischen Nutzendimensionen. Eine isolierte monetäre Bewertung psychographischer Nutzendimensionen ist dagegen nicht möglich. Andererseits sind aber ökonomische Wirkungen bei Messebeteiligungen infolge der Zurechnungsproblematik zumeist nicht quantifizierbar. Insofern ist im Zusammenhang mit den zielbezogenen Nutzendimensionen von einem Dilemma der Effizienzbewertung von Messebeteiligungen zu sprechen.

Eine Lösung dieses Dilemmas ist nach Ansicht des Verfassers nur durch Einbeziehung von Vergleichsobjekten möglich. Dies bedeutet jedoch strenggenommen, daß im Rahmen einer Effizienzbewertung auf der Basis zielbezogener Nutzendimensionen auf eine Überprüfung der notwendigen Bedingung für die Effizienz verzichtet werden muß, soweit nicht eindeutig zurechenbare, ökonomische Nutzendimensionen existieren. Für die Effizienzbewertung kommt somit der Suche nach geeigneten Vergleichsobjekten (hinreichende Bedingung) zentrale Bedeutung zu. 
Hinsichtlich der Vergleichsobjekte ist zunächst zwischen intra- und inter-instrumentellen Vergleichen zu unterscheiden (vgl. Abb. 26). Bei intra-instrumentellen Vergleichen fungieren andere Messebeteiligungen als Vergleichsobjekte. Grundsätzlich kommen dafür sowohl unternehmenseigene, aus zeitlich vorgelagerten Perioden stammende, wie auch Messebeteiligungen anderer Unternehmen in Frage. Dienen eigene Messebeteiligungen als Vergleichsobjekt, so handelt es sich um Zeitvergleiche. Demgegenüber stellen Vergleiche auf Basis unternehmensfremder Messebeteiligungen Betriebsvergleiche dar. Implizite Probleme von Betriebsvergleichen sind $i$. d. R. die unzureichende Datenbasis sowie die Notwendigkeit einer weitestgehend kongruenten Unternehmensstruktur und Umweltsituation. ${ }^{561}$

Neben intra-instrumenteller Vergleiche auf Basis alternativer Messebeteiligungen können grundsätzlich auch andere Kommunikationsmaßnahmen als Vergleichsobjekte dienen. Auch im Rahmen dieser inter-instrumentellen Vergleiche sind wiederum Zeit- und Betriebsvergleiche möglich. Die Suche nach geeigneten Vergleichsobjekten gestaltet sich hierbei jedoch noch schwieriger als dies bei intrainstrumentellen Vergleichen der Fall ist. Daher besitzen inter-instrumentelle Betriebsvergleiche in Folge der unzureichenden Daten- und Vergleichsbasis eine nur untergeordnete Bedeutung.

561 Vgl. Mellerowicz, K., Kontrolle in der Absatzwirtschaft, in: Tietz, B. (Hrsg.), HWAHandwörterbuch der Absatzwirtschaft, Stuttgart 1974, Sp. 1104-1116. 


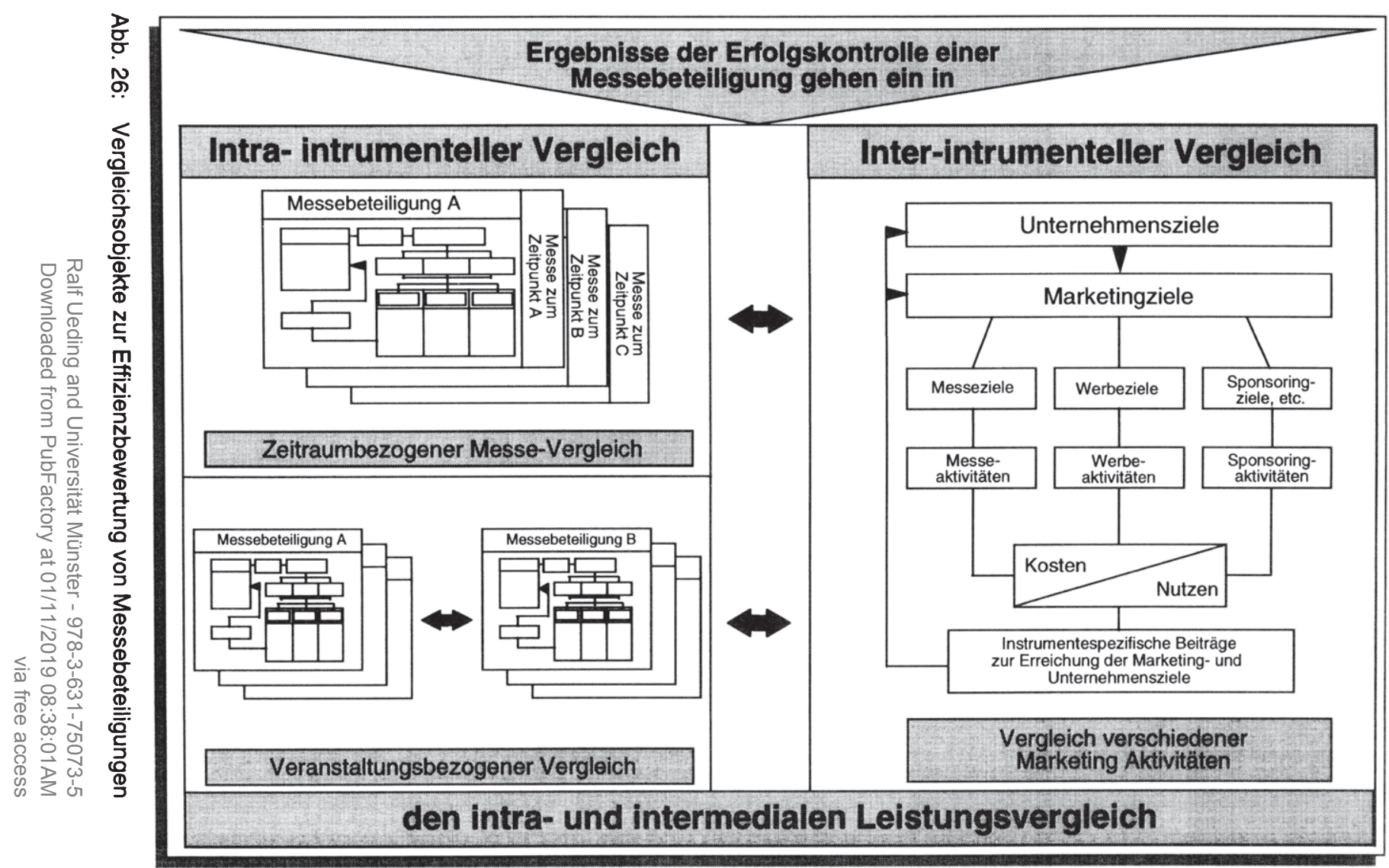


Darüber hinaus ist an ein potentielles Vergleichsobjekt die (idealtypische) Anforderung der Ziel- und Zielgruppenkongruenz zu stellen. Nur wenn bei identischen Zielgruppen identische Wirkungen angestrebt werden, ist eine adäquate Vergleichsbasis gegeben.

Dem eigentlichen Bewertungsprozeß sollte eine strukturierte Zusammenfassung aller für die zu bewertende Messebeteiligung identifizierten Kosten- und Nutzendimensionen vorausgehen. An die Ermittlung der Gesamtkosten der zu bewertenden Messebeteiligung schließt sich zunächst die Erfassung aller unmittelbar zielbezogenen Nutzendimensionen an.$^{562} \mathrm{Da}$ bei Messebeteiligungen mehrere Ziele parallel verfolgt werden, hängt die relative Bedeutung eines erzielten Nutzens von der jeweiligen Zielpriorität ab. Eine Gesamtbeurteilung des Nutzens einer Messebeteiligung entsteht dabei durch die Aggregation der mit hoher Priorität verfolgten Einzelzielsetzungen. Die Einzelzielsetzungen sind dabei zunächst mit den individuellen Zielerreichungsgraden $z u$ verknüpfen, um in einem zweiten Schritt die derart gewichteten Zielerreichungsgrade in einen Nutzenbeitrag des Gesamtzielsystems zu überführen.

Ein solches Vorgehen soll für die messespezifischen Grundhaltungen exemplarisch dargestellt werden. Ausgangspunkt der Überlegung stellt dabei die Ermittlung der mit hoher Priorität verfolgten Einzelzielsetzungen dar ${ }^{563}$. Für diese Zielsetzungen wurden die jeweiligen Zielerreichungsgrade ermittelt und mit den zugehörigen Zielbedeutungen gewichtet ${ }^{564}$. Die resultierenden Nutzenbeiträge wurden schließlich zu einem Messebeteiligungs-Nutzen-Index aggregiert. Die entsprechenden grundhaltungsspezifischen Ergebnisse sind in Abb. 27 wiedergegeben.

562 Darüber hinaus ist eine Integration mittelbar zielbezogener Nutzendimensionen (im Sinne einer unmittelbare Erfassung des erzielten Nutzens erfolgte.

563 Dabei wurden solche Zielsetzungen berücksichtigt, die von den befragten Austellern mit einer Zielwichtigkeit von mindestens 4 auf einer 5-dstufigen Ratingskala bewertet wurden.

564 Somit ließ sich für jedes der priorisierten Ziel ein Nutzenindex mit einem maximalen Wert von 25 ermitteln. 


\section{Messebeteiligungen-Nutzen-Index}

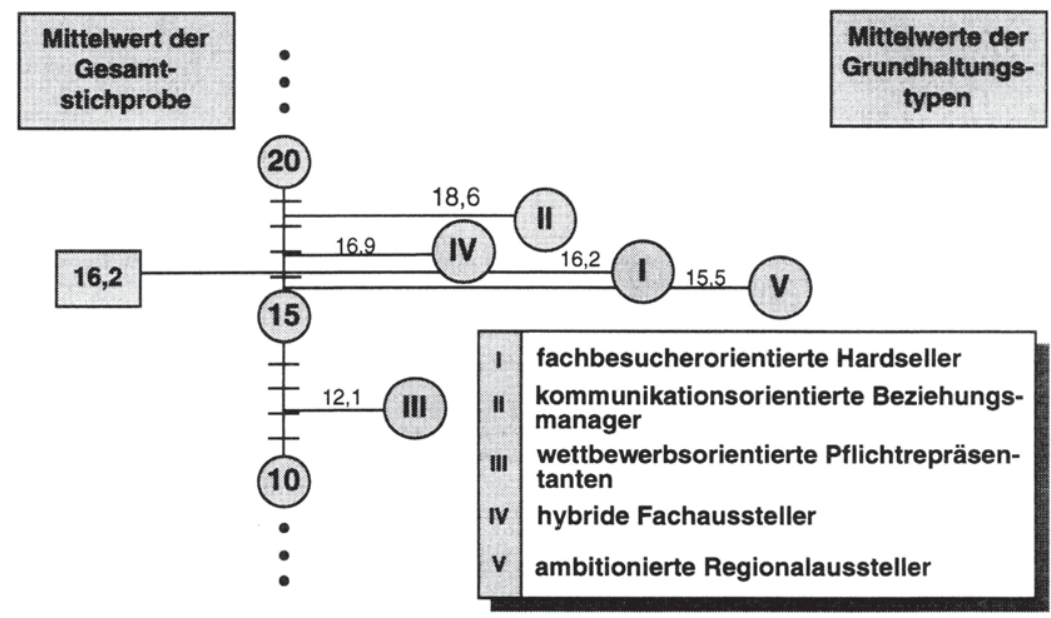

Abb. 27: Grundhaltungspezifische Darstellung des Messebeteiligungs-NutzenIndex

Im Verleich mit den Befunden im Rahmen des exemplarisch durchgeführten Messebeteiligungs-Audits, lassen sich interessante Erkenntnisse ableiten. So stufen mit den kommunikationsorientierten Beziehungsmanagern und den hybriden Fachausstellern gerade jene ausstellenden Unternehmen ihre Messebeteiligung als besonders nutzenstiftend ein, bei denen im Rahmen des MessebeteiligungsAudits deutliche Defizite diagnostiziert werden konnten. Vor dem Hintergrund der Auditing- Ergebnisse lassen sich damit diese Nutzengrößen nur sehr eingeschränkt interpretieren. Im Gegensatz dazu sind die durchschnittlichen Nutzenwerte der fachbesucherorientierten Hardseller sowie der ambitionierten Regionalaussteller hinsichtlich der Auditing-Ergebnisse durchaus als realistisch anzusehen und deuten zudem auf eine eher vorsichtige Bewertung des Nutzens ihrer Messebeteiligungen hin.

Sofern für die Nutzenbewertung keine geeigneten Vergleichsobjekte - etwa bei einem signifikanten Verstoß gegen die Anforderung der Ziel- und Zielgruppenkongruenz - existieren, erscheint eine Nutzenquantifizierung allerdings kaum sinnvoll. Statt dessen empfiehlt sich in derartigen Situationen die Einbeziehung von Experten anderer Kommunikationsbereiche in den Prozeß der Effizienzbewertung, 
um auf diese Weise zumindest zu einer qualitativen Bewertung des erzielten Nutzens im Sinne eines subjektiven Vergleichs mit dem Nutzen alternativer Mittelverwendungen zu gelangen.

Im Ergebnis führt die Nutzenerfassung schließlich zu einem quantitativen Gesamtnutzen ergänzt um qualitative Nutzenbewertungen bei Fehlen geeigneter Vergleichsobjekte. Dieses „Nutzenbündel“ ist in einem letzten Schritt in Relation zu den Gesamtkosten der Messebeteiligung zu setzen, um eine endgültige Entscheidung bezüglich der Effizienz zu ermöglichen. Da die Nutzenbewertung unter Rückgriff auf andere Kommunikationsmaßnahmen erfolgt, ist zudem eine implizite Berücksichtigung alternativer Mittelverwendungen im Sinne der Opportunität gewährleistet.

Insgesamt gilt es, die beschriebenen Ansätze zur Kontrolle von Messebeteiligungen einem weiteren Konkretisierungsprozeß zu unterziehen. Hierzu kann die vorliegende Arbeit lediglich erste Strukturierungshinweise liefern. Als Hauptaufgabe der weiteren Forschungsbemühungen kann daher die Quantifizierung der Nutzendimensionen angeführt werden. Damit sind bereits Implikationen angesprochen, die sich aus dem vorliegenden Untersuchungsergebnis ableiten lassen.

\section{Implikationen für die weiterführende Forschung zum Management von Messebeteiligungen sowie für ausstellende Unternehmen und Messe- veranstalter}

Basierend auf den ermittelten Untersuchungsergebnissen lassen sich einige Implikationen sowohl für die weiterführende Forschung wie auch für die Unternehmenspraxis ableiten. Die Implikationen im Bereich der Wissenschaft und Forschung können wie folgt zusammengefaßt werden:

(1) Die Ausführungen eingangs der vorliegenden Untersuchung haben verdeutlicht, daß in der Vergangenheit verschiedene Entwicklungsstufen des Messemarketing zu verzeichnen waren. In diesem Zusammenhang ist davon auszugehen, daß auch zukünftig weitere Verschiebungen der grundsätzlichen Ausgestaltung von Messen zu erwarten sind. Daher erscheint es sinnvoll und notwendig, die im Rahmen dieser Arbeit herausgestellten Erkenntnisse in regelmäßigen Abständen zu überprüfen und einem erneuten Validierungsprozeß zuzuführen. Dies gilt um so mehr, als auch eine zunehmende Pro- 
fessionalisierung des Management von Messebeteiligungen und damit eine Veränderung der Zusammensetzung der verschiedenen Grundhaltungstypen zu enwarten ist.

(2) Innerhalb der verschiedenen Grundhaltungstypen werden sehr unterschiedliche Instrumentarien der (Erfolgs-)kontrolle eingesetzt. Dabei konnte ein insgesamt unbefriedigender Einsatz der Kontrollinstrumente sowohl in qualitativer wie auch quantitativer Hinsicht konstatiert werden. Dieses Defizit erscheint um so bedeutender, als das Messen im Wettbewerb mit anderen möglichen Kommunikationsinstrumenten einem zunehmenden Rechtfertigungsdruck ausgesetzt sind. Die Marketingwissenschaft ist hier aufgerufen, eine signifikante Verbesserung der eingesetzten Kontrollmechanismen und prozesse im Sinne eines integrierten Messecontrolling zu initiieren. Neben dem angesprochenen Rechtfertigungszwang spricht aber eine weitere Notwendigkeit für derartige Forschungsaktivitäten. Hier sind insbesondere zeitliche Aspekte in den Vordergrund zu stellen. Daher müssen Kontrollinstrumente so weiterentwicklelt werden, daß sie bereits während der Messevorbereitung und in der Messephase selbst eingesetzt werden, um so unmittelbare Korrekturinformationen für das Messemanagement zu liefern.

(3) Zusätzlich erscheint es wünschenswert, eine höhere Vergleichbarkeit des Erfolgs von Messebeteiligungen im Sinne der oben beschriebenen interinstrumentellen Vergleiche auf andere Kommunikationsinstrumente herzustellen. Zum einen sind insbesondere zwei Fragestellungen von Interesse. Dabei ist $\mathrm{zu}$ untersuchen, inwieweit sich auch bei anderen Kommunikationsinstrumenten identische Grundhaltungstypen nachweisen lassen. Zum anderen ist der Frage nachzugehen, ob sich die in dieser Untersuchung festgestellten Erfolgseinschätzungen der einzelnen Grundhaltungen auch in anderen Bereichen der marktgerichteten Unternehmenskommunikation wiederfinden lassen. Darüber hinaus können auf diese Weise Wirkungseffekte, die allein auf einzelne Instrumente zurückzuführen sind, besser abgeschätzt und quantifiziert werden.

(4) Die vorliegende Untersuchung zeichnet sich durch einen bewußt nationalen Fokus der befragten Unternehmen aus. Vor dem Hintergrund einer zunehmenden Globalisierung von Messebeteiligungen werden die befragten Unternehmen aber zunehmend auf Wettbewerber aus anderen Ländern treffen. Daher scheint es sinnvoll, die vorliegende Untersuchung auf weitere 
Länder auszudehnen, nicht zuletzt um eine internationale Validierung der Ergebnisse zu ermöglichen oder aber auf unterschiedliche Kontextfaktoren zu treffen, die entscheidende Hinweise für die Ausgestaltung des Management der deutschen Unternehmen aufzeigen.

(5) Wenngleich ein Vorteil dieser Arbeit darin lag, daß das Management von Messebeteiligungen in vielen Facetten in die Analyse einging, ist es darüber hinaus für zukünftige Forschungsvorhaben wünschenswert, die untersuchten Variablen auch zeitablaufbezogen zu erfassen. Längsschnittanalysen ermöglichen zudem die Beschreibung und Erklärung des Management von Messebeteiligungen der Grundhaltungstypen im Zeitablauf. Dabei ist insbesondere die Konstanz der Realisierung eines bestimmten Grundhaltungstyps im Zeitablauf zu analysieren. Zudem sind die Erfolgsauswirkungen der grundhaltungsspezifischen Ausgestaltung von Messebeteiligungen zeitraumbezogen zu überprüfen.

(6) Weiterhin zeichnet sich die vorliegende Arbeit durch den fast ausschließlichen Einbezug qualitativer Daten aus. Dies erscheint vor dem Hintergrund des verfolgten Analyseziels gerechtfertigt. Andererseits ist es wünschenswert, die im Rahmen der Untersuchung zweifellos vorliegenden Subjektivismen durch Gegenüberstellung mit ergänzenden quantitativen Daten zu validieren.

Trotz des hohen Stellenwertes von Messen im Rahmen der Kommunikationspolitik von Unternehmen zeichnen sich die Prozesse des Management von Messen vielfach durch ein weitgehend intuitives und unstrukturiertes Vorgehen aus. Vor diesem Hintergrund lassen sich zentrale Implikationen hinsichtlich des Messemanagement in der Unternehmenspraxis ableiten:

(1) Die Ergebnisse der vorliegenden Untersuchung sind geeignet, der Ausgestaltung der Partnerschaft zwischen Ausstellern und Messegesellschaften neue Impulse zu geben. Einige der gefundenen Grundhaltungstypen zeichnen sich durch Defizite im Messemanagement auf. Hier ist zu hinterfragen, in welchem Umfang Messegesellschaften als Dienstleistungsanbieter Unterstützung anbieten können und wie derartige Partnerschaften organisatorisch auszugestalten sind. 
(2) Die bereits dargestellten Defizite im Controllingbereich, durch die sich einige der ermittelten Grundhaltungstypen wie etwa der „kommunikations-orientierte Beziehungsmanager" auszeichnen, ziehen die Forderung nach sich, grundsätzliche Umstrukturierungen einzuleiten. Es ist zu vermuten, daß Kontrollmechanismen vielfach unterbleiben, um sich einem Erfolgsdruck zu entziehen. Hier ist die Unternehmensführung gefordert, den Einsatz notwendiger Instrumente zu initiieren und somit eine Ermittlung der Effizienz von Messeauftritten erst zu ermöglichen. Eine Konzentration auf rein messespezifische Kontrollinstrumente scheint hier aber nur bedingt zielführend. Vielmehr erscheint es angezeigt, auf einer höheren Aggregationsebene Kennzahlen zu ermitteln, die einen Vergleich des Messeeinsatzes mit anderen Kommunikationsinstrumenten im Rahmen eines integrierten KommunikationsControllings erlauben.

(3) Aufbauend auf diesen Defiziten im Controllingbereich muß auch ein dezidierter Katalog von Gegensteuerungsmaßnahmen verbindlich festgelegt werden. In diesem Zusammenhang bemißt sich die Sinnhaftigkeit von Kontrollaktivitäten ausschließlich am Nutzen der ermittelten Informationen und dieser wiederum anhand des Erfolges bzw. der Erfolgswahrscheinlichkeit der initiierten Maßnahmen.

(4) Grundsätzlich stellt sich für Unternehmen mit Messebeteiligungen die Frage nach der Konsequenz aus den vorliegenden Untersuchungsergebnissen. Hier ist individuell zu entscheiden, ob die jeweils vorliegende Kombination aus externen Einflußgrößen und internen Managemententscheidungen sowie die daraus resultierende Einstufung in eines der Grundhaltungscluster bewußt angestrebt oder vielmehr der Ausdruck eines wenig zielgerichteten Management ist. Im letztgenannten Fall erscheint es angezeigt, grundsätzliche Praktiken im Rahmen des Messeengagements zu überdenken und ggf. unter strenger Berücksichtigung der Unternehmensziele zu restrukturieren. Aufbauend auf den Untersuchungsergebnissen kann hier die organisatorische Einbindung der Messebeteiligung als kritische Stellgröße genannt werden.

Insgesamt wird deutlich, daß von der wirtschaftswissenschaftlichen Forschung eine Reihe von Fragestellungen zu beantworten ist, die gegenwärtig und zukünftig für die Problemstellungen in der Unternehmenspraxis von besonderer Relevanz sind. Eine fundierte Beantwortung dieser Fragen erscheint um so bedeutender, als ein 
steigender Stellenwert von Messebeteiligungen bei gleichzeitiger Betonung von Effizienzkriterien in vielen Wirtschaftsbereichen zu erwarten ist. 
Gesamtverzeichnis des Anhangs

Anhang 1: Übersicht über die geführten Expertengespräche.................. 271

Anhang 2: Verzeichnis der Teilnehmer an den Expertenworkshops des AUMA e.V. am 8. Mai 1995

und 14. Dezember 1995........................................................ 273

Anhang 3: Ergänzende Tabellen und Abbildungen.................................. 274

Anhang 4: SPSS-Listings der durchgeführten Faktoren-, Cluster- und Diskriminanzanalysen...................................... 280

Anhang 5: Fragebogen für die schriftliche Befragung von ausstellenden Unternehmen. 


\section{Anhang 1: Übersicht über die geführten Expertengespräche}

\begin{tabular}{|c|c|c|c|}
\hline $\begin{array}{l}\text { Unternehmen, } \\
\text { Ort }\end{array}$ & $\begin{array}{l}\text { Gesprächs- } \\
\text { partner }\end{array}$ & $\begin{array}{l}\text { Funktions- } \\
\text { bezeichnung }\end{array}$ & $\begin{array}{l}\text { Gesprächs } \\
\text { termin }\end{array}$ \\
\hline $\begin{array}{l}\text { Mannesmann } \\
\text { Werbegesell- } \\
\text { schaft mbH, } \\
\text { Düsseldorf }\end{array}$ & Herr Kloke & Managing Director & 15.03 .95 \\
\hline $\begin{array}{l}\text { gardeur } \\
\text { Dieter Janssen } \\
\text { GmbH \& Co. KG, } \\
\text { Mönchengladbach }\end{array}$ & Herr Dr. Roesner & Geschäftsführer & 15.03 .95 \\
\hline $\begin{array}{l}\text { Mercedes-Benz AG, } \\
\text { Stuttgart }\end{array}$ & Herr v. Sanden & $\begin{array}{l}\text { Marketing Kommunikation } \\
\text { PKW, Leiter Ausstellungen } \\
\text { und Messen }\end{array}$ & 17.03 .95 \\
\hline $\begin{array}{l}\text { Hüls AG, } \\
\text { Marl }\end{array}$ & $\begin{array}{l}\text { Herr Dr. Ferley } \\
\text { Frau Nussbeutel }\end{array}$ & $\begin{array}{l}\text { Leiter Marktkommunikation } \\
\text { Marktkommunikation }\end{array}$ & 20.03 .95 \\
\hline $\begin{array}{l}\text { Siemens AG, } \\
\text { Nürnberg }\end{array}$ & Herr Schimmang & $\begin{array}{l}\text { Abteilungsleiter Messen } \\
\text { und Ausstellungen }\end{array}$ & 21.03 .95 \\
\hline $\begin{array}{l}\text { Rosenthal AG, } \\
\text { Selb }\end{array}$ & Herr Farasin & Leiter Marketing & 21.03 .95 \\
\hline $\begin{array}{l}\text { Schöninger GHM } \\
\text { Ges. mbH, } \\
\text { Luhe-Wildenau }\end{array}$ & Herr Wirtz & Geschäftsführer i. R. & 21.03 .95 \\
\hline $\begin{array}{l}\text { AEG Aktien- } \\
\text { gesellschaft, } \\
\text { Frankfurt }\end{array}$ & Herr Müller & $\begin{array}{l}\text { Konzernbevollmächtigter } \\
\text { Messen und Ausstellungen }\end{array}$ & 22.03 .95 \\
\hline $\begin{array}{l}\text { Fa. August Hoff, } \\
\text { Nürnberg }\end{array}$ & Herr Röhrich & Verkaufsleitung & 24.03 .95 \\
\hline $\begin{array}{l}\text { ABB Kommu- } \\
\text { nikations-GmbH, } \\
\text { Mannheim }\end{array}$ & Frau Dr. Derieth & $\begin{array}{l}\text { Assist. der Geschäfts- } \\
\text { führung }\end{array}$ & 27.03.95 \\
\hline $\begin{array}{l}\text { VBW - Vereinigte } \\
\text { Beckersche Werk- } \\
\text { zeugfabriken } \\
\text { GmbH \& Co. KG, } \\
\text { Remscheid }\end{array}$ & Herr Krugmann & Prokurist & 28.03 .95 \\
\hline
\end{tabular}




$\begin{array}{llll}\begin{array}{l}\text { Unternehmen, } \\ \text { Ort }\end{array} & \begin{array}{l}\text { Gesprächs- } \\ \text { partner }\end{array} & \begin{array}{l}\text { Funktions- } \\ \text { bezeichnung }\end{array} & \begin{array}{l}\text { Gesprächs- } \\ \text { termin }\end{array}\end{array}$

AEG Haushaltsgeräte AG, Nürnberg

Thyssen AG, Düsseldorf

\section{BASF Aktien-} gesellschaft, Ludwigshafen

debis Marketing Services GmbH, Frankfurt

Gelszus Messe-Marktforschung $\mathrm{GmbH}$
Herr Schweitzer

Marketingleitung

31.03 .95

Herr Schmitz

Leiter Konzernwerbung

04.04 .95

Herr John

Herr Fuchs

Herr Gödert

Herr Pietsch

Leiter Gruppe Messen

Strategische Planung

Messeorganisation

Marketing Consultant

08.04.95

Herr Wilbrink

Messe-Consultant

13.10 .95 


\section{Anhang 2: Verzeichnis der Teilnehmer an den Expertenworkshops des AUMA e. V. am 8. Mai 1995 und 14. Dezember 1995}

Teilnehmerliste Workshop "Ziele und Nutzen von Messebeteiligungen" am 8. Mai 1995

Herr Dr. Boerner
Frau Dr. Derieth
Herr Dr. Ferley
Herr John
Herr Kloke
Herr Kötter
Herr Krugmann
Herr Prof. Dr. Dr. h.c. Meffert
Herr Merbold
Herr Dr. Neven
Frau Nussbeutel
Herr Schmitz
Herr Ueding

AUMA e.V., Köln
ABB, Mannheim
Hüls AG, Marl
BASF AG, Ludwigshafen
Mannesmann, Düsseldorf
AUMA e.V., Köln
VBW, Remscheid
Universität Münster
Siemens AG, München
AUMA e.V., Köln
Hüls AG, Marl
Thyssen AG, Düsseldorf
Universität Münster

Teilnehmerliste Workshop "Ziele und Nutzen von Messebeteiligungen" am 14. Dezember 1995

Herr Dr. Ferley

Herr Greiner

Herr Hellenkamp

Herr John

Herr Kötter

Herr Maßlich

Herr Prof. Dr. Dr. h.c. Meffert

Herr Merbold

Herr Dr. Neven

Herr Robertz

Herr Schmitz

Herr Ueding
Hüls AG, Marl

ABB, Mannheim

Krupp AG. Essen

BASF AG, Ludwigshafen

AUMA e.V., Köln

AEG Daimler Benz Industrie AG,

Frankfurt a. M.

Universität Münster

Siemens AG, München

AUMA e.V., Köln

Universität Münster

Thyssen AG, Düsseldorf

Universität Münster 


\section{Anhang 3: Ergänzende Tabellen und Abbildungen}

\section{Tabellen}

Tabelle A1: Häufigkeitsverteilung der clusterbildenden Merkmale in Cluster I

Tabelle A2: Häufigkeitsverteilung der clusterbildenden Merkmale in Cluster II

Tabelle A3: Häufigkeitsverteilung der clusterbildenden Merkmale in Cluster III

Tabelle A4: Häufigkeitsverteilung der clusterbildenden Merkmale in Cluster IV

Tabelle A5: Häufigkeitsverteilung der clusterbildenden Merkmale in Cluster V 


\begin{tabular}{|c|c|c|c|c|c|c|c|c|}
\hline \multirow{3}{*}{ 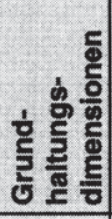 } & \multirow[b]{3}{*}{ Variablen } & \multicolumn{7}{|c|}{ Cluster I ( $n=100)$} \\
\hline & & \multirow{8}{*}{$\begin{array}{l}\text { sehr } \\
\text { gering }\end{array}$} & \multicolumn{5}{|c|}{ Wichtigkeit } & \multirow{8}{*}{$\begin{array}{l}\text { sehr } \\
\text { groß }\end{array}$} \\
\hline & & & 1 & 2 & 3 & 4 & 5 & \\
\hline \multirow{6}{*}{ 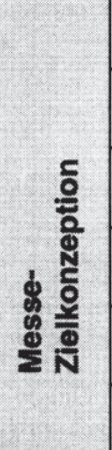 } & Informationsziele & & 1,0 & 37,0 & 44,0 & 16,0 & 2,0 & \\
\hline & Motivationsziele & & 15,0 & 9,0 & 33,0 & 22,0 & 21,0 & \\
\hline & $\begin{array}{l}\text { marktbezogene } \\
\text { Beeinflussungsziele }\end{array}$ & & - & 1,0 & 12,0 & 59,0 & 28,0 & \\
\hline & $\begin{array}{l}\text { anspruchsgruppenbe- } \\
\text { zogene Beeinfl.-ziele }\end{array}$ & & 18,0 & 34,0 & 31,0 & 16,0 & 1,0 & \\
\hline & Verkaufsziele & & 1,0 & 4,0 & 19,0 & 25,0 & 51,0 & \\
\hline & Kontaktziele & & - & 1,0 & 11,0 & 36,0 & 52,0 & \\
\hline \multirow{6}{*}{ 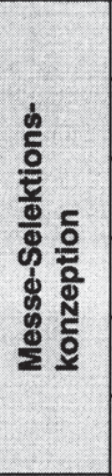 } & $\begin{array}{l}\text { Ausstellungs- } \\
\text { programm }\end{array}$ & $\begin{array}{c}\text { homo- } \\
\text { gen }\end{array}$ & 20,0 & 37,0 & 26,0 & 7,0 & 10,0 & $\begin{array}{c}\text { hetero- } \\
\text { gen }\end{array}$ \\
\hline & $\begin{array}{l}\text { Grundorientierung } \\
\text { der Messe }\end{array}$ & $\begin{array}{l}\text { ver- } \\
\text { kaufs- } \\
\text { orien- } \\
\text { tiert } \\
\end{array}$ & 33,0 & 38,0 & 25,0 & 4,0 & - & $\begin{array}{l}\text { info.- } \\
\text { orien- } \\
\text { tiert }\end{array}$ \\
\hline & $\begin{array}{l}\text { fachliche Orientierung } \\
\text { der Messe-Besucher }\end{array}$ & $\begin{array}{l}\text { Fach- } \\
\text { pub. }\end{array}$ & 66,0 & 21,0 & 8,0 & 5,0 & - & $\begin{array}{l}\text { Privat- } \\
\text { pub. }\end{array}$ \\
\hline & $\begin{array}{l}\text { geogr. Einzugsbereich } \\
\text { der Besucher }\end{array}$ & $\begin{array}{l}\text { national, } \\
\text { regional }\end{array}$ & - & 6,0 & 15,0 & 29,0 & 50,0 & \begin{tabular}{|c|} 
inter- \\
national
\end{tabular} \\
\hline & $\begin{array}{l}\text { geogr. Einzugsbereich } \\
\text { der Aussteller }\end{array}$ & $\begin{array}{l}\text { national, } \\
\text { regional }\end{array}$ & - & 2,0 & 13,0 & 31,0 & 54,0 & $\begin{array}{c}\text { inter- } \\
\text { national }\end{array}$ \\
\hline & $\begin{array}{l}\text { Durchführung von Be- } \\
\text { gleitveranstaltungen }\end{array}$ & viele & 3,0 & 20,0 & 31,0 & 27,0 & 19,0 & keine \\
\hline \multirow{4}{*}{ 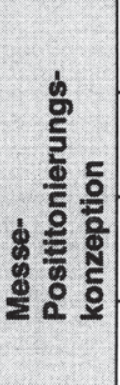 } & $\begin{array}{l}\text { Produkt- bzw. Sorti- } \\
\text { mentsorientiertierung }\end{array}$ & $\begin{array}{l}\text { trifft gar } \\
\text { nicht zu }\end{array}$ & 2,0 & 3,0 & 6,0 & 36,0 & 53,0 & \multirow[t]{4}{*}{$\begin{array}{l}\text { triftt } \\
\text { sehr zu }\end{array}$} \\
\hline & $\begin{array}{l}\text { Problemlösungs- bzw. } \\
\text { Nutzenorientierung }\end{array}$ & & 13,0 & 35,0 & 20,0 & 19,0 & 13,0 & \\
\hline & $\begin{array}{l}\text { Kommunikations- bzw } \\
\text { Erlebnisorientierung }\end{array}$ & & 17,0 & 32,0 & 21,0 & 25,0 & 5,0 & \\
\hline & $\begin{array}{l}\text { Wettbewerbs- } \\
\text { orientierung }\end{array}$ & & 40,0 & 29,0 & 19,0 & 7,0 & 5,0 & \\
\hline
\end{tabular}




\begin{tabular}{|c|c|c|c|c|c|c|c|c|}
\hline \multirow{3}{*}{ 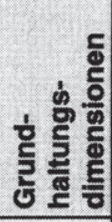 } & \multirow[b]{3}{*}{ Variablen } & \multicolumn{7}{|c|}{ Cluster II (n=155) } \\
\hline & & \multirow{8}{*}{$\begin{array}{l}\text { sehr } \\
\text { gering }\end{array}$} & \multicolumn{5}{|c|}{ Wichtigkeit } & \multirow{8}{*}{$\begin{array}{l}\text { sehr } \\
\text { groß }\end{array}$} \\
\hline & & & 1 & 2 & 3 & 4 & 5 & \\
\hline \multirow{6}{*}{ 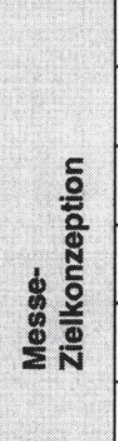 } & Informationsziele & & - & 23,2 & 57,4 & 14,9 & 4,5 & \\
\hline & Motivationsziele & & 4,5 & 8,4 & 31,6 & 29,7 & 25,8 & \\
\hline & $\begin{array}{l}\text { marktbezogene } \\
\text { Beeinflussungsziele }\end{array}$ & & - & 0,6 & 11,0 & 54,2 & 34,2 & \\
\hline & $\begin{array}{l}\text { anspruchsgruppenbe- } \\
\text { zogene Beeinfl.-ziele }\end{array}$ & & 2,5 & 24,0 & 44,5 & 25,1 & 3,9 & \\
\hline & Verkaufsziele & & - & 9,7 & 30,3 & 38,7 & 21,3 & \\
\hline & Kontaktziele & & - & 1,3 & 4,5 & 43,9 & 50,3 & \\
\hline \multirow{6}{*}{ 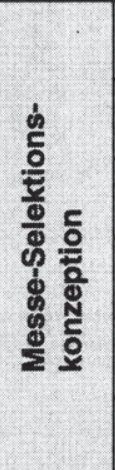 } & $\begin{array}{l}\text { Ausstellungs- } \\
\text { programm }\end{array}$ & $\begin{array}{c}\text { homo- } \\
\text { gen }\end{array}$ & 9,7 & 22,6 & 25,2 & 25,2 & 17,4 & $\begin{array}{l}\text { hetero- } \\
\text { gen }\end{array}$ \\
\hline & $\begin{array}{l}\text { Grundorientierung } \\
\text { der Messe }\end{array}$ & $\begin{array}{l}\text { ver-s } \\
\text { kauffs- } \\
\text { orien- } \\
\text { tiert } \\
\end{array}$ & 0,6 & 8,4 & 18,0 & 46,5 & 26,5 & $\begin{array}{l}\text { info.- } \\
\text { orien- } \\
\text { tiert }\end{array}$ \\
\hline & $\begin{array}{l}\text { fachliche Orientierung } \\
\text { der Messe-Besucher }\end{array}$ & $\begin{array}{l}\text { Fach- } \\
\text { pub. }\end{array}$ & 42,6 & 34,8 & 17,4 & 5,2 & - & $\begin{array}{l}\text { Privat- } \\
\text { pub. }\end{array}$ \\
\hline & $\begin{array}{l}\text { geogr. Einzugsbereich } \\
\text { der Besucher }\end{array}$ & $\begin{array}{l}\text { national, } \\
\text { regional }\end{array}$ & - & 0,6 & 7,1 & 36,2 & 56,1 & $\begin{array}{l}\text { inter- } \\
\text { national }\end{array}$ \\
\hline & $\begin{array}{l}\text { geogr. Einzugsbereich } \\
\text { der Aussteller }\end{array}$ & $\begin{array}{l}\text { national, } \\
\text { regional }\end{array}$ & 1,3 & - & 6,5 & 31,0 & 61,2 & $\begin{array}{c}\text { inter- } \\
\text { national }\end{array}$ \\
\hline & $\begin{array}{l}\text { Durchführung von Be- } \\
\text { gleitveranstaltungen }\end{array}$ & viele & 22,6 & 51,0 & 20,6 & 5,8 & - & keine \\
\hline \multirow{4}{*}{ 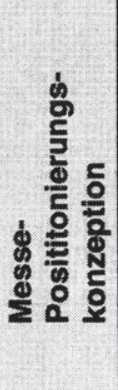 } & $\begin{array}{l}\text { Produkt- bzw. Sorti- } \\
\text { mentsorientiertierung }\end{array}$ & $\begin{array}{l}\text { triftt gar } \\
\text { nicht zu }\end{array}$ & 4,5 & 9,0 & 19,4 & 34,2 & 32,9 & \multirow[t]{4}{*}{$\begin{array}{l}\text { triftt } \\
\text { sehr zu }\end{array}$} \\
\hline & $\begin{array}{l}\text { Problemlösungs- bzw. } \\
\text { Nutzenorientierung }\end{array}$ & & 3,2 & 7,7 & 15,5 & 39,4 & 34,2 & \\
\hline & $\begin{array}{l}\text { Kommunikations- bzw } \\
\text { Erlebnisorientierung }\end{array}$ & & 5,8 & 18,7 & 24,5 & 33,5 & 17,4 & \\
\hline & $\begin{array}{l}\text { Wettbewerbs- } \\
\text { orientierung }\end{array}$ & & 49,7 & 34,8 & 11,6 & 3,2 & 0,6 & \\
\hline
\end{tabular}




\begin{tabular}{|c|c|c|c|c|c|c|c|c|}
\hline & & \multicolumn{7}{|c|}{ Cluster III $\quad(n=87)$} \\
\hline 돌로 & & \multirow{8}{*}{$\begin{array}{l}\text { sehr } \\
\text { gering }\end{array}$} & \multicolumn{5}{|c|}{ Wichtigkeit } & \multirow{8}{*}{$\begin{array}{l}\text { sehr } \\
\text { groß }\end{array}$} \\
\hline 허을 & Variablen & & 1 & 2 & 3 & 4 & 5 & \\
\hline \multirow{6}{*}{ 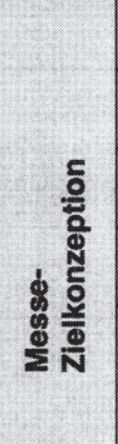 } & Informationsziele & & 1,1 & 36,8 & 51,8 & 10,3 & - & \\
\hline & Motivationsziele & & 6,9 & 14,9 & 29,9 & 31,0 & 17,2 & \\
\hline & $\begin{array}{l}\text { marktbezogene } \\
\text { Beeinflussungsziele }\end{array}$ & & - & 1,1 & 17,3 & 58,6 & 23,0 & \\
\hline & $\begin{array}{l}\text { anspruchsgruppenbe- } \\
\text { zogene Beeinfl.-ziele }\end{array}$ & & 9,2 & 41,4 & 39,1 & 9,2 & 1,1 & \\
\hline & Verkaufsziele & & 1,1 & 11,5 & 23,0 & 35,7 & 28,7 & \\
\hline & Kontaktziele & & - & - & 17,2 & 57,5 & 25,3 & \\
\hline \multirow{6}{*}{ 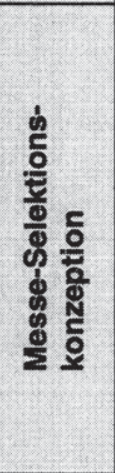 } & $\begin{array}{l}\text { Ausstellungs- } \\
\text { programm }\end{array}$ & $\begin{array}{c}\text { homo- } \\
\text { gen }\end{array}$ & 2,3 & 9,2 & 23,0 & 29,9 & 35,6 & $\begin{array}{l}\text { hetero- } \\
\text { gen }\end{array}$ \\
\hline & $\begin{array}{l}\text { Grundorientierung } \\
\text { der Messe }\end{array}$ & $\begin{array}{l}\text { ver- } \\
\text { kauts- } \\
\text { oriven- } \\
\text { tiert }\end{array}$ & 9,2 & 14,9 & 27,6 & 37,9 & 10,3 & $\begin{array}{l}\text { info.- } \\
\text { orien- } \\
\text { tiert }\end{array}$ \\
\hline & $\begin{array}{l}\text { fachliche Orientierung } \\
\text { der Messe-Besucher }\end{array}$ & $\begin{array}{l}\text { Fach- } \\
\text { pub. }\end{array}$ & - & 8,0 & 35,6 & 36,8 & 19,5 & $\begin{array}{l}\text { Privat- } \\
\text { pub. }\end{array}$ \\
\hline & $\begin{array}{l}\text { geogr. Einzugsbereich } \\
\text { der Besucher }\end{array}$ & $\begin{array}{l}\text { national, } \\
\text { regional }\end{array}$ & 18,4 & 28,7 & 26,4 & 23,0 & 3,4 & $\begin{array}{l}\text { inter- } \\
\text { national }\end{array}$ \\
\hline & $\begin{array}{l}\text { geogr. Einzugsbereich } \\
\text { der Aussteller }\end{array}$ & $\begin{array}{l}\text { national, } \\
\text { regional }\end{array}$ & 8,0 & 17,2 & 32,2 & 31,0 & 11,5 & $\begin{array}{l}\text { inter- } \\
\text { national }\end{array}$ \\
\hline & $\begin{array}{l}\text { Durchführung von Be- } \\
\text { gleitveranstaltungen }\end{array}$ & viele & 12,6 & 18,4 & 36,8 & 24,1 & 8,0 & keine \\
\hline \multirow{4}{*}{ 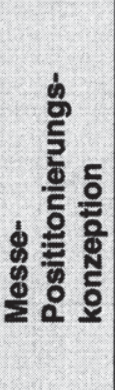 } & $\begin{array}{l}\text { Produkt- bzw. Sorti- } \\
\text { mentsorientiertierung }\end{array}$ & $\begin{array}{l}\text { trifft gar } \\
\text { nicht zu }\end{array}$ & 6,9 & 17,2 & 10,3 & 35,6 & 29,9 & \multirow[t]{4}{*}{$\begin{array}{l}\text { triftt } \\
\text { sehr zu }\end{array}$} \\
\hline & $\begin{array}{l}\text { Problemlösungs- bzw. } \\
\text { Nutzenorientierung }\end{array}$ & & 9,2 & 21,8 & 21,8 & 32,2 & 14,9 & \\
\hline & $\begin{array}{l}\text { Kommunikations- bzw. } \\
\text { Erlebnisorientierung }\end{array}$ & & 16,1 & 21,8 & 34,5 & 21,8 & 5,7 & \\
\hline & $\begin{array}{l}\text { Wettbewerbs- } \\
\text { orientierung }\end{array}$ & & 32,2 & 34,5 & 19,5 & 9,2 & 4,6 & \\
\hline
\end{tabular}




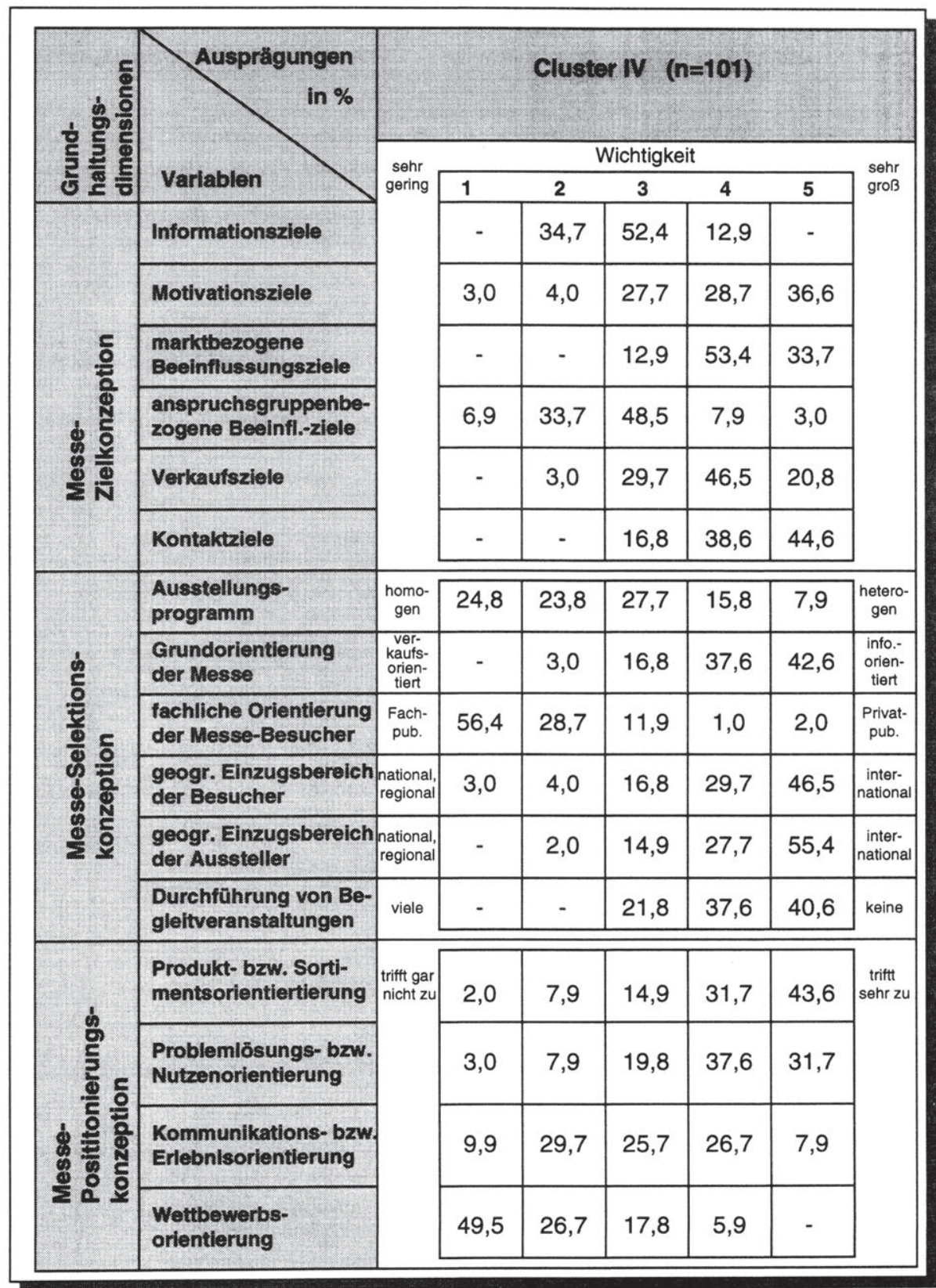

Tabelle A4: Häufigkeitsverteilung der clusterbildenden Merkmale in Cluster IV 


\begin{tabular}{|c|c|c|c|c|c|c|c|c|}
\hline \multirow{3}{*}{ 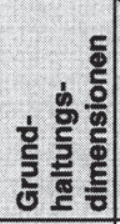 } & \multirow[b]{3}{*}{ Variablen } & \multicolumn{7}{|c|}{ Cluster V $(n=98)$} \\
\hline & & \multirow{8}{*}{$\begin{array}{l}\text { sehr } \\
\text { gering }\end{array}$} & \multicolumn{5}{|c|}{ Wichtigkeit } & \multirow{8}{*}{$\begin{array}{l}\text { sehr } \\
\text { groß }\end{array}$} \\
\hline & & & 1 & 2 & 3 & 4 & 5 & \\
\hline \multirow{6}{*}{ 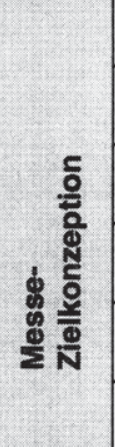 } & Informationsziele & & - & 17,3 & 48,0 & 31,6 & 3,1 & \\
\hline & Motivationsziele & & 1,0 & 4,1 & 23,5 & 26,5 & 44,9 & \\
\hline & $\begin{array}{l}\text { marktbezogene } \\
\text { Beeinflussungsziele }\end{array}$ & & - & 1,0 & 9,2 & 45,9 & 43,9 & \\
\hline & $\begin{array}{l}\text { anspruchsgruppenbe- } \\
\text { zogene Beeinfl.-ziele }\end{array}$ & & 5,1 & 22,5 & 36,7 & 34,7 & 1,0 & \\
\hline & Verkaufsziele & & - & 5,1 & 14,2 & 40,9 & 39,8 & \\
\hline & Kontaktziele & & - & - & 7,1 & 58,2 & 34,7 & \\
\hline \multirow{6}{*}{ 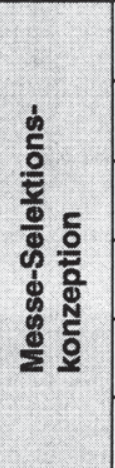 } & $\begin{array}{l}\text { Ausstellungs- } \\
\text { programm }\end{array}$ & $\begin{array}{c}\text { homo- } \\
\text { gen }\end{array}$ & 16,3 & 36,7 & 28,6 & 12,2 & 6,1 & $\begin{array}{c}\text { hetero- } \\
\text { gen }\end{array}$ \\
\hline & $\begin{array}{l}\text { Grundorientierung } \\
\text { der Messe }\end{array}$ & $\begin{array}{l}\text { ver- } \\
\text { kaufs- } \\
\text { orien- } \\
\text { tiert }\end{array}$ & 15,3 & 9,2 & 34,7 & 30,6 & 10,2 & $\begin{array}{l}\text { info.- } \\
\text { orien- } \\
\text { tiert }\end{array}$ \\
\hline & $\begin{array}{l}\text { fachliche Orientierung } \\
\text { der Messe-Besucher }\end{array}$ & $\begin{array}{l}\text { Fach- } \\
\text { pub. }\end{array}$ & 56,1 & 32,7 & 9,2 & 1,0 & 1,0 & $\begin{array}{l}\text { Privat- } \\
\text { pub. }\end{array}$ \\
\hline & $\begin{array}{l}\text { geogr. Einzugsbereich } \\
\text { der Besucher }\end{array}$ & $\begin{array}{l}\text { national, } \\
\text { regional }\end{array}$ & 24,5 & 45,9 & 29,6 & - & - & $\begin{array}{c}\text { inter- } \\
\text { national }\end{array}$ \\
\hline & $\begin{array}{l}\text { geogr. Einzugsbereich } \\
\text { der Aussteller }\end{array}$ & $\begin{array}{l}\text { national, } \\
\text { regional }\end{array}$ & 10,2 & 36,7 & 32,7 & 14,3 & 6,1 & $\begin{array}{c}\text { inter- } \\
\text { national }\end{array}$ \\
\hline & $\begin{array}{l}\text { Durchführung von Be- } \\
\text { gleitveranstaltungen }\end{array}$ & viele & 8,2 & 20,4 & 25,5 & 20,4 & 25,5 & keine \\
\hline \multirow{4}{*}{ 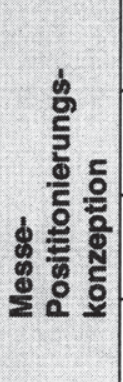 } & $\begin{array}{l}\text { Produkt- bzw. Sorti- } \\
\text { mentsorientiertierung }\end{array}$ & $\begin{array}{l}\text { trifft gar } \\
\text { nicht zu }\end{array}$ & 3,1 & 3,1 & 19,4 & 40,8 & 33,7 & \multirow[t]{4}{*}{$\begin{array}{l}\text { triftt } \\
\text { sehr zu }\end{array}$} \\
\hline & $\begin{array}{l}\text { Problemlösungs- bzw. } \\
\text { Nutzenorientierung }\end{array}$ & & 1,0 & 13,3 & 21,4 & 38,8 & 25,5 & \\
\hline & $\begin{array}{l}\text { Kommunikations- bzw, } \\
\text { Erlebnisorientierung }\end{array}$ & & 5,1 & 25,5 & 31,6 & 31,6 & 6,1 & \\
\hline & $\begin{array}{l}\text { Wettbewerbs- } \\
\text { orientierung }\end{array}$ & & 45,9 & 29,6 & 22,4 & 2,0 & - & \\
\hline
\end{tabular}




\section{Anhang 4: SPSS-Listings der durchgeführten Faktoren-, Cluster- und Diskriminanzanalysen}

1. ....Konfirmatorische Faktorenanalyse für das Modell der MesseZielkonzeption

2. ....Fusionierungsprozeß des Ward-Verfahrens zur Ermittlung der messespezifischen Grundhaltungstypen

3......Diskriminanzanalyse für die ermittleten Grundhaltungstypen: .Verfahrensspezifikation, Diskriminanzfunktionen und Klassifikationsmatrix 282

4......Schrittweise Diskriminanzanalyse für die ermittleten Grundhaltungstypen: Verfahrensspezifikation, Diskriminanzfunktionen und Klassifikationsmatrix

5......Explorative Faktorenanalyse für die Variablen der Markt- und Wettbewerbssituation

6......Fusionierungsprozeß des Ward-Verfahrens zur Ermittlung von Situationsclustern

7......Partionierende Clusteranalyse zur Ermittlung der Mittelwerte der Situationscluster

8......Diskriminanzanalyse für die ermittelten Situationstypen:

.Diskriminanzfunktionen und Klassifikationsmatrix. 288

9......Diskriminanzanalyse für die ermittleten Einflußfaktoren: Verfahrensspezifikation und Diskriminanzfunktionen 289 


\title{
1. Konfirmatorische Faktorenanalyse für das Modell der Messe-Zlelkonzeption
}

\section{Globalwerte}

\author{
CHI-SQUARE WITH 243 DEGREES OF FREEDOM $=1393.56(P=.000)$ \\ GOODNESS OF FIT INDEX $=.932$ \\ ADJUSTED GOODNESS OF FIT INDEX $=.915$ \\ ROOT MEAN SQUARE RESIDUAL $=.075$
}

\section{Standardized Solution}

LAMBDA $\mathrm{X}$

\begin{tabular}{|c|c|c|c|c|c|c|}
\hline & ZIEL1 & ZIEL2 & ZIEL $3 \mathrm{~A}$ & ZIEL3B & ZIEL $4 \mathrm{~A}$ & ZIEL4B \\
\hline FD8HEU_1 & .70 & .00 & .00 & .00 & .00 & .00 \\
\hline FD8HEU_2 & .48 & .00 & .00 & .00 & .00 & .00 \\
\hline FD8HEU_3 & .46 & .00 & .00 & .00 & .00 & .00 \\
\hline FD8HE_20 & .55 & .00 & .00 & .00 & .00 & .00 \\
\hline FD8HE_21 & .47 & .00 & .00 & .00 & .00 & .00 \\
\hline FD8HE_22 & .52 & .00 & .00 & .00 & .00 & .00 \\
\hline FD8HE_23 & .61 & .00 & .00 & .00 & .00 & .00 \\
\hline FD8HE_24 & .42 & .00 & .00 & .00 & .00 & .00 \\
\hline FD8HE_25 & .50 & .00 & .00 & .00 & .00 & .00 \\
\hline FD8HE_19 & .00 & 1.00 & .00 & .00 & .00 & .00 \\
\hline FD8HE_10 & .00 & .00 & .70 & .00 & .00 & .00 \\
\hline FD8HE_11 & .00 & .00 & .62 & .00 & .00 & .00 \\
\hline FD8HE_12 & .00 & .00 & .51 & .00 & .00 & .00 \\
\hline FD8HE_13 & .00 & .00 & .71 & .00 & .00 & .00 \\
\hline FD8HE_14 & .00 & .00 & .57 & .00 & .00 & .00 \\
\hline FD8HE_15 & .00 & .00 & .64 & .00 & .00 & .00 \\
\hline FD8HE_16 & .00 & .00 & .00 & .70 & .00 & .00 \\
\hline FD8HE_17 & .00 & .00 & .00 & .49 & .00 & .00 \\
\hline FD8HE_18 & .00 & .00 & .00 & .60 & .00 & .00 \\
\hline FD8HEU_5 & .00 & .00 & .00 & .00 & .70 & .00 \\
\hline FD8HEU_6 6 & .00 & .00 & .00 & .00 & .82 & .00 \\
\hline FD8HEU_7 & .00 & .00 & .00 & .00 & .00 & .70 \\
\hline FD8HEU_8 & .00 & .00 & .00 & .00 & .00 & .69 \\
\hline FD8HEU_9 & .00 & .00 & .00 & .00 & .00 & .51 \\
\hline
\end{tabular}

PHI

\begin{tabular}{|c|c|c|c|c|c|c|}
\hline & ZIEL1 & ZIEL2 & ZIEL $3 A$ & ZIEL $3 B$ & ZIEL $4 \mathrm{~A}$ & ZIEL 4B \\
\hline ZIEL1 & 1.00 & & & & & \\
\hline ZIEL2 & .48 & 1.00 & & & & \\
\hline ZIEL $3 A$ & .48 & .41 & 1.00 & & & \\
\hline ZIEL $3 B$ & .57 & .35 & .49 & 1.00 & & \\
\hline ZIEL $4 \mathrm{~A}$ & .23 & .17 & .25 & -.08 & 1.00 & \\
\hline ZIEL 4B & .33 & .18 & .47 & .20 & .34 & 1.00 \\
\hline
\end{tabular}


2. Fusionierungsprozeß des Ward-Verfahrens zur Ermittlung der messespezifischen Grundhaltungstypen

\section{HIERARCHICAL CLUSTER ANALYSIS}

Squared Euclidean measure used

Agglomeration Schedule using Ward Method (CONT.)

\begin{tabular}{|c|c|c|c|c|c|c|}
\hline & Clusters & Combined & & Stage Cluster & 1st Appears & Next \\
\hline Stage & Cluster 1 & Cluster 2 & Coefficient & Cluster 1 & Cluster 2 & Stage \\
\hline 528 & 3 & 36 & 6635,478516 & 516 & 521 & 530 \\
\hline 530 & 3 & 8 & 6842,295898 & 528 & 514 & 535 \\
\hline 531 & 14 & 46 & 7016,712402 & 515 & 523 & 533 \\
\hline 532 & 4 & 11 & 7202,685059 & 518 & 527 & 536 \\
\hline 533 & 14 & 45 & 7397,363770 & 531 & 519 & 539 \\
\hline 534 & 1 & 7 & 7596,894043 & 511 & 529 & 537 \\
\hline 535 & 3 & 43 & 7800,367676 & 530 & 512 & 538 \\
\hline 536 & 4 & 12 & 8017,927734 & 532 & 526 & 538 \\
\hline 537 & 1 & 2 & 8279,014648 & 534 & 525 & 539 \\
\hline 538 & 3 & 4 & 8584,002930 & 535 & 536 & 540 \\
\hline 539 & 1 & 14 & 8921,348633 & 537 & 533 & 540 \\
\hline 540 & 1 & 3 & 9809,906250 & 539 & 538 & 0 \\
\hline
\end{tabular}

3. Diskriminanzanalyse für die ermittleten Grundhaltungstypen: Verfahrensspezifikation, Diskriminanzfunktionen und Klassifikationsmatrix

\section{Verfahrensspezifikation}

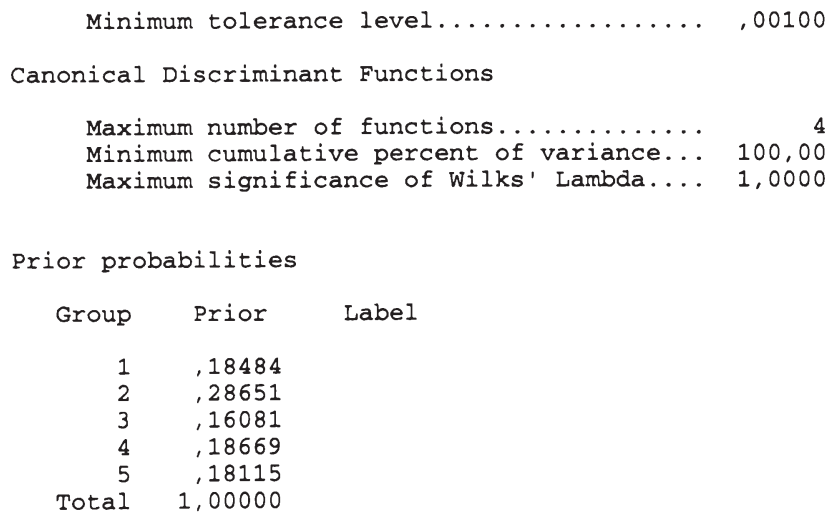




\section{Canonical Discriminant Functions}

\begin{tabular}{|c|c|c|c|c|c|c|c|c|c|}
\hline $\mathrm{FCn}$ & Eigenvalue & $\begin{array}{c}\text { Pct of } \\
\text { Variance }\end{array}$ & $\begin{array}{l}\text { Cum } \\
\text { Pct }\end{array}$ & $\begin{array}{c}\text { Canonical } \\
\text { Corr }\end{array}$ & $\begin{array}{r}\text { After } \\
\text { FCn }\end{array}$ & $\begin{array}{l}\text { Wilks' } \\
\text { Lambda }\end{array}$ & Chi-square & $d f$ & Sig \\
\hline & & & & & 0 & .057640 & 1510,947 & 64 & .0000 \\
\hline 1 * & 1,6381 & 37,91 & 37,91 & .7880 & 1 & .152060 & 997,302 & 45 &, 0000 \\
\hline 2 * & 1,2724 & 29,44 & 67,35 & .7483 & 2 & 345538 & 562,675 & 28 & .0000 \\
\hline 3 * &, 8274 & 19,15 & 86,49 & .6729 & 3 & .631425 & 243,451 & 13 & .0000 \\
\hline 4 * &, 5837 & 13,51 & 100,00 &, 6071 & : & & & & \\
\hline
\end{tabular}

* Marks the 4 canonical discriminant functions remaining in the analysis.

\section{Classification results}

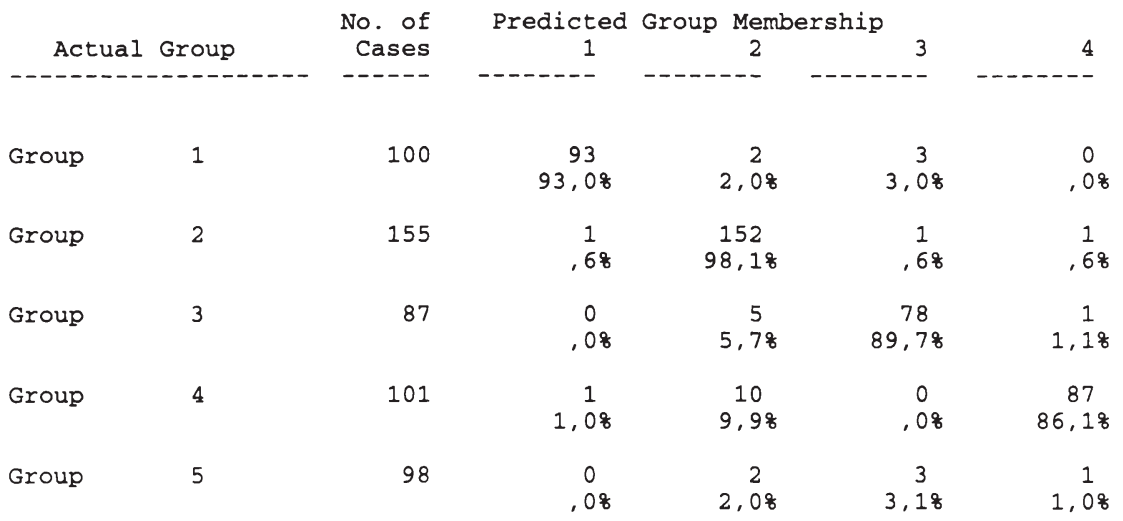

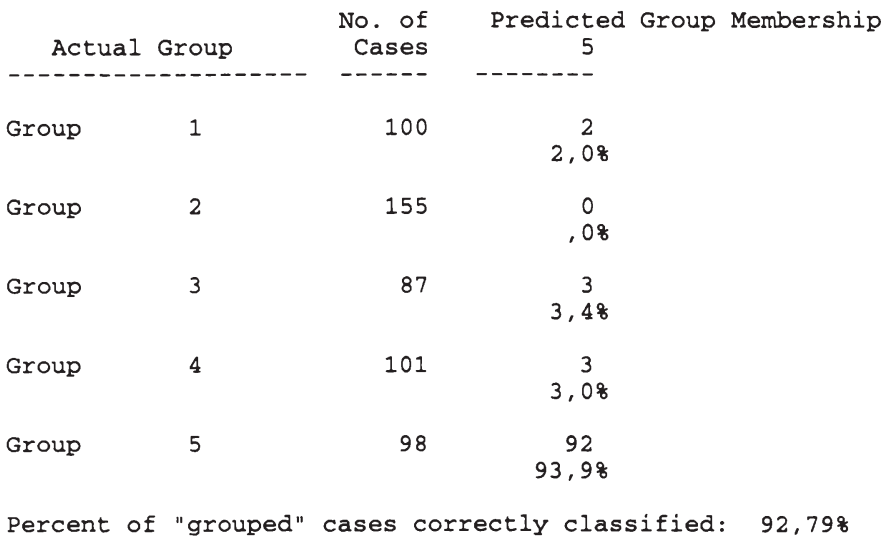


Legende:

Group 1: fachbesucherorientierte Hardseller

Group 2: kommunikationsorientierte Beziehungsmanager

Group 3: wettbewerbsorientierte Pflichtrepräsentanten

Group 4: hybride Fachaussteller

Group 5: ambitionierte Regionalaussteller

\section{Schrittweise Diskriminanzanalyse für die ermittleten Grundhaltungstypen: Verfahrensspezifikation, Diskriminanzfunktionen und Klassifikationsmatrix}

\section{Verfahrensspezifikation}

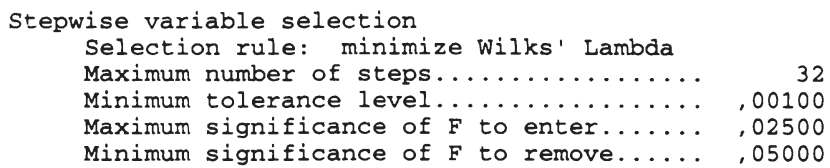

Canonical Discriminant Functions

Maximum number of functions........... 4

Minimum cumulative percent of variance... 100,00

Maximum significance of Wilks' Lambda.... 1,0000

Prior probabilities

$\begin{array}{rrr}\text { Group } & \text { Prior } & \text { Label } \\ 1 & , 18484 \\ 2 & , 28651 \\ 3 & , 16081 \\ 4 & , 18669 \\ 5 & , 18115 \\ \text { Total } & 1,00000\end{array}$

\section{Summary Table}

\begin{tabular}{rlrrll}
\multicolumn{2}{c}{ Action } & Vars & Wilks' \\
Step Entered Removed & in & Lambda & Sig. Label \\
& & 1 &, 45489 &, 0000 & Einzug Besucher \\
2 & FD6MAD & 2 &, 27981 &, 0000 & Messebesucher \\
3 & FD6MAB & 3 &, 17477 &, 0000 & Grundorient. der Messe \\
4 & FD6MAF & 4 &, 11790 &, 0000 & Begleitveranstaltungen \\
5 & FD6MAA & 5 &, 10247 &, 0000 & Ausstellungsprog. \\
6 & FD7B & 6 &, 09025 &, 0000 & Nutzenorientierung \\
7 & FD6MAE & 7 &, 08106 &, 0000 & Einzug Aussteller \\
8 & ZIEL4A & 8 &, 07471 &, 0000 & Verkaufsziele \\
9 & ZIEL2 & 9 &, 06912 &, 0000 & Motivationsziele \\
10 & ZIEL3B & 10 &, 06511 &, 0000 & anspruchsgr.-bez. Beeinfluss.-ziele \\
11 & FD7D & 11 &, 06241 &, 0000 & Wettb.-orient. \\
12 & FD7C & 12 &, 06016 &, 0000 & Kom./Erl.-orient.
\end{tabular}




\section{Canonical Discriminant Functions}

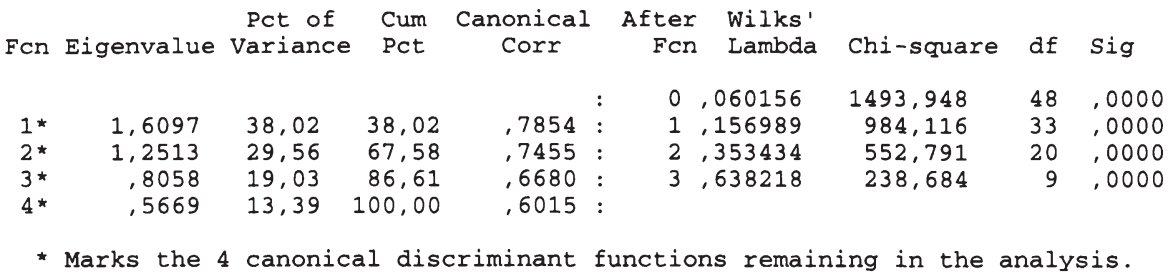

\section{Standardized canonical discriminant function coefficients}

\begin{tabular}{lrrrr} 
& Func 1 & Func 2 & Func & \multicolumn{1}{l}{ Func 4} \\
ZIEL2 &,- 04582 &, 03466 &, 37905 &, 11580 \\
ZIEL3B &,- 09055 &, 03799 &, 14314 &,- 34875 \\
ZIEL4A &, 05589 &,- 38391 &,- 09152 &,- 09195 \\
FD6MAA &,- 17110 &, 42425 &,- 20504 &,- 05689 \\
FD6MAB &, 11256 &, 47195 &, 55625 &, 35523 \\
FD6MAC &,- 24411 &, 54790 &,- 49056 &, 44536 \\
FD6MAD &, 70362 &, 11814 &,- 22169 &, 15690 \\
FD6MAE &, 41755 &, 00791 &,- 14097 &, 13370 \\
FD6MAF &,- 05747 &,- 42296 &, 15989 &, 82051 \\
FD7B &, 05528 &, 21372 &, 34456 &,- 05164 \\
FD7C &, 09989 &, 20108 &, 01315 &,- 16556 \\
FD7D &,- 08069 &,- 09182 &,- 20373 &, 20006
\end{tabular}

\section{Classification results}

\begin{tabular}{|c|c|c|c|c|c|c|}
\hline $\begin{array}{l}\text { No. of } \\
\text { Actual }\end{array}$ & $\begin{array}{l}\text { Predicted } \\
\text { Group }\end{array}$ & $\begin{array}{c}\text { Group Membe } \\
\text { Cases }\end{array}$ & ership & 2 & 3 & 4 \\
\hline Group & 1 & 100 & $\begin{array}{c}92 \\
92,08\end{array}$ & 2,2 & $\begin{array}{c}3 \\
3,08\end{array}$ & $\begin{array}{l}0 \\
.08\end{array}$ \\
\hline Group & 2 & 155 & $\frac{1}{68}$ & $\begin{array}{c}150 \\
96,88\end{array}$ & $\begin{array}{l}1 \\
.68\end{array}$ & $\begin{array}{c}3 \\
1,9 \%\end{array}$ \\
\hline Group & 3 & 87 & $\begin{array}{l}0 \\
.08\end{array}$ & $\begin{array}{c}4 \\
4,68\end{array}$ & $\begin{array}{c}78 \\
89,78\end{array}$ & $\begin{array}{c}2 \\
2,38\end{array}$ \\
\hline Group & 4 & 101 & 1,08 & $\begin{array}{c}9 \\
8,98\end{array}$ & $\begin{array}{l}0 \\
.08\end{array}$ & $\begin{array}{c}86 \\
85,18\end{array}$ \\
\hline Group & 5 & 98 & $\begin{array}{l}0 \\
.08\end{array}$ & 2,08 & $\begin{array}{c}2 \\
2,08\end{array}$ & $\begin{array}{c}1 \\
1,08\end{array}$ \\
\hline
\end{tabular}




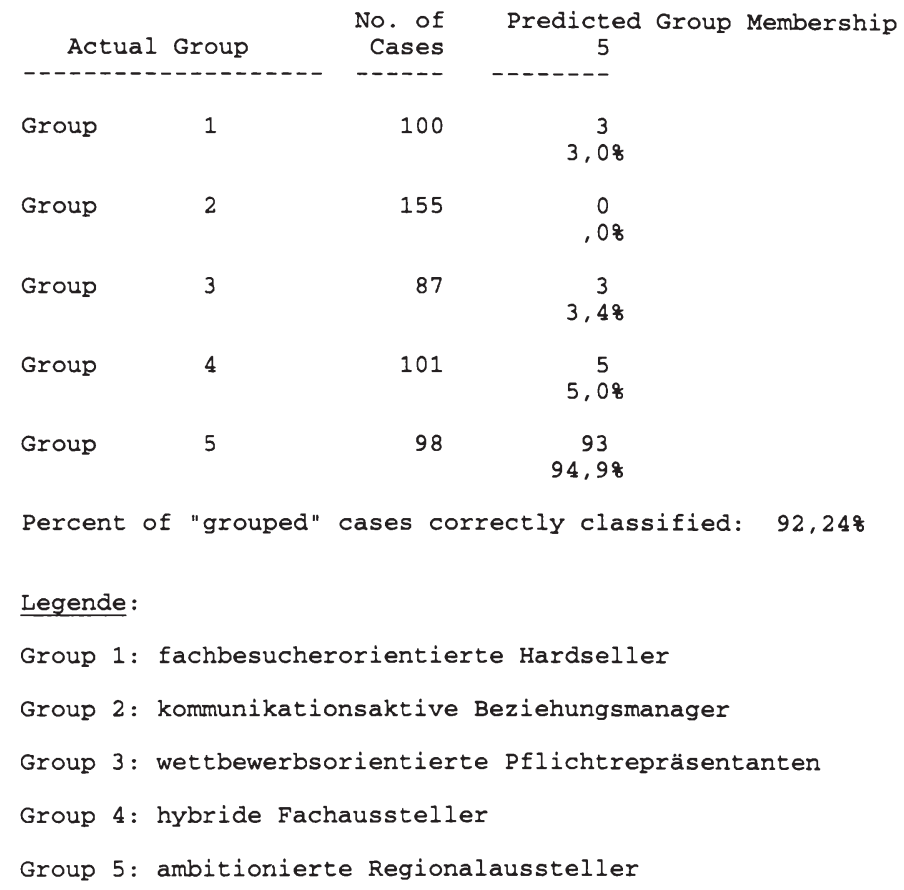

\section{Explorative Faktorenanalyse für die Variablen der Markt- und Wettbe- werbssituation}

\section{Verfahrensspezifikation}

Analysis number 1 Listwise deletion of cases with missing values

Extraction 1 for analysis 1, Principal Components Analysis (PC)

\section{Final Statistics:}

\begin{tabular}{|c|c|c|c|c|c|}
\hline Variable & Communality & * Factor & Eigenvalue & Pct of Var & Cum Pct \\
\hline FD1A &, 50838 & $\star$ & 2,18306 & 19,8 & 19,8 \\
\hline FD1B & .43480 & 2 & 1,59890 & 14,5 & 34,4 \\
\hline FD1C &, 53021 & 3 & 1,30876 & 11,9 & 46,3 \\
\hline FD1D &, 63649 & 4 & 1,04922 & 9,5 & 55,8 \\
\hline FD1E &, 66088 & $\star$ & & & \\
\hline FD1F & .36078 & $\star$ & & & \\
\hline FD1G & .72454 & $\star$ & & & \\
\hline FD1H &, 62613 & $\star$ & & & \\
\hline FD1I &, 56777 & $\star$ & & & \\
\hline FD1J &, 66836 & $\star$ & & & \\
\hline FD1K & 42161 & $\star$ & & & \\
\hline
\end{tabular}




\section{Rotated Factor Matrix:}

\begin{tabular}{|c|c|c|c|c|}
\hline & Factor 1 & Factor & Factor & Factor \\
\hline FD1J &, 81578 &,- 00227 &,- 02466 &,- 04744 \\
\hline FD1I &, 64951 & .00922 &, 15604 &,- 38419 \\
\hline FD1K &, 64688 &, 22375 &, 27855 &,- 30700 \\
\hline FD1D &,- 00104 &, 69491 & .38830 & .05300 \\
\hline FD1E &,- 07969 &, 80154 &,- 10687 &,- 02532 \\
\hline FD1G &, 24871 &,- 00783 &, 79370 &, 18074 \\
\hline FD1C &,- 06511 &, 12910 & .71314 & -.02706 \\
\hline FD1A &, 01567 &,- 23228 & .66701 &, 09633 \\
\hline FD1B & .01149 &, 42873 &, 66494 &, 18624 \\
\hline FD1H &, 11442 &, 05549 & .01403 &, 78087 \\
\hline FD1F & , 29892 &, 14873 &,- 06186 &,- 59546 \\
\hline
\end{tabular}

\section{Fusionierungsprozeß des Ward-Verfahrens zur Ermittlung von Situations- clustern}

\section{HIERARCHICAL CLUSTER ANALYSIS}

Squared Euclidean measure used

Agglomeration Schedule using Ward Method (CONT.)

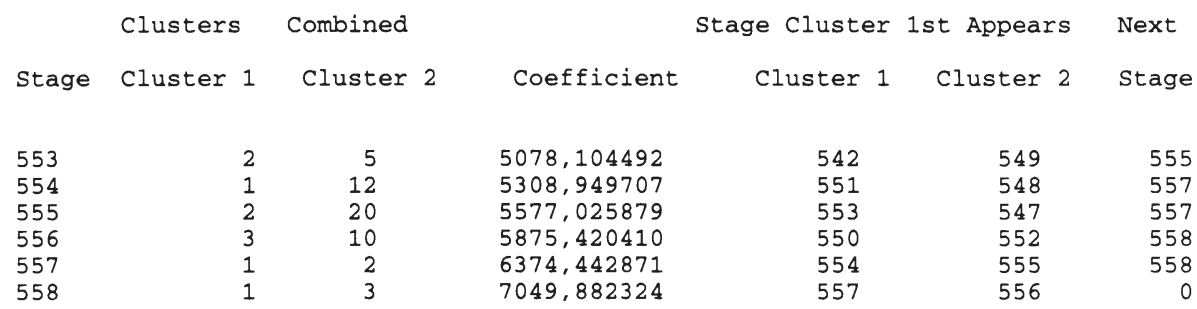


7. Partionierende Clusteranalyse zur Ermittlung der Mittelwerte der Situationscluster

Final Cluster Centers.

$\begin{array}{cllll}\text { Cluster } & \text { FD1A } & \text { FD1B } & \text { FD1C } & \text { FD1D } \\ 1 & & & & \\ 2 & 4,3333 & 4,1542 & 4,2637 & 3,8209 \\ 3 & 4,4756 & 3,8889 & 4,0933 & 3,1911 \\ & 4,0301 & 3,3985 & 1,9323 & 2,6316 \\ \text { Cluster } & \text { FD1E } & \text { FD1F } & \text { FD1G } & \text { FD1H } \\ 1 & 3,1990 & 3,4478 & 2,9154 & 3,3035 \\ 2 & 2,4356 & 2,3600 & 2,7956 & 2,4222 \\ 3 & 2,4135 & 2,4812 & 2,4436 & 2,9549 \\ & & & & \\ \text { Cluster } & \text { FD1I } & \text { FD1J } & \text { FD1K } & \\ & & & & \\ 1 & 4,2886 & 3,4229 & 3,5423 & \\ 2 & 3,0711 & 2,3067 & 2,5378 & \\ 3 & 3,8271 & 3,0977 & 2,4737 & \end{array}$

\section{Number of Cases in each Cluster.}

$\begin{array}{crr}\text { Cluster } & \text { unweighted cases } & \text { weighted cases } \\ 1 & 201,0 & 201,0 \\ 2 & 225,0 & 225,0 \\ 3 & 133,0 & 133,0 \\ \text { Missing } & 31 & \\ \text { Valid cases } & 559,0 & 559,0\end{array}$

\section{Diskriminanzanalyse für die ermittelten Situationstypen: Diskriminanzfunk- tionen und Klassifikationsmatrix}

\section{Canonical Discriminant Functions}

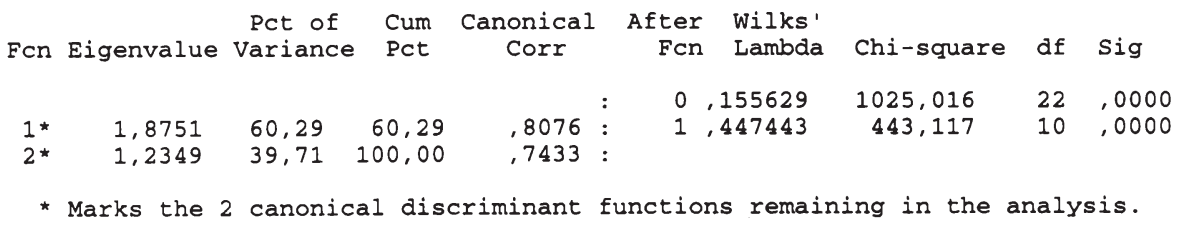


Classification results

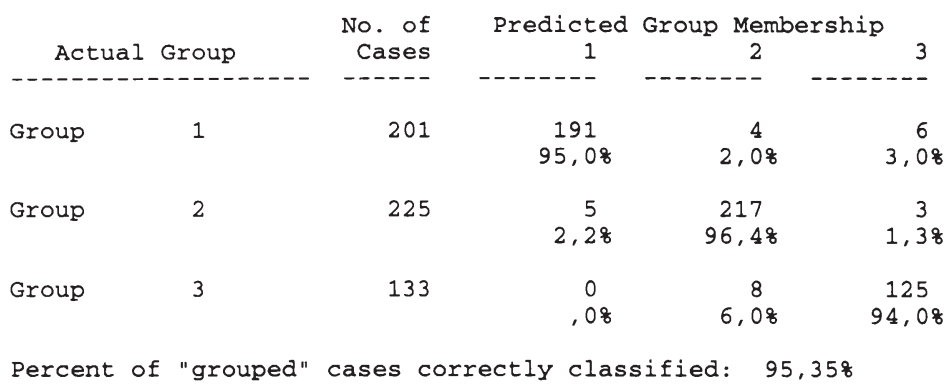

\section{Classification processing summary}

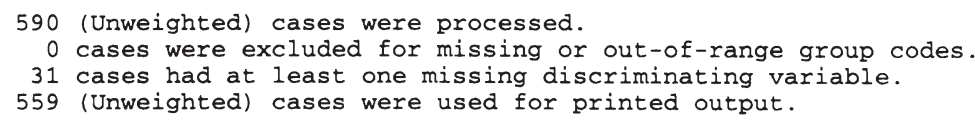

9. Diskriminanzanalyse für die ermittleten Einflußfaktoren: Verfahrensspezifikation und Diskriminanzfunktionen

\section{Verfahrensspezifikation}

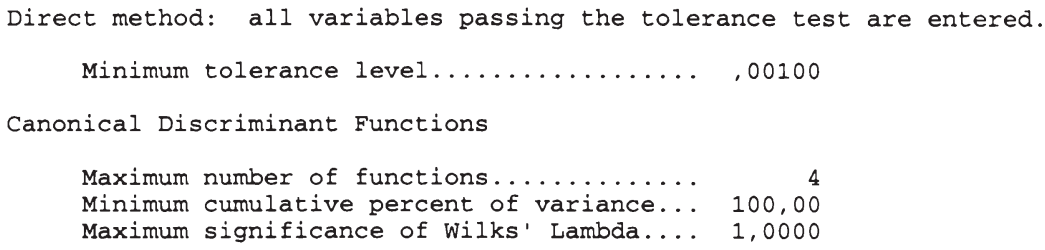




\section{Canonical Discriminant Functions}

\begin{tabular}{|c|c|c|c|c|c|c|c|c|c|}
\hline FCn & Eigenvalue & $\begin{array}{c}\text { Pct of } \\
\text { Variance }\end{array}$ & $\begin{array}{l}\text { Cum } \\
\text { Pct }\end{array}$ & $\begin{array}{c}\text { Canonical } \\
\text { Corr }\end{array}$ & $\begin{array}{r}\text { After } \\
\text { FCn }\end{array}$ & $\begin{array}{l}\text { Wilks' } \\
\text { Lambda }\end{array}$ & Chi-square & $d f$ & Sig \\
\hline & & & & & 0 & .067321 & 485,378 & 80 & .0000 \\
\hline 1 * & 1,1749 & 41,59 & 41,59 &, 8859 & 1 & & 209,848 & 57 & .0000 \\
\hline 2 * & 1,1338 & 31,81 & 73,40 & .7435 & 2 & , 496837 & 151,011 & 36 & .0049 \\
\hline 3 * &, 5062 & 13,36 & 86,76 &, 6307 & 3 &, 547235 & 75,397 & 17 & .0086 \\
\hline & 4557 & 32, & 00,00 & 6297 & & & & & \\
\hline
\end{tabular}

* Marks the 4 canonical discriminant functions remaining in the analysis

\section{Standardized canonical discriminant function coefficients}

\begin{tabular}{|c|c|c|c|c|}
\hline & Func & Func 2 & Func & Func \\
\hline FD1A &, 22959 &, 19471 &,- 21684 &,- 16161 \\
\hline FD1B &,- 10833 &,- 12406 &,- 10781 &, 14389 \\
\hline FD1C &, 18168 &,- 06964 &,- 36234 &,- 10911 \\
\hline FD1D & , 18109 &, 11442 &,- 05905 & .36123 \\
\hline FD1E &, 13841 &,- 25954 &,- 04245 & .10689 \\
\hline FD1F &,- 28216 &,- 10820 &,- 13462 & .18917 \\
\hline FD1G & -.02304 & . 19969 & .10383 & .07404 \\
\hline FD1H & .03749 & -.03293 &,- 11312 & .28275 \\
\hline FD1I &, 06242 & , 11369 &,- 26472 & .20729 \\
\hline FD1J &, 11733 & .22001 & .32914 & .24090 \\
\hline FD1K & .07268 &,- 14857 & -.08581 & .01601 \\
\hline FD2HEUTK &,- 01661 & .63981 &,- 25933 & -.08709 \\
\hline FD $3 A$ &, 11582 &, 12262 &, 44267 & .08190 \\
\hline FD3B &, 28225 &,- 06453 &, 44817 & .28410 \\
\hline FD3C &,- 03579 & -.05007 & .04210 & .30544 \\
\hline FD3D &, 14294 & .36169 &, 36543 & .12320 \\
\hline FD4HEUTD &,- 27664 & .05099 &, 08067 & .36938 \\
\hline FD4FUNKT &, 11692 &, 11613 &, 33787 & -.25949 \\
\hline FINFOC &, 32561 &,- 33721 & .04927 & .02583 \\
\hline BRANCHE &,- 52807 &,- 09354 & .04714 &,- 02581 \\
\hline
\end{tabular}




\section{Classification results}

\begin{tabular}{|c|c|c|c|c|c|c|}
\hline Act & Group & $\begin{array}{l}\text { No. of } \\
\text { Cases }\end{array}$ & $\begin{array}{c}\text { Predicted } \\
1\end{array}$ & $\begin{array}{c}\text { Group Membe } \\
2\end{array}$ & ip & 4 \\
\hline Group & 1 & 100 & $\begin{array}{c}88 \\
88,0 \%\end{array}$ & $\begin{array}{l}0 \\
.08\end{array}$ & 12,08 & $\begin{array}{c}0 \\
, 08\end{array}$ \\
\hline Group & 2 & 155 & 2,18 & $\begin{array}{l}145 \\
93,98\end{array}$ & $\begin{array}{l}0 \\
.08\end{array}$ & $\begin{array}{l}0 \\
, 08\end{array}$ \\
\hline Group & 3 & 87 & 10,28 & $\begin{array}{l}0 \\
.08\end{array}$ & $\begin{array}{c}72 \\
83,08\end{array}$ & $\begin{array}{c}3 \\
3,18\end{array}$ \\
\hline Group & 4 & 101 & $\begin{array}{l}0 \\
.08\end{array}$ & 12,98 & $\begin{array}{c}3 \\
3,28\end{array}$ & $\begin{array}{c}83 \\
82,18\end{array}$ \\
\hline Group & 5 & 98 & $\begin{array}{c}3 \\
3,18\end{array}$ & $\begin{array}{c}4 \\
4,28\end{array}$ & $\begin{array}{c}2 \\
2,18\end{array}$ & $\begin{array}{l}0 \\
08\end{array}$ \\
\hline
\end{tabular}

No. of Predicted Group Membership

Actual Group $\quad$ Cases

$\begin{array}{lccc}\text { Group } & 1 & 100 & 0 \\ \text { Group } & 2 & 155 & 7 \\ \text { Group } & 3 & 87 & 4,18 \\ \text { Group } & 4 & 101 & 3,48 \\ \text { Group } & 5 & 98 & 2,88 \\ & & & 89 \\ & & & 90,98\end{array}$

Percent of "grouped" cases correctly classified: 87,63\%

\section{Classification processing summary}

590 (Unweighted) cases were processed.

0 cases were excluded for missing or out-of-range group codes.

49 cases had at least one missing discriminating variable.

541 (Unweighted) cases were used for printed output.

\section{Legende:}

Group 1: fachbesucherorientierte Hardseller

Group 2: kommunikationsorientierte Beziehungsmanager

Group 3: wettbewerbsorientierte Pflichtrepräsentanten

Group 4: hybride Fachaussteller

Group 5: ambitionierte Regionalaussteller 
Anhang 5: Fragebogen für die schriftliche Befragung von ausstellenden Unternehmen 


\title{
Westfälische Wilhelms-Universität Münster \\ Institut für Marketing
}

Prof. Dr. Dr. h.c. H. Meffert

\section{Forschungsprojekt}

\section{"Nutzen von Messebeteiligungen"}

\author{
- Fragebogen I -
}

zu Messebeteiligungen in Deutschland

Bitte beachten Sie folgende Bearbeitungshinweise:

- dieser Fragebogen bezieht sich, soweit nicht auf etwas anderes verwiesen wird, ausschließlich auf Beteiligungen deutscher Unternehmen oder einzelner Geschäftsbereiche an Messen in Deutschland

- in die Beantwortung des Fragebogens können ggfs. auch mehrere Personen einbezogen werden

- $\quad$ wir bitten Sie, den Fragebogen auch dann zurückzusenden, wenn Sie einzelne Fragen nicht beantwortet haben; eine Frankierung des beiliegenden Rückumschlages ist nicht notwendig

- bei Rückfragen steht Ihnen Herr Dipl.-Kfm. Ralf Ueding, Tel.: 0251/5 6472 bzw. 832931 gerne zur Verfügung

Universität Münster - Institut für Marketing - Universitätsstraße 14-16 - 48143 Münster

Telefon: 0251 - 832931 - Telefax: $0251-838356$ 
1. Unternehmen bzw. einzelne Geschäftsbereiche, die sich an Messen beteiligen, befinden sich in den unterschiedlichsten Marktsituationen. Geben Sie bitte zunăchst an, inwieweit die folgenden Aussagen für den Markt zutreffen, in dem lhr Unternehmen bzw. Geschäftsbereich tätig ist. Sofern in dem von Ihnen bearbeiteten Markt regionale Unterschiede gelten, bitten wir Sie, Durchschnittswerte anzugeben. Dabei bedeutet " 1 " "Die Aussage trift gar nicht zu" und " 5 " "Die Aussage trifft sehr zu". Mit den übrigen Ziffern können Sie Ihre Einschätzung abstufen.

Die Aussage:

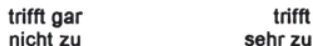

in unserem Markt herrscht ein intensiver Preiswettbewerb

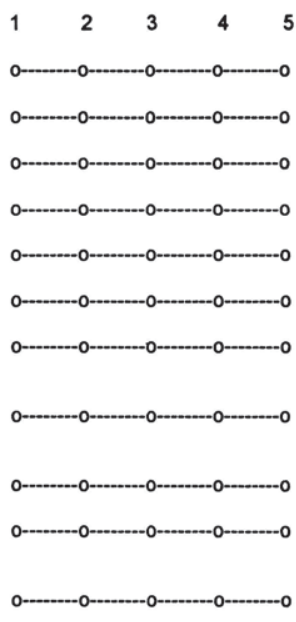

die Rahmenbedingungen in unserem Markt verăndern sich laufend (Fertigungstechnologien, Rechtsvorschriften etc.)

2. Im folgenden haben wir neben der Messebeteiligung weitere Marketinginstrumente zusammengestellt, die Sie möglicherweise im deutschen Markt nutzen. Welche Bedeutung nehmen die einzelnen Instrumente ein? Wie wichtig sind dabei die Marketinginstrumente heute (Teil a), und wie schätzen Sie die Situation für die Zukunft ein (Teil b)?

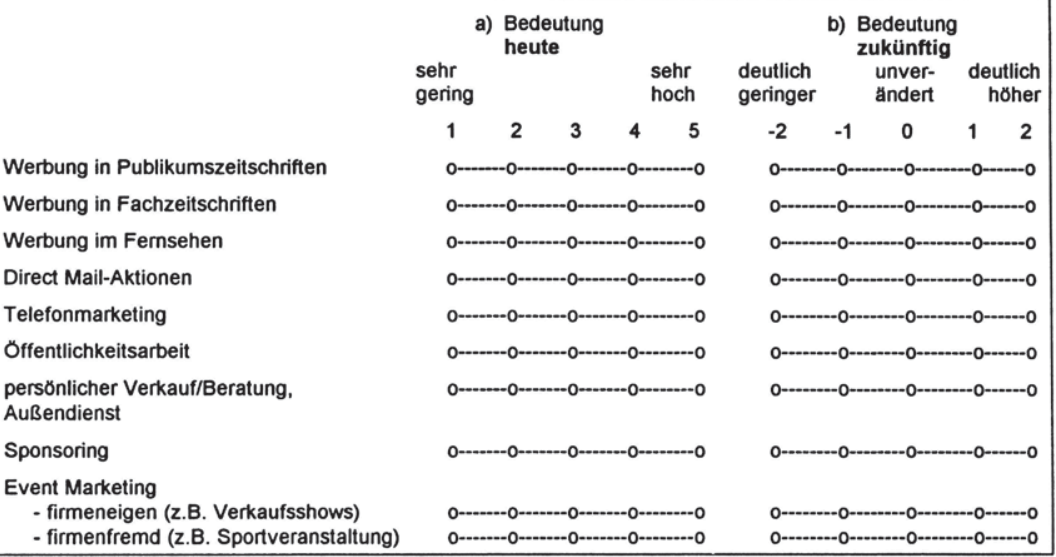




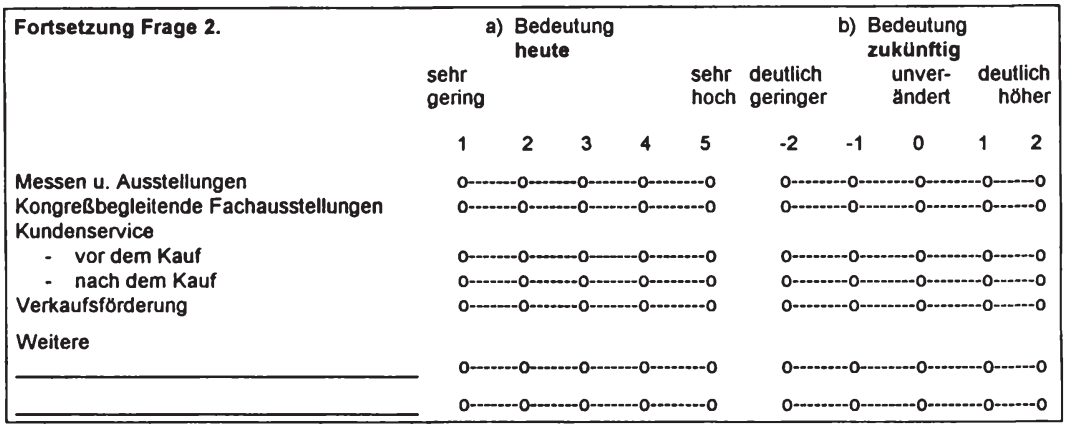

3. Neben der Möglichkeit, jedes Marketinginstrument einzeln und unabhängig von den anderen Instrumenten zu planen und einzusetzen, kann man den Instrumenteeinsatz auch i.S. eines integrierten Konzeptes aufeinander abgestimmt durchführen. Wir möchten gerne wissen, in welcher Weise Sie Ihre Messebeteiligungen in Deutschland planen.

Die Aussage:

trifft gar trifft nicht zu sehr zu

Messebeteiligungen werden völlig unabhăngig von den anderen $\begin{array}{lllll}1 & 2 & 3 & 4 & 5\end{array}$ Marketingaktivitäten geplant und durchgeführt

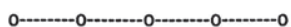

es besteht ein übergeordnetes Marketingkonzept.

in das auch die Messebeteiligungen integriert sind

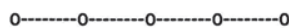

Messebeteiligungen werden insbesondere in ihrem zeitlichen Einsatz mit den anderen Instrumenten koordiniert

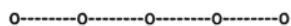

wir achten vor allem auf die formale Übereinstimmung von Messeauftritt und den anderen Marketingaktivitäten

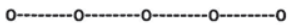

sonstiges

4. An wie vielen Messen hat sich Ihr Unternehmen/Geschäftsbereich als Aussteller im Durchschnitt der letzten Jahre beteiligt, und wie wird die künftige Messebeteiligung sein? Geben Sie bitte auch an, wer heute die Entscheidungen für die wichtigsten Messebeteiligungen fällt.

Messebeteiligung im Jahresdurchschnitt bis heute zukünftig

in Deutschland

im Ausland

an

(Wenn Sie sich auch an ausländischen Messen beteiligen, möchten wir Sie bitten, im Anschluß an diese Befragung kurz einige Fragen auf dem beiliegenden gelben Fragebogen II zu beantwortenl)

Die Beteiligungsentscheidungen werden heute überwiegend von gefălit. (Abteilung/Unternehmensfunktion)

5. Wie wird sich die Höhe des Budgets für Messebeteiligungen in Deutschland in Ihrem Unternehmen / Geschäftsbereich in Zukunft entwickeln?

Das Messebudget wird zukünftig

$\begin{array}{lll}\text { deutlich } & \text { unver- } & \text { deutlich } \\ \text { geringer } & \text { ändert } & \text { höher }\end{array}$

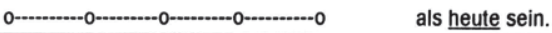


6. Wenn Sie einmal versuchen, den Schwerpunkt Ihrer Messebeteiligungen in Deutschland anzugeben (soliten dies mehr als 3 Messen sein, so konzentrieren Sie sich auf die wichtigsten 3 ), welches Veranstaltungsmuster hatten die Messen, und wie schätzen Sie den Erfolg Ihrer Messebeteiligung jeweils ein? Bitte nennen Sie uns zunächst den Namen und den Ort der Messe.

Für Messebeteiligung A: Name der Messe: Ort:

Im Hinblick auf

das Ausstellungsprogramm

die Grundorientierung

der Messe

die Messebesucher

den Einzugsbereich der

Messebesucher

den Einzugsbereich bei

Ausstellern

die Durchführung von

Begleitveranstaltungen auf

der Messe (z.B. Seminare)

den generellen Erfolg ihrer

Messebeteiligung

Für Messebeteiligung B: Name der Messe:

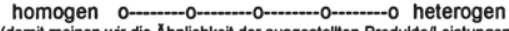
(damit meinen wir die Ahnlichkeit der ausgestellten Produkte/Leistungen)

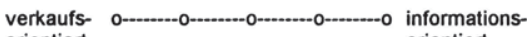

orientiert orientiert

Fachpublikum 0-1-0 0 Privatpublikum

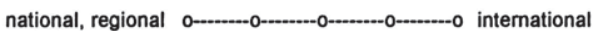

national, regional 0 -

viele Begleit- 0-1-0

veranstaltungen veranstaltung

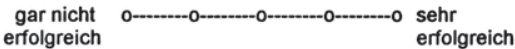
Ort:

Im Hinblick auf

das Ausstellungsprogramm

die Grundorientierung

der Messe

die Messebesucher

den Einzugsbereich der

Messebesucher

den Einzugsbereich bei

Ausstellern

die Durchführung von

Begleitveranstaltungen auf

der Messe (z.B. Seminare)

den generellen Erfolg ihrer

Messebeteiligung

Für Messebeteiligung C: Name der Messe: (damit meinen wir die Âhnlichkelt der ausgestellten Produkte/Leistungen)

verkaufs- 0-1-0 0 informations-

orientiert orientiert

Fachpublikum 0-1-0 0 Privatpublikum

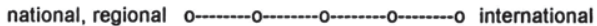

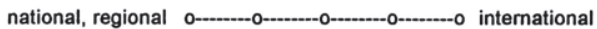

viele Begleit- 0-1-0

veranstaltungen veranstaltung

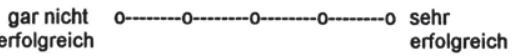

Im Hinblick auf

das Ausstellungsprogramm

die Grundorientierung

der Messe

die Messebesucher

den Einzugsbereich der

Messebesucher

den Einzugsbereich bei

Ausstellern

die Durchführung von

Begleitveranstaltungen auf

der Messe (z.B. Seminare)

den generellen Erfolg ihrer Messebeteiligung

Ort:

\begin{tabular}{|c|c|c|}
\hline $\begin{array}{c}\text { homogen } \\
\text { (damit meinen }\end{array}$ & vir die Áhnlichkelt der ausgestellten Prod & $\begin{array}{l}\text { heterogen } \\
\text { ukte /Leistungen) }\end{array}$ \\
\hline $\begin{array}{l}\text { verkaufs- } \\
\text { orientiert }\end{array}$ & o- 0 & $\begin{array}{l}\text { informations- } \\
\text { orientiert }\end{array}$ \\
\hline Fachpublikum & - & Privatpublikum \\
\hline national, regional & 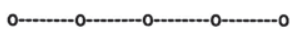 & international \\
\hline national, regional & o- 0 & international \\
\hline $\begin{array}{l}\text { viele Begleit- } \\
\text { veranstaltungen }\end{array}$ & o- 0 & $\begin{array}{l}\text { keine Begleit- } \\
\text { veranstaltung }\end{array}$ \\
\hline $\begin{array}{l}\text { gar nicht } \\
\text { erfolgreich }\end{array}$ & 0 & $\begin{array}{l}\text { sehr } \\
\text { erfolgreich }\end{array}$ \\
\hline
\end{tabular}


7. Für jede Messebeteiligung bestehen bestimmte Grundorientierungen, die sich in einem konkreten Messe-/Ausstellungsstil widerspiegeln. Bitte geben Sie für die nachfolgend genannten Statements jeweils an, ob diese Aussagen für lhre Messestände auf deutschen Messen zutreffen. (Mehrfachnennungen möglich)

durch

eine starke Produkt-bzw. Sortimentsorientierung

eine starke Problemlosungs- bzw. Nutzenorientierung aus Sicht unserer Kunden

trifft gar

nicht zu

\section{1}

2

3

sehr zu

eine starke Kommunikations- bzw. Erlebnisorientierung

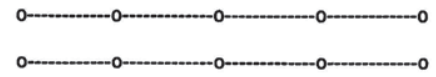

in unserer Messestandgestaltung orientieren wir uns in einem starken Maße auch an den Messekonzepten unserer Wettbewerber

bei der Gestaltung unserer Messestände sind wir stark kostenorientiert, d. h. wir versuchen, mit den geringst möglichen Kosten einen angemessenen Messeauftritt zu realisieren

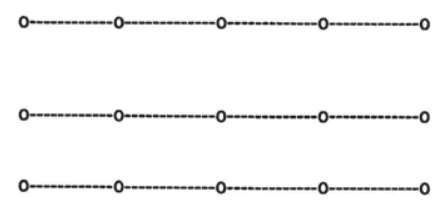

8. Die Ziele einer Messebeteiligung sind i.d.R. vielfältig und meist werden mehrere Ziele verfolgt. Bitte geben Sie für jedes der nachfolgend angeführten Messeziele an, welche Wichtigkeit es für den Schwerpunkt Ihrer Messebeteiligungen in Deutschland heute hat bzw. in Zukunft besitzen wird. In welchem Maße werden Ihre Zielsetzungen heute überwiegend erreicht?

Kennenlernen neuer Mărkte

Kennenlemen neuer Wettbewerber

Kennenlernen neuer Lieferanten

Anbahnung von Gemeinschaftsunternehmen

Verkaufsabschlüsse

Verkaufsanbahnung/Nachmessegeschăft

Kontaktpflege mit bestehenden Kunden

Kontaktbelebung mit ehemaligen Kunden

Kontaktaufnahme mit potentiellen Kunden

Erhohung des Bekanntheitsgrades

des Unternehmens

Erhohung des Bekanntheitsgrades von Produkten und Leistungen

Darstellung der Produkt- bzw. Leistungskompetenz

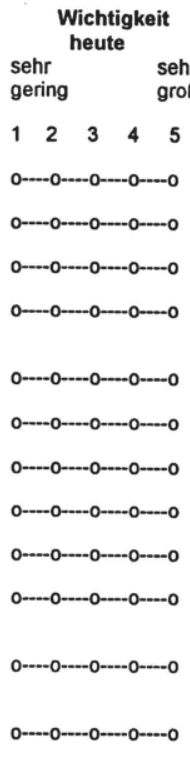

Grad der Zielerreichung heute gar nicht

voll

$\begin{array}{lllll}1 & 2 & 3 & 4 & 5\end{array}$

0

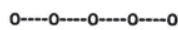

0

0

0

0

0

0---o--o-no

0

0

0-1-0

0

0

0

0 0

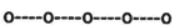

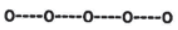

0

0

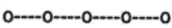

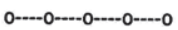

0

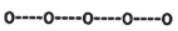

0 


\begin{tabular}{|c|c|c|c|}
\hline \multirow[t]{3}{*}{ Fortsetzung Frage 8.} & $\begin{array}{l}\text { Wichtigkeit } \\
\text { heute }\end{array}$ & $\begin{array}{l}\text { Wichtigkeit } \\
\text { in Zukunft }\end{array}$ & $\begin{array}{l}\text { Grad der Ziel- } \\
\text { erreichung heute }\end{array}$ \\
\hline & $\begin{array}{ll}\text { sehr } & \text { sehr } \\
\text { gering } & \text { groß }\end{array}$ & $\begin{array}{ll}\text { sehr } & \text { sehr } \\
\text { gering } & \text { groß }\end{array}$ & \multirow{2}{*}{$\begin{array}{lllll}\begin{array}{l}\text { gar } \\
\text { nicht }\end{array} & & & \text { voll } \\
1 & 2 & 3 & 4 & 5\end{array}$} \\
\hline & 12 & 12 & \\
\hline Verbesserung Unternehmensimage & $0-0-0-0-0$ & $0-\cdots 0-0-0$ & $0-\cdots-0-\cdots-0-0-0$ \\
\hline - emotionale Profilierung & $0-0-0-0-0-0$ & $0-0-0-0-0-0=0$ & $0-0-0-0-0=0$ \\
\hline - rationale Profilierung & $0-0-0-0-0-0$ & $0-0-0-\cdots 0-0-0$ & $0-0-0-0-0$ \\
\hline $\begin{array}{l}\text { Einstellungsănderungen ggù. kontrovers } \\
\text { diskutierten Branchenproblemen }\end{array}$ & $0-0-0-0-0$ & $0-0-0-0-0=0$ & $0-0-0-0-0$ \\
\hline $\begin{array}{l}\text { Beziehungspflege bzw. -aufbau } \\
\text { mit Medienvertretem }\end{array}$ & $0-0-0-0-0-0$ & $0--0---0--0-0$ & $0-\cdots 0---0---0--\infty 0$ \\
\hline $\begin{array}{l}\text { Beziehungspflege bzw. -aufbau mit } \\
\text { Reprăsentanten versch. Institutionen } \\
\text { (z. B. Staat, Verbănde etc.) }\end{array}$ & $0-0-0-0-0-0$ & $0--0-0-0-0-\infty=0$ & $0--0--0-0-0$ \\
\hline Motivation der eigenen Mitarbeiter & $0-0-0-0$ & 0----0----0----0---o & $0-\cdots-0--0-0-0$ \\
\hline Konkurrenzbeobachtung & 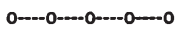 & 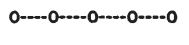 & 0 \\
\hline Mitarbeitersuche & 0 & 0--0----0----0----o & $0-\cdots 0-0-0-0$ \\
\hline Aufbau von Vertriebswegen & $0-0-0-0-0-0$ & 0----0----0----0---o & $0--0----0---0-\cdots$ \\
\hline $\begin{array}{l}\text { Erkennen von Trends bei } \\
\text { Kundenprăferenzen }\end{array}$ & $0-0-0-0-0$ & $0-\cdots-0-0-0-0$ & $0-0-0-0-0-0$ \\
\hline $\begin{array}{l}\text { Erfahrungsaustausch auf begleitenden } \\
\text { Fachveranstaltungen und Kongressen }\end{array}$ & 0 & 0----0----0-----o----0 & $0-\cdots-0--0---0-0$ \\
\hline $\begin{array}{l}\text { Überprufung der Marktreife } \\
\text { eigener Produkte und Leistungen }\end{array}$ & $0---0-3-0-0-0$ & 0----0----o-----o----o & $0-\cdots 0-\cdots 0--0-\cdots 0$ \\
\hline Sonstige Ziele & & & \\
\hline & $0-\cdots 0-\cdots 0-\cdots 0-\cdots$ & $0---0 \cdots-\cdots 0$ & $0-\cdots 0-\cdots 0--0-0$ \\
\hline & $0--0-0-0--0=0$ & $0--0--0-0-0$ & $0-\cdots-0-0--0-0$ \\
\hline
\end{tabular}

9. Im folgenden haben wir Möglichkeiten zur Konkretisierung der Messezielsetzungen aufgelistet. Welche der angeführten Varianten verwenden Sie im allgemeinen? (Mehrfachnennungen möglich!)

Die Messeziele werden wie folgt festgelegt:

- für Gruppen von Messen

- für einzelne Messen

- für jeden einzelnen Messetag einer Messe

- für Zielgruppen, die auf der Messe erwartet werden

- für die ausgestellten Produkt- bzw. Leistungsbereiche Leistungsbereiche

- die Messeziele werden nach der angestrebten Hohe des Zielerreichungsgrades konkretisiert

- die Messeziele werden nicht weiter konkretisiert

$$
\text { nie selten } \begin{gathered}
\text { manch- hăufig immer } \\
\text { mal }
\end{gathered}
$$
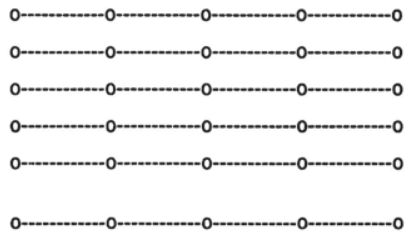

0 
10. Hat Ihr Unternehmen/Geschäftsbereich bereits Untersuchungen durchgeführt, um festzustellen, ob die angestrebten Messezielsetzungen erreicht wurden? Wenn ja, wie häufig?
ja, immer
o (weiter mit Frage 12)
ja, aber nur bei ausgewăhlten Veranstaltungen
o
(weiter mit Frage 12)
nein
- (weiter mit Frage 11)

11. Viele Unternehmen nehmen keine Überprüfungen des Messenutzens vor. Wir möchten Sie bitten, eine Einschätzung der Gründe für dieses Verhalten aus der Sicht Ihres Unternehmens I Geschäftsbereiches zu geben.

Die Überprüfung der Erreichung

unserer Messeziele unterbleibt, weil

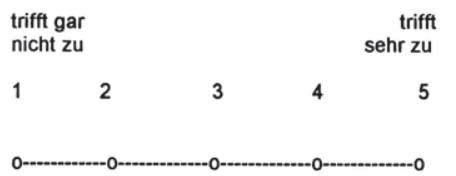

konkrete Nutzenüberlegungen bei uns weniger wichtig sind, da Messen für das

Unternehmen generell als unverzichtbar gelten

- die Personalkapazităten für die Durchführung von Messenutzenuntersuchungen nicht vorhanden sind

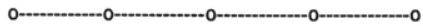

- der zusătzliche Erkenntnisgewinn angesichts der zu erwartenden Mehrkosten nicht als ausreichend betrachtet wird

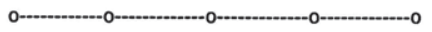

- das methodische Know-how fehlt

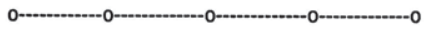

- der Stellenwert von Messen im Unternehmen als zu gering angesehen wird

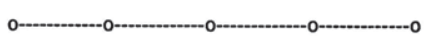

- sie auf den Widerstand des Standpersonals stరBt, das sich kontrolliert fühlt

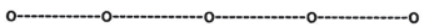

- Messeuntersuchungen den Ablauf der Messeaktivităten stören

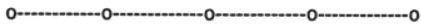

- sonstige Gründe: 

12. Welche der folgenden Methoden zur Kontrolle der Zielerreichung (Nutzenmessung) wendet
Ihr Unternehmen an? Dabei ist es uns wichtig zu erfahren, ob Ihnen die Verfahren bekannt
sind und wie intensiv diese in Ihrem Unternehmen /Geschäftsbereich angewendet werden.

das Verfahren ist

Zăhlung der personlichen Kontakte insgesamt

Zăhlung der Standbesucher insgesamt

Analyse der Kontakte im Zeitablauf einer Messe

Zăhlung des abgegebenen bzw. angeforderten Informationsmaterials

Kennziffernanalysen (z.B. Kontakte/Mitarbeiter u. a.) o

Auswertung von

- formalisierten Gesprăchsprotokollen

- nicht formalisierten Gesprăchsprotokollen

Messekontaktbewertung (z.B. bzgl. Zielgruppenerreichung und Gesprăchsinhalten)

Umsatzkontrolle

Budgetkontrolle

Standbesucherbefragung

Nachbefragungen der Standbesucher

nach Beendigung der Messe

Standbesucherbeobachtung

Wegeverlaufsanalyse

Standpersonalbefragung

Resonanzanalyse auf Einladungsaktionen

Messekonzepttest

Vergleich von Messe-Ergebnissen über mehrere Jahre

Vergleich von Messe-Ergebnissen mit

anderen Messeveranstaltungen

Vergleich von Ergebnissen mit anderen

Marketingaktivităten

Auswertung der Messebesucheranalysen

des Veranstalters

Auswertung des FKM-Besucherstrukturtests

Sonstige:

0

o

o

o

o
Einsatz des Verfahrens

nie selten manch- häufig immer
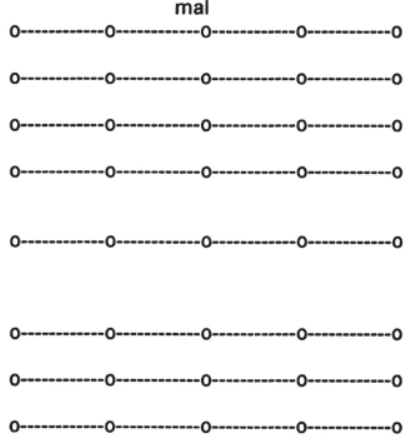

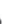
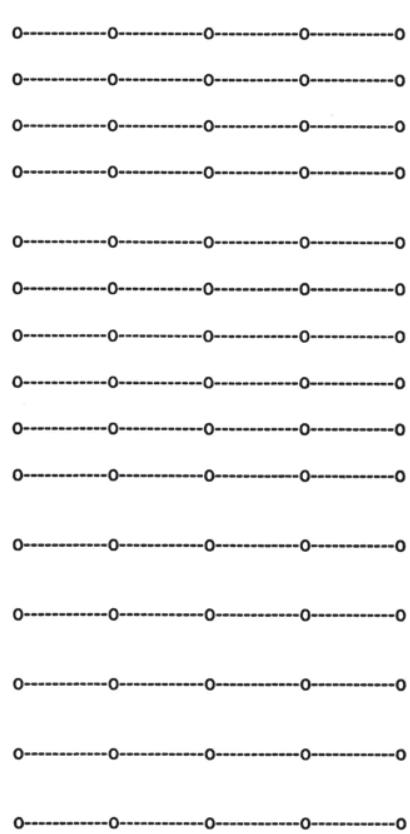

$0-0-0$ 
13. Wie zufrieden sind Sie mit den Methoden zur Kontrolle der Zielerreichung? Wir möchten Sie bitten, den Grad Ihrer Zufriedenheit mit denjenigen der aufgeführten Verfahren anzugeben. die Sie manchmal, häufig oder immer nutzen.

Zăhlung der persőnlichen Kontakte insgesamt

Zählung der Standbesucher insgesamt

Analyse der Kontakte im Zeitablauf einer Messe

Zăhlung des abgegebenen bzw. angeforderten

Informationsmaterials

Kennziffernanalysen (z.B. Kontakte/Mitarbeiter u. ă.)

Auswertung von

- formalisierten Gesprăchsprotokollen

- nicht formalisierten Gesprächsprotokollen

Messekontaktbewertung (z.B. bzgl. Zielgruppenerreichung und Gesprăchsinhalten)

Umsatzkontrolle

Budgetkontrolle

Standbesucherbefragung

schriftliche Nachbefragungen der Standbesucher nach Beendigung der Messe

Standbesucherbeobachtung

Wegeverlaufsanalyse

Standpersonalbefragung

Resonanzanalyse auf Einladungsaktionen

Messekonzepttest

Vergleich von Messe-Ergebnissen über

mehrere Jahre

Vergleich von Messe-Ergebnissen mit anderen Messeveranstaltungen

Vergleich von Ergebnissen mit anderen

Marketingaktivităten

Auswertung der Messebesucheranalysen des Veranstalters

Auswertung des FKM-Besucherstrukturtests

Sonstige:
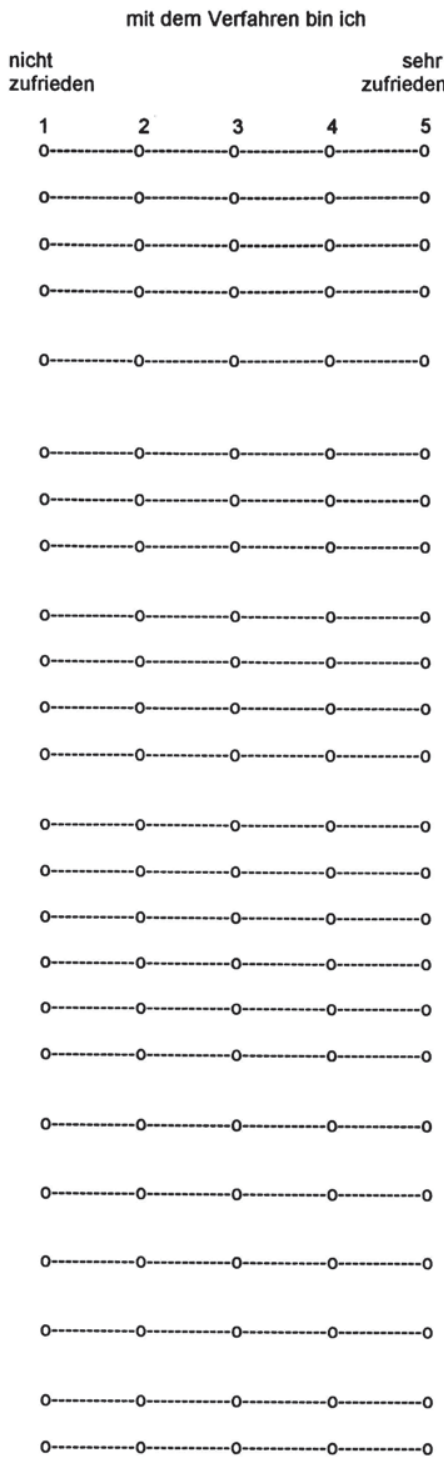
14. Was sind die wesentlichsten Ursachen für Ihre Unzufriedenheit mit einzelnen in Frage 13 genannten Verfahren zur Kontrolle der Zielerreichung? Bitte nennen Sie uns Ihre Hauptkritikpunkte unter Angabe des betreffenden Verfahrens!

Verfahren:

Kritik:

15. Bitte nennen Sie in kurzen Stichworten einige Gründe für die Anwendung der von Ihnen häufig eingesetzten Verfahren (auch Verfahren, die in den Fragen 12 und 13 nicht genannt wurden).

Verfahren:

Anwendungsgründe:

16. Wenn Sie einmal versuchen, den Anteil am gesamten Messebuget zu quantifizieren, der für die Messung der Zielerreichung Ihrer deutschen Messebeteiligungen verwendet wird, wie hoch ist der Anteil in Ihrem Unternehmen/Geschäftsbereich heute, und wie wird er in Zukunft sein?

Anteil der Kontrolle der Zielerreichung am Messebudget (Deutschland) heute:

Anteil der Kontrolle der Zielerreichung am Messebudget (Deutschland) in Zukunft:

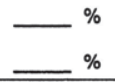

17. Setzen Sie in Deutschland im Rahmen der Messung der Zielerreichung Ihrer Messebeteiligungen externe Dienstleister ein?

$\begin{array}{llll}\text { nie selten } & \begin{array}{l}\text { manch- hăufig } \\ \text { mal }\end{array} & \text { immer } \\ 0 & & \end{array}$

18. Wie ist die organisatorische Ausgestaltung Ihrer Messebeteiligungen in Deutschland, und in welchem Rahmen finden externe Dienstleister Berücksichtigung? Bitte geben Sie an, inwieweit folgende Aussagen zutreffen.

Die Aussage:

- die Messebeteiligung wird komplett durch uns selbst geplant und abgewickelt

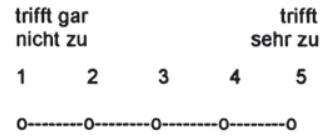

- wir koordinieren und bestimmen den inhaltlichen Schwerpunkt der Messebeteiligung; die operativen Tătigkeiten vergeben wir an externe Dienstleister

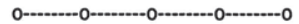

(z. B. Standbau, Catering, Standservices)

- bei uns existiert eine zentrale Messeabteilung die den Messeauftritt plant und uns Vorschläge für die Auswahl der Exponate macht

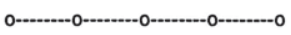

- wir verfügen über eine interne MesseserviceOrganisation, die nach unseren Vorgaben die Messebeteiligung gestaltet

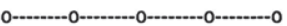


19. Die Erreichung der Messeziele ist im wesentlichen von den Aktivitäten des ausstellenden Unternehmens abhängig. Dabei können die Aktivitäten der Vor-Messe-, der Messe- und der Nach-Messe-Phase zugeordnet werden. Welche der nachfolgenden phasenspezifischen Maßnahmen nutzen Sie im allgemeinen, wenn Sie eine Messebeteiligung in Deutschland planen?

\section{Vor-Messe-Phase}

- Vor-Messe-Werbung durch Anzeigen in Fachzeitschriften

- Abfassung von Messevorberichten für die Presse

- Messeeinladungen für Pressevertreter

- Verschickung von persönlichen Einladungen (Direct Mail)

- an aktuelle Kunden

- an potentielle Kunden

- Terminabsprachen durch Außendienst

- Eintrag im Messekatalog

- Anzeige im Messekatalog

- Qualifizierung des Standpersonals

-- durch externe Trainer

-- durch interne Führungskräfte

sonstiges:

\section{Messe-Phase}

- Werbeaktionen auf der Messe

(z. B. Verteilung Handzettel, Luftballons o.ă.)

- Außenwerbung auf dem Messegelănde

- Nutzung des Messeinformationssystems für Besucher

- Produkt Shows (Produkt in Aktion)

- Video- bzw. Filmvorführungen

- Präsenz der Unternehmensleitung auf der Messe

- Einsatz von Prominenten auf dem Messestand (z. B. Künstler, Sportler)

- Einsatz von Musikgruppen auf dem Messestand

- Abhaltung von Pressekonferenzen

- Beteiligung bzw. Ausrichtung von Zusatzveranstaltungen (Fachvorträge, Symposien)

- Ausrichtung festlicher Rahmenveranstaltungen sonstiges:

\section{Nach-Messe-Phase}

- Systematische Weiterverfolgung der Messekontakte

- Nach-Messe-Werbung mit den Messeergebnissen des Unternehmens/Geschăftsbereiches

- Abfassung von internen Messeberichten

- Abfassung von Messeberichten für die Presse

sonstiges: nie selten manch- hăufig immer mal
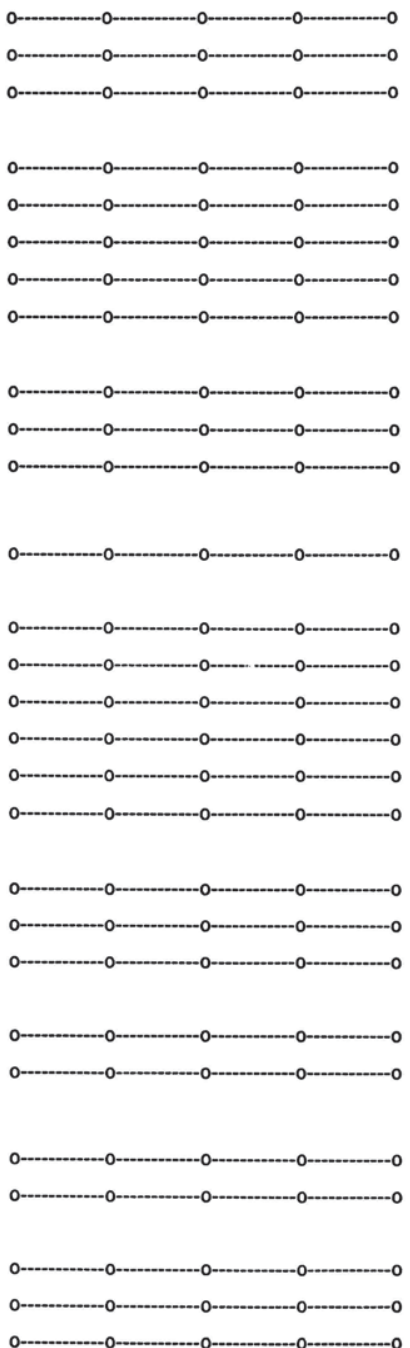
20. Nutzen Sie im Rahmen der Vor-Messe-, der Messe-, oder der Nach-Messe-Phase die unterstützenden Dienstleistungsangebote der Messeveranstalter für Aussteller?

$\begin{array}{lccccc}\text { unbe- } & \text { nie selten } & \begin{array}{l}\text { manch- } \\ \text { mal } \\ \text { kannt }\end{array} & 0 & \text { hăufig } & \text { immer } \\ \text { Vor-Messe-Phase } & 0 & 0 & 0 & 0 & 0 \\ \text { Messe-Phase } & 0 & 0 & 0 & 0\end{array}$

21. Wie werden die Ergebnisse der Nutzenmessung von Messebeteiligungen in Ihrem Unternehmen/Geschäftsbereich aufbereitet? Bitte geben Sie an, welche der folgenden Aussagen zutreffen.

Die Aussage:

trifft gar
nicht zu

trifft

Die Ergebnisse der Nutzenmessung

der Messebeteiligung werden

- der Unternehmensleitung prăsentiert

- den am Messestand beteiligten Mitarbeitern präsentiert

- den betroffenen Abteilungen (z.B. Verkauf, Außendienst) auf Anfrage zur Verfügung gestellt

- zu einem internen Messebericht verdichtet, der allen interessierten Abteilungen zur Verfügung gestellt wird

- im Unternehmen i.d.R. nicht weiter diskutiert

sonstiges:
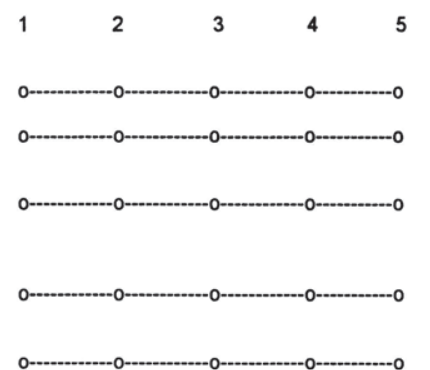

0

22. Welche konkreten Auswirkungen haben die Ergebnisse der Nutzenmessung von Messebeteiligungen in Ihrem Unternehmen/Geschäftsbereich? Bitte geben Sie an, welche der folgenden Aussagen zutreffen.

Die Aussage:

trifft gar

Die Ergebnisse der Nutzenmessung

der Messebeteiligung werden

$\begin{array}{lllll}1 & 2 & 3 & 4 & 5\end{array}$

- zur Beurteilung des weiteren Engagements bzgl. der bewerteten Messeveranstaltung herangezogen

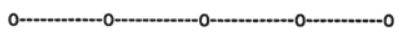

- zur Überprüfung des gesamten Messe-Engagements des Unternehmens genutzt

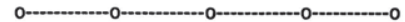

- zur Diskussion mit den betroffenen Messegesellschaften genutzt

- zur Beurteilung der Standmitarbeiter herangezogen

- zur Überarbeitung der Standkonzeption bzgl. weiterer Messeeinsătze genutzt

- selten als Basis zukünftiger Messe-Entscheidungen eingesetzt

sonstiges:

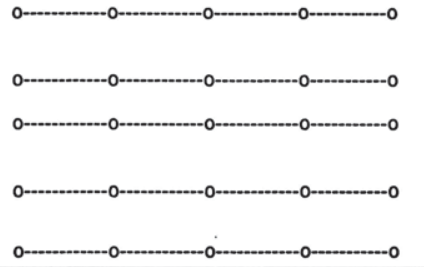


23. Abschließend wollen wir Ihnen noch einige Fragen stellen, die sich auf Ihr Unternehmen $/$ Ihren Geschäftsbereich beziehen. Sie sind für die Auswertung des Fragebogens von wesentlicher Bedeutung.

a) In welcher Anbieterbranche ist Ihr Unternehmen/Geschäftsbereich tätig?

- Land- und Forstwirtschaft, Fischerei

- Energiewirtschaft, Wasserversorgung, Bergbau

- Chemische Industrie, Mineralölverarbeitung

- Kunststoff-, Gummi- und Asbestverarbeitung

- Gewinnung und Verarbeitung von Steinen und Erden

- Feinkeramik, Glasgewerbe

- Metallerzeugung und Verarbeitung

- Stahl-, Maschinen- und Fahrzeugbau

- Holz-, Papier- und Druckgewerbe

- Leder, Textil- und Bekleidungsgewerbe

- Elektrotechnik, Feinmechanik

- Nahrungs- und Genußmittelgewerbe

- Baugewerbe

- Groß- und Einzelhandel

o Verkehr, Nachrichtenübermittlung

- Kreditinstitute, Versicherungsgewerbe

- Dienstleistungen, Freie Berufe

o sonstiges:

b) Wie hoch war 1994 der Umsatz Ihres Unternehmens / Geschäftsbereiches?

$\begin{array}{cccccc}\text { unter } & 10 \text { Mio. bis } & 50 \text { Mio. bis } & 100 \text { Mio. bis } & 250 \text { Mio. bis } & \begin{array}{c}\text { über } \\ \text { 10 Mio. DM }\end{array} \\ & 50 \text { Mio. DM } & 100 \text { Mio. DM } & 250 \text { Mio. DM } & 500 \text { Mio. DM } & 500 \text { Mio. DM } \\ 0 & & \end{array}$

c) Wie hoch war 1994 die Anzahl der Beschäftigten in Ihrem Unternehmen/Geschäftsbereich?

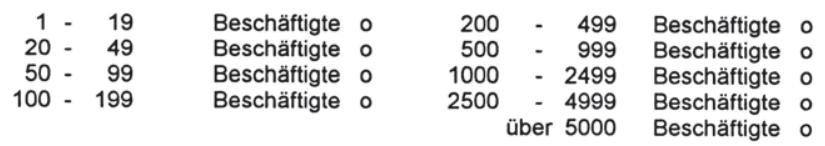

d) Welche Funktion bekleiden Sie im Unternehmen/Geschäftsbereich?

\begin{tabular}{ll} 
InhaberNorstand/Geschäftsführer & 0 \\
Leiter Vertrieb/Marketing & 0 \\
Mitarbeiter Vertrieb/Marketing & 0 \\
Leiter Werbung & 0 \\
Mitarbeiter Werbung & 0 \\
sonstige Funktion & \\
\hline
\end{tabular}

\section{Vielen Dank für Ihre freundliche Unterstützung!}

Ifd. $\mathrm{Nr}$ : 


\section{Literaturverzeichnis}

Aaker, D. A., Shansby, J. G.

Achrol, R. S., Reve, T.,

Stern, L. W.

Adam, D.,

Adamowsky, S.

Amon, P.,

Andritzky, K.,

Antonoff, R.

Aries, L. A.

Arnold, U.

Ausstellungs- und MesseAusschuss der Deutschen Wirtschaft e. V. (AUMA) (Hrsg.)

Ausstellungs- und MesseAusschuss der Deutschen Wirtschaft e. V. (AUMA) (Hrsg.)

Ausstellungs- und MesseAusschuss der Deutschen Wirtschaft e. V.

(AUMA) (Hrsg.)

Ausstellungs- und MesseAusschuss der Deutschen Wirtschaft e. V. (AUMA) (Hrsg.)
Positioning Your Product, in: Business Horizons, Vol. 25, No. 3, 1982, S. 56-62.

The Environment of Marketing Channel Dyads: A Framework for Comparative Analysis, in: JoM, Vol. 47, No. 3, 1983, S. 57.

Planung und Entscheidung: Modelle - Ziele Methoden; mit Fallstudien und Lösungen, 4. Aufl., Wiesbaden 1996.

Prüfung der Organisation, in: Grochla, E. (Hrsg.), HWO - Handwörterbuch der Organisation, Stuttgart 1973, Sp. 1371-1377.

Messen + Ausstellungen: Messe-Ziele exakt formulieren und kontrollieren, in: Marketing Journal, Heft 1, 1991, S. 56 - 60.

Die Operationalisierbarkeit von Theorien zum Konsumentenverhalten, Berlin 1977.

Cl-Report '92: Das Jahrbuch vorbildlicher Cl, Darmstadt 1992.

Bereiten Sie inr Standpersonal intensiv auf das Messe-Verkaufsgespräch vor, in: Marketing Journal, Heft 4, 1986, S. 365-368.

Zur Informationsüberlastung von Konsumenten, in: JAVF, Heft 4, 1989, S. 387-401.

Die Messewirtschaft 1995/96, Bergisch Gladbach 1996.

Erfolg auf Auslandsmessen, Bergisch Gladbach 1994.

AUMA-Handbuch Messeplatz Deutschland '97, Bergisch Gladbach 1996.

AUMA-Handbuch Regionale Ausstellungen '97, Bergisch Gladbach 1996. 
Ausstellungs- und MesseAusschuss der Deutschen Wirtschaft e. V. (AUMA) (Hrsg.)

Ausstellungs- und MesseAusschuss der Deutschen Wirtschaft e. V. (AUMA) (Hrsg.)

Ausstellungs- und MesseAusschuss der Deutschen Wirtschaft e. V. (AUMA) (Hrsg.)

Ausstellungs- und MesseAusschuss der Deutschen Wirtschaft e. V. (AUMA), (Hrsg.)

Bachmeir, H. M.

Backhaus, $\mathrm{K}$. et al.

Backhaus, $\mathrm{K}$.

Backhaus, $\mathrm{K}$. Backhaus, $\mathrm{K}$.

Bagozzi, R. P.

Balderjahn, I.

Bamberg, G., Coenenberg, A. G.

Bauer, $F$.

Becker, J.
Fachmessen made in Germany '97, Bergisch Gladbach 1996.

Die Messekosten der deutschen Aussteller, Bergisch Gladbach 1994.

Erfolgreiche Messebeteiligung Made in Germany, Bonn 1995.

Handbuch Messeplatz Deutschland `96, Köln 1995.

Marktforschung auf Messen, in: Meyer, P. W. (Hrsg.), Arbeitspapiere zur Schriftenreihe Schwerpunkt Marketing der Universität Augsburg, Bd. 23, Augsburg 1989.

Multivariate Analysemethoden: Eine anwendungsorientierte Einführung, 8. Aufl., Berlin u. a. 1996.

Der entscheidungs- und verhaltensorientierte Ansatz in der Investitionsgütenwerbung, in: Rost, D., Strothmann, K.-H. (Hrsg.), Handbuch Werbung für Investitionsgüter, Wiesbaden 1983, S. 49-72.

Investitionsgütermarketing, 4. Aufl., München 1995.

Messen als Institutionen der Informationspolitik, in: Strothmann, K.-H., Busche, M. (Hrsg.), Handbuch Messemarketing, Wiesbaden 1992, S. 83-97.

Causal Modeling: A General Method for Developing and Testing Theories in Consumer Research, Monroe 1981.

Das umweltbewußte Konsumentenverhalten. Eine empirische Studie, Berlin 1986.

Betriebswirtschaftliche Entscheidungslehre, 3. Aufl., München 1991.

Datenanalyse mit SPSS, Berlin u. a. 1984.

Konzeptionelle Grundfragen der Positionierung, in: Tomczak, T., Rudolph, Th., Roosdorp, A. (Hrsg.), Positionierung: Kernentscheidung des Marketing, St. Gallen 1996, S. 13-23. 
Becker, J.

Becker, J.

Bednarczuk, $\mathrm{P}$.

Behrens, G.

Behrens, K. C.

Belz, Chr.

Benad, G.

Berekoven, L.,

Eckert, W.,

Ellenrieder, $P$.

Berger, $M$.

Berghäuser, B.

Berghäuser, B.

Berghäuser, B.

Bergs, S.

Birkigt, K.,

Stadler, M.

Bleymüller, J.
Marketing-Konzeption - Grundlagen des strategischen Marketing-Managements, 4. Aufl., München 1992.

Marketing-Konzeption - Grundlagen des strategischen Marketing-Managements, 5. Aufl., München 1993.

Strategische Kommunikationspolitik für Markenartikel in der Konsumgüterindustrie - Gestaltung und organisatorische Umsetzung, Offenbach 1990.

Das Wahrnehmungsverhalten des Konsumenten, Frankfurt 1982.

Absatzwerbung, Wiesbaden 1963.

Unternehmenspositionen mit integrierter Kommunikation besetzen, in: Tomczak, T., Rudolph, Th., Roosdorp, A. (Hrsg.), Positionierung: Kernentscheidung des Marketing, St. Gallen 1996, S. 122-131.

Messestandanalyse - Ergebnisse von Befragungen auf 40 Messeständen bei 4800 Personen, Düsseldorf 1984.

Marktforschung: methodische Grundlagen und praktische Anwendung. 6. Aufl., Wiesbaden 1993.

Energieverschwendung in der Kommunikation, in: Marketing Joumal, Heft 6, 1988, S. 638-639.

Messen als Entscheidungsproblem, in: Messen als Marketing-Instrument, Deutsche Werbewissenschaftliche Gesellschaft e.V. (Hrsg.), Bonn 1983, S. 5-33.

Messen und Ausstellungen: Entscheidungshilfen für die "richtige" Beteiligung, in: Marketing Journal, Heft 2, 1983, S. 166-168.

So lassen sich Messen messen, in: Marketing Journal, Nr. 9, 1976, S. 61-66.

Optimalität bei Clusteranalysen: Experimente zur Bewertung numerischer Klassifikationsverfahren, Münster 1980.

Corporate Identity - Grundlagen, in: Birkigt, K., Stadler, M., Funck, H.J., (Hrsg.), Corporate Identity. Grundlagen, Funktionen, Fallbeispiele, 5. Aufl., Landsberg/Lech 1992, S. 11-59.

Multivariate Analyse für Wirtschafts-wissenschaftler, Manuskript, Münster 1989. 
Blohm, $H$.

Böcker, K.

Böhler, $\mathrm{H}$.

Bolz, N.

Bosch, K.

Brose, P.

Brosius, G.

Bruhn, M.

Bruhn, M.

Bruhn, M., Zimmermann, A.

Bühner, R.

Buzzell, R. D.

Cavanaugh, S.

Ceyp, M.

Chandler, A. D.

Chapman, E. A.
Messen und Ausstellungen als Mittel der Absatzförderung in der Schweizerischen Maschinenindustrie, Freiburg, Winterthur 1960.

Marketing-Kontrolle, München 1987.

Marktforschung, Stuttgart u. a. 1985.

Individualität als Medieneffekt, in: planung \& analyse, Heft 1, 1996, S. 9-11.

Statistik-Taschenbuch, München, Wien 1992.

Konzeption, Varianten und Perspektiven der Kontingenztheorie, in: Journal für Betriebswirtschaft, 34. Jg, Heft 5, 1984, S. 230-241.

SPSS/PC+ Advanced Statistics and Tables Einführung und praktische Beispiele, Hamburg u. a. 1989.

Integrierte Unternehmenskommunikation, 2. Aufl., Stuttgart 1995.

Neuere Entwicklungen in der Integrierten Kommunikation, in: Thexis, Heft 3, 1996, S. 12 -16.

Integrierte Kommunikationsarbeit in deutschen Unternehmen - Ergebnisse einer Unternehmensbefragung, in: Bruhn, M. Dahlhoff, D. (Hrsg.), Effizientes Kommunikationsmanagement, Konzepte, Beispiele und Erfahrungen aus der integrierten Unternehmenskommunikation, Stuttgart 1993.

Strategie und Organisation, Wiesbaden 1985.

Is Vertical Integration Profitable?, in: HBR, 61. Jg., January-February 1983, S. 92-102.

Setting Objectives and Evaluating the Effectiveness of Trade Show Exhibits, in: JoM, October 1976, S. 100-103.

Ökologieorientierte Profilierung im vertikalen Marketing - dargestellt am Beispiel der Elektrobranche, in: Meffert, H. (Hrsg.), Schriften zu Marketing und Management, Bd. 32, Frankfurt a. M. u. a. 1996.

Strategy and Structure. Chapters in the History of Industrial Enterprise, Cambridge 1962.

Exhibit Marketing: A Survival Guide for Managers, New York u. a. 1987. 
Child, J.

Christman, C.

Cunningham, M. T., White, J. G.

Dallmer, $\mathrm{H}$.

Demuth, A.

Detroy, E. N.

Deutsche Messe AG (Hrsg.)

Diehl, J. M.

Diller, $\mathrm{H}$.

Dinkelbach, $\mathrm{W}$.

Dostal, G.

Doyle, P.,

Gidengil, B. Z.

Dudley, J.W.

Ebert, $\mathrm{D}$.

Ehrmann, $\mathrm{H}$.

Esch, F.-R., Levermann, S.
Organizational Structure, Environment and Performance: The Role of Strategic Choice, in: Sociology, Vol. 6, No. 1, 1972, S. 3-10.

The Complete Handbook of Profitable Trade Show Exhibiting, Englewood Cliffs 1991.

The Role of Exhibitions in Industrial Marketing - An Evaluation of the International Machine Tool Exhibition, in: Industrial Marketing Management, No. 3, 1974, S. 238-251.

System des Direct-Marketing - Entwicklung und Zukunftsperspektiven, in: Dallmer, H. (Hrsg.), Handbuch Direct-Marketing, 6. Aufl., Wiesbaden 1991, S. 3-16.

Corporate Communications, in: Bruhn, M. (Hrsg.), Handbuch des Marketing, München 1989.

So organisieren Sie eine verkaufsaktive Messe, in: asw, 15. Jg., Nr. 21/22, 1982, S. 120-125.

Das transparente Medium - Aussteller- und Besucher-Analysen Hannover Messe '94, Hannover 1994.

Varianzanalyse, Frankfurt a. M. 1977.

Beziehungsmanagement und Marketing, in: Backhaus, K., Diller, H. (Hrsg.), Dokumentation des 1. Workshops der Arbeitsgruppe "Beziehungsmanagement" der wissenschaftlichen Kommission für Marketing im Verband der Hochschullehrer für Betriebswirtschaftslehre, Münster, Nürnberg 1994, S. 1-7.

Ziele, Zielvariablen und Zielfunktionen, in: DBW, Heft 1, 1978, S. 51-58.

Messen und Ausstellungen: So decken Sie die Stärken und Schwächen Ihrer Messe-Beteiligung auf, in: Marketing Journal, Heft 2, 1987, S. 140-144.

A Review of In-Store Experiments, in: JoR, No. 2, 1977 , S. 47-62.

Successful Exhibiting, Holbrook 1991.

Weltweite Entwicklungstendenzen im Messewesen, in: Strothmann, K.-H., Busche, M. (Hrsg.), Hand buch Messemarketing, Wiesbaden 1992.

Marketing-Controlling, 2. Aufl., Ludwigs-hafen 1995.

Positionierung als Grundlage des strategischen Kundenmanagements, in: Thexis, Heft 3, 1995, S. 8-12. 
Exner, W. F.

Fahrmeir, L., Häußler, W., Tutz, G.

Feige, St.

Fischer, A.

Fischer, M.

Fischer, W.

FKM-Gesellschaft zur

Freiwilligen Kontrolle von Messe- und Ausstellungszahlen e. V. (Hrsg.)

Fließ, S.

Földy, R.

Frese, E.

Fritsch, S.

Fritz, W.

Fröhder, St.

Fronhoff, B.

Fuchs, W. A.

Funke, K.,
Der Aussteller und die Ausstellungen - Erörterungen vor allem über die Messe von Wien 1873, Weimar 1873.

Diskriminanzanalyse, in: Fahrmeir, L., Hamerle, A. (Hrsg.), Multivariate statistische Verfahren, Berlin, New York 1984, S. 301-327.

Handelsorientierte Markenführung - Strategien zur Profilierung von Konsumgüterherstellern beim Handel, Frankfurt a. M. u. a. 1996.

Messen als Chance - Erfahrungen einer Unternehmung, in: Thexis, Heft 2, 1989, S. 21-23.

Make-or-Buy-Entscheidungen im Marketing, Wiesbaden 1993.

Zur Geschichte der Messen in Europa, in: Strothmann, K.-H., Busche, M. (Hrsg.), Handbuch Messemarketing, Wiesbaden 1992, S. 3-14.

Bericht 1990, Bergisch Gladbach 1991.

Messeselektion, Entscheidungskriterien für Investitionsgüteranbieter, Wiesbaden 1994.

Die Messe als Markt. Beteiligungsstrategien für Aussteller, Wien 1990.

Grundlagen der Organisation: Konzept, Prinzipien, Strukturen, 5. Aufl., Wiesbaden 1993.

Prognosemodell für Konsumgütermessen, Berlin 1979.

Marketing-Management und Unternehmenserfolg: Grundlagen und Ergebnisse einer empirischen Untersuchung, 2. Aufl., Stuttgart 1995.

Was passiert wirklich auf der Messe? - zweifeln Sie mal wieder, in: Marketing Journal, Heft 1, 1995, S. 47-48.

Die Gestaltung von Marketingstrategien, Köln 1986.

Die Messe als Kommunikationsprozeß, in: Werbeforschung \& Praxis, Heft 3, 1995, S. 99-101.

Messeentscheidungen, Handlungsalternativen und Informationsbedarf, Frankfurt a.M., Bern, New York 1986, S. 354 ff. 
Funke, $\mathrm{K}$.

Gelszus Messe-Marktforschung $\mathrm{GmbH}$ (Hrsg.)

Gelszus Messe-Marktforschung GmbH (Hrsg.)

Gelszus, R. H.

Gesamtverband Werbeagenturen (GWA) (Hrsg.)

Geschka, H., Eggert-Kipfstuhl, K.

Gesellschaft zur freiwilligen Kontrolle von Messe- und Ausstellungszahlen (FKM) (Hrsg.)

Gesellschaft zur freiwilligen Kontrolle von Messe- und Ausstellungszahlen (FKM) (Hrsg.)

Gluth, $\mathrm{H}$.

Goehrmann, K.

Gopalakrishna S., Williams J.

Gopalakrishna, S., Lilien, J. D., Sequeira, I. K.

Goschmann, K.
Ausstelleraktivitäten auf Messen - Eine kritische Analyse einschlägiger Darstellungen und Systematisierungsansätze in der Literatur, in: Arbeitsbericht Nr. 03/86 des Instituts für Wirtschaftswissenschaften der RWTH Aachen, Aachen 1986.

Mehr Erfolg auf Messen durch Messewirkungskontrolle, Dortmund 1994.

Mehr Erfolg auf Messen - Wie Sie Ihren Erfolg messen und neue Potentiale entdecken, o.O., o. J.

Messen und Ausstellungen: Vergessen Sie die Messe-Wirkungskontrolle nicht, in: Marketing Journal, Heft 2, 1985, S. 172-177.

Werbung und Markterfolg. Eine empirische Untersuchung auf der Grundlage von Expertenschätzungen im Markenartikelbereich, Frankfurt a. M. 1992.

Innovationsbedarfserfassung, in: Tomczak, T., Reinecke, S. (Hrsg.), Marktforschung, St. Gallen 1994, S. 127-129.

Bericht 1995, Bergisch Gladbach 1996.

Bericht 1995, Köln 1996.

Gewerbeordnung (GewO) in der Fassung vom 01.01.1987, Titel IV §§ 64, 65.

Keine schwarzen Messen - Erfolgskontrolle mit den Methoden der empirischen Sozialforschung, in: Verlagsbeilage zur FAZ vom 23.01.1996, S. B7.

Vortrag anläßlich des Besuchs von Mitgliedern der Wissenschaftlichen Gesellschaft für Marketing und Unternehmensführung e. V. auf der CEBIT HOME, Hannover, am 29.08.1996.

Identifying Important Factors in Lead Efficiency at Trade Shows, in: Trade Show Bureau (Ed.), Report No. SM 23, Denver 1993.

Do Trade Shows Pay Off?, in: JoM, Vol. 59. July 1995, S. 75-83.

Die erfolgreiche Beteiligung an Messen und Ausstellungen von A-Z, Landsberg/Lech 1988. 
Gräbener, W.

Griffith, R. W.

Groth, C.

Groth, C.

Groth, C.

Groth, C., Lentz, I.

Grotz-Martin, S.

Gutenberg, E.

Gutenberg, E.

Haeberle K. E.

Haedrich, G.

Gussek, F.

Tomczak, T.

Haedrich, G., Tomczak, $\mathrm{T}$.

Hagen, R.

Hamel, G.,

Prahalad, C. K.

Hammann, P.

Erichson, B.
Die Messepolitik als Marketinginstrument dargestellt am Beispiel von Investitionsgüterproduzenten, Göttingen 1981.

Management, 2. Aufl., Boston 1987.

Das Leistungspotential von Fachmessen, in: DWG Deutsche Werbewissenschaftliche Gesellschaft $e$. V. (Hrsg.), Messen als Marketing-Instrument, Bonn 1983, S. 57-71.

Determinanten der Veranstaltungspolitik von Messegesellschaften, in: Strothmann, K.-H., Busche, M. (Hrsg.), Handbuch Messemarketing, Wiesbaden 1992, S. 157-178.

Die Wegeanalyse - Ein Instrument zur Verhaltensforschung bei Messeveranstaltungen und ähnlichen speziellen Märkten, in: GfK Mitteilungen zur Markt- und Absatzforschung, Heft 3, 1986, S. 87-90.

Die Messe als Dreh- und Angelpunkt: Multifunktionales Instrument für erfolgreiches Marketing, Landsberg/Lech 1993.

Informations-Qualität und Informationsakzeptanz in Entscheidungsprozessen, Saarbrücken 1976.

Die Unternehmung als Gegenstand betriebswirtschaftlicher Theoriebildung, Frankfurt a. M. 1967.

Grundlagen der Betriebswirtschaftslehre, Bd 1: Die Produktion, 24. Aufl., Berlin u. a. 1983.

Erfolg auf Messen und Ausstellungen: Handbuch für Teilnahme, Organisation, Gestaltung, Technik, Stuttgart 1967.

Differenzierte Marktbearbeitung und Markterfolg im Reiseveranstaltermarkt der Bundesrepublik Deutschland, in Marketing ZFP, Nr. 1, 1989, S. 1118.

Strategische Markenführung, Bern, Stuttgart 1990.

Erfolgskontrolle auf Verbrauchermessen - dargestellt am Beispiel der Analyse des Informationsstandes des Deutschen Bundestages, in: planung und analyse, Heft 7, 1983, S. 292-296.

So spürten Unternehmen neue Märkte auf, in: Harvard Manager, Heft 2, 1992, S. 44-51.

Marktforschung, Stuttgart, New York 1978. 
Haseloff, O.W.

Hassmann, V.

Hauschildt, J.

Head, J.

Heil, E. B.

Heinen, E.

Heinen, E.

Henkel, H. O.

Hentschel, M.

Hildebrandt, L.

Hildenbrand, W.

Hilker, J.

Hoche, K.

Hofer, C. W.

Hoffmann, J.
Die Reduktion der Komplexität: Messen heute Kommunikation, Information, Entscheider, Köln 1980.

Fachmessen: Mehr Zeit für Ihre Kunden, in: Sales Profi, Heft 1, 1996, S. 18-19.

Zielsysteme, in: Grochla, E. (Hrsg.), Handbuch der Organisation, 2. Aufl., Stuttgart 1984, S. 2419-2430.

Positioning, Objectives and Selection Factors for Trade Shows, in: Trade Show Bureau (Ed.), Report No. MC24, Denver 1992.

Entwicklung und Ausgestaltung des Messe- und Ausstellungswesens in Deutschland nach dem zweiten Weltkrieg, Nürnberg 1966.

Einführung in die Betriebswirtschaftslehre, 9. Aufl., Wiesbaden 1985.

Grundlagen betriebswirtschaftlicher Entscheidungen - Das Zielsystem der Unternehmung, 3. Aufl., Wiesbaden 1976.

Neue Funktionen der Messen, in: Der Handel, Heft 11, 1995.

So können auch mittelständische Unternehmen auf Auslands-Messen gehen, in: Marketing Journal, 20. Jg., Heft 3, 1987, S. 248-253.

Kausalanalytische Validierung in der Marketingforschung, in: Marketing ZFP, 6. Jg., 1984, S. 45-49.

Informationsmarketing in der Kommunikation zwischen Hersteller und Handelsvertreter, Frankfurt a. M. 1983.

Marketingimplementierung: Grundlagen und Umsetzung am Beispiel ostdeutscher Unternehmen, Wiesbaden 1993.

Handbuch für Aussteller, 111 Messetips, München 1974.

Schendel, D., Strategy Formulation: Analytical Concepts, St. Paul u.a. 1978.

Mobile Messe - Kreuz und quer, in: Wirtschaftswoche, Nr. 1/2, vom 4.1.1996, S. 72-74. 
Horn, H.-R.

Hossinger, H.-P.

Huckemann,M., ter Weiler, D. S.

Hugger, P. F.

Jaspersen, $H$.

Jaspersen, $H$.

Jaspert, F.

Jaspert, F.

Jöreskog, K. G. Sörbom, D.

Josef, F.

Jurkovich, R.

Kast, F.

Rosenzweig, J.

Khandwalla, $P$.

Kieser, A.

Kubicek, $\mathrm{H}$.
Category Management aus Sicht der Unternehmenspraxis (Zusammenfassung eines Vortrages), in: Meffert, H., Wagner, H., Backhaus, K. (Hrsg.), Category Management - neue Herausforderung im vertikalen Marketing? - Dokumentationspapier der Wissenschaftlichen Gesellschaft für Marketing und Unternehmensführung e. V. Nr. 97, Münster 1995.

Pretests in der Marktforschung - Die Validität von Pretestverfahren unter besonderer Berücksichtigung der Tachistoskopie, Würzburg, Wien 1982.

Messen meßbar machen. Die 5 trojanischen Pferde des Messe-Marketing, Neuwied, Kriftel, Berlin 1995.

Nutzen-Kosten-Analyse der regionalwirtschaftlichen Auswirkungen von Messen und Ausstellungen, München 1986.

Mehr Messe-Transparenz: Hüls, in: Marketing Journal, Heft 2, 1977, S. 169-173.

Messen beschicken oder Anzeigen schalten? Eine Antwort mit Zahlen, in: Marketing Journal, Heft 5, 1978, S. 456-462.

Das synergetische Potential ist groß - die Messe als Teil der betrieblichen Absatzpolitik, in: Küffner, G., Mortsiefer, J. (Hrsg.), Messeplatz Europa, Messe als Bestandteil des betrieblichen Marketings, Frankfurt a. M. 1990, S. 36-50.

Marketinginstrument Messe: Das synergetische Potential ist groß, in: BddW, Nr. 52, vom 14.03.1989, S. 7.

LISREL 7, A Guide to the Programme and Applications, 2. Aufl., Chicago 1989.

Effizienzsteigerung durch Messekontrolle: Wie man aus Fehlern lernen kann, in: MesseMarkt, o. Nr., Februar 1986, S. 56-57.

A Core Typology of Organizational Environments, in: Administrative Science Quarterly, Vol. 19, o. Nr., 1974 , S. 380-385.

Organization and Management: A Contingency Approach, Tokio 1970.

The Design of Organizations, New York u. a. 1977.

Organisation, 3. Aufl., Berlin, New York 1992. 
Kijewski, V.

Yoon, E.

Young, G.

Kirchgeorg, M.

Kirsch, W.

Knoblich, $\mathrm{H}$.

Koch, K.-W.

Kohle, G. U.

Köhler, R.

Korndörfer, W.

Kotler, Ph., Bliemel, F.

Kotler, Ph., Bliemel, F.

Kotler, Ph., Gregor, W. Rodgers, W.

Kroeber-Riel, W.

Kroeber-Riel, W.

Kroeber-Riel, W.

Kroeber-Riel, W.

Kroeber-Riel, W.

Kroeber-Riel, W.
How Exhibitors Select Trade Shows, in: IMM, Vol. 22, 1993, S. 296-299.

Ökologieorientiertes Unternehmensverhalten: Typologien und Erklärungsansätze auf empirischer Grundlage, Wiesbaden 1990.

Entscheidungsprozesse, Entscheidungen in Organisationen, Bd. 3, Wiesbaden 1971.

Die typologische Methode in der Betriebswirtschaftslehre, in WiSt, Heft 4, 1972, S. 141-147.

Messemarkt kommt ins Rollen, in: Der Handel, Heft 11, 1995, S. 26.

Von der "Messe-Einladung“ zur Einladung mit Konzept, in: Marketing Journal, Heft 5, 1991, S. 460-466.

Kommunikations-Management im Unternehmen, in: Berndt, R., Hermanns, A. (Hrsg.), Handbuch Marketing-Kommunikation, Wiesbaden 1993, S. 93-112.

Unternehmensführungslehre, 5. Aufl., Wiesbaden 1986.

Marketing-Management, 6. Aufl., Stuttgart 1994.

Marketing-Management, 8. Aufl., Stuttgart 1995.

The Marketing Audit comes of Age, in: SMR, Vol. 19, Winter 1977, S. 25-45.

Bildkommunikation - Imagerystrategien für die Werbung, München 1993.

Erlebnisbetontes Marketing, in: Belz, C. (Hrsg.), Realisierung des Marketing, Savosa, St. Gallen 1986, S. 1137-1151.

Informationsüberlastung durch Massenmedien in Deutschland, in: DBW, Heft 3, 1987, S. 257-264.

Integrierte Kommunikation, in: BddW, vom 03.06.1991, S. 7.

Kommunikation im Zeitalter der Informationsüberlastung, in: Marketing ZFP, 10. Jg., Nr. 3, 1988 , S. 182-189.

Strategie und Technik der Werbung. Verhaltenswissenschaftliche Ansätze, 4. Aufl., Stuttgart u.a. 1993. 
Krone, K., Huber, B.,

Krueger, J.

Kubicek, $\mathrm{H}$.

Kühn, R.

Kühn, R.

Kühn, R.

Küng, $P$.

Kupsch, P.

Kustenaar, J.

Laakmann, K.

Lehnert, St.

Leicher, R.

Liebl, F.

Lingenfelder, M., Schneider, W.

Linssen, $\mathrm{H}$.
Messen als Instrument der Unternehmenspolitik, in: Strothmann, K.-H., Busche, M. (Hrsg.), Handbuch Messemarketing, Wiesbaden 1992, S. 143-153.

Messen und Ausstellungen: Die Kontakt-Kosten sichtbar machen, in: Marketing Journal, Heft 5, 1984 , S. 484-489.

Heuristische Bezugsrahmen und heuristisch angelegte Forschungsdesigns als Elemente einer Konstruktionsstrategie empirischer Forschung, in: Köhler, R. (Hrsg.), Empirische und handlungstheoretische Forschungskonzeptionen in der Betriebswirtschaftslehre, Stuttgart 1977, S. 3-36.

Angebotspositionierung als Ansatz zur Präzisierung von Wettbewerbsstrategien, in: Tomczak, $T$., Rudolph, Th., Roosdorp, A. (Hrsg.), Positionierung: Kernentscheidung des Marketing, St. Gallen 1996, S. 112-121.

Marketing - Analyse und Strategie, Zürich 1995.

Marketing-Mix, in: Poth, L. G. (Hrsg.), Marketing, Band 3, Neuwied 1979, S. 40-62.

Der Einsatz des Verkaufs an Messen, in: VM international, Heft 3, 1990, S. 45-49.

Unternehmensziele, Stuttgart, New York 1979.

Messehandbuch - ein Leitfaden für Messe-beteiligungen, Bern, Stuttgart 1983.

Value-Added Services als Profilierungsinstrument im Wettbewerb: Analyse, Generierung und Bewertung, Frankfurt a. M. u. a. 1995.

Die Bedeutung von Kontingenzansätzen für das strategische Management, Frankfurt a.M., Bern, New York 1983.

Die Manöverkritik. Unerläßlicher Bestandteil der Messenacharbeit, in: Ausstellungs- und MesseAusschuss der Deutschen Wirtschaft e. V. (Hrsg.), Literatursammlung des AUMA, Köln o. J., S. 79.

Strategische Frühaufklärung: Trends - Issues Stakeholders, München, Wien, Oldenbourg 1996.

Die Kundenzufriedenheit - Bedeutung, Meßkonzept und empirische Befunde, in: Arbeitspapier des Instituts für Marketing der Universität Mannheim, Nr. 80, Mannheim 1990.

Interdependenzen im absatzpolitischen Instrumentarium der Unternehmung. Ein Beitrag zur optimalen Kombination der Absatzmittel, Berlin 1975. 
Lohmaier, P.

Lückmann, R.

Maurer, E.

Mauthe, K. D.

McCombs, M. E., Shaw, D.L.

McDonald, M.H.B.

McQuail, R., Blumler, J. G., Brown, J. R.

Meffert, $\mathrm{H}$.

Meffert, $\mathrm{H}$.

Meffert, $\mathrm{H}$.

Meffert, $\mathrm{H}$.

Meffert, $\mathrm{H}$.

Meffert, $\mathrm{H}$.

Meffert, $\mathrm{H}$.

Meffert, $\mathrm{H}$.

Meffert, $\mathrm{H}$.
Die Messe - Bedeutung und Problematik aus der Sicht der Aussteller, in: Arbeitspapier des Instituts für Absatzwirtschaft der Universität Augsburg $\mathrm{Nr}$. 1/85, Augsburg 1985.

Messewirtschaft 1995: Schub durch Flaggschiffe, in: HB, Nr. 11, vom 16.01.1996, Sonderteil Internationale Messen, S. B1.

Missa profana. Geschichte und Morphologie der Messen und Fachausstellungen, Stuttgart 1973.

Strategische Analyse, München 1984.

The Agenda Setting Function of the Media, in: Public Opinion Quaterly, No. 36, 1972, S. 176-187.

The Marketing Audit - Translating Marketing Theory into Practice, Oxford, London u.a. 1993.

The Television Audience: A Revised Perspective, in: McQuail, R. (Ed.), Sociology of Mass Communication, Harmondsworth 1972, S. 135-165.

Die Leistungsfähigkeit der entscheidungs- und systemorientierten Marketinglehre, in: von Kortzfleisch, G. (Hrsg.), Wissenschaftsprogramm und Ausbildungsziele der Betriebswirtschaftslehre, Berlin 1971, S. 167-187.

Unternehmensziele, in: Schöttle, K. M. (Hrsg.), Jahrbuch des Marketing, Essen, 1971, S. 22-34.

Marketing, in: Management-Enzyklopädie, Bd. 4, München 1971, S. 392.

Das System des Kommunikations-Mix, Münster 1979.

Integrierte Marktkommunikation, Münster o.J.

Zur Bedeutung von Konkurrenzstrategien im Marketing, in: Marketing ZFP, 7. Jg., Heft 1, 1985, S. 13-19.

Marketing. Grundlagen der Absatzpolitik, 7. Aufl.,Wiesbaden 1986.

Messen und Ausstellungen als Marketinginstrument, in: Verlag Wirtschaft und Finanzen (Hrsg.), Messen als Marketinginstrument, Düsseldorf 1988, S. 8-30.

Strategische Unternehmensführung und Marketing: Beiträge zur marktorientierten Unternehmenspolitik, Wiesbaden 1988. 
Meffert, $\mathrm{H}$.

Meffert, $\mathrm{H}$.

Meffert, $\mathrm{H}$.

Meffert, $\mathrm{H}$.

Meffert, $\mathrm{H}$.

Meffert, $H$.

Meffert, $\mathrm{H}$.

Meffert, $\mathrm{H}$.

Meffert, $\mathrm{H}$.

Meffert, $\mathrm{H}$.
Einführungs- und Schlußworte anläßlich des 16 . Münsteraner Führungsgesprächs am 13./14. März 1989, in: Meffert, H., Wagner, H., Backhaus, K. (Hrsg.), Messemarketing - Bestandsaufnahme und Perspektiven, Dokumentationspapier Nr. 52 der Wissenschaftlichen Gesellschaft für Marketing und Unternehmensführung e.V., Münster 1989, S. 1-5 sowie S. 68-70.

Marketingstrategien in unterschiedlichen Marktsituationen, in: Bruhn, M. (Hrsg.), Handbuch des Marketing, München 1989.

Marketingforschung und Käuferverhalten, 2. Aufl., Wiesbaden 1992.

Messen und Ausstellungen als Marketinginstrument, in: Goehrmann, K. E. (Hrsg.), Politmarketing auf Messen, Düsseldorf 1993, S. 60-83.

Marktorientierte Unternehmensführung im Umbruch: Entwicklungsperspektiven des Marketing in Wissenschaft und Praxis, in: Bruhn, M., Meffert, $H_{\text {., }}$ Wehrle, F. (Hrsg.), Marktorientierte Unternehmensführung im Umbruch, Stuttgart 1994, S. 3-39.

Einführung in die Problemstellung, in: Meffert, $H_{\text {., }}$ Wagner, H., Backhaus, K. (Hrsg.), Beziehungsmarketing - neue Wege zur Kundenbindung, Workshop, Dokumentationspapier Nr. 90 der Wissenschaftlichen Gesellschaft für Marketing und Unternehmensführung e.V., Münster 1994, S. 1-5.

Marketing-Management: Analyse - Strategie Implementierung, Wiesbaden 1994.

Marketing: Retrospektive und Perspektive der marktorientierten Unternehmensführung, in: Wissenschaftliche Gesellschaft für Marketing und Unternehmensführung e. V. (Hrsg.), Marketing im Dialog - neue Herausforderungen an die marktorientierte Führung, Münster 1995, S. 1-13.

Messen als Marketinginstrument, in: BayerischSchwäbische Wirtschaft, Heft 11, 1995, S. 25-27

Eröffnungsrede anläßlich des 30 . Münsteraner Führungsgesprächs am 22./23. Februar 1996 in Gütersloh, in: Meffert, H., Wagner, H., Backhaus, K. (Hrsg.), Mediale Welt von morgen - neue Chancen für das Marketing?, Dokumentationspapier Nr. 104 der Wissenschaftlichen Gesellschaft für Marketing und Unternehmensführung e. V., Münster 1996, S. $1-5$. 
Meffert, H., Gass, C.

Meffert, $\mathrm{H}$. Steffenhagen, $\mathrm{H}$.

Meffert, H., Ueding, R.

Mellerowicz, K.

Merbold, C.

Merdian, M.

Meurer, J.

Meyer, A.

Miller, D.,

Friesen, P. H.

Miller, D.,

Friesen, P. H.

Mintzberg, $\mathrm{H}$.

Möller, $\mathrm{H}$.

Mortsiefer, J.
Messen und Ausstellungen im System des Kommunikationsmix, in: Meffert, H. (Hrsg.), Arbeitspapier des Instituts für Marketing der Universität Münster, Nr. 33, Münster 1985.

Marketingprognosemodelle - Quantitative Grundlagen des Marketing, Stuttgart 1977.

Ziele und Nutzen von Messebeteiligungen, in: Ausstellungs- und Messe-Ausschuss der Deutschen Wirtschaft ө. V. (AUMA) (Hrsg.), AUMA Edition Nr. 4, 1996.

Kontrolle in der Absatzwirtschaft, in: Tietz, B. (Hrsg.), HWA - Handwörterbuch der Absatzwirtschaft, Stuttgart 1974, Sp. 1104-1116.

Strukturerhebungen noch die Ausnahme - Transparenz läßt weiter sehr zu wünschen übrig, in: $\mathrm{HB}, \mathrm{Nr}$. 235, vom 07.12.1983, S. 17.

Die Tragfähigkeit des Systemansatzes und der typologischen Definitionsform für die Darstellung von IG-Messen unter Berücksichtigung einer sozialwissenschaftlich verstandenen Kommunikationstheorie, Berlin 1983.

Führung von Franchisesystemen - Erklärungsansätze, Verhaltens- und Erfolgswirkungen auf der Grundlage einer empirischen Führungstypologie, Wiesbaden 1997.

Produktdifferenzierung, in: Marketing ZFP, Heft 2, 1985, S. 99.

Innovation in Conservative and Entrepreneurial Firms: Two Models of Strategic Momentum, in: SMJ, Vol. 3, No. 1, 1982, S. 1-7.

Momentum and Revolution in Organizational Adaption, in: Academy of Management Journal, Vol. 23, No. 4, 1980, S. 591-603.

The Structuring of Organizations. A Synthesis of the Research, Englewood Cliffs 1979.

Das deutsche Messe- und Ausstellungswesen, Berlin 1989.

Die Intuition alleine reicht nicht - Messebeteiligungen auf der Basis eines Nutzungs-Konzeptes, in: Küffner, G., Mortsiefer, J. (Hrsg.), Messeplatz Europa. Messen als Bestandteil des betrieblichen Marketings, Frankfurt a.M. 1990, S. 11-22. 
Mortsiefer, J.

Mortsiefer, J.

Mortsiefer, J.

Mortsiefer, J.

Mühlbacher, H., Dreher, A., Gabriel-Ritter, A.

Müller, K.

Naumann, C.

Naumann, C.

Naumann, C.

Naumann, C.

Neglein, H.-G.

Niemeier, J.
Die Vorbereitung entscheidet über den Erfolg Planung und Vorbereitung einer Messebeteiligung, in: Küffner, G., Mortsiefer, J. (Hrsg.), Messeplatz Europa. Messen als Bestandteil des betrieblichen Marketings, Frankfurt a.M. 1990, S. 51-61.

Informationen sammeln und auswerten - Die Abwicklung einer Messebeteiligung, in: Küffner, G., Mortsiefer, J. (Hrsg.), Messeplatz Europa. Messen als Bestandteil des betrieblichen Marketings, Frankfurt a.M. 1990, S. 62-72.

Messen und Ausstellungen als Mittel der Absatzpolitik mittelständischer Herstellerbetriebe, Göttingen 1986;

Messen und Ausstellungen als Mittel der Absatzpolitik mittelständischer Herstellerbetriebe, Göttingen 1986.

Strategische Positionierung - Grundpfeiler des Marketings in komplexen und dynamischen Umwelten, in: DBW, 56. Jg, Heft 2, 1996, S. 203219.

Die Messepolitik im Marketing der handwerklichen Zulieferer, Göttingen 1985.

Die Arbeit nach der Arbeit - Messe-Nachbereitung und Erfolgskontrolle, in: Küffner, G., Mortsiefer, J. (Hrsg.), Messeplatz Europa, Messe als Bestandteil des betrieblichen Marketings, Frankfurt a. M. 1990, S. 73-86.

Erfolgreich auf der Messe: Handbuch für die Beteiligung an Messen und Ausstellungen, Heidelberg 1993.

Ist es eine Messe wert? - Den Messeerfolg organisieren und kontrollieren, in: BddW, Nr. 170, vom 06.09.1982, S. 7.

Was war die letzte Messe wert? - Kontaktwertmessung nach Punkten, in: $\mathrm{m}+\mathrm{a}-$ report, 55. Jg., Heft 1, 1974, S. 15-17.

Das Messewesen in Deutschland, in: Strothmann, K.-H., Busche, M. (Hrsg.), Handbuch Messemarketing, Wiesbaden 1992, S. 15-28.

Wettbewerbsumwelt und interne Konfiguration: Theoretische Ansätze und empirische Prüfung, Frankfurt a. M., Bern, New York 1986. 
Nieschlag, R.

Dichtl, E., Hörschgen, H.,

Nieschlag, R.

Dichtl, E., Hörschgen, H.,

Norusis, M. J.

Norusis, M. J.

o. V.

o. V.

o. V.

o. V.

o. V.

o. V.

o. V.

o. V.

o.V.

o.V.

o.V.

o.V.

o.V.

o.V.

o.V.
Marketing, 15. Aufl., Berlin 1988.

Marketing, 17. Aufl., Berlin 1994.

SPSS Professional Statistics 6.1., Chicago 1994.

SPSS/PC + Statistics 4.0, Chicago 1990.

Das deutsche Messewachstum kommt heute aus dem Ausland, in: FAZ, Nr. 280, vom 1.12.1995, S. 30.

Impulsgeber für Warenströme, in: ZAW-service, Nr. 185,1995 , S. 18-20.

Klasse statt Masse, in: Focus, Nr. 42, 1995, S. 102.

Messe-Marketing - Face-to-Face ist Trumpf, in: Pro, Heft 9, 1995, S. 26-30.

Messen bleiben wichtiges Instrument, in: Handelsblatt, vom 16.01.1997, S. 24.

Stabile Großwetterlage für klassische Werbung, in: HB, Nr. 207, vom 26.10.1995, S. 12.

Virtueller Rundgang, in: Markt und Mittelstand, Heft 4, 1996, S. 90-91.

Zuwachs im Intemationalen, in: $\mathrm{m}+$ a report, März 1996, S. 23-24.

Bewegung bei den Riesen, in: asw, Heft 8, 1996, S. 102-105.

Erfolgskontrolle - Umstrittene Methode, in: FAMA intern, Heft 2, 1985, S. 12.

Messe mit Effekt, in: asw, Heft 1, 1992, S. 76-77.

Mitarbeiterschulung - Hymne in der Halle: Unternehmen bereiten sich mit ungewöhnlichen Methoden auf den Messeauftritt vor, in: Wirtschaftswoche, Heft 1/2, vom 5.1.1995, S. 70-72.

Services der Messegesellschaften, in: Marketing Journal, Heft 1, 1991, S. 61.

Verschärfter Kampf um die Marktpositionen, in: pro, Heft 7-8, 1995, S. 32-36.

Wirkungskontrollen für Messebeteiligungen, in: Fachverband Messe- und Ausstellungsbau e.V. (FAMAB) (Hrsg.), Jahresbericht `95, S. 21. 

o.V.
o.V.,
o.V.
o.V.

Ob, K. D.

Patt, P.-J.

Peemöller, V.

Peters, M.

Pfohl, H.-C., Braun, G. E.

Pidun, R.

Porter, M. E.

Prinz Wittgenstein, $P$.

Raffée, $H$.

Raffée, $\mathrm{H}$.

Raffeé, $H_{\text {., }}$ Wiedmann, K.-P.

Ramser, H.-J.
Wo liegt die Zukunft der deutschen Messen?, in: Der Handel, Heft 11, 1995, S. 29-30.

Messe Frankfurt sieht inr Wachstumsfeld im Ausland, in: HB, Nr. 233, vom 1./2.12.1995, S. 17.

Messe oder Event, in: asw, Heft 8, 1996, S. 108111.

Messen bleiben wichtiges Instrument, in: HB, vom 16.01.1997, S. 24.

Methodologie der Sozialwissenschaften, Reinbek 1970.

Strategische Erfolgsfaktoren im Einzelhandel: Eine empirische Analyse am Beispiel des Bekleidungsfachhandels, in: Meffert, H. (Hrsg.), Schriften zu Marketing und Management, Bd. 16, Frankfurt a. M., Berlin u. a. 1988.

Organisationsprüfung, in: Management-Enzyklopädie, Bd. 7, München 1975, Sp. 2556-2565.

Mehr Messetransparenz durch neue Ansätze der Messeerfolgskontrolle, in: m+a report, Heft 2, 1980, S. 18-23.

Entscheidungstheorie. Normative und deskriptive Grundlagen des Entscheidens, Landsberg/Lech 1981.

Messen und Ausstellungen: Messe-Promotion einfach faszinierend und glaubwürdig, in: Marketing Journal, Heft 1, 1990, S. 70-71.

Wettbewerbsstrategien, Frankfurt a.M. 1992.

Vorwort, in: Ausstellungs- und Messe-Ausschuss der Deutschen Wirtschaft e. V. (Hrsg.), Erfolgreiche Messebeteiligung Made in Germany, Bonn 1995.

Integrierte Kommunikation, in: Werbeforschung \& Praxis, Heft 3, 1991, S. 87-90.

Messen als Herausforderung für die MarketingTheorie, in: Deutsche Werbewissenschaftliche Gesellschaft e.V. (Hrsg.), Messen als MarketingInstrument, Bonn 1983, S. 73-96.

Corporate Identity als strategische Basis der Marketingkommunikation, in: Berndt, R., Hermanns, A. (Hrsg.),Handbuch Kommunikation, Wiesbaden 1993 , S. 43-67.

Eigenerstellung oder Fremdbezug von Leistungen, in: Kern, W. (Hrsg.), Handwörterbuch der Produktion, Stuttgart 1979, Sp. 435-450. 
Rasche, C.,

Wolfrum, B.

Rasche, H. O.

Rehorn, J.

Rehorn, J.,

Reuther, K. H.

Ries, A.,

Trout, J.

Roloff, E.

Rominski, D.

Rominski, D.

Rossiter, J., Percy, L.

Rosson, Ph.,

Seringhaus, F.H.R.

Rost, D.

Rost, D.

Rost, D.

Rost, D.

Rost, D.

Rost, D.

Roth, G. D.
Ressourcenorientierte Unternehmensführung, in: DBW, 54. Jg., Heft 4, 1994, S. 503-510.

Wie man Messe-Erfolge programmiert, PraktikerCheckliste, Heiligenhaus 1980.

Markttests, Neuwied 1977.

Labortestmarkt und Minitestmarkt - Alternativen zum Prägen von Marktchancen, in: asw, Heft 9, 1977, S. 51-60.

Die betriebswirtschaftliche Bedeutung der Messen und Ausstellungen für die Konsumgüterindustrie, München 1956.

Positioning: The Battle in Your Mind, New York 1986.

Messen und Medien. Ein sozialpsychologischer Ansatz zur Öffentlichkeitsarbeit, Wiesbaden 1992.

Neue Ausstellerwünsche, in: asw, Heft 6, 1995, S. 102-106.

Neue Besucherwünsche, in: asw, Heft 6, 1995, S. 96-100.

Advertising Theory and Practice, New York 1994.

Reaching Export Objectives through International Trade Fairs, in: Trade Show Bureau (Ed.), Report No. IN 6, Denver 1991, S. 2.

Ausstellen oder Aussteigen?, in: asw, Heft 1, 1982, S. 46-54.

Die Rolle der Messen im Marketing, in: Schöttle, K. M. (Hrsg.), Jahrbuch des Marketing, 4. Ausg., Wiesbaden 1986, S. 137-145.

Maßstäbe zur Beurteilung der Effizienz von Messen, in: VDI-Nachrichten, Nr. 115, 1973, S. 333-338.

Messen als Kommunikations-Problem, in: DWG Deutsche Werbewissenschaftliche Gesellschaft $\theta$. V. (Hrsg.), Messen als Marketing-Instrument, Bonn 1983, S.35-56.

Transparenz schafft Effizienz, in: asw, Heft 9, 1983, S. 68-72.

Messetransparenz - ein Imperativ, in: asw, Heft 8, 1984, S. 30-32.

Messen und Ausstellungen verkaufswirksam planen und durchführen, Landsberg/Lech 1981. 
Roth, G. D.

Rudolph, Th.

Sachs, L.

Saliger, E.

Schanz, G.

Scheitlin, V.

Schenk, M.

Scherrer, M.

Schmalen, H., Wagner, A.

Schmitz, U.

Schober, P. M.

Scholz, Chr.

Schönemann, K.

Schoop, K., Sandt, B.

Schreyögg, G.

Schubö, W. et al.
Messen und Märkte. Aus der Geschichte des Handels: Von der Warenmesse zur Mustermesse, München 1965.

Positionierungs- und Profilierungsstrategien im europäischen Einzelhandel, St. Gallen 1993.

Angewandte Statistik: Planung und Auswertung, Methoden und Modelle, 4. Aufl., Berlin, Heidelberg, New York 1974.

Betriebswirtschaftliche Entscheidungstheorie. Eine Einführung in die Logik individueller und kollektiver Entscheidungen, 2. Aufl., München, Wien 1988.

Organisationsgestaltung, Struktur und Verhalten, München 1982.

Messebeteiligung erfolgreich gestalten, St. Gallen, Stuttgart, Steyr 1984.

Wirkungen der Werbekommunikation, Köln u. a. 1990 .

Integrierte Kommunikation - das große Schlagwort, in: Horizont, Nr. 48, vom 30.11.1990, S. 46.

Empirische Untersuchung zur Messebeteiligung von Unternehmen, Arbeitspapier, o. Nr., Passau 1990.

Messepolitik eines Unternehmens der Investitionsgüterindustrie, in: Strothmann, K.-H., Busche, M. (Hrsg.), Handbuch Messemarketing, Wiesbaden 1992, S. 383-396.

Messen und Ausstellungen: Teilnehmen oder wegbleiben? - Eine kleine Entscheidungshilfe; in: Marketing Journal, 21. Jg., Heft 4, 1988, S. 400402.

Strategisches Management, Berlin, New York 1987.

Messen und Kataloge: Die Sache mit den Katalogen, in: Der Fremdenverkehr, Heft 2, 1989, S. 16.

Die Messeerfolgskontrolle, in: Tietz, B., Die Werbung - Handbuch der Kommunikations- und Werbewirtschaft, Band 2, Landsberg/Lech 1982, S. 1664-1679.

Umwelt, Technologie und Organisationsstruktur, Bern, Stuttgart 1978.

SPSS: Handbuch der Programmversionen 4.0 und SPSS-X 3.0, Stuttgart, New York 1991. 
Schürmann, U.

Schwenzner, J. M., Andersen, E.

Schwenzner, J. M.

Schwickert, E.,

Kindt, M.,

Stallmann, $\mathrm{H}$.

Schwickert, E.

Selinski, $H$.

Selinski, $H$.

Selinski, $\mathrm{H}$.

Selinski, H., Sperling, U. A.

Selinski, $H$.

Siskind, B.

Spiegel Verlag (Hrsg.)

Spiegel-Verlag (Hrsg.)

Spryß, W. M.
Erfolgsfaktoren der Werbung im Produktlebenszyklus - Ein Beitrag zur Werbewirkungsforschung, in: Meffert, H. (Hrsg.), Schriften zu Marketing und Management, Bd. 19, Frankfurt a. M., Berlin u. a. 1993.

Der Messe-Test, in: Wagner, K. (Hrsg.), Allgemeines Statistisches Archiv, Bd. 39, München 1955, S. 25-30.

Messen und Ausstellungen im Kreuzfeuer der Begriffe Reichweitenanalyse und Messeerfolgskontrolle, in: GfK-Mitteilungen zur Markt- und Absatzforschung, 18. Jg., Heft 1, 1972, S. 10-20.

Messeauswahl und -gestaltung, in: Rost, D., Strothmann, K.-H. (Hrsg.), Handbuch Werbung für Investitionsgüter, Wiesbaden 1983, S. 222-230.

Transparenz der Besucher kommt zu kurz, in: HB, vom 24.11.1982, S. 16.

Begleitveranstaltungen von Messen, in: Strothmann, K.-H., Busche, M. (Hrsg.), Handbuch Messemarketing, Wiesbaden 1992, S. 485-500.

Messe- und Kongreßforschung (Teil II), in: planung und analyse, Heft 5, 1984, S. 212-215.

Messe- und Kongressmarketing, Berlin 1983.

Marketinginstrument Messe: Arbeitsbuch für Studium und Praxis, Köln 1995.

Wirkungsrelevante Merkmale der Messestände für Investitionsgüter: Ergebnisse einer empirischen Untersuchung auf der Hannover-Messe 1977, in: Strothmann, K.-H. (Hrsg.), Arbeitspapier des Instituts für Markt- und Verbrauchsforschung, Nr. 8, Berlin 1978.

The Successful Exhibitor's Handbook. Trade Show Techniques for Beginners and Pros, 2. Ed., Vancouver, Bellingham 1994.

Messen und Messebesucher in Deutschland, Hamburg 1992.

Der Entscheidungsprozeß bei Investitionsgütern, Hamburg 1982.

Für Standmitarbeiter gibt es viele Stolpersteine, in: FAZ (Hrsg.), Verlagsbeilage, Heft 20, vom 25.01.1994, S. 8. 
Spryß, W. M.

Spryß, W. M.

Spryß, W. M.

Spryß, W. M., Nerb, G.,

Reuter, J.

Stadler, M.,

Seeger, F.,

Raithel, A.

Staeger, $\mathrm{H}$.

Staehle, W. H.

Steffenhagen, $\mathrm{H}$.

Steffenhagen, $\mathrm{H}$.,

Funke, K.

Steger, U., Winter, M.

Steinhausen, D., Langer, $\mathrm{K}$.

Steinle, C.

Steinmann, H., Schreyögg, G.

Stibi, H. D.

Stiege, J.
Messen und Ausstellungen - Mit dem „MesseDiagramm" vorbereiten - von den Zielen bis zum Controlling, in: Marketing Journal, Heft 4, 1987, S. 368-370.

Mit Forschung Lücken füllen, in Verlagsbeilage der FAZ, Nr. 20, vom 25.01.1994, S. B2.

Einzelaspekte der Messe- und Ausstellungswerbung, in: Tietz, B. (Hrsg.), Die Werbung Handbuch der Kommunikations- und Werbewirtschaft, Bd. 2, Landsberg/Lech 1982, S. 1639-1662.

Messeziele - Messeeffizienz, Münster-Sarmsheim 1994.

Psychologie der Wahrnehmung, 2. Aufl., München 1977.

Messen und Ausstellungen: Auf dem Weg in das Jahr 2000 - Konsequenzen für Aussteller, Veranstalter und insbesondere Messebauer, in: Marketing Journal, Heft 1, 1989, S. 52-56.

Management: Eine verhaltenswissenschaftliche Perspektive, 4. Aufl., München 1989.

Ansätze der Werbewirkungsforschung, in: Marketing ZFP, 6. Jg., Heft 2, 1984, S. 77-88.

Messen und Ausstellungen: Formulieren Sie präzise Messe-Ziele, die später auch nachprüfbar sind, in: Marketing Journal, Heft 6, 1986, S. 546-551.

Strategische Früherkennung zur Antizipation ökologisch motivierter Marktveränderungen, in: DBW, 56. Jg., Heft 5, 1996, S. 607-629.

Clusteranalyse: Einführung in Methoden und Verfahren der automatischen Klassifikation, Berlin, New York 1986.

Führung, Grundlagen, Prozesse und Modelle der Führung in der Unternehmung, Stuttgart 1978.

Management: Grundlagen der Unternehmensführung; Konzepte, Funktionen, Praxisfälle, Wiesbaden 1990.

Messen und Ausstellungen - Auf dem Weg ins Kommunikations-Mix, in: Marketing Journal, Heft 1, 1983 , S. 64-68.

Messebeteiligungen sollen gründlich bewertet werden, in: BddW, vom 24.04.95, S. 1. 
Strothmann, K.-H.

Strothmann, K.-H.

Strothmann, K.-H.

Strothmann, K.-H.

Strothmann, K.-H.

Strothmann, K.-H., Roloff, E.

Strothmann, K.-H.

Strothmann, K.-H.

Strothmann, K.-H.

Strothmann, K.-H., Busche, M. (Hrsg.)

Szeliga, $M$.

Szyperski, N.

Winand, U.

Taeger, M.
Die Instrument-Integration im Messe- und Kongreßwesen - Messestand-Konzeption und Begleitveranstaltung, in: Rost, D., Strothmann, K.-H. (Hrsg.), Handbuch der Werbung für Investitionsgüter, Wiesbaden 1983, S. 219-231.

Die Messe- und Ausstellungswerbung im Überblick, in: Tietz, B. (Hrsg.), Die Werbung - Handbuch der Kommunikations- und Werbewirtschaft, Bd. 2, Landsberg/Lech 1982, S. 1620-1638.

Historischer Abriß über die Entstehung von Messen, in: Strothmann,K.-H. (Hrsg.), Arbeitspapier des Instituts für Markt- und Verbrauchsforschung der Freien Universität Berlin, Berlin 1979/80.

Im Verhalten unterscheiden sich Messe-Besucher beachtlich, in: Marketing Journal, Heft 2, 1992, 1015.

Messeforschung, in: DWG Deutsche Werbewissenschaftliche Gesellschaft e.V. (Hrsg.), Messen als Marketing-Instrument, Bonn 1983, S. 97-107.

Charakterisierung und Arten von Messen, in: Berndt, R., Hermanns, A. (Hrsg.), Handbuch Marketing-Kommunikation, Wiesbaden 1993, S. 708-723.

Segmentorientierte Messepolitik, in: Strothmann, K.H., Busche, M. (Hrsg.), Handbuch Messemarketing, Wiesbaden 1992, S. 100-114.

Suchen Sie das Gespräch mit dem Standbesucher, in: Marketing Journal, Heft 4, 1989, S. 388-391.

Verbundveranstaltungen des Messe- und Kongress wesens im Investitionsgüter-Marketing, in: Rost, D., Strothmann, K.-H. (Hrsg.), Handbuch Werbung für Investitionsgüter, Wiesbaden 1983, S. 399-425.

Handbuch Messemarketing, Wiesbaden 1992

Push und Pull in der Markenpolitik - Ein Beitrag zur modellgestützten Marketingplanung am Beispiel des Reifenmarktes, in: Meffert, H. (Hrsg.), Schriften zum Marketing und Management, Bd. 29, Frankfurt a. M., Berlin u. a. 1996.

Grundbegriffe der Unternehmensplanung, Stuttgart 1980.

Messemarketing - Marketing-Mix von Messe-gesellschaften unter Berücksichtigung wettbewerbs-politischer Rahmenbedingungen, Göttingen 1993. 
Täger, U. C., Ziegler, $R$.

Tanner, J. F., Chonko, L. B.

Tauberer, A., Wartenberg, W.

Tietz, B.

Tietz, B.

Tomczak, T,

Reinecke, S.

Tomczak, T., Müller, $F$., Müller, R.

Tomczak, T., Roosdorp, A

Trade Show Bureau (Ed.)

Trommsdorff, $\mathrm{V}$.

Ueding, $\mathbf{R}$.

Vogt, R. E.

Wagner, G.

Walter, $P$.
Die Bedeutung von Messen und Ausstellungen in der Bundesrepublik Deutschland für den Inlandsund Auslandsabsatz in ausgewählten Branchen, in: ifo-Institut für Wirtschaftsforschung e. V. (Hrsg.), ifoStudien zu Handels- und Dienstleistungsfragen, Nr. 25, S. 51.

Trade Show Objectives, Management, and Staffing Practices, in: IMM, Vol. 24, 1995, S. 257-264.

Serviceleistungen von Messegesellschaften, in: Strothmann, K.-H., Busche, M. (Hrsg.), Handbuch Messemarketing, Wiesbaden 1992, S. 235-248.

Bildung und Verwendung von Typen in der Betriebswirtschaftslehre, dargestellt am Beispiel der Typologie der Messen und Ausstellungen, Köln, Opladen 1960.

Das Konzept des integrierten Kommunikations-Mix, in: Tietz, B. (Hrsg.), Die Werbung - Handbuch der Kommunikations- und Werbewirtschaft, Bd. 3, Landsberg/Lech 1982, S. 2265-2297.

Die Rolle der Positionierung im strategischen Marketing, in: Thommen, J. P. (Hrsg.), Management-Kompetenz, Zürich, S. 499-517.

Nicht-Klassiker der Kommunikation: Erfolgsreserven im Erklärungswettbewerb, in: Tomczak, T., Müller, F., Müller, R. (Hrsg.), Die Nicht-Klassiker der Unternehmenskommunikation, St. Gallen 1995, S. 12-21.

Positionierung - Neue Herausforderungen verlangen neue Ansätze, in: Tomczak, T., Rudolph, Th., Roosdorp, A. (Hrsg.), Positionierung: Kernentscheidung des Marketing, St. Gallen 1996, S. 26-41.

The Power of Trade Shows, Denver 1992.

Positionierung, in: Tietz, B. (Hrsg.), Handbuch des Marketing, 2. Aufl., Stuttgart 1995, Sp. 2056.

Event-Marketing, in: Meffert, $H_{\text {., }}$ Lexikon der aktuellen Marketing-Begriffe, Wien 1994, S. 59-63.

Worauf vor, während und nach der Messe zu achten ist, in: Verkauf + Marketing, Heft 2, 1987, S. 8-10.

Innovatives Messe-Team-Training, in: FAZ (Hrsg.), Verlagsbeilage, Heft 20, vom 25.01.1994, S. 5.

Glaubwürdigkeit und Kontinuität sind das oberste Gebot, in: BddW, vom 07.09.1990, S. 4. 
Webster, F. E.

Wedel, P., Graf von

Weinberg, $P$.

Weinhold-Stünzi, $\mathrm{H}$.

Weintraub, D. K.

Weishäupl, G.

Welge, M. K.

Welge, M. K.

Wenge, H.-U.

Müller, $A$.

Wenge, H.-U.

Wettwer, B.

Wild, J.

Williams, J. D.

Gopalakrishna,S.,

Cox, J. M.

Wind, $Y$.

Winkler, K.-A.

Winnen, R.
Wind Y., Organizational Buying Behavior, Englewood Cliffs 1972.

Messen - vom Markt zum Marketing, Frankfurt 1977.

Erlebnismarketing, München 1992.

Die Rolle der Messen im Marketing der neunziger Jahre, in: Thexis, Heft 2, 1989, S. 1-3.

Trade Show Exhibiting: The Insider's Guide for Entrepreneurs, 0.O. 1991.

Die Messe als Kommunikationsmedium - unter besonderer Berücksichtigung der Öffentlichkeitsarbeit und Werbung einer Messegesellschaft, München 1980, S. $64-76$.

Unternehmensführung, Bd 1, Stuttgart 1985.

Unternehmensführung, Bd. 2, Stuttgart 1987.

Das Management von Messe-Beteiligungen, in: Berndt, R., Hermanns, A. (Hrsg.), Handbuch Marketing-Kommunikation, Wiesbaden 1993, S. 725-745.

Planung von Messebeteiligungen, in: Strothmann, K.-H., Busche, M. (Hrsg.), Handbuch Messemarketing, Wiesbaden 1992, S. 287-302.

Warmer Regen - Die Messestandorte investieren Milliarden in ihre Zukunft, in: Wirtschaftswoche, $\mathrm{Nr}$. 1/2, vom 4.1.1996, S. 62-65.

Grundlagen der Unternehmensplanung, 4. Aufl., Hamburg 1982.

Trade Show Guidelines for Smaller Firms, in: IMM, Vol. 22, 1993, S. 316-324.

Positioning Analysis and Strategy, in: Day, G. S., Weitz, B., Wensley, R. (Ed.), The Interface of Marketing and Strategy, Greenwich 1990, S. 387412.

Kongreß mit Ausstellung, in: Strothmann, K.-H., Busche, M. (Hrsg.), Handbuch Messemarketing, Wiesbaden 1992, S. 502 ff.

Entscheidungshilfen für den Mittelstand - Die Bedeutung der Messeberatung nimmt zu, in: BddW, Nr. 65, vom 04.04.1989, S. 7. 
Winnen, R.

Winnen, R., Beuster, A.

Winter, St. F., Wengler, St.

Wöllenstein, St.

Yang, C.

Zahner, W.

Zerres, R.

Zotter, R.

Zurlino, F.
Entscheidungshilfen für den Mittelstand - Die Bedeutung der Messeberatung nimmt $z u$, in: Küffner, G., Mortsiefer, J. (Hrsg.), Messeplatz Europa, Messe als Bestandteil des betrieblichen Marketings, Frankfurt a. M. 1990, S. 145-153.

Kontrolle des Messeerfolgs, in: Strothmann, K.-H., Busche, M. (Hrsg.), Handbuch Messemarketing, Wiesbaden 1992, S. 378-385.

Das Unternehmen „Deutschland" darstellen, in: BddW, Nr. 27, vom 7.2.1989, S. 7.

Betriebstypenprofilierung in vertraglichen Vertriebssystemen: eine Analyse von Einflußfaktoren und Erfolgswirkungen auf der Grundlage eines Vertragshändlersystems im Automobilhandel, in: Meffert, $\mathrm{H}$. (Hrsg.), Schriften zu Marketing und Management, Bd. 28, Frankfurt a.M., Berlin u. a. 1996.

Determining Advertising Budgets, in: Yang, C. (Ed.), Handbook of Marketing Research, New York u. a. 1974, S. 165-187.

Directmail - Sein Einsatz im Marketing, Bern 1991.

Ausstellen und Gewinnen. Der Kompass für die erfolgreiche Messepraxis, Woldert 1992.

Erfolgreiches Messemarketing. Leitfaden für Analyse, Planung, Durchführung, Nachbearbeitung, Erfolgskontrolle bei Messebeteiligungen im In- und Ausland, Zürich 1993.

Zukunftsorientierung von Industrieunternehmen durch strategische Früherkennung, München, Wien 1995. 


\section{SCHRIFTEN ZUM MARKETING}

Band 1 Friedrich Wehrle: Strategische Marketingplanung in Warenhäusern. Anwendung der Portfolio-Methode. 1981. 2. Auflage. 1984.

Band 2 Jürgen Althans: Die Übertragbarkeit von Werbekonzeptionen auf internationale Märkte. Analyse und Exploration auf der Grundlage einer Befragung bei europaweit tätigen Werbeagenturen. 1982.

Band 3 Günter Kimmeskamp: Die Rollenbeurteilung von Handelsvertretungen. Eine empirische Untersuchung zur Einschätzung des Dienstleistungsangebotes durch Industrie und Handel. 1982.

Band 4 Manfred Bruhn: Konsumentenzufriedenheit und Beschwerden. Erklärungsansätze und Ergebnisse einer empirischen Untersuchung in ausgewählten Konsumbereichen. 1982.

Band 5 Heribert Meffert (Hrsg.): Kundendienst-Management. Entwicklungsstand und Entscheidungsprobleme der Kundendienstpolitik. 1982.

Band 6 Ralf Becker: Die Beurteilung von Handelsvertretern und Reisenden durch Hersteller und Kunden. Eine empirische Untersuchung zum Vergleich der Funktionen und Leistungen. 1982.

Band 7 Gerd Schnetkamp: Einstellungen und Involvement als Bestimmungsfaktoren des sozialen Verhaltens. Eine empirische Analyse am Beispiel der Organspendebereitschaft in der Bundesrepublik Deutschland. 1982.

Band 8 Stephan Bentz: Kennzahlensysteme zur Erfolgskontrolle des Verkaufs und der MarketingLogistik. Entwicklung und Anwendung in der Konsumgüterindustrie. 1983.

Band 9 Jan Honsel: Das Kaufverhalten im Antiquitätenmarkt. Eine empirische Analyse der Kaufmotive, ihrer Bestimmungsfaktoren und Verhaltenswirkungen. 1984.

\section{SCHRIFTEN ZU MARKETING UND MANAGEMENT}

Band 10 Matthias Krups: Marketing innovativer Dienstleistungen am Beispiel elektronischer Wirtschaftsinformationsdienste. 1985.

Band 11 Bernd Faehsler: Emotionale Grundhaltungen als Einflußfaktoren des Käuferverhaltens. Eine empirische Analyse der Beziehungen zwischen emotionalen Grundhaltungen und ausgewählten Konsumstrukturen. 1986.

Band 12 Ernst-Otto Thiesing: Strategische Marketingplanung in filialisierten Universalbanken. Integrierte Filial- und Kundengruppenstrategien auf der Grundlage erfolgsbeeinflussender Schlüsselfaktoren. 1986.

Band 13 Rainer Landwehr: Standardisierung der internationalen Werbeplanung. Eine Untersuchung der Prozeßstandardisierung am Beispiel der Werbebudgetierung im Automobilmarkt. 1988.

Band 14 Paul-Josef Patt: Strategische Erfolgsfaktoren im Einzelhandel. Eine empirische Analyse am Beispiel des Bekleidungsfachhandels. 1988. 2. Auflage. 1990.

Band 15 Elisabeth Tolle: Der Einfluß ablenkender Tätigkeiten auf die Werbewirkung. Bestimmungsfaktoren der Art und Höhe von Ablenkungseffekten bei Rundfunkspots. 1988.

Band 16 Hanns Ostmeier: Ökologieorientierte Produktinnovationen. Eine empirische Analyse unter besonderer Berücksichtigung ihrer Erfolgseinschätzung. 1990.

Band 17 Bernd Büker: Qualitätsbeurteilung investiver Dienstleistungen. Operationalisierungsansätze an einem empirischen Beispiel zentraler EDV-Dienste. 1991.

Band 18 Kerstin Ch. Monhemius: Umweltbewußtes Kaufverhalten von Konsumenten. Ein Beitrag zur Operationalisierung, Erklärung und Typologie des Verhaltens in der Kaufsituation. 1993. 
Band 19 Uwe Schürmann: Erfolgsfaktoren der Werbung im Produktlebenszyklus. Ein Beitrag zur Werbewirkungsforschung. 1993.

Band 20 Ralf Birkelbach: Qualitätsmanagement in Dienstleistungscentern. Konzeption und typenspezifische Ausgestaltung unter besonderer Berücksichtigung von Verkehrsflughäfen. 1993.

Band 21 Simone Frömbling. Zielgruppenmarketing im Fremdenverkehr von Regionen. Ein Beitrag zur Marktsegmentierung auf der Grundlage von Werten, Motiven und Einstellungen. 1993.

Band 22 Marcus Poggenpohl: Verbundanalyse im Einzelhandel auf der Grundlage von Kundenkarteninformationen. Eine empirische Untersuchung von Verbundbeziehungen zwischen Abteilungen. 1994.

Band 23 Kai Bauche: Segmentierung von Kundendienstleistungen auf investiven Märkten. Dargestellt am Beispiel von Personal Computern. 1994.

Band 24 Ewald Werthmöller: Räumliche Identität als Aufgabenfeld des Städte- und Regionenmarketing. Ein Beitrag zur Fundierung des Placemarketing. 1995.

Band 25 Nicolaus Müller: Marketingstrategien in High-Tech-Märkten. Typologisierung, Ausgestaltungsformen und Einflußfaktoren auf der Grundlage strategischer Gruppen. 1995.

Band 26 Nicolaus Henke: Wettbewerbsvorteile durch Integration von Geschäftsaktivitäten. Ein zeitablaufbezogener wettbewerbsstrategischer Analyseansatz unter besonderer Berücksichtigung des Einsatzes von Kommunikations- und Informationssystemen (KIS). 1995.

Band 27 Kai Laakmann: Value-Added Services als Profilierungsinstrument im Wettbewerb. Analyse, Generierung und Bewertung. 1995.

Band 28 Stephan Wöllenstein: Betriebstypenprofilierung in vertraglichen Vertriebssystemen. Eine Analyse von Einflußfaktoren und Erfolgswirkungen auf der Grundlage eines Vertragshändlersystems im Automobilhandel. 1996.

Band 29 Michael Szeliga: Push und Pull in der Markenpolitik. Ein Beitrag zur modellgestützten Marketingplanung am Beispiel des Reifenmarktes. 1996.

Band 30 Hans-Ulrich Schröder: Globales Produktmanagement. Eine empirische Analyse des Instrumenteeinsatzes in ausgewählten Branchen der Konsumgüterindustrie. 1996.

Band 31 Peter Lensker: Planung und Implementierung standardisierter vs. differenzierter Sortimentsstrategien in Filialbetrieben des Einzelhandels. 1996.

Band 32 Michael H. Ceyp: Ökologieorientierte Profilierung im vertikalen Marketing. Dargestellt am Beispiel der Elektrobranche. 1996.

Band 34 Ralf Ueding: Management von Messebeteiligungen. Identifikation und Erklärung messespezifischer Grundhaltungen auf der Basis einer empirischen Untersuchung. 1998.

Band 35 Andreas Siefke: Zufriedenheit mit Dienstleistungen. Ein phasenorientierter Ansatz zur Operationalisierung und Erklärung der Kundenzufriedenheit im Verkehrsbereich auf empirischer Basis. 1998. 
Peter Lensker

\section{Planung und Implementierung standardisierter vs. differenzierter Sortimentsstrategien in Filial- betrieben des Einzelhandels}

Frankfurt/M., Berlin, Bern, New York, Paris, Wien, 1996. XVIII, 159 S., 23 Abb. Schriften zu Marketing und Management.

(2) Herausgegeben von Heribert Meffert. Bd. 31

10 ISBN 3-631-30373-4 · br. DM 65.-*

Die Verknüpfung der Planung und Implementierung von Marketingstrategien stellt insbesondere im Einzelhandel eine wenig beachtete Problemstellung

(1) dar. Häufig liegt hier jedoch ein Hauptgrund für das Scheitern von Strategiekonzepten in der Praxis. Der Verfasser untersucht diesen Problembereich am Beispiel von Strategiealternativen im Spannungsfeld von Sortimentsstandardisierung und -differenzierung. Durch die Abgrenzung verschiedener Modularstrategien sowie spezifischer Instrumente zur Verknüpfung von Strategieplanung und -implementierung werden Ansatzpunkte zur Realisierung einer Synthese von Kosten- und Leistungsorientierung in Filialbetrieben aufgezeigt.

Aus dem Inhalt: Standardisierungsentscheidungen in der Marketingplanung von Filialbetrieben · Sortimentsstrategien im Spannungsfeld von Standardisierung und Differenzierung - Modulare Sortimentsstrategien . Einflußfaktoren der Strategiewahl - Verknüpfung der Strategieplanung und implementierung · Modulsysteme · Fallbeispiele

Frankfurt/M - Berlin · Bern · New York · Paris · Wien

Auslieferung: Verlag Peter Lang AG

Jupiterstr. 15, CH-3000 Bern 15

Telefax (004131) 9402131

*inklusive Mehrwertsteuer

Preisänderungen vorbehalten 